University of Louisville

ThinkIR: The University of Louisville's Institutional Repository

Electronic Theses and Dissertations

$5-2017$

\title{
A multi-period facility location model for service organizations operating in a competitive environment.
}

Sven W. Brose

University of Louisville

Follow this and additional works at: https://ir.library.louisville.edu/etd

Part of the Business Administration, Management, and Operations Commons, Industrial Engineering Commons, Operational Research Commons, Operations and Supply Chain Management Commons, Other Operations Research, Systems Engineering and Industrial Engineering Commons, and the Real Estate Commons

\section{Recommended Citation}

Brose, Sven W., "A multi-period facility location model for service organizations operating in a competitive environment." (2017). Electronic Theses and Dissertations. Paper 2716.

https://doi.org/10.18297/etd/2716

This Doctoral Dissertation is brought to you for free and open access by ThinkIR: The University of Louisville's Institutional Repository. It has been accepted for inclusion in Electronic Theses and Dissertations by an authorized administrator of ThinkIR: The University of Louisville's Institutional Repository. This title appears here courtesy of the author, who has retained all other copyrights. For more information, please contact thinkir@louisville.edu. 


\title{
A MULTI-PERIOD FACILITY LOCATION MODEL FOR SERVICE ORGANIZATIONS OPERATING IN A COMPETITIVE ENVIRONMENT
}

\author{
By \\ Sven W. Brose \\ Diplom-Wirtschaftsingenieur, Technische Universitat Dortmund, Germany 2008
}

\author{
A Dissertation \\ Submitted to the J.B. Speed School of Engineering \\ of the University of Louisville \\ in Partial Fulfillment of the Requirements \\ for the Degree of
}

Doctor of Philosophy in Industrial Engineering

Department of Industrial Engineering

Speed School of Engineering University of Louisville

Louisville, Kentucky

May 2017 

A MULTI-PERIOD FACILITY LOCATION MODEL FOR SERVICE ORGANIZATIONS OPERATING IN A COMPETITIVE ENVIRONMENT

\author{
By \\ Sven W. Brose \\ Diplom-Wirtschaftsingenieur, Technische Universitat Dortmund, Germany 2008
}

A Dissertation Approved on

$\operatorname{March} 27^{\text {th }}, 2017$

by the following Dissertation Committee:

Dr. Sunderesh S. Heragu

Dr. Gail Depuy

Dr. John S. Usher

Dr. Mahesh Gupta 


\section{ACKNOWLEDGEMENTS}

This dissertation would not have been possible without the support of some special persons.

First of all I want to thank my advisor, Professor Sunderesh Heragu, who gave me the direction for exploring the topic and the full support during my research. It has been a great pleasure working with him and I am looking forward to continuing the research on this challenging topic.

I also want to thank my friends and fellow students Volker Johannhörster, Gerhard Koch and Joseph Khoury, who gave me motivation and engaged in intense discussions which made the entire dissertation process much easier.

I would probably still work on the last database issues without the help of Chenna Vamsikrishna.

Much of the technical implementation would not have been possible without the support of Dirk Stähler. His contribution to this work was extraordinary especially on the last mile. 
Many thanks also to Malcom Usher, who critically checked the technical writing and filed off the German language influence on the writing.

Furthermore I want to thank life partner - Carina, who accepted every minute I spent on writing up my dissertation without reservation and for taking control of everything else, especially our two daughters Sophia and Luisa, while I was working on my research topic.

Finally I give thanks to my parents who always believed in me and encouraged me to pursue my future career by giving me the freedom of choosing my field of study, by discussing every sentence critically and giving me any motivation I needed. 


\begin{abstract}
A MULTI-PERIOD FACILITY LOCATION MODEL FOR SERVICE ORGANIZATIONS OPERATING IN A COMPETITIVE ENVIRONMENT
\end{abstract}

Sven W. Brose

March $27^{\text {th }}, 2017$

Facility location is an important problem faced by companies in many industries. Finding an optimal location of facilities and determining their size involves the consideration of many factors including: proximity to customers and suppliers, availability of skilled employees and support services, and cost-related factors, for example, construction or leasing costs, utility costs, taxes, availability of support services, and others.

In addition, the demand of the surrounding region plays an important role in location decisions. A high population density may not necessarily cause a proportional demand for products or services. The demography of a region could dictate the demand for products and this, in turn affects a facility's size and location. 
The location of a company's competitors also affects the location of that company's facilities. Just because a competitor has a facility in a particular location does not necessarily mean the company must also locate its facilities there. Local restrictions such as laws or subsidies and taxes play an important role in a facility location decision. Infrastructural aspects also play a role in the location decision. A facility that has good connections to highways, air transportation, and railroads is more attractive to those with infrastructural bottlenecks.

Developed countries may not necessarily have higher demand for products, compared to emerging markets. In fact, the opposite could be true. Massive declines in sales have caused a deep impact on the economic survival of traditional industries in the last two decades, both in the United States and Europe. In some industries, the focus has now shifted towards the closure of facilities because markets are saturated and new business models have caused changes in business practices. How long to operate a facility in a market that is declining is thus a strategic question. Reports on closures of drugstores or building centers are part of the daily news in Germany. Furthermore, the growth of ecommerce has changed businesses completely and will dominate the development of business process automation.

Developed countries are facing demographic changes, including declining populations, and increase in immigration - this causes significant changes in markets and demands and in the product mix companies must offer. 
Demand often is seasonal. Renting outdoor equipment is of interest for companies during the summer, but not in other seasons.

Location decisions are sometimes not based on a careful, rational and systematic analysis, rather a political decision meant to satisfy multiple parties.

Another important aspect in facility location modeling is that many models focus on the current demand, and do not adequately consider future demand. However, while making location decisions in an industry in decline, carefully and accurately considering future demand is especially important and the question in focus is to shrink or close down certain facilities with the objective of keeping a certain market share or maximizing profit, especially in a competitive environment.

Finally, strategic aspects of a company have significant influence on a facility and its location. For example, the types and level of service offered impacts customers' patronage.

Although numerous factors influence the location of a facility, existing models do not take all of them into consideration.

This thesis develops a multi-period, mathematical programming location model for facilities operating in a competitive environment. The objective is to maximize the profit in a variable demand environment. The solution of the problem can either be done in a robust or in a dynamic manner. A robust solution methodology forecasts the demand for 
all the periods in the planning horizon and provides a robust solution that may be suboptimal for any given period, but optimal when all the periods are taken into consideration. A problem with this approach is that it requires accurate forecast of demand for multiple periods into the future, which is not easy especially when the number of periods is large or the length of each period is long. The dynamic solution methodology circumvents this problem by only considering the demand for the next period in making location decisions. The drawback of this approach is that it may call for frequent closing of existing facilities and opening of new or previously closed facilities and the cost of doing so may be rather high in many environments. However, the cost of opening and closing facilities may not be as high as the benefits of doing so for many service organizations. 


\section{TABLE OF CONTENTS}

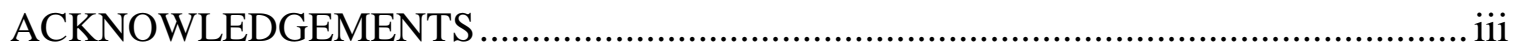

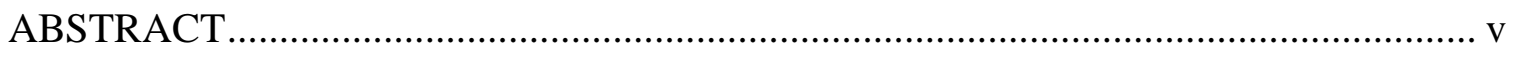

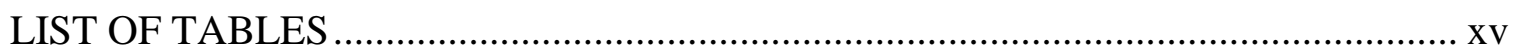

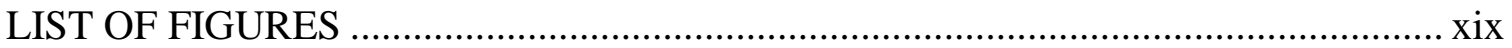

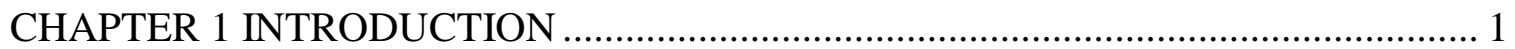

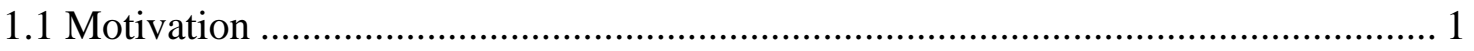

1.2 A multi-period facility location model for service organizations operating in a

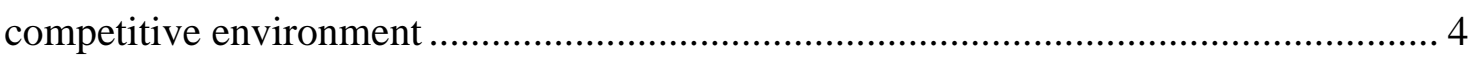

1.3 Scope of this dissertation ............................................................................... 5

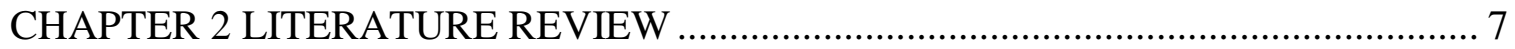

2.1 Papers on classification schemes for facility location .................................... 7

2.2 A classification scheme for facility location models focusing on opening, closing, and capacity decisions incorporating competition.................................................... 12

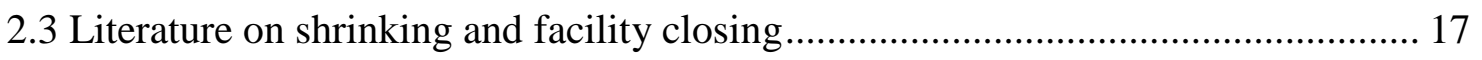


2.4 Literature on expanding or opening new facilities and on variable demand

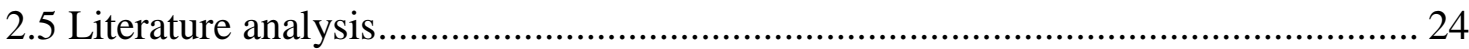

CHAPTER 3 MODELS FOR MULTI-PERIOD FACILITY LOCATION .................... 26

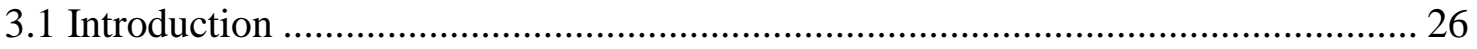

3.2 Model 1: Single product model ..................................................................... 27

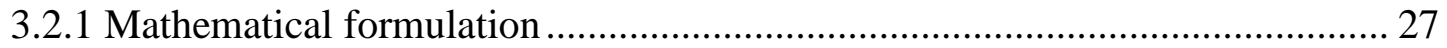

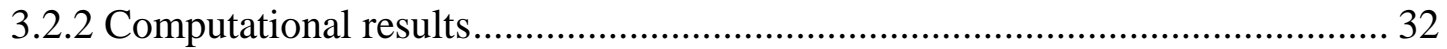

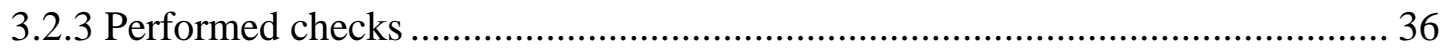

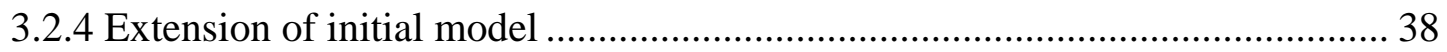

3.2.5 Retrieving the relaxed solution from the model ......................................... 41

3.2.6 Solving the model in dynamic manner.................................................. 42

3.3 Model 2: Allowing multiple products and additional constraints ......................... 59

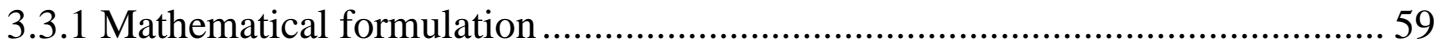

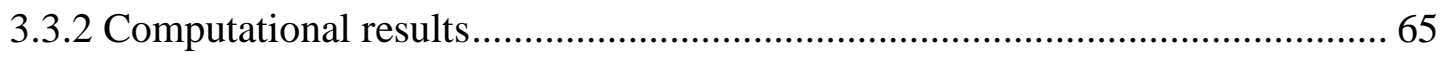

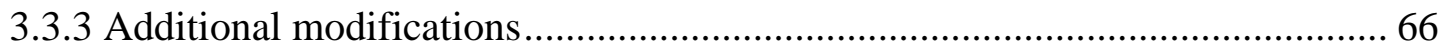

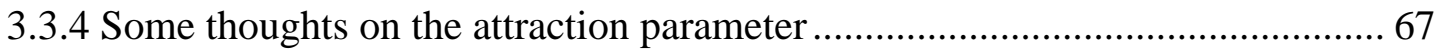


3.4 Summary 
5.1 Introduction 101

5.2 Modification of algorithm 102

5.3 Technical challenges. 104

5.4 Computational results 105

5.4.1 Solution times. 105

5.4.2 The choice of temperature and cooling rate 108

5.5 Pre-processing 112

5.6 Conclusion 115

CHAPTER 6 CASE STUDY 116

6.1 Introduction 116

6.2 About Dortmunder Volksbank

6.3 The model 119

6.3.1 Gathering model data and database implementation 119

6.3.2 Mathematical formulation 122

6.3.3 Computational results 131

6.3.4 Applying simulated annealing 141

6.4 Conclusion 144 
7.2.1 Extension of the attraction function 
CURRICULUM VITA 


\section{LIST OF TABLES}

Table 1 Test data for model 1 with 3 facilities, 3 demand points, and 3 time periods.... 32

Table 2 Variables and runtimes for different datasets 39

Table 3 Objective function values for different model sizes both for mixed integer model and relaxed model 41

Table 4 Results for constant data for dynamic and robust approach 47

Table 5 Results for alternating demand for dynamic and robust approach. 51

Table 6 Results for alternating prices for dynamic and robust approach 55

Table 7 Variables and runtimes for different datasets for extended model 65

Table 8 Database view "modeldata_mp_10_view" 69

Table 9 Database table "modeldata_mp_10_initials_cap" 70

Table 10 Database table "modeldata_mp_10_initials" 70

Table 11 Database table "modeldata_mp_10_facilities_npr" 70

Table 12 The model's bounds for the extended model. 71 
Table 13 Database view MP_5_DEMANDS_P01_VIEW

Table 14 Database view MP_5_FACILITIES_P02_VIEW 78

Table 15 Database view MP_5_INITIALS_CAP_P03_VIEW ................................... 78

Table 16 Solving times for $5 \times 5 \times 5 \times 5$ sub problems in seconds ................................ 79

Table 17 Results tableau for opening status of facilities depending on the sub problem 80

Table 18 Result tableau for sub problems with five products 83

Table 19 Selection of all lines with open status equals 0. 84

Table 20 Objective function value for $5 \times 5 \times 5 \times 5$ model using different selection strategies

Table 21 Results tableau for 10x10x10x10 model and its sub problems 86

Table 22 Results tableau with at least three facilities open for 10x10x10x10 model...... 90

Table 23 Results tableau with at least eight facilities open for 10x10x10x10 model...... 95

Table 24 Objective function values depending on selection strategy 99

Table 25 Computational times for each step of the SA algorithm with respect to problem size for the mixed-integer model 106

Table 26 Computational times for each step of the SA algorithm with respect to problem size for the relaxed model. 106 
Table 27 Potential solving times for all configurations 108

Table 28 Annealing steps depending on the choice of cooling rate and temperature .... 109

Table 29 Means and standard deviations for objective function values using different initial configurations 113

Table $30 \mathrm{p}$-values for $t$-test on means for $5 \times 5 \times 5 \times 5$ 114

Table $31 \mathrm{p}$-values for $t$-test on means for 10x10x10x10 114

Table 32 Database view " CS_COMPETITORS_VIEW".... 121

Table 33 Database table " CS_ATTRACTIVITY_VIEW" 121

Table 34 Database table "CASE_STUDY_DATA_INITIALS_CAP". 121

Table 35 Database table "CASE_STUDY_INITIALS"..... 121

Table 36 Database table "CS_PRODUCT_VIEW" 121

Table 37 Database table "CASE_STUDY_DATA_FACILITIES_NPR" 121

Table 38 Database table "CS_DEMAND_VIEW" 122

Table 39 Open status for sub problems. 134

Table 40 Open status for sub-problems. 137

Table 41 Open status for potential new locations 141

Table 42 Data used for first test - keeping demand and prices constant over time 167 
Table 43 Data used for second test - Alternating demand and keeping prices constant over time 175

Table 44 Data used for third test - Alternating prices and keeping demand constant over time 176

Table 45 Data used for forth test - Contantly sinking demand 176

Table 46 Initial capacity of facilities 201

Table 47 prices and variable cost 202

Table 48 expansion, shrinkage, opening, closing and fixed cost 206

Table 49 Demand data for case study for all 13 products 207

Table 50 Competitors with highest attraction with respect to demand point 245

Table 51 Attraction value for Dortmunder Volksbank's facilities with respect to demand points 283 


\section{LIST OF FIGURES}

Figure 1: Solver status for model with 100 locations, 100 demand points and 100 time

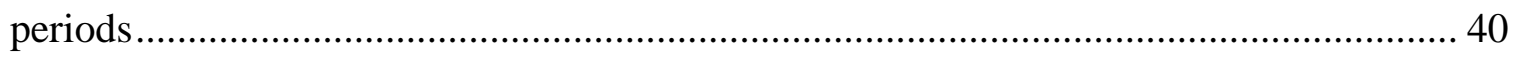

Figure 2 LINGO error message for 150x150x150 model....................................... 41

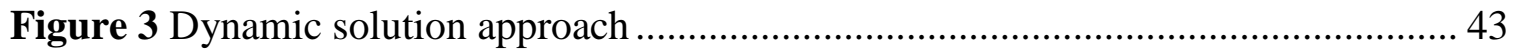

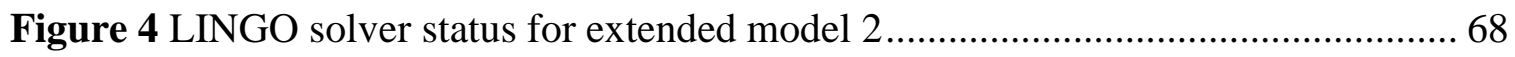

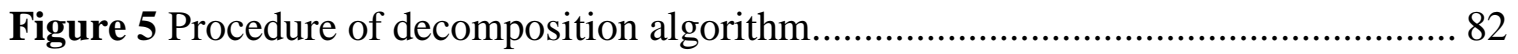

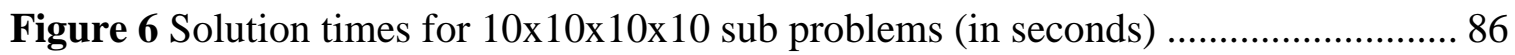

Figure 7 Average runtime per annealing step for the relaxed model......................... 107

Figure 8 Simulated annealing for $10 \times 10 \times 10 \times 10$ model temperature $10^{\wedge} 6$ and cooling

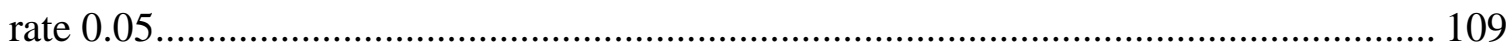

Figure 9 Simulated annealing for 10x10x10x10 model temperature 100 and cooling rate 0.001 110

Figure 10 Simulated annealing for $10 \times 10 \times 10 \times 10$ model temperature $10^{\wedge} 7$ and cooling rate 0.01 111 
Figure 11 Simulated annealing for 10x10x10x10 model temperature 10,000 and cooling rate 0.01

Figure 12 City of Dortmund and its districts (Stadt Dortmund, 3/Dez - Stabsstelle Dortmunder Statistik, 2015) 118

Figure 13 Procedure for solving sub-problems in case study 133

Figure 14 Scenario 1: Facility configuration and assigned demands in period 1 135

Figure 15 Scenario 1: Facility configuration and assigned demands in period 10 136

Figure 16 Scenario 2: Facility configuration and assigned demands in period 1 138

Figure 17 Scenario 2: Facility configuration and assigned demands in period 5 139

Figure 18 Scenario 2: Facility configuration and assigned demands in period 10 140 


\section{CHAPTER 1}

\section{INTRODUCTION}

Location problems deal with the allocation of resources in space. According to Brandeau \& Chiu, 1989 the general location paradigm deals with one or more facilities, called servers, serving a set of customers that are distributed in a specific manner in a region. Location decisions are critical and strategic and must be made thoroughly because very often these require high investments (Klose and Drexl, 2005).

\subsection{Motivation}

Empty parking lots? Useless supermarkets? A McKinsey study states that onlineshopping for commodities will become more and more prevalent and that it will force the closure of commodity markets (n-tv Nachrichtenfernsehen GmbH, 2013). This study provided an outlook on a possible future - but it also dealt with the past which has shown that facility closure or shrinking has always been a concern for planners and needs to be conducted thoroughly.

There are numerous examples illustrating the necessity of facility shrinking or closure. Schlecker (Alter, 2012), General Motors (in Germany especially Adam Opel AG) 
(Süddeutsche Zeitung, 2013), airlines (particularly the regional market) (Döring, 2014) or building centers like Praktiker AG (Dierig and Seidel, 2013) are just a few of them.

Another topical example is Deutsche Bank. Customers use the bank's branches increasingly less often. Deutsche Bank is now planning a massive restructuring of its branches network (Spiegel Online, 2016).

The closing-down of facilities is an operational problem that occurs frequently and not something that occurs rarely (http://plantclosings.com, 2012).

Apart from political decisions, industrial and society trends also influence location decisions and will impact discussions and decisions in location theory.

Numerous new business models have been created during the last years. The influence of e-commerce is omnipresent. Old business models are constantly challenged. For example there are some general stores in Germany, such as Karstadt (Karstadt, 2006), that failed to change their business model recently and had to close many locations during the last few years. Extremely short periods for bringing products and services to markets and a highly competitive environment make companies permanently rethink their location strategies and associated decisions. In some cases, electronic commerce has led to shop closings because more and more products are sold via the Internet.

In contrast to Europe and the United States, previously depressed economic regions are beginning to prosper and generate high demand for consumer products. The effect is that 
companies are interested in investing in those regions such as India, China and even parts of Africa. A majority of the demand for many products is from those regions/areas and not necessarily from the saturated markets in developed countries.

The employment cost in Europe and the US are much higher than they are in countries such as India, China or Eastern Europe. Thus it has been and still is attractive for companies to move production capacities into regions with lower labor cost. Another societal trend that must be mentioned is that there is a movement towards possessing things for a shorter period of time instead of owning them for lifetime. Rental business is becoming more and more prevalent. In Germany "car sharing" has become more and more prevalent in cities. Renting is especially interesting for people who do not want to invest money into things they hardly ever use. As rental companies must keep their offering up to date, facility location is an important topic for them.

All over the world, large cities tend to grow even larger. An urban concentration takes place and causes rural exodus. A widespread distribution network with rather small facilities is very often replaced by large facilities that serve certain demand. Even financial service companies such as banks or insurances concentrate their businesses. For Example Hypo Vereinsbank is currently undergoing such a strategic change by rationalization of its branches and moving to online banking.

Companies face an enormous pressure in bringing products to market. Life cycles for products have become shorter, thus the time to return on investment has reduced. A 
strong competitive environment meets very low distinction in the range of products and services offered.

\subsection{A multi-period facility location model for service organizations operating in a competitive environment}

Very often, decisions on facility location are made without considering the competitors' behavior or by taking the time horizon into account. Subsidies or political interests are sometimes the factors considered in making location decisions, instead of a sound analysis involving the numerous factors that impact location decisions. In many cases companies realize very late that their choice of location is not optimal. This dissertation develops a model which enables companies to select sites for their businesses according to their strategy. The model analyzes the strategic position of the company and forms a guideline for the decision. It investigates which facilities should be closed, (re) opened, shrunk or expanded. If facilities are to shrink or to expand, the model also determines their new capacities. It shows the impact on the market share and takes the costs for closure and opening into consideration.

A number of papers deal with location theory and its applications, but a few have been written for modeling a competitive environment in the case of declining demand. Existing papers in this area of research are mostly static in nature, do not offer multiperiod approaches, nor do they incorporate the behavior of competitors in the market. 
Problems addressed in the model are:

1) The set of facilities that must be reduced in size, and the corresponding size

2) The set of facilities that must be expanded in size, and the corresponding size

3) The set of facilities that must be closed

4) The set of facilities that must be opened

5) The set of demand locations that will be assigned to a company's own facilities

Two possible settings are discussed.

1) Robust solution: The demand for all periods is known at the beginning. The model then is solved only once in a way that the solution is robust for all periods in the planning horizon. This requires more accuracy in demand forecast.

2) Dynamic solution: Beginning with an initial solution, the model is resolved whenever there is a significant change in demand or other parameters. With such an approach, the cost of closing or opening facilities and moving from one solution in one period to another in the next, must be considered.

To solve the problem mathematically, mixed-integer programming models will be developed and heuristic procedures will be applied.

\subsection{Scope of this dissertation}

This dissertation contains a review of the literature on location theory. It begins with an overview on the basis of locating theory, explains the classification of location models 
and takes parameters that influence the choice of locations into consideration. Models for the closing-down and shrinking of facilities are presented as well as models that deal with a high variation of demands

The dissertation also encapsulates the development of the mathematical model for this problem and extends the model in a separate sub chapter. Alternate solution approaches are presented and a decomposition approach is developed. Additionally, a modified simulated annealing procedure is applied to the model.

A case study is carried out and the results are discussed. Finally steps for future research are presented. 


\section{CHAPTER 2}

\section{LITERATURE REVIEW}

This chapter reviews the literature on facility location. Location optimization has been studied since the beginning of the $19^{\text {th }}$ century. The early papers focused on a more economic level in the theory of land usage see (von Thünen, 1910) or in the theory of central locations (Christaller, 1933). Location theory on a business level was formally introduced by Weber (1909). Isard (1956) extended Weber's work. Others also started working on location problems by considering the problem of locating two competing vendors along a straight line e.g. Hotelling (1929). A number of different researchers have studied the location problem from multiple perspectives and have published various papers (Aboolian et al. 2006), (Akinc \& Khumuwala, 1977), (Ashtiani et al. 2013), (Chandrasekaran \& Daughety, 1981), (Cooper, 1972), (Drezner et al. 1985), (Drezner T. , 1998), (Drezner T. , 1994), (Efroymson \& Ray, 1966), (Elzinga \& Hearn, 1972), (Geoffrion, 1975), (Goldman, 1971), (Hotelling, 1929), (Plastria F. , 2005), (ReVelle et al. 2008), (van Roy \& Erlenkotter, 1982), (Weber, 1909).

\subsection{Papers on classification schemes for facility location}

In order to get a more organized view of the scope of the literature, a set of relevant papers are classified in this chapter. First existing classification schemes are introduced. 
Second, these schemes are used to set up a taxonomy to classify the models on facility location and to get a deeper insight into the topic especially on competitive location models and models in the field of expansion, shrinking, opening and closing. Multiple researchers have set up classification schemes for facility location models.

Papers on location theory have been classified in Francis et al. (1983). They distinguish between four classes of problems, continuous planar, discrete planar, mixed planar, and discrete network problems.

Daskin (Daskin et al. 2008) splits location models into analytical models, continuous models, network models and discrete models. According to Daskin, (2008), analytical models are not applicable for certain problems. The main differences according to this classification are to be found in the decision space (market) (Haase \& Hoppe, 2008). (Klose \& Drexl, 2005) do the same clustering as they distinguish between continuous location models, network models and mixed-integer programming models. The latter two types of models actually are discrete optimization models, so this classification has much in common with the classifications mentioned above. They narrow down the mixedinteger programming models into (1) single- vs. multi-stage models, (2) uncapacitated vs. capacitated models, (3) single- vs. multi-product models, (4) static vs. dynamic models, (5) deterministic vs. stochastic models, (6) models with and without routing options included, and (6) single- vs. multi-objective models.

A further categorization splits location models into static and deterministic, continuous and stochastic problems, (Owen \& Daskin, 1998). Static and deterministic problems can 
be broken down into median-based-models, covering based-models, center models and other models. As the covering models focus on designing public services they might not be practical for business applications, but because those models play an important part in location theory, they will be at least sketched.

The p-center problem locates $p$ facilities having the goal of minimizing the maximum distance between a demand point and its nearest facility. Thus the p-center problem is also called the minimax problem. It is probably one of the most known problems in this category. For business applications the median-problems are much more applicable, among these is the p-median model. The p-median problem, introduced by Hakimi (1965), minimizes the total demand-weighted travel distance between the demand points and the facilities. In contrast to the p-median model, the covering problem addresses a certain service level. A demand is covered, if it can be served in a certain time or distance. This measure describes the desired service level. The objective of the covering problem is to minimize the total cost for reaching that service level.

Uncertainty of the future is part of any model as it is the strategic nature of facility location problems, (Owen \& Daskin, 1998). Dynamic location models consider an extended planning horizon and generate robust location decisions. Longer planning horizons and uncertainties in facility location are addressed by dynamic location models. Although the dynamic location models consider an extended planning horizon, they are static in nature, because all parameters are defined in advance and possible parameter changes in later time periods are not considered. 
Stochastic models assume that the input parameters are mostly unknown in real-world applications. Owen and Daskin (1998) split the models into two different approaches, which are the probabilistic approach and the scenario planning approach. The former uses stochastic tools, the latter considers scenarios in a what-if setting.

Brandeau and Chiu (1989) presented an overview of representative problems and established a classification scheme, which appears to be more general. Their work contains a survey of more than 50 papers with problems in location theory. Their classification is divided into three characteristics which are

- The objective of the model

- The decision variables

- The system parameters.

Hamacher and Nickel (1998) introduced a 5-paramater classification using a formalization based on queuing theory. It takes five parameters into account and was built on the research of Eiselt et al. (1993), (Eiselt \& Laporte, 1989), who focused on competitive location models. They indicate that their taxonomy has been a useful tool in designing and structuring lectures and research papers. The benefit of their classification is that not only classes of specific location problems are described, (Hamacher \& Nickel, 1998). The taxonomy uses five parameters to describe the underlying problem. The structure reminds one of Kendall's notation used in queuing theory, (Kendall, 1953). These parameters are 
- P1: Information about the number and type of new facilities

- P2: Type of the location model with respect to the decision space

- P3: Description of particulars of the specific location model

- P4: The relation between new and existing facilities

- P5: Description of the objective function,

A subset of symbols for each position is introduced with respect to continuous, network and discrete location models. Although the classification can be applied to a large variety of location models, this approach does not take a facility expansion or shrinkage into consideration.

Haase and Hoppe (2008) expanded the scheme by including additional parameters for competitive location models. The resulting taxonomy consists of six parameters using a similar notation as described above, which are

- Characteristics of competition

- Characteristics of decision space

- Modeling of demand or market share

- Pricing strategy

- Objective

- Additional parameters.

Haase and Hoppe (2008) mention that only a few authors have discussed the shrinking or closing of facilities. 
Some more specialized classification schemes can be found by Handler and Mirchandani (1973) in a 4-position scheme, applicable to network location models with objective functions of the center type. Carrizosa et al. (1995) developed a 6-position scheme for the classification of planar models, referring mainly to the Weber Problem.

Very little attention has been paid in the literature to closing or relocation of facilities. Revelle et al. (2005) give a first overview on papers both for a competitive and a noncompetitive setting, but do not set up a classification scheme. Finally they end up with two static models for the shrinkage problem.

\subsection{A classification scheme for facility location models focusing on opening, closing, and capacity decisions incorporating competition}

According to the different taxonomies described above, it is necessary to set up a scheme that addresses the objective of the model in this dissertation in order to get an expanded and more diversified view on the body of literature. For a classification of the papers, the following parameters will be used for the literature review.

\section{1) Decision space}

The decision space is essential for the location problem and its formulation. It can be either continuous or discrete. In the case of a continuous decision space the variables used in the model are continuous. Continuous models are most likely used for site generation. The underlying decision space is of geographic nature. The feasible solution can be found inside the given the polygon, (Plastria F. , 2002). The 
underlying network is known in discrete network location models. Thus the question is whether or not to locate a facility on a certain node on a network. Typical examples are the set covering location model and the maximal covering location problem.

\section{2) Objective}

The objective of the model plays an important part. Objectives can be profit or market share maximization, cost-minimization, achieving a certain service level or coverage, maximizing the market share or be based on a certain covering level. Very often several objectives are tried to be combined. The underlying decision space is essential for the objective. Current et al. mention that multiple shareholders with very often contradictory objectives are involved in location decisions, (Plastria F. , 2002).

\section{3) Distance measuring}

Most of the models use distances as parameters that influence customer choices. The region is very often assumed to be a plane. The willingness of a customer to travel to a facility is increasing as the distance decreases ${ }^{1}$. Though customers patronize facilities that are closer instead of using those far away. The distance between a customer $i$ (demand point) and a facility $j$ is denoted by $d_{i j}$. The probability of patronizing a facility with higher distances is lower than that for a closer facility. This is why the attractiveness of a facility decreases while the distance increases.

\footnotetext{
${ }^{1}$ Some authors use travel time instead of distance (Huff, 1964)
} 
Analyses have found out an inversely proportional relationship between the attraction of a facility and the distance of a customer and the facility, (Plastria F. , 2002), (Drezner \& Eiselt, 2002). Distances can then be given as Euclidean, rectangular, max, hexagonal or minowski distance. In some cases distances are only part of a general parameter that describes the level of attractiveness of a certain facility.

4) Time inorporation

Some models are static in nature and only consider a single time period. Others use an extended time horizon and multiple periods, are considered dynamic, and dynamic location models. Manne (1967) researched dynamic location models and pointed out the importance of the time horizons in his studies. Ballou (1968) emphasizes that future time decisions cannot be neglected in location analyses. Though the model can be defined as dynamic, this must not necessarily mean that the solution approach is dynamic. Mostly, the solution approach for such models is robust.

\section{5) Competition}

Competitive location models are used to consider multiple players in a model. Most models in facility location do not consider competitors. This is the difference between location-choice models, which assume that customers decide which facility they use and location-allocation models. In location-allocation models, each facility is assigned to one or more demand points. 


\section{6) Consumers and demand}

In a competitive setting, it is important to understand how the market share of a facility can be estimated and influenced. Drezner and Eiselt (2002) mention that it depends on three factors which are

\section{- Customer characteristics}

- Facility attributes

- The spatial separation of customers and facilities.

Thus it is important to consider all these factors and decide whether or not to incorporate competition in a model. Drezner and Eiselt (2002) set up a customer choice process and list the main factors for patronage of a certain facility. When considering a utility function, the assumption is that customers react rationally and the customers' decisions depend on logic. According to Drezner and Eiselt (2002), some authors have questioned the complete rationality. Customers do not always know the prices in each store for each product before shopping, but it is to be assumed that they might have a rough idea of the price for grocery products and this influences their behavior. Ho et al. (1998) mention some other aspects for store loyalty which is habitual store visit behavior and the concept of basket size thresholds. They explain that customers will patronize nearby stores for small quantities with a higher probability though the prices might be higher. For higher quantities it is assumed that customers will accept longer drives, (Drezner \& Eiselt, 2002). A study by Drezner and Furihata (2000) also states that distance is not correlated with mall patronage in Japan and the U.S. Without having empirical evidence for correlation - the same observation can be made by airports. People tend 
to patronize airports especially because of the offered destinations and the ticket prices. (www.airliners.de, 2014)

7) Single / Multiple product

This parameter indicates whether a single or a multiple product setting is described within the problem formulation. A multiple product setting makes the problem even more complex. Most of the existing models do not consider a multiple products scenario.

8) Homogenous / heterogeneous facilities

Some models are set up as a heterogeneous facility design / layout, to give an example no different sizes for the facilities are considered, the facilities do not differ in quality or in service like opening hours. Such factors can have significant influence on the customer patronage of a facility. Thus factors such as size, service

quality and product quality may be considered. Some authors have developed a utilization function that takes all parameters together, (Bloomstein et al. 1980), (Burnett, 1973), (Drezner T. , 1994), (Drezner \& Furihata, 2000).

\section{9) Constraints}

Each model has a unique set of relevant constraints. Such constraints can be capacity, subsidies, taxes and so on. The general assumption in a model is an unlimited capacity of the facilities which is quite distant from reality. When designing facilities, capacity restrictions play an important part - production capacities, land restrictions or even transportation restrictions might to be considered. Gourdin et al. (2002) 
mention capacitation constraints for telecommunication networks. Capacity restrictions are usually modeled as constraints in the mathematical model. For shrinking or closing purposes further constraints can also be a certain service level that should be kept as well as budget constraints or an exclusive set of facilities that have to be kept open.

10) Opening and closing This parameter indicates whether opening and / or closing of facilities is relevant for the model.

11) Solution methods applied

Solution methods can be either (linear) programming techniques or heuristics. As many models become very large, the use of heuristics is necessary.

\subsection{Literature on shrinking and facility closing}

Only few papers have been published on shrinking or closing of facilities, in contrast to expanding. A good starting point is to be found in a model proposed by Klincewicz et al. (1988). They propose a discrete network model with the objective of minimizing the total discounted costs over a given planning horizon which indicates that it is a dynamic approach. Instead of distances, costs play an important part in the model. The model allows the usage of concave operating costs. Competitors are not considered, though it is to be assumed that the model should be applied to business and not to public services. The demands are considered by arbitrary demand patterns. This allows one to model 
different demands in different time periods. As it is possible to shrink or expand facilities in this model, this allows modelling facilities with different sizes. The constraints in the model focus primarily on the capacity of the facilities and the demand. To incorporate the arbitrary demand patterns Klincewicz et al. (1988) allow both contraction and expansion as well as closing and opening of locations. For solving the model, the authors start with a myopic, initial solution and then apply a heuristic. Overall this model is very powerful and already incorporates a couple of questions that should be addressed in this dissertation, but it does not take competitors into consideration.

Melachrinoudis and Min (2000) published a paper focusing on supply chain management objectives. They consider a phase-out and relocation situation of a hybrid, two-echelon plant/warehousing facility with respect to changes in business environments. The decision space is discrete as the possible locations are known in advance. The objective of the model is to maximize the total profit in a given time horizon with respect to location incentives. Distances are measured in time (minutes). Competitors are not considered. The demands of the customers in certain periods are known as well and are not influenced by the location decision. Melachrinoudis and Min (2000) do not consider special product types. Instead they use units to model both capacity and demand. Because the goal is to relocate the facility, there is no difference made between homogenous and heterogeneous facilities. The major constraints of the model pertain to production capacity and demand satisfaction. The model either closes or opens exactly one facility. The model then computes where and when to open and close. The model is solved by using LINGO. 
Another paper published by Wang et al. (2003) considers a budget constrained location problem with opening and closing of facilities. The model is formulated in a discrete decision space. A set of nodes of existing and possible locations is given. The objective of the model is to minimize the total weighted travel distance for customers. Distance is measured in Euclidean space, but the distances are also weighted such that a certain demand node could become more important than another. The model is a single planning period model. Because the objective is to minimize the total weighted travel distance, the customers' competitors are not considered in the model. The consumers are modeled using demand nodes. A consumer can be ranked higher by assigning a larger weight. As capacity does not play a part in the model, the demand is not considered in case of units. All facilities serve the same product and are equal. The model uses two major constraints, first a budget constraint for opening and closing of facilities and second the total number of open facilities desired. Facilities may be closed down or opened. As the problem is NP-hard, the authors developed three heuristics.

Zhang and Rushton (2008) developed a model that uses a discrete decision space to optimize the size and the location of facilities in a competitive environment with the objective of maximizing the spatial utility of users. Distances are measured in Euclidean space. The authors consider only one time period. In contrast to the models mentioned previously, the location of competitors is considered. The demand is also known. Zhang and Rushton (2008) consider a single product. Facilities can differ in size, while larger branches attract more customers. The model incorporates such constraints as budget and size. Locations can either be opened or closed. Heuristics are used to solve the model. 
ReVelle et al. (2005) considered closing facilities in their model. They propose two models, one for firms with competition and another for firms without competition. As this dissertation will provide a model for firms with competition, only the characteristics of the former are described. The model uses a decision space in a network of discrete points. The assumption is to retain a given number of facilities losing as little market share to the competitors as possible. Thus, the model objective is to minimize the market share lost. Distances are Euclidean and the model does not consider multiple time periods, but takes competition into account. A demand point is lost to a competitor when the distance of one's own nearest facility is larger than the distance of the nearest competitor. The demand is also known in advance. The model uses both single products and homogenous facilities. The authors considered only closure of a fixed number of facilities. The model is solved using LINGO.

Bi-level models are of competitive nature and incorporate the reaction of the follower. A first model deals with the problem of locating new facilities in a market where competitors are already operating (Küçükaydin et al., 2012). The new facilities should be such that the profit is maximized. For this purpose, the authors introduce an attraction index. According to the bi-level approach, the competitor can react by adjusting both the attractiveness of existing facilities or by opening or closing others in order to maximize the profit. The distance measure is Euclidean. Multiple time periods are not considered explicitly, due to the bi-level approach. The consumers demand for a homogeneous product is modeled by the buying power at the demand points. Because it is possible to adjust the attractiveness of each facility, the model works with heterogeneous facilities. In this model it is not possible to adjust the attractiveness. For the existing company in 
the market, it is possible to open or close facilities. The authors use a heuristic approach for solving the model.

Küçükaydin et al. (2011) proposed another, similar model, allowing the adjustment of the attractiveness of the follower, but not allowing the closing or opening of facilities. The assignment of demand points to certain facilities is determined by the attraction index.

A further bi-level approach is proposed by Drezner and Drezner, (1998). They considered a model for a company entering a market knowing that a future competitor is expected to enter the market. They state that the location for one's own facility should be chosen such that it is optimal after the market entry of the new competitor. The model objective is to maximize the market share. The model uses a continuous decision space with distance correction to ensure that a facility will not be located directly on a demand point. The distance measure is Euclidean. In this bi-level approach, only single time periods are considered. Demand is measured in buying power and is aggregated at pre-specified demand points. The model does not consider multiple products. As the model deals with several existing facilities having different attractiveness levels, the facilities are completely heterogeneous. It is not possible to influence the attractiveness level in the model directly. The model does not incorporate closing or opening decisions. The authors introduce three solution approaches: the brute force approach, the pseudo-mathematical programming approach, and the gradient search approach.

A paper for bank networks was published by Ruiz-Hermández et al. (2014). The paper contains closing down as well as long term operations' costs. It also assumes that it 
sometimes might be necessary to resize branches. The model is set up for a competitive environment and takes a ceding market share into account. Distances are Euclidean. The model does not include multiple time periods. The customers are known, capacities may vary at the locations and facilities can have different sizes. The problem is solved using the CPLEX algorithm.

A paper by Bhaumik (2010) introduces the de-location problem with the objective of closing a pre-specified number of facilities and assigning demands to the remaining facilities. The objective of the model is to minimize the total cost of serving the demand nodes, assuming reassignment might cause a higher distribution cost. In the model distances are not explicitly considered, but costs are given for all assignment pairs. It is assumed that the cost is based on distances. In his paper, Bhaumik (2010) did not extend the model to multiple time periods, but the model could be computed period by period. Competition is not considered as the focus is on cost minimization. The demands of the nodes are not known. Facilities in the model can be assumed to be homogenous because Bhaumik (2010) did not consider any differences in terms of operational costs or attractiveness. The model postulates that demand is not lost though closure of facilities. The only impact is that there will be higher distribution cost due to the new assignments. A linear solver was used to run the model. 


\subsection{Literature on expanding or opening new facilities and on variable demand}

Küçükaydin et al. (2011) proposed another model that focused on competitive facility location in a discrete environment. A new market entrant should locate facilities and to compete with a set of existing facilities. They aggregate demand at certain demand points in the plane. Küçükaydin et al.'s (2011) model takes both attractiveness and distance into account. Patronage depends on these two parameters. The attractiveness level can be influenced by each facility. The mixed-integer non-linear model's objective is to maximize the profit. The model is static in nature, as the attractiveness level is set only once and not changed over time. For solving a branch and bound algorithm is applied.

Dasci \& Laporte (2005) analyzed a model dealing with demand uncertainty and the corresponding capacity acquisition. The model uses a market area approach which massively simplifies the mathematical model behind it. The approach should be considered as complementary to existing conventional solution procedures. The model points out that location and capacity decisions may differ substantially.

The challenges of variable demand are especially considered in a paper proposed by Arrondo et al. (2014). The model incorporates competition in a planar location design. The paper develops a heuristic that deals with the increasing complexity of variation in demand. Attraction is also incorporated in the model using a simple additive utility function. The objective of the model is to maximize the profit. The influence of variable 
demand on the overall result was demonstrated in a paper published by Redondo et al. (2011).

\subsection{Literature analysis}

There is much literature on facility location. A variety of papers addresses many different location problems, both in a competitive and in a non-competitive environment. Starting with the Weber problem the basics for facility have been laid. Several papers have been published for classifying problems on facility location. Brandeau et al. (1989) propose a very general classification scheme. Hamacher and Nickel (1998) published a paper on a more detailed scheme. In this dissertation, a taxonomy was developed based on the work of Hamacher and Nickel (1998) and Haase and Hoppe (2008). Our taxonomy considers eleven parameters which are the decision space, the model's objective, distance measuring, time incorporation, the characteristics of competition, the modeling of demand or market share, singe or multiple products, facility types, the incorporation of opening and closing and solution methods. Drezner and Hamacher (2002) have published a book on location analysis that addresses most aspects of location theory. It covers both continuous and discrete location models, addresses the public and the private sector and takes the modeling of demand into account. In the later chapters applications and further research topics are addressed. When dealing with the shrinking or closing of facility it becomes clear very quickly that only little attention has been paid to that area. Applying

our taxonomy also shows that the objectives of the models in papers dealing with the shrinking or closing of facilities do mostly focus on special use cases (Melanchrinoudis \& Min, 2000), (Ruiz-Hermández et al. 2014), (Bhaumik, 2010). Most of them do not 
consider competition or multi periods in their models. A few bi-level models have been developed during the last years. Bi-level models are suitable for modeling competitive settings by using an approach from game theory, but they do not consider multiple periods. Finally it is very hard to solve them. For most of the models Heuristics need to be applied.

This dissertation focuses on a model both considering multiple time periods, competition, shrinking and closing as well as opening and expanding of facilities. The initial model is proposed in the following chapter and extended later. 


\section{CHAPTER 3}

\section{MODELS FOR MULTI-PERIOD FACILITY LOCATION}

\subsection{Introduction}

Most location models in literature either do not consider multiple time periods or neglect competition in the model. The model introduced in this chapter attempts to overcome this limitation. It is based on a competitive, multi-period environment and not only takes downsizing or closing of certain facilities into consideration, but also assumes that a downsizing or closing of one facility might require the expansion of others. Contraction of an existing facility is also considered as an option. Furthermore, competitors are explicitly incorporated in our model. The demands are assumed to vary over a chosen time horizon.

First, the model with a single product is demonstrated. Second the model is then extended by allowing multiple products as well as the addition of several constraints, because the single product assumption limits the applicability. Both models are solved and computational results are provided. 


\subsection{Model 1: Single product model}

The first proposed model considers an existing set of facilities operating in a competitive environment in a market with discrete demand points. The model considers two different scenarios. First the demand is known for all periods in advance. This is solved using a robust approach. Secondly an approach for solving the model period-by-period i applied, as demand can be predicted more accurately only for the next period. The two approaches

are then compared. Operating a facility incurs a fixed cost. Closing and opening of facilities will incur a cost just as expansion or contraction. Finally, the model takes the competitive environment into consideration. This is done by using an attractiveness parameter which indicates the attractiveness of a certain facility. The model postulates that the facility with the highest attraction will be assigned to a demand point, if it is open. The highest attractiveness might also be at a competitor's site. The prices for the homogenous product can differ between the facilities as well as between time periods. A facility will be operating with a certain capacity in each period.

\subsubsection{Mathematical formulation}

\section{Indexes}

$i \quad$ Set of facilities

$j \quad$ Set of demands

$t \quad$ Set of time periods 


\section{Model parameters}

$\begin{array}{ll}\text { initialCapacity }_{i} & \text { The initial capacity of facility } i \text { in period } 1 \\ \text { initialOpen }_{i} & \text { The initial status of facility } i \text { in period } 1 \\ \text { price }_{i t} & \text { The price for selling a single unit at facility } i \text { in period } t \\ \text { variableCost }_{i t} & \text { The unit cost for selling a single unit at facility } i \text { in period } t \\ \text { fixCost }_{i t} & \text { The cost for having open facility } i \text { in period } t \\ \text { openingCost }_{i t} & \text { The cost for opening facility } i \text { in period } t \\ \text { closingCost }_{i t} & \text { The cost for closing facility } i \text { in period } t \\ \text { attraction }_{i j t} & \text { The attraction value of a company's own facility } i \text { with } \\ & \text { respect to demand node } j \text { in period } t \\ \text { competitorsAttraction }_{j t} & \text { The highest attraction of competitors for demand node } j \text { in } \\ & \text { period } t\end{array}$

expansionCost $_{i t} \quad$ Fixed cost for expansion of facility $i$ in period $t$

shrinkage Cost Fix $_{i t} \quad$ Fixed for contraction of facility $i$ in period $t$

unitExpansionCost $_{i t} \quad$ Unit (variable) cost for expansion of facility $i$ in period $t$

unitShrinkageCost $_{i t} \quad$ Unit (variable) cost for contraction of facility $i$ in period $t$

demand $_{j t} \quad$ The demand at demand node $j$ in period $t$

assignment $_{i j t}=\left\{\begin{array}{l}1, \text { ifattraction }_{i j t} \geq \text { competitorsAttraction }_{j t} \\ 0, \text { else }\end{array}\right.$

This parameter can be set to 1 if the attractiveness of a company's own facility $i$ relative to a demand node $j$ is greater or equal than the highest attractiveness of the competitor for the same node $j$ in period $t$. It is used for the assignment of facilities to a demand node. 


\section{Model variables}

\begin{tabular}{|c|c|}
\hline open $_{i t}$ & $=\left\{\begin{array}{l}1, \text { if facility i remains open in } t \\
0, \text { if facility } i \text { is closed in } t\end{array}\right.$ \\
\hline opened $_{i t}$ & $=\left\{\begin{array}{l}1, \text { if faciltiy } i \text { is opened in } t \\
0, \text { else }\end{array}\right.$ \\
\hline closed $_{i t}$ & $=\left\{\begin{array}{l}1, \text { if faciltiy } i \text { is closed in } t \\
0, \text { else }\end{array}\right.$ \\
\hline expanded $_{i t}$ & $=\left\{\begin{array}{l}1, \text { if facility } i \text { is expanded in } t \\
0, \text { else }\end{array}\right.$ \\
\hline shrunk $_{i t}$ & $=\left\{\begin{array}{l}1, \text { if facility } i \text { is shrunk in } t \\
0, \text { else }\end{array}\right.$ \\
\hline$u P l u s_{i t}$ & Expansion in units at facility $i$ in $t$ \\
\hline uMinus $_{i t}$ & Contraction in units at facility $i$ in $t$ \\
\hline totalCapacity $_{i t}$ & Maximum capacity in units at $i$ in period $t$ \\
\hline usedCapacity $_{i t}$ & Capacity utilized in units at $i$ in period $t$ \\
\hline
\end{tabular}

\section{Objective function}

$\operatorname{Max}_{i} \sum_{t}\left(\right.$ usedCapacity $_{i t} *\left(\right.$ price $_{i t}-$ variableCost $\left._{i t}\right)-$ fix $_{\text {Cost }}$ $_{i t} *$ open $_{i t}-$ closingCost $_{i t} *$ closed $_{i t}-$ opening Cost $_{i t} *$ opened $\left._{i t}\right)-\sum_{i} \sum_{t}$ expansionCost $_{i t} *$ expanded $_{i t}-\sum_{i} \sum_{t}$ unitExpansionCost $_{i t} *$ uPlus $_{i t}-\sum_{i} \sum_{t}$ shrinkageCost $_{i t} *$ shrunk $_{i t}-\sum_{i} \sum_{t}$ unitShrinkageCost $_{i t} *$ Minus $_{i t}$ 
Subject to

1) open $_{i 1}=$ initialOpen $_{i}, \quad \forall i$

2) totalCapacity $_{i 1}=$ initialCapacity $_{i}, \quad \forall i$

3) usedCapacity $_{i t} \leq$ totalCapacity $_{i t}, \quad \forall i, t$

4) usedCapacity $_{i t} \leq \sum_{j}$ assignment $_{i j t} *$ demand $_{j t}, \quad \forall i, t$

5) totalCapacity $_{i t+1}=$ totalCapacity $_{i t}+u$ Plus $_{i t}-$ MMinus $_{i t}, \quad \forall i, t$

6) open $_{i t+1}=$ open $_{i t}+$ opened $_{i t}-$ closed $_{i t}, \forall i, t$

7) $\quad M *$ expanded $_{i t} \geq u$ Plus $_{i t}, \forall i, t$

8) $\quad M * \operatorname{shrunk}_{i t} \geq u$ Minus $_{i t}, \forall i, t$

9) totalCapacity $_{i t} \geq 0, \forall i, t$

10) totalCapacity $_{i t} \geq u$ Minus $_{i t}, \forall i, t$

11) expanded $_{i t} \leq$ open $_{i t}, \forall i, t$

12) shrunk $_{i t} \leq$ open $_{i t}, \forall i, t$

13) $M *$ open $_{i t} \geq$ usedCapacity $_{i t}, \forall i, t$

14) $\sum_{i}$ assignment $_{i j t} \leq 1, \forall j, t$

15) assignement $_{i j t}$, expanded $_{i t}$, shrunk $_{i t}$, open $_{i t}$, opened $_{i t}$, closed $_{i t} \in$ $\{0 ; 1\}, \forall i, j, t$ 
16) uPlus $_{i t}$, uMinus $_{i t}$, totalCapacity $_{i t}$, usedCapacity $_{i t} \in \mathbb{N}, \forall i, t$

\section{Explanation}

The objective function maximizes the profit. It consists of six terms. The first term computes the net profit. The second term includes the fixed cost component when a facility is open. The third and fourth terms are for assigning a closing or opening cost that occurs when a facility is opened or closed, respectively. The last two terms consider the expansion or contraction costs. Constraints (1) and (2) are used to specify the open or closed status of each facility as well as the initial capacity. The usage must never be greater than the capacity. This is ensured by constraint (3). Constraint (4) addresses the considering that the usage cannot exceed the assigned demands. The capacity of the facilities might vary over time. Constraint (5) computes the capacity for the following period by adding the units of expansion or subtracting the units of shrinkage from the current capacity. Constraint (6) is a key constraint and is used for setting the status of a facility to open or close. Constraint (7) sets expanded $i t$ to 1 if the facility is expanded. Constraint (8) does the same for the contraction case. Constraint (9) ensures that the capacity will never become negative and constraint (10) states that the capacity will always be greater or equal than a potential contraction. Constraints (11) and (12) assert that only open facilities can be expanded or contracted, respectively. Finally, constraint (13) ensures that capacity will only be used if a facility is open and (14) postulates to assign a certain demand only to one of available facilities and only once. 


\subsubsection{Computational results}

To demonstrate the validity of the model, it is first solved using a small problem set three facilities, three demand locations and three periods - in LINGO solver. The corresponding LINGO model is in Appendix A. The following fictitious data was used and read from an Excel file.

Table 1 Test data for model 1 with 3 facilities, 3 demand points, and 3 time periods

\begin{tabular}{|cc|}
\hline Initial capacity & \\
initialCapacity & \\
Facility $(i)$ & initialCapacity \\
1 & 500 \\
2 & 500 \\
3 & 500 \\
\hline
\end{tabular}

\begin{tabular}{|cc|}
\hline Opensettings for period 1 & \\
initialOpen & \\
Facility $(i)$ & initalOpen \\
1 & 1 \\
2 & 1 \\
3 & 1 \\
\hline
\end{tabular}

\begin{tabular}{|c|c|c|}
\hline \multicolumn{3}{|c|}{ Price for selling one unit at $i$ in $t$} \\
\hline price & & \\
\hline Facility $(i)$ & 1 & 3 \\
\hline
\end{tabular}




\begin{tabular}{|llll|}
1 & 5,000 & 5,000 & 5,000 \\
2 & 5,000 & 5,000 & 5,000 \\
3 & 5,000 & 5,000 & 5,000 \\
\hline
\end{tabular}

\begin{tabular}{|cccc|}
\hline Variable cost for selling in period $t$ at $i$ \\
variableCost \\
Facility $(i)$ & 1 & 2 & 3 \\
1 & 20 & 20 & 50,000 \\
2 & 20 & 20 & 20 \\
3 & 20 & 20 & 20 \\
\hline
\end{tabular}

\begin{tabular}{|cccc|}
\hline Unit cost for expanding $i$ in $t$ & & \\
unitExpansionCost & & & \\
Facility $(i)$ & 1 & 2 & 3 \\
1 & 5 & 5 & 5 \\
2 & 5 & 5 & 5 \\
3 & 5 & 5 & 5 \\
\hline
\end{tabular}

\begin{tabular}{|cccc|}
\hline Unit cost for shrinking $i$ in $t$ & & \\
unitShrinkageCost & & & \\
Facility $(i)$ & 1 & 2 & 3 \\
1 & 3 & 3 & 3 \\
2 & 3 & 3 & 3 \\
3 & 3 & 3 & 3 \\
\hline
\end{tabular}




\begin{tabular}{|cccc|}
\hline Expanding cost for $i$ in $t$ & & \\
expansionCost & & & \\
Facility $(i)$ & 1 & 2 & 3 \\
1 & 10 & 11 & 10 \\
2 & 20 & 10 & 10 \\
3 & 10 & 5 & 20 \\
\hline
\end{tabular}

\begin{tabular}{|cccc|}
\hline $\begin{array}{c}\text { Contraction cost for } i \text { in } t \\
\text { shrinkageCost }\end{array}$ & Period $(t)$ & \\
Facility $(i)$ & 1 & 2 & 3 \\
1 & 5 & 5 & 5 \\
2 & 5 & 3 & 10 \\
3 & 10 & 10 & 10 \\
\hline
\end{tabular}

\begin{tabular}{|cccc|}
\hline $\begin{array}{c}\text { Demand at } j \text { in period } t \\
\text { demand }\end{array}$ & \multicolumn{3}{c|}{ Period $(\mathrm{t})$} \\
Demand location $(j)$ & 1 & 2 & 3 \\
1 & 1,00000 & 0 & 50,0000 \\
2 & 1,00000 & 0 & 50,0000 \\
3 & 1,00000 & 0 & 50,0000 \\
\hline
\end{tabular}

Attraction value for facility $i$ for demand point $j$ in period $t$

attraction

Demand location $(j)$

1

2

3 


\begin{tabular}{|cccccccccc|} 
& & Period $(t)$ & \multicolumn{3}{c}{ Period $(t)$} & \multicolumn{3}{c|}{ Period $(t)$} \\
& & & & & & & \\
Facility $(i)$ & 1 & 2 & 3 & 1 & 2 & 3 & 1 & 2 & 3 \\
1 & 1 & 2 & 3 & 1 & 2 & 3 & 1 & 2 & 3 \\
2 & 2 & 2 & 2 & 2 & 10 & 2 & 2 & 2 & 2 \\
3 & 3 & 2 & 1 & 3 & 2 & 1 & 3 & 30 & 1 \\
\hline
\end{tabular}

\begin{tabular}{|cccc|}
\hline \multicolumn{3}{|l}{ Highest attraction value of competitor } \\
competitorsAttraction & \multicolumn{3}{c|}{ Period $(t)$} \\
Demand location $(j)$ & 1 & 2 & 3 \\
1 & 1 & 1 & 1 \\
2 & 1 & 50 & 1 \\
3 & 1 & 1 & 1 \\
\hline
\end{tabular}

\begin{tabular}{|cccc|}
\hline \multicolumn{4}{|c|}{ Fix cost for running facility $i$ in period $t$} \\
fixCost & \multicolumn{3}{c}{ Period (t) } \\
Facility $(i)$ & 2 & 3 \\
1 & 10 & 50,000 & 50,000 \\
2 & 10 & 50,000 & 50,000 \\
3 & 10 & 50,000 & 50,000 \\
\hline
\end{tabular}

\begin{tabular}{|c|c|c|c|}
\hline \multicolumn{4}{|c|}{ Opening cost for $i$ in $t$} \\
\hline openingCost & & io & \\
\hline Facility $(i)$ & 1 & 2 & 3 \\
\hline 1 & 1 & 1 & 1 \\
\hline 2 & 1 & 1 & 1 \\
\hline
\end{tabular}




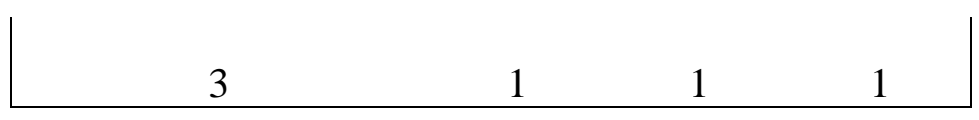

\begin{tabular}{|cccc|}
\hline Closing cost for $i$ in $t$ & & & \\
closingCost & & & \\
Facility $(i)$ & 1 & 2 & 3 \\
1 & 1 & 1 & 1 \\
2 & 1 & 600,000 & 1 \\
3 & 1 & 1 & 1 \\
\hline
\end{tabular}

The model was solved in less than a second by running the LINGO solver on an Intel Core 2.7 GHz CPU with 4GB RAM on Windows 64Bit OS.

\subsubsection{Performed checks}

The model was tested with two additional data sets. Again, the solutions were obtained in less than one second using the same software and hardware. Several tests were applied to confirm the model is working correctly. All tests were conducted on all three different data sets and are listed below.

1. Test: If assignment $_{i j t}=1$, is usedCapacity $i t>0$ ?

This test was conducted to confirm that a facility only has used capacity if the corresponding assignment variable is set to 1 .

2. If assignemnt $_{i j t}=1$, is attraction $_{i j t} \geq$ competitorsAttraction $_{j t}$ ? 
This condition states that an assignment will only be made if attraction of own facility with respect to demand point is greater or equal than the attraction of all competitors in period $t$.

3. If expanded ${ }_{i t}=1$, is $u$ Plus $_{i t}>0$ ?

If a facility is expanded the variable $u$ Plus indicating the amount of expansion must be positive.

4. If $\operatorname{shrunk}_{i t}=1$, is uMinus $>0$ ?

If a facility is contracted, the variable uMinus indicating the amount of contraction needs to be positive.

5. If expanded $_{i t}=1$, is capacity $_{i t+1}=$ capacity $_{i t}+u$ Plus $_{i t}$ ?

If a facility is expanded in period $t$, the capacity of the facility in the preceding period must be expanded by $u$ Plus $s_{i t}$ units.

6. If shrunk $_{i t}=1$, is capacity $_{i t+1}=$ capacity $_{i t}-$ uMinus $_{i t}$ ?

If a facility is contracted in period $t$, the capacity of the facility in the preceding period must be reduced by $u$ Minus $_{i t}$ units.

7. If closed $_{i t}=1$, is open $i t+1=0$ ?

If a facility is closed at the end of period $t$, it must be closed in the preceding period. 
8. If opened $_{i t}=1$, is open $i t+1=1$ ?

If a facility is opened at the end of period $t$, it must be open in the preceding period.

9. If used $_{i t}>0$, is open $i t=1$ ?

This test was conducted to confirm that a facility only has used capacity if it is open.

All tests showed positive results and confirmed the model was working the expected way. The only remark that must be made here is that " $\mathrm{M}$ " is an arbitrarily large positive number, larger than the highest potential capacity. The highest potential capacity will never exceed the demand at all demand points in any period.

\subsubsection{Extension of initial model}

To get a better understanding of the model's behavior, several additional scenarios were constructed. First, the number of demand locations was extended to 10. The problem was solved quickly. Second, the time periods were extended to 10. Again, a solution of the problem could be obtained in about 1 second. Third, the number of facilities was also extended to 10. For the first set of data, the model could not be solved even after $\mathrm{x}$ hours of computation time. For two subsequent data sets, the optimal solution could be determined in a few seconds. 
Another larger data set with 100 facilities, 100 demand points, and 100 time periods was generated. The complexity of the data increased exponentially and working with Excel turned out to be cumbersome, but the model with 1,089,800 variables was solved in about 2.5 minutes.

The following table summarizes the solution times for the multiple datasets that were used to solve Model 1.

Table 2 Variables and runtimes for different datasets

$\begin{array}{ccc}\text { Dataset } & \text { Number of variables } & \text { Elapsed runtime } \\ 3 \times 3 \times 3 & 102 & <1 \mathrm{sec} . \\ 5 \times 5 \times 5 & 340 & <1 \mathrm{sec} \\ 10 \times 10 \times 10^{*} & 1,880 & 30-250 \mathrm{sec} . \\ 100 \times 100 \times 100 & 1,089,800 & \text { n.a. } \\ \text { *Depending on the data } & \end{array}$




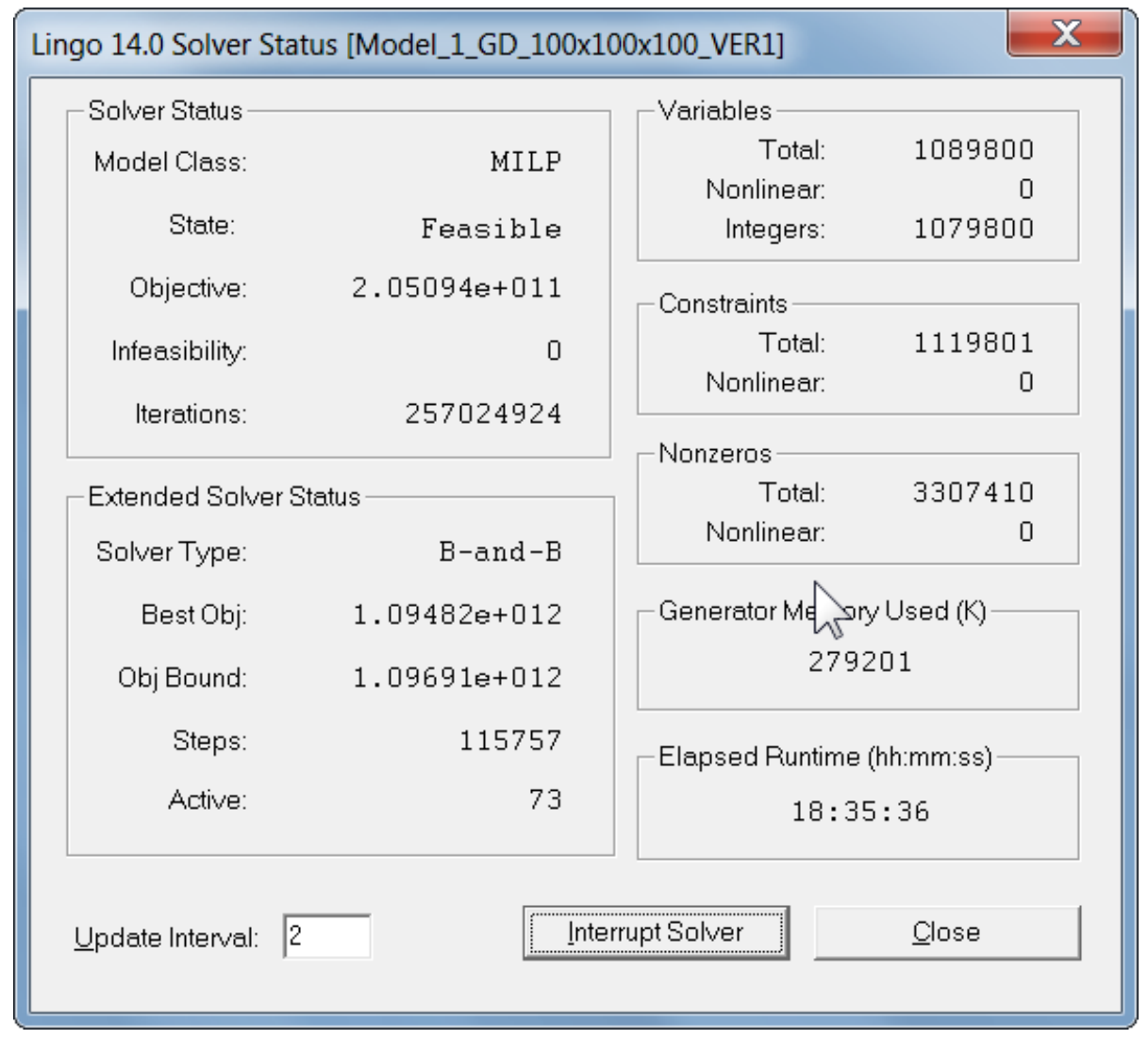

Figure 1: Solver status for model with 100 locations, 100 demand points and 100 time periods

In order to gage the problem sizes that can be solved using standard software, several problem instances were generated. The 100x100x100 scenario could not be solved within a reasonable time. A $150 \times 150 \times 150$ scenario with more than 3.5 million variables, resulted in an LINGO Buffer error. 


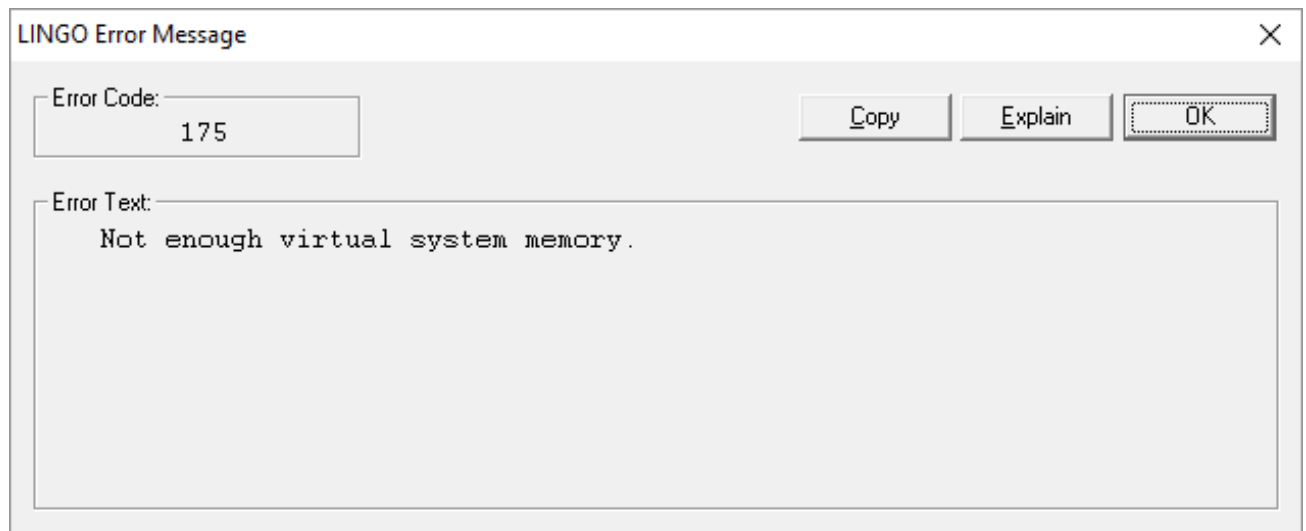

Figure 2 LINGO error message for 150x150x150 model

\subsubsection{Retrieving the relaxed solution from the model}

To obtain an upper bound for the solution of the model, all binary and integer constraints were removed from the model. The following results were obtained.

Table 3 Objective function values for different model sizes both for mixed integer model and relaxed model

\begin{tabular}{ccc} 
Data Set & \multicolumn{2}{c}{ Objective function value } \\
& $\begin{array}{cc}\text { Regular model } \\
5 \times 5 \times 5\end{array}$ & $\begin{array}{c}\text { Relaxed model } \\
5.50502 \mathrm{E} 08\end{array}$ \\
$10 \times 10 \times 10$ & $4.18601 \mathrm{E} 08$ & $4.18671 \mathrm{E} 8$ \\
$100 \times 100 \times 100^{2}$ & $2.05094 \mathrm{E} 11$ & $1.10398 \mathrm{E} 12$
\end{tabular}

The results of the objective function values (OFVs) show that the relaxed model solutions

\footnotetext{
${ }^{2}$ Objective function value of a feasible but not of the optimal solution
} 
provide an upper bound that is relatively close the OFV of the optimal solution for the first two (smaller) problem instances.

\subsubsection{Solving the model in dynamic manner}

It is relatively easier to forecast demands in the short term, than for the long term. Thus, the model was also solved using a dynamic approach; this means the problem is solved for each period considering only the demand for that period. A dynamic solution will result in more frequent opening and closing of facilities due to the myopic approach. An analysis was performed, whether a robust solution (solving the model once for the entire planning horizon, even though the demand forecasts, especially for the later periods in the planning horizon, may be inaccurate) or a dynamic approach (solving the model once in each period and using that solution and the next period's forecast demand to determine the next period's solution) will result in a better solution. With respect to the model and its mathematical formulation, a single period based approach was not implementable as the open status and current capacity is always known for the initial period. Thus, the consecutive period was included in the model and the all variables were determined for that period. After obtaining the variables values, the next run was begun using the just generated variable values as initial parameters. Figure 3 demonstrates the procedure. 


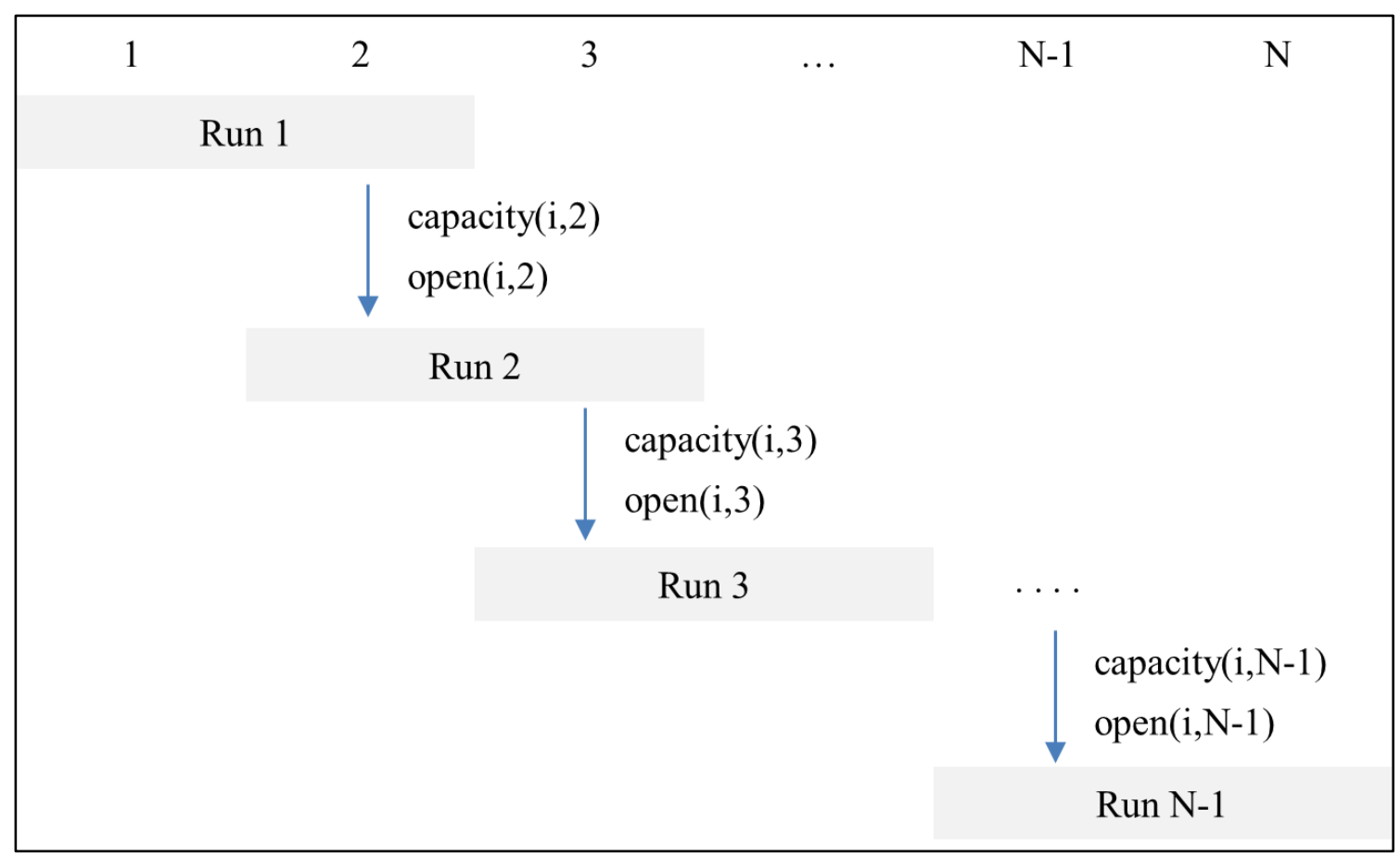

Figure 3 Dynamic solution approach

The dynamic solution was implemented using LINGO scripting. The scripting made it possible to change the relevant variables in the model. The following script shows the implementation for the model with ten facilities, ten demand points and ten time periods. The corresponding model is shown in Appendix B.

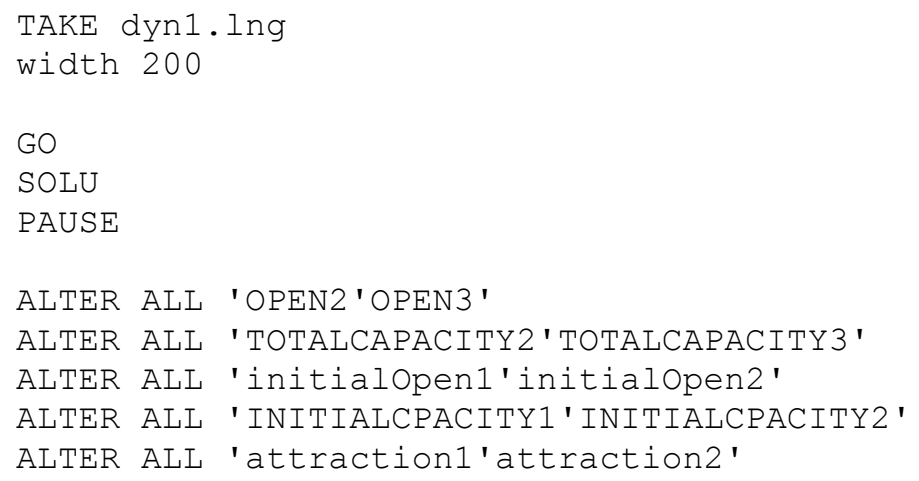




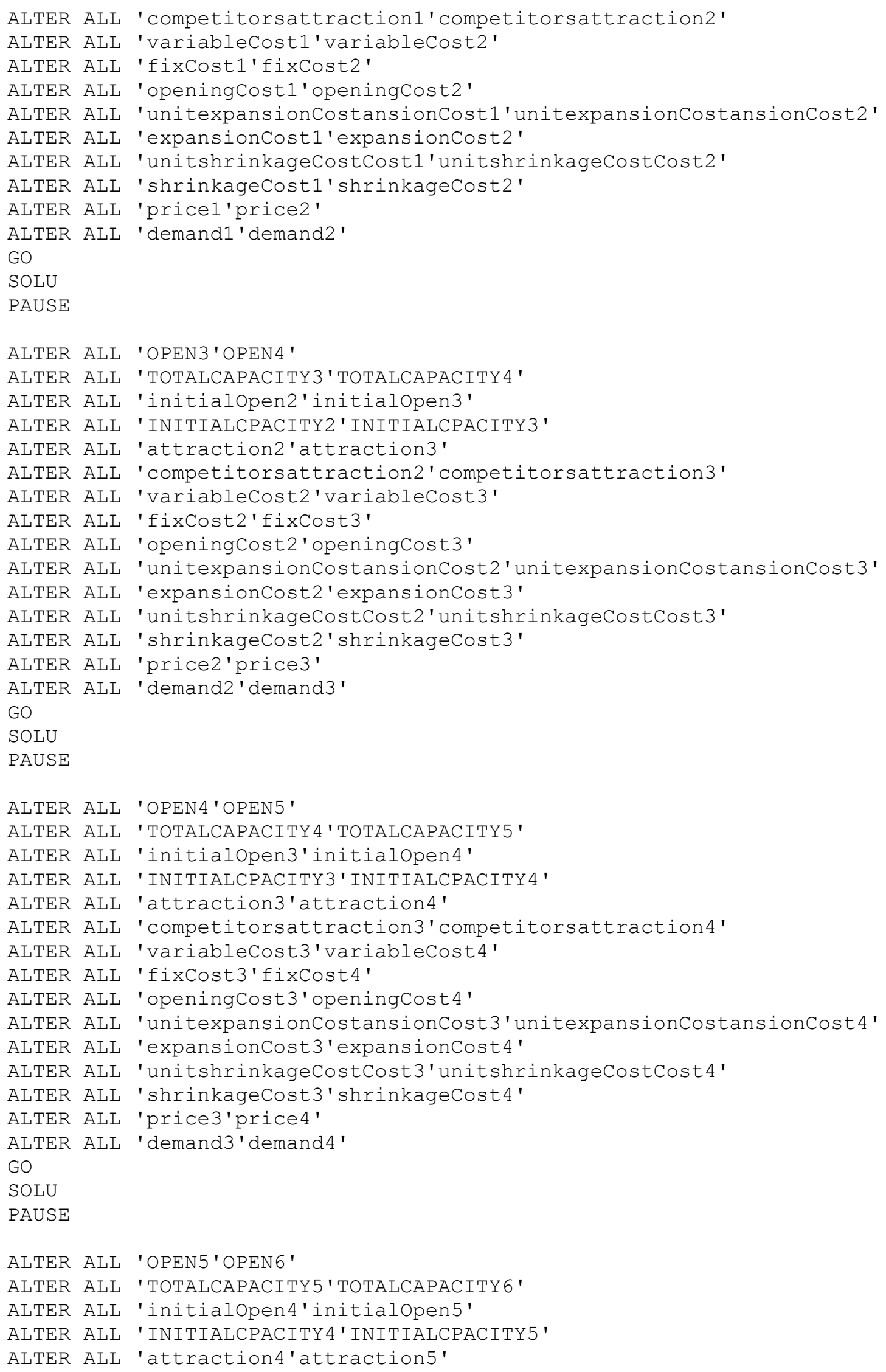




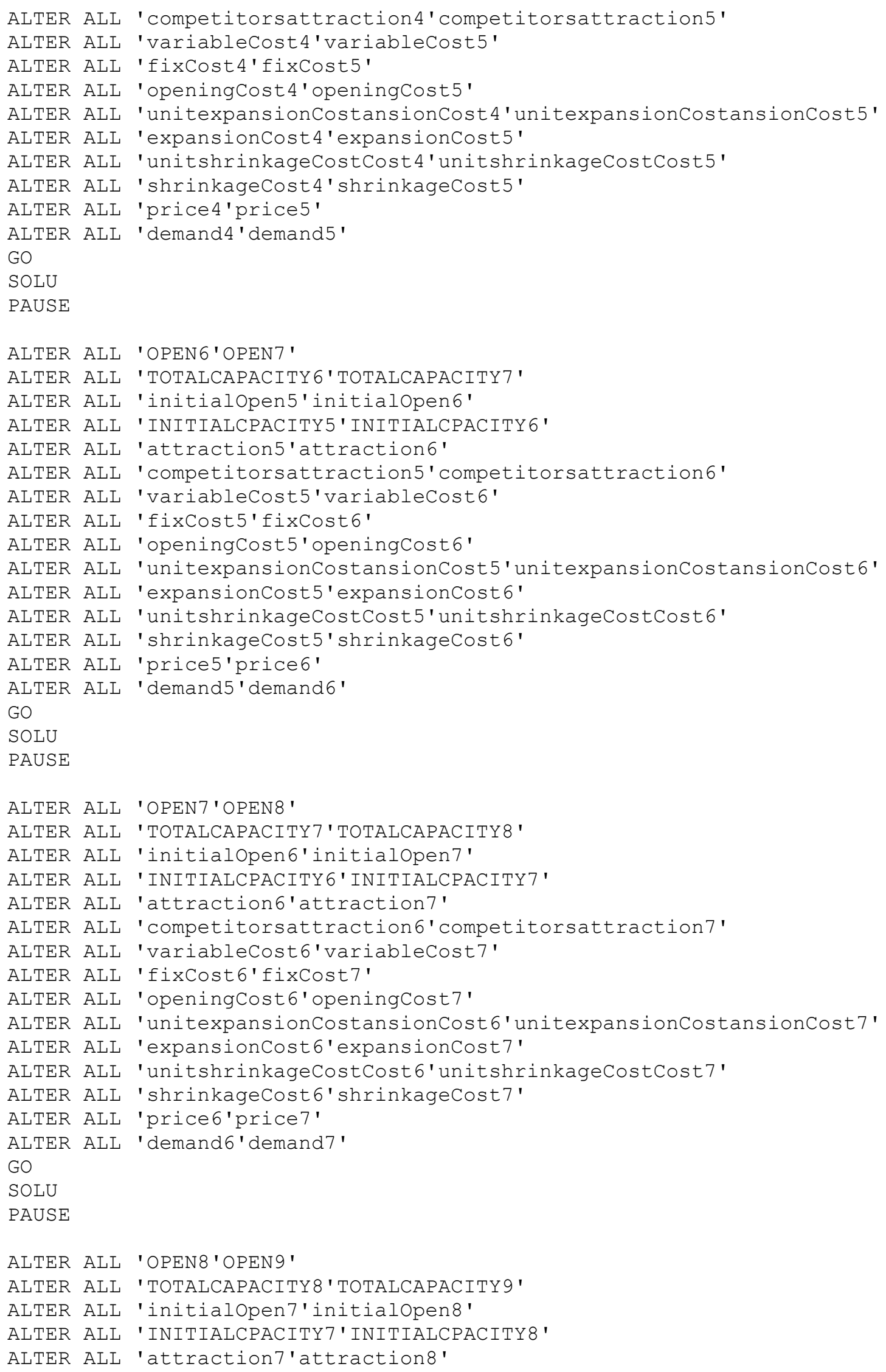




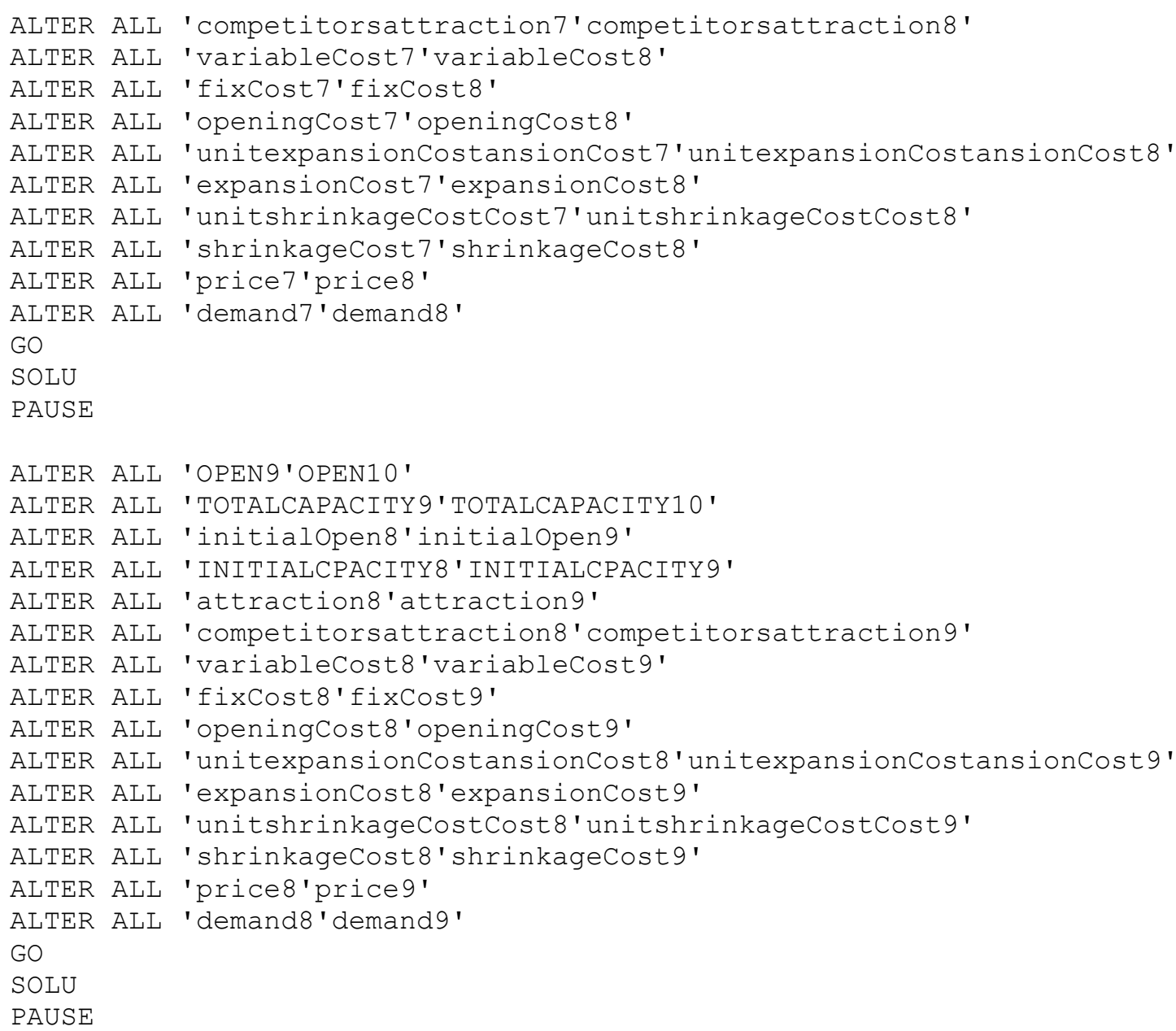

Several checks were performed to explore the models' behavior and to detect the differences of the two approaches (robust versus dynamic).

1. Demand data constant over time horizon

The two approaches show a difference both in terms of capacity and the opening status for facility 1 . The robust solution contains an extension of the capacities; the dynamic one keeps the capacity on the initial level for all periods, due to the fact that the expansion cost cannot be offset by net profit from one period. The opening status is the same for both models. The following table shows the results. 
Table 4 Results for constant data for dynamic and robust approach

\begin{tabular}{|c|c|c|c|c|c|}
\hline \multirow[t]{2}{*}{ Facility } & \multirow[t]{2}{*}{ Period } & \multicolumn{2}{|c|}{ Open status } & \multicolumn{2}{|c|}{ Capacity } \\
\hline & & Robust & Dynamic & Robust & Dynamic \\
\hline 1 & 1 & 1 & 1 & 1,000 & 1,000 \\
\hline 2 & 1 & 1 & 1 & 1,000 & 1,000 \\
\hline 3 & 1 & 1 & 1 & 1,000 & 1,000 \\
\hline 4 & 1 & 1 & 1 & 1,000 & 1,000 \\
\hline 5 & 1 & 1 & 1 & 1,000 & 1,000 \\
\hline 6 & 1 & 1 & 1 & 1,000 & 1,000 \\
\hline 7 & 1 & 1 & 1 & 1,000 & 1,000 \\
\hline 8 & 1 & 1 & 1 & 1,000 & 1,000 \\
\hline 9 & 1 & 1 & 1 & 1,000 & 1,000 \\
\hline 10 & 1 & 1 & 1 & 1,000 & 1,000 \\
\hline 1 & 2 & 0 & 1 & 1,000 & 1,000 \\
\hline 2 & 2 & 1 & 1 & 20,000 & 1,000 \\
\hline 3 & 2 & 1 & 1 & 20,000 & 1,000 \\
\hline 4 & 2 & 1 & 1 & 20,000 & 1,000 \\
\hline 5 & 2 & 1 & 1 & 20,000 & 1,000 \\
\hline 6 & 2 & 1 & 1 & 20,000 & 1,000 \\
\hline 7 & 2 & 1 & 1 & 20,000 & 1,000 \\
\hline 8 & 2 & 1 & 1 & 20,000 & 1,000 \\
\hline 9 & 2 & 1 & 1 & 20,000 & 1,000 \\
\hline 10 & 2 & 1 & 1 & 20,000 & 1,000 \\
\hline 1 & 3 & 0 & 1 & 1,000 & 1,000 \\
\hline 2 & 3 & 1 & 1 & 10,000 & 1,000 \\
\hline 3 & 3 & 1 & 1 & 10,000 & 1,000 \\
\hline 4 & 3 & 1 & 1 & 10,000 & 1,000 \\
\hline 5 & 3 & 1 & 1 & 10,000 & 1,000 \\
\hline 6 & 3 & 1 & 1 & 10,000 & 1,000 \\
\hline 7 & 3 & 1 & 1 & 10,000 & 1,000 \\
\hline 8 & 3 & 1 & 1 & 10,000 & 1,000 \\
\hline
\end{tabular}




\begin{tabular}{|c|c|c|c|c|c|}
\hline 9 & 3 & 1 & 1 & 10,000 & 1,000 \\
\hline 10 & 3 & 1 & 1 & 10,000 & 1,000 \\
\hline 1 & 4 & 0 & 1 & 1,000 & 1,000 \\
\hline 2 & 4 & 1 & 1 & 10,000 & 1,000 \\
\hline 3 & 4 & 1 & 1 & 10,000 & 1,000 \\
\hline 4 & 4 & 1 & 1 & 10,000 & 1,000 \\
\hline 5 & 4 & 1 & 1 & 10,000 & 1,000 \\
\hline 6 & 4 & 1 & 1 & 10,000 & 1,000 \\
\hline 7 & 4 & 1 & 1 & 10,000 & 1,000 \\
\hline 8 & 4 & 1 & 1 & 10,000 & 1,000 \\
\hline 9 & 4 & 1 & 1 & 10,000 & 1,000 \\
\hline 10 & 4 & 1 & 1 & 10,000 & 1,000 \\
\hline 1 & 5 & 0 & 1 & 1,000 & 1,000 \\
\hline 2 & 5 & 1 & 1 & 10,000 & 1,000 \\
\hline 3 & 5 & 1 & 1 & 10,000 & 1,000 \\
\hline 4 & 5 & 1 & 1 & 10,000 & 1,000 \\
\hline 5 & 5 & 1 & 1 & 10,000 & 1,000 \\
\hline 6 & 5 & 1 & 1 & 10,000 & 1,000 \\
\hline 7 & 5 & 1 & 1 & 10,000 & 1,000 \\
\hline 8 & 5 & 1 & 1 & 10,000 & 1,000 \\
\hline 9 & 5 & 1 & 1 & 10,000 & 1,000 \\
\hline 10 & 5 & 1 & 1 & 10,000 & 1,000 \\
\hline 1 & 6 & 0 & 1 & 1,000 & 1,000 \\
\hline 2 & 6 & 1 & 1 & 10,000 & 1,000 \\
\hline 3 & 6 & 1 & 1 & 10,000 & 1,000 \\
\hline 4 & 6 & 1 & 1 & 10,000 & 1,000 \\
\hline 5 & 6 & 1 & 1 & 10,000 & 1,000 \\
\hline 6 & 6 & 1 & 1 & 10,000 & 1,000 \\
\hline 7 & 6 & 1 & 1 & 10,000 & 1,000 \\
\hline 8 & 6 & 1 & 1 & 10,000 & 1,000 \\
\hline 9 & 6 & 1 & 1 & 10,000 & 1,000 \\
\hline 10 & 6 & 1 & 1 & 10,000 & 1,000 \\
\hline
\end{tabular}




\begin{tabular}{|c|c|c|c|c|c|}
\hline 1 & 7 & 0 & 1 & 1,000 & 1,000 \\
\hline 2 & 7 & 1 & 1 & 10,000 & 1,000 \\
\hline 3 & 7 & 1 & 1 & 10,000 & 1,000 \\
\hline 4 & 7 & 1 & 1 & 10,000 & 1,000 \\
\hline 5 & 7 & 1 & 1 & 10,000 & 1,000 \\
\hline 6 & 7 & 1 & 1 & 10,000 & 1,000 \\
\hline 7 & 7 & 1 & 1 & 10,000 & 1,000 \\
\hline 8 & 7 & 1 & 1 & 10,000 & 1,000 \\
\hline 9 & 7 & 1 & 1 & 10,000 & 1,000 \\
\hline 10 & 7 & 1 & 1 & 10,000 & 1,000 \\
\hline 1 & 8 & 0 & 1 & 1,000 & 1,000 \\
\hline 2 & 8 & 1 & 1 & 10,000 & 1,000 \\
\hline 3 & 8 & 1 & 1 & 10,000 & 1,000 \\
\hline 4 & 8 & 1 & 1 & 10,000 & 1,000 \\
\hline 5 & 8 & 1 & 1 & 10,000 & 1,000 \\
\hline 6 & 8 & 1 & 1 & 10,000 & 1,000 \\
\hline 7 & 8 & 1 & 1 & 10,000 & 1,000 \\
\hline 8 & 8 & 1 & 1 & 10,000 & 1,000 \\
\hline 9 & 8 & 1 & 1 & 10,000 & 1,000 \\
\hline 10 & 8 & 1 & 1 & 10,000 & 1,000 \\
\hline 1 & 9 & 0 & 1 & 1,000 & 1,000 \\
\hline 2 & 9 & 1 & 1 & 10,000 & 1,000 \\
\hline 3 & 9 & 1 & 1 & 10,000 & 1,000 \\
\hline 4 & 9 & 1 & 1 & 10,000 & 1,000 \\
\hline 5 & 9 & 1 & 1 & 10,000 & 1,000 \\
\hline 6 & 9 & 1 & 1 & 10,000 & 1,000 \\
\hline 7 & 9 & 1 & 1 & 10,000 & 1,000 \\
\hline 8 & 9 & 1 & 1 & 10,000 & 1,000 \\
\hline 9 & 9 & 1 & 1 & 10,000 & 1,000 \\
\hline 10 & 9 & 1 & 1 & 10,000 & 1,000 \\
\hline 1 & 10 & 0 & 1 & 1,000 & 1,000 \\
\hline 2 & 10 & 1 & 1 & 10,000 & 1,000 \\
\hline
\end{tabular}




$\begin{array}{llllll}3 & 10 & 1 & 1 & 10,000 & 1,000 \\ 4 & 10 & 1 & 1 & 10,000 & 1,000 \\ 5 & 10 & 1 & 1 & 10,000 & 1,000 \\ 6 & 10 & 1 & 1 & 10,000 & 1,000 \\ 7 & 10 & 1 & 1 & 10,000 & 1,000 \\ 8 & 10 & 1 & 1 & 10,000 & 1,000 \\ 9 & 10 & 1 & 1 & 10,000 & 1,000 \\ 10 & 10 & 1 & 1 & 10,000 & 1,000\end{array}$

2. Variable demand data

Demand can be estimated more accurately in the short-run, but it is difficult to do so for periods far into the feature due to a variety of factors including changes in trends, customer preferences, seasonality and others. Because the dynamic model only considers the current and next periods, the solution provided by the two models will be different. The dynamic approach closes a facility immediately if there is no demand in the next period and if the fixed costs are significant. A disadvantage of the dynamic model is that it makes myopic decisions based on the just next period's demand. For example, it may close a facility because there is no demand in the next period and opens it again in a subsequent future period when the demand is high. Once it is closed, it will only be opened if the costs can be offset by demand in the next period. The robust approach shows a different solution because demand for multiple periods into the future is assumed to be known.

A comparison of objective function values is difficult as the dynamic 
approach always generates an objective value for two consecutive periods. The following table shows the difference for open status and total capacity for the robust approach and the dynamic approach, respectively using a model size of 10x10x10 with a fixed cost of 50,000.

Table 5 Results for alternating demand for dynamic and robust approach

\begin{tabular}{|c|c|c|c|c|c|}
\hline \multirow[t]{2}{*}{ Facility } & \multirow[t]{2}{*}{ Period } & \multicolumn{2}{|c|}{ Open status } & \multicolumn{2}{|c|}{ Capacity } \\
\hline & & Robust & Dynamic & Robust & Dynamic \\
\hline 1 & 1 & 1 & 1 & 1.000 & 1.000 \\
\hline 2 & 1 & 1 & 1 & 1.000 & 1.000 \\
\hline 3 & 1 & 1 & 1 & 1.000 & 1.000 \\
\hline 4 & 1 & 1 & 1 & 1.000 & 1.000 \\
\hline 5 & 1 & 1 & 1 & 1.000 & 1.000 \\
\hline 6 & 1 & 1 & 1 & 1.000 & 1.000 \\
\hline 7 & 1 & 1 & 1 & 1.000 & 1.000 \\
\hline 8 & 1 & 1 & 1 & 1.000 & 1.000 \\
\hline 9 & 1 & 1 & 1 & 1.000 & 1.000 \\
\hline 10 & 1 & 1 & 1 & 1.000 & 1.000 \\
\hline 1 & 2 & 0 & 0 & 1.000 & 1.000 \\
\hline 2 & 2 & 0 & 0 & 20000 & 1.000 \\
\hline 3 & 2 & 0 & 0 & 10000 & 1.000 \\
\hline 4 & 2 & 0 & 0 & 10000 & 1.000 \\
\hline 5 & 2 & 0 & 0 & 10000 & 1.000 \\
\hline 6 & 2 & 0 & 0 & 10000 & 1.000 \\
\hline 7 & 2 & 0 & 0 & 10000 & 1.000 \\
\hline 8 & 2 & 0 & 0 & 10000 & 1.000 \\
\hline 9 & 2 & 0 & 0 & 10000 & 1.000 \\
\hline 10 & 2 & 0 & 0 & 10000 & 1.000 \\
\hline 1 & 3 & 0 & 0 & 1.000 & 1.000 \\
\hline
\end{tabular}




\begin{tabular}{|c|c|c|c|c|c|}
\hline 2 & 3 & 1 & 1 & 20000 & 1.000 \\
\hline 3 & 3 & 1 & 1 & 10000 & 1.000 \\
\hline 4 & 3 & 1 & 1 & 10000 & 1.000 \\
\hline 5 & 3 & 1 & 1 & 10000 & 1.000 \\
\hline 6 & 3 & 1 & 1 & 10000 & 1.000 \\
\hline 7 & 3 & 1 & 1 & 10000 & 1.000 \\
\hline 8 & 3 & 1 & 1 & 10000 & 1.000 \\
\hline 9 & 3 & 1 & 1 & 10000 & 1.000 \\
\hline 10 & 3 & 1 & 1 & 10000 & 1.000 \\
\hline 1 & 4 & 0 & 0 & 1.000 & 1.000 \\
\hline 2 & 4 & 0 & 0 & 20000 & 1.000 \\
\hline 3 & 4 & 0 & 0 & 10000 & 1.000 \\
\hline 4 & 4 & 0 & 0 & 10000 & 1.000 \\
\hline 5 & 4 & 0 & 0 & 10000 & 1.000 \\
\hline 6 & 4 & 0 & 0 & 10000 & 1.000 \\
\hline 7 & 4 & 0 & 0 & 10000 & 1.000 \\
\hline 8 & 4 & 0 & 0 & 10000 & 1.000 \\
\hline 9 & 4 & 0 & 0 & 10000 & 1.000 \\
\hline 10 & 4 & 0 & 0 & 10000 & 1.000 \\
\hline 1 & 5 & 0 & 0 & 1.000 & 1.000 \\
\hline 2 & 5 & 1 & 1 & 20000 & 1.000 \\
\hline 3 & 5 & 1 & 1 & 10000 & 1.000 \\
\hline 4 & 5 & 1 & 1 & 10000 & 1.000 \\
\hline 5 & 5 & 1 & 1 & 10000 & 1.000 \\
\hline 6 & 5 & 1 & 1 & 10000 & 1.000 \\
\hline 7 & 5 & 1 & 1 & 10000 & 1.000 \\
\hline 8 & 5 & 1 & 1 & 10000 & 1.000 \\
\hline 9 & 5 & 1 & 1 & 10000 & 1.000 \\
\hline 10 & 5 & 1 & 1 & 10000 & 1.000 \\
\hline 1 & 6 & 0 & 0 & 1.000 & 1.000 \\
\hline 2 & 6 & 0 & 0 & 20000 & 1.000 \\
\hline 3 & 6 & 0 & 0 & 10000 & 1.000 \\
\hline
\end{tabular}




\begin{tabular}{|c|c|c|c|c|}
\hline 4 & 6 & 0 & 0 & 10000 \\
\hline 5 & 6 & 0 & 0 & 10000 \\
\hline 6 & 6 & 0 & 0 & 10000 \\
\hline 7 & 6 & 0 & 0 & 10000 \\
\hline 8 & 6 & 0 & 0 & 10000 \\
\hline 9 & 6 & 0 & 0 & 10000 \\
\hline 10 & 6 & 0 & 0 & 10000 \\
\hline 1 & 7 & 0 & 1 & 1.000 \\
\hline 2 & 7 & 1 & 1 & 20000 \\
\hline 3 & 7 & 1 & 1 & 10000 \\
\hline 4 & 7 & 1 & 1 & 10000 \\
\hline 5 & 7 & 1 & 1 & 10000 \\
\hline 6 & 7 & 1 & 1 & 10000 \\
\hline 7 & 7 & 1 & 1 & 10000 \\
\hline 8 & 7 & 1 & 1 & 10000 \\
\hline 9 & 7 & 1 & 1 & 10000 \\
\hline 10 & 7 & 1 & 1 & 10000 \\
\hline 1 & 8 & 0 & 0 & 1.000 \\
\hline 2 & 8 & 0 & 0 & 20000 \\
\hline 3 & 8 & 0 & 0 & 10000 \\
\hline 4 & 8 & 0 & 0 & 10000 \\
\hline 5 & 8 & 0 & 0 & 10000 \\
\hline 6 & 8 & 0 & 0 & 10000 \\
\hline 7 & 8 & 0 & 0 & 10000 \\
\hline 8 & 8 & 0 & 0 & 10000 \\
\hline 9 & 8 & 0 & 0 & 10000 \\
\hline 10 & 8 & 0 & 0 & 10000 \\
\hline 1 & 9 & 0 & 1 & 1.000 \\
\hline 2 & 9 & 1 & 1 & 20000 \\
\hline 3 & 9 & 1 & 1 & 10000 \\
\hline 4 & 9 & 1 & 1 & 10000 \\
\hline 5 & 9 & 1 & 1 & 10000 \\
\hline
\end{tabular}




$\begin{array}{llllll}6 & 9 & 1 & 1 & 10000 & 1.000 \\ 7 & 9 & 1 & 1 & 10000 & 1.000 \\ 8 & 9 & 1 & 1 & 10000 & 1.000 \\ 9 & 9 & 1 & 1 & 10000 & 1.000 \\ 10 & 9 & 1 & 1 & 10000 & 1.000 \\ 1 & 10 & 0 & 0 & 1.000 & 1.000 \\ 2 & 10 & 0 & 0 & 20000 & 1.000 \\ 3 & 10 & 0 & 0 & 10000 & 1.000 \\ 4 & 10 & 0 & 0 & 10000 & 1.000 \\ 5 & 10 & 0 & 0 & 10000 & 1.000 \\ 6 & 10 & 0 & 0 & 10000 & 1.000 \\ 7 & 10 & 0 & 0 & 10000 & 1.000 \\ 8 & 10 & 0 & 0 & 10000 & 1.000 \\ 9 & 10 & 0 & 0 & 10000 & 1.000 \\ 10 & 10 & 0 & 0 & 10000 & 1.000\end{array}$

3. Variability in net profit

A variability in net profit shows an expansion of capacity in the robust approach. The facility is closed down when net profit decreases and opened again when the net profit increases again. The dynamic approach keeps the facilities closed. Lowering opening and closing costs influences the behavior. If the opening and closing costs are relatively low, facilities will be closed and reopened. The following table shows the results for high opening, closing and fixed costs. Demand is assigned only to one single facility in periods when net profit is high enough to cover the variable cost. 
Table 6 Results for alternating prices for dynamic and robust approach

\begin{tabular}{|c|c|c|c|c|c|}
\hline \multirow[t]{2}{*}{ Facility } & \multirow[t]{2}{*}{ Period } & \multicolumn{2}{|c|}{ Open status } & \multicolumn{2}{|c|}{ Capacity } \\
\hline & & Robust & Dynamic & Robust & Dynamic \\
\hline 1 & 1 & 1 & 1 & 1.000 & 1,000 \\
\hline 2 & 1 & 1 & 1 & 1.000 & 1,000 \\
\hline 3 & 1 & 1 & 1 & 1.000 & 1,000 \\
\hline 4 & 1 & 1 & 1 & 1.000 & 1,000 \\
\hline 5 & 1 & 1 & 1 & 1.000 & 1,000 \\
\hline 6 & 1 & 1 & 1 & 1.000 & 1,000 \\
\hline 7 & 1 & 1 & 1 & 1.000 & 1,000 \\
\hline 8 & 1 & 1 & 1 & 1.000 & 1,000 \\
\hline 9 & 1 & 1 & 1 & 1.000 & 1,000 \\
\hline 10 & 1 & 1 & 1 & 1.000 & 1,000 \\
\hline 1 & 2 & 0 & 0 & 1.000 & 1,000 \\
\hline 2 & 2 & 0 & 0 & 1000000 & 1,000 \\
\hline 3 & 2 & 0 & 0 & 1.000 & 1,000 \\
\hline 4 & 2 & 0 & 0 & 1.000 & 1,000 \\
\hline 5 & 2 & 0 & 0 & 1.000 & 1,000 \\
\hline 6 & 2 & 0 & 0 & 1.000 & 1,000 \\
\hline 7 & 2 & 0 & 0 & 1.000 & 1,000 \\
\hline 8 & 2 & 0 & 0 & 1.000 & 1,000 \\
\hline 9 & 2 & 0 & 0 & 1.000 & 1,000 \\
\hline 10 & 2 & 0 & 0 & 1.000 & 1,000 \\
\hline 1 & 3 & 0 & 0 & 1.000 & 1,000 \\
\hline 2 & 3 & 1 & 0 & 1000000 & 1,000 \\
\hline 3 & 3 & 0 & 0 & 1.000 & 1,000 \\
\hline 4 & 3 & 0 & 0 & 1.000 & 1,000 \\
\hline 5 & 3 & 0 & 0 & 1.000 & 1,000 \\
\hline 6 & 3 & 0 & 0 & 1.000 & 1,000 \\
\hline 7 & 3 & 0 & 0 & 1.000 & 1,000 \\
\hline 8 & 3 & 0 & 0 & 1.000 & 1,000 \\
\hline
\end{tabular}




\begin{tabular}{|c|c|c|c|c|c|}
\hline 9 & 3 & 0 & 0 & 1.000 & 1,000 \\
\hline 10 & 3 & 0 & 0 & 1.000 & 1,000 \\
\hline 1 & 4 & 0 & 0 & 1.000 & 1,000 \\
\hline 2 & 4 & 0 & 0 & 1000000 & 1,000 \\
\hline 3 & 4 & 0 & 0 & 1.000 & 1,000 \\
\hline 4 & 4 & 0 & 0 & 1.000 & 1,000 \\
\hline 5 & 4 & 0 & 0 & 1.000 & 1,000 \\
\hline 6 & 4 & 0 & 0 & 1.000 & 1,000 \\
\hline 7 & 4 & 0 & 0 & 1.000 & 1,000 \\
\hline 8 & 4 & 0 & 0 & 1.000 & 1,000 \\
\hline 9 & 4 & 0 & 0 & 1.000 & 1,000 \\
\hline 10 & 4 & 0 & 0 & 1.000 & 1,000 \\
\hline 1 & 5 & 0 & 0 & 1.000 & 1,000 \\
\hline 2 & 5 & 1 & 0 & 1000000 & 1,000 \\
\hline 3 & 5 & 0 & 0 & 1.000 & 1,000 \\
\hline 4 & 5 & 0 & 0 & 1.000 & 1,000 \\
\hline 5 & 5 & 0 & 0 & 1.000 & 1,000 \\
\hline 6 & 5 & 0 & 0 & 1.000 & 1,000 \\
\hline 7 & 5 & 0 & 0 & 1.000 & 1,000 \\
\hline 8 & 5 & 0 & 0 & 1.000 & 1,000 \\
\hline 9 & 5 & 0 & 0 & 1.000 & 1,000 \\
\hline 10 & 5 & 0 & 0 & 1.000 & 1,000 \\
\hline 1 & 6 & 0 & 0 & 1.000 & 1,000 \\
\hline 2 & 6 & 0 & 0 & 1000000 & 1,000 \\
\hline 3 & 6 & 0 & 0 & 1.000 & 1,000 \\
\hline 4 & 6 & 0 & 0 & 1.000 & 1,000 \\
\hline 5 & 6 & 0 & 0 & 1.000 & 1,000 \\
\hline 6 & 6 & 0 & 0 & 1.000 & 1,000 \\
\hline 7 & 6 & 0 & 0 & 1.000 & 1,000 \\
\hline 8 & 6 & 0 & 0 & 1.000 & 1,000 \\
\hline 9 & 6 & 0 & 0 & 1.000 & 1,000 \\
\hline 10 & 6 & 0 & 0 & 1.000 & 1,000 \\
\hline
\end{tabular}




\begin{tabular}{|c|c|c|c|c|c|}
\hline 1 & 7 & 0 & 0 & 1.000 & 1,000 \\
\hline 2 & 7 & 1 & 0 & 1000000 & 1,000 \\
\hline 3 & 7 & 0 & 0 & 1.000 & 1,000 \\
\hline 4 & 7 & 0 & 0 & 1.000 & 1,000 \\
\hline 5 & 7 & 0 & 0 & 1.000 & 1,000 \\
\hline 6 & 7 & 0 & 0 & 1.000 & 1,000 \\
\hline 7 & 7 & 0 & 0 & 1.000 & 1,000 \\
\hline 8 & 7 & 0 & 0 & 1.000 & 1,000 \\
\hline 9 & 7 & 0 & 0 & 1.000 & 1,000 \\
\hline 10 & 7 & 0 & 0 & 1.000 & 1,000 \\
\hline 1 & 8 & 0 & 0 & 1.000 & 1,000 \\
\hline 2 & 8 & 0 & 0 & 1000000 & 1,000 \\
\hline 3 & 8 & 0 & 0 & 1.000 & 1,000 \\
\hline 4 & 8 & 0 & 0 & 1.000 & 1,000 \\
\hline 5 & 8 & 0 & 0 & 1.000 & 1,000 \\
\hline 6 & 8 & 0 & 0 & 1.000 & 1,000 \\
\hline 7 & 8 & 0 & 0 & 1.000 & 1,000 \\
\hline 8 & 8 & 0 & 0 & 1.000 & 1,000 \\
\hline 9 & 8 & 0 & 0 & 1.000 & 1,000 \\
\hline 10 & 8 & 0 & 0 & 1.000 & 1,000 \\
\hline 1 & 9 & 0 & 0 & 1.000 & 1,000 \\
\hline 2 & 9 & 1 & 0 & 1000000 & 1,000 \\
\hline 3 & 9 & 0 & 0 & 1.000 & 1,000 \\
\hline 4 & 9 & 0 & 0 & 1.000 & 1,000 \\
\hline 5 & 9 & 0 & 0 & 1.000 & 1,000 \\
\hline 6 & 9 & 0 & 0 & 1.000 & 1,000 \\
\hline 7 & 9 & 0 & 0 & 1.000 & 1,000 \\
\hline 8 & 9 & 0 & 0 & 1.000 & 1,000 \\
\hline 9 & 9 & 0 & 0 & 1.000 & 1,000 \\
\hline 10 & 9 & 0 & 0 & 1.000 & 1,000 \\
\hline 1 & 10 & 0 & 0 & 1.000 & 1,000 \\
\hline 2 & 10 & 0 & 0 & 1000000 & 1,000 \\
\hline
\end{tabular}




$\begin{array}{llllll}3 & 10 & 0 & 0 & 1.000 & 1,000 \\ 4 & 10 & 0 & 0 & 1.000 & 1,000 \\ 5 & 10 & 0 & 0 & 1.000 & 1,000 \\ 6 & 10 & 0 & 0 & 1.000 & 1,000 \\ 7 & 10 & 0 & 0 & 1.000 & 1,000 \\ 8 & 10 & 0 & 0 & 1.000 & 1,000 \\ 9 & 10 & 0 & 0 & 1.000 & 1,000 \\ 10 & 10 & 0 & 0 & 1.000 & 1,000\end{array}$

4. Demand is constantly decreasing

The behavior of the model in terms of constantly decreasing demand again depends on the net profIt. If the net profit is relatively high, the robust model extends the capacity to be able to satisfy higher demand in the earlier periods. All facilities are kept open the entire planning horizon. If net profit is relatively low, then those facilities with highest cost are closed first. The dynamic model shows a different behavior. Though there is a higher demand than capacity at the beginning, no expansion is made, even in terms of higher net profit. If net profit decreases, the model closes only that facility with the smallest net profit first. Later all facilities will be closed at the same time.

In a nutshell the dynamic model is much more sensitive towards variability in demand. The better knowledge of future demands will enable to satisfy higher demands in earlier periods when using the robust approach.

The initial data used for the model is shown in Appendix C. 


\subsection{Model 2: Allowing multiple products and additional constraints}

Considering multiple products resulted in a significant increase in the number of variables. The second improvement was to add additional constraints to the model in order to allow stepwise cost. This was necessary, because a facility will be shrunk only if operation with higher capacity is more expensive than operation with lower capacity. Another modification that was tested was to add budget constraints for the expansion of the model. When applying it to the real-world, it is to be expected that the model will always need to be modified to suit the situation at hand.

The subsequent subchapters describe the mathematical formulation of the model and display mathematical results. As the complexity of the data grew, an additional subchapter for database setup and the corresponding challenges is added to this dissertation.

\subsubsection{Mathematical formulation}

\section{Indexes}

$i \quad$ Set of facilities

$j \quad$ Set of demand

$t \quad$ Set of time periods

$p \quad$ Set of products 


\section{Model parameters}

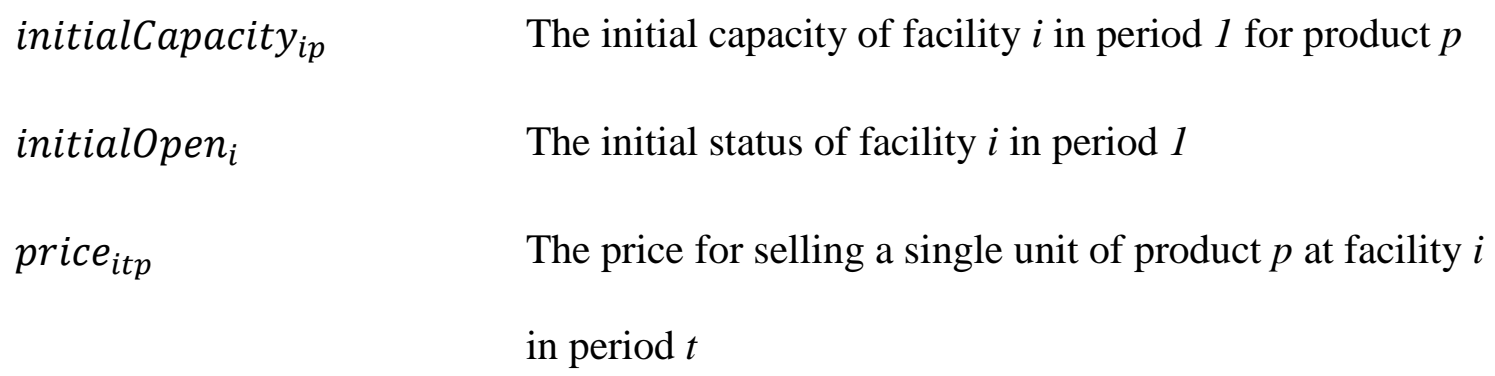

The initial capacity of facility $i$ in period 1 for product $p$

The initial status of facility $i$ in period 1

The price for selling a single unit of product $p$ at facility $i$ in period $t$

The unit cost for selling a single unit of product $p$ at facility $i$ in period $t$

The cost for having open facility $i$ in period $t$

The cost for opening facility $i$ in period $t$

The cost for closing facility $i$ in period $t$

Attraction value for product $p$ of a company's own facility $i$ with respect to demand node $j$ in period $t$

The highest attraction for product $p$ of competitors for demand node $j$ in period $t$

Fix cost for expansion of facility $i$ in period $t$

Fix cost for contraction of facility $i$ in period $t$

Unit (variable) cost for expansion of product $p$ at facility $i$ in period $t$

Unit (variable) cost for contraing of product $p$ at facility $i$ in period $t$

Demand for product $p$ at demand node $j$ in period $t$ 
step $<X>_{i t p}$

stepCost $<X>_{i t p}$

assignment $_{i j t p}$
The step (capacity) when a modification of fix cost is necessary. As there might be several steps, $\mathrm{X}$ indicates the number of the step.

The additional fixed cost for a certain step if the capacity is expanded for the corresponding product $p$ at facility $i$ in period $t$

$=\left\{\begin{array}{l}1, \text { ifattraction }_{i j t p} \geq \operatorname{comp}_{j t p} \\ 0, \text { else }\end{array}\right.$

This parameter is set to one if the attraction for a product $p$ of a company's own facility $i$ relative to a demand node $j$ is greater or equal than the highest attraction for that or a competitive product of the competitor with respect to node $j$ in period $t$. It is used for the assignment of products and facilities to a demand node in that period.

\section{Model variables}

$$
\begin{array}{ll}
\text { open }_{\text {it }} & =\left\{\begin{array}{l}
1, \text { if facility i remains open in } t \\
0, \text { if facility } i \text { is closed in } t
\end{array}\right. \\
\text { opened }_{i t} & =\left\{\begin{array}{l}
1, \text { if faciltiy } i \text { is opened in } t \\
0, \text { else }
\end{array}\right. \\
& =\left\{\begin{array}{l}
1, \text { if faciltiy } i \text { is closed in } t \\
0, \text { else }
\end{array}\right. \\
\text { closed }_{i t} & =\left\{\begin{array}{l}
1, \text { if facility } i \text { is expanded in } t \text { for product } p \\
0, \text { else }
\end{array}\right. \\
\text { expanded }_{i t p} & =\left\{\begin{array}{l}
1, \text { if facility } i \text { is shrunk in } t \text { for product } p \\
0, \text { else }
\end{array}\right.
\end{array}
$$


PPlus $_{i t p}$

uMinus $_{i t p}$

totalCapacity $_{i t p}$

usedCapacity $_{\text {itp }}$

${\text { totalFix } \text { Cost }_{i t}}$

threshold $<X>_{i t p}$

thresholdSwitch $\left\langle X>_{i t p}\right.$
Expansion of product $p$ in units at facility $i$ in $t$

Contraction of product $p$ in units at facility $i$ in $t$

Maximum capacity in units of product $p$ at $i$ in period $t$

Capacity utilized in units of product $p$ at $i$ in period $t$

The fix cost for operating facility $i$ in period $t$ depending on the step-wise cost function

The variable that is indicating whether the costs for a certain threshold in terms of capacity have to be incorporated in the model. $\mathrm{X}$ is again used for the corresponding step

This variable is used for modeling purposes in order to set the threshold correctly for facility $i$ in period $t$ and product $p$

\section{Objective function}

$$
\begin{aligned}
& \max _{i} \sum_{t} \sum_{p}\left(\text { usedCapacity }_{i t p} *\left(\text { price }_{i t p}-\text { variableCost }_{i t p}\right)-\text { totalFixCost }_{i t}-\right. \\
& \text { closingCost } \left._{i t} * \text { closed }_{i t}-\text { openingCost }_{i t} * \text { opened }_{i t}\right)-\sum_{i} \sum_{t} \sum_{p} \text { expansionCost }_{i t} * \\
& \text { expanded }_{i t p}-\sum_{i} \sum_{t} \sum_{p} \text { unitExpansionCost }_{i t p} * \text { uPlus }_{i t p}- \\
& \sum_{i} \sum_{t} \sum_{p} \text { shrinkageCost }_{i t} * \text { shrunk }_{i t p}-\sum_{i} \sum_{t} \sum_{p} \text { unitShrinkageCost }_{i t p} * \text { uMinus }_{i t p}
\end{aligned}
$$

\section{Subject to}

1) open $_{i 1}=$ initialOpen $_{i}, \quad \forall i$ 
2) totalCapacity $_{i 1 \mathrm{p}}=$ initialCapacity $_{i p}, \quad \forall i, p$

3) usedCapacity $_{i t p} \leq$ totalCapacity $_{i t p}, \quad \forall i, t, p$

4) usedCapacity $_{i t p} \leq \sum_{j}$ assignment $_{i j t p} *$ demand $_{j t p}, \quad \forall i, t, p$

5) totalCapacity $_{i t+1 \mathrm{p}}=$ totalCapacity $_{i t p}+u$ Plus $_{i t p}-$ uMinus $_{i t p}, \quad \forall i, t, p$

6) open $_{i t+1}=$ open $_{i t}+$ opened $_{i t}-$ closed $_{i t}, \forall i, t$

7) $\quad M *$ expanded $_{i t p} \geq u$ Plus $_{i t p}, \forall i, t, p$

8) $\quad M *$ shrunk $_{i t p} \geq$ uMinus $_{i t p}, \forall i, t, p$

9) totalCapacity $_{i t p} \geq 0, \forall i, \mathrm{t}, \mathrm{p}$

10) totalCapacity $_{i t p} \geq$ uMinus $_{i t p}, \forall i, t, p$

11) expanded $_{i t p} \leq$ open $_{i t p}, \forall i, t, p$

12) shrunk $_{i t p} \leq$ open $_{i t}, \forall i, t, p$

13) $\quad M *$ open $_{i t} \geq$ usedCapacity $_{i t}, \forall i, t$

14) $\sum_{i}$ assignment $_{i j t} \leq 1, \forall j, t$

15) $\mathrm{M} *$ threshold $\left.^{\mathrm{a}}\langle X\rangle_{i t p} \leq\left(\text { totalCapacity }_{i t p} / \text { step }<X+1\right\rangle_{i t p}\right)-1, \forall i, t, p$ where $1 \leq X \leq N$

16) threshold $\left\langle X>_{i t p}+\right.$ open $_{i t}+$ thresholdSwitch $\left\langle X>_{i t p} \leq 2, \forall i, t, p\right.$ where $1 \leq X \leq N$

17) threshold $\langle X\rangle_{i t p}$-thresholdSwitch $\langle X\rangle_{i t p} \geq 0$ 
18) totalFixCost $_{i t}=$ open $_{i t} *$ fixCost $_{i t}+\sum_{p}\left(\right.$ stepCost $_{i t p} *($ threshold $1-$ thresholdSwitch $\left.1_{i t p}\right)+$ stepCost $2_{i t p}\left(\right.$ threshold $2-$ thresholdSwitch $\left.2_{i t p}\right)+\ldots$

22) assignment $_{i j t p}$, expanded ${ }_{i t p}$, shrunk $_{i t p}$, open $_{i t}$, opened $_{i t}$, closed $_{i t}$ $\in\{0 ; 1\}, \forall i, j, t, p$

23) uPlus $_{\text {itp }}$, uMinus $_{i t p}$, totalCapacity $_{i t p}$, usedCapacity $_{\text {itp }} \in \mathbb{N}, \forall i, t$

The objective function maximizes the profit. It consists of six terms. The first term computes the net profit. The second term includes the fixed cost component when a facility is open, which is also determined by the stepwise cost function. The third and fourth terms are for assigning a closing or opening cost, respectively. The last two terms consider the expansion and contraction costs. Constraints (1) and (2) are used to initialize the open status of each facility as well as the initial capacity. The usage must never be greater than the capacity. This is ensured by constraint (3). Constraint (4) ensures that the usage cannot exceed the assigned demands. The capacity of the facilities might vary over time. Constraint (5) computes the capacity for the next period by adding the units of expansion or subtracting the units of contraction from the current capacity. Constraint (6) is a key constraint and is used for setting the status of a facility to open or close. Constraint (7) sets expanded exp $_{i t}$ to $l$ if the facility is expanded with respect to product $p$. Constraint (8) does the same for the contraction case. Constraint (9) ensures that the capacity will never become negative and constraint (10) states that the capacity will always be greater or equal than a potential shrinkage. Constraints (11) and (12) assert that only open facilities can be contracted or expanded, respectively. Constraint (13) ensures that capacity will only be used if a facility is open. Constraint (14) guarantees a 
maximum of one assignment per demand. Constraints (15), (16), (17) are used to determine the values for modification of the total cost function due to a change in capacity. Finally the total fixed costs are calculated in constraint (18).

\subsubsection{Computational results}

The model was solved with five different settings and a LINGO solver. The corresponding LINGO Model is provided in Appendix D . Fictitious data was used for tests and read from the oracle database.

Table 7 Variables and runtimes for different datasets for extended model

$\begin{array}{ccc}\text { Data Set } & \text { Number of variables } & \text { Elapsed runtime } \\ 3 \times 3 \times 3 \times 3 & 657 & <1 \mathrm{sec} . \\ 5 \times 5 \times 5 \times 5 & 3,945 & 22 \mathrm{sec} . \\ 10 \times 10 \times 10 \times 10 & 30,290 & \text { n.a. } \\ 50 \times 50 \times 50 \times 50 & 8,757,450 & \text { error } \\ 50 \times 50 \times 12 \times 50 & 2,099,250 & \text { n.a. }\end{array}$

The model can be solved relatively easily for specific problem instances with 1.7 million variables. Larger models need too much memory, and the LINGO solver crashed when these problems were solved. Although the model could be solved optimally for some very large problem instances, for example, those with 1 million variables, there were other 
problems that were much smaller that could not be solved optimally. The ability of the solver to solve problems depends upon the specific dataset used, whether or not the data matrix is sparse and other factors.

\subsubsection{Additional modifications}

The complexity of the model was raised by adding more constraints. The budget constraint provides a limited budget for expansion in a given period. A second constraint limits the capacity to a maximum and also requires a certain minimum capacity of a certain product. In real world problems, expansion to infinity is not possible, thus the maximum size of a facility will limit the potential expansion to a certain level. The LINGO model was also modified. The corresponding file is in Appendix E.

\section{Additional parameters}

expansionBudget $_{i t p} \quad$ Available budget for expansion at facility $i$ in period $t$ for product $p$

minimumCapacity $_{i t p}$ The minimum capacity of product $p$ at facility $i$ in period $t$ maximumCapacity $_{i t p}$ The maximum capacity of product $p$ at facility $i$ in period $t$ 


\section{Additional constraints}

a) $\quad \sum_{i} \sum_{p}\left(\right.$ expanded $_{i t p} *$ expansionCost $_{i t}+u$ Plus $_{i t p} *$ unitExpansionCost $\left._{i t p}\right)$

$$
\leq \text { expansionBudget }_{i t p}, \forall t
$$

b) totalCapacity $_{i t p} \leq$ maximumCapacity $_{i t p}, \forall i, t, p$

c) totalCapacity $_{i t p} \geq$ minimumCapacity $_{i t p}, \forall i, t, p$

\subsubsection{Some thoughts on the attraction parameter}

Allowing multiple products in the model raised the question whether the attraction parameter is to be considered as a facility-based or a product-based factor. The facilitybased option would not allow a product-related consideration afterwards and the overall attraction of a facility very much depends on the product mix. The extended model considers a product-based attraction. This results in an assignment of a specific product at a facility only if the attraction value is higher than that of the competitors with the highest attraction for that product. A facility-related attraction can either be realized by setting all parameters to the same value for all products at the relevant facility or by removing the product indexation for the attraction parameter.

\subsubsection{Computational results}

Several tests for solving the extended model were performed. The tests were again performed on different model sizes. The model extensions added complexity to the model. 
Solving the model for $10 \times 10 \times 10 \times 10$ could not be carried out in a reasonable time. The LINGO solver had to be interrupted after 100 hours. Smaller problem instances were solved quickly.

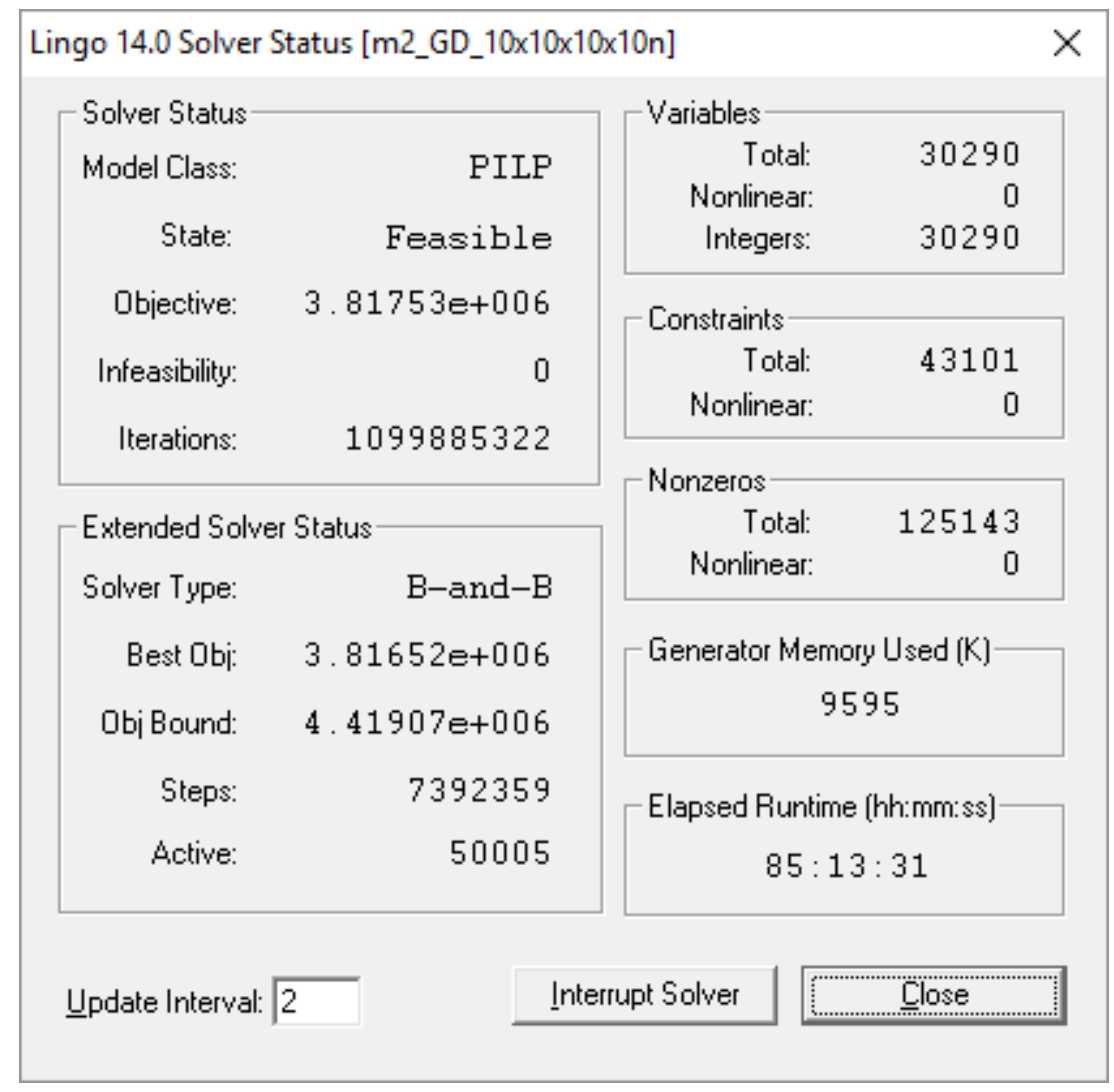

Figure 4 LINGO solver status for extended model 2

\subsubsection{Complexity in real world applications}

Real world application settings are very much depending on the industry. For retail businesses, a network of 50 or more facilities is typical and a forecast for 5 years (60 months) is reasonable. Even the number of demand locations will exceed 50. Financial institutions such as banks or insurance companies will also have several facilities. Big 
banks or insurance companies can have hundreds or thousands of locations. A time horizon of 5-10 years is often considered in the location analysis process. The product mix very often varies and is not limited to capacities. Production companies do usually not have dozens of facilities, but very often a very high variety of products or even product groups. Thus, real-world applications will exceed the size of the problems discussed in this dissertation and different approaches for solution generation are required.

\subsubsection{Technical implementation and corresponding challenges}

Utilizing Excel for a large model was not appropriate, requiring the data to be input from a database. An ODBC connection to an Oracle Database was established and a data structure both for the model input and output was created. Writing to a databases requires the table to have exactly the number of required rows. The setup of the tables is also very important as the data is read in exactly the order of the indexes. A change in the order will result in a wrong initialization of the model. This turned out to be a significant issue when views are created from the data as the order of the data might get mixed up. For each model, four different database tables are used. The following tables display the structure.

Table 8 Database view "modeldata_mp_10_view"

$\begin{array}{lll} & \text { COLUMN_NAME } & \text { DATA_TYPE } \\ 1 & \text { VARIABLECOST } & \text { FLOAT }(20) \\ 2 & \text { DEMAND } & \text { FLOAT }(20) \\ 3 & \text { ATTRACTIVITY } & \text { FLOAT }(20) \\ 4 & \text { COMPETITORSATTRACTION } & \text { FLOAT }(20) \\ 5 & \text { INDEXI } & \text { NUMBER(3) } \\ 6 & \text { PRICE } & \text { FLOAT }(20) \\ 7 & \text { INDEXJ } & \text { NUMBER(3) }\end{array}$



8 INDEXT
NUMBER(3)
9 INDEXP
$\operatorname{NUMBER}(3)$
10 UNITSHRINKAGECOST
FLOAT(126)
11 UNITEXPANSIONCOST
FLOAT(126)

Table 9 Database table "modeldata_mp_10_initials_cap"

$\begin{array}{ll}\text { COLUMN_NAME } & \text { DATA_TYPE } \\ \text { INITIALCAP } & \text { FLOAT } \\ \text { INDEXI } & \text { FLOAT } \\ \text { INDEXP } & \text { FLOAT }\end{array}$

Table 10 Database table "modeldata_mp_10_initials"

$\begin{array}{lll} & \text { COLUMN_NAME } & \text { DATA_TYPE } \\ 1 & \text { INITIALOPEN } & \text { FLOAT }\end{array}$

Table 11 Database table "modeldata_mp_10_facilities_npr"

$\begin{array}{lll} & \text { COLUMN_NAME } & \text { DATA_TY } \\ 1 & \text { EXPANSIONCOST } & \text { FLOAT } \\ 2 & \text { SHRINKAGECOST } & \text { FLOAT } \\ 3 & \text { FIXCOST } & \text { FLOAT } \\ 4 & \text { OPENINGCOST } & \text { FLOAT } \\ 5 & \text { CLOSINGCOST } & \text { FLOAT }\end{array}$

Table 8 contains all product relevant data. It is created as database view to ensure the correct order of the data. The view is created from a database table containing exactly the 
same data. Table 9 is used for initialization of capacities in period 1 for product $p$ at facility $i$.

Table 10 provides the open status for each facility in period 1 .

Table 11 contains all not product related data, such as fix cost, opening, closing, expansion and shrinkage cost information for the relevant facilities and periods.

The database turned out to be much faster in data access than Excel files.

\subsubsection{Retrieving the model's bounds}

In order to get a bound on the optimal OFV, the relaxed problem was solved by removing all binary and integer constraints from the variables. The following table shows the relaxed solutions and the corresponding objective function values if available for the model. This is only useful as a first estimate. If the model is not too big (i.e., starting LINGO solver is possible but will not generate a solution) we were able to retrieve at least some upper bounds for orientation for the non-relaxed problem. Using the data from the models above the following bounds were obtained. It shows that only for the $5 \times 5 \times 5 \times 5$ model the relaxed solution is close to the solution for the original problem.

Table 12 The model's bounds for the extended model

\begin{tabular}{c|cc} 
Model & Original problem & Relaxed solution \\
\hline $3 \times 3 \times 3 \times 3$ & $2.41038 \mathrm{E} 10$ & $1.70044 \mathrm{E} 9$
\end{tabular}




\begin{tabular}{c|cr}
$5 \times 5 \times 5 \times 5$ & $2.95213 \mathrm{E} 8$ & $2.98255 \mathrm{E} 8$ \\
\hline $10 \times 10 \times 10 \times 10$ & Not available & Not available \\
& & \\
$50 \times 50 \times 50 \times 50$ & Not available & Not available
\end{tabular}

\subsubsection{Modification of parameters}

In order to get a better understanding of the models behaviour, several parameter modifications were made. All tests were performed on the 5x5x5x5 model.

1. Starting with too big initial capacity

The first modification was starting with a much too high initial capacity. Much too high was given by multiplying the cumulated demand of one product at all demand points over all periods by five. As the fix cost function will automatically adjust the fix cost to the corresponding facility size this modification causes a massive cost explosion. The change was made at first for one single product at one facility, than for all products at one facility and finally for all products at all facilities.

The model's behaviour is very much depending on the underlying cost structure. If operating costs are too high the solver will immediately close down the facilities. If the difference is not too big, the facility's capacity with respect to that certain product is shrunk.

2. Letting the variable cost explode 
A second modification was made by a change in the variable cost. The variable costs were changed over time and raised progressively. Again this modification was made at first for only one single product at one facility, second for all products at one facility and finally for all products at all facilities.

The first observation was that the solving time for the problem rose significantly. All other problems with $5 \times 5 \times 5 \times 5$ dimension could be solved within 2 minutes. The current problem took almost 40 minutes to be solved. In period one the first shrinkage is to be observed. All facilities are finally closed when the variable cost exceed the price.

3. Letting opening cost explode

A similar modification was made to the opening cost. The opening cost were set to the fivefold of the net profit made over all products made in that period. This modification was made for one, two and three periods at two facilities. After running the model it could be observed that despite of higher demand than capacity the facilities were not expanded. This is obvious as it is the objective of the model to maximize the profit over all time periods. If there are only some facilities assigned very high expansion cost, the demand is transferred to those facilities which have either not been open before or can be expanded for a price that is very much below. 


\subsection{Summary}

A single product model has been developed and extended. The first part of this chapter has demonstrated that the model provides reasonable results. It is necessary to extend the model in such a way that multiple products are taken into consideration. This extension makes the model much more complex. This complexity requires a technology for data management that covers larger data than excel can. Thus an oracle database has been used for further computations. Several tests were performed and have shown that it is not possible to solve the model with standard software for problem sizes that are expected in real world applications. A dynamic solving procedure could be a first try to get an approximate solution but it will neglect the advantages of the original model, taking multiple time periods into consideration. For models using fewer $(<5)$ time periods this approach could be appropriate. Different solution approaches have to be developed to get either a good approximate solution or better to find a way to get the optimal solution for bigger problems that will arise for retail companies, financial companies, rentals, logistic companies and so on. A heuristic based on a decomposition approach therefore will be developed in the next chapter and will be applied to the model. 


\section{CHAPTER 4}

\section{A DECOMPOSITION APPROACH}

\subsection{Introduction}

Trials to solve the model using LINGO have shown that it is too complex to be solved using standard software. The complexity increased when multiple products were introduced in the model. The dynamic solution approach has already shown that splitting up the problem into smaller sub problems is a realistic approach for gathering a solution, but will result in different solutions than a robust approach and likely not lead to optimal results. Thus another decomposition approach is developed. The original problem is decomposed into sub-problems such that each sub-problem is for a specific product. A disadvantage of this approach is that it solves each sub-problem in isolation and ignores the fact that the products compete for the same set of resources. However, it allows us to develop separate solutions - one for each product - that can be combined in a subsequent step to include resource and capacity constraints that impact each of the sub-problems. The following section depicts how the problem was decomposed and the technical implementation. Then the computational results are provided and the results are combined so as to provide a solution the original problem. 


\subsection{Decomposition and database realization}

The model was decomposed by products. This resulted in a sub model for each of the

products. The mathematical model looked like the original model, only the number of facilities was set to 1 (refer to 3.3 Model 2: Allowing multiple products and additional constraints). The following code fragment shows the modification for a $5 \times 5 \times 5 \times 5$ model.

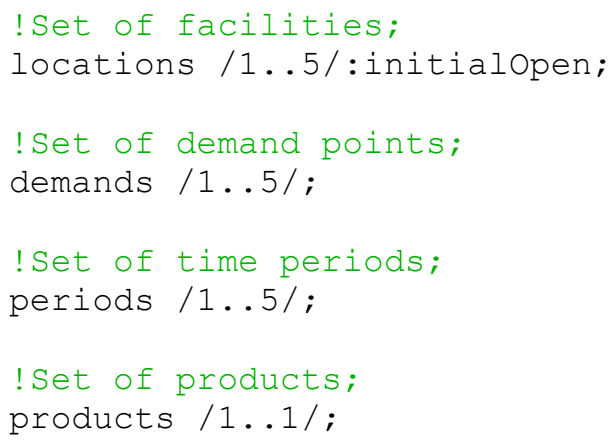

In order to be able to compare the results, the data used in Chapter 3 was used for the decomposed problem. For the sub problem data, database views were created. In order to be able to work on the correct data set, the original data was enriched by columns containing the indices of the relevant row. This made it possible to work on the correct order of the sub data sets, because first trials have shown that the creation of the views resulted in a different order of the data than expected. The following SQL statements show the view creation and the corresponding tables depict the data structure for the sub problems.

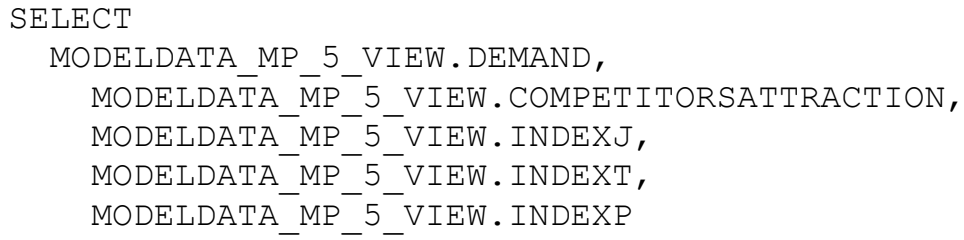




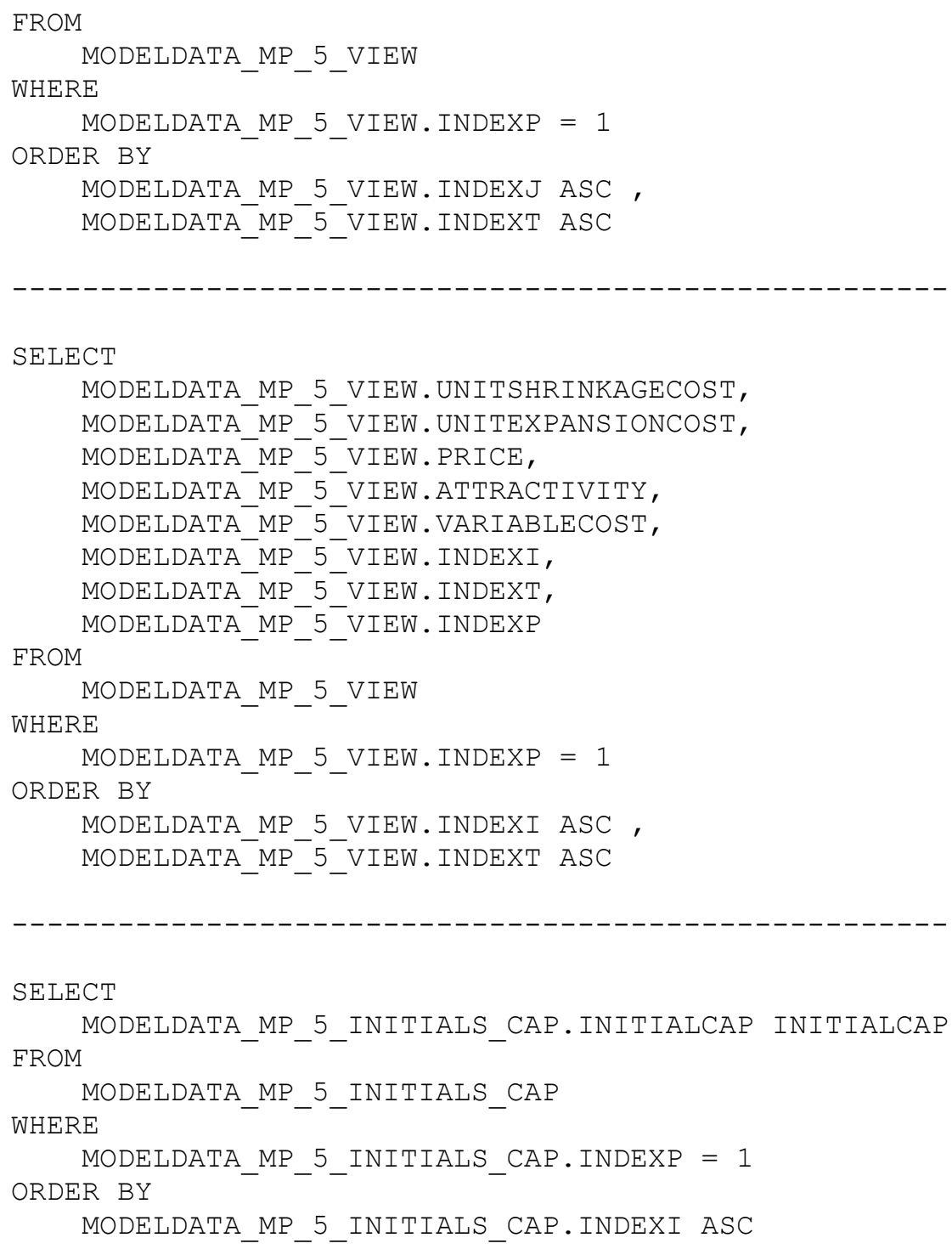

Table 13 Database view MP_5_DEMANDS_P01_VIEW

$\begin{array}{lll} & \text { COLUMN_NAME } & \text { DATA_TYPE } \\ 1 & \text { DEMAND } & \text { FLOAT(20) } \\ 2 & \text { COMPETITORSATTRACTION } & \text { FLOAT(20) } \\ 3 & \text { INDEXJ } & \text { NUMBER(3) } \\ 4 & \text { INDEXT } & \text { NUMBER(3) } \\ 5 & \text { INDEXP } & \text { NUMBER(3) }\end{array}$


Table 14 Database view MP_5_FACILITIES_P02_VIEW

1 UNITEXPANSIONCOST FLOAT(126)

2 PRICE FLOAT(20)

3 ATTRACTIVITY FLOAT(20)

4 VARIABLECOST FLOAT(20)

5 INDEXI

6 UNITSHRINKAGECOST FLOAT(126)

7 INDEXT NUMBER(3)

8 INDEXP NUMBER(3)

Table 15 Database view MP_5_INITIALS_CAP_P03_VIEW

$\begin{array}{ll}\text { MP_5_INITIALS_CAP_P01_VIEW } \\ 1 & \text { INITIALCAP } \\ \end{array}$

All statements and tables refer to the $5 \times 5 \times 5 \times 5$ problem. The data structure for problems of other sizes is identical. Table 12 contains the demands for the relevant product (product 1 in this case) for each period and the corresponding demand point. The table also contains the value for competitors' attraction levels. Table 13 contains all variables depending on the facility as unitexpansioncost, unitshrinkagecost, price, attractivity and variablecost. The last created view is Table 14. It contains the initial capacities for the product. 


\subsection{Computational results}

All models were solved separately using LINGO. The results for all variables were written in the database. The solution time for each sub problem differs. This difference is only recognizable for very large problems.

Table 16 Solving times for $5 \times 5 \times 5 \times 5$ sub problems in seconds

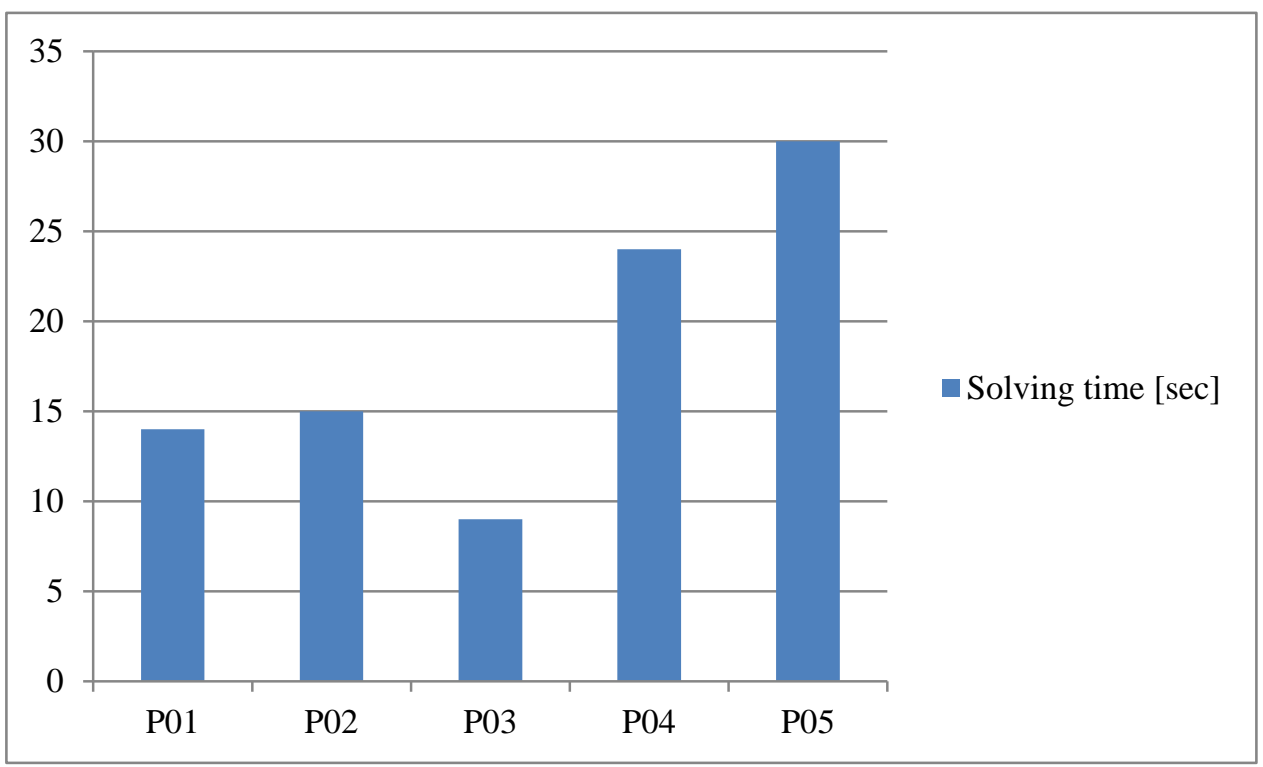

For each product, the value for open status was written into a database table. The corresponding indices can be found in the columns indexi and indext. A value of 0 means facility $i$ was closed in period $t$ in the corresponding sub model.

Table 17 shows the opening status for certain products for various time periods. 
Table 17 Results tableau for opening status of facilities depending on the sub problem

\begin{tabular}{|ccccc|cc|}
\hline P01 & P02 & P03 & P04 & P05 & indexi & indext \\
\hline 1 & 1 & 1 & 1 & 1 & 1 & 1 \\
0 & 1 & 1 & 1 & 1 & 1 & 2 \\
0 & 0 & 0 & 1 & 1 & 1 & 3 \\
0 & 0 & 0 & 0 & 0 & 1 & 4 \\
0 & 0 & 0 & 0 & 0 & 1 & 5 \\
1 & 1 & 1 & 1 & 1 & 2 & 1 \\
0 & 0 & 0 & 0 & 0 & 2 & 2 \\
0 & 1 & 0 & 0 & 0 & 2 & 3 \\
0 & 0 & 0 & 0 & 0 & 2 & 4 \\
0 & 0 & 0 & 0 & 0 & 2 & 5 \\
1 & 1 & 1 & 1 & 1 & 3 & 1 \\
1 & 0 & 0 & 0 & 0 & 3 & 2 \\
0 & 0 & 1 & 0 & 0 & 3 & 3 \\
0 & 0 & 0 & 0 & 0 & 3 & 4 \\
0 & 0 & 0 & 0 & 0 & 3 & 5 \\
1 & 1 & 1 & 1 & 1 & 4 & 1 \\
0 & 0 & 0 & 0 & 0 & 4 & 2 \\
0 & 0 & 0 & 0 & 0 & 4 & 3 \\
0 & 0 & 0 & 0 & 0 & 4 & 4 \\
0 & 0 & 0 & 0 & 0 & 4 & 5 \\
1 & 1 & 1 & 1 & 1 & 5 & 1 \\
1 & 0 & 0 & 0 & 0 & 5 & 2 \\
1 & 0 & 0 & 0 & 0 & 5 & 3 \\
0 & 0 & 0 & 0 & 0 & 5 & 4 \\
0 & 0 & 0 & 0 & 0 & 5 & 5 \\
\hline
\end{tabular}

\subsection{Combining different solutions}

After solving all the sub-problems separately, the results had to be combined and applied to the original problem. In order to get a better understanding of which procedure is best, different selection procedures were used. The selection strategies were tested on the models of size $5 \times 5 \times 5 \times 5$ and $10 \times 10 \times 10 \times 10$. The first strategy selects all facilities where the status is open for all sub problems with respect to a certain period. Selecting all 
closed facilities was not included. The following strategies select those facilities where at least some number of sub-problems requires the facility to be open in a certain period

The relevant information was added as constraint to the original model and the model was solved. The results were compared with the original problem. The following flow chart shows the general procedure of the decomposition algorithm. 


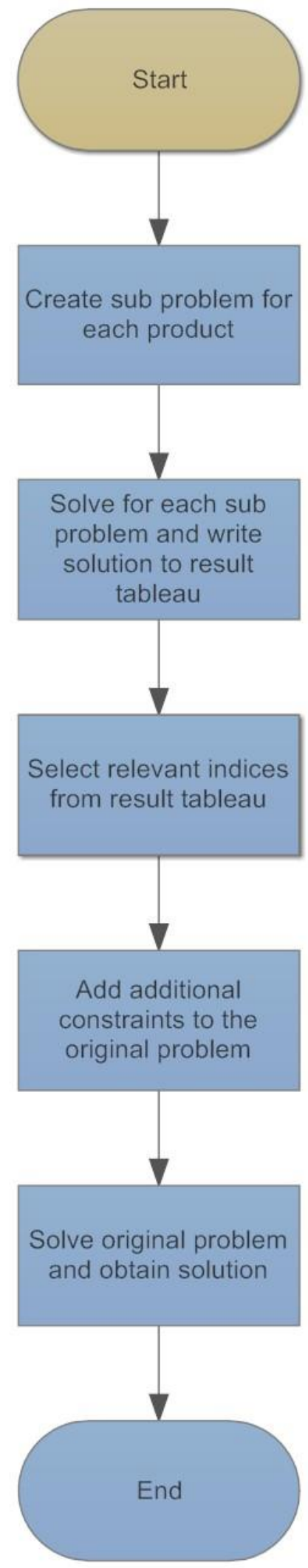

Figure 5 Procedure of decomposition algorithm 


\subsubsection{Selection strategy 1: Select if open for all products}

First, the facilities that were open for all the sub-problems were selected. Facilities that were closed in all the sub-problems were not considered in the first run. The lines corresponding to the open facilities are marked in Table 18.

Table 18 Result tableau for sub problems with five products

\begin{tabular}{|ccccc|cc|}
\hline P01 & P02 & P03 & P04 & P05 & indexi & indext \\
\hline 1 & 1 & 1 & 1 & 1 & 1 & 1 \\
0 & 1 & 1 & 1 & 1 & 1 & 2 \\
0 & 0 & 0 & 1 & 1 & 1 & 3 \\
0 & 0 & 0 & 0 & 0 & 1 & 4 \\
0 & 0 & 0 & 0 & 0 & 1 & 5 \\
1 & 1 & 1 & 1 & 1 & 2 & 1 \\
0 & 0 & 0 & 0 & 0 & 2 & 2 \\
0 & 1 & 0 & 0 & 0 & 2 & 3 \\
0 & 0 & 0 & 0 & 0 & 2 & 4 \\
0 & 0 & 0 & 0 & 0 & 2 & 5 \\
1 & 1 & 1 & 1 & 1 & 3 & 1 \\
1 & 0 & 0 & 0 & 0 & 3 & 2 \\
0 & 0 & 1 & 0 & 0 & 3 & 3 \\
0 & 0 & 0 & 0 & 0 & 3 & 4 \\
0 & 0 & 0 & 0 & 0 & 3 & 5 \\
1 & 1 & 1 & 1 & 1 & 4 & 1 \\
0 & 0 & 0 & 0 & 0 & 4 & 2 \\
0 & 0 & 0 & 0 & 0 & 4 & 3 \\
0 & 0 & 0 & 0 & 0 & 4 & 4 \\
0 & 0 & 0 & 0 & 0 & 4 & 5 \\
1 & 1 & 1 & 1 & 1 & 5 & 1 \\
1 & 0 & 0 & 0 & 0 & 5 & 2 \\
1 & 0 & 0 & 0 & 0 & 5 & 3 \\
0 & 0 & 0 & 0 & 0 & 5 & 4 \\
0 & 0 & 0 & 0 & 0 & 5 & 5 \\
\hline
\end{tabular}

Because only the lines containing " 1 " as entries are initialized with theses values, there was no need to add constraints to the model. 
Another run was made by selecting all the lines where the open status for all products equals zero with respect to a facility and period. The corresponding lines are marked in blue.

Table 19 Selection of all lines with open status equals 0

\begin{tabular}{|ccccc|cc|}
\hline P01 & P02 & P03 & P04 & P05 & indexi & indext \\
1 & 1 & 1 & 1 & 1 & 1 & 1 \\
0 & 1 & 1 & 1 & 1 & 1 & 2 \\
0 & 0 & 0 & 1 & 1 & 1 & 3 \\
0 & 0 & 0 & 0 & 0 & 1 & 4 \\
0 & 0 & 0 & 0 & 0 & 1 & 5 \\
1 & 1 & 1 & 1 & 1 & 2 & 1 \\
0 & 0 & 0 & 0 & 0 & 2 & 2 \\
0 & 1 & 0 & 0 & 0 & 2 & 3 \\
0 & 0 & 0 & 0 & 0 & 2 & 4 \\
0 & 0 & 0 & 0 & 0 & 2 & 5 \\
1 & 1 & 1 & 1 & 1 & 3 & 1 \\
1 & 0 & 0 & 0 & 0 & 3 & 2 \\
0 & 0 & 1 & 0 & 0 & 3 & 3 \\
0 & 0 & 0 & 0 & 0 & 3 & 4 \\
0 & 0 & 0 & 0 & 0 & 3 & 5 \\
1 & 1 & 1 & 1 & 1 & 4 & 1 \\
0 & 0 & 0 & 0 & 0 & 4 & 2 \\
0 & 0 & 0 & 0 & 0 & 4 & 3 \\
0 & 0 & 0 & 0 & 0 & 4 & 4 \\
0 & 0 & 0 & 0 & 0 & 4 & 5 \\
1 & 1 & 1 & 1 & 1 & 5 & 1 \\
1 & 0 & 0 & 0 & 0 & 5 & 2 \\
1 & 0 & 0 & 0 & 0 & 5 & 3 \\
0 & 0 & 0 & 0 & 0 & 5 & 4 \\
0 & 0 & 0 & 0 & 0 & 5 & 5 \\
\hline
\end{tabular}

This selection resulted in the addition of the following constraints to the model.

open $(1,4)=0 ;$
open $(1,5)=0 ;$
open $(2,2)=0 ;$ 


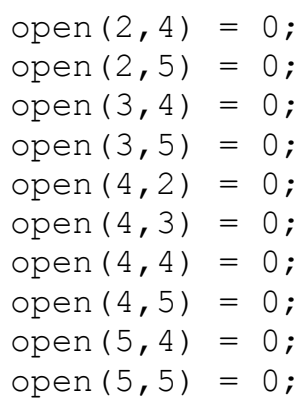

The model was solved and a solution could be obtained. Because the $5 \times 5 \times 5 \times 5$ problem is small enough to be solved optimally for the original (non-decomposed) model, we were able to compare the OFV of the suboptimal decomposition approach with that of the optimal solution.

Table 20 Objective function value for $5 \times 5 \times 5 \times 5$ model using different selection strategies

\begin{tabular}{|c|c|c|}
\hline Selection strategy & 1 & 0 \\
\hline Original model & \multicolumn{2}{|c|}{ 2.95213E08 } \\
\hline All values & Not available & 2.95213E08 \\
\hline At least one & 2.95044E08 & $1.82156 \mathrm{E} 08$ \\
\hline At least two & 2.95213E08 & $2.69256 \mathrm{E} 08$ \\
\hline At least three & Not available & $2.69256 \mathrm{E} 08$ \\
\hline At least four & Not available & $2.81741 \mathrm{E} 08$ \\
\hline
\end{tabular}

The decomposition procedure was also applied to the model size of 10x10x10x10. The 
complexity for the data structure grew, but the sub problems could be solved quickly. The solution times for each of the different sub problems were less than 35 seconds.

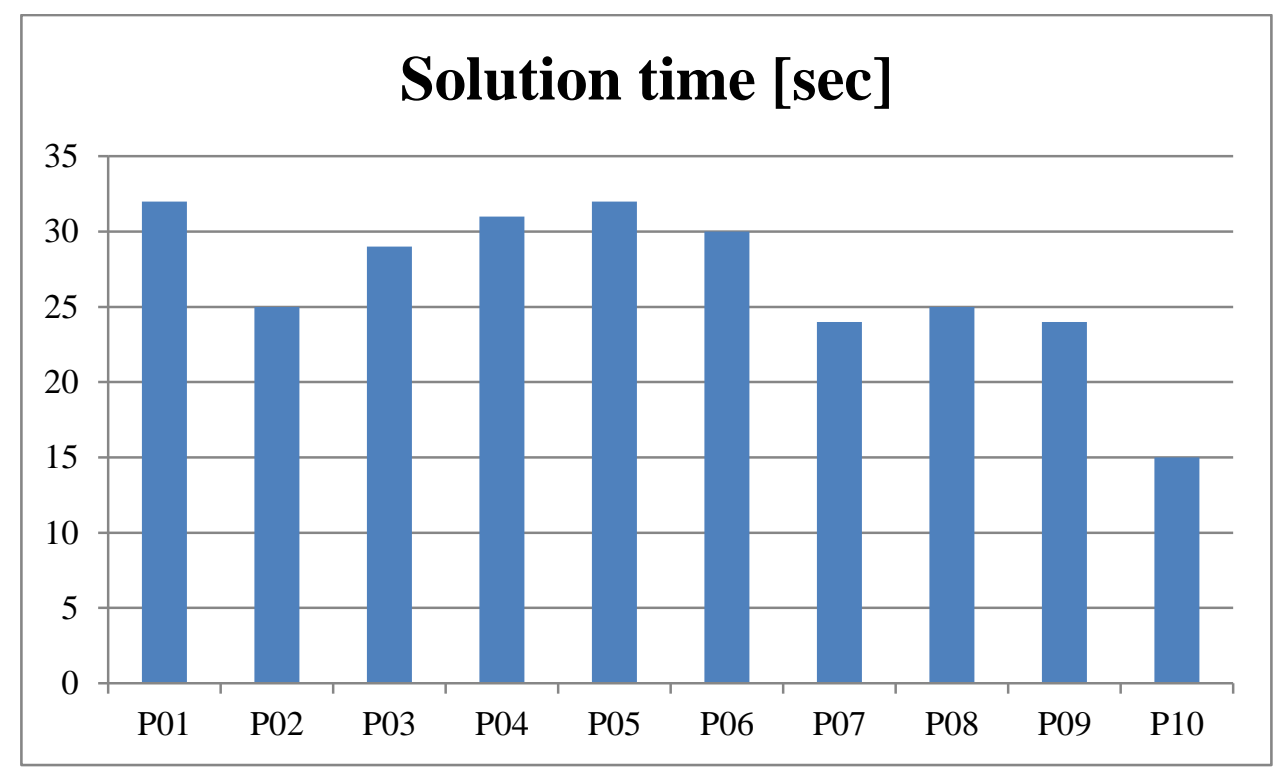

Figure 6 Solution times for 10x10x10x10 sub problems (in seconds)

Table 21 Results tableau for 10x10x10x10 model and its sub problems

\begin{tabular}{|cccccccccc|cc|}
\hline P01 & P02 & P03 & P04 & P05 & P06 & P07 & P08 & P09 & P10 & indexi & indext \\
\hline 1 & 1 & 1 & 1 & 1 & 1 & 1 & 1 & 1 & 1 & 1 & 1 \\
1 & 1 & 1 & 1 & 1 & 0 & 0 & 0 & 0 & 0 & 1 & 2 \\
1 & 0 & 1 & 1 & 1 & 0 & 0 & 0 & 0 & 0 & 1 & 3 \\
0 & 0 & 0 & 0 & 0 & 0 & 0 & 0 & 0 & 0 & 1 & 4 \\
0 & 0 & 0 & 0 & 0 & 0 & 0 & 0 & 0 & 0 & 1 & 5 \\
0 & 0 & 0 & 0 & 0 & 0 & 0 & 0 & 0 & 0 & 1 & 6 \\
0 & 0 & 0 & 0 & 0 & 0 & 0 & 0 & 0 & 0 & 1 & 7 \\
0 & 0 & 0 & 0 & 0 & 0 & 0 & 1 & 0 & 0 & 1 & 8 \\
0 & 0 & 0 & 0 & 0 & 0 & 0 & 0 & 0 & 0 & 1 & 9 \\
0 & 0 & 0 & 0 & 0 & 0 & 0 & 0 & 0 & 0 & 1 & 10 \\
1 & 1 & 1 & 1 & 1 & 1 & 1 & 1 & 1 & 1 & 2 & 1 \\
0 & 0 & 0 & 0 & 1 & 0 & 0 & 0 & 0 & 0 & 2 & 2 \\
0 & 1 & 1 & 1 & 1 & 0 & 0 & 0 & 0 & 0 & 2 & 3
\end{tabular}




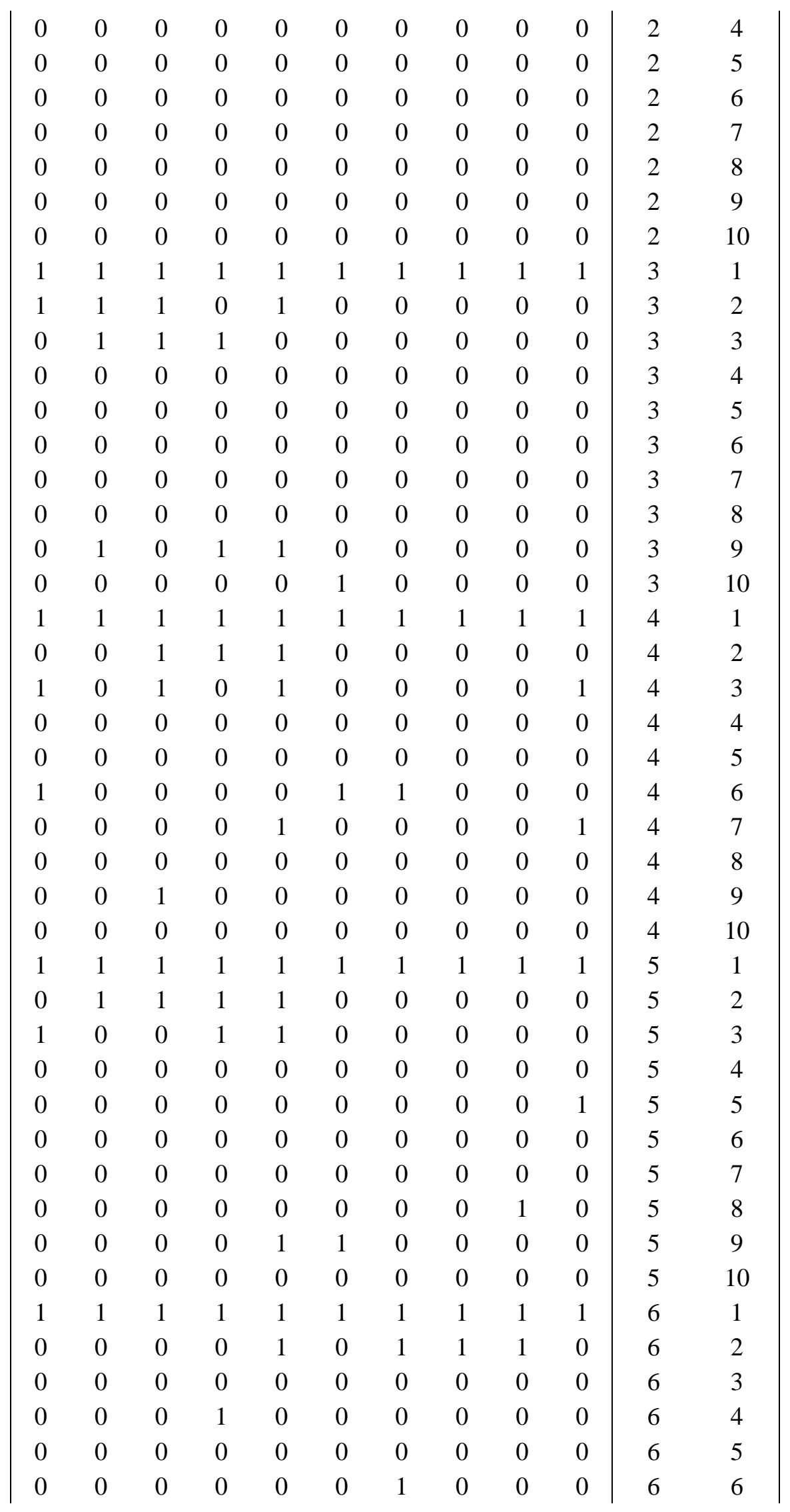




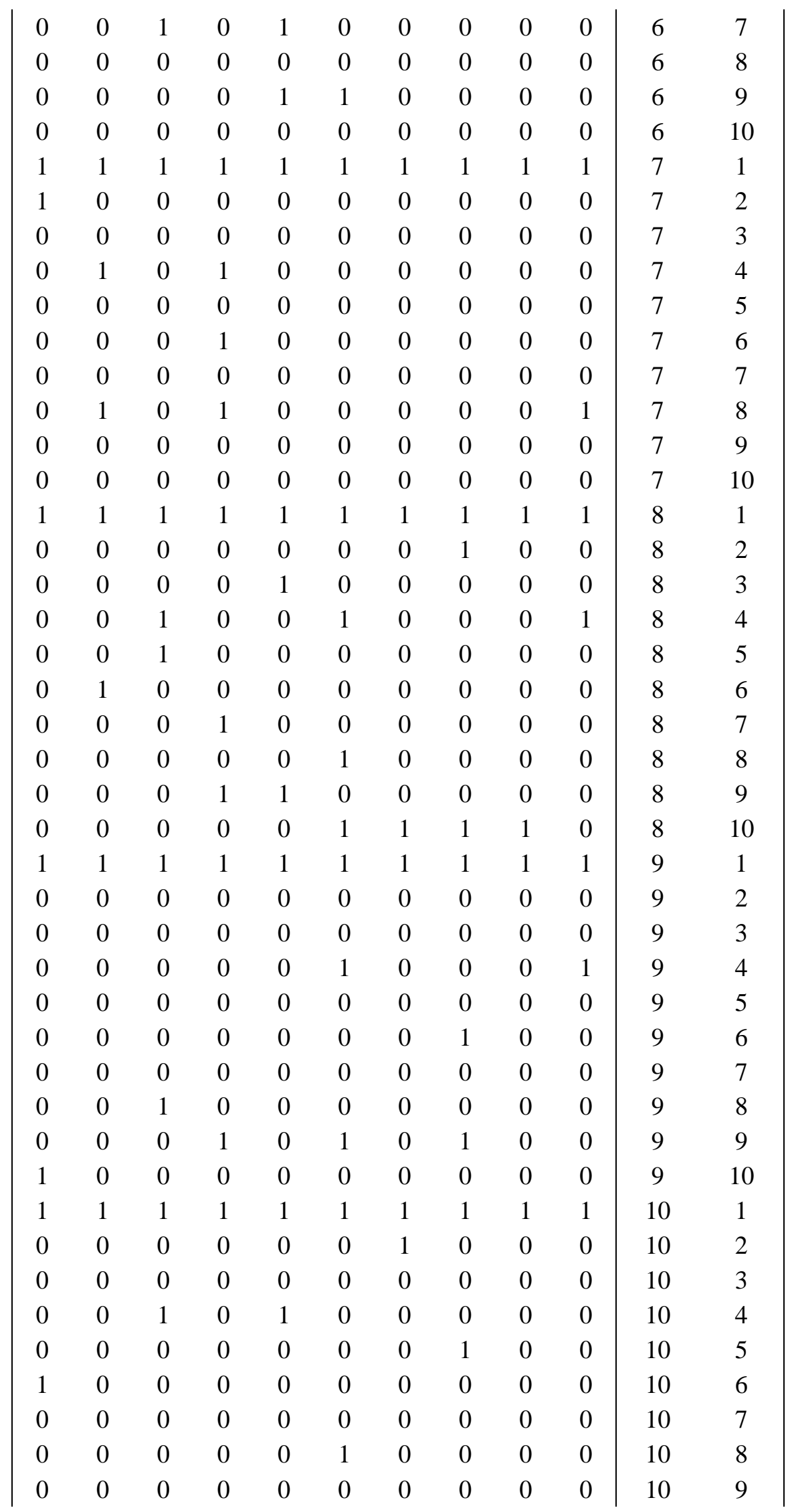




\begin{tabular}{|llllllllll|ll}
0 & 0 & 0 & 0 & 0 & 0 & 0 & 0 & 0 & 0 & 10 & 10 \\
\hline
\end{tabular}

A selection of lines where all sub problems show an open status returned an empty result.

Though there are 10 lines with all 1's, these values result from initialization and are already set. So the selection of all lines with only zeros was made and the following constraints were added to the model.

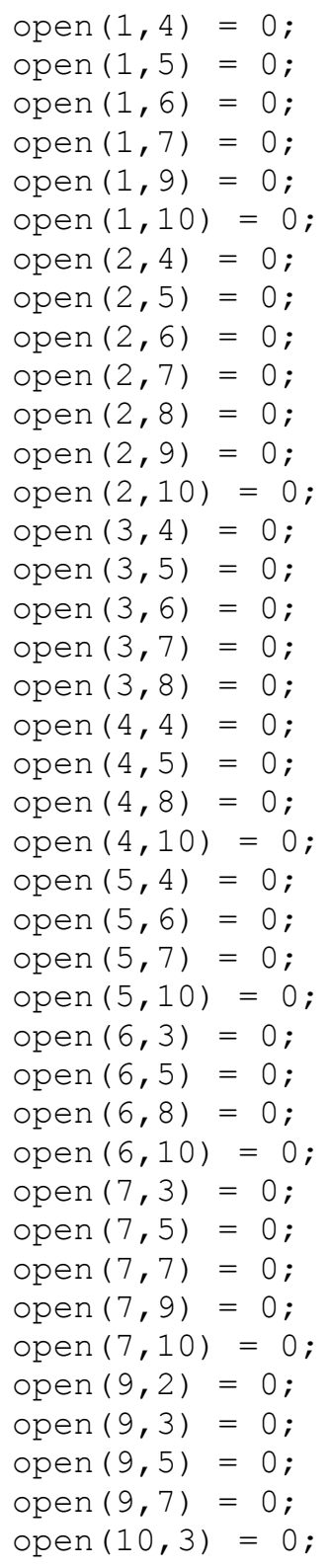


open $(10,7)=0$;

open $(10,9)=0 ;$

open $(10,10)=0$;

The complexity of the model was reduced by 43 variables (30,247 variables) but a solution could be obtained quickly. Comparing the original problem solution's objective function value to the solution including the result of the sub problems, the objective function value is the same as value from the original problem (2.61799E8).

Three more selection strategies were tested.

\subsubsection{Selection where at least three facilities are open / closed}

In real applications one might not want to solve all subproblems. Thus, the next selection was made when at least three facilities were declared to be open (grey shadowed) or closed (in italics) as shown in Table 22.

Table 22 Results tableau with at least three facilities open for 10x10x10x10 model

\begin{tabular}{|ccccc|ccccc|cc|}
\hline P01 & P02 & P03 & P04 & P05 & P06 & P07 & P08 & P09 & P10 & indexi & indext \\
\hline 1 & 1 & 1 & 1 & 1 & 1 & 1 & 1 & 1 & 1 & 1 & 1 \\
1 & 1 & 1 & 1 & 1 & 0 & 0 & 0 & 0 & 0 & 1 & 2 \\
1 & 0 & 1 & 1 & 1 & 0 & 0 & 0 & 0 & 0 & 1 & 3 \\
0 & 0 & 0 & 0 & 0 & 0 & 0 & 0 & 0 & 0 & 1 & 4 \\
0 & 0 & 0 & 0 & 0 & 0 & 0 & 0 & 0 & 0 & 1 & 5 \\
0 & 0 & 0 & 0 & 0 & 0 & 0 & 0 & 0 & 0 & 1 & 6 \\
0 & 0 & 0 & 0 & 0 & 0 & 0 & 0 & 0 & 0 & 1 & 7 \\
0 & 0 & 0 & 0 & 0 & 0 & 0 & 1 & 0 & 0 & 1 & 8 \\
0 & 0 & 0 & 0 & 0 & 0 & 0 & 0 & 0 & 0 & 1 & 9 \\
0 & 0 & 0 & 0 & 0 & 0 & 0 & 0 & 0 & 0 & 1 & 10 \\
1 & 1 & 1 & 1 & 1 & 1 & 1 & 1 & 1 & 1 & 2 & 1 \\
0 & 0 & 0 & 0 & 1 & 0 & 0 & 0 & 0 & 0 & 2 & 2 \\
0 & 1 & 1 & 1 & 1 & 0 & 0 & 0 & 0 & 0 & 2 & 3 \\
0 & 0 & 0 & 0 & 0 & 0 & 0 & 0 & 0 & 0 & 2 & 4
\end{tabular}




\begin{tabular}{|c|c|c|c|c|c|c|c|c|c|c|c|}
\hline 0 & 0 & 0 & 0 & 0 & 0 & 0 & 0 & 0 & 0 & 2 & 5 \\
\hline 0 & 0 & 0 & 0 & 0 & 0 & 0 & 0 & 0 & 0 & 2 & 6 \\
\hline 0 & 0 & 0 & 0 & 0 & 0 & 0 & 0 & 0 & 0 & 2 & 7 \\
\hline 0 & 0 & 0 & 0 & 0 & 0 & 0 & 0 & 0 & 0 & 2 & 8 \\
\hline 0 & 0 & 0 & 0 & 0 & 0 & 0 & 0 & 0 & 0 & 2 & 9 \\
\hline 0 & 0 & 0 & 0 & 0 & 0 & 0 & 0 & 0 & 0 & 2 & 10 \\
\hline 1 & 1 & 1 & 1 & 1 & 1 & 1 & 1 & 1 & 1 & 3 & 1 \\
\hline 1 & 1 & 1 & 0 & 1 & 0 & 0 & 0 & 0 & 0 & 3 & 2 \\
\hline 0 & 1 & 1 & 1 & 0 & 0 & 0 & 0 & 0 & 0 & 3 & 3 \\
\hline 0 & 0 & 0 & 0 & 0 & 0 & 0 & 0 & 0 & 0 & 3 & 4 \\
\hline 0 & 0 & 0 & 0 & 0 & 0 & 0 & 0 & 0 & 0 & 3 & 5 \\
\hline 0 & 0 & 0 & 0 & 0 & 0 & 0 & 0 & 0 & 0 & 3 & 6 \\
\hline 0 & 0 & 0 & 0 & 0 & 0 & 0 & 0 & 0 & 0 & 3 & 7 \\
\hline 0 & 0 & 0 & 0 & 0 & 0 & 0 & 0 & 0 & 0 & 3 & 8 \\
\hline 0 & 1 & 0 & 1 & 1 & 0 & 0 & 0 & 0 & 0 & 3 & 9 \\
\hline 0 & 0 & 0 & 0 & 0 & 1 & 0 & 0 & 0 & 0 & 3 & 10 \\
\hline 1 & 1 & 1 & 1 & 1 & 1 & 1 & 1 & 1 & 1 & 4 & 1 \\
\hline 0 & 0 & 1 & 1 & 1 & 0 & 0 & 0 & 0 & 0 & 4 & 2 \\
\hline 1 & 0 & 1 & 0 & 1 & 0 & 0 & 0 & 0 & 1 & 4 & 3 \\
\hline 0 & 0 & 0 & 0 & 0 & 0 & 0 & 0 & 0 & 0 & 4 & 4 \\
\hline 0 & 0 & 0 & 0 & 0 & 0 & 0 & 0 & 0 & 0 & 4 & 5 \\
\hline 1 & 0 & 0 & 0 & 0 & 1 & 1 & 0 & 0 & 0 & 4 & 6 \\
\hline 0 & 0 & 0 & 0 & 1 & 0 & 0 & 0 & 0 & 1 & 4 & 7 \\
\hline 0 & 0 & 0 & 0 & 0 & 0 & 0 & 0 & 0 & 0 & 4 & 8 \\
\hline 0 & 0 & 1 & 0 & 0 & 0 & 0 & 0 & 0 & 0 & 4 & 9 \\
\hline 0 & 0 & 0 & 0 & 0 & 0 & 0 & 0 & 0 & 0 & 4 & 10 \\
\hline 1 & 1 & 1 & 1 & 1 & 1 & 1 & 1 & 1 & 1 & 5 & 1 \\
\hline 0 & 1 & 1 & 1 & 1 & 0 & 0 & 0 & 0 & 0 & 5 & 2 \\
\hline 1 & 0 & 0 & 1 & 1 & 0 & 0 & 0 & 0 & 0 & 5 & 3 \\
\hline 0 & 0 & 0 & 0 & 0 & 0 & 0 & 0 & 0 & 0 & 5 & 4 \\
\hline 0 & 0 & 0 & 0 & 0 & 0 & 0 & 0 & 0 & 1 & 5 & 5 \\
\hline 0 & 0 & 0 & 0 & 0 & 0 & 0 & 0 & 0 & 0 & 5 & 6 \\
\hline 0 & 0 & 0 & 0 & 0 & 0 & 0 & 0 & 0 & 0 & 5 & 7 \\
\hline 0 & 0 & 0 & 0 & 0 & 0 & 0 & 0 & 1 & 0 & 5 & 8 \\
\hline 0 & 0 & 0 & 0 & 1 & 1 & 0 & 0 & 0 & 0 & 5 & 9 \\
\hline 0 & 0 & 0 & 0 & 0 & 0 & 0 & 0 & 0 & 0 & 5 & 10 \\
\hline 1 & 1 & 1 & 1 & 1 & 1 & 1 & 1 & 1 & 1 & 6 & 1 \\
\hline 0 & 0 & 0 & 0 & 1 & 0 & 1 & 1 & 1 & 0 & 6 & 2 \\
\hline 0 & 0 & 0 & 0 & 0 & 0 & 0 & 0 & 0 & 0 & 6 & 3 \\
\hline 0 & 0 & 0 & 1 & 0 & 0 & 0 & 0 & 0 & 0 & 6 & 4 \\
\hline 0 & 0 & 0 & 0 & 0 & 0 & 0 & 0 & 0 & 0 & 6 & 5 \\
\hline 0 & 0 & 0 & 0 & 0 & 0 & 1 & 0 & 0 & 0 & 6 & 6 \\
\hline 0 & 0 & 1 & 0 & 1 & 0 & 0 & 0 & 0 & 0 & 6 & 7 \\
\hline 0 & 0 & 0 & 0 & 0 & 0 & 0 & 0 & 0 & 0 & 6 & 8 \\
\hline 0 & 0 & 0 & 0 & 1 & 1 & 0 & 0 & 0 & 0 & 6 & 9 \\
\hline
\end{tabular}




\begin{tabular}{|c|c|c|c|c|c|c|c|c|c|c|c|}
\hline 0 & 0 & 0 & 0 & 0 & 0 & 0 & 0 & 0 & 0 & 6 & 10 \\
\hline 1 & 1 & 1 & 1 & 1 & 1 & 1 & 1 & 1 & 1 & 7 & 1 \\
\hline 1 & 0 & 0 & 0 & 0 & 0 & 0 & 0 & 0 & 0 & 7 & 2 \\
\hline 0 & 0 & 0 & 0 & 0 & 0 & 0 & 0 & 0 & 0 & 7 & 3 \\
\hline 0 & 1 & 0 & 1 & 0 & 0 & 0 & 0 & 0 & 0 & 7 & 4 \\
\hline 0 & 0 & 0 & 0 & 0 & 0 & 0 & 0 & 0 & 0 & 7 & 5 \\
\hline 0 & 0 & 0 & 1 & 0 & 0 & 0 & 0 & 0 & 0 & 7 & 6 \\
\hline 0 & 0 & 0 & 0 & 0 & 0 & 0 & 0 & 0 & 0 & 7 & 7 \\
\hline 0 & 1 & 0 & 1 & 0 & 0 & 0 & 0 & 0 & 1 & 7 & 8 \\
\hline 0 & 0 & 0 & 0 & 0 & 0 & 0 & 0 & 0 & 0 & 7 & 9 \\
\hline 0 & 0 & 0 & 0 & 0 & 0 & 0 & 0 & 0 & 0 & 7 & 10 \\
\hline 1 & 1 & 1 & 1 & 1 & 1 & 1 & 1 & 1 & 1 & 8 & 1 \\
\hline 0 & 0 & 0 & 0 & 0 & 0 & 0 & 1 & 0 & 0 & 8 & 2 \\
\hline 0 & 0 & 0 & 0 & 1 & 0 & 0 & 0 & 0 & 0 & 8 & 3 \\
\hline 0 & 0 & 1 & 0 & 0 & 1 & 0 & 0 & 0 & 1 & 8 & 4 \\
\hline 0 & 0 & 1 & 0 & 0 & 0 & 0 & 0 & 0 & 0 & 8 & 5 \\
\hline 0 & 1 & 0 & 0 & 0 & 0 & 0 & 0 & 0 & 0 & 8 & 6 \\
\hline 0 & 0 & 0 & 1 & 0 & 0 & 0 & 0 & 0 & 0 & 8 & 7 \\
\hline 0 & 0 & 0 & 0 & 0 & 1 & 0 & 0 & 0 & 0 & 8 & 8 \\
\hline 0 & 0 & 0 & 1 & 1 & 0 & 0 & 0 & 0 & 0 & 8 & 9 \\
\hline 0 & 0 & 0 & 0 & 0 & 1 & 1 & 1 & 1 & 0 & 8 & 10 \\
\hline 1 & 1 & 1 & 1 & 1 & 1 & 1 & 1 & 1 & 1 & 9 & 1 \\
\hline 0 & 0 & 0 & 0 & 0 & 0 & 0 & 0 & 0 & 0 & 9 & 2 \\
\hline 0 & 0 & 0 & 0 & 0 & 0 & 0 & 0 & 0 & 0 & 9 & 3 \\
\hline 0 & 0 & 0 & 0 & 0 & 1 & 0 & 0 & 0 & 1 & 9 & 4 \\
\hline 0 & 0 & 0 & 0 & 0 & 0 & 0 & 0 & 0 & 0 & 9 & 5 \\
\hline 0 & 0 & 0 & 0 & 0 & 0 & 0 & 1 & 0 & 0 & 9 & 6 \\
\hline 0 & 0 & 0 & 0 & 0 & 0 & 0 & 0 & 0 & 0 & 9 & 7 \\
\hline 0 & 0 & 1 & 0 & 0 & 0 & 0 & 0 & 0 & 0 & 9 & 8 \\
\hline 0 & 0 & 0 & 1 & 0 & 1 & 0 & 1 & 0 & 0 & 9 & 9 \\
\hline 1 & 0 & 0 & 0 & 0 & 0 & 0 & 0 & 0 & 0 & 9 & 10 \\
\hline 1 & 1 & 1 & 1 & 1 & 1 & 1 & 1 & 1 & 1 & 10 & 1 \\
\hline 0 & 0 & 0 & 0 & 0 & 0 & 1 & 0 & 0 & 0 & 10 & 2 \\
\hline 0 & 0 & 0 & 0 & 0 & 0 & 0 & 0 & 0 & 0 & 10 & 3 \\
\hline 0 & 0 & 1 & 0 & 1 & 0 & 0 & 0 & 0 & 0 & 10 & 4 \\
\hline 0 & 0 & 0 & 0 & 0 & 0 & 0 & 1 & 0 & 0 & 10 & 5 \\
\hline 1 & 0 & 0 & 0 & 0 & 0 & 0 & 0 & 0 & 0 & 10 & 6 \\
\hline 0 & 0 & 0 & 0 & 0 & 0 & 0 & 0 & 0 & 0 & 10 & 7 \\
\hline 0 & 0 & 0 & 0 & 0 & 1 & 0 & 0 & 0 & 0 & 10 & 8 \\
\hline 0 & 0 & 0 & 0 & 0 & 0 & 0 & 0 & 0 & 0 & 10 & 9 \\
\hline 0 & 0 & 0 & 0 & 0 & 0 & 0 & 0 & 0 & 0 & 10 & 10 \\
\hline
\end{tabular}

The following constraints were added to the model with at least three facilities open. 


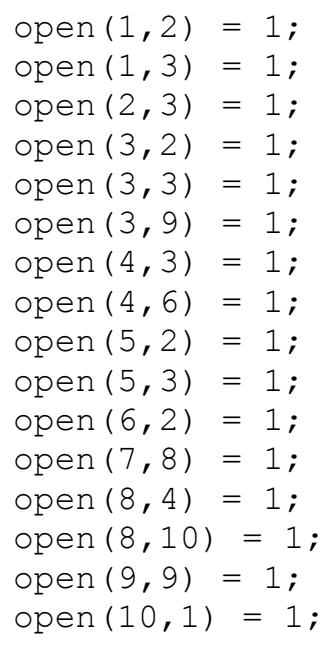

The objective function value did not change (2.61799E08).

Adding constraints for closed facilities resulted in closing down all facilities after the first (initial) period.

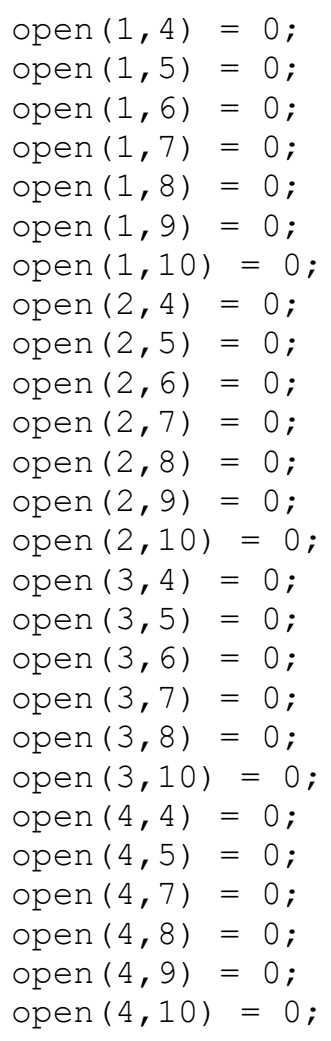




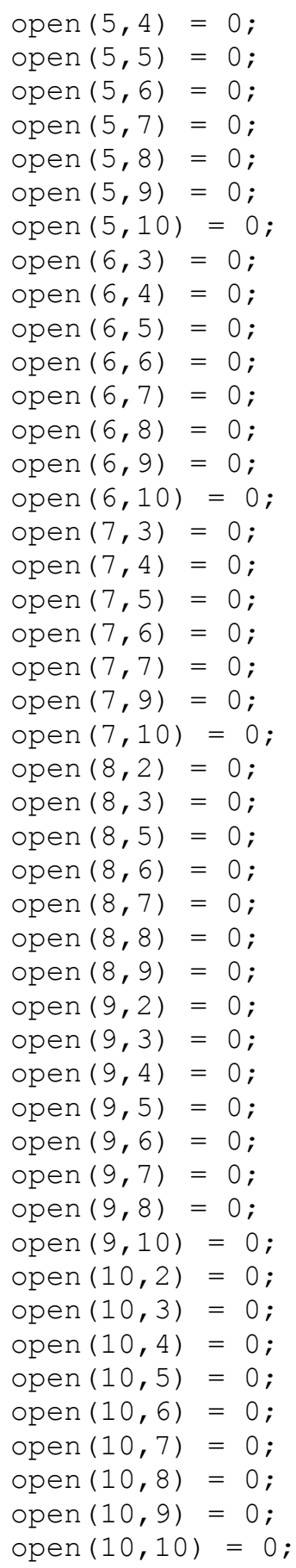

The model was solved and the objective function value fell down rapidly (1.5499*1E08).

\subsubsection{Selection where at least five facilities are open / closed}

The same procedure was made selecting where at least five facilities were open or closed. 
The following constraint was added to the model

$\operatorname{open}(1,2)=1 ;$

The objective function value was the same as for the original problem. Another run for 0line was not made because the relevant lines are exactly the same as for the selection strategy "at least three".

\subsubsection{Selection where at least eight facilities are open /closed}

This selection was not possible with the data, as there is no row with at least eight " 1 " entries. Selecting the lines with at least eight "0" entries yields the result shown in Table 23.

Table 23 Results tableau with at least eight facilities open for 10x10x10x10 model

\begin{tabular}{|ccccc|ccccc|cc|}
\hline P01 & P02 & P03 & P04 & P05 & P06 & P07 & P08 & P09 & P10 & indexi & indext \\
\hline 1 & 1 & 1 & 1 & 1 & 1 & 1 & 1 & 1 & 1 & 1 & 1 \\
1 & 1 & 1 & 1 & 1 & 0 & 0 & 0 & 0 & 0 & 1 & 2 \\
1 & 0 & 1 & 1 & 1 & 0 & 0 & 0 & 0 & 0 & 1 & 3 \\
0 & 0 & 0 & 0 & 0 & 0 & 0 & 0 & 0 & 0 & 1 & 4 \\
0 & 0 & 0 & 0 & 0 & 0 & 0 & 0 & 0 & 0 & 1 & 5 \\
0 & 0 & 0 & 0 & 0 & 0 & 0 & 0 & 0 & 0 & 1 & 6 \\
0 & 0 & 0 & 0 & 0 & 0 & 0 & 0 & 0 & 0 & 1 & 7 \\
0 & 0 & 0 & 0 & 0 & 0 & 0 & 1 & 0 & 0 & 1 & 8 \\
0 & 0 & 0 & 0 & 0 & 0 & 0 & 0 & 0 & 0 & 1 & 9 \\
0 & 0 & 0 & 0 & 0 & 0 & 0 & 0 & 0 & 0 & 1 & 10 \\
1 & 1 & 1 & 1 & 1 & 1 & 1 & 1 & 1 & 1 & 2 & 1 \\
0 & 0 & 0 & 0 & 1 & 0 & 0 & 0 & 0 & 0 & 2 & 2 \\
0 & 1 & 1 & 1 & 1 & 0 & 0 & 0 & 0 & 0 & 2 & 3 \\
0 & 0 & 0 & 0 & 0 & 0 & 0 & 0 & 0 & 0 & 2 & 4 \\
0 & 0 & 0 & 0 & 0 & 0 & 0 & 0 & 0 & 0 & 2 & 5 \\
0 & 0 & 0 & 0 & 0 & 0 & 0 & 0 & 0 & 0 & 2 & 6 \\
0 & 0 & 0 & 0 & 0 & 0 & 0 & 0 & 0 & 0 & 2 & 7 \\
0 & 0 & 0 & 0 & 0 & 0 & 0 & 0 & 0 & 0 & 2 & 8
\end{tabular}




\begin{tabular}{|c|c|c|c|c|c|c|c|c|c|c|c|}
\hline 0 & 0 & 0 & 0 & 0 & 0 & 0 & 0 & 0 & 0 & 2 & 9 \\
\hline 0 & 0 & 0 & 0 & 0 & 0 & 0 & 0 & 0 & 0 & 2 & 10 \\
\hline 1 & 1 & 1 & 1 & 1 & 1 & 1 & 1 & 1 & 1 & 3 & 1 \\
\hline 1 & 1 & 1 & 0 & 1 & 0 & 0 & 0 & 0 & 0 & 3 & 2 \\
\hline 0 & 1 & 1 & 1 & 0 & 0 & 0 & 0 & 0 & 0 & 3 & 3 \\
\hline 0 & 0 & 0 & 0 & 0 & 0 & 0 & 0 & 0 & 0 & 3 & 4 \\
\hline 0 & 0 & 0 & 0 & 0 & 0 & 0 & 0 & 0 & 0 & 3 & 5 \\
\hline 0 & 0 & 0 & 0 & 0 & 0 & 0 & 0 & 0 & 0 & 3 & 6 \\
\hline 0 & 0 & 0 & 0 & 0 & 0 & 0 & 0 & 0 & 0 & 3 & 7 \\
\hline 0 & 0 & 0 & 0 & 0 & 0 & 0 & 0 & 0 & 0 & 3 & 8 \\
\hline 0 & 1 & 0 & 1 & 1 & 0 & 0 & 0 & 0 & 0 & 3 & 9 \\
\hline 0 & 0 & 0 & 0 & 0 & 1 & 0 & 0 & 0 & 0 & 3 & 10 \\
\hline 1 & 1 & 1 & 1 & 1 & 1 & 1 & 1 & 1 & 1 & 4 & 1 \\
\hline 0 & 0 & 1 & 1 & 1 & 0 & 0 & 0 & 0 & 0 & 4 & 2 \\
\hline 1 & 0 & 1 & 0 & 1 & 0 & 0 & 0 & 0 & 1 & 4 & 3 \\
\hline 0 & 0 & 0 & 0 & 0 & 0 & 0 & 0 & 0 & 0 & 4 & 4 \\
\hline 0 & 0 & 0 & 0 & 0 & 0 & 0 & 0 & 0 & 0 & 4 & 5 \\
\hline 1 & 0 & 0 & 0 & 0 & 1 & 1 & 0 & 0 & 0 & 4 & 6 \\
\hline 0 & 0 & 0 & 0 & 1 & 0 & 0 & 0 & 0 & 1 & 4 & 7 \\
\hline 0 & 0 & 0 & 0 & 0 & 0 & 0 & 0 & 0 & 0 & 4 & 8 \\
\hline 0 & 0 & 1 & 0 & 0 & 0 & 0 & 0 & 0 & 0 & 4 & 9 \\
\hline 0 & 0 & 0 & 0 & 0 & 0 & 0 & 0 & 0 & 0 & 4 & 10 \\
\hline 1 & 1 & 1 & 1 & 1 & 1 & 1 & 1 & 1 & 1 & 5 & 1 \\
\hline 0 & 1 & 1 & 1 & 1 & 0 & 0 & 0 & 0 & 0 & 5 & 2 \\
\hline 1 & 0 & 0 & 1 & 1 & 0 & 0 & 0 & 0 & 0 & 5 & 3 \\
\hline 0 & 0 & 0 & 0 & 0 & 0 & 0 & 0 & 0 & 0 & 5 & 4 \\
\hline 0 & 0 & 0 & 0 & 0 & 0 & 0 & 0 & 0 & 1 & 5 & 5 \\
\hline 0 & 0 & 0 & 0 & 0 & 0 & 0 & 0 & 0 & 0 & 5 & 6 \\
\hline 0 & 0 & 0 & 0 & 0 & 0 & 0 & 0 & 0 & 0 & 5 & 7 \\
\hline 0 & 0 & 0 & 0 & 0 & 0 & 0 & 0 & 1 & 0 & 5 & 8 \\
\hline 0 & 0 & 0 & 0 & 1 & 1 & 0 & 0 & 0 & 0 & 5 & 9 \\
\hline 0 & 0 & 0 & 0 & 0 & 0 & 0 & 0 & 0 & 0 & 5 & 10 \\
\hline 1 & 1 & 1 & 1 & 1 & 1 & 1 & 1 & 1 & 1 & 6 & 1 \\
\hline 0 & 0 & 0 & 0 & 1 & 0 & 1 & 1 & 1 & 0 & 6 & 2 \\
\hline 0 & 0 & 0 & 0 & 0 & 0 & 0 & 0 & 0 & 0 & 6 & 3 \\
\hline 0 & 0 & 0 & 1 & 0 & 0 & 0 & 0 & 0 & 0 & 6 & 4 \\
\hline 0 & 0 & 0 & 0 & 0 & 0 & 0 & 0 & 0 & 0 & 6 & 5 \\
\hline 0 & 0 & 0 & 0 & 0 & 0 & 1 & 0 & 0 & 0 & 6 & 6 \\
\hline 0 & 0 & 1 & 0 & 1 & 0 & 0 & 0 & 0 & 0 & 6 & 7 \\
\hline 0 & 0 & 0 & 0 & 0 & 0 & 0 & 0 & 0 & 0 & 6 & 8 \\
\hline 0 & 0 & 0 & 0 & 1 & 1 & 0 & 0 & 0 & 0 & 6 & 9 \\
\hline 0 & 0 & 0 & 0 & 0 & 0 & 0 & 0 & 0 & 0 & 6 & 10 \\
\hline 1 & 1 & 1 & 1 & 1 & 1 & 1 & 1 & 1 & 1 & 7 & 1 \\
\hline 1 & 0 & 0 & 0 & 0 & 0 & 0 & 0 & 0 & 0 & 7 & 2 \\
\hline 0 & 0 & 0 & 0 & 0 & 0 & 0 & 0 & 0 & 0 & 7 & 3 \\
\hline
\end{tabular}




\begin{tabular}{|c|c|c|c|c|c|c|c|c|c|c|c|}
\hline 0 & 1 & 0 & 1 & 0 & 0 & 0 & 0 & 0 & 0 & 7 & 4 \\
\hline 0 & 0 & 0 & 0 & 0 & 0 & 0 & 0 & 0 & 0 & 7 & 5 \\
\hline 0 & 0 & 0 & 1 & 0 & 0 & 0 & 0 & 0 & 0 & 7 & 6 \\
\hline 0 & 0 & 0 & 0 & 0 & 0 & 0 & 0 & 0 & 0 & 7 & 7 \\
\hline 0 & 1 & 0 & 1 & 0 & 0 & 0 & 0 & 0 & 1 & 7 & 8 \\
\hline 0 & 0 & 0 & 0 & 0 & 0 & 0 & 0 & 0 & 0 & 7 & 9 \\
\hline 0 & 0 & 0 & 0 & 0 & 0 & 0 & 0 & 0 & 0 & 7 & 10 \\
\hline 1 & 1 & 1 & 1 & 1 & 1 & 1 & 1 & 1 & 1 & 8 & 1 \\
\hline 0 & 0 & 0 & 0 & 0 & 0 & 0 & 1 & 0 & 0 & 8 & 2 \\
\hline 0 & 0 & 0 & 0 & 1 & 0 & 0 & 0 & 0 & 0 & 8 & 3 \\
\hline 0 & 0 & 1 & 0 & 0 & 1 & 0 & 0 & 0 & 1 & 8 & 4 \\
\hline 0 & 0 & 1 & 0 & 0 & 0 & 0 & 0 & 0 & 0 & 8 & 5 \\
\hline 0 & 1 & 0 & 0 & 0 & 0 & 0 & 0 & 0 & 0 & 8 & 6 \\
\hline 0 & 0 & 0 & 1 & 0 & 0 & 0 & 0 & 0 & 0 & 8 & 7 \\
\hline 0 & 0 & 0 & 0 & 0 & 1 & 0 & 0 & 0 & 0 & 8 & 8 \\
\hline 0 & 0 & 0 & 1 & 1 & 0 & 0 & 0 & 0 & 0 & 8 & 9 \\
\hline 0 & 0 & 0 & 0 & 0 & 1 & 1 & 1 & 1 & 0 & 8 & 10 \\
\hline 1 & 1 & 1 & 1 & 1 & 1 & 1 & 1 & 1 & 1 & 9 & 1 \\
\hline 0 & 0 & 0 & 0 & 0 & 0 & 0 & 0 & 0 & 0 & 9 & 2 \\
\hline 0 & 0 & 0 & 0 & 0 & 0 & 0 & 0 & 0 & 0 & 9 & 3 \\
\hline 0 & 0 & 0 & 0 & 0 & 1 & 0 & 0 & 0 & 1 & 9 & 4 \\
\hline 0 & 0 & 0 & 0 & 0 & 0 & 0 & 0 & 0 & 0 & 9 & 5 \\
\hline 0 & 0 & 0 & 0 & 0 & 0 & 0 & 1 & 0 & 0 & 9 & 6 \\
\hline 0 & 0 & 0 & 0 & 0 & 0 & 0 & 0 & 0 & 0 & 9 & 7 \\
\hline 0 & 0 & 1 & 0 & 0 & 0 & 0 & 0 & 0 & 0 & 9 & 8 \\
\hline 0 & 0 & 0 & 1 & 0 & 1 & 0 & 1 & 0 & 0 & 9 & 9 \\
\hline 1 & 0 & 0 & 0 & 0 & 0 & 0 & 0 & 0 & 0 & 9 & 10 \\
\hline 1 & 1 & 1 & 1 & 1 & 1 & 1 & 1 & 1 & 1 & 10 & 1 \\
\hline 0 & 0 & 0 & 0 & 0 & 0 & 1 & 0 & 0 & 0 & 10 & 2 \\
\hline 0 & 0 & 0 & 0 & 0 & 0 & 0 & 0 & 0 & 0 & 10 & 3 \\
\hline 0 & 0 & 1 & 0 & 1 & 0 & 0 & 0 & 0 & 0 & 10 & 4 \\
\hline 0 & 0 & 0 & 0 & 0 & 0 & 0 & 1 & 0 & 0 & 10 & 5 \\
\hline 1 & 0 & 0 & 0 & 0 & 0 & 0 & 0 & 0 & 0 & 10 & 6 \\
\hline 0 & 0 & 0 & 0 & 0 & 0 & 0 & 0 & 0 & 0 & 10 & 7 \\
\hline 0 & 0 & 0 & 0 & 0 & 1 & 0 & 0 & 0 & 0 & 10 & 8 \\
\hline 0 & 0 & 0 & 0 & 0 & 0 & 0 & 0 & 0 & 0 & 10 & 9 \\
\hline 0 & 0 & 0 & 0 & 0 & 0 & 0 & 0 & 0 & 0 & 10 & 10 \\
\hline
\end{tabular}

A selection of at least eight was not possible, as there were no relevant lines. For the selection of at least eight entries with 0 , the following constraints were added.

$\operatorname{open}(1,4)=0$;

open $(1,5)=0$; 


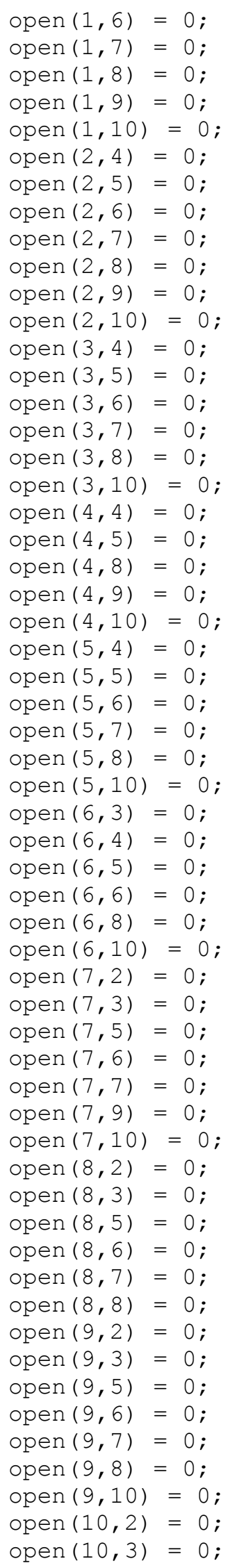




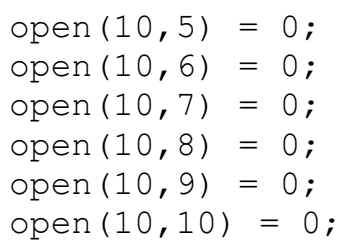

The following table shows the objective function values of the different selection strategies for the 10x10x10x10 model.

Table 24 Objective function values depending on selection strategy

\begin{tabular}{lcc} 
Selection strategy & 1 & 0 \\
Original model & \multicolumn{2}{c}{$2.61799 \mathrm{E} 08$} \\
\hline All values & Not available & $2.61799 \mathrm{E} 08$ \\
\hline At least three & $2.61799 \mathrm{E} 08$ & $1.549897 \mathrm{E} 08$ \\
& & \\
At least five & $2.61799 \mathrm{E} 08$ & $1.549897 \mathrm{E} 08$ \\
& & \\
At least eight & Not available & $2.60496 \mathrm{E} 08$
\end{tabular}

The table shows that it is better to focus on open facilities and not on closed facilities for the objective function value. For a good result it is also better to have knowledge about as many products as possible. If there is a high number of closed facilities, the selection of those might be a good approach. 


\subsection{Summary}

The decomposition approach reduces the complexity of the original model significantly. Depending on the selection strategy, different results are obtained. Based on our experimentation with the $5 \times 5 \times 5 \times 5$ problem (for which an optimal solution can be obtained), the sub-problem solutions lead to the optimal solution of the original problem, if the selection is made for those facilities where all sub problems require the facilities to be opened or closed respectively. With respect to the 10x10x10x10 problem, the results were different. In general, it is important to look at the data carefully, because selecting "open" and adding open constraints to the original problem provide better results than focusing on closed facilities. The more facilities we know that are open for the relevant sub problems, the better the objective function value. Sometimes the problems might become so large that a fully decomposition is too time consuming, In that case a selection based on a subset of products could be made to get some good results.

The solving times of the sub problem and the modified original problem are fast. For larger problems, this procedure is time consuming as the data has to be prepared and each sub problem has to be solved separately. Finally, the decomposition approach provides an algorithm for obtaining an optimal solution from the original problem with some effort. It must be checked whether a preprocessing or an alternative decomposition approach will generate a better result. 


\section{CHAPTER 5}

\section{A SIMULATED ANNEALING ALGORITHM}

\subsection{Introduction}

In real world applications, companies deal with many more than 10 products. Therefore, it is important to try alternatives in solving the optimization problem, as a decomposition approach is likely to be ineffective when we have a large number of products and there is significant interaction amongst them. Simulated annealing (SA) is known to be an effective approach for large-scale combinatorial optimization problems. It is a heuristic technique for finding an approximate solution, when it is not possible to find an optimal solution due to computational complexity. SA replicates the gradual cooling of molten material. Slow annealing ensures that atoms have enough time to find a stable position to build crystals. A state of low energy close to the optimum is reached. Translated to the optimization problem, the SA explores better solutions gradually ensuring a sufficient number of solutions are evaluated at each temperature epoch. Not only does the algorithm move to better solutions like a steepest descent 2-opt algorithm, it also moves to a worse solution with a certain probability that depends upon how much worse that solution is (Press, Teukolsky, Vetterling, \& Flannery, 2007). SA is applied to solve the models in Chpaters 3 and 4. 


\subsection{Modification of algorithm}

In the SA algorithm applied to solve to the facility location problem considered in this dissertation, an array with a dimension corresponding to the product of the number of facilities and time periods considered is used. It is named configuration and contains binary values 0 (facility is closed in the corresponding time period) and 1 (facility is open). In each step of the SA algorithm, the value of only one element in the array is changed - from 0 to 1 or vice-versa. The array is then passed to the LINGO solver and the objective value is obtained. The rest of the procedure is the same as that proposed in the original annealing algorithm.

The following pseudo code demonstrates how the algorithm works.

Algorithm simulatedAnnealing (temperature, cooling Rate)

\section{Begin}

Step 1, Initialization

startConfiguration $=$ createNewConfiguration $($ facilities, periods $)$;

bestEnergy = getEnergy(startConfiguration);

bestConfiguration $=$ startConfiguration;

currentConfiguration $=$ startConfiguration;

currentEnergy = bestEnergy;

Step 2, Annealing

While temperature $>1$ Do

neighborConfiguration $=$ createNewConfiguration (currentConfiguration $)$; neighborEnergy = getEnergy(neighborConfiguration); 
If getAcceptanceProbability > random number Then

currentConfiguration $=$ neighborConfiguration;

currentEnergy = neighborEnergy;

End If

If currentEnergy > bestEnergy Then

bestConfiguration $=$ currentConfiguration;

bestEnergy = currentEnergy;

\section{End If}

temperature $*=1$-coolingRate;

\section{End While}

Return bestEnergy, bestConfiguration;

\section{End}

Procedure getAcceptanceProbability (energy, newEnergy, temperature)

Begin Procedure

If newEnergy > energy then

Return 1.0

Else

$$
\text { Return } e^{\frac{-(\text { energy-newEnergy })}{\text { temperature }}}
$$

\section{End if}

\section{End Procedure}

Procedure createNewConfiguration (currentConfiguration)

indexi $=$ RandonNumber ${ }^{*}$ facilities;

indext $=0$; 
While indext $=0$ Do

indext $=$ RandonNumber ${ }^{*}$ periods;

End While

newConfiguration = currentConfiguration;

if newConfiguration[indexi][indext $]=0$ ) Then

newConfiguration[indexi] [indext $]=1$;

Else If

newConfiguration $[$ indexi] $[$ indext $]=0$;

End If

return newConfiguration;

\section{End Procedure}

Procedure getEnergy(configuration)

Load the LINGO model;

Retrieve variable indices;

For each variableIndex Do

Add constraint from configuration;

\section{End For}

Solve the model;

Return objective function value;

\section{End Procedure}

The corresponding JAVA source code can be found in Appendix F.

\subsection{Technical challenges}

Using the LINDO API in Java raised several technical challenges. The model needed to be saved as an MPS file in advance so that the API could read it. For the computation the 
LINDO API with unlimited variables is necessary. To be able to change the model's constraints, it was necessary to obtain the variable indices. This was possible using one of the functions provided in LINDO. Additionally several JAVA error messages had be resolved, especially setting the PATH environment was difficult. Finally the source code was written using Eclipse with SDK 8.

\subsection{Computational results}

The annealing algorithm was tested on four different problem instances $(5 \times 5 \times 5 \times 5$, 10x10x10x10, 50x50x50x50 and 50x50x12x50). Mixed integer problems could be solved for problems with fewer than 4,000 variables. Relaxing the model resulted in durations of less than 100 milliseconds for each step for smaller models, while it took up to ten hours for the largest models.

\subsubsection{Solution times}

As expected, the solution times were highly dependent on the problem sizes. First, the SA algorithm was applied to the mixed-integer problem, and then to the relaxed model.

The following table shows the average computational times for each annealing step for the mixed-integer case. The SA algorithm can barely be applied to a problem of size $5 \times 5 \times 5 \times 5$. The standard deviation of the solution times for the mixed-integer problems is larger than the mean which indicates a strong dependence of the configuration. 
Table 25 Computational times for each step of the SA algorithm with respect to problem size for the mixed-integer model

$\begin{array}{lccc} & \text { Mean } & \text { Standard deviation } & \text { \# of variables } \\ & & {\left[\text { sec } * \mathbf{1 0}^{-\mathbf{3}}\right]} & \\ 5 \times 5 \times 5 \times 5 & 14740967 & 3,945 \\ 10 \times 10 \times 10 \times 10 & \text { n.a. } & \text { n.a. } & 30,290 \\ 50 \times 50 \times 12 \times 50 & \text { n.a. } & \text { n.a. } & 2,099,850 \\ 50 \times 50 \times 50 \times 50 & \text { n.a. } & \text { n.a. } & 8,757,450\end{array}$

The application of the SA algorithm works much better for the relaxed model. This shows that application of the annealing algorithm is a good option for model sizes up to 2 million variables when using the relaxed model.

Table 26 Computational times for each step of the SA algorithm with respect to problem size for the relaxed model

\begin{tabular}{lccc} 
& \multicolumn{1}{c}{ Mean } & Standard deviation & \# of variables \\
& {$\left[\boldsymbol{s e c} * \mathbf{1 0}^{-\mathbf{3}}\right]$} & {$\left[\boldsymbol{s e c} * \mathbf{1 0}^{-\mathbf{3}}\right]$} & \\
\hline $5 \times 5 \times 5 \times 5$ & 87 & 8 & 3,945 \\
\hline $10 \times 10 \times 10 \times 10$ & 806 & 67 & 30,290 \\
\hline $50 \times 50 \times 12 \times 50$ & $34,917,794$ & n.a. & $2,099,850$
\end{tabular}


The results show again that large models require considerable solution time for each step, although the specific data also influences runtime. Therefore the right choice of temperature and cooling rate is of great importance. The 50x50x50x50 model could only be run after a RAM extension to the computer from $8 \mathrm{~GB}$ to $16 \mathrm{~GB}$ was made as the application required too much working memory. This shows that even for the SA algorithm applied to the relaxed model, there are technical restrictions of the problem size that can be solved.

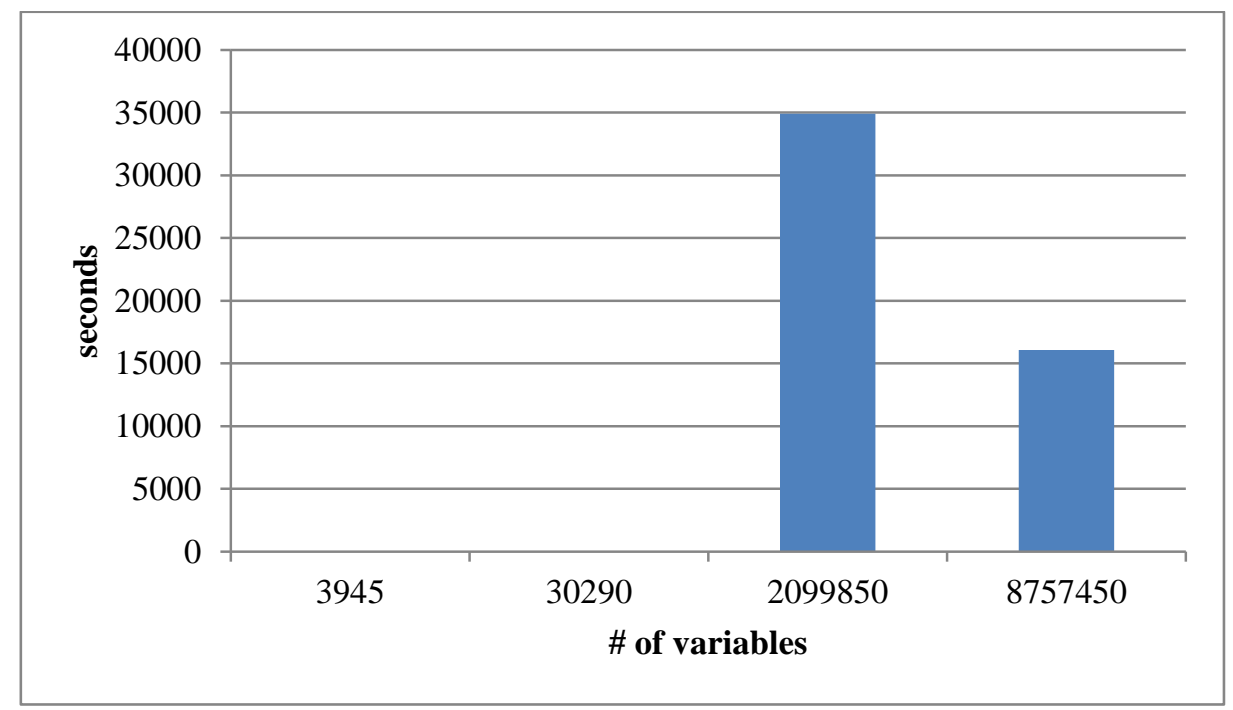

Figure 7 Average runtime per annealing step for the relaxed model 


\subsubsection{The choice of temperature and cooling rate}

For each model, potentially $2^{\text {locations*(periods-1) }}$ configurations are possible. Taking the different problem sizes considered, this would result in the following solution times if all possible configurations had to be tested to obtain the optimal solution.

Table 27 Potential solving times for all configurations

\begin{tabular}{lcc} 
& Potential configurations & Total solving time \\
\hline $5 \times 5 \times 5 \times 5$ & $2^{5 *(5-1)}=1,048,576$ & 3.6 hours \\
\hline $10 \times 10 \times 10 \times 10$ & $2^{10 *(10-1)}$ & Too big to display \\
\hline $50 \times 50 \times 12 \times 50$ & $2^{50 *(12-1)}$ & \\
\hline $50 \times 50 \times 50 \times 50$ & $2^{50 *(50-1)}$ & Too big to display
\end{tabular}

It is obvious that it is not possible to test all these configurations. Therefore, the SA algorithm was applied. A higher temperature will cause both a longer run of the entire annealing procedure and a higher probability accepting lower energy values at the beginning. The cooling rate also influences the length of the annealing procedure. Several runs with different energy levels were done. The following table shows the number of corresponding steps. 
Table 28 Annealing steps depending on the choice of cooling rate and temperature

\begin{tabular}{|c|c|c|c|c|c|c|c|}
\hline & \multicolumn{7}{|c|}{ Temperature } \\
\hline & 100 & 1,000 & 10,000 & 100,000 & $1,000,000$ & $10,000,000$ & $100,000,000$ \\
\hline 0.001 & 4,603 & 6,905 & 9,206 & 11,508 & 13,809 & 16,111 & 18,412 \\
\hline 0.01 & 459 & 688 & 917 & 1,146 & 1,375 & 1,604 & 1,866 \\
\hline 0.05 & 90 & 135 & 180 & 225 & 270 & 315 & 360 \\
\hline 0.1 & 44 & 66 & 88 & 110 & 132 & 153 & 175 \\
\hline 0.2 & 21 & 31 & 42 & 52 & 62 & 73 & 83 \\
\hline 0.25 & 7 & 10 & 14 & 17 & 20 & 24 & 27 \\
\hline 0.75 & 4 & 5 & 7 & 9 & 10 & 12 & 14 \\
\hline
\end{tabular}

As the number of steps grows rapidly with lower cooling rates, attention was particularly drawn to the change of energy levels. The following figures show the development of the energy levels over time for the 10x10x10x10 model with different settings of temperature and cooling rate.

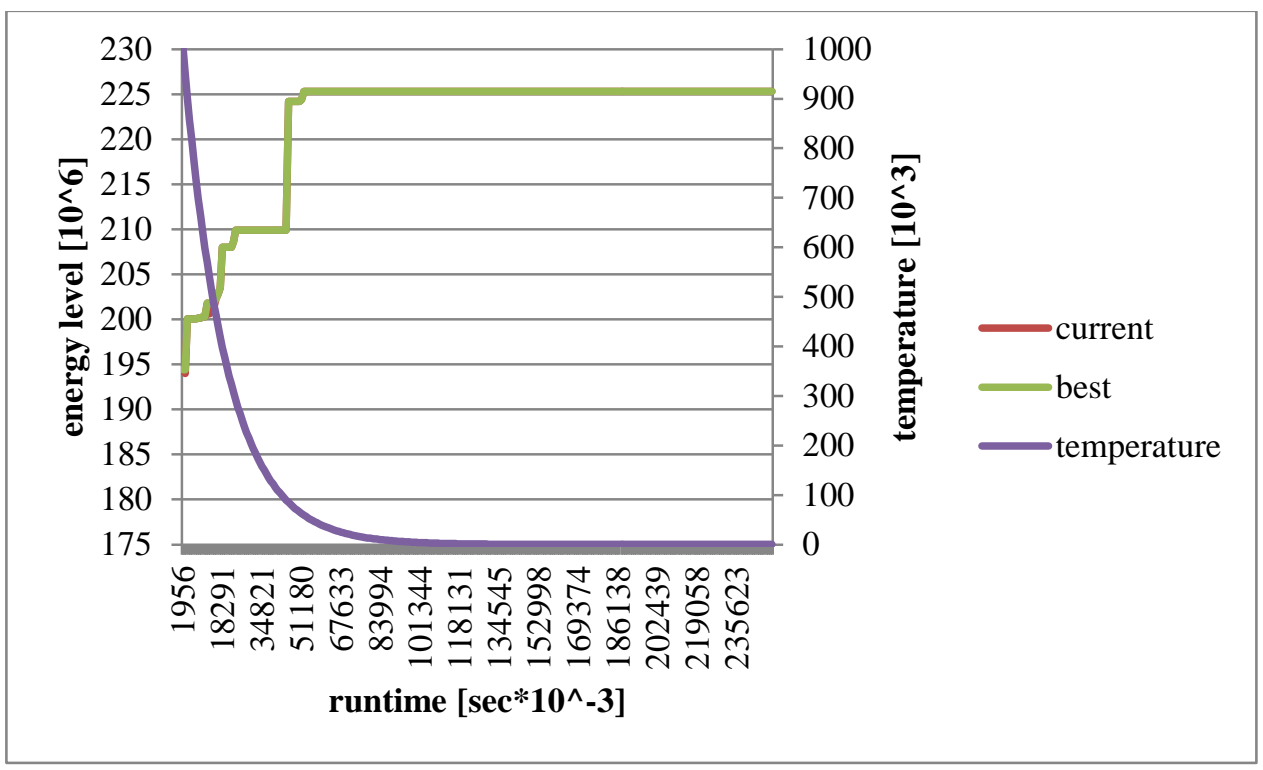

Figure 8 Simulated annealing for $10 \times 10 \times 10 \times 10$ model temperature $10^{\wedge} 6$ and cooling rate 0.05 


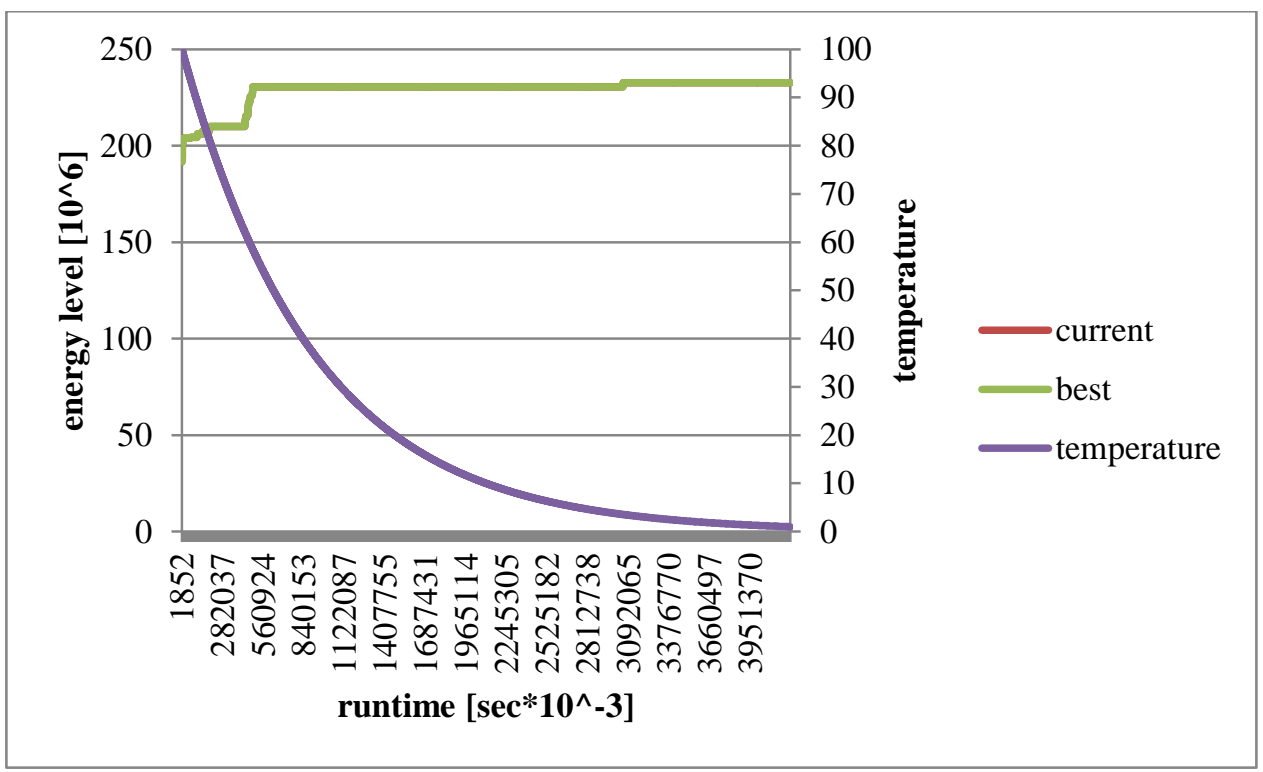

Figure 9 Simulated annealing for 10x10x10x10 model temperature 100 and cooling rate 0.001 


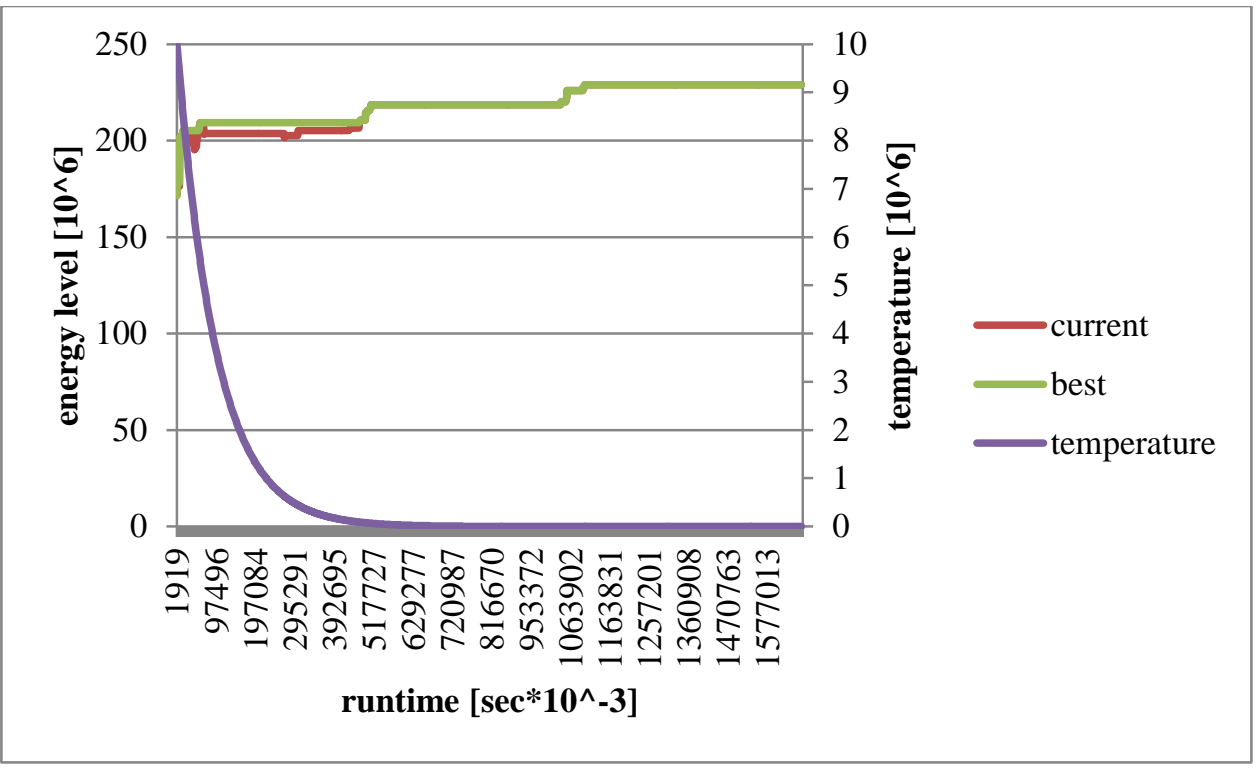

Figure 10 Simulated annealing for $10 \times 10 \times 10 \times 10$ model temperature $10^{\wedge} 7$ and cooling rate 0.01

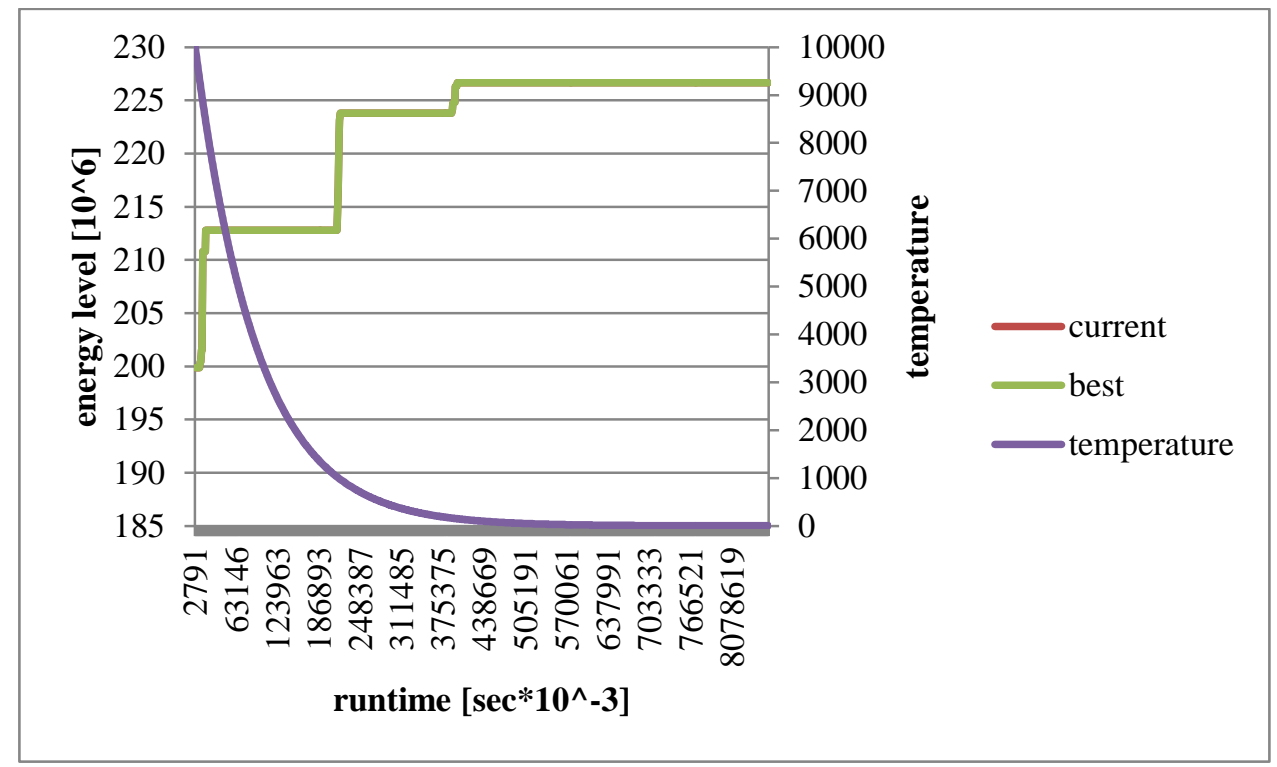

Figure 11 Simulated annealing for 10x10x10x10 model temperature 10,000 and cooling rate 0.01 
These charts have been created for all the different combinations (temperature / cooling rate) They demonstrate that the best results for the SA algorithm are to be found when the temperature is about $1 / 10^{\text {th }}$ of the objective function value and a cooling rate of $1 \%$. Lower cooling rates $(<<0.01)$ will only cause a longer run of the annealing algorithm without significant improvement of the objective function value.

\subsection{Pre-processing}

Comparing the results of the annealing algorithm to the results from the decomposition approach, it is obvious that the objective function values are worse. A preprocessing test was made to improve the objective function value. The following preprocessing approaches were carried out.

1. Setting all facilities to open.

2. Setting all facilities to close.

3. Random opening or closing the facilities.

4. Setting those facilities to open in a period during which the net profit is positive for more than half of the products in that period.

5. Setting those facilities to open in a period during which the attraction for more than half of the products is greater than the attraction of competitor in that period.

The SA algorithm was started after the preprocessing with a temperature of $10,000,000$ and cooling rate 0.01 using both the $5 \times 5 \times 5 \times 5$ and the $10 \times 10 \times 10 \times 10$ problem instances. Each of the different approaches was run 10 times for both models. The objective 
function values were obtained and mean and standard deviation were computed. A twosample $t$-test was performed to test for significant difference in the energy levels. The following table shows the results for the means and standard deviations for both models and the pre-processing approach. For the $5 \times 5 \times 5 \times 5$ problem, the values did not improve after the sixteenth and second annealing step for the net profit and attraction pre-setting, respectively. Therefore the best value obtained were taken from the simulation.

Table 29 Means and standard deviations for objective function values using different initial configurations

\begin{tabular}{|c|c|c|c|c|}
\hline & \multicolumn{2}{|c|}{$5 \times 5 \times 5 \times 5$} & \multicolumn{2}{|c|}{$10 \times 10 \times 10 \times 10$} \\
\hline & Mean & Standard deviation & Mean & Standard deviation \\
\hline All open & $298,470,150$ & 0 & $224,786,154$ & $2,750,762$ \\
\hline All closed & $297,812,850$ & $1,562,038$ & $222,801,956$ & 512,0944 \\
\hline Random & $298,328,881$ & 57,201 & $223,662,044$ & $4,487,364$ \\
\hline Net profit & $298,355,119$ & 17,236 & $242,092,760$ & 429,700 \\
\hline Attraction & $298,337,036$ & 49,097 & $224,586,090$ & $2,475,947$ \\
\hline
\end{tabular}

Because the objective function values did not differ greatly for the $5 \times 5 \times 5 \times 5$ problem, no further tests were performed. The values for the $10 \times 10 \times 10 \times 10$ showed a difference. A $t$ test on the mean was performed. 
Table $30 \mathrm{p}$-values for $t$-test on means for $5 \times 5 \times 5 \times 5$

\begin{tabular}{|c|c|c|c|c|c|}
\hline p-value & All open & All closed & Random & Net profit & Attraction \\
\hline All open & & $23.85 \%$ & $0.00 \%$ & $0.00 \%$ & $0.00 \%$ \\
\hline All closed & & & $34.79 \%$ & $32.49 \%$ & $34.06 \%$ \\
\hline Random & & & & $21.70 \%$ & $74.95 \%$ \\
\hline Net profit & & & & & $31.95 \%$ \\
\hline Attraction & & & & & \\
\hline
\end{tabular}

Table $31 \mathrm{p}$-values for $t$-test on means for 10x10x10x10

\begin{tabular}{|c|c|c|c|c|c|}
\hline p-value & All open & All closed & Random & Net profit & Attraction \\
\hline All open & & $32.45 \%$ & $53.21 \%$ & $0.00 \%$ & $87.31 \%$ \\
\hline All closed & & & $70.94 \%$ & $0.00 \%$ & $36.53 \%$ \\
\hline Random & & & & $0.00 \%$ & $59.71 \%$ \\
\hline Net profit & & & & & $0.00 \%$ \\
\hline Attraction & & & & & \\
\hline
\end{tabular}


The $t$-test shows that there is a significance difference in the objective function values when using a start configuration based on the net profit. The value turns out to be more than $30 \%$ better in this model.

\subsection{Conclusion}

Simulated annealing is used in general to obtain fast and good solutions. The original algorithm had to be modified. Several modifications were made and the simulation was run on different model sizes. First, the mixed integer model was solved. The model works up to a size of about 4,000 variables. Second the algorithm was applied to the relaxed model. The limitations of the simulation are essentially determined by the values of temperature, cooling rate, time periods and facilities. Therefore it is better to use the annealing when a model has relatively fewer time periods. The algorithm provides a good approach for finding better solutions, knowing not to have reached the global optimum, but getting a near-optimal solution. A guideline for selecting the cooling rate and temperature can be derived from the results, in addition to the observation that selection of a good initial configuration also helps to improve the objective function value. 


\section{CHAPTER 6}

\section{CASE STUDY}

\subsection{Introduction}

Banking business has changed significantly in the last two decades. Internet banking, low interest rates, and monetary policies have brought many changes to the banking industry. Branch banking has lost market share and is faced with operational costs that are higher than cost in direct banking business. The cooperative financial associations also have similar problems. High operational costs are especially attributed to facilities and employees. On the other hand for distribution purposes, a high density of facilities is the key for business, especially for financial products requiring personal consulting. Almost all banks in Germany are currently reviewing their facility strategy - their strategy for spatial distribution. The model developed in this dissertation is applied to the facility network of Dortmunder Volksbank. Several scenarios are considered and a recommendation is developd based on the results. The next section describes the situation of Dortmunder Volksbank with respect to the German and the local market conditions including the objective of the bank's facility configuration. Next the model is developed. It is based on spatial data and data of the facilities as well on the products that are offered. 


\subsection{About Dortmunder Volksbank}

Dortmunder Volksbank has a history of more than 100 years. The bank's business is built on four pillars.

1. Direct contact with customers in facilities

2. Consultancy on special topics

3. Online banking and brokerage

4. ATMs and service terminals.

Dortmunder Volksbank currently operates 61 facilities in 5 different cities. 33 are located in a core area of Dortmund city. A set of additional ATMs is also available. With a balance sheet total of 6,321 billion EUR in 2015 , the bank is ranked $11^{\text {th }}$ out of 1,045 cooperative banks in Germany.

The goal of this case study is to define the strategic facility configuration for Dortmunder Volksbank for the next 10 years. The bank has already started an initiative of facility restructuring, including the closure of three facilities. Currently the bank operates 33 facilities in the Dortmund region, but several mergers have made the bank grow in smaller cities around Dortmund. Originally Dortmund had 36 facilities but the recent closure of three, has resulted in the current 33. The location of ATMs and self-service terminals are not included in the model, but should be part of an additional analysis, beyond the scope of this thesis. 13 typical financial products are included and it is assumed that all employees are qualified equally and no sepcial qualifications are 
necessary for selling the products. It is also assumed that each employee has a capacity to sell 600 products a year. Facilities are rented and fixed costs are paid for rent. Costs occur for additional employees. The number of products sold per year and facilities were set to the capacity of the model. Demand occurs at 170 demand locations, which are the different districts of the city.

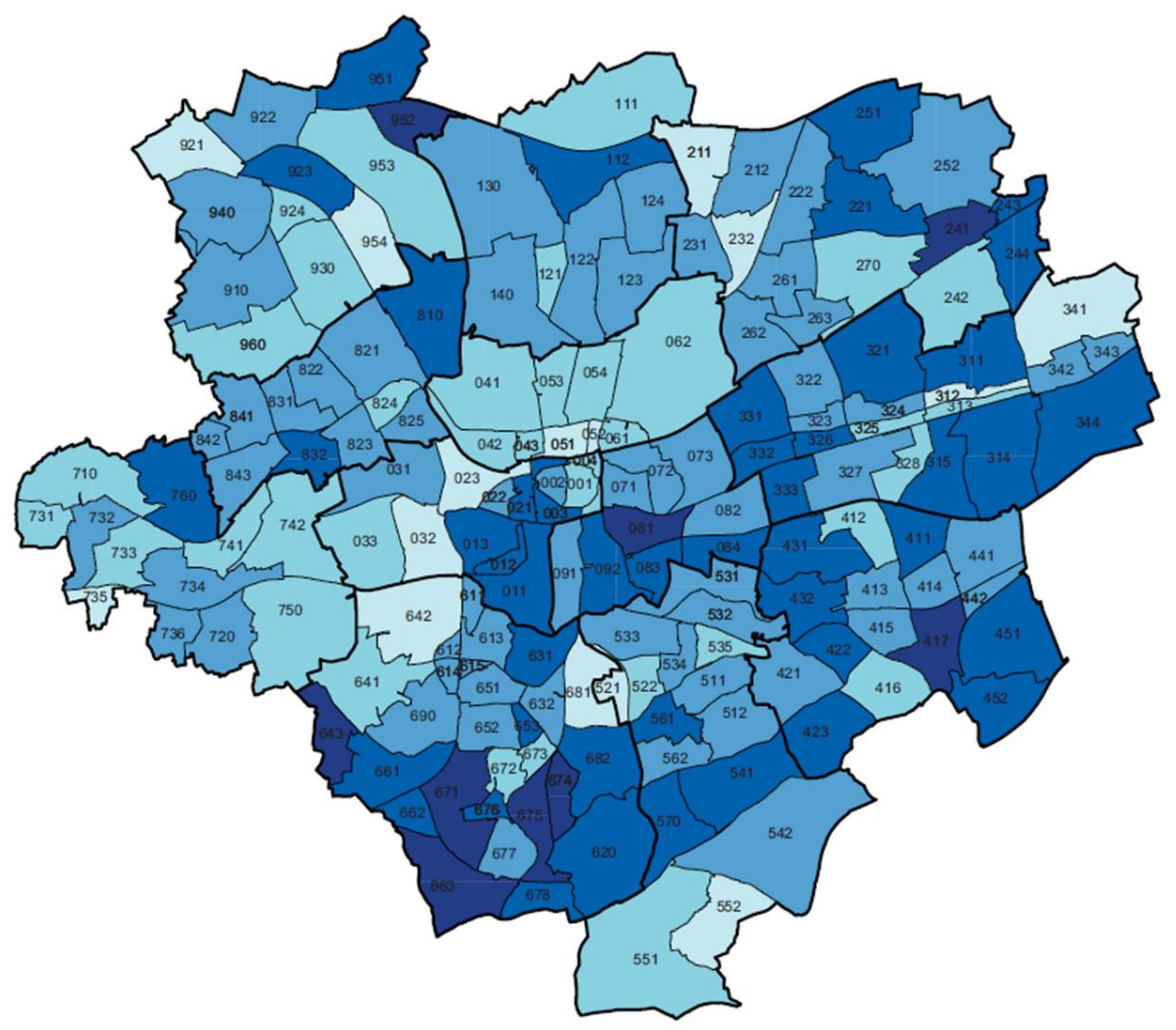

Figure 12 City of Dortmund and its districts (Stadt Dortmund, 3/Dez - Stabsstelle Dortmunder Statistik, 2015)

Three different scenarios were carried out.

Scenario 1: The model run was carried out for all 36 locations, including those already closed. Additional facilities were not included. 
Scenario 2: The model run was performed for all 33 existing facilities. Additional facilities were not included.

Scenario 3: The model was run with all 33 existing facilities. 10 potential new locations were included.

\subsection{The model}

\subsubsection{Gathering model data and database implementation}

1) Demand data

Demand points have geographical coordinates. These coordinates were obtained manually from Google maps. Demands were taken from statistical data provided by the administration of the city of Dortmund (Stadt Dortmund, 3/Dez - Stabsstelle Dortmunder Statistik, 2015) and (Eckert, 2014). The sources contain relevant information on migration, changes in population, (un)employment rates, age structure, affinity towards certain banking products, amount of money invested in banking products and the need to be consulted for investment decisions.

2) Location data

All facilities have certain location data, such as rent, the number of employees and geographic coordinates. This data was taken to determine the fixed cost, opening cost, closing cost, expansion cost, shrinking cost and finally the attraction parameter. The 
attraction parameter was generated by using the inverse of the squared (driving) distance between facility $i$ and demand location $j$. The matrix was created using Google API. The script used for the generation ${ }^{3}$ was coded in Ruby. Appendix $G$ has the script for generating the distance information.

\section{3) Product data}

The 13 relevant products are checking accounts, saving accounts, cash value life insurances, building savings agreements, mortgaging, call money, funds, fixed term deposits, savings plans, stocks, fixed-interest securities, gold and certificates. The demand data can be found in Appenix $\mathrm{H}$.

\section{4) Competitors data}

Seven competitors with a total of 68 facilities are incorporated in the model. The highest attraction parameter with respect to a demand point was determined. Competitors are Sparkasse, Deutsche Bank, Targo Bank and Commerzbank. The relevant data can be found in Appendix H Data for case study.

The data was loaded into seven data base tables. The database structure used for the other models had to be modified as the number of locations, periods, demands and products were not equal. The data base structure is listed below.

\footnotetext{
${ }^{3}$ https://developers.google.com/maps/?hl=en
} 
Table 32 Database view " CS_COMPETITORS_VIEW"

$\begin{array}{ccc} & \text { COLUMN_NAME } & \text { DATA_TYPE } \\ 1 & \text { COMPETITORSATTRACTION } & \text { FLOAT(126) } \\ 2 & \text { INDEXJ } & \text { NUMBER(3) } \\ 3 & \text { INDEXT } & \text { NUMBER(3) }\end{array}$

Table 33 Database table " CS_ATTRACTIVITY_VIEW"

$\begin{array}{ccc} & \text { COLUMN_NAME } & \text { DATA_TYPE } \\ 1 & \text { ATTRACTIVITY } & \text { NUMBER(30) } \\ 2 & \text { INDEXI } & \text { NUMBER(3) } \\ 3 & \text { INDEXJ } & \text { NUMBER(3) } \\ 4 & \text { INDEXT } & \text { NUMBER(3) }\end{array}$

Table 34 Database table "CASE_STUDY_DATA_INITIALS_CAP"

$\begin{array}{ccc} & \text { COLUMN_NAME } & \text { DATA_TYPE } \\ 1 & \text { INITIALCAP } & \text { FLOAT } \\ 2 & \text { INDEXI } & \text { FLOAT }\end{array}$

Table 35 Database table "CASE_STUDY_INITIALS"

$\begin{array}{llc} & \text { COLUMN_NAME } & \text { DATA_TYPE } \\ 1 & \text { INITIALOPEN } & \text { FLOAT }\end{array}$

Table 36 Database table "CS_PRODUCT_VIEW"

$\begin{array}{lcc} & \text { COLUMN_NAME } & \text { DATA_TYPE } \\ 1 & \text { PRICE } & \text { FLOAT(20) } \\ 2 & \text { VARIABLECOST } & \text { FLOAT(20) } \\ 3 & \text { INDEXI } & \text { NUMBER(3) } \\ 4 & \text { INDEXT } & \text { NUMBER(3) } \\ 5 & \text { INDEXP } & \text { NUMBER(3) } \\ 6 & \text { UNITSHRINKAGECOST } & \text { FLOAT(126) } \\ 7 & \text { UNITEXPANSIONCOST } & \text { FLOAT(126) }\end{array}$

Table 37 Database table "CASE_STUDY_DATA_FACILITIES_NPR"

$\begin{array}{ccc} & \text { COLUMN_NAME } & \text { DATA_TYPE } \\ 1 & \text { EXPANSIONCOST } & \text { FLOAT }\end{array}$




$\begin{array}{lcc}2 & \text { SHRINKAGECOST } & \text { FLOAT } \\ 3 & \text { FIXCOST } & \text { FLOAT } \\ 4 & \text { OPENINGCOST } & \text { FLOAT } \\ 5 & \text { CLOSINGCOST } & \text { FLOAT } \\ 6 & \text { NAME } & \text { VARCHAR2(60 BYTE) }\end{array}$

Table 38 Database table "CS_DEMAND_VIEW" COLUMN_NAME DATA_TYPE

1

DEMAND NUMBER(30)

2 INDEXP

NUMBER(3)

INDEXT

NUMBER(3)

4

INDEXJ

NUMBER(3)

\subsubsection{Mathematical formulation}

\section{Indexes}

$i \quad$ Set of facilities

j Set of demand nodes

$t \quad$ Set of time periods

$p \quad$ Set of products 


\section{Model parameters}

initialCapacity $_{i}$

initialOpen $_{i}$

price $_{i t p}$

variableCost $_{i t p}$ $\operatorname{fix}_{\operatorname{Cost}}$

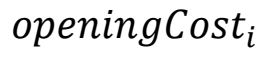

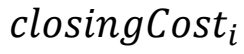

attraction $_{i j t}$
The initial capacity of facility $i$ in period 1 . The capacity represents the number of personal consultations that can be carried out

The initial status of facility $i$ in period 1

The price for selling one unit of product $p$ at facility $i$ in period $t$

The unit cost for selling product $p$ at facility $i$ in period $t$

The cost for having open facility $i$ - assumed to be constant over all periods. The fix cost component contains

especially the rent for the office space

The cost for opening facility $i$. It is assumed that opening cost will not change over time

The cost for closing facility $i$. It is assumed that closing cost will not change over time

Attraction value of own facility $i$ with respect to demand

node $j$ in period $t$ computed by $\frac{1}{\mathrm{~d}^{2}}$ where $\mathrm{d}$ represents the driving distance in minutes form demand node $j$ to facility $i$ in period $t$ 
competitorsAttraction $_{j t}$ expansionCost $_{i}$
shrinkageCost $_{i}$
unitExpansionCost
unitp
step $<$ X $_{\text {it }}$

stepCost $<X>_{i t}$

$\operatorname{assignment}_{i j t}$
The highest attraction of competitors for demand node $j$ in period $t$. The value is computed using the same formula as for the attraction value

Fixed cost for expansion of facility $i$. The value represents basically recruiting cost and training.

Fixed cost for shrinking of facility $i$. This value contains cost for compensation

Unit (variable) cost for expansion of product $p$ at facility $i$ in period $t$, assumed to be 0

Unit (variable) cost for shrinking of product $p$ at facility $i$ in period $t$, assumed to be 0

Demand for product $p$ at demand node $j$ in period $t$ The step-wise (capacity) when a modification of fix cost is necessary. The model incorporates ten potential steps, each representing an additional employee for the relevant facility.

The additional fix cost for a certain step if the capacity is expanded for the corresponding product $p$ at facility $i$ in period $t$, representing the annual wage

$=\left\{\begin{array}{l}1, \text { ifattraction }_{i j t} \geq \operatorname{comp}_{j t} \\ 0, \text { else }\end{array}\right.$ 
This parameter is set to one if the attraction of a company's own facility $i$ relative to a demand node $j$ is greater or equal than the highest attraction of the competitor with respect to node $j$ in period $t$. It is used for the assignment of facilities to a demand node in that period.

\section{Model variables}

\begin{tabular}{|c|c|}
\hline open $_{i t}$ & $=\left\{\begin{array}{l}1, \text { if facility } i \text { remains open in } t \\
0, \text { if facility } i \text { is closed in } t\end{array}\right.$ \\
\hline opened $_{i t}$ & $=\left\{\begin{array}{l}1, \text { if faciltiy } i \text { is opened in } t \\
0, \text { else }\end{array}\right.$ \\
\hline closed $_{i t}$ & $=\left\{\begin{array}{l}1, \text { if faciltiy } i \text { is closed in } t \\
0, \text { else }\end{array}\right.$ \\
\hline $\operatorname{expanded}_{i t}$ & $=\left\{\begin{array}{l}1, \text { if facility } i \text { is expanded in } t \\
0, \text { else }\end{array}\right.$ \\
\hline shrunk $_{i t}$ & $=\left\{\begin{array}{l}1, \text { if facility } i \text { is shrunk in } t \\
0, \text { else }\end{array}\right.$ \\
\hline$u P l u s_{i t}$ & Expansion of capacity at facility $i$ in $t$ \\
\hline uMinus $_{i t}$ & Shrinkage of capacity at facility $i$ in $t$ \\
\hline totalCapacity $_{i t}$ & Maximum capacity at $i$ in period $t$ \\
\hline usedCapacity $_{i t p}$ & Used capacity in units of product $p$ at $i$ in period $t$ \\
\hline totalFix Cost $_{i t}$ & The fixed cost for operating facility $i$ in period $t$ \\
\hline
\end{tabular}


threshold $\langle X\rangle_{i t}$

\begin{abstract}
threshold $<X>$ it
\end{abstract}

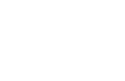

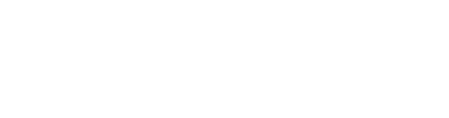

thresholdSwitch $\left\langle X>_{i t}\right.$

The variable that is indicating whether the costs for a certain threshold in terms of capacity have to be incorporated in the model. $\mathrm{X}$ is used for the corresponding step.

This variable is used for modeling purposesto set the threshold correctly for facility $i$ in period $t$.

\section{Objective function}

Subject to

1) open $_{i 1}=$ initialOpen $_{i}, \forall i$

2) totalCapacity $_{i 1}=$ initialCapacity $_{i}, \forall i$

3a) $\operatorname{step} 1_{i t}=600, \quad \forall i, t$

3b) step $_{i t}=1200, \quad \forall i, t$

3c) $s t e p 3_{i t}=1800, \quad \forall i, t$

3d) step $_{i t}=2400, \quad \forall i, t$ 
3e) step $_{i t}=3000, \quad \forall i, t$

3f) $\quad$ step $_{i t}=3600, \quad \forall i, t$

3g) $\quad$ step $7_{i t}=4200, \quad \forall i, t$

3h) step $_{i t}=4800, \quad \forall i, t$

3i) $\quad$ step $9_{i t}=5400, \quad \forall i, t$

3j) $\quad$ step $10_{i t}=6000, \quad \forall i, t$

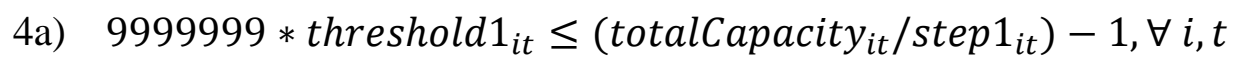

4b) $9999999 *$ threshold $_{i t} \leq\left(\right.$ totalCapacity $_{i t} /$ step $\left._{i t}\right)-1, \forall i, t$

4c) $9999999 *$ threshold $_{i t} \leq\left(\right.$ totalCapacity $_{i t} /$ step $\left._{i t}\right)-1, \forall i, t$

4d) $9999999 *$ threshold $_{i t} \leq\left(\right.$ totalCapacity $_{i t} /$ step $\left._{i t}\right)-1, \forall i, t$

4e) $9999999 *$ threshold $_{i t} \leq\left(\right.$ totalCapacity $_{i t} /$ step $\left.5_{i t}\right)-1, \forall i, t$

4f) $9999999 *$ threshold6 $_{i t} \leq\left(\right.$ totalCapacity $_{i t} /$ step $\left._{i t}\right)-1, \forall i, t$

4g) $9999999 *$ threshold $_{i t} \leq\left(\right.$ totalCapacity $_{i t} /$ step $\left._{i t}\right)-1, \forall i, t$

4h) $9999999 *$ threshold $_{i t} \leq\left(\right.$ totalCapacity $_{i t} /$ step $\left._{i t}\right)-1, \forall i, t$

4i) $9999999 *$ threshold $_{i t} \leq\left(\right.$ totalCapacity $_{i t} /$ step $\left.{ }_{i t}\right)-1, \forall i, t$

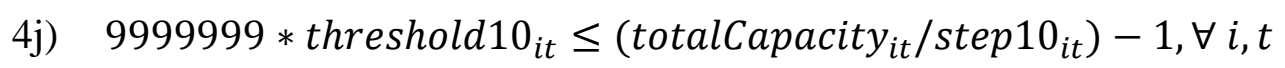

5a) threshold $1_{i t}+$ open $_{i t}+$ thresholdSwitch $1_{i t} \leq 2, \forall i, t$

5b) threshold $_{i t}+$ open $_{i t}+$ thresholdSwitch $2_{i t} \leq 2, \forall i, t$

5c) threshold $_{i t}+$ open $_{i t}+$ thresholdSwitch $_{i t} \leq 2, \forall i, t$

5d) threshold4 $_{i t}+$ open $_{i t}+$ thresholdSwitch4 $_{i t} \leq 2, \forall i, t$ 
5e) threshold5 $_{i t}+$ open $_{i t}+$ thresholdSwitch $_{i t} \leq 2, \forall i, t$

5f) threshold6 $_{i t}+$ open $_{i t}+$ thresholdSwitch $_{i t} \leq 2, \forall i, t$

5g) threshold7 $_{i t}+$ open $_{i t}+$ thresholdSwitch $_{i t} \leq 2, \forall i, t$

5h) threshold $_{i t}+$ open $_{i t}+$ thresholdSwitch $8_{i t} \leq 2, \forall i, t$

5i) threshold $_{i t}+$ open $_{i t}+$ thresholdSwitch $_{i t} \leq 2, \forall i, t$

5j) threshold $10_{i t}+$ open $_{i t}+$ thresholdSwitch $10_{i t} \leq 2, \forall i, t$

6a) threshold $1_{i t}-$ thresholdSwitch $1_{i t} \geq 0, \forall i, t$

6b) threshold $2_{i t}-$ thresholdSwitch $2_{i t} \geq 0, \forall i, t$

6c) threshold $3_{i t}-$ thresholdSwitch $3_{i t} \geq 0, \forall i, t$

6d) threshold $_{i t}-$ thresholdSwitch $_{i t} \geq 0, \forall i, t$

6e) threshold $5_{i t}-$ thresholdSwitch $5_{i t} \geq 0, \forall i, t$

6f) threshold6 ${ }_{i t}-$ thresholdSwitch6 $_{i t} \geq 0, \forall i, t$

6g) threshold7 $_{i t}-$ thresholdSwitch $_{i t} \geq 0, \forall i, t$

6h) threshold $8_{i t}-$ thresholdSwitch $_{i t} \geq 0, \forall i, t$

6i) threshold $9_{i t}-$ thresholdSwitch $_{i t} \geq 0, \forall i, t$

6j) threshold $10_{i t}-$ thresholdSwitch $10_{i t} \geq 0, \forall i, t$ 
7) totalFixCost

$$
\begin{aligned}
& =\text { open }_{i t} * \text { fixCost }_{i}+\text { stepCost }_{i t} *\left(\text { threshold } 1_{i t}\right. \\
& \left.- \text { thresholdSwitch } 1_{i t}\right)+ \text { stepCost } 2_{i t} *\left(\text { threshold } 2_{i t}\right. \\
& \left.- \text { thresholdSwitch } 2_{i t}\right)+\operatorname{stepCost} 3_{i t} *\left(\text { threshold } 3_{i t}\right. \\
& \left.- \text { thresholdSwitch } 3_{i t}\right)+\operatorname{stepCost} 4_{i t} *\left(\text { threshold } 4_{i t}\right. \\
& \left.- \text { thresholdSwitch } 4_{i t}\right)+\operatorname{stepCost} 5_{i t} *\left(\text { threshold } 5_{i t}\right. \\
& \left.- \text { thresholdSwitch } 5_{i t}\right)+\operatorname{stepCost} 6_{i t} *\left(\text { threshold } 6_{i t}\right. \\
& \left.- \text { thresholdSwitch } 6_{i t}\right)+\operatorname{stepCost} 7_{i t} *\left(\text { threshold } 7_{i t}\right. \\
& \left.- \text { thresholdSwitch } 7_{i t}\right)+\operatorname{stepCost} 8_{i t} *\left(\text { threshold } 8_{i t}\right. \\
& \left.- \text { thresholdSwitch } 8_{i t}\right)+\operatorname{stepCost} 9_{i t} *\left(\text { threshold } 9_{i t}\right. \\
& \left.- \text { thresholdSwitch } 9_{i t}\right)+\operatorname{stepCost~} 10_{i t} *\left(\text { threshold } 1_{0 i t}\right. \\
& \left.- \text { thresholdSwitch } 10_{i t}\right), \forall i, t
\end{aligned}
$$

7)

$$
\sum_{p=1}^{13} \text { usedCapacity }_{i t p} \leq \text { totalCapacity }_{i t}, \forall i, t
$$

9)

$$
\text { usedCapacity }_{i t p} \leq \sum_{j=1}^{170} \text { assignment }_{i j t} * \text { demand }_{j t p}, \quad \forall i, t, p
$$

10) totalCapacity $_{i t+1}=$ totalCapacity $_{i t}+u$ Plus $_{i t}-u$ Minus $_{i t}, \quad \forall i, t$

11) open $_{i t+1}=$ open $_{i t}+$ opened $_{i t}-$ closed $_{i t}, \forall i, t$

12) $9999999 *$ expanded $_{i t} \geq u$ Plus $_{i t}, \forall i, t$

13) $9999999 * \operatorname{shrunk}_{i t} \geq$ uMinus $_{i t}, \forall i, t$

14) totalCapacity $_{i t} \geq$ uMinus $_{i t}, \forall i, t, p$ 
16) shrunk $_{i t p} \leq$ open $_{i t}, \forall i, t, p$

17) $9999999 *$ open $_{i t} \geq$ usedCapacity $_{i t}, \forall i, t$

18) $\sum_{i=1}^{36}$ assignment $_{i j t} \leq 1 \forall j, t$

19) assignment $_{i j t p}$, expanded ${ }_{i t p}$, shrunk $_{i t p}$, open $_{i t}$, opened $_{i t}$, closed $_{i t}$

20) uPlus $_{i t p}$, uMinus $_{i t p}, \quad$ totalCapacity $_{i t p}, \quad$ usedCapacity $_{i t p} \in \mathbb{N}, \forall i, t$

The objective function maximizes the profit. It consists of six terms. The first term computes the net profit. The second term includes the fixed cost component when a facility is open, which is also determined by the step cost function. The third and fourth terms are for assigning a closing or opening cost that occurs if a facility is opened or closed, respectively. The last two terms consider the expansion or shrinkage cost. Constraints (1) and (2) are used to initialize the open status of each facility as well as the initial capacity. Constraint (3), (4), (5) and (6) are used for the step cost function. The total cost occurring in the relevant period $t$ at facility $i$ is calculated in constraint (7). Usage must never be greater than the capacity. This is ensured by constraint (8). This constraint is modified slightly such that all products are competing for the whole capacity in a facility. Constraint (9) addresses the usage again given that usage must never exceed the assigned demands. The capacity of the facilities might vary over time. Constraint (10) computes the capacity for the following period by adding the units of expansion or subtracting the units of shrinkage from the current. Constraint (11) is a key constraint and 
is used for setting the status of a facility to open or close. Constraint (12) sets expanded $_{i t}$ to 1 if the facility is expanded. Constraint (13) does the same for the contraction case. Constraint (14) ensures that the capacity will never become negative. Constraints (15) and (16) assert that only open facilities can be contracted or expanded, respectively. Constraint (17) ensures that capacity will only be used if a facility is open. Constraint (18) guarantees a maximum of one assignment per demand.

The corresponding LINGO model can be found in Appendix I LINGO file for case study model.

\subsubsection{Computational results}

Scenario 1: The problem uses 76,239 variables. It could not be solved in a reasonable time. First a decomposition approach was applied by splitting up the problem into 13 subproblems and trying to solve for each product separately. The decomposition did not greatly reduce complexity. Each of the sub-models still consists of 71,919 variables because most of the complexity is derived by facilities and demand points, and not products. As discussed in Chapter 4, decomposition by demand will adjust the capacity of the facilities to the demand of the certain demand point. Thus, a decomposition approach by facilities was applied. 36 sub-models were created, except for facility $1(2,111$ variables) consisting of 2,120 variables. Solving times for each of the sub-models differ greatly. Several sub-models could were not solved in less than a day. The model assumes a facility cannot be reopened again once it has been closed. Thus 10 potential solutions for the open status are possible. Each of the configurations was applied to the sub-model 
by adding additional constraints for the opening status, the solutions were obtained and the configuration with the highest objective function value was taken.

\begin{tabular}{|ccccccccccc|}
\hline & \multicolumn{10}{c|}{ period } \\
\hline Configuration & $\mathbf{1}$ & $\mathbf{2}$ & $\mathbf{3}$ & $\mathbf{4}$ & $\mathbf{5}$ & $\mathbf{6}$ & $\mathbf{7}$ & $\mathbf{8}$ & $\mathbf{9}$ & $\mathbf{1 0}$ \\
\hline 1 & 1 & 1 & 1 & 1 & 1 & 1 & 1 & 1 & 1 & 1 \\
\hline 2 & 1 & 1 & 1 & 1 & 1 & 1 & 1 & 1 & 1 & 0 \\
\hline 3 & 1 & 1 & 1 & 1 & 1 & 1 & 1 & 1 & 0 & 0 \\
\hline 4 & 1 & 1 & 1 & 1 & 1 & 1 & 1 & 0 & 0 & 0 \\
\hline 5 & 1 & 1 & 1 & 1 & 1 & 1 & 0 & 0 & 0 & 0 \\
\hline 6 & 1 & 1 & 1 & 1 & 1 & 0 & 0 & 0 & 0 & 0 \\
\hline 7 & 1 & 1 & 1 & 1 & 0 & 0 & 0 & 0 & 0 & 0 \\
\hline 8 & 1 & 1 & 1 & 0 & 0 & 0 & 0 & 0 & 0 & 0 \\
\hline 9 & 1 & 1 & 0 & 0 & 0 & 0 & 0 & 0 & 0 & 0 \\
\hline 10 & 1 & 0 & 0 & 0 & 0 & 0 & 0 & 0 & 0 & 0 \\
\hline
\end{tabular}

The following flow chart describes the procedure. 


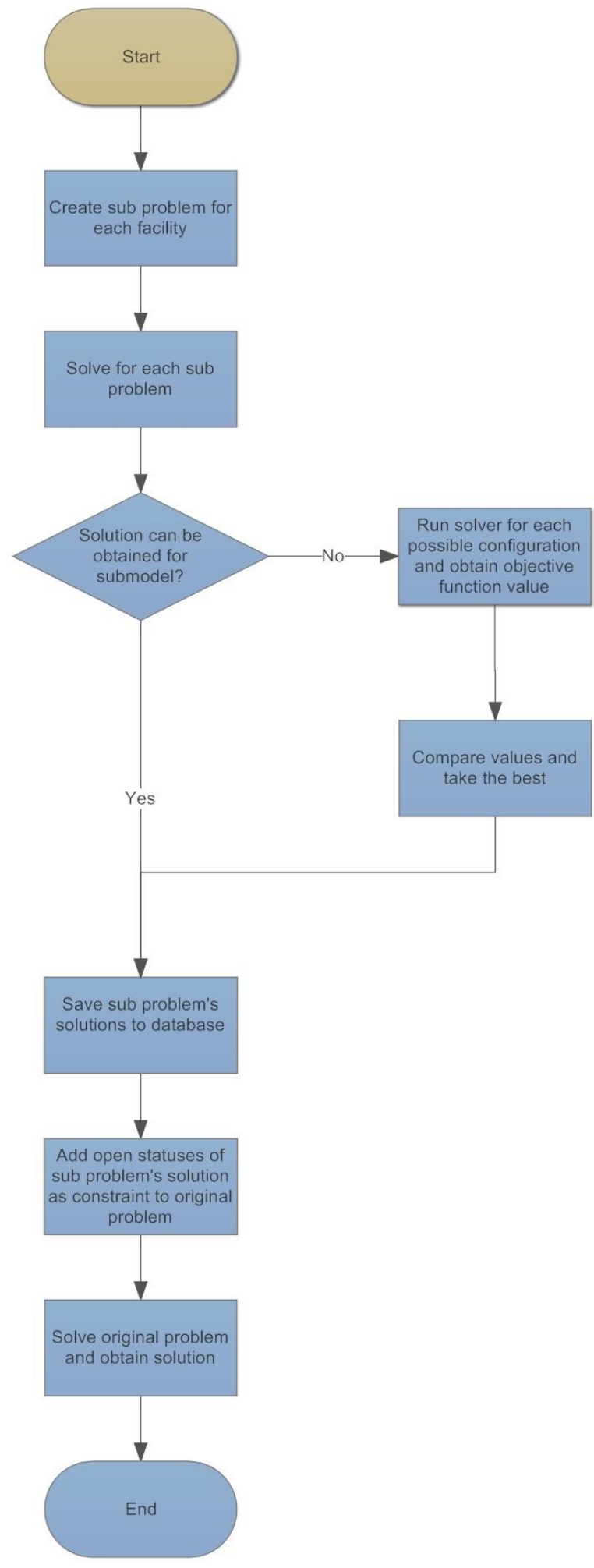

Figure 13 Procedure for solving sub-problems in case study 
The solutions for the sub-problems were obtained. The results for the corresponding open statuses were written into the database.

Table 39 Open status for sub problems

$\begin{array}{lllllllllll}\text { INDEXT } & 1 & 2 & 3 & 4 & 5 & 6 & 7 & 8 & 9 & 10 \\ \text { F01 } & 1 & 1 & 1 & 1 & 1 & 1 & 1 & 1 & 1 & 1 \\ \text { F02 } & 1 & 1 & 1 & 1 & 1 & 1 & 1 & 1 & 1 & 1 \\ \text { F03 } & 1 & 1 & 1 & 1 & 1 & 1 & 1 & 1 & 1 & 1 \\ \text { F04 } & 1 & 1 & 1 & 1 & 1 & 1 & 1 & 1 & 1 & 1 \\ \text { F05 } & 1 & 1 & 1 & 1 & 1 & 1 & 1 & 1 & 1 & 1 \\ \text { F06 } & 1 & 1 & 1 & 1 & 1 & 1 & 1 & 1 & 1 & 1 \\ \text { F07 } & 1 & 1 & 1 & 1 & 1 & 1 & 1 & 1 & 1 & 1 \\ \text { F08 } & 1 & 1 & 1 & 1 & 1 & 1 & 1 & 1 & 1 & 1 \\ \text { F09 } & 1 & 1 & 1 & 1 & 1 & 1 & 1 & 1 & 1 & 1 \\ \text { F10 } & 1 & 1 & 1 & 1 & 1 & 1 & 1 & 1 & 1 & 1 \\ \text { F11 } & 1 & 1 & 1 & 1 & 1 & 1 & 1 & 1 & 1 & 1 \\ \text { F12 } & 1 & 1 & 1 & 1 & 1 & 1 & 1 & 1 & 1 & 1 \\ \text { F13 } & 1 & 1 & 1 & 1 & 1 & 1 & 1 & 1 & 1 & 1 \\ \text { F14 } & 1 & 1 & 1 & 1 & 1 & 1 & 1 & 1 & 0 & 0 \\ \text { F15 } & 1 & 0 & 0 & 0 & 0 & 0 & 0 & 0 & 0 & 0 \\ \text { F16 } & 1 & 0 & 0 & 0 & 0 & 0 & 0 & 0 & 0 & 0 \\ \text { F17 } & 1 & 1 & 1 & 1 & 1 & 1 & 1 & 1 & 1 & 1 \\ \text { F18 } & 1 & 1 & 1 & 1 & 1 & 1 & 1 & 1 & 1 & 1 \\ \text { F19 } & 1 & 1 & 1 & 1 & 1 & 1 & 1 & 1 & 1 & 1 \\ \text { F20 } & 1 & 0 & 0 & 0 & 0 & 0 & 0 & 0 & 0 & 0 \\ \text { F21 } & 1 & 0 & 0 & 0 & 0 & 0 & 0 & 0 & 0 & 0 \\ \text { F22 } & 1 & 0 & 0 & 0 & 0 & 0 & 0 & 0 & 0 & 0 \\ \text { F23 } & 1 & 1 & 1 & 1 & 1 & 1 & 1 & 1 & 1 & 1 \\ \text { F24 } & 1 & 1 & 1 & 1 & 1 & 1 & 1 & 1 & 1 & 1 \\ \text { F25 } & 1 & 0 & 0 & 0 & 0 & 0 & 0 & 0 & 0 & 0 \\ \text { F26 } & 1 & 1 & 1 & 1 & 1 & 1 & 1 & 1 & 1 & 1 \\ \text { F27 } & 1 & 1 & 1 & 1 & 1 & 1 & 1 & 1 & 1 & 1 \\ \text { F28 } & 1 & 1 & 1 & 1 & 1 & 1 & 1 & 1 & 1 & 1 \\ \text { F29 } & 1 & 1 & 1 & 1 & 1 & 1 & 1 & 1 & 1 & 1 \\ \text { F30 } & 1 & 1 & 1 & 1 & 1 & 1 & 1 & 1 & 1 & 1 \\ \text { F31 } & 1 & 1 & 1 & 1 & 1 & 1 & 1 & 1 & 1 & 1 \\ \text { F32 } & 1 & 1 & 1 & 1 & 1 & 1 & 1 & 1 & 1 & 1 \\ \text { F33 } & 1 & 0 & 0 & 0 & 0 & 0 & 0 & 0 & 0 & 0 \\ \text { F34 } & 1 & 1 & 1 & 1 & 1 & 1 & 1 & 1 & 1 & 1 \\ \text { F35 } & 1 & 1 & 1 & 1 & 1 & 1 & 1 & 1 & 1 & 1 \\ \text { F36 } & 1 & 1 & 1 & 1 & 1 & 1 & 1 & 1 & 1 & 1\end{array}$


The open statuses for all 36 sub-problems were added to the original problem and the original problem was solved. The following graphs illustrate the facility configuration in period 1 and in period 10. The locations are colored in blue, demand points are colored violet. Assignments are illustrated by the use of edges. Grey colored nodes point out closed facilities. The size of the nodes visualizes the total capacity. The figures were created by means Gephi ${ }^{4}$.

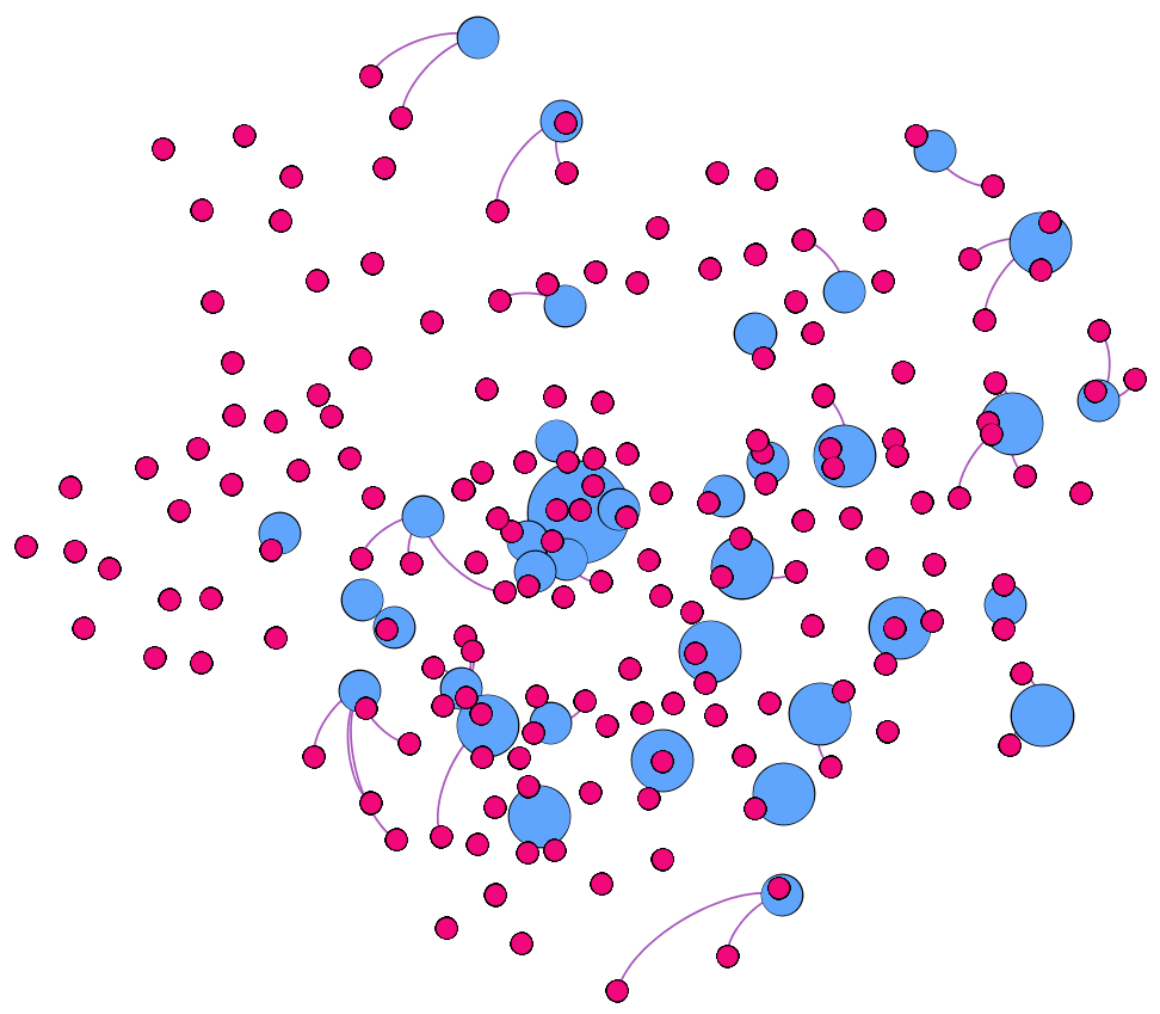

Figure 14 Scenario 1: Facility configuration and assigned demands in period 1

\footnotetext{
${ }^{4}$ https://gephi.org/
} 


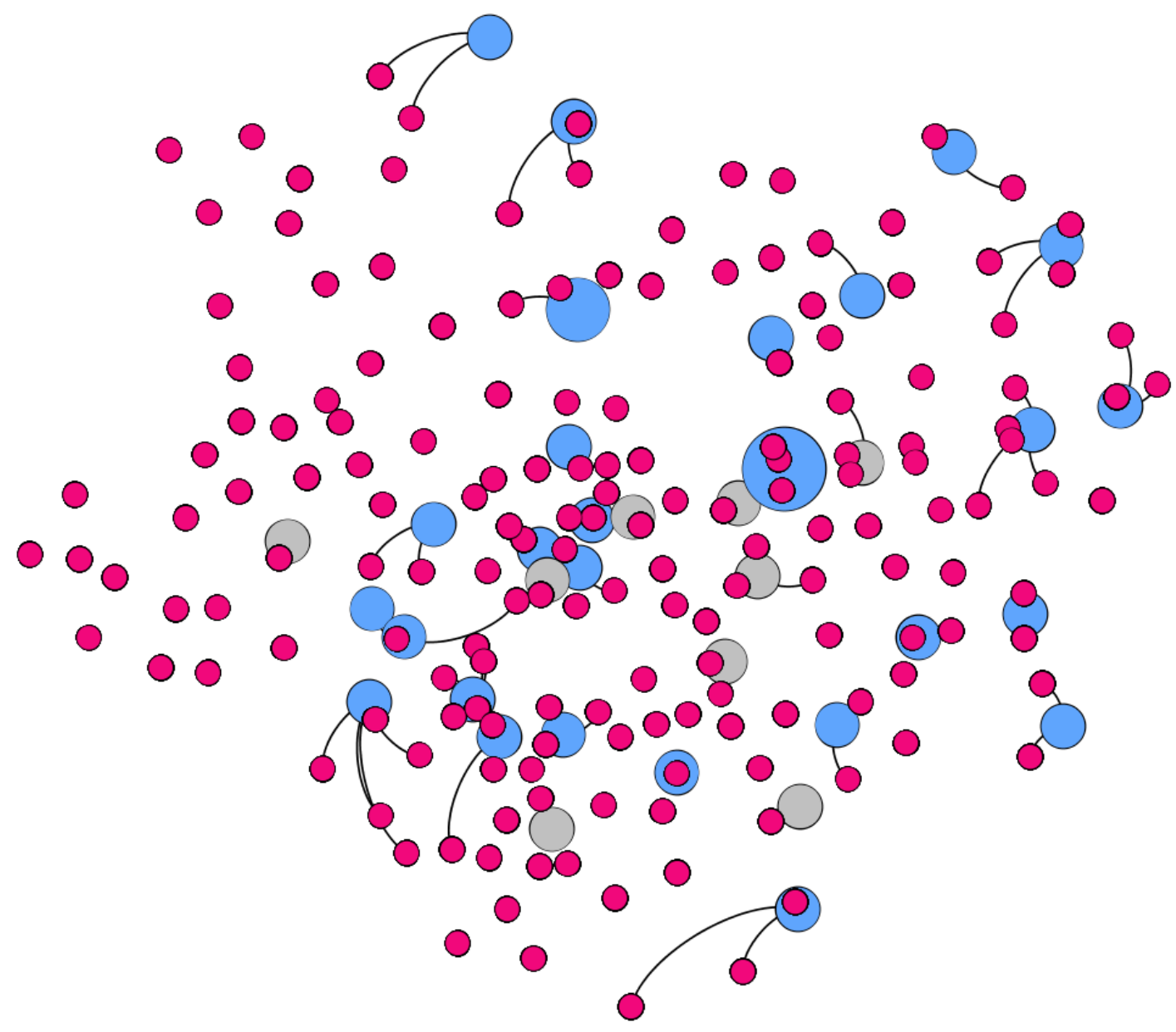

Figure 15 Scenario 1: Facility configuration and assigned demands in period 10

The model closes down 8 facilities in the sub-models. As the results of the sub-models require certain facilities to remain open, several facilities are contracted in the complete model and operated with the lowest capacity possible. The closing decisions differ from those made by Dortmunder Volksbank. The relevant facilities are still assigned demands even in period 10 of the model. 
Scenario 2: The problem uses 69,885 variables. It could also not be solved in a reasonable amount of time. The open status of the relevant 33 remaining facilities was taken from the decomposition made for Scenario 1. The model was run including the solutions of the sub-problems. A solution was obtained in less than 5 minutes. Demand assignment differs over the periods. The facility sizes change over time and are almost all reduced to the minimum size by the end of period 10 .

Table 40 Open status for sub-problems

\begin{tabular}{|c|c|c|c|c|c|c|c|c|c|}
\hline $\begin{array}{l}\text { INDEXT } \\
\text { F01 }\end{array}$ & $\begin{array}{l}1 \\
1\end{array}$ & & $\begin{array}{l}3 \\
1\end{array}$ & & & & & & 1 \\
\hline F02 & 1 & 1 & 1 & 1 & & & & & 1 \\
\hline F03 & 1 & 1 & 1 & 1 & 1 & 1 & & 1 & 1 \\
\hline F04 & 1 & 1 & 1 & 1 & 1 & 1 & 1 & 1 & 1 \\
\hline F05 & 1 & & & 1 & 1 & 1 & 1 & 1 & 1 \\
\hline F06 & 1 & 1 & & 1 & 1 & 1 & 1 & 1 & 1 \\
\hline F07 & 1 & 1 & & 1 & 1 & 1 & 1 & 1 & 1 \\
\hline F08 & 1 & 1 & 1 & 1 & 1 & 1 & 1 & 1 & 1 \\
\hline F09 & 1 & 1 & 1 & 1 & 1 & 1 & 1 & 1 & 1 \\
\hline F10 & 1 & 1 & 1 & 1 & 1 & 1 & 1 & 1 & 1 \\
\hline F11 & 1 & 1 & 1 & 1 & 1 & 1 & 1 & 1 & 1 \\
\hline $\mathrm{F} 12$ & 1 & 1 & 1 & 1 & 1 & 1 & 1 & 1 & 1 \\
\hline F13 & 1 & 1 & 1 & 1 & 1 & 1 & 1 & 1 & 1 \\
\hline F14 & 1 & 1 & 1 & 1 & 1 & 1 & 1 & 1 & 0 \\
\hline F15 & 1 & 0 & 0 & 0 & 0 & 0 & 0 & 0 & 0 \\
\hline F16 & 1 & 0 & 0 & 0 & 0 & 0 & 0 & 0 & 0 \\
\hline F17 & 1 & 1 & 1 & 1 & 1 & 1 & 1 & 1 & 1 \\
\hline F18 & 1 & 1 & 1 & 1 & 1 & 1 & 1 & 1 & 1 \\
\hline F19 & 1 & 1 & 1 & 1 & 1 & 1 & 1 & 1 & 1 \\
\hline F20 & 1 & 0 & 0 & 0 & 0 & 0 & 0 & 0 & 0 \\
\hline F21 & 1 & 0 & 0 & 0 & 0 & 0 & 0 & 0 & 0 \\
\hline F22 & 1 & 0 & 0 & 0 & 0 & 0 & 0 & 0 & 0 \\
\hline F23 & 1 & 1 & 1 & 1 & 1 & 1 & 1 & 1 & 1 \\
\hline F24 & 1 & 1 & 1 & 1 & 1 & 1 & 1 & 1 & 1 \\
\hline F25 & 1 & 0 & 0 & 0 & 0 & 0 & 0 & 0 & 0 \\
\hline F26 & 1 & 1 & 1 & 1 & 1 & 1 & & 1 & 1 \\
\hline F27 & 1 & 1 & 1 & 1 & 1 & 1 & & 1 & 1 \\
\hline F28 & 1 & 1 & 1 & 1 & 1 & 1 & & & 1 \\
\hline F29 & 1 & 1 & 1 & 1 & 1 & 1 & & & 1 \\
\hline
\end{tabular}




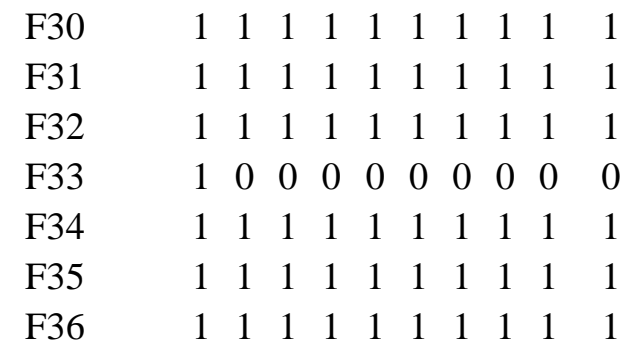

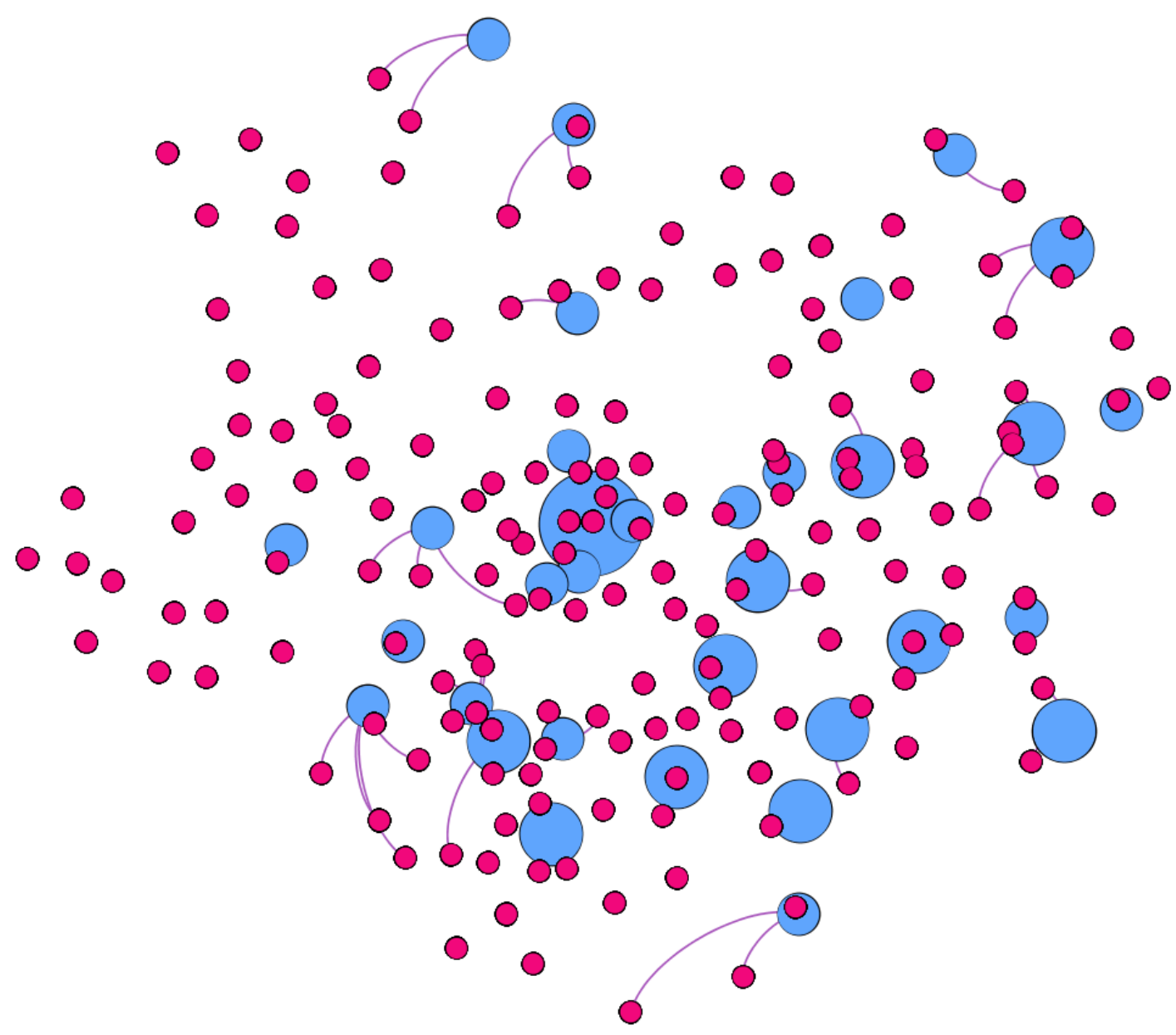

Figure 16 Scenario 2: Facility configuration and assigned demands in period 1 


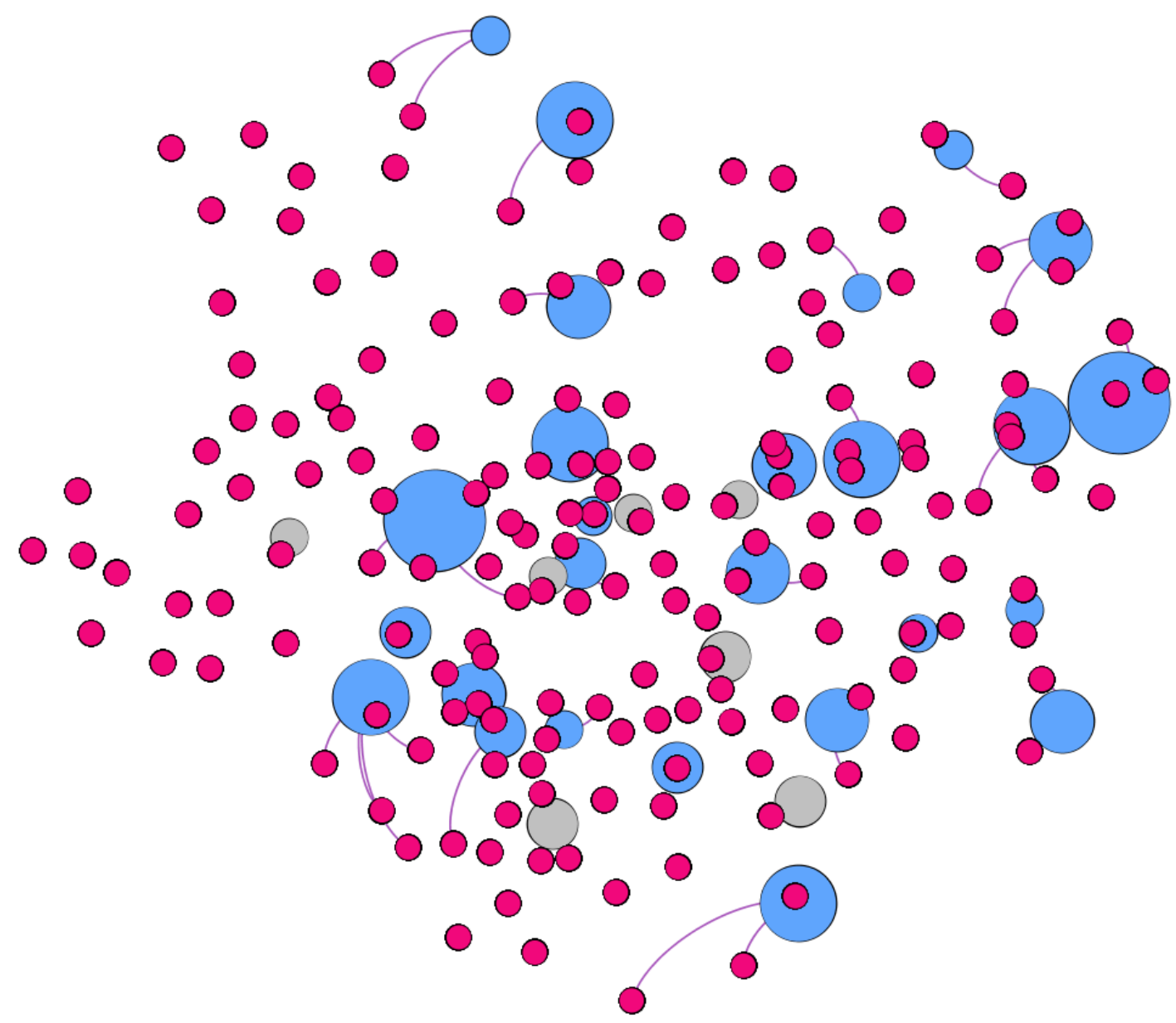

Figure 17 Scenario 2: Facility configuration and assigned demands in period 5 


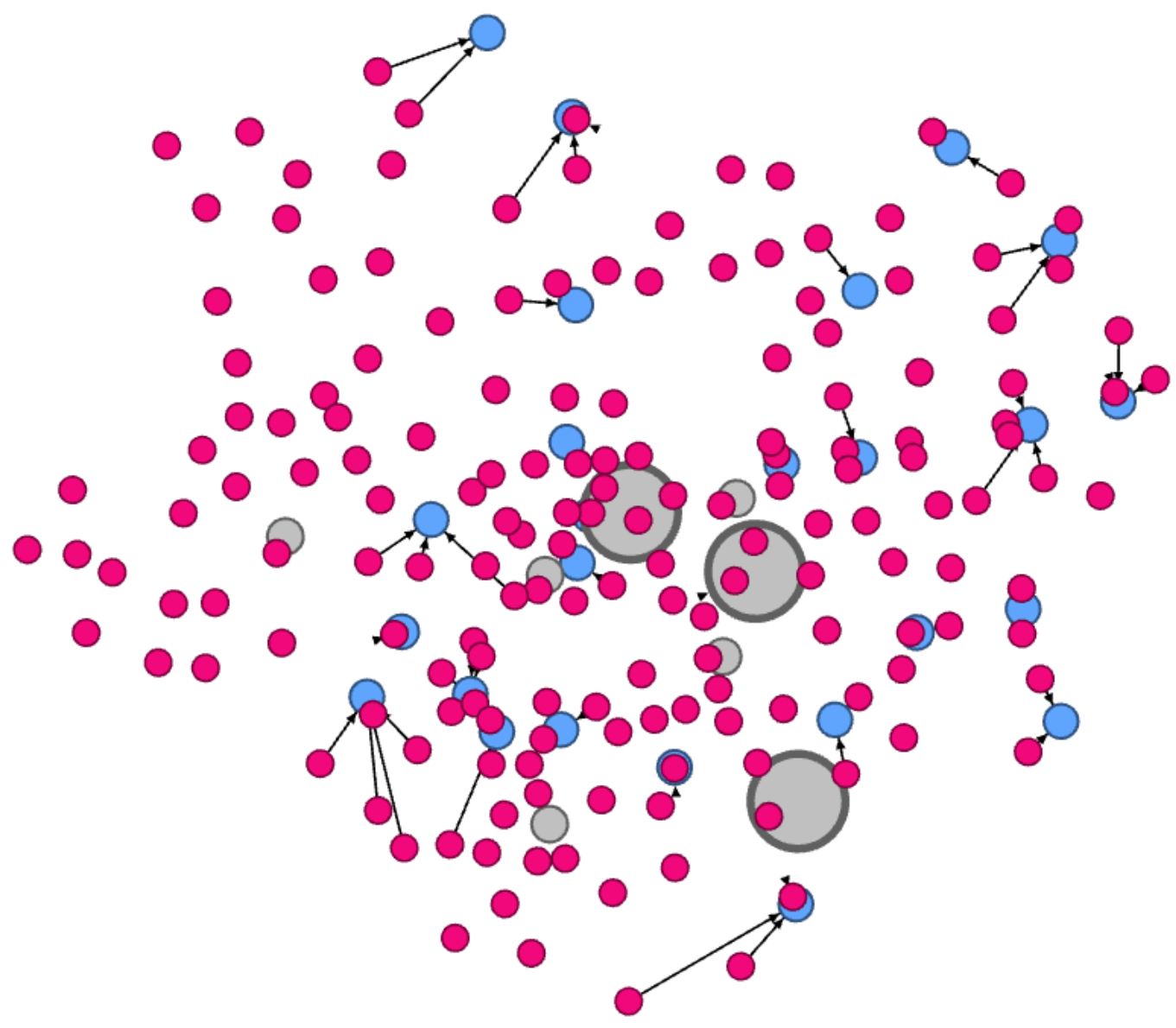

Figure 18 Scenario 2: Facility configuration and assigned demands in period 10

Scenario 3: The problem consists of 91,065 variables. A feasible solution was gathered using the decomposition and LINGO. The following table shows the result of the subproblems for the additional locations. 
Table 41 Open status for potential new locations

\begin{tabular}{|l|lllllllllll|}
\hline INDEXT & \multicolumn{1}{c}{2} & \multicolumn{1}{c}{3} & \multicolumn{1}{c}{5} & 5 & 7 & 8 & 9 & 10 \\
\hline NL 1 & 1 & 1 & 1 & 1 & 1 & 1 & 1 & 1 & 1 & 1 \\
\hline NL 2 & 1 & 0 & 0 & 0 & 0 & 0 & 0 & 0 & 0 & 0 \\
\hline NL 3 & 1 & 1 & 1 & 1 & 1 & 1 & 1 & 1 & 1 & 1 \\
\hline NL 4 & 1 & 1 & 1 & 1 & 1 & 1 & 1 & 1 & 1 & 1 \\
\hline NL 5 & 1 & 0 & 0 & 0 & 0 & 0 & 0 & 0 & 0 & 0 \\
\hline NL 6 & 1 & 1 & 1 & 1 & 1 & 1 & 1 & 1 & 1 & 1 \\
\hline NL 7 & 1 & 1 & 1 & 1 & 1 & 1 & 1 & 1 & 1 & 1 \\
\hline NL 8 & 1 & 1 & 1 & 1 & 1 & 1 & 1 & 1 & 1 & 1 \\
\hline NL 9 & 1 & 1 & 1 & 1 & 1 & 1 & 1 & 1 & 1 & 1 \\
\hline NL 10 & 1 & 1 & 1 & 1 & 1 & 1 & 1 & 1 & 1 & 1 \\
\hline
\end{tabular}

The feasible solution shows an objective function value of $0.31 \mathrm{E} 08$ which is better than the objective function value of the value for scenario 2, also ending up with low capacities for the new facilities.

\subsubsection{Applying simulated annealing}

Another attempt to solve the problem was made using simulated annealing. The SA algorithm developed in Chapter 5 allows a facility to be reopened again if it has previously been closed. The banking application does not allow a facility to be opened if it was closed in all the following periods. The SA algorithm had to be modified respectively.

The following pseudo code demonstrates how the adjusted algorithm works.

Algorithm simulatedAnnealing (temperature, cooling Rate)

Begin

Step 1, Initialization 
startConfiguration $=$ feasibleConfiguration;

bestEnergy = getEnergy(startConfiguration);

bestConfiguration $=$ startConfiguration;

currentConfiguration $=$ startConfiguration;

currentEnergy = bestEnergy;

Step 2, Annealing

While temperature $>1$ Do

neighborConfiguration $=$ createNewConfiguration $($ currentConfiguration $)$; neighborEnergy = getEnergy(neighborConfiguration);

If getAcceptanceProbability > random number Then

currentConfiguration $=$ neighborConfiguration;

currentEnergy = neighborEnergy;

\section{End If}

If currentEnergy > bestEnergy Then

bestConfiguration $=$ currentConfiguration;

bestEnergy = currentEnergy;

\section{End If}

temperature *=1-coolingRate;

\section{End While}

Return bestEnergy, bestConfiguration;

\section{End}

Procedure getAcceptanceProbability (energy, newEnergy, temperature)

Begin Procedure 
If newEnergy > energy then

Return 1.0

Else

$$
\text { Return } e^{\frac{-(\text { energy-newEnergy })}{\text { temperature }}}
$$

End if

\section{End Procedure}

Procedure createNewConfiguration (currentConfiguration)

indexi $=$ RandonNumber $*$ facilities;

indext $=0$;

While indext $=0$ Do

indext $=$ RandonNumber $*$ periods;

\section{End While}

newConfiguration $=$ currentConfiguration;

For each $\mathrm{t} 1:=$ indext; $\mathrm{t} 1<$ periods Do

newConfiguration[indexi][ $\mathrm{t} 1]=0$;

\section{End For}

For each $\mathrm{t} 2:=$ indext; $\mathrm{t} 1<=$ indext Do

newConfiguration[indexi] $[\mathrm{t} 2]=1$;

\section{End For}

return newConfiguration;

\section{End Procedure}

\section{Procedure getEnergy(configuration)}

Load the LINGO model;

Retrieve variable indices;

For each variableIndex Do 
Add constraint from configuration;

\section{End For}

Solve the model;

Return objective function value;

\section{End Procedure}

The corresponding JAVA code can be found in Appendix J.

Solving the problem was not possible within 100 hours. In order to get an upper bound, the relaxed model was passed to the SA algorithm. The SA algorithm was run using two different initial configurations. The first declared all facilities open for all periods. The second declared all facilities closed for all peridos except period $1 .$. The headquarter $(i=$ 1) was also declared to be open for all periods.

The SA algorithm was run with a temperature of $30,000,000$ and a cooling rate of 0.01 . It is obvious that the objective function value having all facilities open is much better than starting with having all facilities except the headquarter closed after the first period.

\subsection{Conclusion}

Dortmunder Volksbank is faced with a significant change of business and as a consequence, their entire facility landscape. The results show that the facility model is complex. Closure decisions in the past are not confirmed by the model. The complete model depends on the competition. As soon as a competitor is removed, the model shows 
a different behavior. The same thing happens after closure of one of the bank's own facility.

Solving the model for each facility using a decomposition approach could be run in a reasonable time. It is important to combine the solutions carefully. Solving for each facility resulted in assigning several demands more than once. Thus, it could be part of an additional analysis to compose the solutions of the sub-problems in a different way.

Keeping the option for opening additional facilities gives the entire model more degrees of freedom. If a facility has to be closed, in most cases the best objective value can be obtained when the closure is done as soon as possible.

Simulated annealing is not easy to apply to the model, as the problem is complex. The algorithm had to be modified when a facility is not re-opened.

Furthermore it is to be expected that customers will patronize a facility not only because of its proximity. Several other aspects need to be considered and are currently not incorporated in the model. Finally the model can show certain recommendations for the facility settings. 


\section{CHAPTER 7}

\section{CONCLUSIONS}

\subsection{Contributions}

This dissertation provides several contributions.

1) First, the models presented provide an extension of existing facility location models that can be applied to a variety of location problems in commercial and industry sectors that need to make their decisions considering future time periods and competitors. An initial model focusing on a single product was developed. The model was extended by including multiple products. The developed heuristic shows multiple options for solving the problem including the advantages and disadvantages, respectively. The JAVA code and LINGO fragments thus developed can be used to provide easy access for related problems.

2) The case study shows the application of the problem in a real world environment. Slight modifications make it easy to customize the model to the area of interest. Given the real data of Dortmunder Volksbank, several solution approaches and scenarios give detailed advice for the bank's facility decisions. 


\subsection{Further Works}

The research in this dissertation gives direction to further topics that are of great interest and can be very useful for the daily application of the model.

\subsubsection{Extension of the attraction function}

The attraction parameter plays a central role in the assignment of a facility. The model takes it as a given parameter. The case study computes the parameter using the distances. Facility attraction depends on several factors. The model can be extended by setting up an attraction function which incorporates additional parameters and may change over time - depending on the decisions made by the model. A utility function can be used for model purposes.

\subsubsection{Incorporating additional constraints}

The current model uses several constraints. With respect to certain applications, additional constraints may become necessary, such as subsidies, taxes, space limitations, laws, etc. The development of these constraints will make the model more realistic, but for every problem the model needs to be adjusted to the relevant use case.

\subsubsection{Combination of solutions}

Taking additional analysis into account - such as the location of ATMs, the combination of analyses will give the model and other analyses additional value. The location of ATMs was not incorporated in the case study, but it is of greater interest to find out 
where they may located best. A facility location will undoubtedly also influence the location of an ATM. Dependency of the two models is to be expected. 


\section{REFERENCES}

Aboolian, R., Berman, O., \& Krass, D. (2006). Competitive facility location model with concave demand. European Journal of Operations Research, 181(2).

Akinc, U., \& Khumuwala, B. M. (1977). An Efficient Branch and Bound Algorithm for the Capacitated Warehouse Location Problem. Management Science, 23, pp. 585594.

Alter, P. D. (2012, April). Schlecker, oder: Geiz ist dumm - Aufstieg und Absturz eines Milliardärs. Heilbronn.

Arrondo, A., Fernández, J., Redondo, J. L., \& Ortigosa, P. M. (2014). An approach for solving competitive location problems with variable demand using multicore systems. Optim Lett, pp. 555-567.

Ashtiani, M. G., Makui, A., \& Ramezanian, R. (2013). A robust model for a leaderfollower competitive facility location problem in a discrete space. Applied Mathematial Modelling, 37(1-2), pp. 62-71.

Ballou, R. H. (1968). Dynamic warehouse location analysis. Journal of Marketing, 5(3), pp. 271-276.

Bhaumik, P. K. (2010). Optimal shrinking of the distribution chain: the facilities delocation decision. International Journal of Systems Science, 41(3), pp. 271-280. 
Bloomstein, H., Nijkamp, P., \& van Veenendaal, W. (1980). Shopping Perceptions and Preferences: A Multidimensional Attractiveness Analysis of Consumer and Enterpreneurial Attitudes. Economic Geography, 56, pp. 155-174.

Brandeau, M. L., \& Chiu, S. S. (1989). An overview of representativ problems in location research. Management Science, 35(6).

Burnett, K. P. (1973). The Dimensions of Alternatives in Spatial Choice Processes. Geographical Analysis, 5, pp. 181-204.

Carrizosa, E., Conde, E., Munoz-Marquez, M., \& Puerto, J. (1995). The generalized weber problem with expected distances. 29(1), pp. 35-57.

Chandrasekaran, R., \& Daughety, A. (1981). Location on Tree Networks: P-Centre and P-Dispersion Problems. Mathematics of Operations Research, 6, pp. 50-57.

Christaller, W. (1933). Die zentralen Orte in Süddeutschland.

Cooper, L. L. (1972). The Transportation-Location Problem. Operations Research, 20(1), pp. 94-108.

Dasci, A., \& Laporte, G. (2005). An analytical approach to the facility location and capacity acquisition problem under demand uncertainty. (O. R. Ltd., Ed.) Journal of the Operational Research Society(56), pp. 397-405. 
Daskin, M. S. (2008, 3 28). What You Should Know About Location Modeling. Naval Research Logistics, 55(4), pp. 283-294.

Dierig, C., \& Seidel, H. (2013, 07 11). WELT. Retrieved from welt.de: https://www.welt.de/wirtschaft/article117956191/20-Prozent-auf-alles-brachPraktiker-das-Genick.html

Döring, T. (2014, 02 12). Handelsblatt. Retrieved from handelsblatt.de: http://www.handelsblatt.com/unternehmen/handel-konsumgueter/neuefluggesellschaften-die-wiedergeburt-der-regional-airlines/9464606.html

Drezner, T. (1994). Locationg a single new facility among existing, unequally attractive facilities. Journal of Regional Science, 34(2), pp. 237-252.

Drezner, T. $(1998,7)$. Location of multiple retail facilities with limited budget constraints — in continuous space. Journal of Retailing and Consumer Services, 5(3), pp. 173-184.

Drezner, T., \& Drezner, Z. (1998). Facility location in anticipation of future competition. Location Science, 6(1-4), pp. 155-173.

Drezner, T., \& Eiselt, H. A. (2002). Consumers in Competitive Location Models. (Z. Drezner, \& H. W. Hamacher, Eds.) Facility Location - Applications and Theory, pp. 151-169. 
Drezner, T., \& Furihata, T. (2000). Components of attractivenss of shopping malls - U.S. and Japan. Working Papaer, College of Business and Economics, California State University-Fullerton.

Drezner, Z., \& Hamacher, H. W. (2002). Facility Location. Berlin, Heidelberg: Springer Verlag.

Drezner, Z., Steiner, G., \& Wesolowsky, G. O. (1985). One-Facility Location with Rectilinear Tour Distances. Naval Research Logistics, 32(3), pp. 391-405.

Eckert, D. $(2014,10$ 07). Welt.de. Retrieved 12 2016, from https://www.welt.de/finanzen/geldanlage/article133000571/Geld-der-Deutschenvergammelt-auf-Girokonten.html

Efroymson, M. A., \& Ray, T. L. (1966). A Branch-Bound Algorithm for Plant Location. Operations Research, 17(3), pp. 361-368.

Eiselt, H. A., \& Laporte, G. (1989). Competitive spatial models. European Journal of Operational Research, 39(3), pp. 231-242.

Eiselt, H. A., Laporte, G., \& Thisse, J.-F. (1993). Competitive Location Models: A Framework and Bibliography. Transportation Science, 27(1), pp. 44-54.

Elzinga, J., \& Hearn, D. W. (1972). Geometrical Solutions for Some Minimax Location Problems. Transportation Science, 6, pp. 379-394. 
Francis, R. L., McGinnis, L. F., \& White, J. A. (1983). Locational Analysis. European Journal of Operational Research, 12, pp. 220-252.

Geoffrion, A. M. (1975). A Guide to Computer-Assisted Methods for Distribution Systems Planning. Sloan Management Rev., 16, pp. 17-41.

Goldman, A. J. (1971). Optimal Location in Simple Networks. Transportation Science, 5, pp. 212-221.

Gourdin, E., Labbé, M., \& Yaman, H. (2002). Telecommunication and Location. (Z. Drezner, \& H. W. Hamacher, Eds.) Facility Location - Applications and Theory, pp. 275-302.

Haase, K., \& Hoppe, M. (2008). Standortplanung unter Wettbewerb. Technische Universtität Dresden. Dresden: Die Professeron des Instituts für Wirtschaft und Verkehr.

Hakimi, S. L. (1965). Optimum distribution of switching centers in a communication network and some related graph theoretic problems. Operations Research 3, 13, pp. $462-475$.

Hamacher, H. W., \& Nickel, S. (1998). Classification of location models. Location Science, 6(1), pp. 229-242. 
Handler, G., \& Mirchandani, P. (1973). Location on networks: Theory and algorithms. Cambridge: MIT Press.

Ho, T.-H., Tang, C. S., \& Bell, D. R. (1998). Rational Shopping Behavior and the Option Value of Variable Pricing. Management Science, 44, pp. 145-160.

Hotelling, H. (1929). Stability in competition. The Economic Journal, 39, pp. 41-57.

http://plantclosings.com. (2012). Retrieved from Plant Closing News: http://plantclosings.com

Huff, D. L. (1964). Defining and estimating a trade area. Journal of Marketing, 28(3), pp. 34-38.

Isard, W. (1956). Location and Space-economy; a General Theory Relating to Industrial Location, Market Areas, Land Use, Trade, and Urban Structure.

Karstadt. (2006). Schaufenster Karstadt. Einblicke in 125 Jahre. Essen.

Kendall, D. G. (1953). Stochastic Processes Occurring in the Theory of Queues and their Analysis by the Method of the Imbedded Markov Chain. The Annals of Mathematical Statistics, 24(3), pp. 338-354.

Klincewicz, J. G., Luss, H., \& Yu, C.-S. (1988). A large-scale multilocation capacity planning model. European Journal of Operational Research, 34(2), pp. 178-190. 
Klose, A., \& Drexl, A. (2005). Facility location models for distribution system design. European Journal of Operation Research.

Küçükaydin , H., Aras, N., \& Altinel, I. K. (2012). A leader-follower game in competitive facility location. Computers \& Operations Research, 39(2), pp. 437448.

Küçükaydin, H., Aras, N., \& Altinel, I. (2011). A discrete cmpetitive facility location model with varibale attractiveness. Journal of the Operational Research Society(62), pp. 1726-1741.

Küçükaydin, H., Aras, N., \& Altinel, I. K. (2011). Competitive facility location problem with attractiveness adjustment of the follower: A bilevel programming model and its solution. European Journal of Operational research, 208(3), pp. 206-220.

Manne, A. S. (1967). Investments for Capacity Expansion. Cambridge, MA: MIT Press.

Melanchrinoudis, E., \& Min, H. (2000). The dynamic relocation and phase-out of a hybrid, two-echelon plant/warehousing facility: A multiple objective approach. European Journal of Operational Research, 123(1), pp. 1-15.

n-tv Nachrichtenfernsehen GmbH. (2013, 10 27). www.n-tv.de. Retrieved from n-tv.de: http://www.n-tv.de/wirtschaft/McKinsey-warnt-Einzelhaendler-article... 
Owen, S. H., \& Daskin, M. A. (1998). Strategic facility location: A review. European Journal of Operations Research, 111(3).

Plastria, F. (2002). Continous Covering Location Problems. (Z. Drezner, \& H. W. Hamacher, Eds.) Facility Location, pp. 37-79.

Plastria, F. (2005). Avoiding cannibalisation and/or competitor reaction in planar single facility location. Journal of the Operations Research Society of Japan, 48(2), pp. 148-157.

Press, W. H., Teukolsky, S. A., Vetterling, W. T., \& Flannery, B. P. (2007). Numerical Recipes in C. Cambridge University Press.

Redondo, J. L., Fernande'z, J., Arrondo, A. G., Garc1'a, I., \& Ortigosa, P. M. (2011, March 21). Fixed or variable demand? Does it matter when locating a facility? Omega - The international journal of Management Science, pp. 9-20.

ReVelle, C. S., \& H., A. E. (2005). Location analysis: A synthesis and survey. European Journal of Operations Research, 165(1).

ReVelle, C. S., Scholssberg, M., \& Williams, J. (2008). Solving the maximal covering location problem with heuristic concentration. Computers and Operations Research, 35(2), pp. 427-435. 
ReVelle, C., Murray, A. T., \& Serra, D. (2005). Location models for ceding market share and shrinking services. The International Journal Of Management Science, 36(5).

Ruiz-Hermández, D., Delgado-Gómez, D., \& López-Pascual, J. (2014). Restructuring bank networks after mergers and acquisitions: A capacitated delocation model for closing and resizing branches. Computers and Operational Research.

Spiegel Online. (2016, 07 17). Retrieved from Diese Deutsche-Bank-Filialen werden geschlossen: http://www.spiegel.de/wirtschaft/unternehmen/deutsche-bank-diesefilialen-werden-geschlossen-a-1103401.html

Stadt Dortmund, 3/Dez - Stabsstelle Dortmunder Statistik. (2015). Statistikatlas Dortmunder Stadtteile. Dortmund.

Süddeutsche Zeitung. (2013, 04 26). Retrieved from sueddeutschede: http://www.sueddeutsche.de/wirtschaft/autobauer-opel-schliesst-werk-bochumkomplett-1.1659323

van Roy, T. J., \& Erlenkotter, D. (1982). A Dual-Based Procedure for Dynamic Facility Location. Management Science, 28, pp. 1091-1105.

von Thünen, J. H. (1910). Der isolierte Staat in Beziehung auf Landwirtschaft und Nationalökonomie. Jena: Verlag von Gustav Fischer. 
Wang, Q., Batta, R., Bhadury, J., \& Rump, C. M. (2003). Budget constrained location problem with opening and closing of facilities. Computers \& Operations Research, 30(13), pp. 2047-2069.

Weber, A. (1909). Über den Standort der Industrie.

www.airliners.de. (2014, $01 \quad$ 10). Retrieved from www.airliners.de: http://www.airliners.de/fast-jeder-vierte-passagier-in-dortmund-ist-eingeschaeftsreisender/31136\#disqus_thread

Zhang, L., \& Rushton, G. (2008). Optimizing the size and locations of facilities in competitive multi-site service systems. Computers \& Operations Research, 35(2), pp. 327-338. 


\section{Appendix A The Lingo File of model 1}

Model :

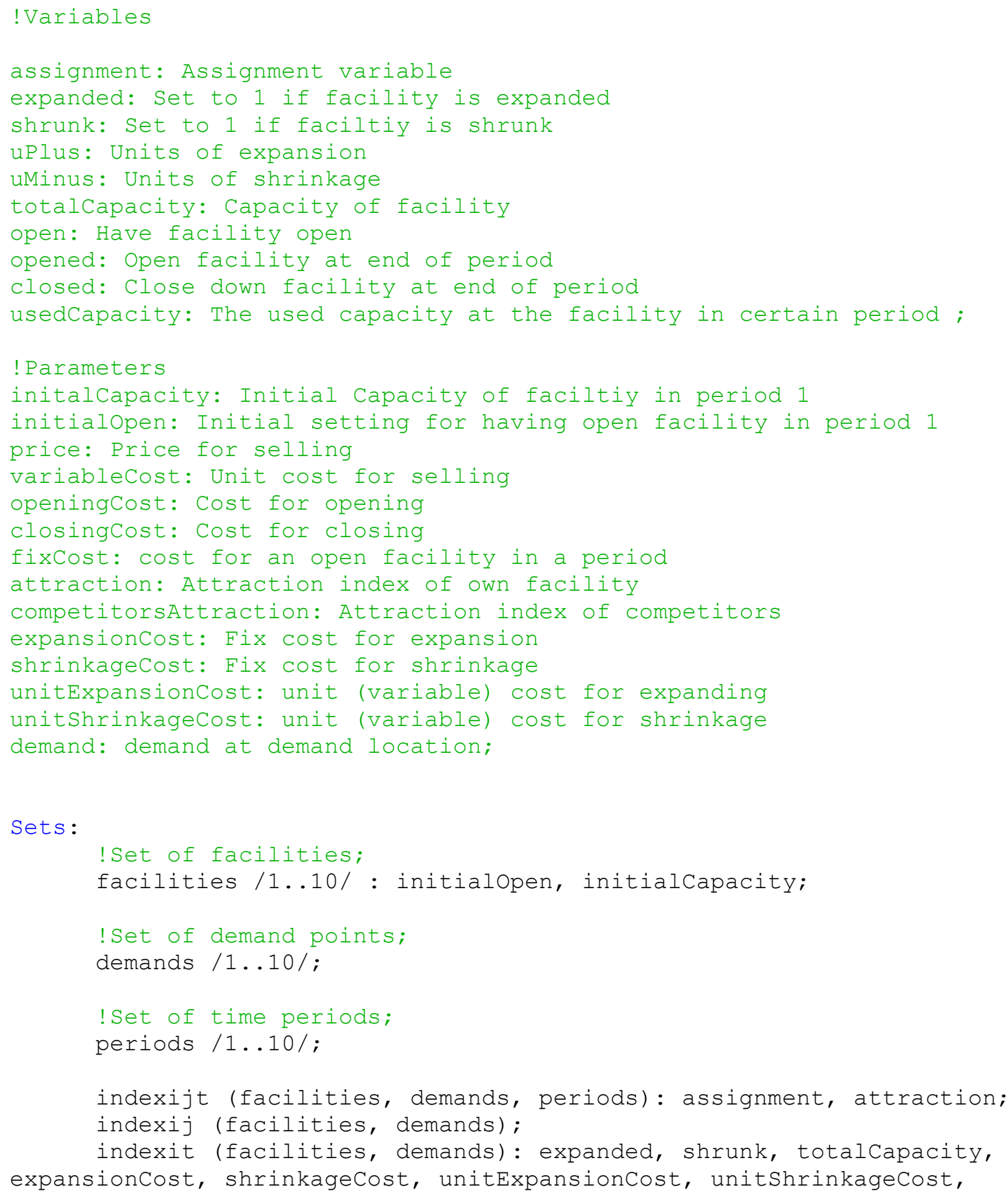


uplus, uMinus, usedCapacity, price, variablecost, fixcost, openingCost, closingCost, open, opened, closed;

indexjt (demands, periods): competitorsAttraction, demand;

Endsets

afor (facilities (i): defor (demands $(j)$ : afor (periods (t) :

abin (assignment ( $i, j, t))$;

abin (expanded $(i, t))$;

abin ( shrunk $(i, t))$;

abin (open $(i, t))$;

abin (opened $(i, t))$;

abin (closed $(i, t))$;

@gin (uPlus $(i, t))$;

@gin (uMinus $(i, t))$;

@gin (totalCapacity $(i, t))$;

))) ;

Data:

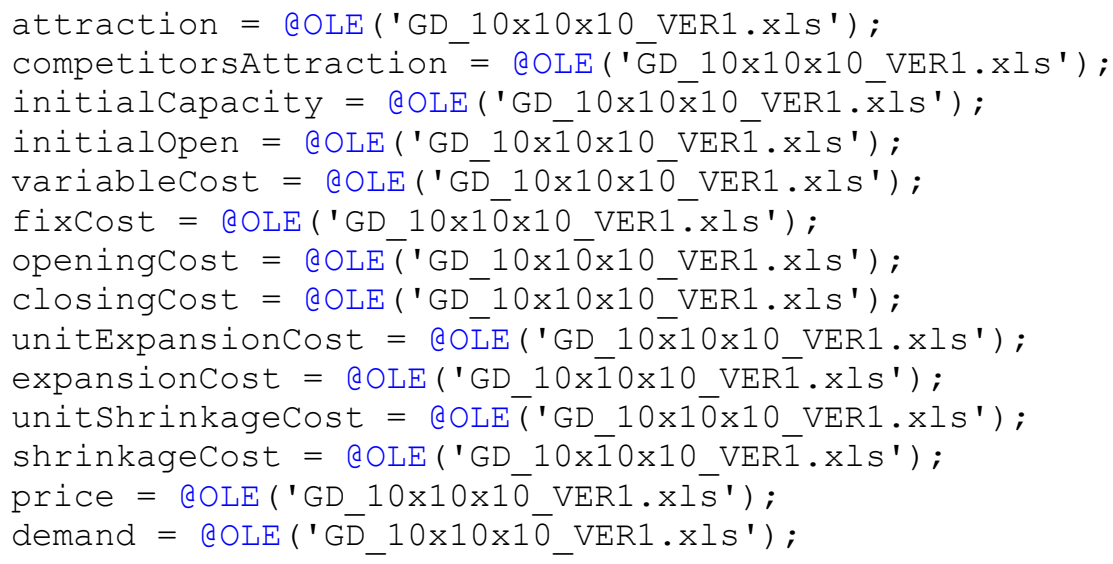

enddata

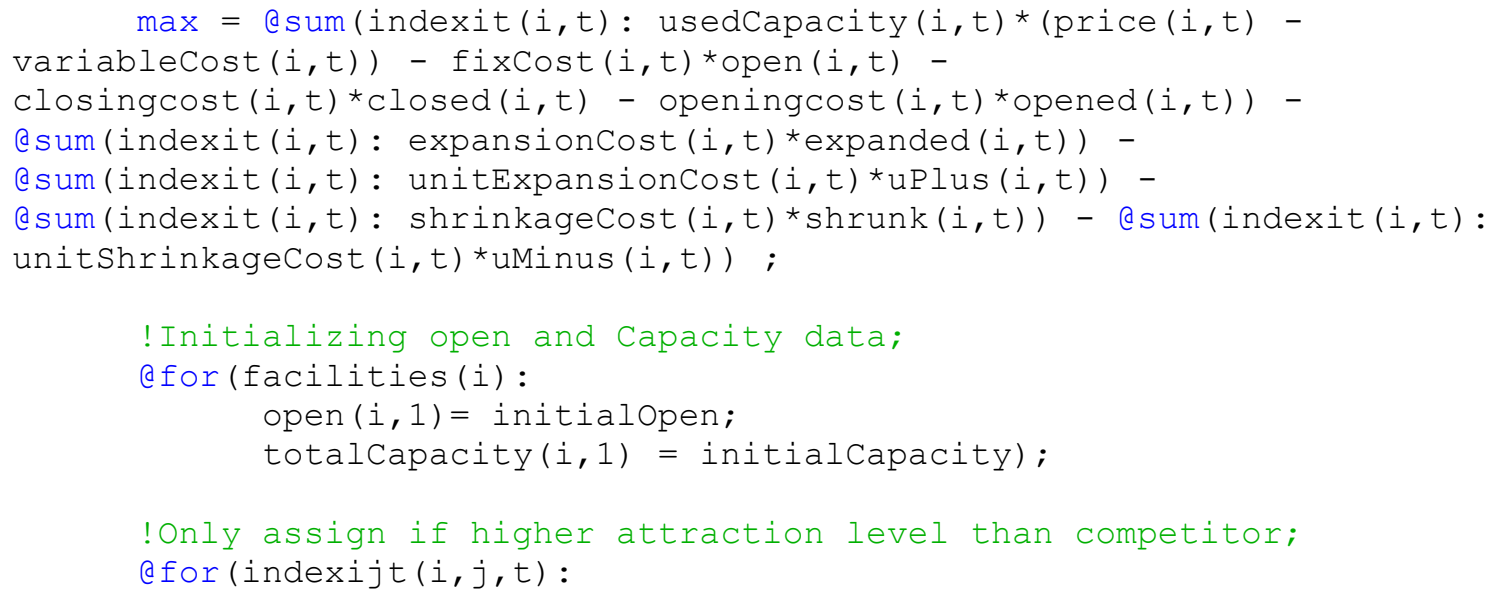


(assignment $(i, j, t))$ * (attraction $(i, j, t)-$

competitorsAttraction $(j, t))>=0)$;

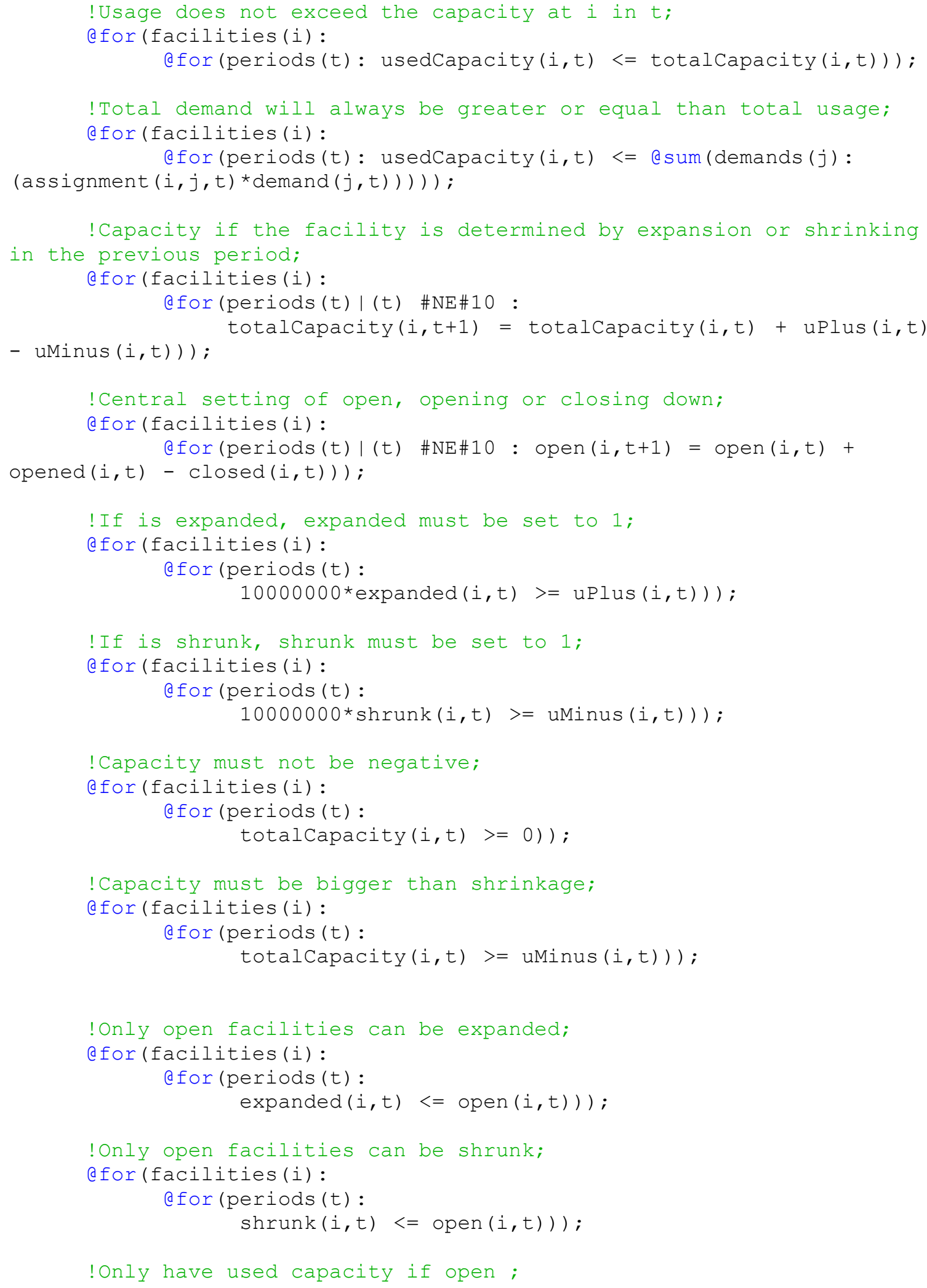




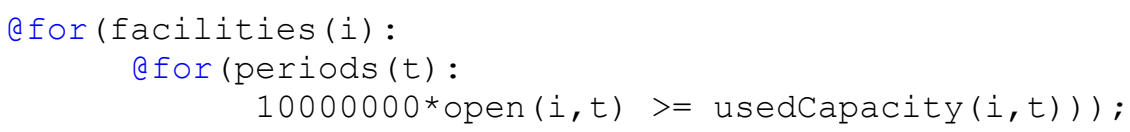




\section{Appendix B The Lingo file of model 1 using a dynamic approach}

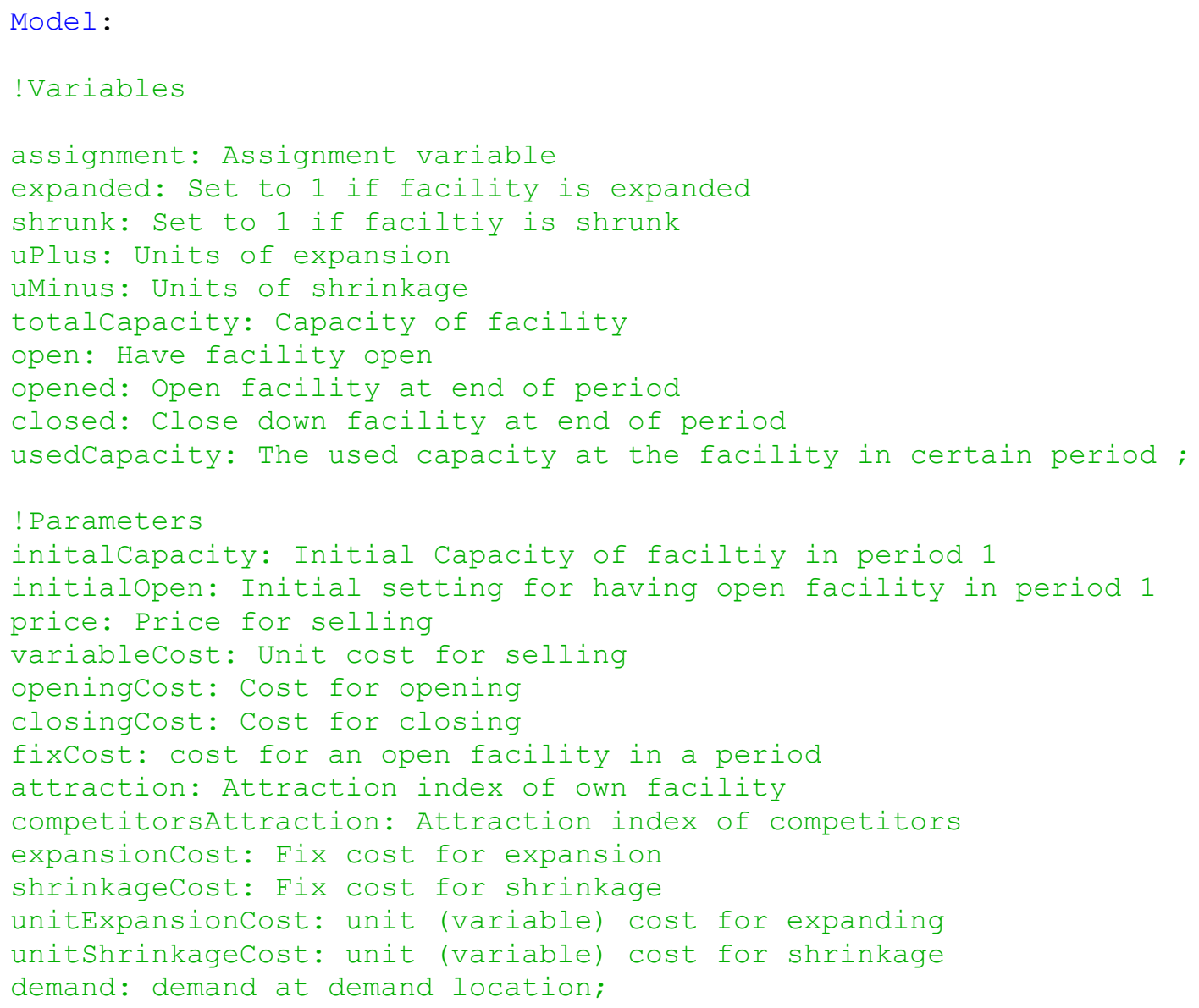


uPlus, uMinus, usedCapacity, pricel, variablecost1, fixcost1, openingCost1, closingCost1, open, opened, closed;

indexjt (demands, periods): competitorsattraction1, demand1;

Endsets

afor (facilities (i): defor (demands $(j)$ :

afor (periods (t):

abin (assignment ( $i, j, t))$;

abin (expanded $(i, t))$;

abin $(\operatorname{shrunk}(i, t))$;

abin (open $(i, t))$;

abin (opened $(i, t))$;

abin (closed $(i, t))$;

@gin (uPlus $(i, t))$;

@gin (uMinus $(i, t))$;

@gin (totalCapacity $(i, t))$;

))) ;

Data:

attraction 1 = @OLE ('GD 10x10x10 VER1 dynamic.xls');

competitorsAttraction ${ }^{-}=$QOLE ('GD_10ㅈ10x10_VER1_dynamic.xls');

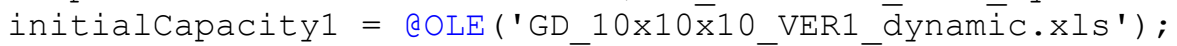

initialopen $1=\operatorname{QOLE}\left({ }^{\mathrm{G} D} 10 \times \overline{1} 0 \times 10 \mathrm{VER} \overline{1}\right.$ dynāmic.xls');

variableCost $1=$ aOLE ('GD $10 \times 10 \times 1 \overline{0}$ VER $\overline{1}$ dynamic.xls');

fixCost1 = @OLE('GD $10 \times 1 \overline{0} \times 10$ VER1 dynamic.xls');

openingCost $1=$ @OLE ('GD_10x1 $\overline{0} \times 10$ VER1_dynamic.xls') ;

closingCost $1=\operatorname{QOLE}\left(\mathrm{GD}^{-} 10 \times 10 \times 10^{-} \mathrm{VER} 1\right.$ - dynamic.xls');

unitExpansionCost $1=$ QOLE ('GD_10 $\overline{\mathrm{L}} 10 \times 1 \overline{0}$ VER1_dynamic.xls');

expansioncost $1=$ QOLE ('GD $10 \times \overline{1} 0 \times 10$ VER $\overline{1}$ dynämic.xls');

unitShrinkageCost $1=$ QOLE ('GD_10x1 $\overline{0} \times 10 \bar{V} E R 1$ dynamic.xls');

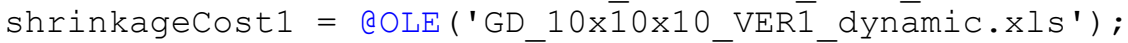

price1 = QOLE('GD_10x10x10_VER1_dyñamic.xls');

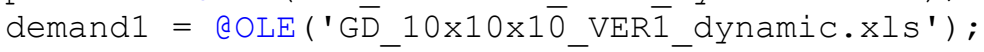

enddata

$\max =$ esum(indexit $(i, t)$ : usedCapacity $(i, t) *(p r i c e 1(i, t) \quad-$

variableCost1 $(i, t))$ - fixCost1 $(i, t) * o p e n(i, t) \quad-$

closingCost $1(i, t) * \operatorname{closed}(i, t)$ - openingCost $1(i, t) * o p e n e d(i, t))$ -

dsum(indexit $(i, t)$ : expansionCost1 $(i, t) * \operatorname{expanded}(i, t))$ -

(asum (indexit (i,t) : unitExpansionCost1 (i,t)*uPlus $(i, t))$ -

@sum(indexit (i,t): shrinkageCost1 (i,t)*shrunk(i,t)) - $a \operatorname{sum}(i n d e x i t(i, t)$ :

unitShrinkageCost $1(i, t) * u \operatorname{Minus}(i, t))$;

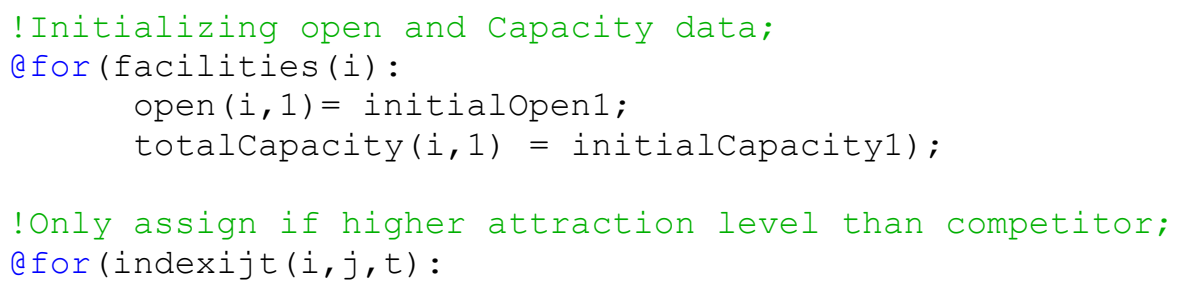


(assignment $(i, j, t)) *(\operatorname{attraction} 1(i, j, t)-$

competitorsattraction $1(j, t))>=0)$;

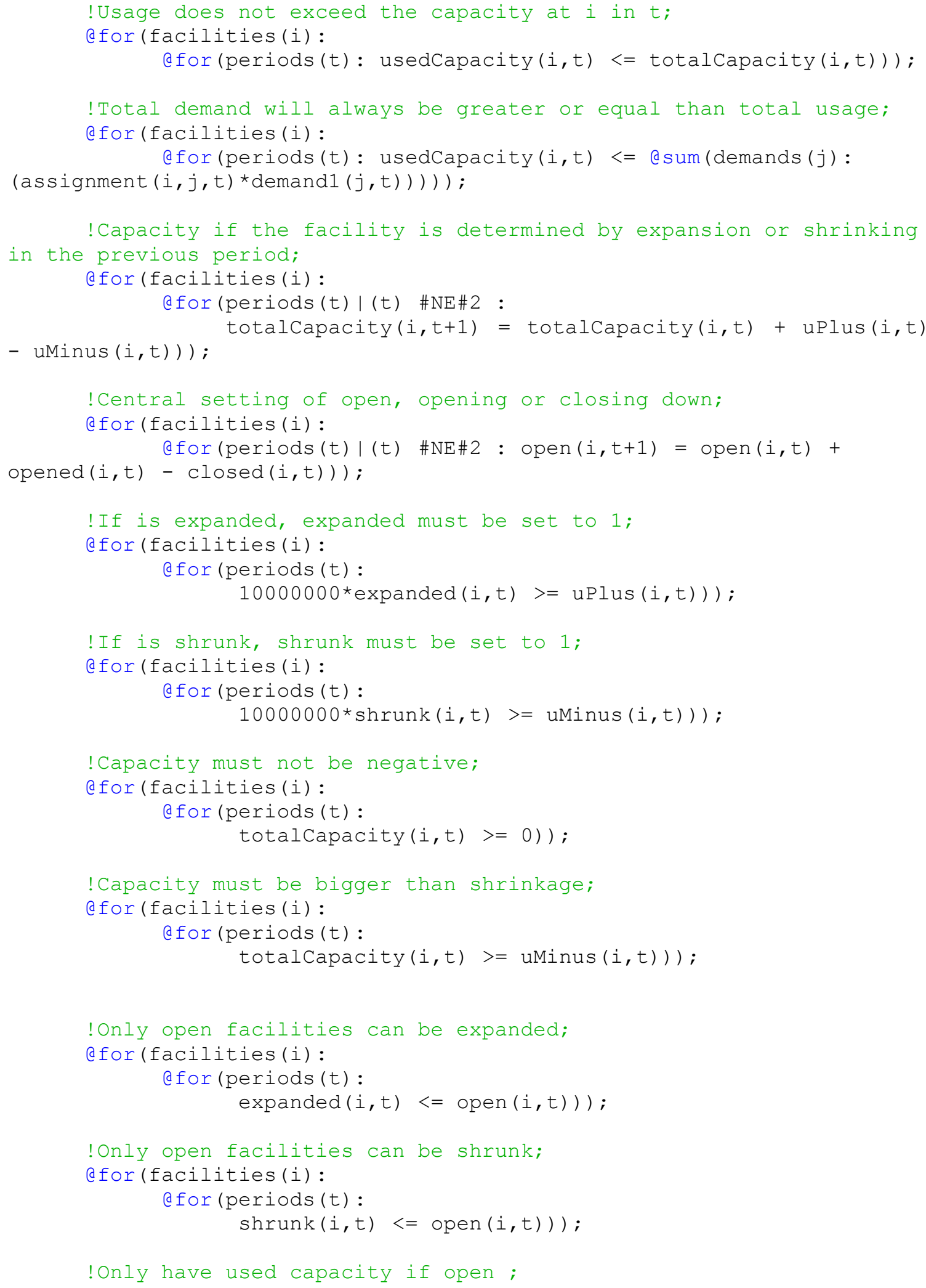




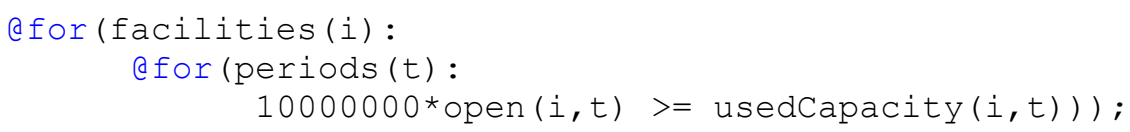

END 


\section{Appendix C Data used for comparison of robust and dynamic solution approach}

Table 42 Data used for first test - keeping demand and prices constant over time

Initial Capacity
\begin{tabular}{|cc|} 
initialCap \\
\hline Facility (i) & InitialCapacity \\
\hline 1 & 5000 \\
\hline 2 & 5000 \\
\hline 3 & 5000 \\
\hline 4 & 5000 \\
\hline 5 & 5000 \\
\hline 6 & 5000 \\
\hline 7 & 5000 \\
\hline 8 & 5000 \\
\hline 9 & 5000 \\
\hline 10 & 5000 \\
\hline
\end{tabular}

Open settings for period 1 initialOpen

\begin{tabular}{|cc|}
\hline Facility (i) & initalOpen \\
\hline 1 & 1 \\
\hline 2 & 1 \\
\hline 3 & 1 \\
\hline 4 & 1 \\
\hline 5 & 1 \\
\hline 6 & 1 \\
\hline 7 & 1 \\
\hline 8 & 1 \\
\hline 9 & 1 \\
\hline 10 & 1 \\
\hline
\end{tabular}




\begin{tabular}{|c|c|c|c|c|c|c|c|c|c|c|}
\hline \multicolumn{11}{|c|}{ Price for selling one unit at $\mathrm{i}$ in $\mathrm{t}$} \\
\hline price & & & & & Per & $(\mathrm{t})$ & & & & \\
\hline \multicolumn{11}{|l|}{ Facilit } \\
\hline y (i) & 1 & 2 & 3 & 4 & 5 & 6 & 7 & 8 & 9 & 10 \\
\hline 1 & 250 & 250 & 250 & 250 & 250 & 250 & 250 & 250 & 250 & 250 \\
\hline 2 & 250 & 250 & 250 & 250 & 250 & 250 & 250 & 250 & 250 & 250 \\
\hline 3 & 250 & 250 & 250 & 250 & 250 & 250 & 250 & 250 & 250 & 250 \\
\hline 4 & 250 & 250 & 250 & 250 & 250 & 250 & 250 & 250 & 250 & 250 \\
\hline 5 & 250 & 250 & 250 & 250 & 250 & 250 & 250 & 250 & 250 & 250 \\
\hline 6 & 250 & 250 & 250 & 250 & 250 & 250 & 250 & 250 & 250 & 250 \\
\hline 7 & 250 & 250 & 250 & 250 & 250 & 250 & 250 & 250 & 250 & 250 \\
\hline 8 & 250 & 250 & 250 & 250 & 250 & 250 & 250 & 250 & 250 & 250 \\
\hline 9 & 250 & 250 & 250 & 250 & 250 & 250 & 250 & 250 & 250 & 250 \\
\hline 10 & 250 & 250 & 250 & 250 & 250 & 250 & 250 & 250 & 250 & 250 \\
\hline
\end{tabular}

$\begin{aligned} & \text { Variable cost for selling in period } \mathrm{t} \\
& \text { variabl } \\
& \text { eCost }\end{aligned}$
\begin{tabular}{|ccccccccccc|c|}
\hline $\begin{array}{c}\text { Facilit } \\
\text { y (i) }\end{array}$ & 1 & 2 & 3 & 4 & 5 & 6 & 7 & 8 & 9 & 10 \\
\hline 1 & 150 & 150 & 150 & 150 & 150 & 150 & 150 & 150 & 150 & 150 \\
\hline 2 & 150 & 150 & 150 & 150 & 150 & 150 & 150 & 150 & 150 & 150 \\
\hline 3 & 150 & 150 & 150 & 150 & 150 & 150 & 150 & 150 & 150 & 150 \\
\hline 4 & 150 & 150 & 150 & 150 & 150 & 150 & 150 & 150 & 150 & 150 \\
\hline 5 & 150 & 150 & 150 & 150 & 150 & 150 & 150 & 150 & 150 & 150 \\
\hline 6 & 150 & 150 & 150 & 150 & 150 & 150 & 150 & 150 & 150 & 150 \\
\hline 7 & 150 & 150 & 150 & 150 & 150 & 150 & 150 & 150 & 150 & 150 \\
\hline 8 & 150 & 150 & 150 & 150 & 150 & 150 & 150 & 150 & 150 & 150 \\
\hline 9 & 150 & 150 & 150 & 150 & 150 & 150 & 150 & 150 & 150 & 150 \\
\hline 10 & 150 & 150 & 150 & 150 & 150 & 150 & 150 & 150 & 150 & 150 \\
\hline
\end{tabular}

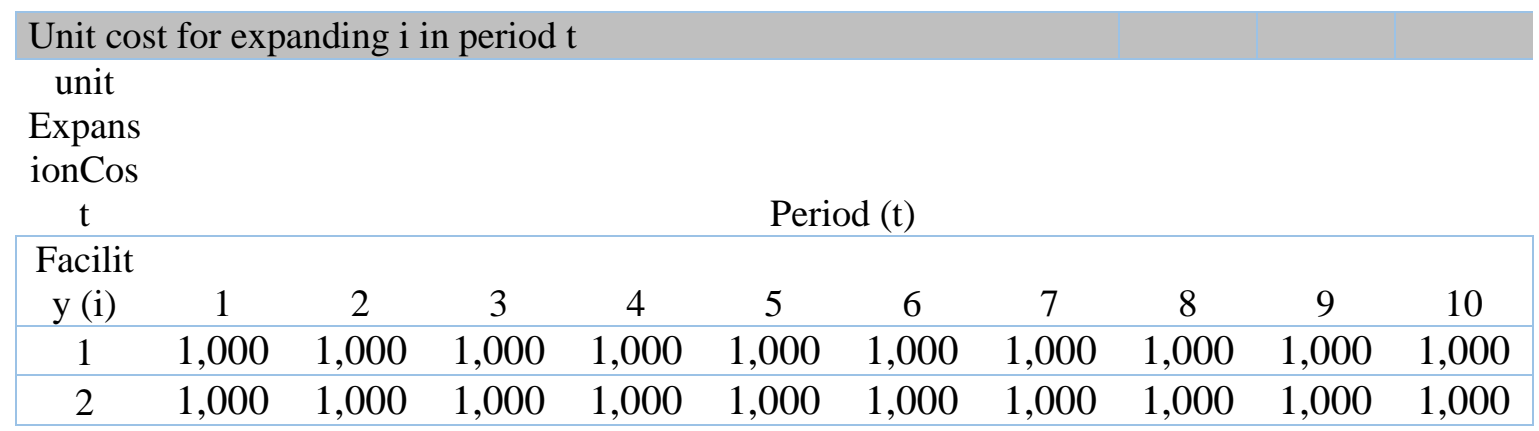




\begin{tabular}{|crrrrrrrrrr|}
\hline 3 & 1,000 & 1,000 & 1,000 & 1,000 & 1,000 & 1,000 & 1,000 & 1,000 & 1,000 & 1,000 \\
\hline 4 & 1,000 & 1,000 & 1,000 & 1,000 & 1,000 & 1,000 & 1,000 & 1,000 & 1,000 & 1,000 \\
\hline 5 & 1,000 & 1,000 & 1,000 & 1,000 & 1,000 & 1,000 & 1,000 & 1,000 & 1,000 & 1,000 \\
\hline 6 & 1,000 & 1,000 & 1,000 & 1,000 & 1,000 & 1,000 & 1,000 & 1,000 & 1,000 & 1,000 \\
\hline 7 & 1,000 & 1,000 & 1,000 & 1,000 & 1,000 & 1,000 & 1,000 & 1,000 & 1,000 & 1,000 \\
\hline 8 & 1,000 & 1,000 & 1,000 & 1,000 & 1,000 & 1,000 & 1,000 & 1,000 & 1,000 & 1,000 \\
\hline 9 & 1,000 & 1,000 & 1,000 & 1,000 & 1,000 & 1,000 & 1,000 & 1,000 & 1,000 & 1,000 \\
\hline 10 & 1,000 & 1,000 & 1,000 & 1,000 & 1,000 & 1,000 & 1,000 & 1,000 & 1,000 & 1,000 \\
\hline
\end{tabular}

\begin{tabular}{|c|c|c|c|c|c|c|c|c|c|c|}
\hline \multicolumn{11}{|c|}{ Unit cost for shrinking $\mathrm{i}$ in period $\mathrm{t}$} \\
\hline $\begin{array}{c}\text { unit } \\
\text { Shrink } \\
\text { ageCos }\end{array}$ & & & & & & & & & & \\
\hline $\mathrm{t}$ & & & & & & & & & & \\
\hline Facilit & & & & & & & & & & \\
\hline y (i) & 1 & 2 & 3 & 4 & 5 & 6 & 7 & 8 & 9 & 10 \\
\hline 1 & 10 & 10 & 10 & 10 & 10 & 10 & 10 & 10 & 10 & 10 \\
\hline 2 & 10 & 10 & 10 & 10 & 10 & 10 & 10 & 10 & 10 & 10 \\
\hline 3 & 10 & 10 & 10 & 10 & 10 & 10 & 10 & 10 & 10 & 10 \\
\hline 4 & 10 & 10 & 10 & 10 & 10 & 10 & 10 & 10 & 10 & 10 \\
\hline 5 & 10 & 10 & 10 & 10 & 10 & 10 & 10 & 10 & 10 & 10 \\
\hline 6 & 10 & 10 & 10 & 10 & 10 & 10 & 10 & 10 & 10 & 10 \\
\hline 7 & 10 & 10 & 10 & 10 & 10 & 10 & 10 & 10 & 10 & 10 \\
\hline 8 & 10 & 10 & 10 & 10 & 10 & 10 & 10 & 10 & 10 & 10 \\
\hline 9 & 10 & 10 & 10 & 10 & 10 & 10 & 10 & 10 & 10 & 10 \\
\hline 10 & 10 & 10 & 10 & 10 & 10 & 10 & 10 & 10 & 10 & 10 \\
\hline
\end{tabular}

\begin{tabular}{|c|c|c|c|c|c|c|c|c|c|c|}
\hline \multicolumn{11}{|c|}{ Expansion cost for $\mathrm{i}$ in $\mathrm{t}$} \\
\hline \multicolumn{11}{|c|}{$\begin{array}{l}\text { expans } \\
\text { ionCos }\end{array}$} \\
\hline $\mathrm{t}$ & \multicolumn{10}{|c|}{ Period $(\mathrm{t})$} \\
\hline Facilit & & & & & & & & & & \\
\hline y (i) & 1 & 2 & 3 & 4 & 5 & 6 & 7 & 8 & 9 & 10 \\
\hline 1 & 5,000 & 5,000 & 5,000 & 5,000 & 5,000 & 5,000 & 5,000 & 5,000 & 5,000 & 5,000 \\
\hline 2 & 5,000 & 5,000 & 5,000 & 5,000 & 5,000 & 5,000 & 5,000 & 5,000 & 5,000 & 5,000 \\
\hline 3 & 5,000 & 5,000 & 5,000 & 5,000 & 5,000 & 5,000 & 5,000 & 5,000 & 5,000 & 5,000 \\
\hline 4 & 5,000 & 5,000 & 5,000 & 5,000 & 5,000 & 5,000 & 5,000 & 5,000 & 5,000 & 5,000 \\
\hline 5 & 5,000 & 5,000 & 5,000 & 5,000 & 5,000 & 5,000 & 5,000 & 5,000 & 5,000 & 5,000 \\
\hline 6 & 5,000 & 5,000 & 5,000 & 5,000 & 5,000 & 5,000 & 5,000 & 5,000 & 5,000 & 5,000 \\
\hline
\end{tabular}




\begin{tabular}{|ccccccccccc|}
\hline 7 & 5,000 & 5,000 & 5,000 & 5,000 & 5,000 & 5,000 & 5,000 & 5,000 & 5,000 & 5,000 \\
\hline 8 & 5,000 & 5,000 & 5,000 & 5,000 & 5,000 & 5,000 & 5,000 & 5,000 & 5,000 & 5,000 \\
\hline 9 & 5,000 & 5,000 & 5,000 & 5,000 & 5,000 & 5,000 & 5,000 & 5,000 & 5,000 & 5,000 \\
\hline 10 & 5,000 & 5,000 & 5,000 & 5,000 & 5,000 & 5,000 & 5,000 & 5,000 & 5,000 & 5,000 \\
\hline
\end{tabular}

$\begin{aligned} & \text { Shrinkage cost for i in t } \\
& \text { shrinka } \\
& \text { geCost }\end{aligned}$
\begin{tabular}{|ccccccccccc|c|}
\begin{tabular}{|c|c} 
Facilit \\
y (i)
\end{tabular} & 1 & 2 & 3 & 4 & 5 & 6 & 7 & 8 & 9 & 10 \\
\hline 1 & 5,000 & 5,000 & 5,000 & 5,000 & 5,000 & 5,000 & 5,000 & 5,000 & 5,000 & 5,000 \\
\hline 2 & 5,000 & 5,000 & 5,000 & 5,000 & 5,000 & 5,000 & 5,000 & 5,000 & 5,000 & 5,000 \\
\hline 3 & 5,000 & 5,000 & 5,000 & 5,000 & 5,000 & 5,000 & 5,000 & 5,000 & 5,000 & 5,000 \\
\hline 4 & 5,000 & 5,000 & 5,000 & 5,000 & 5,000 & 5,000 & 5,000 & 5,000 & 5,000 & 5,000 \\
\hline 5 & 5,000 & 5,000 & 5,000 & 5,000 & 5,000 & 5,000 & 5,000 & 5,000 & 5,000 & 5,000 \\
\hline 6 & 5,000 & 5,000 & 5,000 & 5,000 & 5,000 & 5,000 & 5,000 & 5,000 & 5,000 & 5,000 \\
\hline 7 & 5,000 & 5,000 & 5,000 & 5,000 & 5,000 & 5,000 & 5,000 & 5,000 & 5,000 & 5,000 \\
\hline 8 & 5,000 & 5,000 & 5,000 & 5,000 & 5,000 & 5,000 & 5,000 & 5,000 & 5,000 & 5,000 \\
\hline 9 & 5,000 & 5,000 & 5,000 & 5,000 & 5,000 & 5,000 & 5,000 & 5,000 & 5,000 & 5,000 \\
\hline 10 & 5,000 & 5,000 & 5,000 & 5,000 & 5,000 & 5,000 & 5,000 & 5,000 & 5,000 & 5,000 \\
\hline
\end{tabular}

\begin{tabular}{|c|c|c|c|c|c|c|c|c|c|c|}
\hline \multicolumn{11}{|c|}{ Demand at $\mathrm{j}$ in $\mathrm{t}$} \\
\hline $\begin{array}{l}\text { demand } \\
\text { demand }\end{array}$ & \multicolumn{10}{|c|}{ Period $(\mathrm{t})$} \\
\hline $\begin{array}{l}\text { demand } \\
\text { (j) }\end{array}$ & 1 & 2 & 3 & 4 & 5 & 6 & 7 & 8 & 9 & 10 \\
\hline 1 & 10,000 & 9,000 & 8,000 & 7,000 & 6,000 & 5,000 & 4,000 & 3,000 & 2,000 & 1,000 \\
\hline 2 & 10,000 & 9,000 & 8,000 & 7,000 & 6,000 & 5,000 & 4,000 & 3,000 & 2,000 & 1,000 \\
\hline 3 & 10,000 & 9,000 & 8,000 & 7,000 & 6,000 & 5,000 & 4,000 & 3,000 & 2,000 & 1,000 \\
\hline 4 & 10,000 & 9,000 & 8,000 & 7,000 & 6,000 & 5,000 & 4,000 & 3,000 & 2,000 & 1,000 \\
\hline 5 & 10,000 & 9,000 & 8,000 & 7,000 & 6,000 & 5,000 & 4,000 & 3,000 & 2,000 & 1,000 \\
\hline 6 & 10,000 & 9,000 & 8,000 & 7,000 & 6,000 & 5,000 & 4,000 & 3,000 & 2,000 & 1,000 \\
\hline 7 & 10,000 & 9,000 & 8,000 & 7,000 & 6,000 & 5,000 & 4,000 & 3,000 & 2,000 & 1,000 \\
\hline 8 & 10,000 & 9,000 & 8,000 & 7,000 & 6,000 & 5,000 & 4,000 & 3,000 & 2,000 & 1,000 \\
\hline 9 & 10,000 & 9,000 & 8,000 & 7,000 & 6,000 & 5,000 & 4,000 & 3,000 & 2,000 & 1,000 \\
\hline 10 & 10,000 & 9,000 & 8,000 & 7,000 & 6,000 & 5,000 & 4,000 & 3,000 & 2,000 & 1,000 \\
\hline
\end{tabular}




\begin{tabular}{|c|c|c|c|c|c|c|c|c|c|c|}
\hline \multicolumn{11}{|c|}{ Attraction value for facility $\mathrm{i}$ for demand point $\mathrm{j}$ in period $\mathrm{t}$} \\
\hline & \multicolumn{10}{|c|}{ demand 1} \\
\hline & \multicolumn{10}{|c|}{ Period $(\mathrm{t})$} \\
\hline \multicolumn{11}{|l|}{ facilit } \\
\hline y (i) & 1 & 2 & 3 & 4 & 5 & 6 & 7 & 8 & 9 & 10 \\
\hline 1 & 30 & 30 & 30 & 30 & 30 & 30 & 30 & 30 & 30 & 30 \\
\hline 2 & 80 & 81 & 81 & 81 & 81 & 81 & 81 & 81 & 80 & 80 \\
\hline 3 & 60 & 60 & 60 & 60 & 60 & 60 & 60 & 60 & 60 & 60 \\
\hline 4 & 60 & 60 & 60 & 60 & 60 & 60 & 60 & 60 & 60 & 60 \\
\hline 5 & 60 & 60 & 60 & 60 & 60 & 60 & 60 & 60 & 60 & 60 \\
\hline 6 & 2 & 2 & 2 & 2 & 2 & 2 & 2 & 2 & 2 & 2 \\
\hline 7 & 2 & 2 & 2 & 2 & 2 & 2 & 2 & 2 & 2 & 2 \\
\hline 8 & 2 & 2 & 2 & 2 & 2 & 2 & 2 & 2 & 2 & 2 \\
\hline 9 & 2 & 2 & 2 & 2 & 2 & 2 & 2 & 2 & 2 & 2 \\
\hline \multirow[t]{3}{*}{10} & 2 & 2 & 2 & 2 & 2 & 2 & 2 & 2 & 2 & 2 \\
\hline & \multicolumn{10}{|c|}{ demand 2} \\
\hline & \multicolumn{10}{|c|}{ Period $(\mathrm{t})$} \\
\hline \multicolumn{11}{|l|}{ facilit } \\
\hline y (i) & 1 & 2 & 3 & 4 & 5 & 6 & 7 & 8 & 9 & 10 \\
\hline 1 & 40 & 40 & 40 & 40 & 40 & 40 & 40 & 40 & 40 & 40 \\
\hline 2 & 80 & 80 & 80 & 80 & 80 & 80 & 80 & 80 & 80 & 80 \\
\hline 3 & 60 & 60 & 60 & 60 & 60 & 60 & 60 & 60 & 60 & 60 \\
\hline 4 & 60 & 60 & 60 & 60 & 60 & 60 & 60 & 60 & 60 & 60 \\
\hline 5 & 60 & 60 & 60 & 60 & 60 & 60 & 60 & 60 & 60 & 60 \\
\hline 6 & 2 & 2 & 2 & 2 & 2 & 2 & 2 & 2 & 2 & 2 \\
\hline 7 & 2 & 2 & 2 & 2 & 2 & 2 & 2 & 2 & 2 & 2 \\
\hline 8 & 2 & 2 & 2 & 2 & 2 & 2 & 2 & 2 & 2 & 2 \\
\hline 9 & 2 & 2 & 2 & 2 & 2 & 2 & 2 & 2 & 2 & 2 \\
\hline 10 & 2 & 2 & 2 & 2 & 2 & 2 & 2 & 2 & 2 & 2 \\
\hline & \multicolumn{10}{|c|}{ demand 3} \\
\hline \multicolumn{11}{|c|}{ Period $(\mathrm{t})$} \\
\hline \multicolumn{11}{|l|}{ facilit } \\
\hline y (i) & 1 & 2 & 3 & 4 & 5 & 6 & 7 & 8 & 9 & 10 \\
\hline 1 & 50 & 50 & 50 & 50 & 50 & 50 & 50 & 50 & 50 & 50 \\
\hline 2 & 80 & 80 & 80 & 80 & 80 & 80 & 80 & 80 & 80 & 80 \\
\hline 3 & 60 & 60 & 60 & 60 & 60 & 60 & 60 & 60 & 60 & 60 \\
\hline 4 & 60 & 60 & 60 & 60 & 60 & 60 & 60 & 60 & 60 & 60 \\
\hline 5 & 60 & 60 & 60 & 60 & 60 & 60 & 60 & 60 & 60 & 60 \\
\hline 6 & 2 & 2 & 2 & 2 & 2 & 2 & 2 & 2 & 2 & 2 \\
\hline 7 & 2 & 2 & 2 & 2 & 2 & 2 & 2 & 2 & 2 & 2 \\
\hline 8 & 2 & 2 & 2 & 2 & 2 & 2 & 2 & 2 & 2 & 2 \\
\hline 9 & 2 & 2 & 2 & 2 & 2 & 2 & 2 & 2 & 2 & 2 \\
\hline 10 & 2 & 2 & 2 & 2 & 2 & 2 & 2 & 2 & 2 & 2 \\
\hline & \multicolumn{10}{|c|}{ demand 4} \\
\hline \multicolumn{11}{|c|}{ Period $(\mathrm{t})$} \\
\hline facilit & 1 & 2 & 3 & 4 & 5 & 6 & 7 & 8 & 9 & 10 \\
\hline
\end{tabular}




\begin{tabular}{|ccccccccccc|}
\hline $\mathrm{y}(\mathrm{i})$ & & & & & & & & & & \\
\hline 1 & 60 & 60 & 60 & 60 & 60 & 60 & 60 & 60 & 60 & 60 \\
\hline 2 & 80 & 80 & 80 & 80 & 80 & 80 & 80 & 80 & 80 & 80 \\
\hline 3 & 60 & 60 & 60 & 60 & 60 & 60 & 60 & 60 & 60 & 60 \\
\hline 4 & 60 & 60 & 60 & 60 & 60 & 60 & 60 & 60 & 60 & 60 \\
\hline 5 & 60 & 60 & 60 & 60 & 60 & 60 & 60 & 60 & 60 & 60 \\
\hline 6 & 2 & 2 & 2 & 2 & 2 & 2 & 2 & 2 & 2 & 2 \\
\hline 7 & 2 & 2 & 2 & 2 & 2 & 2 & 2 & 2 & 2 & 2 \\
\hline 8 & 2 & 2 & 2 & 2 & 2 & 2 & 2 & 2 & 2 & 2 \\
\hline 9 & 2 & 2 & 2 & 2 & 2 & 2 & 2 & 2 & 2 & 2 \\
\hline 10 & 2 & 2 & 2 & 2 & 2 & 2 & 2 & 2 & 2 & 2 \\
\hline & & & & & demand 5 & & & & \\
\hline
\end{tabular}

facilit

Period (t)

\begin{tabular}{|ccccccccccc|}
\hline $\mathrm{y} \mathrm{(i)}$ & 1 & 2 & 3 & 4 & 5 & 6 & 7 & 8 & 9 & 10 \\
\hline 1 & 70 & 70 & 70 & 70 & 70 & 70 & 70 & 70 & 70 & 70 \\
\hline 2 & 80 & 80 & 80 & 80 & 80 & 80 & 80 & 80 & 80 & 80 \\
\hline 3 & 60 & 60 & 60 & 60 & 60 & 60 & 60 & 60 & 60 & 60 \\
\hline 4 & 60 & 60 & 60 & 60 & 60 & 60 & 60 & 60 & 60 & 60 \\
\hline 5 & 60 & 60 & 60 & 60 & 60 & 60 & 60 & 60 & 60 & 60 \\
\hline 6 & 2 & 2 & 2 & 2 & 2 & 2 & 2 & 2 & 2 & 2 \\
\hline 7 & 2 & 2 & 2 & 2 & 2 & 2 & 2 & 2 & 2 & 2 \\
\hline 8 & 2 & 2 & 2 & 2 & 2 & 2 & 2 & 2 & 2 & 2 \\
\hline 9 & 2 & 2 & 2 & 2 & 2 & 2 & 2 & 2 & 2 & 2 \\
\hline 10 & 2 & 2 & 2 & 2 & 2 & 2 & 2 & 2 & 2 & 2 \\
\hline
\end{tabular}

Period (t)

\begin{tabular}{|ccccccccccc|}
\hline $\begin{array}{c}\text { facilit } \\
\text { y (i) }\end{array}$ & 1 & 2 & 3 & 4 & 5 & 6 & 7 & 8 & 9 & 10 \\
\hline 1 & 80 & 80 & 80 & 80 & 80 & 80 & 80 & 80 & 80 & 80 \\
\hline 2 & 80 & 80 & 80 & 80 & 80 & 80 & 80 & 80 & 80 & 80 \\
\hline 3 & 60 & 60 & 60 & 60 & 60 & 60 & 60 & 60 & 60 & 60 \\
\hline 4 & 60 & 60 & 60 & 60 & 60 & 60 & 60 & 60 & 60 & 60 \\
\hline 5 & 60 & 60 & 60 & 60 & 60 & 60 & 60 & 60 & 60 & 60 \\
\hline 6 & 2 & 2 & 2 & 2 & 2 & 2 & 2 & 2 & 2 & 2 \\
\hline 7 & 2 & 2 & 2 & 2 & 2 & 2 & 2 & 2 & 2 & 2 \\
\hline 8 & 2 & 2 & 2 & 2 & 2 & 2 & 2 & 2 & 2 & 2 \\
\hline 9 & 2 & 2 & 2 & 2 & 2 & 2 & 2 & 2 & 2 & 2 \\
\hline 10 & 2 & 2 & 2 & 2 & 2 & 2 & 2 & 2 & 2 & 2 \\
\hline & & & & & demand 7 & & & & \\
\hline & & & & & Period (t) & & & & \\
\hline facilit & & & & & & & & & & \\
\hline y (i) & 1 & 2 & 3 & 4 & 5 & 6 & 7 & 8 & 9 & 10 \\
\hline 1 & 90 & 90 & 90 & 90 & 90 & 90 & 90 & 90 & 90 & 90 \\
\hline 2 & 80 & 80 & 80 & 80 & 80 & 80 & 80 & 80 & 80 & 80 \\
\hline 3 & 60 & 60 & 60 & 60 & 60 & 60 & 60 & 60 & 60 & 60 \\
\hline
\end{tabular}




\begin{tabular}{|cccccccccccc|}
\hline 4 & 60 & 60 & 60 & 60 & 60 & 60 & 60 & 60 & 60 & 60 \\
\hline 5 & 60 & 60 & 60 & 60 & 60 & 60 & 60 & 60 & 60 & 60 \\
\hline 6 & 2 & 2 & 2 & 2 & 2 & 2 & 2 & 2 & 2 & 2 \\
\hline 7 & 2 & 2 & 2 & 2 & 2 & 2 & 2 & 2 & 2 & 2 \\
\hline 8 & 2 & 2 & 2 & 2 & 2 & 2 & 2 & 2 & 2 & 2 \\
\hline 9 & 2 & 2 & 2 & 2 & 2 & 2 & 2 & 2 & 2 & 2 \\
\hline 10 & 2 & 2 & 2 & 2 & 2 & 2 & 2 & 2 & 2 & 2 \\
\hline & & & & & demand 8 & & & & \\
\hline
\end{tabular}

\begin{tabular}{|ccccccccccc|}
\hline $\begin{array}{c}\text { facilit } \\
\mathrm{y} \mathrm{(i)}\end{array}$ & 1 & 2 & 3 & 4 & 5 & 6 & 7 & 8 & 9 & 10 \\
\hline 1 & 90 & 90 & 90 & 90 & 90 & 90 & 90 & 90 & 90 & 90 \\
\hline 2 & 80 & 80 & 80 & 80 & 80 & 80 & 80 & 80 & 80 & 80 \\
\hline 3 & 60 & 60 & 60 & 60 & 60 & 60 & 60 & 60 & 60 & 60 \\
\hline 4 & 60 & 60 & 60 & 60 & 60 & 60 & 60 & 60 & 60 & 60 \\
\hline 5 & 60 & 60 & 60 & 60 & 60 & 60 & 60 & 60 & 60 & 60 \\
\hline 6 & 2 & 2 & 2 & 2 & 2 & 2 & 2 & 2 & 2 & 2 \\
\hline 7 & 2 & 2 & 2 & 2 & 2 & 2 & 2 & 2 & 2 & 2 \\
\hline 8 & 2 & 2 & 2 & 2 & 2 & 2 & 2 & 2 & 2 & 2 \\
\hline 9 & 2 & 2 & 2 & 2 & 2 & 2 & 2 & 2 & 2 & 2 \\
\hline 10 & 2 & 2 & 2 & 2 & 2 & 2 & 2 & 2 & 2 & 2 \\
\hline & & & & & demand 9 & & & & \\
\hline
\end{tabular}

\begin{tabular}{|ccccccccccc|}
\hline $\begin{array}{c}\text { facilit } \\
\text { y (i) }\end{array}$ & 1 & 2 & 3 & 4 & 5 & 6 & 7 & 8 & 9 & 10 \\
\hline 1 & 90 & 90 & 90 & 90 & 90 & 90 & 90 & 90 & 90 & 90 \\
\hline 2 & 80 & 80 & 80 & 80 & 80 & 80 & 80 & 80 & 80 & 80 \\
\hline 3 & 60 & 60 & 60 & 60 & 60 & 60 & 60 & 60 & 60 & 60 \\
\hline 4 & 60 & 60 & 60 & 60 & 60 & 60 & 60 & 60 & 60 & 60 \\
\hline 5 & 60 & 60 & 60 & 60 & 60 & 60 & 60 & 60 & 60 & 60 \\
\hline 6 & 2 & 2 & 2 & 2 & 2 & 2 & 2 & 2 & 2 & 2 \\
\hline 7 & 2 & 2 & 2 & 2 & 2 & 2 & 2 & 2 & 2 & 2 \\
\hline 8 & 2 & 2 & 2 & 2 & 2 & 2 & 2 & 2 & 2 & 2 \\
\hline 9 & 2 & 2 & 2 & 2 & 2 & 2 & 2 & 2 & 2 & 2 \\
\hline 10 & 2 & 2 & 2 & 2 & 2 & 2 & 2 & 2 & 2 & 2 \\
\hline
\end{tabular}

Period (t)

\begin{tabular}{|ccccccccccc|}
\hline $\begin{array}{c}\text { facilit } \\
\text { y (i) }\end{array}$ & 1 & 2 & 3 & 4 & 5 & 6 & 7 & 8 & 9 & 10 \\
\hline 1 & 90 & 90 & 90 & 90 & 90 & 90 & 90 & 90 & 90 & 90 \\
\hline 2 & 80 & 80 & 80 & 80 & 80 & 80 & 80 & 80 & 80 & 80 \\
\hline 3 & 60 & 60 & 60 & 60 & 60 & 60 & 60 & 60 & 60 & 60 \\
\hline 4 & 60 & 60 & 60 & 60 & 60 & 60 & 60 & 60 & 60 & 60 \\
\hline 5 & 60 & 60 & 60 & 60 & 60 & 60 & 60 & 60 & 60 & 60 \\
\hline 6 & 2 & 2 & 2 & 2 & 2 & 2 & 2 & 2 & 2 & 2 \\
\hline 7 & 2 & 2 & 2 & 2 & 2 & 2 & 2 & 2 & 2 & 2 \\
\hline
\end{tabular}




\begin{tabular}{|ccccccccccc|}
\hline 8 & 2 & 2 & 2 & 2 & 2 & 2 & 2 & 2 & 2 & 2 \\
\hline 9 & 2 & 2 & 2 & 2 & 2 & 2 & 2 & 2 & 2 & 2 \\
\hline 10 & 2 & 2 & 2 & 2 & 2 & 2 & 2 & 2 & 2 & 2 \\
\hline
\end{tabular}

\begin{tabular}{|c|c|c|c|c|c|c|c|c|c|c|}
\hline \multicolumn{11}{|c|}{ Highest attraction value of competitor for demand $\mathrm{j}$ in period $\mathrm{t}$} \\
\hline $\begin{array}{l}\text { compet } \\
\text { itorsAtt } \\
\text { raction }\end{array}$ & & & & & & & & & \multicolumn{2}{|c|}{ Period $(\mathrm{t})$} \\
\hline $\begin{array}{l}\text { demand } \\
\text { (j) }\end{array}$ & 1 & 2 & 3 & 4 & 5 & 6 & 7 & 8 & 9 & 10 \\
\hline 1 & 53 & 53 & 53 & 53 & 53 & 53 & 53 & 53 & 53 & 53 \\
\hline 2 & 73 & 73 & 73 & 73 & 73 & 73 & 73 & 73 & 73 & 73 \\
\hline 3 & 53 & 53 & 53 & 53 & 53 & 53 & 53 & 53 & 53 & 53 \\
\hline 4 & 79 & 79 & 79 & 79 & 79 & 79 & 79 & 79 & 79 & 79 \\
\hline 5 & 11 & 11 & 11 & 11 & 11 & 11 & 11 & 11 & 11 & 11 \\
\hline 6 & 1 & 1 & 1 & 1 & 1 & 1 & 1 & 1 & 1 & 1 \\
\hline 7 & 1 & 1 & 1 & 1 & 1 & 1 & 1 & 1 & 1 & 1 \\
\hline 8 & 1 & 1 & 1 & 1 & 1 & 1 & 1 & 1 & 1 & 1 \\
\hline 9 & 1 & 1 & 1 & 1 & 1 & 1 & 1 & 1 & 1 & 1 \\
\hline 10 & 1 & 1 & 1 & 1 & 1 & 1 & 1 & 1 & 1 & 1 \\
\hline
\end{tabular}

Fix cost for running facility i in period t
fixCos
t
\begin{tabular}{|cccccccccccc|}
\begin{tabular}{|} 
Facility \\
(i)
\end{tabular} & 1 & 2 & 3 & 4 & 5 & 6 & 7 & 8 & 9 & 10 \\
\hline 1 & 500,000 & 500,000 & 500,000 & 500,000 & 500,000 & 500,000 & 500,000 & 500,000 & 500,000 & 500,000 \\
\hline 2 & 500,000 & 500,000 & 500,000 & 500,000 & 500,000 & 500,000 & 500,000 & 500,000 & 500,000 & 500,000 \\
\hline 3 & 500,000 & 500,000 & 500,000 & 500,000 & 500,000 & 500,000 & 500,000 & 500,000 & 500,000 & 500,000 \\
\hline 4 & 500,000 & 500,000 & 500,000 & 500,000 & 500,000 & 500,000 & 500,000 & 500,000 & 500,000 & 500,000 \\
\hline 5 & 500,000 & 500,000 & 500,000 & 500,000 & 500,000 & 500,000 & 500,000 & 500,000 & 500,000 & 500,000 \\
\hline 6 & 500,000 & 500,000 & 500,000 & 500,000 & 500,000 & 500,000 & 500,000 & 500,000 & 500,000 & 500,000 \\
\hline 7 & 500,000 & 500,000 & 500,000 & 500,000 & 500,000 & 500,000 & 500,000 & 500,000 & 500,000 & 500,000 \\
\hline 8 & 500,000 & 500,000 & 500,000 & 500,000 & 500,000 & 500,000 & 500,000 & 500,000 & 500,000 & 500,000 \\
\hline 9 & 500,000 & 500,000 & 500,000 & 500,000 & 500,000 & 500,000 & 500,000 & 500,000 & 500,000 & 500,000 \\
\hline 10 & 500,000 & 500,000 & 500,000 & 500,000 & 500,000 & 500,000 & 500,000 & 500,000 & 500,000 & 500,000 \\
\hline
\end{tabular}




\begin{tabular}{|c|c|c|c|c|c|c|c|c|c|c|}
\hline \multicolumn{11}{|c|}{ Opening cost for $\mathrm{i}$ in $\mathrm{t}$} \\
\hline \multirow{4}{*}{$\begin{array}{l}\text { opening } \\
\text { Cost } \\
\text { Facility } \\
\text { (i) }\end{array}$} & \multirow{2}{*}{\multicolumn{10}{|c|}{ Period $(\mathrm{t})$}} \\
\hline & & & & & & & & & & \\
\hline & & & & & & & & & & \\
\hline & 1 & 2 & 3 & 4 & 5 & 6 & 7 & 8 & 9 & 10 \\
\hline 1 & 10,000 & 10,000 & 10,000 & 10,000 & 10,000 & 10,000 & 10,000 & 10,000 & 10,000 & 10,000 \\
\hline 2 & 10,000 & 10,000 & 10,000 & 10,000 & 10,000 & 10,000 & 10,000 & 10,000 & 10,000 & 10,000 \\
\hline 3 & 10,000 & 10,000 & 10,000 & 10,000 & 10,000 & 10,000 & 10,000 & 10,000 & 10,000 & 10,000 \\
\hline 4 & 10,000 & 10,000 & 10,000 & 10,000 & 10,000 & 10,000 & 10,000 & 10,000 & 10,000 & 10,000 \\
\hline 5 & 10,000 & 10,000 & 10,000 & 10,000 & 10,000 & 10,000 & 10,000 & 10,000 & 10,000 & 10,000 \\
\hline 6 & 10,000 & 10,000 & 10,000 & 10,000 & 10,000 & 10,000 & 10,000 & 10,000 & 10,000 & 10,000 \\
\hline 7 & 10,000 & 10,000 & 10,000 & 10,000 & 10,000 & 10,000 & 10,000 & 10,000 & 10,000 & 10,000 \\
\hline 8 & 10,000 & 10,000 & 10,000 & 10,000 & 10,000 & 10,000 & 10,000 & 10,000 & 10,000 & 10,000 \\
\hline 9 & 10,000 & 10,000 & 10,000 & 10,000 & 10,000 & 10,000 & 10,000 & 10,000 & 10,000 & 10,000 \\
\hline 10 & 10,000 & 10,000 & 10,000 & 10,000 & 10,000 & 10,000 & 10,000 & 10,000 & 10,000 & 10,000 \\
\hline
\end{tabular}

Closing cost for $\mathrm{i}$ in $\mathrm{t}$ closing

Cost

Period $(\mathrm{t})$

Facility

\begin{tabular}{|ccccccccccc|} 
(i) & 1 & 2 & 3 & 4 & 5 & 6 & 7 & 8 & 9 & 10 \\
\hline 1 & 4,961 & 5,940 & 4,741 & 5,119 & 4,411 & 5,071 & 5,132 & 4,631 & 5,810 & 4,421 \\
\hline 2 & 5,596 & 4,687 & 5,266 & 4,528 & 5,273 & 4,428 & 5,307 & 4,910 & 5,194 & 5,023 \\
\hline 3 & 5,246 & 5,389 & 4,460 & 4,682 & 5,455 & 4,644 & 5,056 & 5,114 & 4,570 & 5,243 \\
\hline 4 & 4,679 & 4,182 & 4,388 & 5,076 & 4,271 & 5,008 & 4,655 & 4,896 & 5,371 & 4,712 \\
\hline 5 & 3,989 & 4,854 & 5,192 & 4,905 & 5,128 & 4,154 & 5,454 & 5,007 & 5,180 & 5,294 \\
\hline 6 & 5,293 & 5,356 & 5,436 & 5,244 & 5,044 & 4,783 & 5,798 & 5,362 & 4,547 & 5,379 \\
\hline 7 & 5,732 & 5,190 & 4,799 & 4,312 & 5,241 & 4,713 & 4,625 & 5,023 & 5,172 & 4,959 \\
\hline 8 & 4,507 & 5,268 & 5,543 & 4,665 & 6,141 & 4,990 & 5,181 & 4,559 & 4,712 & 6,372 \\
\hline 9 & 4,049 & 4,526 & 4,059 & 4,637 & 5,092 & 4,193 & 4,691 & 4,567 & 4,582 & 4,737 \\
\hline 10 & 5,005 & 5,024 & 5,869 & 5,114 & 5,033 & 4,451 & 5,109 & 4,925 & 5,065 & 5,077 \\
\hline
\end{tabular}

Table 43 Data used for second test - Alternating demand and keeping prices constant over time

\begin{tabular}{|c|c|c|c|c|c|c|c|c|c|c|}
\hline \multicolumn{11}{|c|}{ Demand at $\mathrm{j}$ in $\mathrm{t}$} \\
\hline demand & \multirow{2}{*}{\multicolumn{10}{|c|}{ Period $(\mathrm{t})$}} \\
\hline demand & & & & & & & & & & \\
\hline (j) & 1 & 2 & 3 & 4 & 5 & 6 & 7 & 8 & 9 & 10 \\
\hline 1 & 10,000 & 0 & 10,000 & 0 & 10,000 & 0 & 10,000 & 0 & 10,000 & 0 \\
\hline
\end{tabular}




\begin{tabular}{|ccccccccccc|}
\hline 2 & 10,000 & 0 & 10,000 & 0 & 10,000 & 0 & 10,000 & 0 & 10,000 & 0 \\
\hline 3 & 10,000 & 0 & 10,000 & 0 & 10,000 & 0 & 10,000 & 0 & 10,000 & 0 \\
\hline 4 & 10,000 & 0 & 10,000 & 0 & 10,000 & 0 & 10,000 & 0 & 10,000 & 0 \\
\hline 5 & 10,000 & 0 & 10,000 & 0 & 10,000 & 0 & 10,000 & 0 & 10,000 & 0 \\
\hline 6 & 10,000 & 0 & 10,000 & 0 & 10,000 & 0 & 10,000 & 0 & 10,000 & 0 \\
\hline 7 & 10,000 & 0 & 10,000 & 0 & 10,000 & 0 & 10,000 & 0 & 10,000 & 0 \\
\hline 8 & 10,000 & 0 & 10,000 & 0 & 10,000 & 0 & 10,000 & 0 & 10,000 & 0 \\
\hline 9 & 10,000 & 0 & 10,000 & 0 & 10,000 & 0 & 10,000 & 0 & 10,000 & 0 \\
\hline 10 & 10,000 & 0 & 10,000 & 0 & 10,000 & 0 & 10,000 & 0 & 10,000 & 0 \\
\hline
\end{tabular}

Table 44 Data used for third test - Alternating prices and keeping demand constant over time

Price for selling one unit at i in t
price
\begin{tabular}{|ccccccccccc|}
\hline $\begin{array}{c}\text { Facility } \\
\text { (i) }\end{array}$ & 1 & 2 & 3 & 4 & 5 & 6 & 7 & 8 & 9 & 10 \\
\hline 1 & 1,100 & 50 & 1,100 & 50 & 1,100 & 50 & 1,100 & 50 & 1,100 & 50 \\
\hline 2 & 1,100 & 50 & 1,100 & 50 & 1,100 & 50 & 1,100 & 50 & 1,100 & 50 \\
\hline 3 & 1,100 & 50 & 1,100 & 50 & 1,100 & 50 & 1,100 & 50 & 1,100 & 50 \\
\hline 4 & 1,100 & 50 & 1,100 & 50 & 1,100 & 50 & 1,100 & 50 & 1,100 & 50 \\
\hline 5 & 1,100 & 50 & 1,100 & 50 & 1,100 & 50 & 1,100 & 50 & 1,100 & 50 \\
\hline 6 & 1,100 & 50 & 1,100 & 50 & 1,100 & 50 & 1,100 & 50 & 1,100 & 50 \\
\hline 7 & 1,100 & 50 & 1,100 & 50 & 1,100 & 50 & 1,100 & 50 & 1,100 & 50 \\
\hline 8 & 1,100 & 50 & 1,100 & 50 & 1,100 & 50 & 1,100 & 50 & 1,100 & 50 \\
\hline 9 & 1,100 & 50 & 1,100 & 50 & 1,100 & 50 & 1,100 & 50 & 1,100 & 50 \\
\hline 10 & 1,100 & 50 & 1,100 & 50 & 1,100 & 50 & 1,100 & 50 & 1,100 & 50 \\
\hline
\end{tabular}

Table 45 Data used for forth test - Contantly sinking demand

\begin{tabular}{|c|c|c|c|c|c|c|c|c|c|c|}
\hline \multicolumn{11}{|c|}{ Demand at $\mathrm{j}$ in $\mathrm{t}$} \\
\hline \multirow{2}{*}{$\begin{array}{c}\text { demand } \\
\text { demand } \\
\text { (j) }\end{array}$} & \multicolumn{10}{|c|}{ Period (t) } \\
\hline & 1 & 2 & 3 & 4 & 5 & 6 & 7 & 8 & 9 & 10 \\
\hline 1 & 10,000 & 9,000 & 8,000 & 7,000 & 6,000 & 5,000 & 4,000 & 3,000 & 2,000 & 1,000 \\
\hline 2 & 10,000 & 9,000 & 8,000 & 7,000 & 6,000 & 5,000 & 4,000 & 3,000 & 2,000 & 1,000 \\
\hline 3 & 10,000 & 9,000 & 8,000 & 7,000 & 6,000 & 5,000 & 4,000 & 3,000 & 2,000 & 1,000 \\
\hline 4 & 10,000 & 9,000 & 8,000 & 7,000 & 6,000 & 5,000 & 4,000 & 3,000 & 2,000 & 1,000 \\
\hline 5 & 10,000 & 9,000 & 8,000 & 7,000 & 6,000 & 5,000 & 4,000 & 3,000 & 2,000 & 1,000 \\
\hline 6 & 10,000 & 9,000 & 8,000 & 7,000 & 6,000 & 5,000 & 4,000 & 3,000 & 2,000 & 1,000 \\
\hline
\end{tabular}




\begin{tabular}{|ccccccccccc|}
\hline 7 & 10,000 & 9,000 & 8,000 & 7,000 & 6,000 & 5,000 & 4,000 & 3,000 & 2,000 & 1,000 \\
\hline 8 & 10,000 & 9,000 & 8,000 & 7,000 & 6,000 & 5,000 & 4,000 & 3,000 & 2,000 & 1,000 \\
\hline 9 & 10,000 & 9,000 & 8,000 & 7,000 & 6,000 & 5,000 & 4,000 & 3,000 & 2,000 & 1,000 \\
\hline 10 & 10,000 & 9,000 & 8,000 & 7,000 & 6,000 & 5,000 & 4,000 & 3,000 & 2,000 & 1,000 \\
\hline
\end{tabular}




\section{Appendix D The Lingo File of model 2}

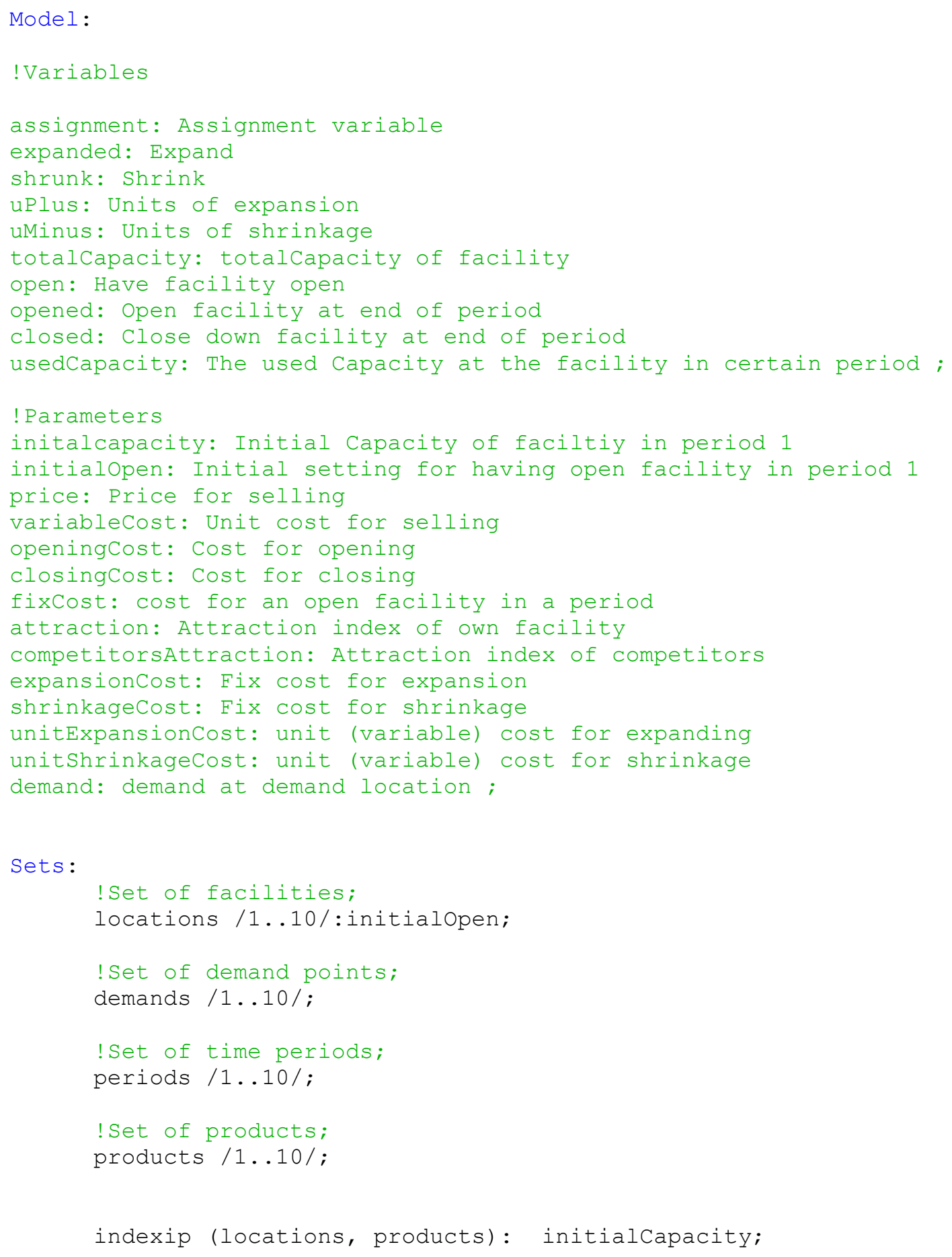


indexijtp (locations, demands,periods,products): assignment; indexijp (locations, demands, products);

indexitp (locations, periods, products): expanded, shrunk, attraction, unitExpansionCost, totalCapacity, unitShrinkageCost, uplus, uMinus, usedCapacity, price, variableCost; demand; indexjtp (demands, periods, products): competitorsAttraction,

indexit (locations, periods): open, opened, closed, fixcost, expansionCost, shrinkageCost, openingCost, closingCost;

Endsets

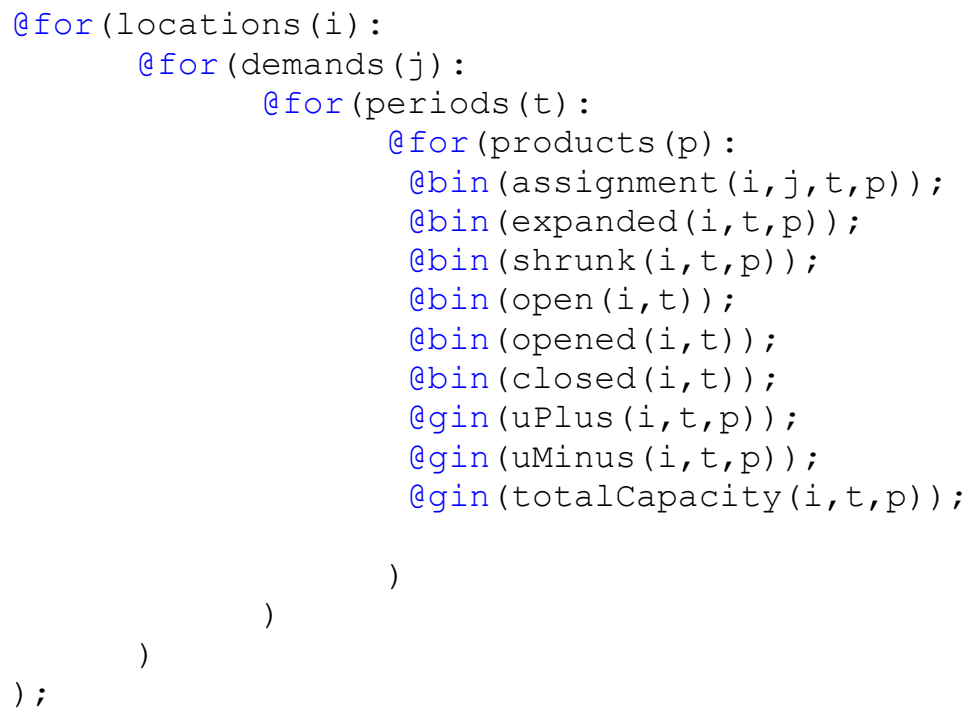

Data :

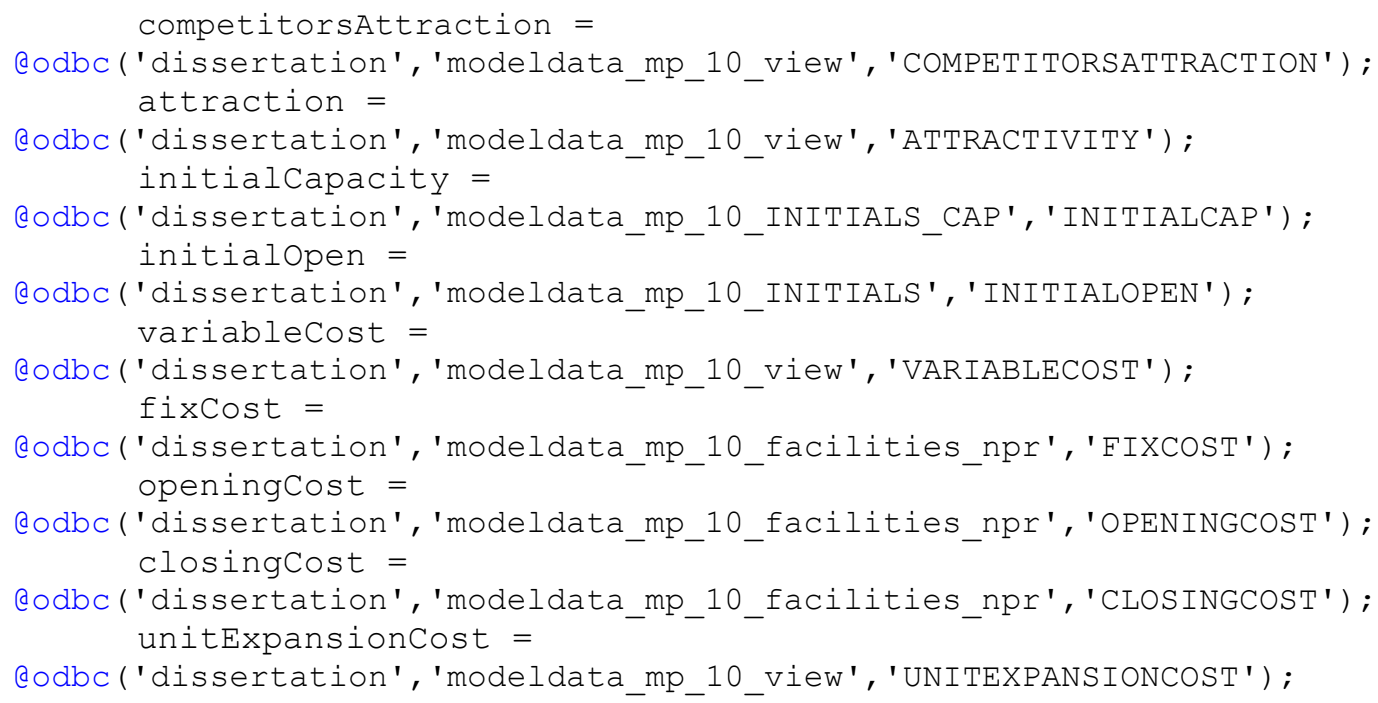




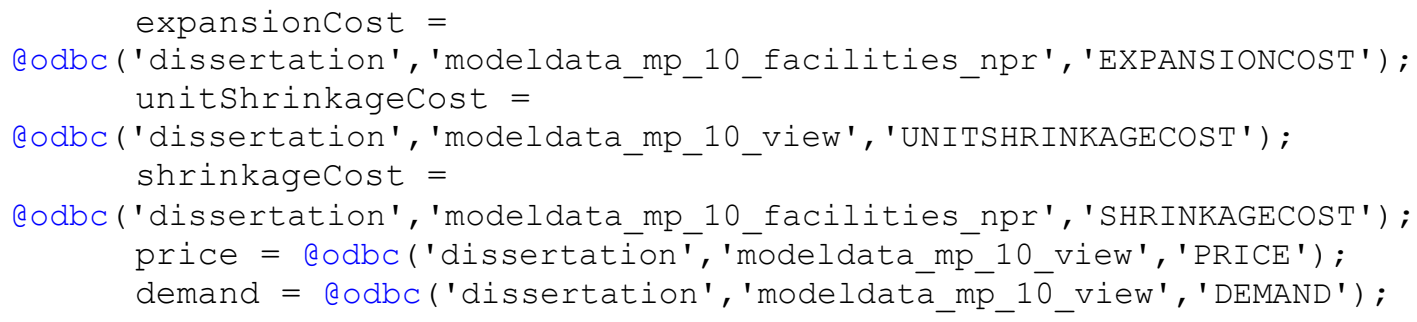




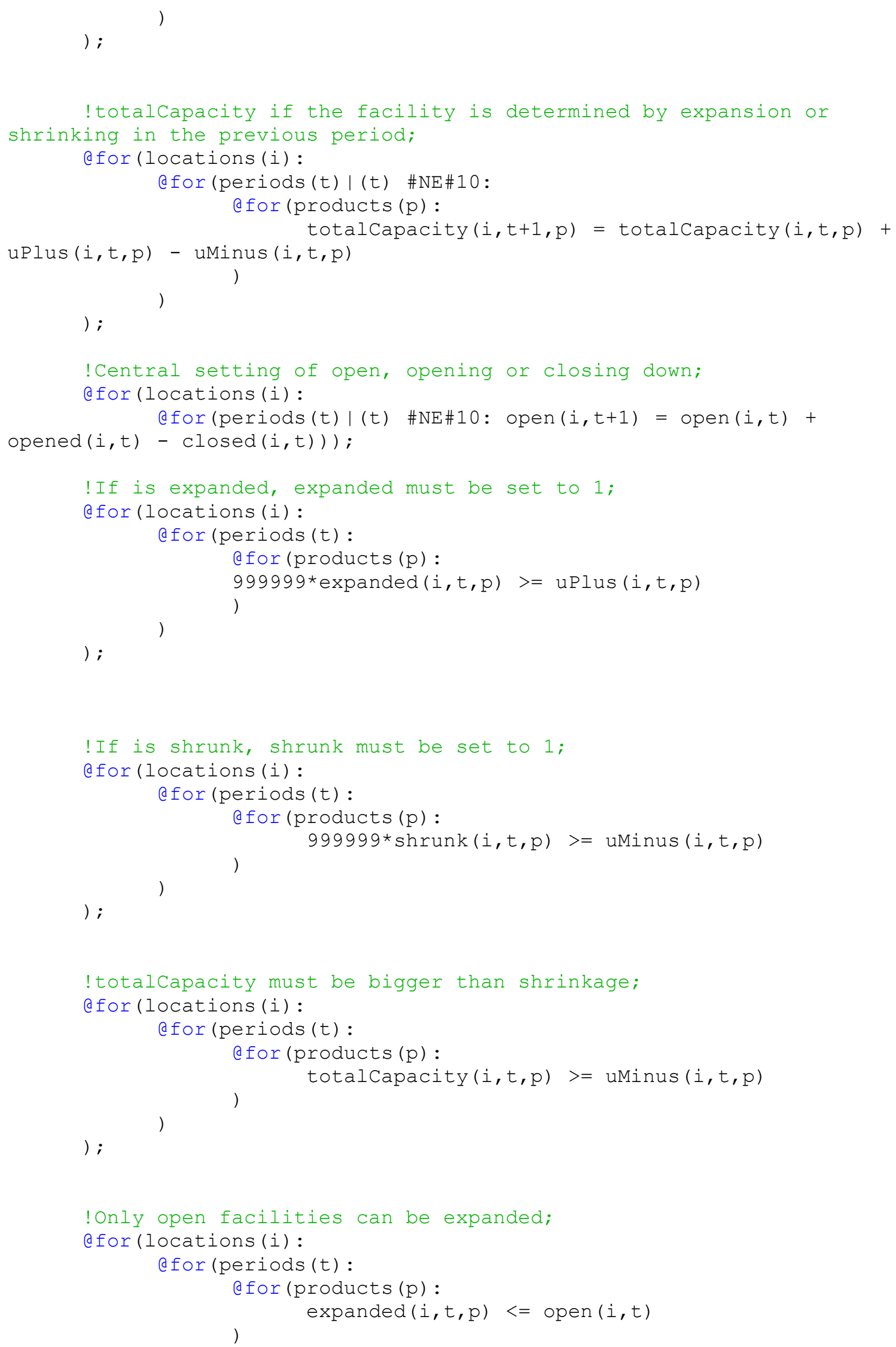




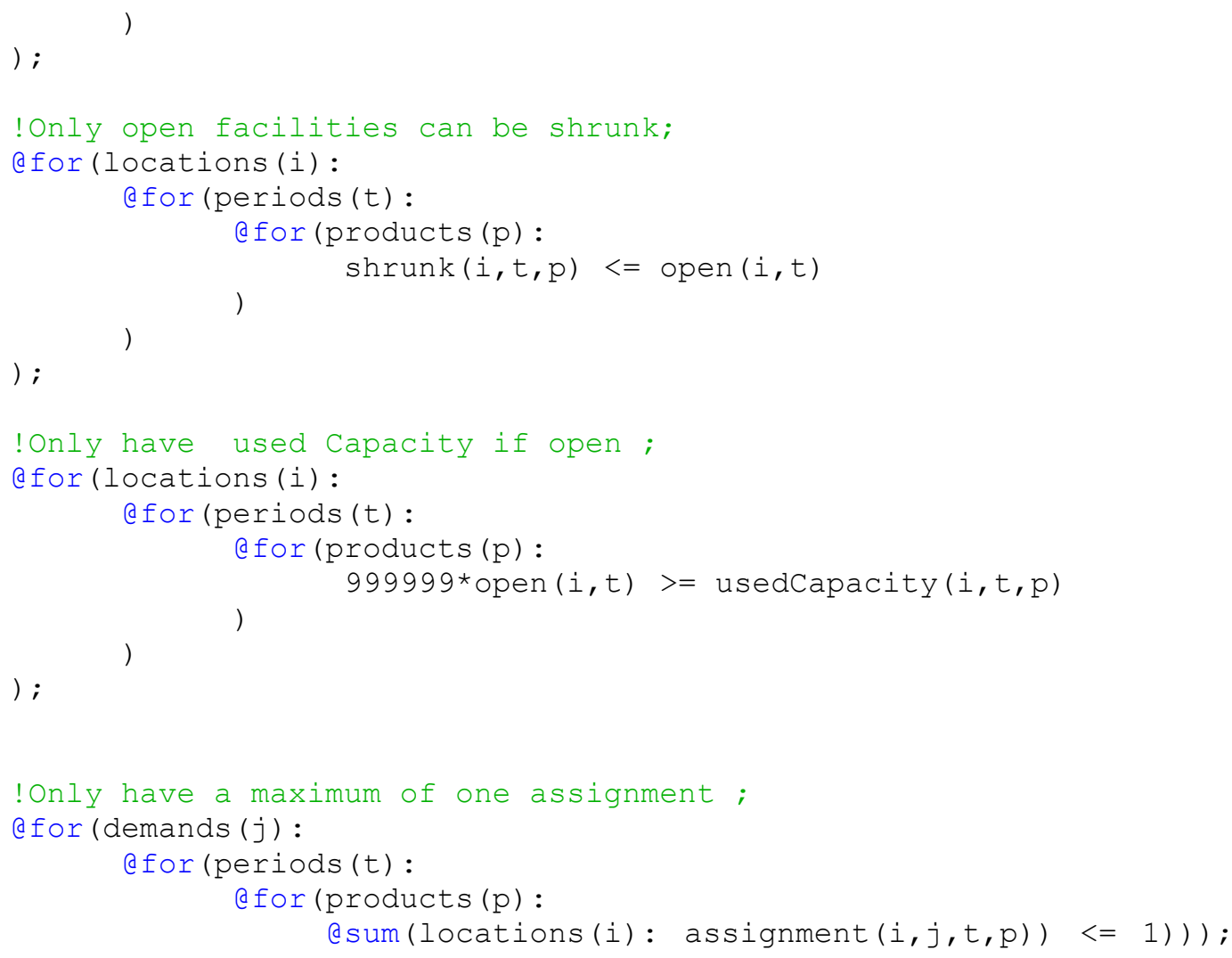

end 


\section{Appendix E The Lingo File of model 2 including extensions}

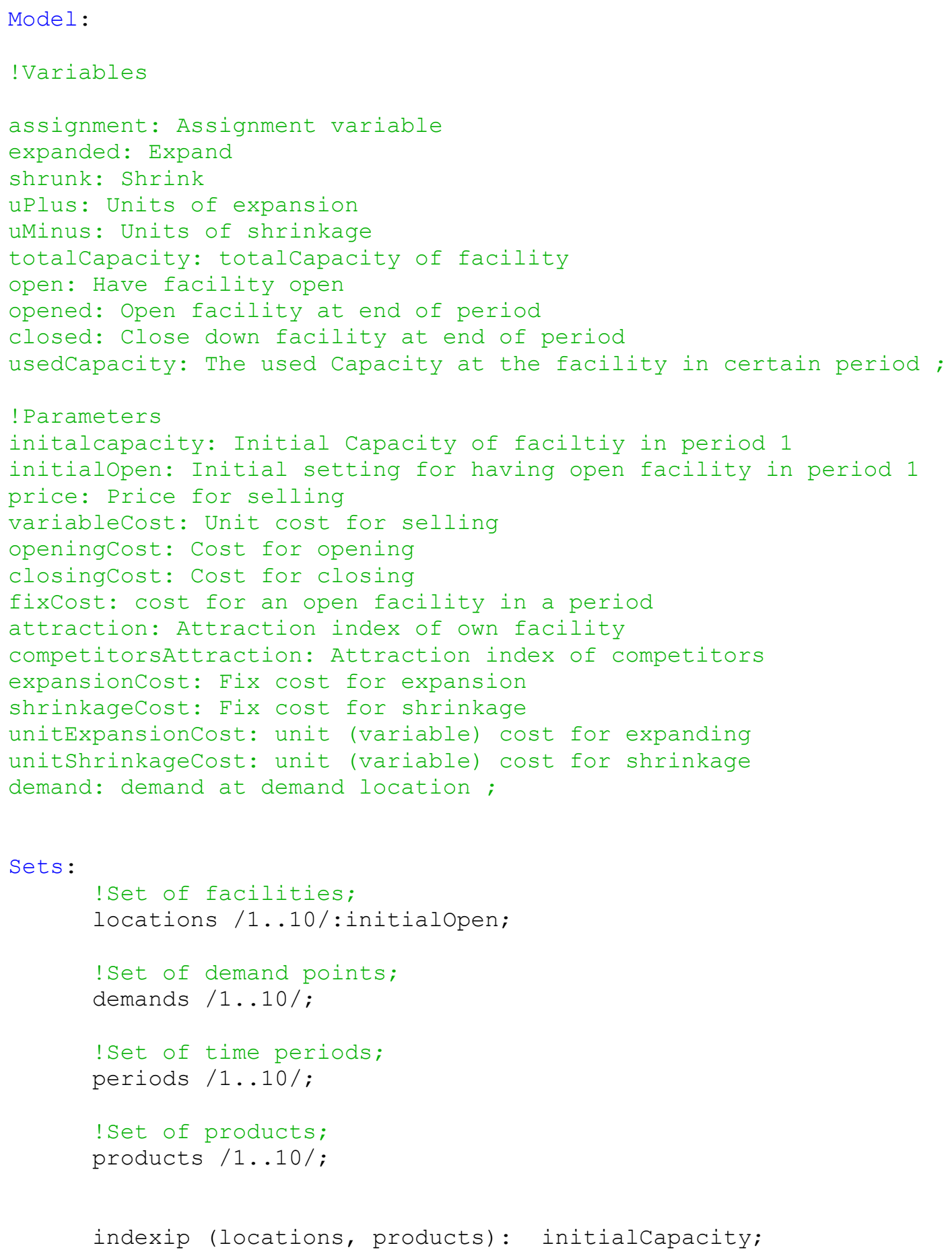




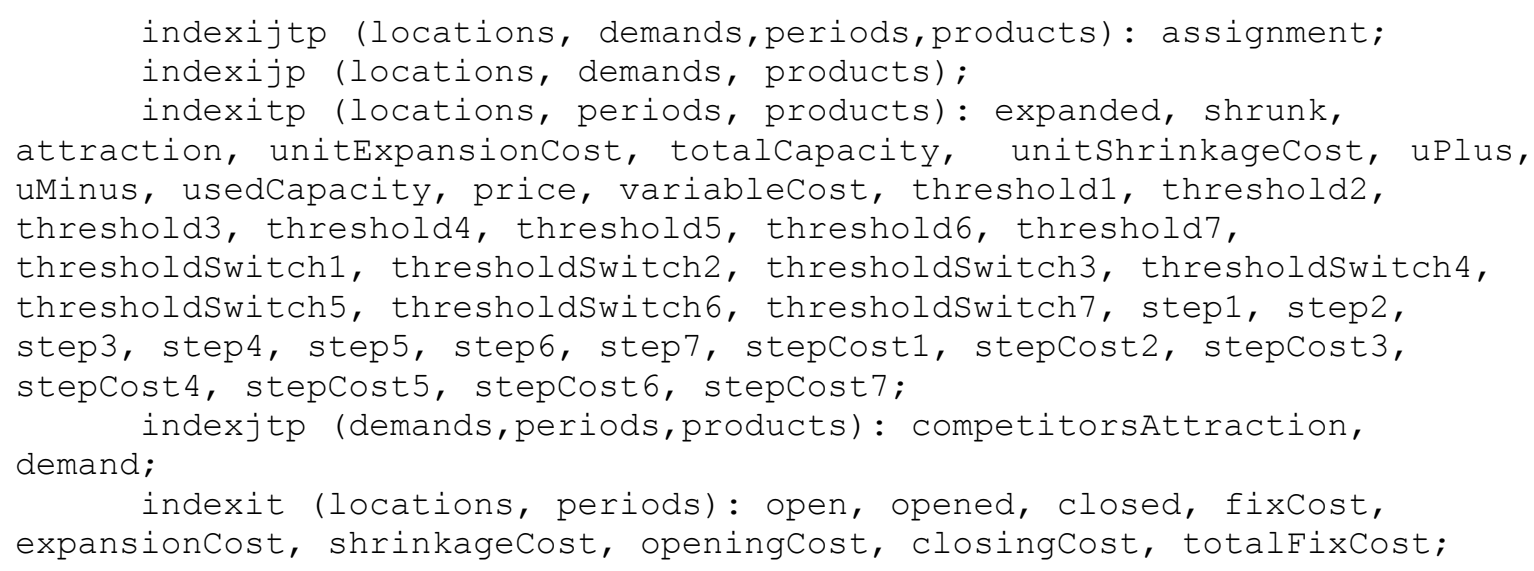

Data: 


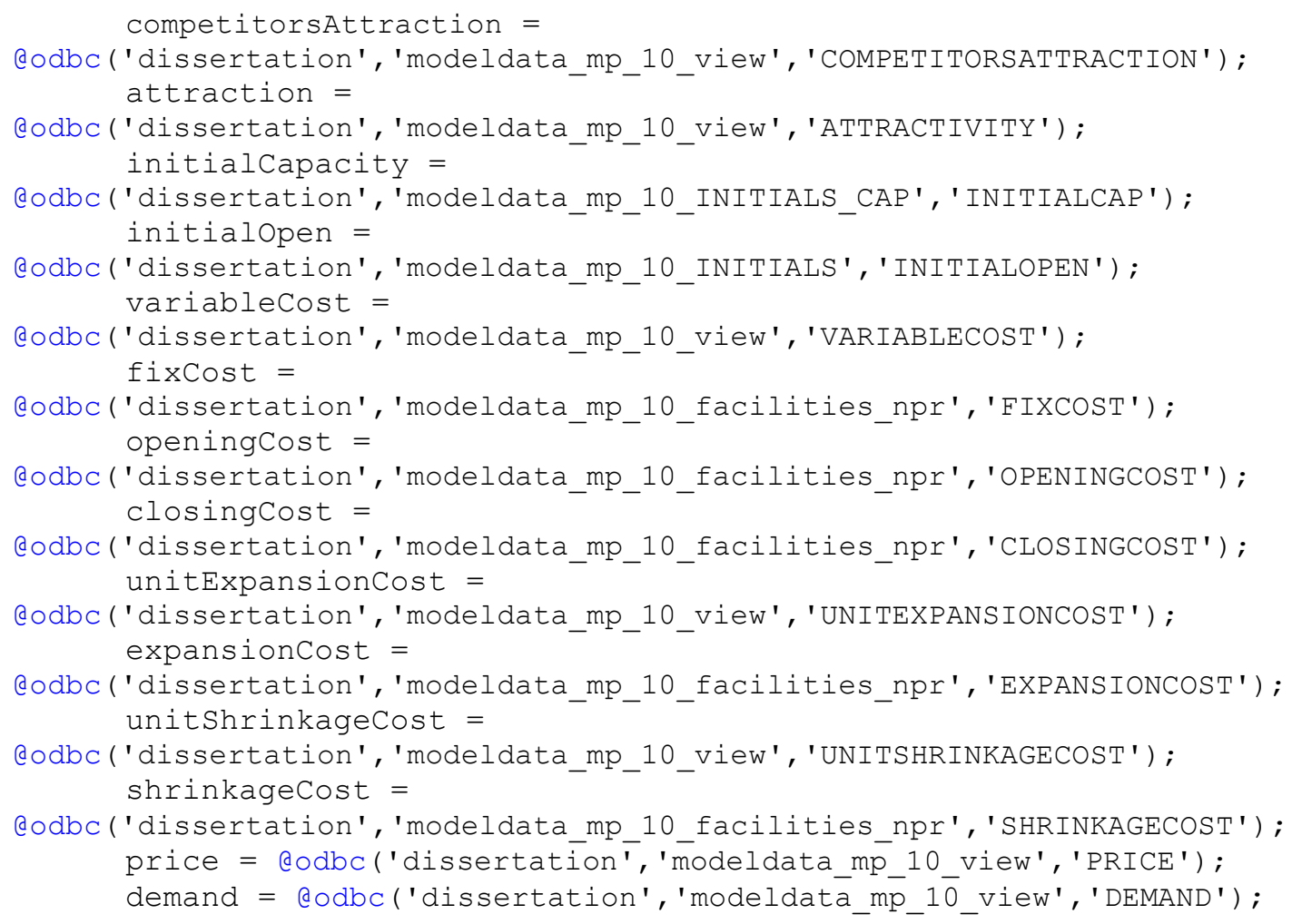

enddata

$\max =$ @sum $(i n d e x i t p(i, t, p): \operatorname{usedCapacity}(i, t, p) *(\operatorname{price}(i, t, p) \quad-$ variableCost $(i, t, p))$ - totalFixCost $(i, t)-\operatorname{closingcost}(i, t){ }^{*} \operatorname{closed}(i, t)$ - openingcost $(i, t) * o p e n e d(i, t))-\operatorname{esum}(i n d e x i t p(i, t, p)$ : expansionCost $(i, t) * \operatorname{expanded}(i, t, p))-$ esum(indexitp $(i, t, p)$ : unitExpansionCost $(i, t, p) * u P l u s(i, t, p))-\operatorname{cum}(i n d e x i t p(i, t, p)$ : shrinkageCost $\left.(i, t){ }^{*} \operatorname{shrunk}(i, t, p)\right)-@ \operatorname{sum}(i n d e x i t p(i, t, p):$ unitShrinkageCost $(i, t, p) * u \operatorname{Minus}(i, t, p))$;

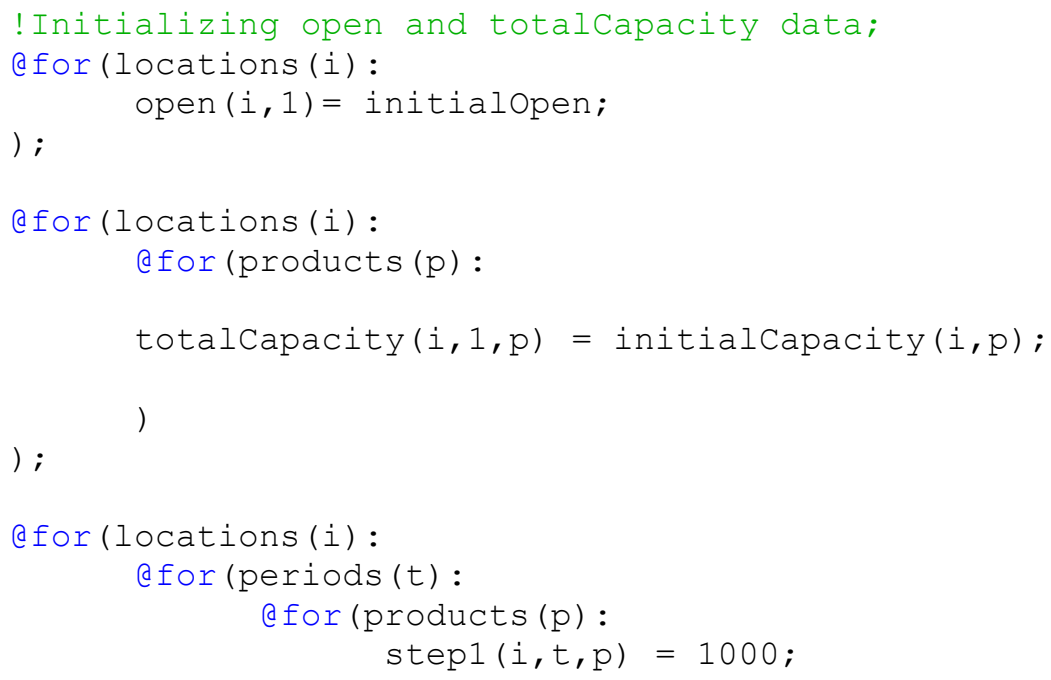




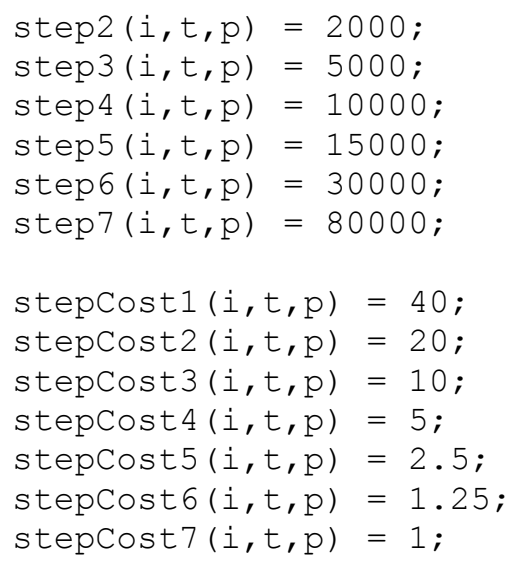




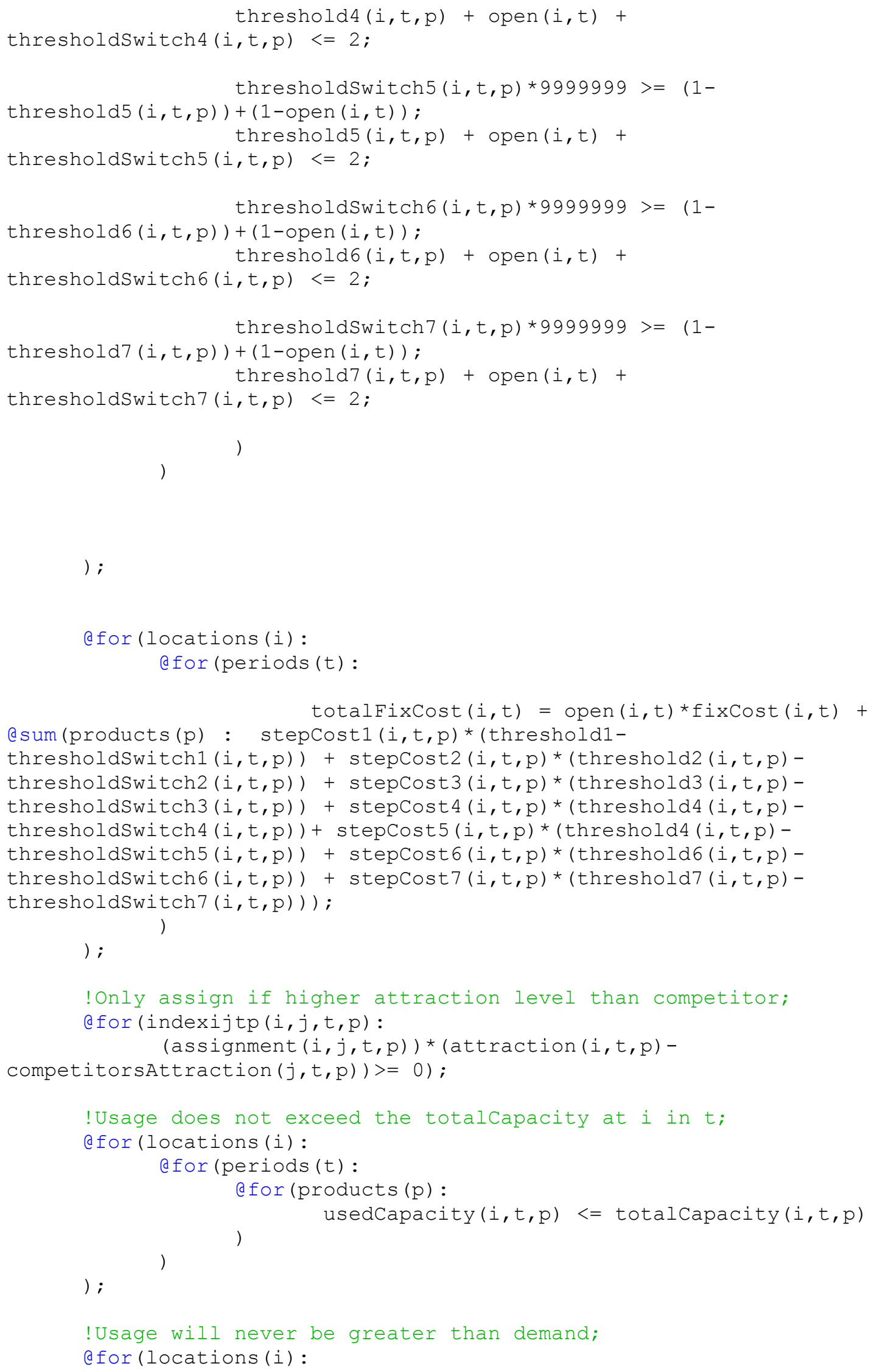




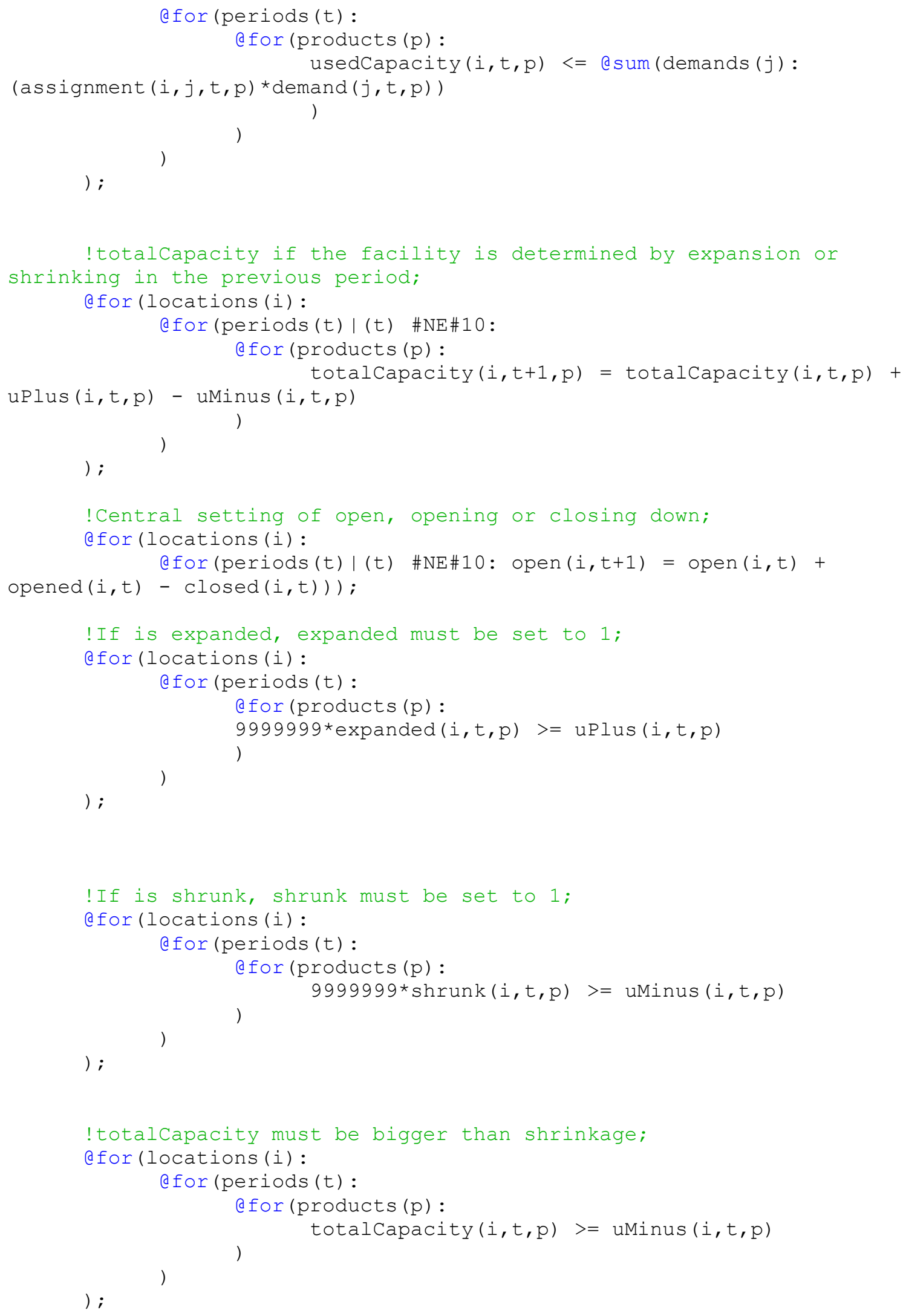




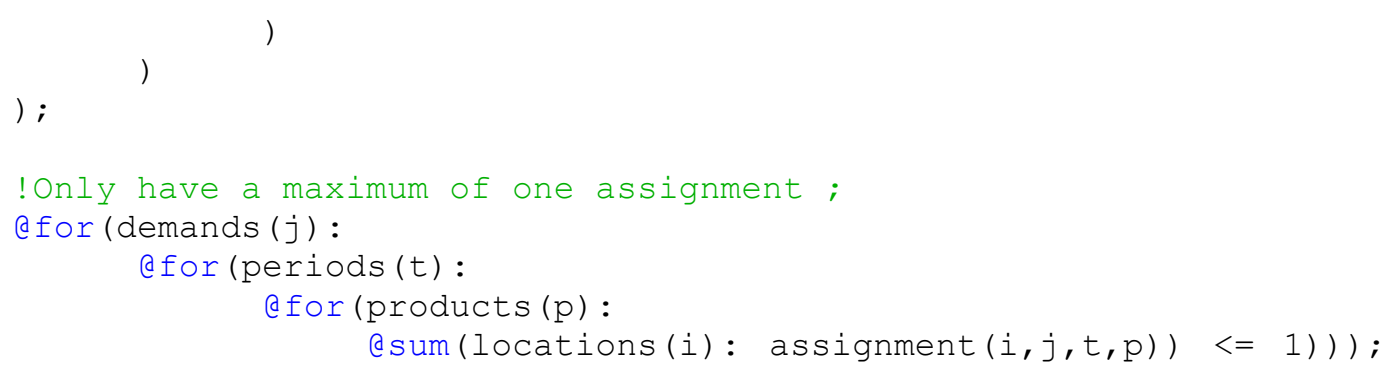

end 


\section{Appendix F The JAVA source code for the simulated annealing}

\section{algorithm}

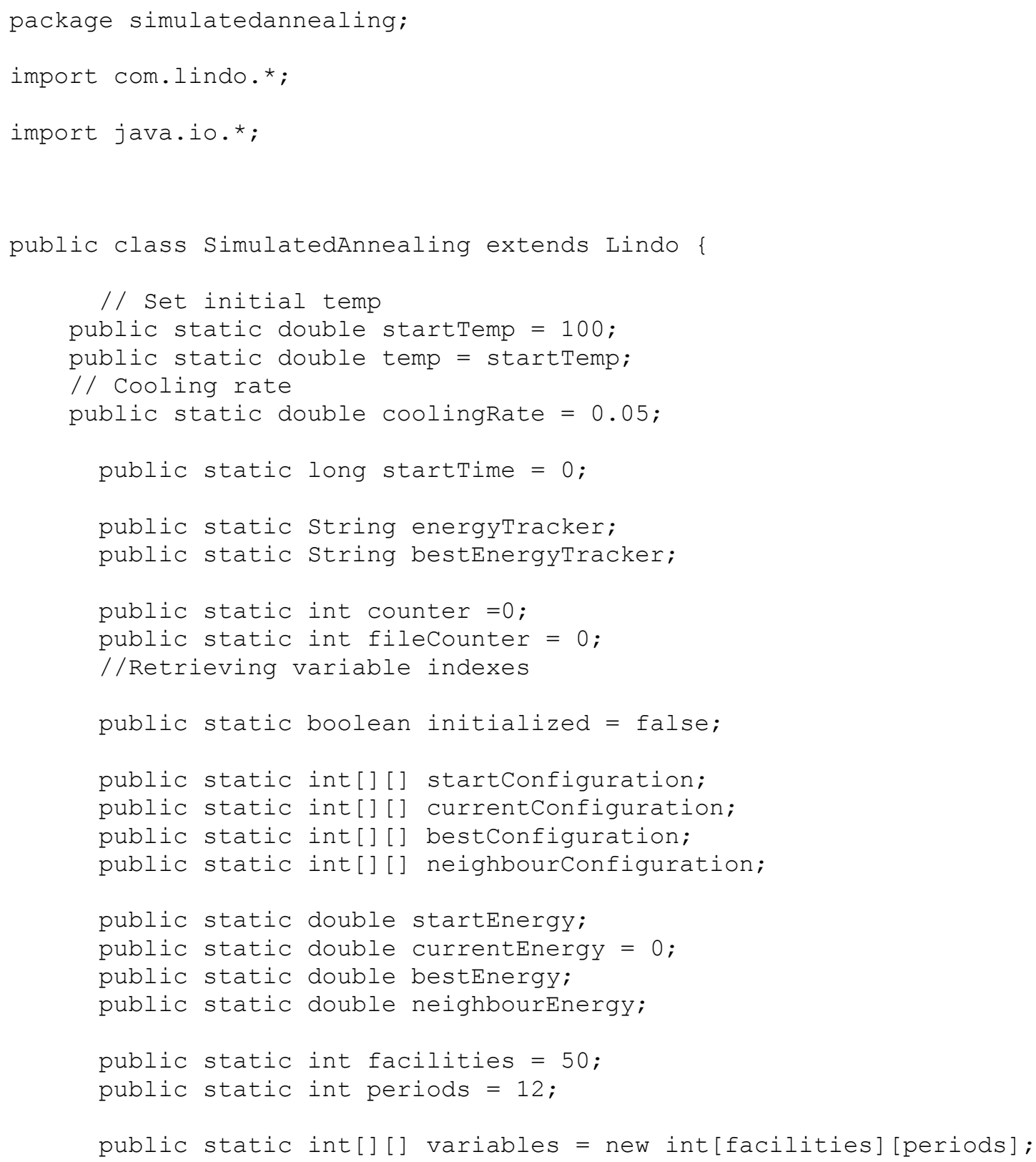


public static String license = "D:/Dropbox/PhD/Dissertation Sven Brose/09_Development/workspace/lib/lindoapi/license/Indapil00.lic";

private static int nErrorCode[] = new int[1];

private static StringBuffer cErrorMessage = new

StringBuffer(LS MAX_ERROR MESSAGE_LENGTH);

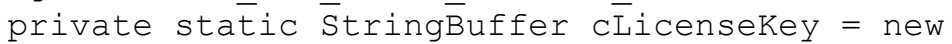

StringBuffer(LS_MAX_ERROR_MESSAGE_LENGTH) ;

double[] [] $\bar{a} r r a \bar{y}=$ nulli;

double[] adC = null;

double[] $\mathrm{adB}=\mathrm{null}$;

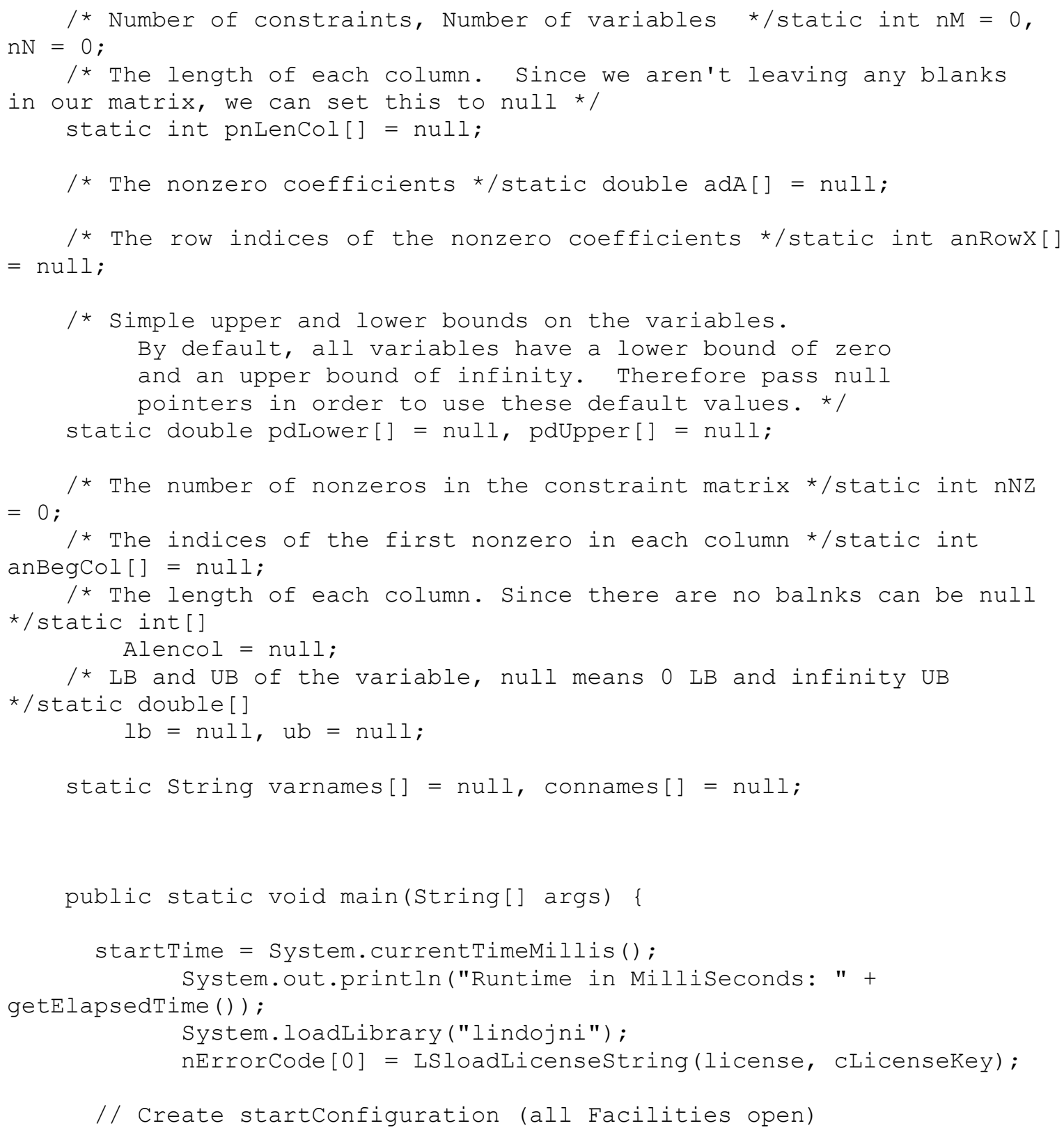




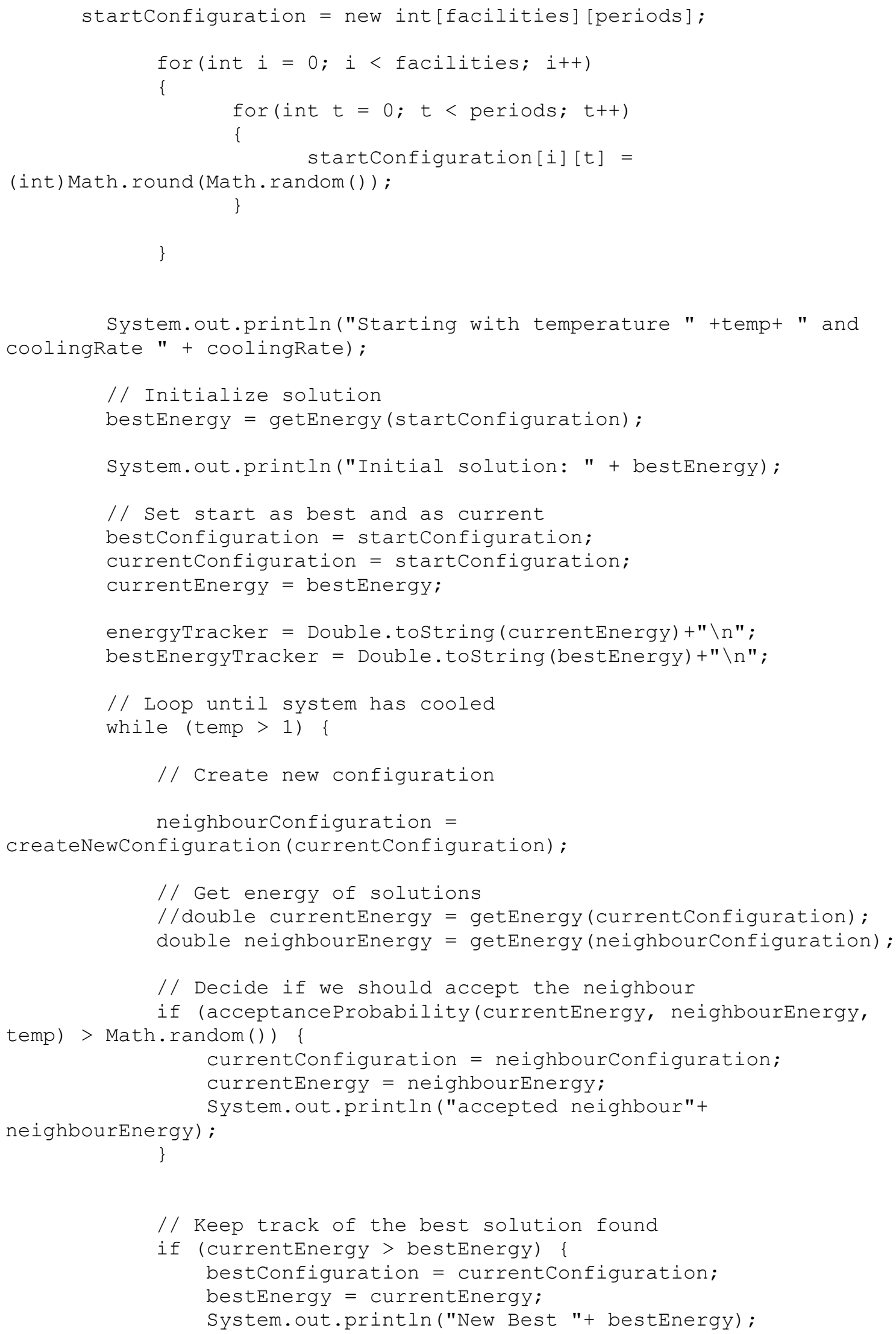




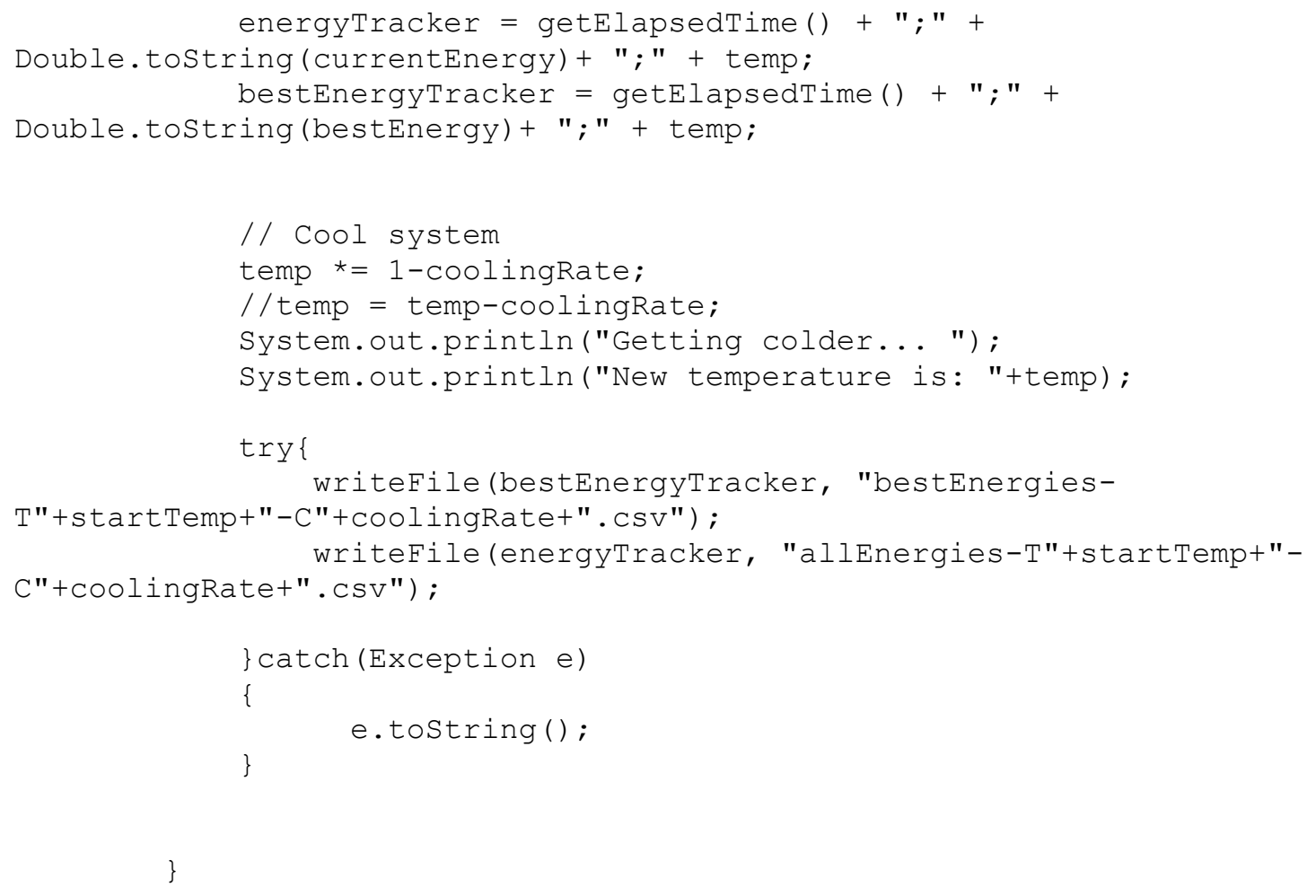




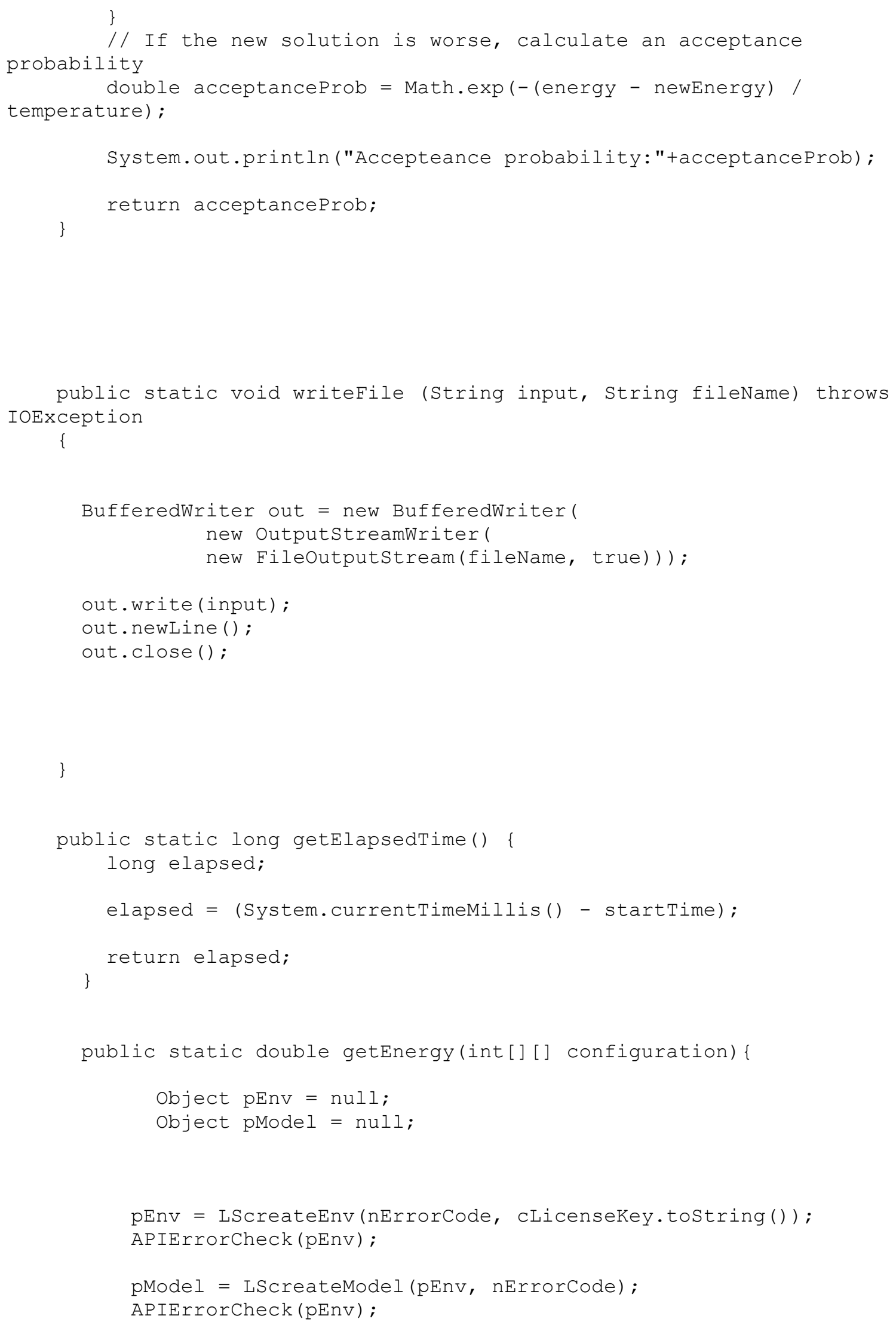


String fileName = "D:/Users/sbrose/Dropbox/PhD/Dissertation Sven Brose/06_Lingo/Models/Model 2/50x50x50x12/50x50x50x12.mps";

//System.out.println("Loading file ... ");

//System.out.println(fileName);

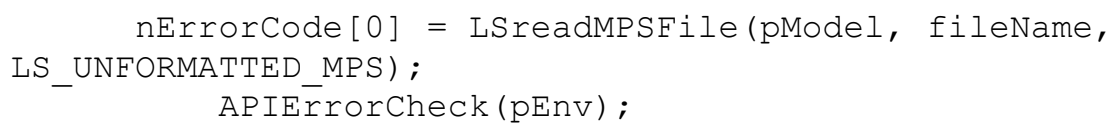




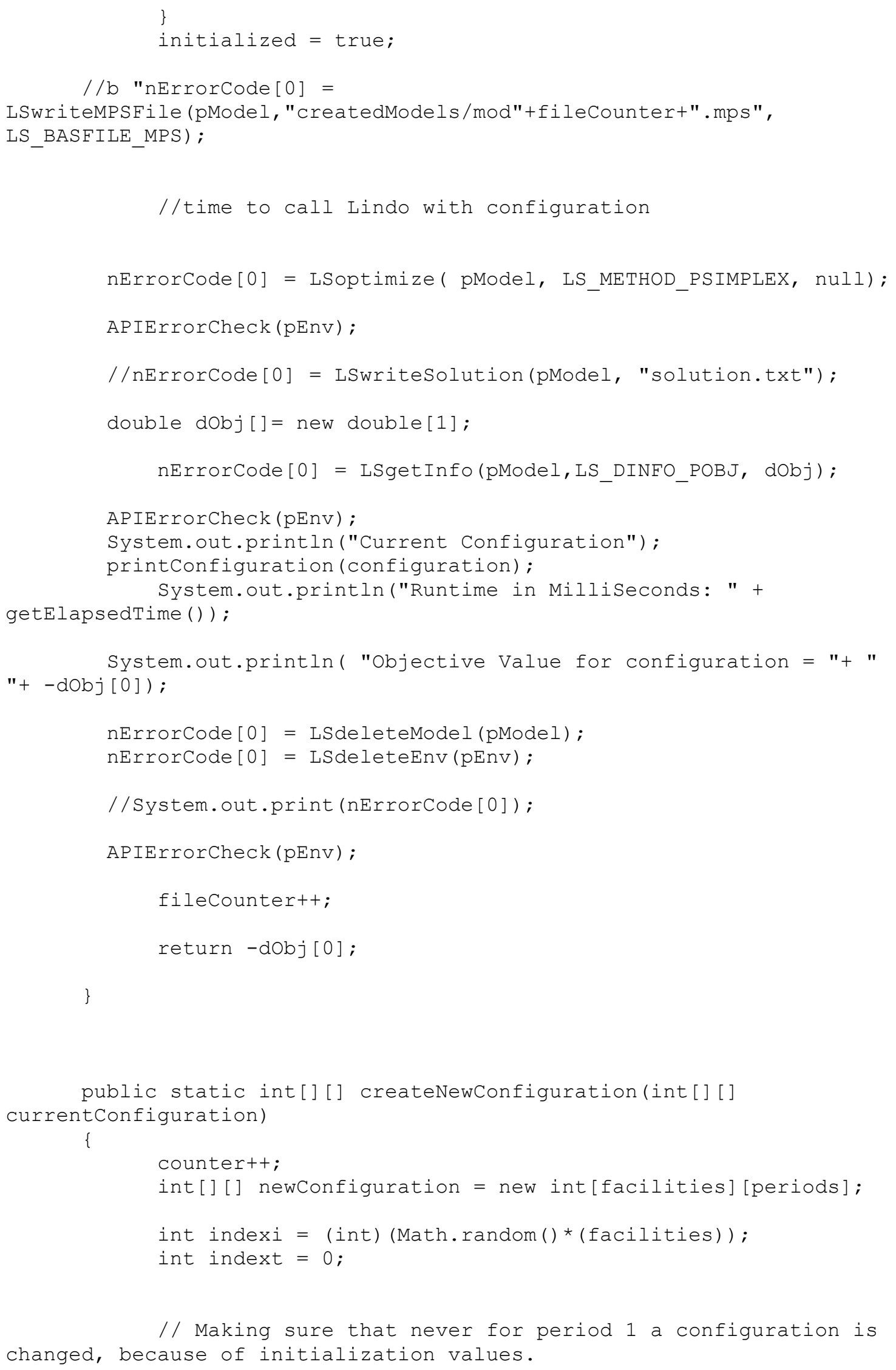




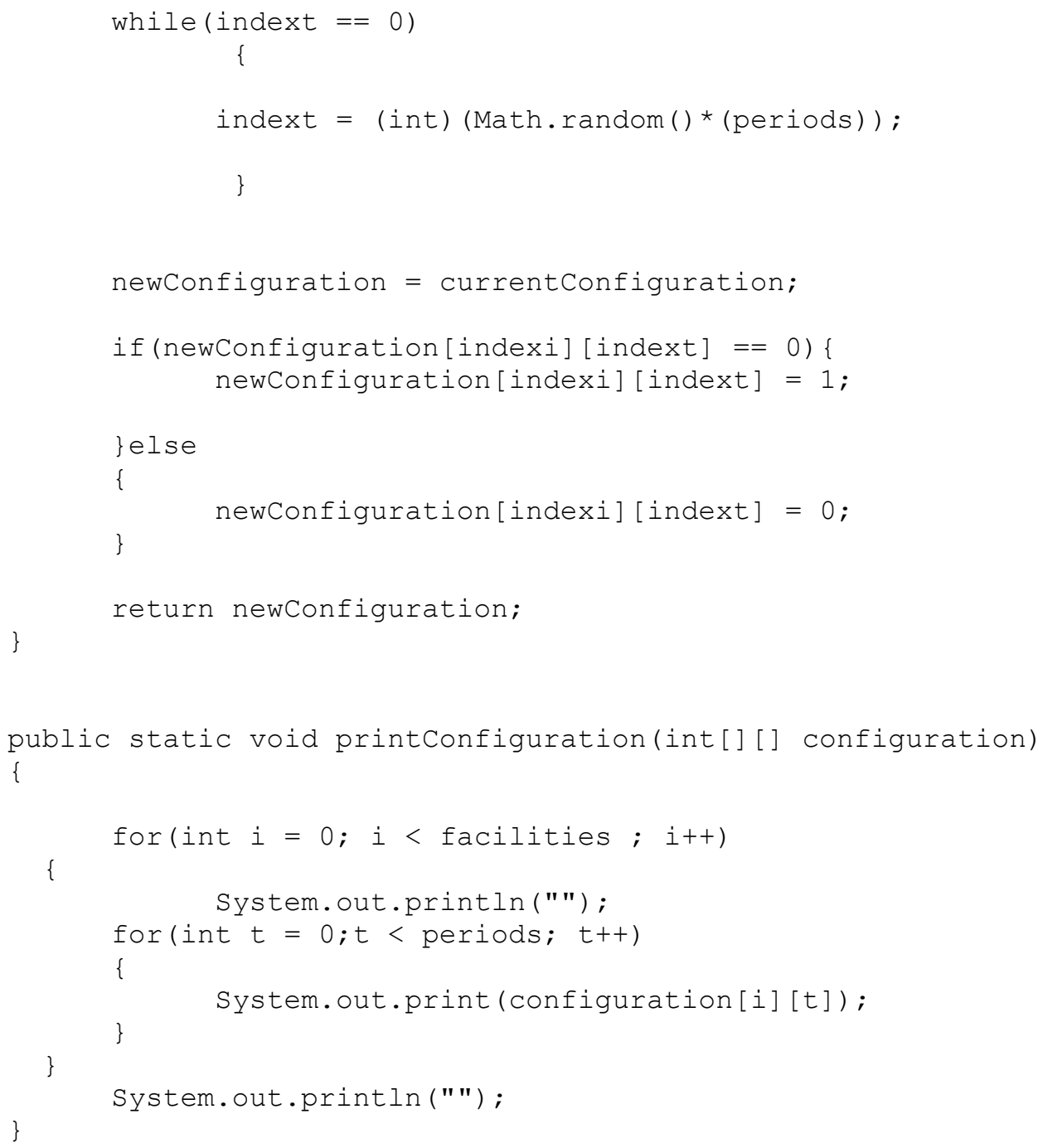




\section{Appendix G Ruby script for gathering distance information}

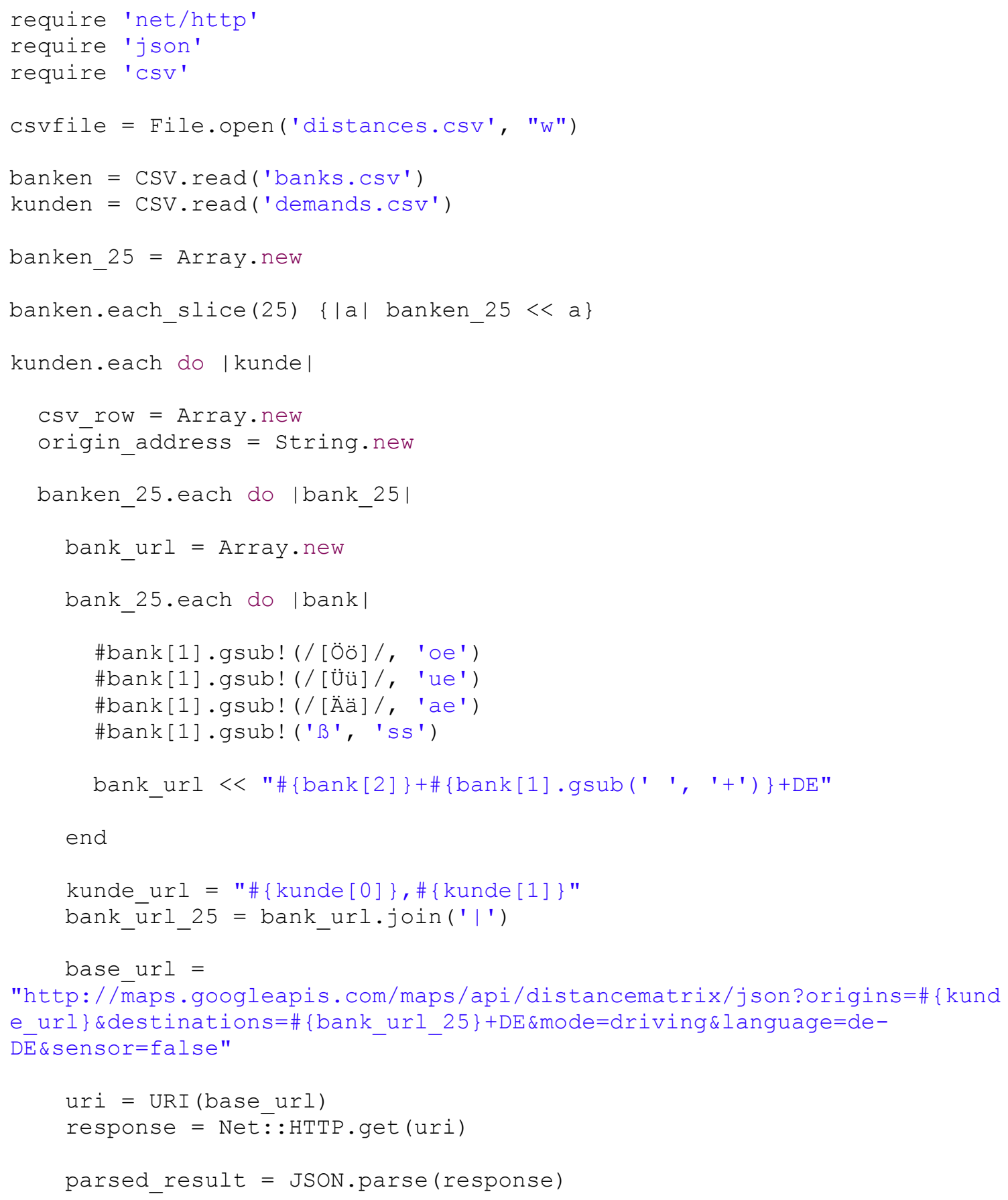




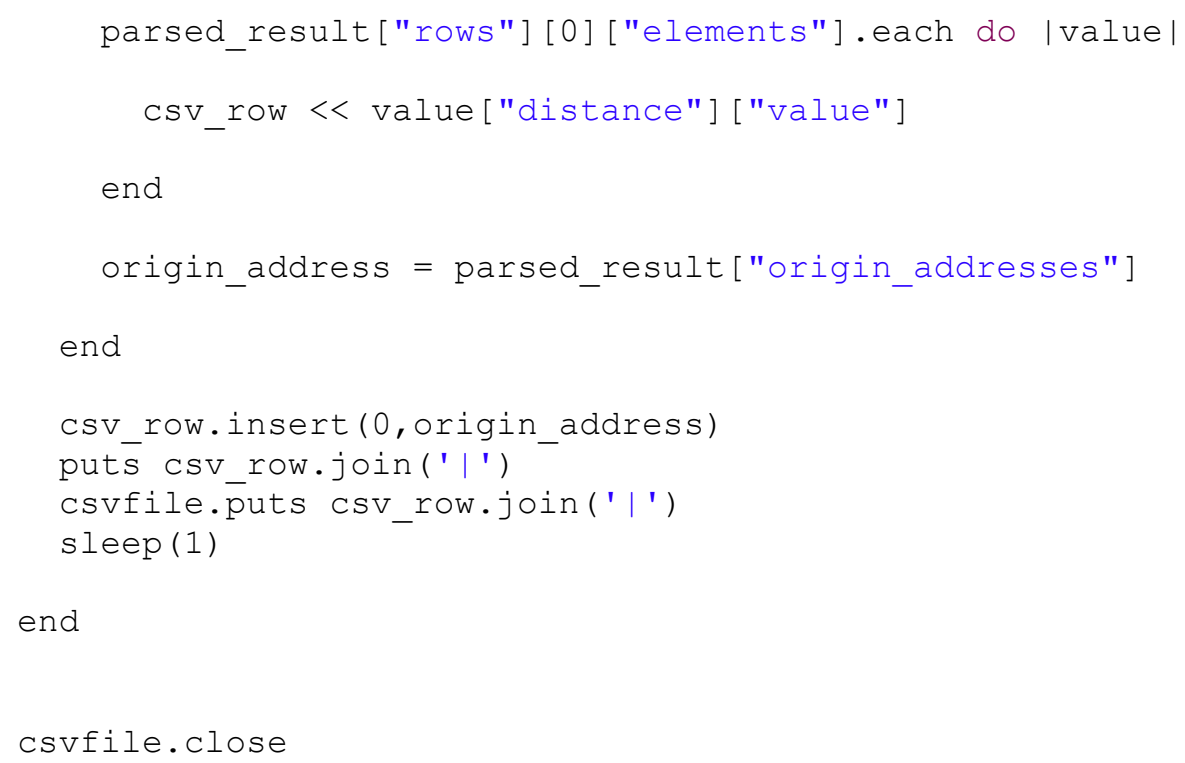




\section{Appendix H Data for case study}

Table 46 Initial capacity of facilities

\begin{tabular}{|c|c|}
\hline facility & capacity \\
\hline 1 & 2400 \\
\hline 2 & 600 \\
\hline 3 & 1200 \\
\hline 4 & 1200 \\
\hline 5 & 600 \\
\hline 6 & 1200 \\
\hline 7 & 1200 \\
\hline 8 & 600 \\
\hline 9 & 600 \\
\hline 10 & 600 \\
\hline 11 & 600 \\
\hline 12 & 600 \\
\hline 13 & 600 \\
\hline 14 & 1200 \\
\hline 15 & 1200 \\
\hline 16 & 1200 \\
\hline 17 & 600 \\
\hline 18 & 1200 \\
\hline 19 & 1200 \\
\hline 20 & 600 \\
\hline 21 & 1200 \\
\hline 22 & 600 \\
\hline 23 & 600 \\
\hline 24 & 1200 \\
\hline 25 & 600 \\
\hline 26 & 600 \\
\hline 27 & 600 \\
\hline 28 & 600 \\
\hline 29 & 600 \\
\hline 30 & 600 \\
\hline 31 & 600 \\
\hline 32 & 600 \\
\hline 33 & 600 \\
\hline 34 & 600 \\
\hline
\end{tabular}




\begin{tabular}{|cc|}
35 & 1200 \\
36 & 600 \\
\hline
\end{tabular}

Table 47 prices and variable cost

\begin{tabular}{|c|c|c|c|}
\hline INDEXT & INDEXP & PRICE & VARIABLECOST \\
\hline 1 & 1 & 24 & 30 \\
\hline 1 & 2 & 10 & 1.1 \\
\hline 1 & 3 & 30 & 27.5 \\
\hline 1 & 4 & 10 & 5.5 \\
\hline 1 & 5 & 1000 & 550 \\
\hline 1 & 6 & 20 & 11 \\
\hline 1 & 7 & 50 & 27.5 \\
\hline 1 & 8 & 20 & 11 \\
\hline 1 & 9 & 10 & 1.1 \\
\hline 1 & 10 & 25 & 11 \\
\hline 1 & 11 & 10 & 5.5 \\
\hline 1 & 12 & 10 & 5.5 \\
\hline 1 & 13 & 10 & 5.5 \\
\hline 2 & 1 & 24 & 30 \\
\hline 2 & 2 & 10 & 1.2 \\
\hline 2 & 3 & 30 & 30 \\
\hline 2 & 4 & 10 & 6 \\
\hline 2 & 5 & 1000 & 600 \\
\hline 2 & 6 & 20 & 12 \\
\hline 2 & 7 & 50 & 30 \\
\hline 2 & 8 & 20 & 12 \\
\hline 2 & 9 & 10 & 1.2 \\
\hline 2 & 10 & 25 & 12 \\
\hline 2 & 11 & 10 & 6 \\
\hline 2 & 12 & 10 & 6 \\
\hline 2 & 13 & 10 & 6 \\
\hline 3 & 1 & 24 & 30 \\
\hline 3 & 2 & 10 & 1.3 \\
\hline 3 & 3 & 30 & 32.5 \\
\hline 3 & 4 & 10 & 6.5 \\
\hline 3 & 5 & 1000 & 650 \\
\hline 3 & 6 & 20 & 13 \\
\hline 3 & 7 & 50 & 32.5 \\
\hline 3 & 8 & 20 & 13 \\
\hline 3 & 9 & 10 & 1.3 \\
\hline 3 & 10 & 25 & 13 \\
\hline 3 & 11 & 10 & 6.5 \\
\hline 3 & 12 & 10 & 6.5 \\
\hline
\end{tabular}




\begin{tabular}{|c|c|c|c|}
\hline 3 & 13 & 10 & 6.5 \\
\hline 4 & 1 & 24 & 30 \\
\hline 4 & 2 & 10 & 1.4 \\
\hline 4 & 3 & 30 & 35 \\
\hline 4 & 4 & 10 & 7 \\
\hline 4 & 5 & 1000 & 700 \\
\hline 4 & 6 & 20 & 14 \\
\hline 4 & 7 & 50 & 35 \\
\hline 4 & 8 & 20 & 14 \\
\hline 4 & 9 & 10 & 1.4 \\
\hline 4 & 10 & 25 & 14 \\
\hline 4 & 11 & 10 & 7 \\
\hline 4 & 12 & 10 & 7 \\
\hline 4 & 13 & 10 & 7 \\
\hline 5 & 1 & 24 & 30 \\
\hline 5 & 2 & 10 & 1.5 \\
\hline 5 & 3 & 30 & 37.5 \\
\hline 5 & 4 & 10 & 7.5 \\
\hline 5 & 5 & 1000 & 750 \\
\hline 5 & 6 & 20 & 15 \\
\hline 5 & 7 & 50 & 37.5 \\
\hline 5 & 8 & 20 & 15 \\
\hline 5 & 9 & 10 & 1.5 \\
\hline 5 & 10 & 25 & 15 \\
\hline 5 & 11 & 10 & 7.5 \\
\hline 5 & 12 & 10 & 7.5 \\
\hline 5 & 13 & 10 & 7.5 \\
\hline 6 & 1 & 24 & 30 \\
\hline 6 & 2 & 10 & 1.6 \\
\hline 6 & 3 & 30 & 40 \\
\hline 6 & 4 & 10 & 8 \\
\hline 6 & 5 & 1000 & 800 \\
\hline 6 & 6 & 20 & 16 \\
\hline 6 & 7 & 50 & 40 \\
\hline 6 & 8 & 20 & 16 \\
\hline 6 & 9 & 10 & 1.6 \\
\hline 6 & 10 & 25 & 16 \\
\hline 6 & 11 & 10 & 8 \\
\hline 6 & 12 & 10 & 8 \\
\hline 6 & 13 & 10 & 8 \\
\hline 7 & 1 & 24 & 30 \\
\hline 7 & 2 & 10 & 1.7 \\
\hline 7 & 3 & 30 & 42.5 \\
\hline 7 & 4 & 10 & 8.5 \\
\hline 7 & 5 & 1000 & 850 \\
\hline
\end{tabular}




\begin{tabular}{|c|c|c|c|}
\hline 7 & 6 & 20 & 17 \\
\hline 7 & 7 & 50 & 42.5 \\
\hline 7 & 8 & 20 & 17 \\
\hline 7 & 9 & 10 & 1.7 \\
\hline 7 & 10 & 25 & 17 \\
\hline 7 & 11 & 10 & 8.5 \\
\hline 7 & 12 & 10 & 8.5 \\
\hline 7 & 13 & 10 & 8.5 \\
\hline 8 & 1 & 24 & 30 \\
\hline 8 & 2 & 10 & 1.8 \\
\hline 8 & 3 & 30 & 45 \\
\hline 8 & 4 & 10 & 9 \\
\hline 8 & 5 & 1000 & 900 \\
\hline 8 & 6 & 20 & 18 \\
\hline 8 & 7 & 50 & 45 \\
\hline 8 & 8 & 20 & 18 \\
\hline 8 & 9 & 10 & 1.8 \\
\hline 8 & 10 & 25 & 18 \\
\hline 8 & 11 & 10 & 9 \\
\hline 8 & 12 & 10 & 9 \\
\hline 8 & 13 & 10 & 9 \\
\hline 9 & 1 & 24 & 30 \\
\hline 9 & 2 & 10 & 1.9 \\
\hline 9 & 3 & 30 & 47.5 \\
\hline 9 & 4 & 10 & 9.5 \\
\hline 9 & 5 & 1000 & 950 \\
\hline 9 & 6 & 20 & 19 \\
\hline 9 & 7 & 50 & 47.5 \\
\hline 9 & 8 & 20 & 19 \\
\hline 9 & 9 & 10 & 1.9 \\
\hline 9 & 10 & 25 & 19 \\
\hline 9 & 11 & 10 & 9.5 \\
\hline 9 & 12 & 10 & 9.5 \\
\hline 9 & 13 & 10 & 9.5 \\
\hline 10 & 1 & 24 & 30 \\
\hline 10 & 2 & 10 & 2 \\
\hline 10 & 3 & 30 & 50 \\
\hline 10 & 4 & 10 & 10 \\
\hline 10 & 5 & 1000 & 1000 \\
\hline 10 & 6 & 20 & 20 \\
\hline 10 & 7 & 50 & 50 \\
\hline 10 & 8 & 20 & 20 \\
\hline 10 & 9 & 10 & 2 \\
\hline 10 & 10 & 25 & 20 \\
\hline 10 & 11 & 10 & 10 \\
\hline \multicolumn{4}{|c|}{204} \\
\hline
\end{tabular}




\begin{tabular}{llll}
10 & 12 & 10 & 10 \\
10 & 13 & 10 & 10 \\
\hline
\end{tabular}


Table 48 expansion, shrinkage, opening, closing and fixed cost

\begin{tabular}{|c|c|c|c|c|c|}
\hline INDEXI & $\begin{array}{l}\text { EXPANSION } \\
\text { COST }\end{array}$ & $\begin{array}{l}\text { SHRINKAGE } \\
\text { COST }\end{array}$ & $\begin{array}{l}\text { FIXED } \\
\text { COST }\end{array}$ & $\begin{array}{l}\text { OPENING } \\
\text { COST }\end{array}$ & $\begin{array}{l}\text { CLOSING } \\
\text { COST }\end{array}$ \\
\hline 1 & 20000 & 25000 & 96000 & 5000000 & 500000 \\
\hline 1 & 20000 & 25000 & 24000 & 100000 & 50000 \\
\hline 1 & 20000 & 25000 & 36000 & 100000 & 50000 \\
\hline 1 & 20000 & 25000 & 24000 & 100000 & 50000 \\
\hline 1 & 20000 & 25000 & 18000 & 100000 & 50000 \\
\hline 1 & 20000 & 25000 & 36000 & 100000 & 50000 \\
\hline 1 & 20000 & 25000 & 24000 & 100000 & 50000 \\
\hline 1 & 20000 & 25000 & 12000 & 100000 & 50000 \\
\hline 1 & 20000 & 25000 & 24000 & 100000 & 50000 \\
\hline 1 & 20000 & 25000 & 18000 & 100000 & 50000 \\
\hline 1 & 20000 & 25000 & 12000 & 100000 & 50000 \\
\hline 1 & 20000 & 25000 & 18000 & 100000 & 50000 \\
\hline 1 & 20000 & 25000 & 12000 & 100000 & 50000 \\
\hline 1 & 20000 & 25000 & 48000 & 100000 & 50000 \\
\hline 1 & 20000 & 25000 & 36000 & 100000 & 50000 \\
\hline 1 & 20000 & 25000 & 36000 & 100000 & 50000 \\
\hline 1 & 20000 & 25000 & 18000 & 100000 & 50000 \\
\hline 1 & 20000 & 25000 & 36000 & 100000 & 50000 \\
\hline 1 & 20000 & 25000 & 24000 & 100000 & 50000 \\
\hline 1 & 20000 & 25000 & 24000 & 100000 & 50000 \\
\hline 1 & 20000 & 25000 & 48000 & 100000 & 50000 \\
\hline 1 & 20000 & 25000 & 12000 & 100000 & 50000 \\
\hline 1 & 20000 & 25000 & 12000 & 100000 & 50000 \\
\hline 1 & 20000 & 25000 & 24000 & 100000 & 50000 \\
\hline 1 & 20000 & 25000 & 12000 & 100000 & 50000 \\
\hline 1 & 20000 & 25000 & 24000 & 100000 & 50000 \\
\hline 1 & 20000 & 25000 & 9600 & 100000 & 50000 \\
\hline 1 & 20000 & 25000 & 24000 & 100000 & 50000 \\
\hline 1 & 20000 & 25000 & 18000 & 100000 & 50000 \\
\hline 1 & 20000 & 25000 & 12000 & 100000 & 50000 \\
\hline 1 & 20000 & 25000 & 12000 & 100000 & 50000 \\
\hline 1 & 20000 & 25000 & 18000 & 100000 & 50000 \\
\hline 1 & 20000 & 25000 & 18000 & 100000 & 50000 \\
\hline 1 & 20000 & 25000 & 24000 & 100000 & 50000 \\
\hline 1 & 20000 & 25000 & 36000 & 100000 & 50000 \\
\hline 1 & 20000 & 25000 & 12000 & 100000 & 50000 \\
\hline
\end{tabular}


Table 49 Demand data for case study for all 13 products

\begin{tabular}{|c|c|c|c|c|c|c|c|c|c|c|c|c|c|c|}
\hline period & demand & 1 & 2 & 3 & 4 & 5 & 6 & 7 & 8 & 9 & 10 & 11 & 12 & 13 \\
\hline 1 & 1 & 2071 & 1225 & 1194 & 1013 & 854 & 881 & 749 & 654 & 557 & 445 & 299 & 207 & 138 \\
\hline 1 & 2 & 53 & 313 & 290 & 242 & 215 & 224 & 188 & 174 & 144 & 117 & 83 & 55 & 40 \\
\hline 1 & 3 & 2283 & 1362 & 1340 & 1144 & 956 & 979 & 832 & 716 & 613 & 488 & 324 & 225 & 149 \\
\hline 1 & 4 & 1473 & 864 & 832 & 704 & 600 & 621 & 531 & 473 & 399 & 321 & 219 & 150 & 100 \\
\hline 1 & 5 & 745 & 2828 & 2712 & 2291 & 1964 & 2030 & 1725 & 1539 & 1297 & 1043 & 713 & 487 & 322 \\
\hline 1 & 6 & 1737 & 1035 & 998 & 848 & 725 & 743 & 622 & 546 & 463 & 371 & 250 & 172 & 117 \\
\hline 1 & 7 & 3766 & 2218 & 2101 & 1770 & 1537 & 1591 & 1342 & 1212 & 1016 & 820 & 567 & 385 & 261 \\
\hline 1 & 8 & 733 & 1628 & 1597 & 1364 & 1144 & 1170 & 982 & 842 & 722 & 574 & 380 & 265 & 180 \\
\hline 1 & 9 & 4127 & 2433 & 2388 & 2040 & 1711 & 1749 & 1503 & 1305 & 1113 & 889 & 595 & 412 & 273 \\
\hline 1 & 10 & 914 & 542 & 543 & 468 & 385 & 390 & 339 & 287 & 248 & 196 & 129 & 90 & 58 \\
\hline 1 & 11 & 2597 & 1510 & 1446 & 1235 & 1068 & 1084 & 916 & 817 & 689 & 554 & 378 & 259 & 178 \\
\hline 1 & 12 & 2868 & 1667 & 1606 & 1381 & 1193 & 1197 & 1024 & 911 & 768 & 8 & 421 & 288 & 196 \\
\hline 1 & 13 & 4139 & 2433 & 2308 & 1968 & 1723 & 1745 & 1473 & 1330 & 1115 & 900 & 621 & 422 & 286 \\
\hline 1 & 14 & 58 & 2812 & 2755 & 2355 & 1980 & 2021 & 1743 & 1521 & 1294 & 1035 & 695 & 480 & 325 \\
\hline 1 & 15 & 2710 & 1590 & 1577 & 1355 & 1125 & 1144 & 989 & 849 & 728 & 579 & 384 & 267 & 177 \\
\hline 1 & 16 & 94 & 1978 & 1949 & 1671 & 1398 & 1422 & 1214 & 1044 & 894 & 712 & 472 & 329 & 224 \\
\hline 1 & 17 & & & 2761 & 2378 & 1989 & & 1739 & 1501 & 1284 & 1023 & 681 & 473 & 315 \\
\hline 1 & 18 & 2374 & 1394 & 1393 & 1203 & 994 & 1003 & 873 & 744 & 640 & 508 & 334 & 234 & 153 \\
\hline 1 & 19 & & 3076 & 3026 & 2599 & 2182 & 2211 & 1905 & 1651 & 1409 & 1125 & 751 & 521 & 349 \\
\hline 1 & 20 & 2624 & 1542 & 1529 & 1318 & 1098 & 1110 & 955 & 819 & 702 & 55 & 370 & 258 & 171 \\
\hline 1 & 21 & 4638 & 2703 & 2672 & 2301 & 1923 & 1944 & 1667 & 1432 & 1227 & 977 & 647 & 451 & 305 \\
\hline 1 & 2 & & & 1291 & & & & 806 & 684 & & & 307 & 215 & 139 \\
\hline 1 & 23 & 5446 & 3221 & 3112 & 2643 & 2251 & 2313 & 1974 & 1747 & 1477 & 1186 & 806 & 553 & 367 \\
\hline 1 & & & & 2579 & 2188 & 1859 & 1915 & 1642 & 1455 & 1230 & 987 & 671 & 460 & 309 \\
\hline 1 & 25 & & 3527 & 3328 & 2815 & 2466 & 2530 & 2066 & 1848 & 1555 & 1252 & 858 & 586 & 423 \\
\hline 1 & 26 & 2506 & 1468 & 1355 & 1137 & 1020 & 1051 & 866 & 801 & 665 & 540 & 380 & 255 & 180 \\
\hline 1 & & & & 2389 & & & & 1524 & 1410 & 11 & & 669 & 449 & 322 \\
\hline 1 & 28 & 2904 & 1696 & 1555 & 1302 & 1176 & 1214 & 984 & 912 & 756 & 615 & 433 & 291 & 210 \\
\hline 1 & & & & & & & 569 & 468 & 444 & 64 & 8 & 214 & 142 & 98 \\
\hline 1 & & & & 2833 & 2405 & 2047 & 2105 & 1781 & 1568 & 1329 & 1065 & 721 & 496 & 331 \\
\hline 1 & 31 & 3372 & 1993 & 1886 & 1590 & 1383 & 1429 & 1187 & 1067 & 896 & 722 & 497 & 338 & 233 \\
\hline 1 & 32 & & 2020 & 1877 & 1610 & 1452 & 1448 & 1209 & 1116 & 926 & 2 & 529 & 355 & 243 \\
\hline 1 & 33 & 342 & 1376 & 1285 & 1098 & 981 & 986 & 825 & 758 & 630 & 511 & 358 & 241 & 165 \\
\hline 1 & 34 & & & 1265 & 1084 & 948 & 954 & 794 & 709 & 597 & 480 & 328 & 224 & 160 \\
\hline 1 & & & & 1382 & 1171 & 1040 & 1059 & 845 & 757 & 637 & 513 & 352 & 240 & 184 \\
\hline 1 & 36 & 7302 & 4241 & 4080 & 3492 & 3009 & 3045 & 2586 & 2297 & 1939 & 1558 & 1061 & 727 & 499 \\
\hline 1 & 37 & 1034 & 601 & 584 & 501 & 428 & 432 & 370 & 326 & 277 & 222 & 150 & 103 & 70 \\
\hline 1 & 38 & 278 & 165 & 153 & 132 & 119 & 118 & 97 & 88 & 74 & 60 & 42 & 28 & 20 \\
\hline 1 & 39 & 3370 & 1977 & 1922 & 1657 & 1416 & 1420 & 1206 & 1055 & 897 & 718 & 483 & 333 & 226 \\
\hline 1 & 40 & & 345 & 325 & 277 & 244 & 247 & 207 & 187 & 157 & 127 & 88 & 60 & 41 \\
\hline 1 & 41 & 3169 & 1834 & 1731 & 1485 & 1313 & 1315 & 1103 & 1000 & 837 & 676 & 468 & 317 & 222 \\
\hline 1 & 42 & 403 & 240 & 226 & 195 & 174 & 172 & 144 & 131 & 110 & 89 & 61 & 42 & 28 \\
\hline
\end{tabular}




\begin{tabular}{|c|c|c|c|c|c|c|c|c|c|c|c|c|c|c|}
\hline 1 & 43 & 1034 & 608 & 563 & 477 & 429 & 435 & 366 & 340 & 282 & 229 & 162 & 108 & 74 \\
\hline 1 & 44 & 1663 & 970 & 906 & 780 & 700 & 695 & 587 & 540 & 449 & 364 & 255 & 172 & 118 \\
\hline 1 & 45 & 767 & 450 & 428 & 366 & 321 & 323 & 270 & 242 & 204 & 164 & 113 & 77 & 53 \\
\hline 1 & 46 & 619 & 364 & 330 & 281 & 260 & 260 & 217 & 206 & 169 & 138 & 99 & 66 & 45 \\
\hline 1 & 47 & 1694 & 984 & 905 & 778 & 711 & 705 & 574 & 531 & 441 & 358 & 252 & 169 & 122 \\
\hline 1 & 48 & 316 & 185 & 178 & 154 & 133 & 133 & 113 & 101 & 85 & 69 & 47 & 32 & 22 \\
\hline 1 & 49 & 1913 & 1141 & 1090 & 944 & 829 & 819 & 680 & 603 & 510 & 409 & 279 & 191 & 130 \\
\hline 1 & 50 & 1709 & 980 & 920 & 787 & 698 & 703 & 569 & 511 & 429 & 346 & 237 & 162 & 121 \\
\hline 1 & 51 & 769 & 457 & 426 & 365 & 328 & 328 & 260 & 233 & 196 & 158 & 108 & 74 & 54 \\
\hline 1 & 52 & 2143 & 1254 & 1141 & 970 & 893 & 898 & 713 & 662 & 548 & 446 & 315 & 211 & 156 \\
\hline 1 & 53 & 2053 & 1189 & 1133 & 972 & 849 & 854 & 676 & 587 & 501 & 400 & 267 & 185 & 142 \\
\hline 1 & 54 & 1008 & 584 & 531 & 451 & 415 & 418 & 349 & 332 & 272 & 223 & 160 & 106 & 74 \\
\hline 1 & 55 & 7111 & 4072 & 3877 & 3346 & 2938 & 2922 & 2482 & 2236 & 1876 & 1513 & 1043 & 709 & 496 \\
\hline 1 & 56 & 1869 & 1089 & 999 & 843 & 765 & 780 & 654 & 616 & 507 & 414 & 295 & 197 & 135 \\
\hline 1 & 57 & 832 & 490 & 462 & 393 & 346 & 351 & 299 & 273 & 228 & 184 & 129 & 87 & 58 \\
\hline 1 & 58 & 679 & 396 & 367 & 312 & 281 & 283 & 236 & 218 & 181 & 147 & 103 & 69 & 49 \\
\hline 1 & 59 & 1751 & 1040 & 982 & 847 & 751 & 746 & 632 & 575 & 480 & 388 & 270 & 183 & 121 \\
\hline 1 & 60 & 497 & 291 & 269 & 228 & 205 & 209 & 176 & 164 & 136 & 110 & 78 & 52 & 36 \\
\hline 1 & 61 & 2229 & 1319 & 1236 & 1065 & 952 & 945 & 800 & 734 & 611 & 495 & 346 & 234 & 156 \\
\hline 1 & 62 & 2791 & 1608 & 1456 & 1222 & 1123 & 1150 & 956 & 911 & 746 & 611 & 440 & 291 & 206 \\
\hline 1 & 63 & 2323 & 1340 & 1253 & 1070 & 954 & 961 & 800 & 731 & 610 & 494 & 344 & 233 & 165 \\
\hline 1 & 64 & 1932 & 1133 & 1040 & 887 & 808 & 811 & 668 & 623 & 515 & 419 & 297 & 199 & 139 \\
\hline 1 & 65 & 612 & 356 & 333 & 284 & 253 & 255 & 214 & 197 & 164 & 133 & 93 & 63 & 43 \\
\hline 1 & 66 & 1345 & 792 & 744 & 631 & 557 & 568 & 480 & 439 & 366 & 297 & 207 & 140 & 94 \\
\hline 1 & 67 & 1253 & 729 & 680 & 581 & 520 & 522 & 442 & 408 & 339 & 275 & 194 & 130 & 89 \\
\hline 1 & 68 & 1934 & 1108 & 1016 & 854 & 773 & 793 & 653 & 610 & 504 & 411 & 291 & 195 & 141 \\
\hline 1 & 69 & 1580 & 918 & 860 & 727 & 643 & 658 & 555 & 509 & 424 & 344 & 240 & 162 & 112 \\
\hline 1 & 70 & 1928 & 1131 & 1037 & 876 & 794 & 809 & 679 & 638 & 526 & 429 & 306 & 204 & 139 \\
\hline 1 & 71 & 1421 & 859 & 857 & 747 & 623 & 618 & 539 & 461 & 396 & 314 & 207 & 145 & 90 \\
\hline 1 & 72 & 3791 & 2180 & 2057 & 1762 & 1556 & 1563 & 1298 & 1170 & 982 & 792 & 546 & 371 & 266 \\
\hline 1 & 73 & 2656 & 1543 & 1432 & 1217 & 1093 & 1105 & 916 & 844 & 701 & 569 & 399 & 269 & 190 \\
\hline 1 & 74 & 1874 & 1084 & 1017 & 870 & 773 & 777 & 647 & 590 & 492 & 398 & 277 & 187 & 132 \\
\hline 1 & 75 & 1338 & 780 & 742 & 635 & 555 & 560 & 474 & 427 & 358 & 289 & 199 & 135 & 93 \\
\hline 1 & 76 & 1610 & 941 & 862 & 733 & 669 & 673 & 562 & 528 & 435 & 355 & 253 & 169 & 116 \\
\hline 1 & 77 & 2168 & 1266 & 1180 & 1005 & 898 & 907 & 766 & 708 & 588 & 477 & 335 & 225 & 154 \\
\hline 1 & 78 & 2467 & 1420 & 1305 & 1102 & 999 & 1017 & 853 & 801 & 660 & 539 & 383 & 256 & 179 \\
\hline 1 & 79 & 1481 & 870 & 805 & 687 & 619 & 623 & 509 & 467 & 389 & 315 & 221 & 149 & 106 \\
\hline 1 & 80 & 2297 & 1347 & 1252 & 1065 & 954 & 965 & 815 & 756 & 627 & 510 & 359 & 241 & 163 \\
\hline 1 & 81 & 2760 & 1631 & 1488 & 1273 & 1174 & 1168 & 977 & 925 & 760 & 621 & 445 & 296 & 200 \\
\hline 1 & 82 & 857 & 504 & 453 & 387 & 363 & 360 & 298 & 286 & 233 & 192 & 138 & 91 & 63 \\
\hline 1 & 83 & 3984 & 2325 & 2136 & 1814 & 1649 & 1665 & 1378 & 1287 & 1063 & 866 & 614 & 410 & 288 \\
\hline 1 & 84 & 2085 & 1237 & 1170 & 1006 & 888 & 887 & 749 & 679 & 568 & 459 & 318 & 216 & 144 \\
\hline 1 & 85 & 1468 & 875 & 819 & 703 & 628 & 627 & 528 & 484 & 403 & 327 & 228 & 154 & 103 \\
\hline 1 & 86 & 3112 & 1831 & 1729 & 1485 & 1314 & 1313 & 1118 & 1019 & 851 & 688 & 479 & 324 & 217 \\
\hline 1 & 87 & 2732 & 1614 & 1542 & 1324 & 1153 & 1158 & 990 & 890 & 747 & 603 & 415 & 282 & 187 \\
\hline
\end{tabular}




\begin{tabular}{|c|c|c|c|c|c|c|c|c|c|c|c|c|c|c|}
\hline 1 & 88 & 2524 & 1469 & 1348 & 1140 & 1035 & 1052 & 861 & 801 & 663 & 540 & 381 & 255 & 183 \\
\hline 1 & 89 & 1206 & 708 & 662 & 562 & 499 & 507 & 410 & 370 & 310 & 250 & 173 & 117 & 85 \\
\hline 1 & 90 & 2228 & 1317 & 1211 & 1040 & 952 & 943 & 776 & 722 & 597 & 486 & 343 & 230 & 160 \\
\hline 1 & 91 & 1538 & 908 & 832 & 712 & 652 & 650 & 535 & 500 & 413 & 337 & 239 & 160 & 111 \\
\hline 1 & 92 & 2982 & 1723 & 1581 & 1329 & 1202 & 1233 & 1015 & 946 & 782 & 637 & 451 & 302 & 216 \\
\hline 1 & 93 & 2430 & 1432 & 1319 & 1127 & 1025 & 1025 & 833 & 768 & 638 & 518 & 364 & 245 & 174 \\
\hline 1 & 94 & 534 & 313 & 299 & 256 & 222 & 225 & 188 & 167 & 141 & 113 & 77 & 53 & 37 \\
\hline 1 & 95 & 496 & 290 & 259 & 217 & 203 & 207 & 157 & 146 & 121 & 99 & 70 & 47 & 37 \\
\hline 1 & 96 & 4834 & 2817 & 2694 & 2296 & 1988 & 2022 & 1708 & 1525 & 1285 & 1034 & 708 & 483 & 332 \\
\hline 1 & 97 & 2208 & 1286 & 1240 & 1057 & 905 & 924 & 766 & 670 & 570 & 456 & 307 & 212 & 150 \\
\hline 1 & 98 & 2080 & 1206 & 1177 & 1004 & 848 & 867 & 734 & 638 & 544 & 434 & 291 & 201 & 140 \\
\hline 1 & 99 & 3697 & 2129 & 2026 & 1713 & 1484 & 1528 & 1294 & 1165 & 978 & 789 & 543 & 370 & 257 \\
\hline 1 & 100 & 1789 & 1005 & 956 & 823 & 722 & 721 & 611 & 550 & 461 & 372 & 256 & 174 & 126 \\
\hline 1 & 101 & 1520 & 898 & 806 & 687 & 644 & 642 & 526 & 503 & 411 & 337 & 243 & 161 & 112 \\
\hline 1 & 102 & 4087 & 2414 & 2232 & 1915 & 1738 & 1729 & 1397 & 1279 & 1066 & 863 & 603 & 407 & 291 \\
\hline 1 & 103 & 586 & 349 & 322 & 275 & 249 & 250 & 200 & 182 & 152 & 123 & 86 & 58 & 42 \\
\hline 1 & 104 & 332 & 198 & 183 & 157 & 142 & 142 & 117 & 108 & 90 & 73 & 51 & 34 & 24 \\
\hline 1 & 105 & 2403 & 1412 & 1322 & 1125 & 1000 & 1012 & 825 & 746 & 625 & 504 & 348 & 237 & 169 \\
\hline 1 & 106 & 2189 & 1282 & 1157 & 978 & 908 & 917 & 739 & 698 & 574 & 469 & 335 & 223 & 161 \\
\hline 1 & 107 & 1564 & 932 & 864 & 737 & 665 & 668 & 546 & 501 & 417 & 338 & 236 & 159 & 111 \\
\hline 1 & 108 & 756 & 451 & 423 & 362 & 322 & 323 & 272 & 248 & 207 & 167 & 117 & 79 & 53 \\
\hline 1 & 109 & 348 & 207 & 195 & 167 & 147 & 148 & 122 & 110 & 92 & 74 & 51 & 35 & 24 \\
\hline 1 & 110 & 2390 & 1403 & 1318 & 1117 & 985 & 1006 & 819 & 735 & 618 & 498 & 342 & 233 & 167 \\
\hline 1 & 111 & 1090 & 641 & 604 & 509 & 447 & 460 & 383 & 347 & 290 & 235 & 162 & 110 & 76 \\
\hline 1 & 112 & 309 & 184 & 175 & 149 & 130 & 132 & 110 & 99 & 83 & 67 & 46 & 31 & 21 \\
\hline 1 & 113 & 1647 & 975 & 911 & 780 & 698 & 699 & 583 & 534 & 445 & 360 & 251 & 170 & 116 \\
\hline 1 & 114 & 575 & 343 & 316 & 268 & 242 & 245 & 205 & 192 & 158 & 129 & 91 & 61 & 41 \\
\hline 1 & 115 & 1938 & 1116 & 991 & 828 & 779 & 797 & 634 & 608 & 497 & 408 & 295 & 195 & 146 \\
\hline 1 & 116 & 2884 & 1715 & 1629 & 1388 & 1213 & 1231 & 1012 & 900 & 760 & 611 & 417 & 285 & 198 \\
\hline 1 & 117 & 2615 & 1585 & 1593 & 1375 & 1129 & 1141 & 987 & 831 & 719 & 569 & 371 & 261 & 165 \\
\hline 1 & 118 & 88 & 52 & 50 & 43 & 38 & 38 & 31 & 28 & 24 & 19 & 13 & 9 & 6 \\
\hline 1 & 119 & 3620 & 2108 & 1995 & 1684 & 1467 & 1512 & 1286 & 1169 & 977 & 790 & 548 & 371 & 252 \\
\hline 1 & 120 & 3311 & 1939 & 1815 & 1538 & 1363 & 1390 & 1161 & 1062 & 885 & 717 & 500 & 338 & 233 \\
\hline 1 & 121 & 730 & 422 & 380 & 322 & 299 & 301 & 247 & 236 & 193 & 158 & 114 & 75 & 54 \\
\hline 1 & 122 & 1257 & 737 & 679 & 582 & 531 & 528 & 443 & 415 & 342 & 279 & 198 & 132 & 90 \\
\hline 1 & 123 & 778 & 463 & 437 & 378 & 337 & 332 & 268 & 238 & 201 & 161 & 110 & 75 & 54 \\
\hline 1 & 124 & 436 & 261 & 247 & 213 & 189 & 187 & 160 & 145 & 121 & 98 & 68 & 46 & 30 \\
\hline 1 & 125 & 143 & 85 & 77 & 65 & 60 & 61 & 51 & 48 & 40 & 32 & 23 & 15 & 10 \\
\hline 1 & 126 & 864 & 503 & 469 & 404 & 364 & 361 & 306 & 283 & 235 & 191 & 134 & 90 & 61 \\
\hline 1 & 127 & 560 & 328 & 289 & 240 & 227 & 235 & 194 & 191 & 155 & 128 & 94 & 61 & 42 \\
\hline 1 & 128 & 1687 & 1000 & 917 & 779 & 710 & 716 & 603 & 569 & 468 & 382 & 273 & 182 & 121 \\
\hline 1 & 129 & 1396 & 825 & 752 & 637 & 584 & 590 & 496 & 471 & 386 & 316 & 227 & 151 & 101 \\
\hline 1 & 130 & 603 & 349 & 311 & 264 & 249 & 249 & 208 & 202 & 164 & 135 & 99 & 65 & 45 \\
\hline 1 & 131 & 565 & 335 & 317 & 271 & 239 & 240 & 205 & 187 & 156 & 126 & 88 & 59 & 39 \\
\hline 1 & 132 & 248 & 147 & 138 & 118 & 106 & 105 & 90 & 83 & 69 & 56 & 39 & 26 & 17 \\
\hline
\end{tabular}




\begin{tabular}{|c|c|c|c|c|c|c|c|c|c|c|c|c|c|c|}
\hline 1 & 133 & 434 & 245 & 169 & 113 & 140 & 173 & 131 & 161 & 119 & 104 & 88 & 53 & 40 \\
\hline 1 & 134 & 3017 & 1780 & 1596 & 1353 & 1266 & 1272 & 1062 & 1026 & 835 & 687 & 499 & 329 & 223 \\
\hline & & 855 & 1099 & 1056 & 909 & 787 & 789 & 678 & 606 & 510 & 411 & 282 & 192 & 126 \\
\hline 1 & 36 & 249 & 1881 & 1802 & 1548 & 1344 & 1350 & 1159 & 1040 & 874 & 705 & 484 & 330 & 224 \\
\hline 1 & 37 & 2184 & 1285 & 1217 & 1040 & 914 & 922 & 787 & 716 & 598 & 484 & 336 & 228 & 151 \\
\hline 1 & 38 & 791 & 466 & 444 & 378 & 329 & 334 & 286 & 258 & 217 & 175 & 121 & 82 & 54 \\
\hline 1 & 39 & 3328 & 1946 & 1813 & 1541 & 1378 & 1395 & 1183 & 1096 & 909 & 739 & 520 & 349 & 236 \\
\hline 1 & 140 & 4265 & 2483 & 2347 & 2005 & 1764 & 1781 & 1520 & 1386 & 1157 & 936 & 651 & 440 & 298 \\
\hline 1 & 141 & 561 & 2740 & 2637 & 2261 & 1949 & 1967 & 1692 & 1511 & 1272 & 1024 & 701 & 479 & 317 \\
\hline 1 & 142 & 1167 & 681 & 646 & 549 & 480 & 488 & 417 & 379 & 317 & 256 & 178 & 120 & 81 \\
\hline 1 & 143 & 354 & 210 & 204 & 175 & 150 & 151 & 130 & 115 & 98 & 78 & 53 & 37 & 24 \\
\hline 1 & 144 & 2377 & 1386 & 1349 & 1157 & 984 & 996 & 860 & 759 & 643 & 515 & 349 & 240 & 160 \\
\hline 1 & 145 & 449 & 2024 & 1953 & 1676 & 1441 & 1454 & 1251 & 1114 & 940 & 755 & 516 & 353 & 234 \\
\hline 1 & 146 & & & 1525 & & 1156 & 1163 & 990 & 908 & 756 & 613 & 428 & 289 & 191 \\
\hline 1 & 147 & 1650 & 977 & 918 & 788 & 702 & 700 & 596 & 547 & 455 & 369 & 258 & 174 & 115 \\
\hline 1 & & & 697 & 672 & & 505 & 501 & 431 & 384 & & & 178 & 122 & 79 \\
\hline 1 & 149 & 653 & 2137 & 2061 & 1773 & 1528 & 1534 & 1321 & 1176 & 992 & 798 & 545 & 373 & 248 \\
\hline 1 & 150 & 614 & 938 & 909 & 780 & 666 & 674 & 581 & 515 & 435 & 349 & 237 & 163 & 109 \\
\hline 1 & & & 604 & 573 & 492 & 433 & 433 & 370 & 336 & 281 & & 158 & 107 & 71 \\
\hline 1 & 152 & 1791 & 1041 & 994 & 847 & 736 & 747 & 640 & 577 & 484 & 391 & 270 & 183 & 124 \\
\hline 1 & & & & & & & 896 & 764 & 698 & & & 28 & 222 & 150 \\
\hline 1 & 154 & 3278 & 1894 & 1772 & 1514 & 1350 & 1358 & 1154 & 1064 & 884 & 718 & 504 & 339 & 233 \\
\hline 1 & 155 & 677 & 395 & 367 & 313 & 282 & 283 & 240 & 223 & 184 & 15 & 106 & 71 & 48 \\
\hline 1 & 156 & & & 1071 & 917 & 799 & & & 623 & 522 & & 291 & 198 & 133 \\
\hline 1 & 157 & 1729 & 993 & 935 & 804 & 714 & 712 & 607 & 556 & 463 & 375 & 262 & 177 & 122 \\
\hline 1 & & & & 1694 & & 1269 & 1273 & & 983 & 825 & & 59 & 312 & 206 \\
\hline 1 & 159 & & 2540 & 2412 & 2072 & 1820 & 1822 & 1558 & 1413 & 1182 & & 662 & 449 & 299 \\
\hline 1 & 160 & 172 & 102 & 97 & 84 & 74 & 73 & 63 & 57 & 48 & 38 & 26 & 18 & 12 \\
\hline 1 & & & & 1078 & 930 & & 821 & 699 & 640 & 533 & & 301 & 203 & 134 \\
\hline 1 & 162 & 2914 & 1706 & 1606 & 1371 & 1212 & 1223 & 1042 & 954 & 795 & 644 & 450 & 304 & 204 \\
\hline 1 & & & & 1227 & & & 901 & 780 & 683 & 580 & 464 & 313 & 216 & 142 \\
\hline 1 & & & & 2024 & 1739 & 1538 & 1538 & 1312 & 1197 & 999 & & 563 & 381 & 262 \\
\hline 1 & 165 & 3736 & 2191 & 2114 & 1817 & 1565 & 1574 & 1355 & 1206 & 1017 & 818 & 559 & 382 & 253 \\
\hline 1 & & 242 & 145 & 140 & 121 & 104 & 104 & 90 & 80 & 67 & & 37 & 25 & 16 \\
\hline 1 & 167 & 99 & 59 & 57 & 50 & 42 & 42 & 37 & 32 & 27 & 2 & 15 & 10 & 7 \\
\hline 1 & & 117 & & 69 & 59 & & 50 & 44 & 38 & 33 & 26 & 18 & 12 & 8 \\
\hline 1 & & 51 & 31 & 29 & 25 & 22 & 22 & 19 & 17 & 14 & 12 & 8 & 6 & 4 \\
\hline 1 & 170 & & & 2210 & 1902 & 1662 & 1662 & 1424 & 1284 & 1077 & 869 & 600 & 408 & 280 \\
\hline 2 & 1 & 2095 & 1201 & 1368 & 1105 & 875 & 904 & 749 & 654 & 557 & 445 & 299 & 207 & 138 \\
\hline 2 & 2 & 544 & 313 & 328 & 264 & 223 & 227 & 188 & 174 & 144 & 117 & 83 & 55 & 40 \\
\hline 2 & 3 & 2294 & 1062 & 1532 & 1271 & 976 & 1022 & 832 & 716 & 613 & & 324 & 225 & 149 \\
\hline 2 & 4 & 1498 & 657 & 948 & 767 & 617 & 625 & 531 & 473 & 399 & 321 & 219 & 150 & 100 \\
\hline 2 & 5 & 4761 & 2828 & 3080 & 2544 & 1986 & 2059 & 1725 & 1539 & 1297 & 1043 & 713 & 487 & 322 \\
\hline 2 & 6 & & 993 & 1123 & 944 & 734 & 774 & 622 & 546 & 463 & 371 & 250 & 172 & 117 \\
\hline 2 & 7 & 3759 & 2706 & 2372 & 1969 & 1565 & 1615 & 1342 & 1212 & 1016 & 820 & 567 & 385 & 261 \\
\hline
\end{tabular}




\begin{tabular}{|c|c|c|c|c|c|c|c|c|c|c|c|c|c|c|}
\hline 2 & 8 & 2723 & 1628 & 1812 & 1508 & 1168 & 1245 & 982 & 842 & 722 & 574 & 380 & 265 & 180 \\
\hline 2 & 9 & 4133 & 2287 & 2706 & 2222 & 1768 & 1782 & 1503 & 1305 & 1113 & 889 & 595 & 412 & 273 \\
\hline 2 & 10 & 921 & 499 & 618 & 497 & 402 & 401 & 339 & 287 & 248 & 196 & 129 & 90 & 58 \\
\hline 2 & 11 & 2592 & 1691 & 1592 & 1352 & 1102 & 1105 & 916 & 817 & 689 & 554 & 378 & 259 & 178 \\
\hline 2 & 12 & 2854 & 1634 & 1736 & 1498 & 1234 & 1207 & 1024 & 911 & 768 & 618 & 421 & 288 & 196 \\
\hline 2 & 13 & 4124 & 2822 & 2527 & 2177 & 1756 & 1771 & 1473 & 1330 & 1115 & 900 & 621 & 422 & 286 \\
\hline 2 & 14 & 4929 & 2531 & 3105 & 2524 & 2079 & 2039 & 1743 & 1521 & 1294 & 1035 & 695 & 480 & 325 \\
\hline 2 & 15 & 2727 & 1526 & 1780 & 1454 & 1180 & 1164 & 989 & 849 & 728 & 579 & 384 & 267 & 177 \\
\hline 2 & 16 & 3403 & 1780 & 2193 & 1792 & 1466 & 1477 & 1214 & 1044 & 894 & 712 & 472 & 329 & 224 \\
\hline 2 & 17 & 4837 & 2404 & 3055 & 2520 & 2086 & 2035 & 1739 & 1501 & 1284 & 1023 & 681 & 473 & 315 \\
\hline 2 & 18 & 2402 & 1394 & 1549 & 1272 & 1050 & 1017 & 873 & 744 & 640 & 508 & 334 & 234 & 153 \\
\hline 2 & 19 & 5301 & 2584 & 3366 & 2781 & 2283 & 2247 & 1905 & 1651 & 1409 & 1125 & 751 & 521 & 349 \\
\hline 2 & 20 & 2635 & 1326 & 1693 & 1406 & 1148 & 1140 & 955 & 819 & 702 & 559 & 370 & 258 & 171 \\
\hline 2 & 21 & 4674 & 2432 & 2961 & 2444 & 2022 & 2006 & 1667 & 1432 & 1227 & 977 & 647 & 451 & 305 \\
\hline 2 & 22 & 2205 & 1160 & 1440 & 1192 & 962 & 949 & 806 & 684 & 589 & 468 & 307 & 215 & 139 \\
\hline 2 & 23 & 5499 & 3157 & 3515 & 2908 & 2299 & 2346 & 1974 & 1747 & 1477 & 1186 & 806 & 553 & 367 \\
\hline 2 & 24 & 4581 & 2453 & 2930 & 2395 & 1921 & 1930 & 1642 & 1455 & 1230 & 987 & 671 & 460 & 309 \\
\hline 2 & 25 & 6068 & 3527 & 3704 & 3126 & 2525 & 2650 & 2066 & 1848 & 1555 & 1252 & 858 & 586 & 423 \\
\hline 2 & 26 & 2519 & 1351 & 1506 & 1275 & 1035 & 1076 & 866 & 801 & 665 & 540 & 380 & 255 & 180 \\
\hline 2 & 27 & 4500 & 2125 & 2599 & 2240 & 1874 & 1902 & 1524 & 1410 & 1169 & 950 & 669 & 449 & 322 \\
\hline 2 & 28 & 2932 & 2035 & 1727 & 1443 & 1194 & 1253 & 984 & 912 & 756 & 615 & 433 & 291 & 210 \\
\hline 2 & 29 & 1342 & 859 & 784 & 670 & 564 & 577 & 468 & 444 & 364 & 298 & 214 & 142 & 98 \\
\hline 2 & 30 & 4935 & 2227 & 3204 & 2671 & 2079 & 2161 & 1781 & 1568 & 1329 & 1065 & 721 & 496 & 331 \\
\hline 2 & 31 & 3417 & 1833 & 2123 & 1772 & 1404 & 1475 & 1187 & 1067 & 896 & 722 & 497 & 338 & 233 \\
\hline 2 & 32 & 3423 & 2465 & 2007 & 1790 & 1470 & 1468 & 1209 & 1116 & 926 & 752 & 529 & 355 & 243 \\
\hline 2 & 33 & 2364 & 1376 & 1388 & 1228 & 997 & 1001 & 825 & 758 & 630 & 511 & 358 & 241 & 165 \\
\hline 2 & 34 & 2311 & 1569 & 1373 & 1176 & 981 & 986 & 794 & 709 & 597 & 480 & 328 & 224 & 160 \\
\hline 2 & 35 & 2583 & 1803 & 1519 & 1283 & 1074 & 1125 & 845 & 757 & 637 & 513 & 352 & 240 & 184 \\
\hline 2 & 36 & 7349 & 3987 & 4475 & 3814 & 3115 & 3098 & 2586 & 2297 & 1939 & 1558 & 1061 & 727 & 499 \\
\hline 2 & 37 & 1025 & 661 & 637 & 548 & 445 & 437 & 370 & 326 & 277 & 222 & 150 & 103 & 70 \\
\hline 2 & 38 & 277 & 158 & 163 & 146 & 120 & 122 & 97 & 88 & 74 & 60 & 42 & 28 & 20 \\
\hline 2 & 39 & 3392 & 2135 & 2075 & 1814 & 1462 & 1460 & 1206 & 1055 & 897 & 718 & 483 & 333 & 226 \\
\hline 2 & 40 & 583 & 469 & 355 & 303 & 249 & 252 & 207 & 187 & 157 & 127 & 88 & 60 & 41 \\
\hline 2 & 41 & 3157 & 2237 & 1862 & 1637 & 1352 & 1339 & 1103 & 1000 & 837 & 676 & 468 & 317 & 222 \\
\hline 2 & 42 & 403 & 273 & 240 & 220 & 175 & 175 & 144 & 131 & 110 & 89 & 61 & 42 & 28 \\
\hline 2 & 43 & 1029 & 912 & 615 & 530 & 435 & 438 & 366 & 340 & 282 & 229 & 162 & 108 & 74 \\
\hline 2 & 44 & 1676 & 815 & 964 & 862 & 715 & 700 & 587 & 540 & 449 & 364 & 255 & 172 & 118 \\
\hline 2 & 45 & 766 & 441 & 465 & 405 & 327 & 332 & 270 & 242 & 204 & 164 & 113 & 77 & 53 \\
\hline 2 & 46 & 612 & 495 & 355 & 313 & 263 & 262 & 217 & 206 & 169 & 138 & 99 & 66 & 45 \\
\hline 2 & 47 & 1700 & 1260 & 962 & 861 & 725 & 726 & 574 & 531 & 441 & 358 & 252 & 169 & 122 \\
\hline 2 & 48 & 313 & 270 & 191 & 169 & 137 & 134 & 113 & 101 & 85 & 69 & 47 & 32 & 22 \\
\hline 2 & 49 & 1912 & 1529 & 1147 & 1055 & 836 & 852 & 680 & 603 & 510 & 409 & 279 & 191 & 130 \\
\hline 2 & 50 & 1668 & 1353 & 997 & 864 & 722 & 739 & 569 & 511 & 429 & 346 & 237 & 162 & 121 \\
\hline 2 & 51 & 767 & 549 & 456 & 408 & 331 & 349 & 260 & 233 & 196 & 158 & 108 & 74 & 54 \\
\hline 2 & 52 & 2159 & 1079 & 1232 & 1080 & 904 & 936 & 713 & 662 & 548 & 446 & 315 & 211 & 156 \\
\hline
\end{tabular}




\begin{tabular}{|c|c|c|c|c|c|c|c|c|c|c|c|c|c|c|}
\hline 2 & 53 & 2032 & 1285 & 1226 & 1068 & 877 & 934 & 676 & 587 & 501 & 400 & 267 & 185 & 142 \\
\hline 2 & 54 & 1009 & 701 & 574 & 499 & 423 & 420 & 349 & 332 & 272 & 223 & 160 & 106 & 74 \\
\hline 2 & & 083 & 4805 & 4124 & 3642 & 3056 & 2950 & 2482 & 2236 & 1876 & 1513 & 1043 & 709 & 496 \\
\hline 2 & 56 & 1853 & 1525 & 1095 & 940 & 778 & 783 & 654 & 616 & 507 & 414 & 295 & 197 & 135 \\
\hline 2 & & 823 & 657 & 506 & 438 & 352 & 352 & 299 & 273 & 228 & 184 & 129 & 87 & 58 \\
\hline 2 & 58 & 676 & 451 & 398 & 349 & 286 & 288 & 236 & 218 & 181 & 147 & 103 & 69 & 49 \\
\hline 2 & 59 & 1764 & 1124 & 1044 & 950 & 758 & 752 & 632 & 575 & 480 & 388 & 270 & 183 & 121 \\
\hline 2 & 60 & 499 & 309 & 295 & 256 & 208 & 209 & 176 & 164 & 136 & 110 & 78 & 52 & 36 \\
\hline 2 & 6 & 2362 & 343 & 1315 & 1194 & 963 & 950 & 800 & 734 & 611 & 495 & 346 & 234 & 156 \\
\hline 2 & 62 & 774 & 1640 & 1601 & 1361 & 1146 & 1158 & 956 & 911 & 746 & 611 & 440 & 291 & 206 \\
\hline 2 & 63 & 337 & 1581 & 1357 & 1177 & 981 & 980 & 800 & 731 & 610 & 494 & 344 & 233 & 165 \\
\hline 2 & 64 & 1918 & 1450 & 1121 & 990 & 819 & 827 & 668 & 623 & 515 & 419 & 297 & 199 & 139 \\
\hline 2 & 65 & 607 & 421 & 361 & 313 & 259 & 259 & 214 & 197 & 164 & 133 & 93 & 63 & 43 \\
\hline 2 & 66 & 1345 & 839 & 819 & 703 & 566 & 571 & 480 & 439 & 366 & 297 & 207 & 140 & 94 \\
\hline 2 & 67 & 276 & 466 & 734 & 642 & 531 & 524 & 442 & 408 & 339 & 275 & 194 & 130 & 89 \\
\hline 2 & 68 & 30 & 975 & 1121 & 947 & 794 & 808 & 653 & 610 & 504 & 411 & 291 & 195 & 141 \\
\hline 2 & 69 & 587 & 937 & 951 & 807 & 658 & 664 & 555 & 509 & 424 & 44 & 240 & 162 & 112 \\
\hline 2 & 7 & 930 & 1153 & 1137 & 977 & 805 & 813 & 679 & 638 & 526 & 429 & 306 & 204 & 139 \\
\hline 2 & 71 & & 1374 & 893 & 837 & 628 & & 539 & 461 & 396 & & 207 & 145 & 90 \\
\hline 2 & 72 & 3765 & 2703 & 2223 & 1929 & 1608 & 1610 & 1298 & 1170 & 982 & 792 & 546 & 371 & 266 \\
\hline 2 & & & & & 1360 & & & 916 & 844 & & & 399 & 269 & 190 \\
\hline 2 & 74 & 1867 & 1171 & 1100 & 963 & 794 & 794 & 647 & 590 & 492 & 398 & 277 & 187 & 132 \\
\hline 2 & 75 & 1326 & 1108 & 807 & 709 & 570 & 567 & 474 & 427 & 358 & 289 & 199 & 135 & 93 \\
\hline 2 & & & & 932 & 817 & 680 & & 562 & 528 & 435 & & 253 & 169 & 116 \\
\hline 2 & 77 & 2128 & 1924 & 1283 & 1102 & 916 & 911 & 766 & 708 & 588 & 477 & 335 & 225 & 154 \\
\hline 2 & 78 & & & & & & & 853 & 801 & 660 & 39 & 383 & 256 & 179 \\
\hline 2 & 79 & & 1148 & 870 & 763 & 628 & & 509 & 467 & 389 & & 221 & 149 & 106 \\
\hline 2 & 80 & 2288 & 1670 & 1363 & 1186 & 969 & 968 & 815 & 756 & 627 & 510 & 359 & 241 & 163 \\
\hline 2 & 81 & & & & 1407 & 1182 & & 977 & 925 & 760 & & 445 & 296 & 200 \\
\hline 2 & 82 & 850 & 645 & 483 & 429 & 366 & 363 & 298 & 286 & 233 & 192 & 138 & 91 & 63 \\
\hline 2 & 83 & & & & 2010 & 1676 & 1691 & 1378 & 1287 & 1063 & 866 & 614 & 410 & 288 \\
\hline 2 & 84 & & 1559 & 1254 & 1119 & & & 749 & 679 & 568 & & 318 & 216 & 144 \\
\hline 2 & 85 & 1459 & 1243 & 877 & 781 & 632 & 634 & 528 & 484 & 403 & 327 & 228 & 154 & 103 \\
\hline 2 & 86 & & & 1853 & 1647 & 1338 & 1316 & 1118 & 1019 & 851 & 688 & 479 & 324 & 217 \\
\hline 2 & 87 & 730 & 2227 & 1667 & 1475 & 1174 & 1162 & 990 & 890 & 747 & 603 & 415 & 282 & 187 \\
\hline 2 & 88 & & & & 1267 & 1053 & 1078 & 861 & 801 & 663 & 540 & 381 & 255 & 183 \\
\hline 2 & 89 & & 807 & 726 & 624 & & & 410 & 370 & 310 & 250 & 173 & 117 & 85 \\
\hline 2 & 90 & 2219 & 1765 & 1286 & 1156 & 959 & 964 & 776 & 722 & 597 & 486 & 343 & 230 & 160 \\
\hline 2 & 91 & 1525 & 1126 & 890 & 793 & 658 & 662 & 535 & 500 & 413 & 337 & 239 & 160 & 111 \\
\hline 2 & 92 & 2995 & 1619 & 1745 & 1471 & 1229 & 1259 & 1015 & 946 & 782 & 637 & 451 & 302 & 216 \\
\hline 2 & 93 & 2424 & 1776 & 1416 & 1248 & 1036 & 1060 & 833 & 768 & 638 & 518 & 364 & 245 & 174 \\
\hline 2 & 94 & 521 & 469 & 326 & 277 & 228 & 232 & 188 & 167 & 141 & 113 & 77 & 53 & 37 \\
\hline 2 & 95 & & 203 & 283 & 242 & 205 & & 157 & 146 & 121 & & 70 & 47 & 37 \\
\hline 2 & 96 & 4881 & 2930 & 2976 & 2519 & 2046 & 2062 & 1708 & 1525 & 1285 & 1034 & 708 & 483 & 332 \\
\hline 2 & 97 & 2205 & 1492 & 1381 & 1159 & 936 & 972 & 766 & 670 & 570 & 456 & 307 & 212 & 150 \\
\hline
\end{tabular}




\begin{tabular}{|c|c|c|c|c|c|c|c|c|c|c|c|c|c|c|}
\hline 2 & 98 & 2077 & 1230 & 1325 & 1094 & 886 & 899 & 734 & 638 & 544 & 434 & 291 & 201 & 140 \\
\hline & 99 & 3679 & 2300 & 2275 & 1880 & 1537 & 1547 & 1294 & 1165 & 978 & 789 & 543 & 370 & 257 \\
\hline 2 & 100 & 785 & 1145 & 1023 & 884 & 760 & 730 & 611 & 550 & 461 & 372 & 256 & 174 & 126 \\
\hline 2 & 01 & 514 & 1257 & 863 & 763 & 648 & 651 & 526 & 503 & 411 & 337 & 243 & 161 & 112 \\
\hline 2 & 02 & 4073 & 3235 & 2381 & 2121 & 1755 & 1800 & 1397 & 1279 & 1066 & 863 & 603 & 407 & 291 \\
\hline 2 & 03 & 581 & 419 & 347 & 302 & 250 & 262 & 200 & 182 & 152 & 123 & 86 & 58 & 42 \\
\hline 2 & 104 & 331 & 293 & 197 & 173 & 142 & 145 & 117 & 108 & 90 & 73 & 51 & 34 & 24 \\
\hline 2 & 105 & 2403 & 1808 & 1442 & 1252 & 1016 & 1055 & 825 & 746 & 625 & 504 & 348 & 237 & 169 \\
\hline 2 & 106 & 2201 & 1436 & 1254 & 1086 & 918 & 943 & 739 & 698 & 574 & 469 & 335 & 223 & 161 \\
\hline 2 & 107 & 552 & 1193 & 933 & 823 & 667 & 690 & 546 & 501 & 417 & 338 & 236 & 159 & 111 \\
\hline 2 & 108 & 756 & 451 & 457 & 399 & 324 & 327 & 272 & 248 & 207 & 167 & 117 & 79 & 53 \\
\hline 2 & 109 & 348 & 240 & 213 & 184 & 148 & 154 & 122 & 110 & 92 & 74 & 51 & 35 & 24 \\
\hline 2 & 110 & 2385 & 1431 & 1457 & 1230 & 1002 & 1054 & 819 & 735 & 618 & 498 & 342 & 233 & 167 \\
\hline 2 & 11 & 1083 & 757 & 675 & 563 & 454 & 471 & 383 & 347 & 290 & 235 & 162 & 110 & 76 \\
\hline 2 & 112 & 312 & 173 & 193 & 165 & 131 & 135 & 110 & 99 & 83 & 67 & 46 & 31 & 21 \\
\hline 2 & 113 & 1657 & 1092 & 979 & 864 & 706 & 712 & 583 & 534 & 445 & 360 & 251 & 170 & 116 \\
\hline 2 & 14 & 574 & 398 & 344 & 295 & 243 & 247 & 205 & 192 & 158 & 129 & 91 & 61 & 41 \\
\hline 2 & 115 & 1960 & 871 & 1086 & 913 & 793 & 821 & 634 & 608 & 497 & 408 & 295 & 195 & 146 \\
\hline 2 & 116 & 2931 & 1441 & 1788 & 1520 & 1225 & 1288 & 1012 & 900 & 760 & 611 & 417 & 285 & 198 \\
\hline 2 & 117 & 2608 & 1712 & 1796 & 1463 & 1134 & 1196 & 987 & 831 & & & 371 & 261 & 165 \\
\hline 2 & 118 & 89 & 28 & 53 & 48 & 38 & 39 & 31 & 28 & 24 & 19 & 13 & 9 & 6 \\
\hline 2 & 9 & & 71 & 2236 & 1858 & 1506 & 1518 & 1286 & 1169 & & 790 & 548 & 371 & 252 \\
\hline 2 & 120 & 3308 & 2210 & 1999 & 1700 & 1388 & 1414 & 1161 & 1062 & 885 & 717 & 500 & 338 & 233 \\
\hline 2 & 121 & 734 & 346 & 411 & 358 & 305 & 306 & 247 & 236 & 193 & 158 & 114 & 75 & 54 \\
\hline 2 & 22 & 1249 & 900 & 724 & 646 & 538 & 531 & 443 & 415 & 342 & 279 & 198 & 132 & 90 \\
\hline 2 & 123 & 792 & 538 & 461 & 425 & 339 & 353 & 268 & 238 & 201 & 161 & 110 & 75 & 54 \\
\hline 2 & 124 & & 292 & 261 & 235 & 190 & 187 & 160 & 145 & 121 & 98 & 68 & 46 & 30 \\
\hline 2 & & & 95 & 84 & 73 & 60 & 61 & 51 & 48 & 40 & 32 & 23 & 15 & 10 \\
\hline 2 & 126 & 857 & 594 & 497 & 446 & 372 & 361 & 306 & 283 & 235 & 191 & 134 & 90 & 61 \\
\hline 2 & & & 335 & 319 & 268 & 229 & 235 & 194 & 191 & 155 & 128 & 94 & 61 & 42 \\
\hline 2 & 128 & 1699 & 1280 & 994 & 857 & 714 & 716 & 603 & 569 & 468 & 382 & 273 & 182 & 121 \\
\hline 2 & 129 & 1384 & & 816 & 703 & 588 & 590 & 496 & 471 & 386 & 316 & 227 & 151 & 101 \\
\hline 2 & & & & 335 & 294 & 253 & 249 & 208 & 202 & & & 99 & 65 & 45 \\
\hline 2 & 131 & 567 & 429 & 343 & 303 & 241 & 240 & 205 & 187 & 156 & 126 & 88 & 59 & 39 \\
\hline 2 & & & 218 & 148 & 131 & 107 & 105 & 90 & 83 & 69 & 56 & 39 & 26 & 17 \\
\hline 2 & & 00 & 191 & 195 & 121 & 141 & 173 & 131 & 161 & 119 & 104 & 88 & 53 & 40 \\
\hline 2 & 134 & & & 1721 & 1485 & 1274 & 1272 & 1062 & 1026 & 835 & 687 & 499 & 329 & 223 \\
\hline 2 & & & 1165 & 1138 & 1008 & 801 & 789 & 678 & 606 & 510 & 411 & 282 & 192 & 126 \\
\hline 2 & 136 & 3242 & 2107 & 1950 & 1694 & 1391 & 1350 & 1159 & 1040 & 874 & 705 & 484 & 330 & 224 \\
\hline 2 & 137 & 2191 & 1182 & 1322 & 1152 & 931 & 922 & 787 & 716 & 598 & 484 & 336 & 228 & 151 \\
\hline 2 & 138 & 785 & 559 & 489 & 421 & 336 & 334 & 286 & 258 & 217 & 175 & 121 & 82 & 54 \\
\hline 2 & & & 2336 & 1976 & 1714 & 1403 & 1395 & 1183 & 1096 & 909 & 739 & 520 & 349 & 236 \\
\hline 2 & 140 & & 3179 & 2556 & 2207 & 1810 & 1781 & 1520 & 1386 & 1157 & 936 & 651 & 440 & 298 \\
\hline 2 & 141 & 4631 & 3452 & 2880 & 2495 & 1998 & 1967 & 1692 & 1511 & 1272 & 1024 & 701 & 479 & 317 \\
\hline 2 & 142 & 1230 & 27 & 712 & 591 & 492 & 488 & 417 & 379 & 317 & 256 & 178 & 120 & 81 \\
\hline
\end{tabular}




\begin{tabular}{|c|c|c|c|c|c|c|c|c|c|c|c|c|c|c|}
\hline 2 & 143 & 352 & 299 & 222 & 195 & 152 & 151 & 130 & 115 & 98 & 78 & 53 & 37 & 24 \\
\hline & 144 & 2390 & 1081 & 1486 & 1264 & 1021 & 996 & 860 & 759 & 643 & 515 & 349 & 240 & 160 \\
\hline 2 & 45 & 154 & 2226 & 2133 & 1841 & 1481 & 1454 & 1251 & 1114 & 940 & 755 & 516 & 353 & 234 \\
\hline 2 & 46 & 43 & 1946 & 1650 & 1446 & 1172 & 1163 & 990 & 908 & 756 & 613 & 428 & 289 & 191 \\
\hline 2 & 147 & 531 & 1407 & 983 & 887 & 710 & 700 & 596 & 547 & 455 & 369 & 258 & 174 & 115 \\
\hline 2 & 148 & 161 & 1004 & 711 & 650 & 510 & 501 & 431 & 384 & 324 & 260 & 178 & 122 & 79 \\
\hline 2 & 149 & 3623 & 2649 & 2231 & 1939 & 1574 & 1534 & 1321 & 1176 & 992 & 798 & 545 & 373 & 248 \\
\hline 2 & 150 & 1615 & 863 & 999 & 850 & 692 & 674 & 581 & 515 & 435 & 349 & 237 & 163 & 109 \\
\hline 2 & 151 & 69 & 350 & 616 & 544 & 441 & 433 & 370 & 336 & 281 & 227 & 158 & 107 & 71 \\
\hline 2 & 152 & 785 & 1125 & 1094 & 928 & 758 & 747 & 640 & 577 & 484 & 391 & 270 & 183 & 124 \\
\hline 2 & 153 & 130 & 1349 & 1280 & 1114 & 909 & 896 & 764 & 698 & 582 & 471 & 328 & 222 & 150 \\
\hline 2 & 154 & 254 & 2235 & 1916 & 1673 & 1387 & 1358 & 1154 & 1064 & 884 & 718 & 504 & 339 & 233 \\
\hline 2 & 155 & 73 & 521 & 395 & 349 & 288 & 283 & 240 & 223 & 184 & & 106 & 71 & 48 \\
\hline 2 & 156 & 1925 & 1369 & 1165 & 1013 & 823 & 806 & 690 & 623 & 522 & 421 & 291 & 198 & 133 \\
\hline 2 & 157 & 08 & 1331 & 999 & 878 & 738 & 712 & 607 & 556 & 463 & 375 & 262 & 177 & 122 \\
\hline 2 & & & 2200 & & & 1294 & & 1091 & 983 & 825 & & 459 & 312 & 206 \\
\hline 2 & 159 & 4307 & 3505 & 2595 & 2294 & 1858 & 1822 & 1558 & 1413 & 1182 & 955 & 662 & 449 & 299 \\
\hline 2 & 160 & 173 & 106 & 103 & 92 & 75 & 73 & 63 & 57 & 48 & 38 & 26 & 18 & 12 \\
\hline 2 & & & 1625 & & 1039 & 834 & 821 & 699 & 640 & 533 & & 301 & 203 & 134 \\
\hline 2 & 162 & 2926 & 1945 & 1745 & 1522 & 1237 & 1223 & 1042 & 954 & 795 & 644 & 450 & 304 & 204 \\
\hline 2 & & & & & & & & 780 & 683 & & & 13 & 216 & 142 \\
\hline 2 & 164 & 3724 & 2187 & 2172 & 1907 & 1590 & 1538 & 1312 & 1197 & 999 & 809 & 563 & 381 & 262 \\
\hline 2 & 165 & 3740 & 2367 & 2294 & 2005 & 1609 & 1574 & 1355 & 1206 & 1017 & 818 & 559 & 382 & 253 \\
\hline 2 & 166 & & 215 & 151 & 134 & 105 & 104 & 90 & 80 & & & & 25 & 16 \\
\hline 2 & 167 & 99 & 47 & 61 & 56 & 43 & 42 & 37 & 32 & 27 & & 15 & 10 & 7 \\
\hline 2 & & & 67 & & 66 & & & 44 & 38 & & & 18 & 12 & 8 \\
\hline 2 & 169 & 51 & 52 & 31 & 28 & 22 & 22 & 19 & 17 & 14 & & 8 & 6 & 4 \\
\hline 2 & 170 & 4082 & 1807 & 2372 & 2076 & 1728 & 1662 & 1424 & 1284 & 1077 & 869 & 600 & 408 & 280 \\
\hline 3 & 1 & & & & 1206 & & & 749 & & & & 299 & 207 & 138 \\
\hline 3 & 2 & & 313 & 370 & 287 & 231 & 229 & 188 & 174 & 144 & 117 & 83 & 55 & 40 \\
\hline 3 & 3 & & & & 1411 & & & 832 & 716 & & & 324 & 225 & 149 \\
\hline 3 & 4 & & 499 & & 835 & & & & 473 & 399 & & 219 & 150 & 100 \\
\hline 3 & 5 & 4777 & 2828 & 3498 & 2825 & 2008 & 2088 & 1725 & 1539 & 1297 & 1043 & 713 & 487 & 322 \\
\hline 3 & 6 & & & 1263 & 1051 & 744 & 806 & 622 & 546 & & & 250 & 172 & 117 \\
\hline 3 & 7 & 752 & 3301 & 2678 & 2190 & 1593 & 1639 & 1342 & 1212 & 1016 & 820 & 567 & 385 & 261 \\
\hline 3 & 8 & & & & 1667 & & 1326 & 982 & 842 & 722 & 574 & 380 & 265 & 180 \\
\hline 3 & 9 & 4139 & 2150 & 3065 & 2420 & 1826 & 1814 & 1503 & 1305 & 1113 & 889 & 595 & 412 & 273 \\
\hline 3 & 10 & & 459 & 704 & 527 & 420 & 412 & 339 & 287 & 248 & 196 & 129 & 90 & 58 \\
\hline 3 & 11 & 587 & 1894 & 1752 & 1480 & 1138 & 1126 & 916 & 817 & 689 & 554 & 378 & 259 & 178 \\
\hline 3 & 12 & 841 & 1601 & 1877 & 1625 & 1277 & 1218 & 1024 & 911 & 768 & 618 & 421 & 288 & 196 \\
\hline 3 & 13 & & 3274 & 2768 & 2408 & 1791 & 1798 & 1473 & 1330 & 1115 & 900 & 621 & 422 & 286 \\
\hline 3 & 14 & 5001 & 2278 & 3499 & 2706 & 2182 & 2057 & 1743 & 1521 & 1294 & 1035 & 695 & 480 & 325 \\
\hline 3 & 15 & & 1465 & 2008 & 1559 & 1237 & 1185 & 989 & 849 & 728 & 579 & 384 & 267 & 177 \\
\hline 3 & 16 & & 1602 & 2466 & 1921 & 1537 & 1533 & 1214 & 1044 & 894 & 712 & 472 & 329 & 224 \\
\hline 3 & 17 & 4888 & 2067 & 3380 & 2670 & 2188 & 2061 & 1739 & 1501 & 1284 & 1023 & 681 & 473 & 315 \\
\hline
\end{tabular}




\begin{tabular}{|c|c|c|c|c|c|c|c|c|c|c|c|c|c|c|}
\hline 3 & 18 & 2429 & 1394 & 1723 & 1346 & 1109 & 1030 & 873 & 744 & 640 & 508 & 334 & 234 & 153 \\
\hline & 19 & 5330 & 2170 & 3744 & 2976 & 2389 & 2283 & 1905 & 1651 & 1409 & 1125 & 751 & 521 & 349 \\
\hline 3 & & 546 & 1141 & 1874 & 1500 & 1200 & 1172 & 955 & 819 & 702 & 559 & 370 & 258 & 171 \\
\hline & & 711 & 2189 & 3280 & 2597 & 2127 & 2071 & 1667 & 1432 & 1227 & 977 & 647 & 451 & 305 \\
\hline 3 & 2 & 2236 & 1044 & 1606 & 1273 & 1007 & 970 & 806 & 684 & 589 & 468 & 307 & 215 & 139 \\
\hline 3 & 3 & 553 & 3093 & 3971 & 3201 & 2348 & 2378 & 1974 & 1747 & 1477 & 1186 & 806 & 553 & 367 \\
\hline 3 & 24 & 4598 & 2257 & 3327 & 2622 & 1985 & 1944 & 1642 & 1455 & 1230 & 987 & 671 & 460 & 309 \\
\hline 3 & 25 & 6088 & 3527 & 4123 & 3471 & 2585 & 2776 & 2066 & 1848 & 1555 & 1252 & 858 & 586 & 423 \\
\hline 3 & 26 & 2532 & 1243 & 1674 & 1431 & 1051 & 1100 & 866 & 801 & 665 & 540 & 380 & 255 & 180 \\
\hline 3 & 27 & 4528 & 1742 & 2826 & 2474 & 1914 & 1950 & 1524 & 1410 & 1169 & 950 & 669 & 449 & 322 \\
\hline 3 & 28 & 960 & 2442 & 1919 & 1600 & 1213 & 1294 & 984 & 912 & 756 & 615 & 433 & 291 & 210 \\
\hline 3 & 29 & 1339 & 927 & 854 & 738 & 568 & 585 & 468 & 444 & 364 & 298 & 214 & 142 & 98 \\
\hline 3 & 30 & 4945 & 1692 & 3625 & 2965 & 2112 & 2220 & 1781 & 1568 & 1329 & 1065 & 721 & 496 & 331 \\
\hline 3 & 31 & 3462 & 1687 & 2390 & 1975 & 1425 & 1521 & 1187 & 1067 & 896 & 722 & 497 & 338 & 233 \\
\hline 3 & 32 & 418 & 3007 & 2147 & 1990 & 1488 & 1488 & 1209 & 1116 & 926 & 752 & 529 & 355 & 243 \\
\hline 3 & 33 & 386 & 1376 & 1500 & 1375 & 1013 & 1015 & 825 & 758 & 630 & 511 & 358 & 241 & 165 \\
\hline 3 & 34 & 316 & 1851 & 1490 & 1275 & 1015 & 1020 & 794 & 709 & 597 & 480 & 328 & 224 & 160 \\
\hline 3 & 35 & 583 & 2200 & 1671 & 1404 & 1110 & 1195 & 845 & 757 & 637 & 513 & 352 & 240 & 184 \\
\hline 3 & 36 & 7396 & 3747 & 4908 & 4166 & 3224 & 3152 & 2586 & 2297 & 1939 & 1558 & 1061 & 727 & 499 \\
\hline 3 & & 1016 & 728 & 696 & 598 & 463 & 441 & 370 & 326 & 277 & 222 & 150 & 103 & 70 \\
\hline 3 & 38 & 276 & 152 & 173 & 163 & 121 & 126 & 97 & 88 & 74 & 60 & 42 & 28 & 20 \\
\hline 3 & 39 & 3413 & 2306 & 2240 & 1986 & 1509 & 1501 & 1206 & 1055 & 897 & 718 & 483 & 333 & 226 \\
\hline 3 & 40 & 579 & 638 & 388 & 330 & 253 & 258 & 207 & 187 & 157 & 127 & 88 & 60 & 41 \\
\hline 3 & 41 & 3145 & 2729 & 2004 & 1805 & 1392 & 1363 & 1103 & 1000 & 837 & 676 & 468 & 317 & 222 \\
\hline 3 & 42 & & 312 & 254 & 248 & 176 & 178 & 144 & 131 & 110 & 89 & 61 & 42 & 28 \\
\hline 3 & 43 & 1025 & 1368 & 671 & 589 & 441 & 441 & 366 & 340 & 282 & 229 & 162 & 108 & 74 \\
\hline 3 & 44 & 1690 & 684 & 1024 & 952 & 730 & 704 & 587 & 540 & 449 & 364 & 255 & 172 & 118 \\
\hline 3 & 45 & & 433 & 505 & 448 & 334 & 341 & 270 & 242 & 204 & 164 & 113 & 77 & 53 \\
\hline 3 & 46 & 605 & 673 & 382 & 348 & 265 & 263 & 217 & 206 & 169 & 138 & 99 & 66 & 45 \\
\hline 3 & 47 & 1706 & 1613 & 1021 & 952 & 739 & 748 & 574 & 531 & 441 & 358 & 252 & 169 & 122 \\
\hline 3 & 48 & & 395 & 205 & 185 & 141 & 136 & 113 & 101 & 85 & 69 & 47 & 32 & 22 \\
\hline 3 & 49 & & 2049 & 1207 & 1179 & 844 & 887 & 680 & 603 & 510 & 409 & 279 & 191 & 130 \\
\hline 3 & 50 & 627 & 1867 & 1079 & 950 & 746 & 777 & 569 & 511 & 429 & 346 & 237 & 162 & 121 \\
\hline 3 & 51 & 764 & 658 & 488 & 455 & 333 & 372 & 260 & 233 & 196 & 158 & 108 & 74 & 54 \\
\hline 3 & 52 & 175 & 928 & 1330 & 1204 & 916 & 977 & 713 & 662 & 548 & 446 & 315 & 211 & 156 \\
\hline 3 & 53 & 011 & 1387 & 1327 & 1174 & 905 & 1022 & 676 & 587 & 501 & 400 & 267 & 185 & 142 \\
\hline 3 & 54 & & 841 & & 553 & 430 & 422 & 349 & 332 & 272 & 223 & 160 & 106 & 74 \\
\hline 3 & 55 & 7054 & 5670 & 4387 & 3965 & 3178 & 2979 & 2482 & 2236 & 1876 & 1513 & 1043 & 709 & 496 \\
\hline 3 & 56 & 1836 & 2135 & 1200 & 1049 & 791 & 786 & 654 & 616 & 507 & 414 & 295 & 197 & $7 \quad 135$ \\
\hline 3 & 57 & 814 & 880 & 554 & 489 & 358 & 353 & 299 & 273 & 228 & 184 & 129 & 87 & 58 \\
\hline 3 & 58 & 673 & 514 & 433 & 390 & 292 & 293 & 236 & 218 & 181 & 147 & 103 & 69 & 49 \\
\hline 3 & 59 & 1777 & 1213 & 1110 & 1065 & 766 & 759 & 632 & 575 & 480 & 388 & 270 & 183 & $\begin{array}{ll}3 & 121\end{array}$ \\
\hline 3 & 60 & 501 & 327 & 324 & 288 & 211 & 210 & 176 & 164 & 136 & 110 & 78 & 52 & 36 \\
\hline 3 & 61 & 2503 & 89 & 1399 & 1340 & 974 & 956 & 800 & 734 & 611 & 495 & 346 & 234 & 156 \\
\hline 3 & 62 & 2758 & 1673 & 1760 & 1516 & 1170 & 1165 & 956 & 911 & 746 & 611 & 440 & 291 & 1206 \\
\hline
\end{tabular}




\begin{tabular}{|c|c|c|c|c|c|c|c|c|c|c|c|c|c|c|}
\hline 3 & 63 & 2352 & 1866 & 1470 & 1295 & 1009 & 1000 & 800 & 731 & 610 & 494 & 344 & 233 & 165 \\
\hline & 64 & 1904 & 1856 & 1209 & 1105 & 830 & 844 & 668 & 623 & 515 & 419 & 297 & 199 & 139 \\
\hline & 65 & 602 & 496 & 392 & 345 & 265 & 262 & 214 & 197 & 164 & 133 & 93 & 63 & 43 \\
\hline 3 & 66 & 346 & 890 & 901 & 783 & 575 & 575 & 480 & 439 & 366 & 297 & 207 & 140 & 94 \\
\hline 3 & 67 & 299 & 299 & 792 & 710 & 543 & 525 & 442 & 408 & 339 & 275 & 194 & 130 & 89 \\
\hline 3 & 68 & 925 & 858 & 1237 & 1049 & 815 & 824 & 653 & 610 & 504 & 411 & 291 & 195 & 141 \\
\hline 3 & 69 & 593 & 955 & 1053 & 897 & 674 & 670 & 555 & 509 & 424 & 344 & 240 & 162 & 112 \\
\hline 3 & 70 & 32 & 1176 & 1246 & 1089 & 816 & 816 & 679 & 638 & 526 & 429 & 306 & 204 & 139 \\
\hline 3 & 71 & +30 & 2198 & 931 & 938 & 633 & 623 & 539 & 461 & 396 & 314 & 207 & 145 & 90 \\
\hline 3 & 72 & 740 & 3351 & 2402 & 2112 & 1662 & 1658 & 1298 & 1170 & 982 & 792 & 546 & 371 & 266 \\
\hline 3 & 73 & 530 & 2005 & 1696 & 1521 & 1140 & 1151 & 916 & 844 & 701 & 569 & 399 & 269 & 190 \\
\hline 3 & 74 & 1860 & 1265 & 1189 & 1068 & 816 & 812 & 647 & 590 & 492 & 398 & 277 & 187 & 132 \\
\hline 3 & 75 & 1314 & 1573 & 877 & 792 & 585 & 575 & 474 & 427 & 358 & 289 & 199 & 135 & 93 \\
\hline 3 & 76 & & 1138 & 1007 & 911 & 690 & 685 & 562 & 528 & 435 & & 253 & 169 & 116 \\
\hline 3 & 77 & 088 & 2924 & 1395 & 1208 & 934 & 915 & 766 & 708 & 588 & 477 & 335 & 225 & 154 \\
\hline 3 & 78 & 53 & 1911 & 1565 & 1341 & 1048 & 1026 & 853 & 801 & 660 & 539 & 383 & 256 & 179 \\
\hline 3 & 79 & 71 & 1515 & 941 & 847 & 637 & 664 & 509 & 467 & 389 & 315 & 221 & 149 & 106 \\
\hline 3 & 80 & 79 & 2071 & 1484 & 1321 & 985 & 971 & 815 & 756 & 627 & 510 & 359 & 241 & 163 \\
\hline 3 & 81 & & 3383 & 1696 & 1555 & 1191 & 1176 & 977 & 925 & & & 445 & 296 & 200 \\
\hline 3 & 82 & 844 & 826 & 516 & 476 & 369 & 366 & 298 & 286 & 233 & 192 & 138 & 91 & 63 \\
\hline 3 & 83 & & 2917 & 2515 & 2228 & 1703 & 1718 & 1378 & 1287 & 1063 & 866 & 614 & 410 & 288 \\
\hline 3 & 84 & 2129 & 1964 & 1344 & 1246 & 909 & 910 & 749 & 679 & 568 & 45 & 318 & 216 & 144 \\
\hline 3 & 85 & 451 & 1764 & 939 & 868 & 635 & 640 & 528 & 484 & 403 & 327 & 228 & 154 & 103 \\
\hline 3 & 86 & & 1687 & 1986 & 1826 & 1362 & 1319 & 1118 & 1019 & & & 479 & 324 & 217 \\
\hline 3 & 87 & 2728 & 3073 & 1803 & 1644 & 1196 & 1167 & 990 & 890 & 747 & 603 & 415 & 282 & 187 \\
\hline 3 & 88 & & 2879 & & 1408 & 1072 & 1104 & 861 & 801 & 663 & & 81 & 255 & 183 \\
\hline 3 & 89 & & 920 & 796 & & & 558 & 410 & & & & 173 & & 85 \\
\hline 3 & 90 & 2210 & 2366 & 1367 & 1285 & 967 & 985 & 776 & 722 & 597 & 486 & 343 & 230 & 160 \\
\hline 3 & 91 & & 1396 & & & & 675 & & 500 & & & 239 & 160 & 111 \\
\hline 3 & 92 & 008 & 1522 & 1927 & 1628 & 1256 & 1285 & 1015 & 946 & 782 & 637 & 451 & 302 & 216 \\
\hline 3 & 93 & & 2202 & 1520 & 1381 & 1048 & 1095 & 833 & 768 & & 518 & 364 & 245 & 174 \\
\hline 3 & 94 & & 704 & & & & 240 & 188 & 167 & & 113 & 77 & & 37 \\
\hline 3 & 95 & 500 & 142 & 309 & 270 & 207 & 235 & 157 & 146 & 121 & 99 & 70 & 47 & 37 \\
\hline 3 & 96 & & 3047 & 3287 & 2763 & 2107 & 2102 & 1708 & 1525 & 1285 & 1034 & 708 & 483 & 332 \\
\hline 3 & 97 & 203 & 1731 & 1537 & 1270 & 968 & 1024 & 766 & 670 & 570 & 456 & 307 & 212 & 150 \\
\hline 3 & 98 & & 1255 & & 1191 & 926 & 933 & 734 & 638 & 544 & 434 & 291 & 201 & 140 \\
\hline 3 & 99 & & 2484 & 2555 & 2064 & 1593 & 1567 & 1294 & 1165 & 978 & 789 & 543 & 370 & 257 \\
\hline 3 & 100 & 1782 & 1306 & 1095 & 949 & 799 & 739 & 611 & 550 & 461 & 372 & 256 & 174 & 126 \\
\hline 3 & 101 & & 1759 & 924 & 847 & 651 & 660 & 526 & 503 & 411 & 337 & 243 & 161 & 112 \\
\hline 3 & 102 & 4059 & 4335 & 2541 & 2350 & 1773 & 1873 & 1397 & 1279 & 1066 & 863 & 603 & 407 & 291 \\
\hline 3 & 103 & 576 & 502 & 374 & 332 & 251 & 275 & 200 & 182 & 152 & 123 & 86 & 58 & 42 \\
\hline 3 & 104 & 330 & 433 & 211 & 192 & 143 & 148 & 117 & 108 & 90 & 73 & 51 & 34 & 24 \\
\hline 3 & 105 & 2402 & 2314 & 1573 & 1394 & 1033 & 1100 & 825 & 746 & 625 & 504 & 348 & 237 & 169 \\
\hline 3 & 106 & 2214 & 1608 & 1360 & 1207 & 928 & 969 & 739 & 698 & 574 & 469 & 335 & 223 & 161 \\
\hline 3 & 107 & 1541 & 1528 & 1008 & 918 & 670 & 712 & 546 & 501 & 417 & 338 & 236 & 159 & 111 \\
\hline
\end{tabular}




\begin{tabular}{|c|c|c|c|c|c|c|c|c|c|c|c|c|c|c|}
\hline 3 & 108 & 755 & 451 & 493 & 440 & 326 & 332 & 272 & 248 & 207 & 167 & 117 & 79 & 53 \\
\hline 3 & 109 & 348 & 278 & 232 & 204 & 150 & 159 & 122 & 110 & 92 & 74 & 51 & 35 & 24 \\
\hline 3 & 110 & 2380 & 1460 & 1610 & 1354 & 1020 & 1104 & 819 & 735 & 618 & 498 & 342 & 233 & 167 \\
\hline 3 & 111 & 1076 & 893 & 754 & 623 & 462 & 483 & 383 & 347 & 290 & 235 & 162 & 110 & 76 \\
\hline 3 & 112 & 315 & 163 & 213 & 183 & 132 & 139 & 110 & 99 & 83 & 67 & 46 & 31 & 21 \\
\hline 3 & 113 & 1667 & 1223 & 1052 & 957 & 713 & 725 & 583 & 534 & 445 & 360 & 251 & 170 & 116 \\
\hline 3 & 114 & 573 & 461 & 375 & 325 & 244 & 250 & 205 & 192 & 158 & 129 & 91 & 61 & 41 \\
\hline 3 & 115 & 1983 & 679 & 1190 & 1007 & 806 & 845 & 634 & 608 & 497 & 408 & 295 & 195 & 146 \\
\hline 3 & 116 & 2978 & 1210 & 1963 & 1665 & 1238 & 1348 & 1012 & 900 & 760 & 611 & 417 & 285 & 198 \\
\hline 3 & 117 & 2601 & 1849 & 2024 & 1556 & 1139 & 1253 & 987 & 831 & 719 & 569 & 371 & 261 & 165 \\
\hline 3 & 118 & 90 & 15 & 56 & 54 & 39 & 40 & 31 & 28 & 24 & 19 & 13 & 9 & 6 \\
\hline 3 & 119 & 3623 & 3137 & 2507 & 2050 & 1545 & 1524 & 1286 & 1169 & 977 & 790 & 548 & 371 & 252 \\
\hline 3 & 120 & 3306 & 2520 & 2202 & 1879 & 1414 & 1439 & 1161 & 1062 & 885 & 717 & 500 & 338 & 233 \\
\hline 3 & 121 & 738 & 283 & 444 & 397 & 311 & 310 & 247 & 236 & 193 & 158 & 114 & 75 & 54 \\
\hline 3 & 122 & 1242 & 1098 & 773 & 717 & 545 & 534 & 443 & 415 & 342 & 279 & 198 & 132 & 90 \\
\hline 3 & 123 & 805 & 624 & 485 & 477 & 342 & 375 & 268 & 238 & 201 & 161 & 110 & 75 & 54 \\
\hline 3 & 124 & 430 & 327 & 276 & 260 & 191 & 187 & 160 & 145 & 121 & 98 & 68 & 46 & 30 \\
\hline 3 & 125 & 140 & 106 & 92 & 82 & 60 & 61 & 51 & 48 & 40 & 32 & 23 & 15 & 10 \\
\hline 3 & 126 & 850 & 701 & 527 & 493 & 379 & 361 & 306 & 283 & 235 & 191 & 134 & 90 & 61 \\
\hline 3 & 127 & 557 & 342 & 351 & 300 & 231 & 235 & 194 & 191 & 155 & 128 & 94 & 61 & 42 \\
\hline 3 & 128 & 1711 & 1638 & 1077 & 943 & 719 & 716 & 603 & 569 & 468 & 382 & 273 & 182 & 121 \\
\hline 3 & 129 & 1373 & 1617 & 886 & 775 & 593 & 590 & 496 & 471 & 386 & 316 & 227 & 151 & 101 \\
\hline 3 & 130 & 588 & 916 & 360 & 328 & 257 & 249 & 208 & 202 & 164 & 135 & 99 & 65 & 45 \\
\hline 3 & 131 & 570 & 549 & 372 & 338 & 244 & 240 & 205 & 187 & 156 & 126 & 88 & 59 & 39 \\
\hline 3 & 132 & 256 & 322 & 158 & 145 & 108 & 105 & 90 & 83 & 69 & 56 & 39 & 26 & 17 \\
\hline 3 & 133 & 423 & 149 & 224 & 129 & 141 & 173 & 131 & 161 & 119 & 104 & 88 & 53 & 40 \\
\hline 3 & 134 & 3030 & 2649 & 1856 & 1631 & 1283 & 1272 & 1062 & 1026 & 835 & 687 & 499 & 329 & 223 \\
\hline 3 & 135 & 1868 & 1235 & 1226 & 1117 & 815 & 789 & 678 & 606 & 510 & 411 & 282 & 192 & 126 \\
\hline 3 & 136 & 3235 & 2360 & 2111 & 1853 & 1440 & 1350 & 1159 & 1040 & 874 & 705 & 484 & 330 & 224 \\
\hline 3 & 137 & 2199 & 1088 & 1436 & 1276 & 949 & 922 & 787 & 716 & 598 & 484 & 336 & 228 & 151 \\
\hline 3 & 138 & 779 & 671 & 538 & 467 & 343 & 334 & 286 & 258 & 217 & 175 & 121 & 82 & 54 \\
\hline 3 & 139 & 3293 & 2803 & 2154 & 1907 & 1429 & 1395 & 1183 & 1096 & 909 & 739 & 520 & 349 & 236 \\
\hline 3 & 140 & 4258 & 4069 & 2783 & 2430 & 1856 & 1781 & 1520 & 1386 & 1157 & 936 & 651 & 440 & 298 \\
\hline 3 & 141 & 4602 & 4350 & 3145 & 2753 & 2048 & 1967 & 1692 & 1511 & 1272 & 1024 & 701 & 479 & 317 \\
\hline 3 & 142 & 1296 & 1 & 786 & 636 & 505 & 488 & 417 & 379 & 317 & 256 & 178 & 120 & 81 \\
\hline 3 & 143 & 350 & 424 & 242 & 217 & 155 & 151 & 130 & 115 & 98 & 78 & 53 & 37 & 24 \\
\hline 3 & 144 & 2404 & 843 & 1638 & 1380 & 1060 & 996 & 860 & 759 & 643 & 515 & 349 & 240 & 160 \\
\hline 3 & 145 & 3459 & 2449 & 2328 & 2022 & 1522 & 1454 & 1251 & 1114 & 940 & 755 & 516 & 353 & 234 \\
\hline 3 & 146 & 2742 & 2335 & 1785 & 1603 & 1187 & 1163 & 990 & 908 & 756 & 613 & 428 & 289 & 191 \\
\hline 3 & 147 & 1613 & 2026 & 1053 & 997 & 718 & 700 & 596 & 547 & 455 & 369 & 258 & 174 & 115 \\
\hline 3 & 148 & 1154 & 1446 & 752 & 726 & 516 & 501 & 431 & 384 & 324 & 260 & 178 & 122 & 79 \\
\hline 3 & 149 & 3593 & 3285 & 2415 & 2121 & 1623 & 1534 & 1321 & 1176 & 992 & 798 & 545 & 373 & 248 \\
\hline 3 & 150 & 1617 & 794 & 1098 & 926 & 719 & 674 & 581 & 515 & 435 & 349 & 237 & 163 & 109 \\
\hline 3 & 151 & 1112 & 203 & 662 & 602 & 450 & 433 & 370 & 336 & 281 & 227 & 158 & 107 & 71 \\
\hline 3 & 152 & 1780 & 1215 & 1205 & 1016 & 781 & 747 & 640 & 577 & 484 & 391 & 270 & 183 & 124 \\
\hline
\end{tabular}




\begin{tabular}{|c|c|c|c|c|c|c|c|c|c|c|c|c|c|c|}
\hline 3 & 153 & 2120 & 1457 & 1391 & 1232 & 930 & 896 & 764 & 698 & 582 & 471 & 328 & 222 & 150 \\
\hline 3 & 154 & 3231 & 2638 & 2073 & 1850 & 1425 & 1358 & 1154 & 1064 & 884 & 718 & 504 & 339 & 233 \\
\hline 3 & 55 & 670 & 688 & 425 & 389 & 293 & 283 & 240 & 223 & 184 & 150 & 106 & 71 & 48 \\
\hline 3 & 56 & 17 & 1671 & 1267 & 1119 & 849 & 806 & 690 & 623 & 522 & 421 & 291 & 198 & 133 \\
\hline 3 & 157 & 587 & 1783 & 1067 & 959 & 762 & 712 & 607 & 556 & 463 & 375 & 262 & 177 & 122 \\
\hline 3 & 158 & 2969 & 2728 & 1976 & 1793 & 1321 & 1273 & 1091 & 983 & 825 & 666 & 459 & 312 & 206 \\
\hline 3 & 159 & 4288 & 4837 & 2792 & 2540 & 1898 & 1822 & 1558 & 1413 & 1182 & 955 & 662 & 449 & 299 \\
\hline 3 & 160 & 174 & 111 & 110 & 100 & 76 & 73 & 63 & 57 & 48 & 38 & 26 & 18 & 12 \\
\hline 3 & 161 & 1911 & 2307 & 1216 & 1160 & 841 & 821 & 699 & 640 & 533 & 432 & 301 & 203 & 134 \\
\hline 3 & 162 & 2939 & 2217 & 1897 & 1690 & 1262 & 1223 & 1042 & 954 & 795 & 644 & 450 & 304 & 204 \\
\hline 3 & 163 & 39 & 1990 & 1455 & 1253 & 967 & 901 & 780 & 683 & 580 & 464 & 313 & 216 & 142 \\
\hline 3 & 164 & 3718 & 2231 & 2331 & 2090 & 1644 & 1538 & 1312 & 1197 & 999 & 809 & 563 & 381 & 262 \\
\hline 3 & 165 & 3745 & 2556 & 2488 & 2211 & 1655 & 1574 & 1355 & 1206 & 1017 & 818 & 559 & 382 & 253 \\
\hline 3 & 166 & 244 & 318 & 162 & 149 & 105 & 104 & 90 & 80 & 67 & & 37 & 25 & 16 \\
\hline 3 & 167 & 99 & 38 & 64 & 62 & 44 & 42 & 37 & 32 & 27 & 2 & 15 & 10 & 7 \\
\hline 3 & & 119 & 65 & 78 & 73 & 52 & 50 & 44 & 38 & . & & 18 & 12 & 8 \\
\hline 3 & 169 & 50 & 89 & 32 & 30 & 22 & 22 & 19 & 17 & 14 & 12 & 8 & & 4 \\
\hline 3 & 170 & 4130 & 1409 & 2545 & 2266 & 1796 & 1662 & 1424 & 1284 & 1077 & 869 & 600 & 408 & 280 \\
\hline 4 & 1 & 2144 & 1153 & 1797 & 1315 & 919 & 953 & 749 & 654 & 55 & & 299 & 207 & 138 \\
\hline 4 & 2 & 528 & 313 & 417 & 312 & 239 & 231 & 188 & 174 & 144 & 117 & 83 & 55 & 40 \\
\hline 4 & 3 & & & & & 1017 & & 832 & 716 & 13 & & 324 & 225 & 149 \\
\hline 4 & 4 & 1549 & 379 & 1230 & 910 & 651 & 633 & 531 & 473 & 399 & 321 & 219 & 150 & 100 \\
\hline 4 & 5 & 794 & 2828 & 3972 & 3137 & 2030 & 2118 & 1725 & 1539 & 1297 & 1043 & 713 & 487 & 322 \\
\hline 4 & 6 & & 915 & 1420 & & 755 & & 622 & 546 & & & 250 & 172 & 117 \\
\hline 4 & 7 & 3746 & 4027 & 3023 & 2436 & 1622 & 1664 & 1342 & 1212 & 1016 & 820 & 567 & 385 & 261 \\
\hline 4 & 8 & & & & & & & 982 & 842 & 722 & & 380 & 265 & 180 \\
\hline 4 & 9 & 144 & 2021 & 3473 & 2636 & 1886 & 1848 & 1503 & 1305 & 1113 & 889 & 595 & 412 & 273 \\
\hline 4 & 10 & 934 & 422 & 802 & 559 & 439 & 423 & 339 & 287 & 248 & 1 & 129 & 90 & 58 \\
\hline 4 & 11 & & & 1929 & & 1174 & & 916 & 817 & & & 378 & 259 & 178 \\
\hline 4 & 12 & 827 & 1569 & 2030 & 1762 & 1322 & 1228 & 1024 & 911 & 768 & 618 & 421 & 288 & 196 \\
\hline 4 & 13 & & & 3032 & & 1826 & 1824 & 1473 & 1330 & 1115 & & 621 & 422 & 286 \\
\hline 4 & 14 & & 2050 & 3943 & 2901 & 2291 & 2075 & 1743 & 1521 & 1294 & 1035 & 695 & 480 & 325 \\
\hline 4 & 15 & & & 2266 & 1673 & 1298 & 1206 & 989 & 849 & 728 & & 384 & 267 & 177 \\
\hline 4 & 16 & & & 2774 & 2059 & 1612 & 1592 & 1214 & 1044 & 894 & & 472 & 329 & 224 \\
\hline 4 & 17 & 4940 & 1778 & 3740 & 2830 & 2295 & 2086 & 1739 & 1501 & 1284 & 1023 & 681 & 473 & 315 \\
\hline 4 & 18 & & 1394 & 1916 & 1424 & 1172 & 1044 & 873 & 744 & 640 & & 334 & 234 & 153 \\
\hline 4 & 19 & 359 & 1823 & 4164 & 3184 & 2500 & 2319 & 1905 & 1651 & 1409 & 1125 & 751 & 521 & 349 \\
\hline 4 & 20 & & & & & 1253 & 1205 & & 819 & 702 & & 370 & 258 & 171 \\
\hline 4 & 21 & & 1970 & 3635 & 2759 & 2237 & 2138 & 1667 & 1432 & 1227 & 977 & 647 & 451 & 305 \\
\hline 4 & 22 & 2268 & 940 & 1792 & 1360 & 1054 & 992 & 806 & 684 & 589 & 468 & 307 & 215 & 139 \\
\hline 4 & 23 & & 3032 & 4485 & 3523 & 2398 & 2412 & 1974 & 1747 & 1477 & 1186 & 806 & 553 & 367 \\
\hline 4 & 24 & 4614 & 2076 & 3779 & 2871 & 2051 & 1959 & 1642 & 1455 & 1230 & 987 & 671 & 460 & 309 \\
\hline 4 & 25 & & 3527 & 4589 & 3854 & 2646 & 2908 & 2066 & 1848 & 1555 & 1252 & 858 & 586 & 423 \\
\hline 4 & 26 & & & 1861 & 1604 & 1066 & 1126 & 866 & 801 & 665 & 540 & 380 & 255 & 180 \\
\hline 4 & 27 & 4557 & 1429 & 3074 & 2732 & 1955 & 1998 & 1524 & 1410 & 1169 & 950 & 669 & 449 & 322 \\
\hline
\end{tabular}




\begin{tabular}{|c|c|c|c|c|c|c|c|c|c|c|c|c|c|c|}
\hline 4 & 28 & 2988 & 2930 & 2132 & 1774 & 1232 & 1336 & 984 & 912 & 756 & 615 & 433 & 291 & 210 \\
\hline 4 & 29 & 1335 & 1001 & 930 & 814 & 572 & 593 & 468 & 444 & 364 & 298 & 214 & 142 & 98 \\
\hline & 0 & 955 & 1286 & 4100 & 3293 & 2145 & 2280 & 1781 & 1568 & 1329 & 1065 & 721 & 496 & 331 \\
\hline 4 & & 3507 & 1552 & 2690 & 2201 & 1446 & 1569 & 1187 & 1067 & 896 & 722 & 497 & 338 & 233 \\
\hline 4 & 2 & 3414 & 3669 & 2295 & 2212 & 1507 & 1509 & 1209 & 1116 & 926 & 752 & 529 & 355 & 243 \\
\hline 4 & 3 & 2409 & 1376 & 1620 & 1538 & 1029 & 1030 & 825 & 758 & 630 & 511 & 358 & 241 & 165 \\
\hline 4 & 34 & 2322 & 2184 & 1616 & 1383 & 1051 & 1055 & 794 & 709 & 597 & 480 & 328 & 224 & 160 \\
\hline 4 & 35 & 583 & 2684 & 1837 & 1538 & 1146 & 1269 & 845 & 757 & 637 & 513 & 352 & 240 & 184 \\
\hline 4 & 36 & 7443 & 3523 & 5383 & 4550 & 3336 & 3207 & 2586 & 2297 & 1939 & 1558 & 1061 & 727 & 499 \\
\hline 4 & 37 & 1007 & 800 & 759 & 654 & 481 & 446 & 370 & 326 & 277 & 222 & 150 & 103 & 70 \\
\hline 4 & 38 & 275 & 146 & 185 & 181 & 123 & 130 & 97 & 88 & 74 & 60 & 42 & 28 & 20 \\
\hline 4 & 39 & 3435 & 2490 & 2419 & 2174 & 1557 & 1543 & 1206 & 1055 & 897 & 718 & 483 & 333 & 226 \\
\hline 4 & 40 & 576 & 867 & 424 & 361 & 257 & 263 & 207 & 187 & 157 & 127 & 88 & 60 & 41 \\
\hline 4 & 41 & 3133 & 3329 & 2156 & 1989 & 1433 & 1388 & 1103 & 1000 & 837 & 676 & 468 & 317 & 222 \\
\hline 4 & 42 & 403 & 355 & 269 & 279 & 178 & 181 & 144 & 131 & 110 & 89 & 61 & 42 & 28 \\
\hline 4 & 43 & 1020 & 2052 & 733 & 654 & 447 & 444 & 366 & 340 & 282 & 229 & 162 & 108 & 74 \\
\hline 4 & 44 & 1703 & 575 & 1089 & 1052 & 745 & 709 & 587 & 540 & 449 & 364 & 255 & 172 & 118 \\
\hline 4 & 45 & 765 & 424 & 548 & 495 & 341 & 350 & 270 & 242 & 204 & 164 & 113 & 77 & 53 \\
\hline 4 & 46 & 598 & 916 & 410 & 387 & 268 & 265 & 217 & 206 & 169 & 138 & 99 & 66 & 45 \\
\hline 4 & 47 & 1711 & 2064 & 1085 & 1054 & 753 & 771 & 574 & 531 & 441 & 358 & 252 & 169 & 122 \\
\hline 4 & 48 & 308 & 577 & 219 & 204 & 145 & 137 & 113 & 101 & 85 & 69 & 47 & 32 & 22 \\
\hline 4 & 49 & 1910 & 2746 & 1271 & 1317 & 852 & 922 & 680 & 603 & 510 & 409 & 279 & 191 & 130 \\
\hline 4 & 50 & 88 & 2576 & 1168 & 1043 & 771 & 817 & 569 & 511 & 429 & 346 & 237 & 162 & 121 \\
\hline 4 & 51 & 762 & 790 & 523 & 508 & 335 & 396 & 260 & 233 & 196 & 158 & 108 & 74 & 54 \\
\hline 4 & 5 & & 798 & 1435 & 1341 & 928 & 1020 & 713 & 662 & 548 & 446 & 315 & 211 & 156 \\
\hline 4 & 53 & 991 & 1498 & 1435 & 1290 & 934 & 1118 & 676 & 587 & 501 & 400 & 267 & 185 & 142 \\
\hline 4 & 54 & 1012 & 1010 & 672 & 613 & 438 & 423 & 349 & 332 & 272 & 223 & 160 & 106 & 74 \\
\hline 4 & 55 & & 6691 & 4667 & 4316 & 3305 & 3007 & 2482 & 2236 & 1876 & 1513 & 1043 & 709 & 496 \\
\hline 4 & 56 & 1820 & 2989 & 1315 & 1170 & 805 & 789 & 654 & 616 & 507 & 414 & 295 & 197 & 135 \\
\hline 4 & 5 & 805 & 1179 & 607 & 546 & 364 & 354 & 299 & 273 & 228 & 184 & 129 & 87 & 58 \\
\hline 4 & 58 & 670 & 586 & 470 & 436 & 298 & 299 & 236 & 218 & 181 & 147 & 103 & 69 & 49 \\
\hline 4 & 59 & 1790 & 1311 & 1180 & 1195 & 773 & 765 & 632 & 575 & 480 & 388 & 270 & 183 & 121 \\
\hline 4 & 60 & & 347 & 356 & 323 & 214 & 211 & 176 & 164 & 136 & & 78 & 52 & 36 \\
\hline 4 & 61 & 2652 & 23 & 1488 & 1504 & 986 & 961 & 800 & 734 & 611 & 495 & 346 & 234 & 156 \\
\hline 4 & 62 & 741 & 1707 & 1935 & 1689 & 1194 & 1173 & 956 & 911 & 746 & 611 & 440 & 291 & 206 \\
\hline 4 & 63 & 366 & 2202 & 1592 & 1425 & 1037 & 1021 & 800 & 731 & 610 & 494 & 344 & 233 & 165 \\
\hline 4 & 64 & 1889 & 2376 & 1303 & 1233 & 841 & 860 & 668 & 623 & 515 & 419 & 297 & 199 & 139 \\
\hline 4 & 65 & 598 & 586 & 425 & 380 & 270 & 265 & 214 & 197 & 164 & 133 & 93 & 63 & 43 \\
\hline 4 & 66 & 1346 & 943 & 992 & 872 & 585 & 579 & 480 & 439 & 366 & 297 & 207 & 140 & 94 \\
\hline 4 & 67 & & 191 & 855 & 785 & 555 & 527 & 442 & 408 & 339 & 275 & 194 & 130 & 89 \\
\hline 4 & 68 & 1921 & 755 & 1365 & 1162 & 837 & 839 & 653 & 610 & 504 & 411 & 291 & 195 & 141 \\
\hline 4 & 69 & 1600 & 974 & 1166 & 996 & 690 & 675 & 555 & 509 & 424 & 344 & 240 & 162 & 112 \\
\hline 4 & 70 & 1934 & 1200 & 1365 & 1214 & 827 & 819 & 679 & 638 & 526 & 429 & 306 & 204 & 139 \\
\hline 4 & 71 & 1435 & 3517 & 969 & 1050 & 639 & 625 & 539 & 461 & 396 & 314 & 207 & 145 & 90 \\
\hline 4 & 72 & 3714 & 4156 & 2596 & 2313 & 1718 & 1707 & 1298 & 1170 & 982 & 792 & 546 & 371 & 266 \\
\hline
\end{tabular}




\begin{tabular}{|c|c|c|c|c|c|c|c|c|c|c|c|c|c|c|}
\hline 4 & 73 & 2616 & 2286 & 1847 & 1700 & 1165 & 1175 & 916 & 844 & 701 & 569 & 399 & 269 & 190 \\
\hline & 74 & 853 & 1366 & 1285 & 1183 & 839 & 830 & 647 & 590 & 492 & 398 & 277 & 187 & 132 \\
\hline & 75 & 302 & 2234 & 954 & 884 & 601 & 583 & 474 & 427 & 358 & 289 & 199 & 135 & 93 \\
\hline 4 & 76 & 576 & 1252 & 1088 & 1015 & 700 & 690 & 562 & 528 & 435 & 355 & 253 & 169 & 116 \\
\hline 4 & 77 & 49 & 4445 & 1517 & 1325 & 952 & 918 & 766 & 708 & 588 & 477 & 335 & 225 & 154 \\
\hline 4 & 78 & 146 & 2217 & 1715 & 1479 & 1073 & 1030 & 853 & 801 & 660 & 539 & 383 & 256 & 179 \\
\hline 4 & 79 & 166 & 2000 & 1017 & 940 & 646 & 685 & 509 & 467 & 389 & 315 & 221 & 149 & 106 \\
\hline 2 & 80 & 270 & 2568 & 1616 & 1472 & 1001 & 974 & 815 & 756 & 627 & 510 & 359 & 241 & 163 \\
\hline 4 & 81 & 2706 & 4872 & 1810 & 1719 & 1199 & 1180 & 977 & 925 & 760 & 621 & 445 & 296 & 200 \\
\hline 4 & 82 & 837 & 1057 & 550 & 528 & 372 & 369 & 298 & 286 & 233 & 192 & 138 & 91 & 63 \\
\hline 4 & 83 & 3979 & 3267 & 2730 & 2469 & 1731 & 1745 & 1378 & 1287 & 1063 & 866 & 614 & 410 & 288 \\
\hline 4 & 84 & 151 & 2475 & 1440 & 1387 & 919 & 921 & 749 & 679 & 568 & 459 & 318 & 216 & 144 \\
\hline 4 & 85 & 143 & 2505 & 1005 & 965 & 639 & 647 & 528 & 484 & 403 & 327 & 228 & 154 & 103 \\
\hline 4 & 86 & & 1620 & 2128 & 2025 & 1386 & 1322 & 1118 & & 851 & 688 & 479 & 324 & 217 \\
\hline 4 & 87 & 725 & 4240 & 1950 & 1833 & 1218 & 1171 & 990 & 890 & 747 & 603 & 415 & 282 & 187 \\
\hline 4 & 88 & 24 & 4031 & 1759 & 1564 & 1091 & 1131 & 861 & 801 & 663 & 540 & 381 & 255 & 183 \\
\hline 4 & 89 & 93 & 1048 & 872 & 768 & 526 & 586 & 410 & 370 & 310 & 250 & 173 & 117 & 85 \\
\hline 4 & 90 & 201 & 3170 & 1453 & 1428 & 975 & 1006 & 776 & 722 & 597 & 486 & 343 & 230 & 160 \\
\hline 4 & 91 & & & & 986 & 670 & 687 & & & & & 239 & 160 & 111 \\
\hline 4 & 92 & 3022 & 1431 & 2128 & 1802 & 1283 & 1311 & 1015 & 946 & 782 & 637 & 451 & 302 & 216 \\
\hline 4 & 93 & & & 1632 & 1530 & 1059 & & & 768 & 638 & 518 & 364 & 245 & 174 \\
\hline 4 & 94 & 497 & 1056 & 389 & 326 & 239 & 248 & 188 & 167 & 141 & 113 & 77 & 53 & 37 \\
\hline 4 & 95 & 502 & 100 & 338 & 301 & 210 & 250 & 157 & 146 & 121 & 99 & 70 & 47 & 37 \\
\hline 4 & 96 & & & 3631 & 3031 & 2169 & 2143 & 1708 & & 1285 & 1034 & 708 & 483 & 332 \\
\hline 4 & 97 & 200 & 2007 & 1712 & 1392 & 1001 & 1078 & 766 & 670 & 570 & 456 & 307 & 212 & 150 \\
\hline 4 & 98 & & & & & 968 & 968 & 734 & 638 & 544 & & 291 & 201 & 140 \\
\hline 4 & 99 & & 2682 & 2869 & 2265 & 1650 & 1587 & 1294 & 1165 & 978 & & 543 & 370 & 257 \\
\hline 4 & 100 & 1778 & 1489 & 1172 & 1019 & 840 & 749 & 611 & 550 & 461 & 372 & 256 & 174 & 126 \\
\hline 4 & & & & & 940 & & 670 & 526 & & & & 243 & 161 & 112 \\
\hline 4 & 102 & 4045 & 5809 & 2711 & 2603 & 1790 & 1949 & 1397 & 1279 & 1066 & 863 & 603 & 407 & 291 \\
\hline 4 & & & 603 & & & & 289 & 200 & & 152 & & 86 & 58 & 42 \\
\hline 4 & & & 641 & 226 & 212 & 144 & & & & & & 51 & 34 & 24 \\
\hline 4 & 105 & 2402 & 2962 & 1717 & 1552 & 1049 & 1147 & 825 & 746 & 625 & 504 & 348 & 237 & 169 \\
\hline 4 & & & 1801 & 1474 & 1341 & 938 & 997 & 739 & 698 & 574 & 469 & 335 & 223 & 161 \\
\hline 4 & 107 & 1530 & 1955 & 1088 & 1024 & 673 & 735 & 546 & 501 & 417 & 338 & 236 & 159 & 111 \\
\hline 4 & & & & & 485 & 328 & 336 & 272 & 248 & 207 & 167 & 117 & 79 & 53 \\
\hline 4 & 109 & & 322 & 253 & 225 & 152 & 165 & 122 & & 92 & 74 & 51 & 35 & 24 \\
\hline 4 & & 2376 & 1489 & 1780 & 1491 & 1039 & 1156 & 819 & 735 & 618 & 498 & 342 & 233 & 167 \\
\hline 4 & & 1069 & 1053 & 843 & 689 & 470 & 495 & 383 & 347 & 290 & 235 & 162 & 110 & 76 \\
\hline 4 & 112 & 319 & 153 & 235 & 203 & 134 & 143 & 110 & 99 & 83 & 67 & 46 & 31 & 21 \\
\hline 4 & & 1677 & 1370 & 1131 & 1059 & 721 & 738 & 583 & 534 & 445 & 360 & 251 & 170 & 116 \\
\hline 4 & 114 & 572 & 535 & 408 & 358 & 245 & 252 & 205 & 192 & 158 & 129 & 91 & 61 & 41 \\
\hline 4 & & & 530 & 1303 & 1110 & 820 & 870 & 634 & 608 & 497 & 408 & 295 & 195 & 146 \\
\hline 4 & 116 & & & 2155 & 1824 & 1251 & 1410 & 1012 & 900 & 760 & 611 & 417 & 285 & 198 \\
\hline 4 & 117 & 2595 & 1997 & 2282 & 1656 & 1144 & 1312 & 987 & 831 & 719 & 569 & 371 & 261 & 165 \\
\hline
\end{tabular}




\begin{tabular}{|c|c|c|c|c|c|c|c|c|c|c|c|c|c|c|}
\hline 4 & 118 & 91 & 8 & 59 & 60 & 39 & 42 & 31 & 28 & 24 & 19 & 13 & 9 & 6 \\
\hline 4 & 119 & 3624 & 3827 & 2811 & 2262 & 1586 & 1530 & 1286 & 1169 & 977 & 790 & 548 & 371 & 252 \\
\hline 4 & 120 & 3303 & 2872 & 2426 & 2077 & 1441 & 1464 & 1161 & 1062 & 885 & 717 & 500 & 338 & 233 \\
\hline 4 & 121 & 741 & 232 & 481 & 441 & 317 & 315 & 247 & 236 & 193 & 158 & 114 & 75 & 54 \\
\hline 4 & 122 & 1235 & 1339 & 824 & 795 & 553 & 537 & 443 & 415 & 342 & 279 & 198 & 132 & 90 \\
\hline 4 & 123 & 819 & 723 & 512 & 535 & 345 & 398 & 268 & 238 & 201 & 161 & 110 & 75 & 54 \\
\hline 4 & 124 & 427 & 366 & 291 & 286 & 192 & 187 & 160 & 145 & 121 & 98 & 68 & 46 & 30 \\
\hline 4 & 125 & 138 & 119 & 100 & 91 & 61 & 61 & 51 & 48 & 40 & 32 & 23 & 15 & 10 \\
\hline 4 & 126 & 843 & 827 & 558 & 544 & 387 & 361 & 306 & 283 & 235 & 191 & 134 & 90 & 61 \\
\hline 4 & 127 & 556 & 348 & 387 & 335 & 232 & 235 & 194 & 191 & 155 & 128 & 94 & 61 & 42 \\
\hline 4 & 128 & 1723 & 2097 & 1168 & 1037 & 723 & 716 & 603 & 569 & 468 & 382 & 273 & 182 & 121 \\
\hline 4 & 129 & 1361 & 2263 & 962 & 855 & 597 & 590 & 496 & 471 & 386 & 316 & 227 & 151 & 101 \\
\hline 4 & 130 & 580 & 1484 & 387 & 366 & 261 & 249 & 208 & 202 & 164 & 135 & 99 & 65 & 45 \\
\hline 4 & 131 & 572 & 703 & 403 & 378 & 247 & 240 & 205 & 187 & 156 & 126 & 88 & 59 & 39 \\
\hline 4 & 132 & 259 & 477 & 169 & 160 & 109 & 105 & 90 & 83 & 69 & 56 & 39 & 26 & 17 \\
\hline 4 & 133 & 418 & 116 & 258 & 138 & 142 & 173 & 131 & 161 & 119 & 104 & 88 & 53 & 40 \\
\hline 4 & 134 & 3037 & 3232 & 2002 & 1791 & 1291 & 1272 & 1062 & 1026 & 835 & 687 & 499 & 329 & 223 \\
\hline 4 & 135 & 1875 & 1309 & 1321 & 1238 & 830 & 789 & 678 & 606 & 510 & 411 & 282 & 192 & 126 \\
\hline 4 & 136 & 3228 & 2643 & 2284 & 2028 & 1491 & 1350 & 1159 & 1040 & 874 & 705 & 484 & 330 & 224 \\
\hline 4 & 137 & 2206 & 1001 & 1560 & 1414 & 966 & 922 & 787 & 716 & 598 & 484 & 336 & 228 & 151 \\
\hline 4 & 138 & 774 & 805 & 593 & 519 & 350 & 334 & 286 & 258 & 217 & 175 & 121 & 82 & 54 \\
\hline 4 & 139 & 3276 & 3363 & 2348 & 2121 & 1455 & 1395 & 1183 & 1096 & 909 & 739 & 520 & 349 & 236 \\
\hline 4 & 140 & 4255 & 5208 & 3030 & 2674 & 1904 & 1781 & 1520 & 1386 & 1157 & 936 & 651 & 440 & 298 \\
\hline 4 & 141 & 4572 & 5481 & 3434 & 3037 & 2100 & 1967 & 1692 & 1511 & 1272 & 1024 & 701 & 479 & 317 \\
\hline 4 & 142 & 1366 & 0 & 866 & 684 & 518 & 488 & 417 & 379 & 317 & 256 & 178 & 120 & 81 \\
\hline 4 & 143 & 349 & 602 & 263 & 241 & 158 & 151 & 130 & 115 & 98 & 78 & 53 & 37 & 24 \\
\hline 4 & 144 & 2417 & 658 & 1805 & 1508 & 1101 & 996 & 860 & 759 & 643 & 515 & 349 & 240 & 160 \\
\hline 4 & 145 & 3464 & 2694 & 2542 & 2221 & 1565 & 1454 & 1251 & 1114 & 940 & 755 & 516 & 353 & 234 \\
\hline 4 & 146 & 2741 & 2802 & 1931 & 1777 & 1203 & 1163 & 990 & 908 & 756 & 613 & 428 & 289 & 191 \\
\hline 4 & 147 & 1594 & 2917 & 1128 & 1122 & 726 & 700 & 596 & 547 & 455 & 369 & 258 & 174 & 115 \\
\hline 4 & 148 & 1146 & 2083 & 795 & 810 & 522 & 501 & 431 & 384 & 324 & 260 & 178 & 122 & 79 \\
\hline 4 & 149 & 3564 & 4074 & 2614 & 2320 & 1672 & 1534 & 1321 & 1176 & 992 & 798 & 545 & 373 & 248 \\
\hline 4 & 150 & 1618 & 730 & 1206 & 1009 & 747 & 674 & 581 & 515 & 435 & 349 & 237 & 163 & 109 \\
\hline 4 & 151 & 1156 & 118 & 712 & 666 & 459 & 433 & 370 & 336 & 281 & 227 & 158 & 107 & 71 \\
\hline 4 & 152 & 1774 & 1312 & 1326 & 1113 & 805 & 747 & 640 & 577 & 484 & 391 & 270 & 183 & 124 \\
\hline 4 & 153 & 2109 & 1573 & 1512 & 1363 & 951 & 896 & 764 & 698 & 582 & 471 & 328 & 222 & 150 \\
\hline 4 & 154 & 3208 & 3113 & 2242 & 2044 & 1463 & 1358 & 1154 & 1064 & 884 & 718 & 504 & 339 & 233 \\
\hline 4 & 155 & 666 & 908 & 457 & 433 & 299 & 283 & 240 & 223 & 184 & 150 & 106 & 71 & 48 \\
\hline 4 & 156 & 1910 & 2038 & 1379 & 1237 & 875 & 806 & 690 & 623 & 522 & 421 & 291 & 198 & 133 \\
\hline 4 & 157 & 1666 & 2390 & 1140 & 1048 & 787 & 712 & 607 & 556 & 463 & 375 & 262 & 177 & 122 \\
\hline 4 & 158 & 2948 & 3383 & 2133 & 1990 & 1347 & 1273 & 1091 & 983 & 825 & 666 & 459 & 312 & 206 \\
\hline 4 & 159 & 4269 & 6675 & 3003 & 2813 & 1938 & 1822 & 1558 & 1413 & 1182 & 955 & 662 & 449 & 299 \\
\hline 4 & 160 & 175 & 115 & 116 & 109 & 77 & 73 & 63 & 57 & 48 & 38 & 26 & 18 & 12 \\
\hline 4 & 161 & 1904 & 3277 & 1291 & 1296 & 848 & 821 & 699 & 640 & 533 & 432 & 301 & 203 & 134 \\
\hline 4 & 162 & 2951 & 2527 & 2062 & 1876 & 1288 & 1223 & 1042 & 954 & 795 & 644 & 450 & 304 & 204 \\
\hline
\end{tabular}




\begin{tabular}{|c|c|c|c|c|c|c|c|c|c|c|c|c|c|c|}
\hline 4 & 163 & 2138 & 2507 & 1584 & 1363 & 1005 & 901 & 780 & 683 & 580 & 464 & 313 & 216 & 142 \\
\hline 4 & 164 & 712 & 2275 & 2501 & 2292 & 1700 & 1538 & 1312 & 1197 & 999 & & 563 & 381 & 262 \\
\hline 2 & 165 & 3749 & 2760 & 2699 & 2439 & 1702 & 1574 & 1355 & 1206 & 1017 & 818 & 559 & 382 & 253 \\
\hline 4 & 166 & 245 & 470 & 175 & 165 & 106 & 104 & 90 & 80 & 67 & 54 & 37 & 25 & 16 \\
\hline 4 & 167 & 99 & 30 & 68 & 70 & 45 & 42 & 37 & 32 & 27 & 22 & 15 & 10 & 7 \\
\hline 4 & 168 & 121 & 62 & 83 & 81 & 52 & 50 & 44 & 38 & 33 & 26 & 18 & 12 & 8 \\
\hline 4 & 169 & 50 & 152 & 34 & 34 & 22 & 22 & 19 & 17 & 14 & 12 & 8 & 6 & 4 \\
\hline 4 & 170 & 4178 & 1099 & 2732 & 2473 & 1867 & 1662 & 1424 & 1284 & 1077 & 869 & 600 & 408 & 280 \\
\hline 5 & 1 & 69 & 1130 & 2059 & 1435 & 942 & 979 & 749 & 654 & 557 & 445 & 299 & 207 & 138 \\
\hline 5 & 2 & 519 & 313 & 471 & 340 & 248 & 234 & 188 & 174 & 144 & 117 & 83 & 55 & 40 \\
\hline 5 & 3 & 2327 & 504 & 2286 & 1741 & 1038 & 1163 & 832 & 716 & 613 & 488 & 324 & 225 & 149 \\
\hline 5 & 4 & 576 & 288 & 1402 & 992 & 668 & 637 & 531 & 473 & 399 & 321 & 219 & 150 & 100 \\
\hline 5 & 5 & 810 & 2828 & 4510 & 3483 & 2053 & 2148 & 1725 & 1539 & 1297 & 1043 & 713 & 487 & 322 \\
\hline 5 & 6 & & 879 & 1597 & 1302 & 765 & 874 & 622 & 546 & 463 & 371 & 250 & 172 & 117 \\
\hline 5 & 7 & 739 & 4913 & 3412 & 2710 & 1651 & 1689 & 1342 & 1212 & 1016 & 820 & 567 & 385 & 261 \\
\hline 5 & 8 & & & & 2038 & 1244 & & 982 & 842 & 722 & & 380 & 265 & 180 \\
\hline 5 & 9 & 150 & 1900 & 3935 & 2871 & 1948 & 1882 & 1503 & 1305 & 1113 & 889 & 595 & 412 & 273 \\
\hline 5 & 10 & 41 & 388 & 913 & 593 & 459 & 435 & 339 & 287 & 248 & 196 & 129 & 90 & 58 \\
\hline 5 & 1 & 576 & 2375 & 2123 & 1774 & 1212 & & 916 & 817 & & & 378 & 259 & 178 \\
\hline 5 & 12 & 2813 & 1538 & 2195 & 1912 & 1368 & 1239 & 1024 & 911 & 768 & 618 & 421 & 288 & 196 \\
\hline 5 & 1 & & & & & & & & & & & 621 & 422 & 286 \\
\hline 5 & 14 & 148 & 1845 & 4444 & 3110 & 2405 & 2093 & 1743 & 1521 & 1294 & 1035 & 695 & 480 & 325 \\
\hline 5 & 15 & 780 & 1350 & 2557 & 1795 & 1361 & 1228 & 989 & 849 & 728 & 579 & 384 & 267 & 177 \\
\hline 5 & 16 & & 1298 & 3120 & 2207 & 1690 & & 1214 & & & & 472 & 329 & 224 \\
\hline 5 & 17 & 4992 & 1529 & 4138 & 2999 & 2407 & 2112 & 1739 & 1501 & 1284 & 1023 & 681 & 473 & 315 \\
\hline 5 & 18 & & & & & & & 873 & 744 & 640 & & 334 & 234 & 153 \\
\hline 5 & 19 & & 1531 & 4631 & 3407 & 2616 & 2356 & 1905 & 1651 & 1409 & 1125 & 751 & 521 & 349 \\
\hline 5 & 20 & 2668 & 844 & 2298 & 1708 & 1310 & 1238 & 955 & 819 & 702 & 559 & 370 & 258 & 171 \\
\hline 5 & 2 & & 1773 & 4027 & 2931 & 2353 & 2207 & 1667 & 1432 & 1227 & & 647 & 451 & 305 \\
\hline 5 & 22 & 299 & 846 & 1999 & 1453 & 1104 & 1014 & 806 & 684 & 589 & 468 & 307 & 215 & 139 \\
\hline 5 & 23 & & & & & 2449 & & 1974 & & 1477 & 1186 & 806 & 553 & 367 \\
\hline 5 & 24 & & & & 3143 & 2119 & 1974 & 1642 & & & & 671 & 460 & 309 \\
\hline 5 & 25 & 130 & 3527 & 5108 & 4280 & 2709 & 3047 & 2066 & 1848 & 1555 & 1252 & 858 & 586 & 423 \\
\hline 5 & 26 & & 1052 & 2069 & 1800 & 1082 & 1152 & 866 & 801 & 665 & & 380 & 255 & 180 \\
\hline 5 & 27 & 587 & 1172 & 3343 & 3017 & 1997 & 2048 & 1524 & 1410 & 1169 & 950 & 669 & 449 & 322 \\
\hline 5 & 28 & & & & 1967 & 1251 & & 984 & 912 & 756 & & 433 & 291 & 210 \\
\hline 5 & 29 & & 1082 & 1013 & 896 & 575 & 601 & 468 & 444 & 364 & & 214 & 142 & 98 \\
\hline 5 & 30 & 4965 & 978 & 4638 & 3656 & 2178 & 2341 & 1781 & 1568 & 1329 & 1065 & 721 & 496 & 331 \\
\hline 5 & 31 & & 1428 & 3028 & 2454 & 1467 & 1619 & 1187 & 1067 & 896 & 722 & 497 & 338 & 233 \\
\hline 5 & 32 & 409 & 4476 & 2455 & 2460 & 1525 & 1530 & 1209 & 1116 & 926 & 752 & 529 & 355 & 243 \\
\hline 5 & 33 & & 1376 & 1751 & 1721 & 1045 & 1045 & 825 & 758 & 630 & & 358 & 241 & 165 \\
\hline 5 & 34 & 327 & 2577 & 1754 & 1499 & 1088 & 1090 & 794 & 709 & 597 & 480 & 328 & 224 & 160 \\
\hline 5 & 35 & & 3275 & 2020 & 1684 & 1184 & 1348 & 845 & 757 & 637 & 513 & 352 & 240 & 184 \\
\hline 5 & 36 & 7491 & 3311 & 5905 & 4970 & 3453 & 3263 & 2586 & 2297 & 1939 & 1558 & 1061 & 727 & 499 \\
\hline 5 & 37 & 998 & 880 & 829 & 714 & 499 & 451 & 370 & 326 & 277 & 222 & 150 & 103 & 70 \\
\hline
\end{tabular}




\begin{tabular}{|c|c|c|c|c|c|c|c|c|c|c|c|c|c|c|}
\hline 5 & 38 & 274 & 140 & 196 & 201 & 124 & 134 & 97 & 88 & 74 & 60 & 42 & 28 & 20 \\
\hline 5 & 39 & 3457 & 2690 & 2612 & 2380 & 1607 & 1586 & 1206 & 1055 & 897 & 718 & 483 & 333 & 226 \\
\hline 5 & 40 & 573 & 1179 & 463 & 394 & 262 & 268 & 207 & 187 & 157 & 127 & 88 & 60 & 41 \\
\hline 5 & 41 & 3121 & 4062 & 2320 & 2193 & 1475 & 1413 & 1103 & 1000 & 837 & 676 & 468 & 317 & 222 \\
\hline 5 & 42 & 403 & 405 & 285 & 314 & 179 & 184 & 144 & 131 & 110 & 89 & 61 & 42 & 28 \\
\hline 5 & 43 & 1016 & 3078 & 800 & 726 & 453 & 447 & 366 & 340 & 282 & 229 & 162 & 108 & 74 \\
\hline 5 & 44 & 1717 & 483 & 1157 & 1163 & 761 & 714 & 587 & 540 & 449 & 364 & 255 & 172 & 118 \\
\hline 5 & 45 & 765 & 415 & 595 & 548 & 348 & 360 & 270 & 242 & 204 & 164 & 113 & 77 & 53 \\
\hline 5 & 46 & 591 & 1245 & 441 & 430 & 271 & 267 & 217 & 206 & 169 & 138 & 99 & 66 & 45 \\
\hline 5 & 47 & 1717 & 2643 & 1152 & 1166 & 768 & 795 & 574 & 531 & 441 & 358 & 252 & 169 & 122 \\
\hline 5 & 48 & 305 & 842 & 235 & 224 & 149 & 139 & 113 & 101 & 85 & 69 & 47 & 32 & 22 \\
\hline 5 & 49 & 1908 & 3680 & 1338 & 1472 & 860 & 960 & 680 & 603 & 510 & 409 & 279 & 191 & 130 \\
\hline 5 & 50 & 1549 & 3555 & 1264 & 1146 & 797 & 859 & 569 & 511 & 429 & 346 & 237 & 162 & 121 \\
\hline 5 & 51 & 759 & 948 & 560 & 568 & 338 & 422 & 260 & 233 & 196 & 158 & 108 & 74 & 54 \\
\hline 5 & 52 & 2207 & 686 & 1549 & 1494 & 939 & 1064 & 713 & 662 & 548 & 446 & 315 & 211 & 156 \\
\hline 5 & 53 & 1971 & 1618 & 1553 & 1418 & 964 & 1224 & 676 & 587 & 501 & 400 & 267 & 185 & 142 \\
\hline 5 & 54 & 1013 & 1212 & 727 & 679 & 446 & 425 & 349 & 332 & 272 & 223 & 160 & 106 & 74 \\
\hline 5 & 55 & 6998 & 7896 & 4965 & 4698 & 3438 & 3036 & 2482 & 2236 & 1876 & 1513 & 1043 & 709 & 496 \\
\hline 5 & 56 & 1804 & 4184 & 1441 & 1306 & 819 & 792 & 654 & 616 & 507 & 414 & 295 & 197 & 135 \\
\hline 5 & 57 & 797 & 1580 & 665 & 608 & 369 & 355 & 299 & 273 & 228 & 184 & 129 & 87 & 58 \\
\hline 5 & 58 & 667 & 668 & 510 & 488 & 304 & 304 & 236 & 218 & 181 & 147 & 103 & 69 & 49 \\
\hline 5 & 59 & 1803 & 1415 & 1254 & 1340 & 781 & 772 & 632 & 575 & 480 & 388 & 270 & 183 & 121 \\
\hline 5 & 60 & 504 & 368 & 390 & 364 & 217 & 211 & 176 & 164 & 136 & 110 & 78 & 52 & 36 \\
\hline 5 & 61 & 2810 & 6 & 1583 & 1687 & 997 & 966 & 800 & 734 & 611 & 495 & 346 & 234 & 156 \\
\hline 5 & 62 & 2725 & 1741 & 2127 & 1882 & 1219 & 1180 & 956 & 911 & 746 & 611 & 440 & 291 & 206 \\
\hline 5 & 63 & 2381 & 2598 & 1725 & 1568 & 1066 & 1041 & 800 & 731 & 610 & 494 & 344 & 233 & 165 \\
\hline 5 & 64 & 1875 & 3042 & 1405 & 1376 & 852 & 877 & 668 & 623 & 515 & 419 & 297 & 199 & 139 \\
\hline 5 & 65 & 593 & 691 & 461 & 419 & 276 & 269 & 214 & 197 & 164 & 133 & 93 & 63 & 43 \\
\hline 5 & 66 & 1346 & 999 & 1092 & 971 & 594 & 583 & 480 & 439 & 366 & 297 & 207 & 140 & 94 \\
\hline 5 & 67 & 1347 & 122 & 923 & 868 & 567 & 529 & 442 & 408 & 339 & 275 & 194 & 130 & 89 \\
\hline 5 & 68 & 1917 & 664 & 1506 & 1288 & 859 & 855 & 653 & 610 & 504 & 411 & 291 & 195 & 141 \\
\hline 5 & 69 & 1607 & 994 & 1291 & 1107 & 707 & 681 & 555 & 509 & 424 & 344 & 240 & 162 & 112 \\
\hline 5 & 70 & 1936 & 1224 & 1496 & 1354 & 838 & 822 & 679 & 638 & 526 & 429 & 306 & 204 & 139 \\
\hline 5 & 71 & 1439 & 5626 & 1010 & 1177 & 644 & 628 & 539 & 461 & 396 & 314 & 207 & 145 & 90 \\
\hline 5 & 72 & 3689 & 5153 & 2805 & 2533 & 1776 & 1758 & 1298 & 1170 & 982 & 792 & 546 & 371 & 266 \\
\hline 5 & 73 & 2603 & 2606 & 2010 & 1901 & 1189 & 1199 & 916 & 844 & 701 & 569 & 399 & 269 & 190 \\
\hline 5 & 74 & 1846 & 1475 & 1390 & 1311 & 862 & 848 & 647 & 590 & 492 & 398 & 277 & 187 & 132 \\
\hline 5 & 75 & 1290 & 3172 & 1038 & 988 & 617 & 590 & 474 & 427 & 358 & 289 & 199 & 135 & 93 \\
\hline 5 & 76 & 1565 & 1377 & 1176 & 1132 & 711 & 696 & 562 & 528 & 435 & 355 & 253 & 169 & 116 \\
\hline 5 & 77 & 2011 & 6756 & 1649 & 1453 & 971 & 922 & 766 & 708 & 588 & 477 & 335 & 225 & 154 \\
\hline 5 & 78 & 2439 & 2572 & 1878 & 1631 & 1099 & 1035 & 853 & 801 & 660 & 539 & 383 & 256 & 179 \\
\hline 5 & 79 & 1461 & 2640 & 1099 & 1045 & 655 & 707 & 509 & 467 & 389 & 315 & 221 & 149 & 106 \\
\hline 5 & 80 & 2260 & 3185 & 1760 & 1640 & 1017 & 977 & 815 & 756 & 627 & 510 & 359 & 241 & 163 \\
\hline 5 & 81 & 2688 & 7015 & 1933 & 1900 & 1208 & 1185 & 977 & 925 & 760 & 621 & 445 & 296 & 200 \\
\hline 5 & 82 & 831 & 1353 & 587 & 586 & 375 & 371 & 298 & 286 & 233 & 192 & 138 & 91 & 63 \\
\hline
\end{tabular}




\begin{tabular}{|c|c|c|c|c|c|c|c|c|c|c|c|c|c|c|}
\hline 5 & 83 & 3978 & 3659 & 2962 & 2736 & 1760 & 1773 & 1378 & 1287 & 1063 & 866 & 614 & 410 & 288 \\
\hline 5 & 84 & 2173 & 3118 & 1544 & 1544 & 930 & 933 & 749 & 679 & 568 & & 318 & 216 & 144 \\
\hline & 85 & +34 & 3558 & 1076 & 1073 & 642 & 654 & 528 & 484 & 403 & 327 & 228 & 154 & 103 \\
\hline 5 & 86 & 256 & 1555 & 2281 & 2245 & 1410 & 1326 & 1118 & 1019 & 851 & 688 & 479 & 324 & 217 \\
\hline 5 & 87 & 723 & 5852 & 2108 & 2043 & 1240 & 1176 & 990 & 890 & 747 & 603 & 415 & 282 & 187 \\
\hline 5 & 88 & 524 & 5644 & 1923 & 1738 & 1111 & 1159 & 861 & 801 & 663 & 540 & 381 & 255 & 183 \\
\hline 5 & 89 & 189 & 1195 & 957 & 853 & 535 & 615 & 410 & 370 & 310 & 250 & 173 & 117 & 85 \\
\hline 5 & 90 & 93 & 4248 & 1544 & 1588 & 983 & 1028 & 776 & 722 & 597 & 486 & 343 & 230 & 160 \\
\hline 5 & 91 & 187 & 2147 & 1089 & 1099 & 675 & 700 & 535 & 500 & 413 & 337 & 239 & 160 & 111 \\
\hline 5 & 92 & 035 & 1345 & 2350 & 1994 & 1311 & 1338 & 1015 & 946 & 782 & 637 & 451 & 302 & 216 \\
\hline 5 & 93 & 2405 & 3385 & 1752 & 1694 & 1070 & 1170 & 833 & 768 & 638 & 518 & 364 & 245 & 174 \\
\hline 5 & 94 & 485 & 1584 & 425 & 354 & 245 & 256 & 188 & 167 & 141 & 113 & 77 & 53 & 37 \\
\hline 5 & 95 & 504 & 70 & 369 & 335 & 212 & 266 & 157 & 146 & 121 & 99 & 70 & 47 & 37 \\
\hline 5 & 96 & 5026 & 3296 & 4011 & 3325 & 2234 & 2185 & 1708 & 1525 & 1285 & 1034 & 708 & 483 & 332 \\
\hline 5 & 97 & 197 & 2329 & 1906 & 1525 & 1035 & 1135 & 766 & 670 & 570 & 456 & 307 & 212 & 150 \\
\hline 5 & 98 & & & & 1412 & 1011 & 1005 & 734 & 638 & & & 291 & 201 & 140 \\
\hline 5 & 99 & 527 & 2897 & 3222 & 2486 & 1709 & 1608 & 1294 & 1165 & 978 & 789 & 543 & 370 & 257 \\
\hline 5 & 100 & 775 & 1697 & 1254 & 1094 & 883 & 758 & 611 & 550 & 461 & 372 & 256 & 174 & 126 \\
\hline 5 & & & & 1058 & 1043 & 659 & 680 & 526 & 503 & & & 243 & 161 & 112 \\
\hline 5 & 102 & 4032 & 7783 & 2893 & 2884 & 1808 & 2029 & 1397 & 1279 & 1066 & 863 & 603 & 407 & 291 \\
\hline 5 & & & & & 400 & 253 & 303 & 200 & & & & 86 & 58 & 42 \\
\hline 5 & 104 & 327 & 949 & 243 & 235 & 145 & 155 & 117 & 108 & 90 & 73 & 51 & 34 & 24 \\
\hline 5 & 105 & 2401 & 3791 & 1873 & 1728 & 1066 & 1195 & 825 & 746 & 625 & 504 & 348 & 237 & 169 \\
\hline 5 & & & & 1598 & 1489 & 948 & 1025 & 739 & 698 & & & 335 & 223 & 161 \\
\hline 5 & 107 & 1518 & 2503 & 1175 & 1143 & 675 & 759 & 546 & 501 & 417 & 338 & 236 & 159 & 111 \\
\hline 5 & & & & & & 330 & 340 & 272 & 248 & & & 117 & 79 & 53 \\
\hline 5 & & & 374 & 275 & 248 & 154 & 171 & 122 & 110 & 92 & 74 & 51 & 35 & 24 \\
\hline 5 & 110 & 2371 & 1519 & 1967 & 1641 & 1057 & 1211 & 819 & 735 & 618 & 498 & 342 & 233 & 167 \\
\hline 5 & & & & 942 & 762 & 478 & 507 & 383 & 347 & & & 162 & 110 & 76 \\
\hline 5 & 112 & 322 & 144 & 260 & 225 & 135 & 146 & 110 & 99 & 83 & 67 & 46 & 31 & 21 \\
\hline 5 & & & & 1215 & 1173 & 728 & 752 & 583 & 534 & & 60 & 251 & 170 & 116 \\
\hline 5 & & & & 445 & & 245 & 254 & 205 & 192 & & & 91 & 61 & 41 \\
\hline 5 & & 2029 & 413 & 1428 & 1223 & 835 & 895 & 634 & 608 & 497 & 408 & 295 & 195 & 146 \\
\hline 5 & & & & 2366 & 1997 & 1264 & 1476 & 1012 & 900 & & 611 & 417 & 285 & 198 \\
\hline 5 & & 2588 & 2157 & 2573 & 1761 & 1149 & 1375 & 987 & 831 & 719 & 569 & 371 & 261 & 165 \\
\hline 5 & & 92 & 4 & 62 & 67 & 39 & 43 & 31 & 28 & 24 & 19 & 13 & 9 & 6 \\
\hline 5 & & & 4669 & 3152 & 2496 & 1627 & 1536 & 1286 & 1169 & & 790 & 548 & 371 & 252 \\
\hline 5 & & 3300 & 3275 & 2673 & 2296 & 1468 & 1490 & 1161 & 1062 & 885 & 717 & 500 & 338 & 233 \\
\hline 5 & & 745 & 191 & 520 & 489 & 322 & 320 & 247 & 236 & 193 & 158 & 114 & 75 & 54 \\
\hline 5 & 122 & 1227 & 1634 & 879 & 882 & 560 & 540 & 443 & 415 & 342 & 279 & 198 & 132 & 90 \\
\hline 5 & & 834 & 839 & 539 & 601 & 348 & 423 & 268 & 238 & 201 & 161 & 110 & 75 & 54 \\
\hline 5 & 124 & 424 & 410 & 307 & 316 & 194 & 187 & 160 & 145 & 121 & 98 & 68 & 46 & 30 \\
\hline 5 & & & 133 & 110 & 102 & 61 & 61 & 51 & 48 & 40 & 32 & 23 & 15 & 10 \\
\hline 5 & 126 & 837 & 975 & 592 & 601 & 396 & 361 & 306 & 283 & 235 & 191 & 134 & 90 & 61 \\
\hline 5 & 127 & 554 & 355 & 426 & 375 & 234 & 235 & 194 & 191 & 155 & 128 & 94 & 61 & 42 \\
\hline
\end{tabular}




\begin{tabular}{|c|c|c|c|c|c|c|c|c|c|c|c|c|c|c|}
\hline 5 & 128 & 1735 & 2684 & 1266 & 1141 & 728 & 716 & 603 & 569 & 468 & 382 & 273 & 182 & 121 \\
\hline 5 & 129 & 1350 & 3168 & 1044 & 943 & 602 & 590 & 496 & 471 & 386 & 316 & 227 & 151 & 101 \\
\hline 5 & 130 & 573 & 2404 & 416 & 409 & 265 & 249 & 208 & 202 & 164 & 135 & 99 & 65 & 45 \\
\hline 5 & 131 & 575 & 900 & 436 & 422 & 250 & 240 & 205 & 187 & 156 & 126 & 88 & 59 & 39 \\
\hline 5 & 132 & 263 & 706 & 182 & 177 & 110 & 105 & 90 & 83 & 69 & 56 & 39 & 26 & 17 \\
\hline 5 & 133 & 413 & 91 & 296 & 148 & 143 & 173 & 131 & 161 & 119 & 104 & 88 & 53 & 40 \\
\hline 5 & 134 & 3044 & 3942 & 2159 & 1966 & 1299 & 1272 & 1062 & 1026 & 835 & 687 & 499 & 329 & 223 \\
\hline 5 & 135 & 1882 & 1387 & 1424 & 1373 & 845 & 789 & 678 & 606 & 510 & 411 & 282 & 192 & 126 \\
\hline 5 & 136 & 3221 & 2960 & 2472 & 2219 & 1544 & 1350 & 1159 & 1040 & 874 & 705 & 484 & 330 & 224 \\
\hline 5 & 137 & 2214 & 921 & 1695 & 1566 & 984 & 922 & 787 & 716 & 598 & 484 & 336 & 228 & 151 \\
\hline 5 & 138 & 768 & 966 & 652 & 577 & 357 & 334 & 286 & 258 & 217 & 175 & 121 & 82 & 54 \\
\hline 5 & 139 & 3259 & 4036 & 2559 & 2359 & 1481 & 1395 & 1183 & 1096 & 909 & 739 & 520 & 349 & 236 \\
\hline 5 & 140 & 4251 & 6666 & 3299 & 2944 & 1953 & 1781 & 1520 & 1386 & 1157 & 936 & 651 & 440 & 298 \\
\hline 5 & 141 & 4543 & 6906 & 3750 & 3351 & 2153 & 1967 & 1692 & 1511 & 1272 & 1024 & 701 & 479 & 317 \\
\hline 5 & 142 & 1439 & 0 & 956 & 736 & 531 & 488 & 417 & 379 & 317 & 256 & 178 & 120 & 81 \\
\hline 5 & 143 & 347 & 855 & 287 & 269 & 160 & 151 & 130 & 115 & 98 & 78 & 53 & 37 & 24 \\
\hline 5 & 144 & 2431 & 513 & 1989 & 1647 & 1143 & 996 & 860 & 759 & 643 & 515 & 349 & 240 & 160 \\
\hline 5 & 145 & 3468 & 2963 & 2776 & 2439 & 1609 & 1454 & 1251 & 1114 & 940 & 755 & 516 & 353 & 234 \\
\hline 5 & 146 & 2740 & 3362 & 2089 & 1969 & 1219 & 1163 & 990 & 908 & 756 & 613 & 428 & 289 & 191 \\
\hline 5 & 147 & 1576 & 4201 & 1208 & 1262 & 734 & 700 & 596 & 547 & 455 & 369 & 258 & 174 & 115 \\
\hline 5 & 148 & 1139 & 2999 & 841 & 905 & 527 & 501 & 431 & 384 & 324 & 260 & 178 & 122 & 79 \\
\hline 5 & 149 & 3535 & 5052 & 2829 & 2538 & 1723 & 1534 & 1321 & 1176 & 992 & 798 & 545 & 373 & 248 \\
\hline 5 & 150 & 1619 & 672 & 1325 & 1100 & 776 & 674 & 581 & 515 & 435 & 349 & 237 & 163 & 109 \\
\hline 5 & 151 & 1203 & 68 & 766 & 736 & 468 & 433 & 370 & 336 & 281 & 227 & 158 & 107 & 71 \\
\hline 5 & 152 & 1768 & 1417 & 1460 & 1218 & 830 & 747 & 640 & 577 & 484 & 391 & 270 & 183 & 124 \\
\hline 5 & 153 & 2099 & 1699 & 1643 & 1509 & 974 & 896 & 764 & 698 & 582 & 471 & 328 & 222 & 150 \\
\hline 5 & 154 & 3185 & 3673 & 2425 & 2260 & 1503 & 1358 & 1154 & 1064 & 884 & 718 & 504 & 339 & 233 \\
\hline 5 & 155 & 663 & 1198 & 492 & 482 & 305 & 283 & 240 & 223 & 184 & 150 & 106 & 71 & 48 \\
\hline 5 & 156 & 1903 & 2487 & 1500 & 1367 & 901 & 806 & 690 & 623 & 522 & 421 & 291 & 198 & 133 \\
\hline 5 & 157 & 1646 & 3202 & 1219 & 1145 & 813 & 712 & 607 & 556 & 463 & 375 & 262 & 177 & 122 \\
\hline 5 & 158 & 2928 & 4194 & 2304 & 2209 & 1374 & 1273 & 1091 & 983 & 825 & 666 & 459 & 312 & 206 \\
\hline 5 & 159 & 4250 & 9211 & 3231 & 3114 & 1979 & 1822 & 1558 & 1413 & 1182 & 955 & 662 & 449 & 299 \\
\hline 5 & 160 & 175 & 120 & 123 & 119 & 78 & 73 & 63 & 57 & 48 & 38 & 26 & 18 & 12 \\
\hline 5 & 161 & 1898 & 4653 & 1371 & 1448 & 856 & 821 & 699 & 640 & 533 & 432 & 301 & 203 & 134 \\
\hline 5 & 162 & 2963 & 2881 & 2242 & 2083 & 1314 & 1223 & 1042 & 954 & 795 & 644 & 450 & 304 & 204 \\
\hline 5 & 163 & 2138 & 3159 & 1725 & 1483 & 1044 & 901 & 780 & 683 & 580 & 464 & 313 & 216 & 142 \\
\hline 5 & 164 & 3706 & 2321 & 2684 & 2513 & 1758 & 1538 & 1312 & 1197 & 999 & 809 & 563 & 381 & 262 \\
\hline 5 & 165 & 3754 & 2981 & 2928 & 2690 & 1750 & 1574 & 1355 & 1206 & 1017 & 818 & 559 & 382 & 253 \\
\hline 5 & 166 & 246 & 696 & 188 & 183 & 107 & 104 & 90 & 80 & 67 & 54 & 37 & 25 & 16 \\
\hline 5 & 167 & 99 & 24 & 72 & 78 & 46 & 42 & 37 & 32 & 27 & 22 & 15 & 10 & 7 \\
\hline 5 & 168 & 122 & 60 & 89 & 89 & 53 & 50 & 44 & 38 & 33 & 26 & 18 & 12 & 8 \\
\hline 5 & 169 & 49 & 258 & 36 & 37 & 22 & 22 & 19 & 17 & 14 & 12 & 8 & 6 & 4 \\
\hline 5 & 170 & 4228 & 857 & 2932 & 2699 & 1940 & 1662 & 1424 & 1284 & 1077 & 869 & 600 & 408 & 280 \\
\hline 6 & 1 & 2194 & 1108 & 2360 & 1565 & 965 & 1005 & 749 & 654 & 557 & 445 & 299 & 207 & 138 \\
\hline 6 & 2 & 511 & 313 & 531 & 370 & 257 & 236 & 188 & 174 & 144 & 117 & 83 & 55 & 40 \\
\hline
\end{tabular}




\begin{tabular}{|c|c|c|c|c|c|c|c|c|c|c|c|c|c|c|}
\hline 6 & 3 & 2338 & 393 & 2612 & 1933 & 1059 & 1214 & 832 & 716 & 613 & 488 & 324 & 225 & 149 \\
\hline 6 & 4 & 1603 & 219 & 1597 & 1081 & 686 & 641 & 531 & 473 & 399 & 321 & 219 & 150 & 100 \\
\hline 6 & 5 & 826 & 2828 & 5122 & 3868 & 2076 & 2178 & 1725 & 1539 & 1297 & 1043 & 713 & 487 & 322 \\
\hline 6 & 6 & 770 & 844 & 1796 & 1449 & 775 & 910 & 622 & 546 & 463 & 371 & 250 & 172 & 117 \\
\hline 6 & 7 & 732 & 5994 & 3851 & 3014 & 1681 & 1714 & 1342 & 1212 & 1016 & 820 & 567 & 385 & 261 \\
\hline 6 & 8 & 684 & 1628 & 3003 & 2253 & 1270 & 1599 & 982 & 842 & 722 & 574 & 380 & 265 & 180 \\
\hline 6 & 9 & 4156 & 1786 & 4458 & 3127 & 2013 & 1916 & 1503 & 1305 & 1113 & 889 & 595 & 412 & 273 \\
\hline 6 & 10 & 947 & 357 & 1039 & 630 & 479 & 446 & 339 & 287 & 248 & 196 & 129 & 90 & 58 \\
\hline 6 & 11 & 2571 & 2660 & 2337 & 1942 & 1251 & 1194 & 916 & 817 & 689 & 554 & 378 & 259 & 178 \\
\hline 6 & 12 & 2800 & 1507 & 2373 & 2073 & 1416 & 1250 & 1024 & 911 & 768 & 618 & 421 & 288 & 196 \\
\hline 6 & 13 & 4065 & 5110 & 3636 & 3257 & 1898 & 1879 & 1473 & 1330 & 1115 & 900 & 621 & 422 & 286 \\
\hline 6 & 14 & 5223 & 1660 & 5008 & 3334 & 2525 & 2112 & 1743 & 1521 & 1294 & 1035 & 695 & 480 & 325 \\
\hline 6 & 15 & 2798 & 1296 & 2885 & 1925 & 1427 & 1250 & 989 & 849 & 728 & 579 & 384 & 267 & 177 \\
\hline 6 & 16 & 438 & 1168 & 3510 & 2366 & 1772 & 1716 & 1214 & 1044 & 894 & 712 & 472 & 329 & 224 \\
\hline 6 & 17 & 5045 & 1315 & 4578 & 3179 & 2524 & 2138 & 1739 & 1501 & 1284 & 1023 & 681 & 473 & 315 \\
\hline 6 & 18 & 515 & 1394 & 2370 & 1593 & 1307 & 1073 & 873 & 744 & 640 & 508 & 334 & 234 & 153 \\
\hline 6 & 19 & 417 & 1286 & 5150 & 3645 & 2737 & 2394 & 1905 & 1651 & 1409 & 1125 & 751 & 521 & 349 \\
\hline 6 & 20 & 680 & 726 & 2544 & 1822 & 1369 & 1273 & 955 & 819 & 702 & 559 & 370 & 258 & 171 \\
\hline 6 & & & 1596 & 4462 & 3114 & 2475 & 2278 & 1667 & 1432 & 1227 & 977 & 647 & 451 & 305 \\
\hline 6 & 22 & 2332 & 761 & 2229 & 1553 & 1155 & 1036 & 806 & 684 & 589 & 468 & 307 & 215 & 139 \\
\hline 6 & & & & & 4267 & 2500 & 2480 & 1974 & & 1477 & 1186 & 806 & 553 & 367 \\
\hline 6 & 24 & 4648 & 1757 & 4875 & 3441 & 2189 & 1989 & 1642 & 1455 & 1230 & 987 & 671 & 460 & 309 \\
\hline 6 & 25 & 6151 & 3527 & 5685 & 4752 & 2774 & 3192 & 2066 & 1848 & 1555 & 1252 & 858 & 586 & 423 \\
\hline 6 & & & 968 & 2300 & 2018 & 1099 & & & & & & 380 & 255 & 180 \\
\hline 6 & 27 & 4616 & 961 & 3636 & 3332 & 2040 & 2100 & 1524 & 1410 & 1169 & 950 & 669 & 449 & 322 \\
\hline 6 & & & & 2632 & 2180 & 1271 & 1424 & 984 & 912 & 56 & 15 & 433 & 291 & 210 \\
\hline 6 & 29 & 329 & 1168 & 1103 & 988 & 579 & 609 & 468 & 444 & & 298 & 214 & 142 & 98 \\
\hline 6 & 30 & 4974 & 743 & 5247 & 4060 & 2212 & 2404 & 1781 & 1568 & 1329 & 1065 & 721 & 496 & 331 \\
\hline 6 & & & & 3408 & & 1489 & & & & & & 497 & 338 & 233 \\
\hline 6 & 32 & 3404 & 5461 & 2625 & 2735 & 1544 & 1552 & 1209 & 1116 & 926 & 752 & 529 & 355 & 243 \\
\hline 6 & 33 & & & 1891 & 1926 & 1061 & & 825 & 758 & 30 & 511 & 358 & 241 & 165 \\
\hline 6 & 34 & & 3041 & 1903 & 1626 & 1126 & 1127 & 794 & & & 480 & 328 & 224 & 160 \\
\hline 6 & 35 & 2583 & 3995 & 2221 & 1844 & 1223 & 1431 & 845 & 757 & 637 & 513 & 352 & 240 & 184 \\
\hline 6 & 36 & 7539 & 3113 & 6476 & 5428 & 3574 & 3319 & 2586 & 2297 & 1939 & 1558 & 1061 & 727 & 499 \\
\hline 6 & 37 & 989 & 968 & 905 & 780 & 519 & 456 & 370 & 326 & 277 & 222 & 150 & 103 & 70 \\
\hline 6 & 38 & & 134 & 209 & 224 & 125 & 138 & 97 & 88 & 74 & 60 & 42 & 28 & 20 \\
\hline 6 & 39 & 3479 & 2905 & 2821 & 2606 & 1658 & 1631 & 1206 & 1055 & & 718 & 483 & 333 & 226 \\
\hline 6 & 40 & 569 & 1604 & 506 & 430 & 266 & 274 & 207 & 187 & 157 & 127 & 88 & 60 & 41 \\
\hline 6 & 41 & 3109 & 4955 & 2496 & 2418 & 1519 & 1439 & 1103 & 1000 & 837 & 676 & 468 & 317 & 222 \\
\hline 6 & 42 & 403 & 462 & 302 & 353 & 181 & 187 & 144 & 131 & 110 & 89 & 61 & 42 & 28 \\
\hline 6 & 43 & 1011 & 4617 & 874 & 806 & 459 & 450 & 366 & 340 & & 229 & 162 & 108 & 74 \\
\hline 6 & 44 & 1731 & 406 & 1230 & 1284 & 776 & 719 & 587 & 540 & 449 & 364 & 255 & 172 & 118 \\
\hline 6 & 45 & 764 & 407 & 647 & 606 & 355 & 369 & 270 & 242 & 204 & 164 & 113 & 77 & 53 \\
\hline 6 & 46 & 584 & 1694 & 474 & 478 & 273 & 268 & 217 & 206 & 169 & 138 & 99 & 66 & 45 \\
\hline 6 & 47 & 1723 & 3382 & 1223 & 1290 & 782 & 819 & 574 & 531 & 441 & 358 & 252 & 169 & 122 \\
\hline
\end{tabular}




\begin{tabular}{|c|c|c|c|c|c|c|c|c|c|c|c|c|c|c|}
\hline 6 & 48 & 303 & 1229 & 252 & 246 & 153 & 140 & 113 & 101 & 85 & 69 & 47 & 32 & 22 \\
\hline 6 & 49 & 1907 & 4931 & 1408 & 1645 & 867 & 998 & 680 & 603 & 510 & 409 & 279 & 191 & 130 \\
\hline 6 & 50 & 1512 & 4906 & 1369 & 1259 & 823 & 904 & 569 & 511 & 429 & 346 & 237 & 162 & 121 \\
\hline 6 & 51 & 757 & 1138 & 600 & 634 & 340 & 449 & 260 & 233 & 196 & 158 & 108 & 74 & 54 \\
\hline 6 & 52 & 2223 & 590 & 1672 & 1664 & 951 & 1110 & 713 & 662 & 548 & 446 & 315 & 211 & 156 \\
\hline 6 & 53 & 1950 & 1748 & 1681 & 1558 & 995 & 1339 & 676 & 587 & 501 & 400 & 267 & 185 & 142 \\
\hline 6 & 54 & 1014 & 1454 & 786 & 752 & 455 & 427 & 349 & 332 & 272 & 223 & 160 & 106 & 74 \\
\hline 6 & 55 & 6970 & 9317 & 5282 & 5115 & 3575 & 3065 & 2482 & 2236 & 1876 & 1513 & 1043 & 709 & 496 \\
\hline 6 & 56 & 1788 & 5858 & 1580 & 1457 & 832 & 795 & 654 & 616 & 507 & 414 & 295 & 197 & 135 \\
\hline 6 & 57 & 788 & 2117 & 728 & 679 & 375 & 356 & 299 & 273 & 228 & 184 & 129 & 87 & 58 \\
\hline 6 & 58 & 664 & 762 & 554 & 545 & 310 & 309 & 236 & 218 & 181 & 147 & 103 & 69 & 49 \\
\hline 6 & 59 & 1817 & 1529 & 1333 & 1503 & 789 & 778 & 632 & 575 & 480 & 388 & 270 & 183 & 121 \\
\hline 6 & 60 & 506 & 390 & 428 & 409 & 220 & 212 & 176 & 164 & 136 & 110 & 78 & 52 & 36 \\
\hline 6 & 61 & 2977 & 2 & 1684 & 1893 & 1009 & 971 & 800 & 734 & 611 & 495 & 346 & 234 & 156 \\
\hline 6 & 62 & 2708 & 1776 & 2339 & 2096 & 1244 & 1188 & 956 & 911 & 746 & 611 & 440 & 291 & 206 \\
\hline 6 & 63 & 2396 & 3066 & 1868 & 1726 & 1096 & 1063 & 800 & 731 & 610 & 494 & 344 & 233 & 165 \\
\hline 6 & 64 & 1862 & 3893 & 1515 & 1536 & 863 & 895 & 668 & 623 & 515 & 419 & 297 & 199 & 139 \\
\hline 6 & 65 & 589 & 816 & 500 & 462 & 282 & 272 & 214 & 197 & 164 & 133 & 93 & 63 & 43 \\
\hline 6 & 66 & 1346 & 1059 & 1202 & 1082 & 604 & 587 & 480 & 439 & 366 & 297 & 207 & 140 & 94 \\
\hline 6 & 67 & 1371 & 78 & 997 & 959 & 580 & 530 & 442 & 408 & 339 & 275 & 194 & 130 & 89 \\
\hline 6 & 68 & 1913 & 585 & 1661 & 1428 & 882 & 871 & 653 & 610 & 504 & 411 & 291 & 195 & 141 \\
\hline 6 & 69 & 1613 & 1014 & 1429 & 1230 & 724 & 687 & 555 & 509 & 424 & 344 & 240 & 162 & 112 \\
\hline 6 & 70 & 1938 & 1248 & 1639 & 1509 & 849 & 826 & 679 & 638 & 526 & 429 & 306 & 204 & 139 \\
\hline 6 & 71 & 1444 & 9002 & 1052 & 1318 & 650 & 630 & 539 & 461 & 396 & 314 & 207 & 145 & 90 \\
\hline 6 & 72 & 3664 & 6390 & 3031 & 2773 & 1836 & 1810 & 1298 & 1170 & 982 & 792 & 546 & 371 & 266 \\
\hline 6 & 73 & 2590 & 2971 & 2188 & 2125 & 1215 & 1224 & 916 & 844 & 701 & 569 & 399 & 269 & 190 \\
\hline 6 & 74 & 1839 & 1593 & 1503 & 1452 & 885 & 867 & 647 & 590 & 492 & 398 & 277 & 187 & 132 \\
\hline 6 & 75 & 1279 & 4505 & 1129 & 1103 & 634 & 598 & 474 & 427 & 358 & 289 & 199 & 135 & 93 \\
\hline 6 & 76 & 1554 & 1515 & 1271 & 1261 & 722 & 702 & 562 & 528 & 435 & 355 & 253 & 169 & 116 \\
\hline 6 & 77 & 1974 & 10269 & 1793 & 1593 & 991 & 926 & 766 & 708 & 588 & 477 & 335 & 225 & 154 \\
\hline 6 & 78 & 2433 & 2983 & 2057 & 1799 & 1126 & 1040 & 853 & 801 & 660 & 539 & 383 & 256 & 179 \\
\hline 6 & 79 & 1456 & 3485 & 1188 & 1160 & 665 & 730 & 509 & 467 & 389 & 315 & 221 & 149 & 106 \\
\hline 6 & 80 & 2251 & 3949 & 1916 & 1827 & 1033 & 980 & 815 & 756 & 627 & 510 & 359 & 241 & 163 \\
\hline 6 & 81 & 2670 & 10102 & 2063 & 2101 & 1216 & 1189 & 977 & 925 & 760 & 621 & 445 & 296 & 200 \\
\hline 6 & 82 & 824 & 1731 & 626 & 650 & 378 & 374 & 298 & 286 & 233 & 192 & 138 & 91 & 63 \\
\hline 6 & 83 & 3976 & 4098 & 3215 & 3032 & 1789 & 1801 & 1378 & 1287 & 1063 & 866 & 614 & 410 & 288 \\
\hline 6 & 84 & 2196 & 3929 & 1655 & 1719 & 941 & 945 & 749 & 679 & 568 & 459 & 318 & 216 & 144 \\
\hline 6 & 85 & 1426 & 5052 & 1152 & 1193 & 646 & 660 & 528 & 484 & 403 & 327 & 228 & 154 & 103 \\
\hline 6 & 86 & 3293 & 1493 & 2445 & 2489 & 1436 & 1329 & 1118 & 1019 & 851 & 688 & 479 & 324 & 217 \\
\hline 6 & 87 & 2721 & 8076 & 2280 & 2277 & 1263 & 1180 & 990 & 890 & 747 & 603 & 415 & 282 & 187 \\
\hline 6 & 88 & 2524 & 7901 & 2101 & 1931 & 1130 & 1188 & 861 & 801 & 663 & 540 & 381 & 255 & 183 \\
\hline 6 & 89 & 1184 & 1362 & 1049 & 946 & 544 & 645 & 410 & 370 & 310 & 250 & 173 & 117 & 85 \\
\hline 6 & 90 & 2184 & 5692 & 1640 & 1765 & 991 & 1051 & 776 & 722 & 597 & 486 & 343 & 230 & 160 \\
\hline 6 & 91 & 1474 & 2662 & 1165 & 1225 & 681 & 713 & 535 & 500 & 413 & 337 & 239 & 160 & 111 \\
\hline 6 & 92 & 3048 & 1264 & 2595 & 2207 & 1340 & 1366 & 1015 & 946 & 782 & 637 & 451 & 302 & 216 \\
\hline
\end{tabular}




\begin{tabular}{|c|c|c|c|c|c|c|c|c|c|c|c|c|c|c|}
\hline 6 & 93 & 2399 & 4198 & 1881 & 1875 & 1082 & 1210 & 833 & 768 & 638 & 518 & 364 & 245 & 174 \\
\hline 6 & 94 & 474 & 2376 & 464 & 383 & 251 & 265 & 188 & 167 & 141 & 113 & 77 & 53 & 37 \\
\hline 6 & 95 & 507 & 49 & 403 & 374 & 214 & 283 & 157 & 146 & 121 & 99 & 70 & 47 & 37 \\
\hline 6 & 96 & 076 & 3427 & 4430 & 3648 & 2300 & 2228 & 1708 & 1525 & 1285 & 1034 & 708 & 483 & 332 \\
\hline 6 & 97 & 195 & 2701 & 2122 & 1672 & 1070 & 1195 & 766 & 670 & 570 & 456 & 307 & 212 & 150 \\
\hline 6 & 98 & 063 & 1332 & 2132 & 1537 & 1057 & 1042 & 734 & 638 & 544 & 434 & 291 & 201 & 140 \\
\hline 6 & 99 & 3609 & 3129 & 3619 & 2729 & 1771 & 1628 & 1294 & 1165 & 978 & 789 & 543 & 370 & 257 \\
\hline 6 & 100 & 1771 & 1935 & 1342 & 1175 & 929 & 768 & 611 & 550 & 461 & 372 & 256 & 174 & 126 \\
\hline 6 & & 1490 & 4827 & 1133 & 1158 & 663 & 689 & 526 & 503 & 411 & & 243 & 161 & 112 \\
\hline 6 & 102 & 4018 & 10430 & 3087 & 3195 & 1826 & 2112 & 1397 & 1279 & 1066 & 863 & 603 & 407 & 291 \\
\hline 6 & 103 & 561 & 868 & 469 & 440 & 254 & 318 & 200 & 182 & 152 & 123 & 86 & 58 & 42 \\
\hline 6 & 104 & 326 & 1404 & 260 & 259 & 146 & 158 & 117 & 108 & 90 & 73 & 51 & 34 & 24 \\
\hline 6 & 05 & 2401 & 4852 & 2043 & 1923 & 1084 & 1246 & 825 & 746 & 625 & 504 & 348 & 237 & 169 \\
\hline 6 & & 251 & 2259 & 1733 & 1654 & 959 & 1054 & 739 & 698 & 574 & 469 & 335 & 223 & 161 \\
\hline 6 & 107 & 1507 & 3204 & 1270 & 1276 & 678 & 783 & 546 & 501 & 417 & 338 & 236 & 159 & 111 \\
\hline 6 & & & 451 & 622 & & 332 & 345 & & 248 & & & 117 & 79 & 53 \\
\hline 6 & 09 & 348 & 434 & 300 & 274 & 155 & 178 & 122 & 110 & 92 & 74 & 51 & 35 & 24 \\
\hline 6 & 10 & 2366 & 1549 & 2174 & 1807 & 1077 & 1268 & 819 & 735 & 618 & 498 & 342 & 233 & 167 \\
\hline 6 & & 1055 & & 1053 & 842 & 486 & 519 & 383 & 347 & & & 162 & 110 & 76 \\
\hline 6 & 112 & 325 & 135 & 287 & 250 & 136 & 150 & 110 & 99 & 83 & 67 & 46 & 31 & 21 \\
\hline 6 & & & & 1306 & 1299 & & & 583 & 534 & & & 251 & 170 & 116 \\
\hline 6 & 114 & 570 & 720 & 485 & 435 & 246 & 256 & 205 & 192 & 158 & 129 & 91 & 61 & 41 \\
\hline 6 & 115 & 2053 & 322 & 1564 & 1349 & 849 & 922 & 634 & 608 & 497 & 408 & 295 & 195 & 146 \\
\hline 6 & & & & 2597 & & 1277 & & & 900 & & & 417 & 285 & 198 \\
\hline 6 & 117 & 2581 & 2329 & 2901 & 1874 & 1154 & 1440 & 987 & 831 & 719 & 569 & 371 & 261 & 165 \\
\hline 6 & & & 2 & 65 & 75 & & 45 & 31 & 28 & 24 & & 13 & 0 & 6 \\
\hline 6 & & 3627 & 5696 & 3534 & 2754 & 1670 & 1542 & 1286 & 1169 & 977 & & 548 & 371 & 252 \\
\hline 6 & 120 & 3298 & 3733 & 2944 & 2538 & 1496 & 1516 & 1161 & 1062 & 885 & 717 & 500 & 338 & 233 \\
\hline 6 & & & & 562 & & & & & 236 & & & 114 & 75 & 54 \\
\hline 6 & 122 & 1220 & 1993 & 938 & 978 & 568 & 542 & 443 & 415 & 342 & 279 & 198 & 132 & 90 \\
\hline 6 & & & & 568 & & & & 268 & 238 & & 161 & 110 & 75 & 54 \\
\hline 6 & & & 459 & 325 & 348 & 195 & 187 & 160 & 145 & & & 68 & 46 & 30 \\
\hline 6 & 125 & 135 & 149 & 120 & 114 & 61 & 61 & 51 & 48 & 40 & 32 & 23 & 15 & 10 \\
\hline 6 & & & & 627 & 663 & 404 & & 306 & 283 & 235 & 191 & 134 & 90 & 61 \\
\hline 6 & 127 & 553 & 363 & 469 & 418 & 236 & 235 & 194 & 191 & 155 & 128 & 94 & 61 & 42 \\
\hline 6 & & & & 1372 & 1255 & & 716 & 603 & 569 & 468 & 382 & 273 & 182 & 121 \\
\hline 6 & & 1338 & 4436 & 1134 & 1040 & 606 & 590 & 496 & 471 & 386 & 316 & 227 & 151 & 101 \\
\hline 6 & & 565 & 3894 & 447 & 456 & 269 & 249 & 208 & 202 & 164 & 13 & 99 & 65 & 45 \\
\hline 6 & & 577 & 1152 & 473 & 472 & 252 & 240 & 205 & 187 & 156 & 126 & 88 & 59 & 39 \\
\hline 6 & 132 & 267 & 1045 & 195 & 196 & 111 & 105 & 90 & 83 & 69 & 56 & 39 & 26 & 17 \\
\hline 6 & & 408 & 71 & 341 & 158 & 143 & 173 & 131 & 161 & 119 & & 88 & 53 & 40 \\
\hline 6 & 134 & 3050 & 4810 & 2328 & 2159 & 1307 & 1272 & 1062 & 1026 & 835 & 687 & 499 & 329 & 223 \\
\hline 6 & & & & 1534 & 1522 & 860 & 789 & 678 & 606 & 510 & 411 & 282 & 192 & 126 \\
\hline 6 & & 3213 & 3315 & 2675 & 2428 & 1598 & 1350 & 1159 & 1040 & 874 & 705 & 484 & 330 & 224 \\
\hline 6 & 137 & 2221 & 847 & 1841 & 1734 & 1003 & 922 & 787 & 716 & 598 & 484 & 336 & 228 & 151 \\
\hline
\end{tabular}




\begin{tabular}{|c|c|c|c|c|c|c|c|c|c|c|c|c|c|c|}
\hline 6 & 138 & 762 & 1159 & 718 & 641 & 364 & 334 & 286 & 258 & 217 & 175 & 121 & 82 & 54 \\
\hline 6 & 139 & 3242 & 4843 & 2789 & 2623 & 1508 & 1395 & 1183 & 1096 & 909 & 739 & 520 & 349 & 236 \\
\hline 6 & 40 & 48 & 8533 & 3592 & 3241 & 2004 & 1781 & 1520 & 1386 & 1157 & 936 & 651 & 440 & 298 \\
\hline 6 & 41 & 514 & 8701 & 4095 & 3698 & 2207 & 1967 & 1692 & 1511 & 1272 & 1024 & 701 & 479 & 317 \\
\hline 6 & 42 & 1517 & 0 & 1054 & 792 & 545 & 488 & 417 & 379 & 317 & 256 & 178 & 120 & 81 \\
\hline 6 & 43 & 345 & 1214 & 313 & 299 & 163 & 151 & 130 & 115 & 98 & 78 & 53 & 37 & 24 \\
\hline 6 & 144 & 2444 & 400 & 2192 & 1799 & 1187 & 996 & 860 & 759 & 643 & 515 & 349 & 240 & 160 \\
\hline 6 & 45 & 173 & 3260 & 3030 & 2679 & 1654 & 1454 & 1251 & 1114 & 940 & 755 & 516 & 353 & 234 \\
\hline 6 & 146 & 739 & 4035 & 2260 & 2183 & 1235 & 1163 & 990 & 908 & 756 & 613 & 428 & 289 & 191 \\
\hline 6 & 147 & 58 & 6050 & 1294 & 1419 & 743 & 700 & 596 & 547 & 455 & 369 & 258 & 174 & 115 \\
\hline 6 & 148 & 32 & 4319 & 890 & 1010 & 533 & 501 & 431 & 384 & 324 & 260 & 178 & 122 & 79 \\
\hline 6 & 49 & 506 & 6264 & 3062 & 2776 & 1776 & 1534 & 1321 & 1176 & 992 & 798 & 545 & 373 & 248 \\
\hline 6 & 150 & 520 & 618 & 1455 & 1199 & 806 & 674 & 581 & 515 & 435 & 349 & 237 & 163 & 109 \\
\hline 6 & 51 & 51 & 40 & 823 & 814 & 477 & 433 & 370 & 336 & 281 & 227 & 158 & 107 & 71 \\
\hline 6 & 152 & 63 & 1530 & 1608 & 1334 & 855 & 747 & 640 & 577 & 484 & 391 & 270 & 183 & 124 \\
\hline 6 & 153 & 089 & 1835 & 1785 & 1669 & 996 & 896 & 764 & 698 & 582 & 471 & 328 & 222 & 150 \\
\hline 6 & 54 & 62 & 4334 & 2623 & 2498 & 1544 & 1358 & 1154 & 1064 & 884 & 718 & 504 & 339 & 233 \\
\hline 6 & 155 & 660 & 1582 & 529 & 537 & 310 & 283 & 240 & 223 & 184 & 150 & 106 & 71 & 48 \\
\hline 6 & 156 & 896 & 3034 & 1632 & 1510 & 929 & 806 & 690 & 623 & 522 & 421 & 291 & 198 & 133 \\
\hline 6 & 57 & & 4291 & & & 840 & 712 & 607 & 556 & 463 & & 262 & 177 & 122 \\
\hline 6 & 158 & 2907 & 5201 & 2487 & 2452 & 1402 & 1273 & 1091 & 983 & 825 & 666 & 459 & 312 & 206 \\
\hline 6 & 159 & 4232 & 12711 & 3476 & 3448 & 2021 & 1822 & 1558 & 1413 & 1182 & 955 & 662 & 449 & 299 \\
\hline 6 & 160 & 176 & 124 & 131 & 129 & 79 & 73 & 63 & 57 & 48 & 38 & 26 & 18 & 12 \\
\hline 6 & 161 & 1892 & 6607 & 1456 & 1618 & 863 & 821 & 699 & 640 & 533 & 432 & 301 & 203 & 134 \\
\hline 6 & 162 & & & & 231 & 1341 & 1223 & 1042 & 954 & 795 & & 450 & 304 & 204 \\
\hline 6 & 163 & 137 & 3980 & 1878 & 1614 & 1084 & 901 & 780 & 683 & 580 & 464 & 313 & 216 & 142 \\
\hline 6 & 164 & 700 & 2367 & 2879 & 2755 & 1818 & 1538 & 1312 & 1197 & 999 & 809 & 563 & 381 & 262 \\
\hline 6 & 165 & & & 3176 & 2967 & 1800 & 1574 & 1355 & 1206 & 1017 & & 559 & 382 & 253 \\
\hline 6 & 166 & 247 & 1030 & 202 & 203 & 107 & 104 & 90 & 80 & 67 & 54 & 37 & 25 & 16 \\
\hline 6 & 167 & 99 & 19 & 77 & 87 & 46 & 42 & 37 & 32 & 27 & 22 & 15 & 10 & 7 \\
\hline 6 & 168 & 123 & 57 & 94 & 99 & 54 & 50 & 44 & 38 & 33 & & 18 & 12 & 8 \\
\hline 6 & 169 & & 438 & 38 & 41 & 22 & 22 & 19 & 17 & 14 & 12 & 8 & 6 & 4 \\
\hline 6 & 170 & & 669 & 3146 & 2945 & 2017 & 1662 & 1424 & 1284 & 1077 & 869 & 600 & 408 & 280 \\
\hline 7 & 1 & 2219 & 1086 & 2704 & 1707 & 989 & 1032 & 749 & 654 & 557 & 445 & 299 & 207 & 138 \\
\hline 7 & 2 & & 313 & 599 & 403 & 266 & 238 & 188 & 174 & 144 & 117 & 83 & 55 & 40 \\
\hline 7 & 3 & 2350 & 307 & 2986 & 2147 & 1081 & 1268 & 832 & 716 & 613 & 488 & 324 & 225 & 149 \\
\hline 7 & 4 & & 167 & & 1177 & 705 & 646 & 531 & 473 & 399 & 321 & 219 & 150 & 100 \\
\hline 7 & 5 & & 2828 & 5817 & 4295 & 2099 & 2209 & 1725 & 1539 & 1297 & 1043 & 713 & 487 & 322 \\
\hline 7 & 6 & 1777 & 810 & 2019 & 1613 & 786 & 948 & 622 & 546 & 463 & 371 & 250 & 172 & 117 \\
\hline 7 & 7 & & 7313 & 4347 & 3353 & 1711 & 1740 & 1342 & 1212 & 1016 & 820 & 567 & 385 & 261 \\
\hline 7 & 8 & 574 & 1628 & 3408 & 2491 & 1297 & 1702 & 982 & 842 & 722 & 574 & 380 & 265 & 180 \\
\hline 7 & 9 & 4162 & 1679 & 5051 & 3406 & 2079 & 1952 & 1503 & 1305 & 1113 & & 595 & 412 & 273 \\
\hline 7 & 10 & & 329 & 1184 & 668 & 501 & 459 & 339 & 287 & 248 & 196 & 129 & 90 & 58 \\
\hline 7 & 11 & 2566 & 2980 & 2573 & 2126 & 1291 & 1217 & 916 & 817 & 689 & 554 & 378 & 259 & 178 \\
\hline 7 & 12 & 2786 & 1477 & 2565 & 2249 & 1466 & 1260 & 1024 & 911 & 768 & 618 & 421 & 288 & 190 \\
\hline
\end{tabular}




\begin{tabular}{|c|c|c|c|c|c|c|c|c|c|c|c|c|c|c|}
\hline 7 & 13 & 4050 & 5928 & 3982 & 3603 & 1935 & 1906 & 1473 & 1330 & 1115 & 900 & 621 & 422 & 286 \\
\hline 7 & & 5299 & 1494 & 5644 & 3574 & 2651 & 2130 & 1743 & 1521 & 1294 & 1035 & 695 & 480 & 325 \\
\hline r & & 316 & 1244 & 3256 & 2065 & 1496 & 1273 & 989 & 849 & 728 & 579 & 384 & 267 & 177 \\
\hline 7 & 10 & 147 & 1051 & 3948 & 2536 & 1858 & 1781 & 1214 & 1044 & 894 & 712 & 472 & 329 & 224 \\
\hline 7 & & 99 & 1131 & 5065 & 3369 & 2647 & 2165 & 1739 & 1501 & 1284 & 1023 & 681 & 473 & 315 \\
\hline 7 & & 544 & 1394 & 2636 & 1685 & 1381 & 1088 & 873 & 744 & 640 & 508 & 334 & 234 & 153 \\
\hline 7 & 19 & 446 & 1080 & 5728 & 3901 & 2864 & 2432 & 1905 & 1651 & 1409 & 1125 & 751 & 521 & 349 \\
\hline 7 & & 691 & 624 & 2817 & 1944 & 1430 & 1308 & 955 & 819 & 702 & 559 & 370 & 258 & 171 \\
\hline 7 & 21 & 359 & 1436 & 4944 & 3308 & 2603 & 2351 & 1667 & 1432 & 1227 & 977 & 647 & 451 & 305 \\
\hline 7 & 22 & 364 & 685 & 2486 & 1659 & 1209 & 1060 & 806 & 684 & 589 & 468 & 307 & 215 & 139 \\
\hline 7 & 23 & 774 & 2853 & 6465 & 4697 & 2553 & 2515 & 1974 & 1747 & 1477 & 1186 & 806 & 553 & 367 \\
\hline 7 & 24 & 664 & 1617 & 5537 & 3767 & 2262 & 2004 & 1642 & 1455 & 1230 & 987 & 671 & 460 & 309 \\
\hline 7 & 25 & 171 & 3527 & 6327 & 5277 & 2840 & 3344 & 2066 & 1848 & 1555 & 1252 & 858 & 586 & 423 \\
\hline 7 & 2 & 85 & 890 & 2556 & 2264 & 1115 & 1205 & 866 & 801 & 665 & 540 & 380 & 255 & 180 \\
\hline 7 & 2 & 645 & 788 & 3954 & 3680 & 2084 & 2152 & 1524 & 1410 & 1169 & 950 & 669 & 449 & 322 \\
\hline 7 & 2 & 75 & & 2924 & 2417 & 1291 & & 984 & 912 & 756 & 615 & 433 & 291 & 210 \\
\hline 7 & 29 & 326 & 1261 & 1202 & 1088 & 583 & 617 & 468 & 444 & 364 & 298 & 214 & 142 & 98 \\
\hline 7 & 30 & 84 & 565 & 5935 & 4508 & 2246 & 2469 & 1781 & 1568 & 1329 & 1065 & 721 & 496 & 331 \\
\hline 7 & 3 & & 1208 & 3836 & 3048 & 1511 & 1723 & 1187 & & 896 & 722 & 497 & 338 & 233 \\
\hline 7 & 32 & 3399 & 6662 & 2807 & 3040 & 1563 & 1574 & 1209 & 1116 & 926 & 752 & 529 & 355 & 243 \\
\hline 7 & & & & & 2155 & & & 825 & 758 & 630 & 511 & 358 & 241 & 165 \\
\hline 7 & 34 & 338 & 3589 & 2065 & 1763 & 1166 & 1166 & 794 & 709 & 597 & 480 & 328 & 224 & 160 \\
\hline 7 & 3. & 583 & 4874 & 2443 & 2019 & 1264 & 1520 & 845 & 757 & 637 & 513 & 352 & 240 & 184 \\
\hline 7 & & & & 7103 & 5928 & 3699 & 3377 & 2586 & 2297 & 1939 & 1558 & 1061 & 727 & 499 \\
\hline 7 & 37 & 981 & 1065 & 988 & 852 & 539 & 461 & 370 & 326 & 277 & 222 & 150 & 103 & 70 \\
\hline 7 & 3 & & & 223 & 249 & 126 & 143 & 97 & 88 & 74 & 60 & 42 & 28 & 20 \\
\hline 7 & 39 & 3501 & 3137 & 3046 & 2853 & 1711 & 1676 & 1206 & 1055 & 897 & & 483 & 333 & 226 \\
\hline 7 & 40 & 566 & 2182 & 553 & 469 & 271 & 279 & 207 & 187 & 157 & 127 & 88 & 60 & 41 \\
\hline 7 & & 3097 & 6046 & 2686 & 2665 & 1564 & 1465 & 1103 & 1000 & 837 & & 468 & 317 & 222 \\
\hline 7 & 42 & 403 & 526 & 319 & 398 & 182 & 191 & 144 & 131 & 110 & 89 & 61 & 42 & 28 \\
\hline 7 & 43 & & & 954 & 895 & & 453 & 366 & 340 & 282 & 229 & 162 & 108 & 74 \\
\hline 7 & 44 & & & 1308 & 1419 & 792 & 723 & & 540 & 449 & & 255 & 172 & 118 \\
\hline 7 & 45 & 763 & 399 & 703 & 670 & 362 & 379 & 270 & 242 & 204 & 164 & 113 & 77 & 53 \\
\hline 7 & 46 & & 2303 & 510 & 531 & 276 & 270 & 217 & 206 & 169 & 138 & 99 & 66 & 45 \\
\hline 7 & 47 & 1729 & 4330 & 1299 & 1427 & 797 & 844 & 574 & 531 & 441 & 358 & 252 & 169 & 122 \\
\hline 7 & 48 & & & & 270 & 157 & 142 & 113 & 101 & 85 & 69 & 47 & 32 & 22 \\
\hline 7 & 49 & & 6607 & 1482 & 1838 & 875 & 1039 & 680 & 603 & 510 & 409 & 279 & 191 & 130 \\
\hline 7 & 50 & 1475 & 6770 & 1482 & 1383 & 851 & 950 & 569 & 511 & 429 & 346 & 237 & 162 & 121 \\
\hline 7 & 51 & 754 & 1365 & 642 & 708 & 342 & 479 & 260 & 233 & 196 & 158 & 108 & 74 & 54 \\
\hline 7 & 52 & 2240 & 507 & 1805 & 1854 & 964 & 1158 & 713 & 662 & 548 & 446 & 315 & 211 & 156 \\
\hline 7 & 53 & & 1888 & 1818 & 1713 & 1027 & 1465 & 676 & 587 & 501 & 400 & 267 & 185 & 142 \\
\hline 7 & 54 & 1015 & 1745 & 850 & 833 & 463 & 429 & 349 & 332 & 272 & 223 & 160 & 106 & 74 \\
\hline 7 & 55 & 6942 & 10994 & 5619 & 5568 & 3718 & 3095 & 2482 & 2236 & 1876 & 1513 & 1043 & 709 & 496 \\
\hline 7 & 56 & 1772 & & 1731 & 1625 & 847 & 798 & 654 & 616 & 507 & 414 & 295 & 197 & 135 \\
\hline 7 & 57 & 780 & 2837 & 798 & 757 & 382 & 357 & 299 & 273 & 228 & 184 & 129 & 87 & \\
\hline
\end{tabular}




\begin{tabular}{|c|c|c|c|c|c|c|c|c|c|c|c|c|c|c|}
\hline 7 & 58 & 661 & 868 & 602 & 609 & 316 & 314 & 236 & 218 & 181 & 147 & 103 & 69 & 49 \\
\hline 7 & 59 & 1830 & 1651 & 1417 & 1686 & 796 & 785 & 632 & 575 & 480 & 388 & 270 & 183 & 121 \\
\hline 7 & 60 & 508 & 413 & 470 & 460 & 224 & 213 & 176 & 164 & 136 & 110 & 78 & 52 & 36 \\
\hline 7 & 61 & 3155 & 0 & 1792 & 2124 & 1021 & 976 & 800 & 734 & 611 & 495 & 346 & 234 & 156 \\
\hline 7 & 62 & 2692 & 1811 & 2571 & 2335 & 1269 & 1195 & 956 & 911 & 746 & 611 & 440 & 291 & 206 \\
\hline 7 & 63 & 2411 & 3618 & 2024 & 1899 & 1127 & 1084 & 800 & 731 & 610 & 494 & 344 & 233 & 165 \\
\hline 7 & 64 & 1848 & 4983 & 1633 & 1715 & 874 & 913 & 668 & 623 & 515 & 419 & 297 & 199 & 139 \\
\hline 7 & 65 & 584 & 962 & 543 & 509 & 288 & 276 & 214 & 197 & 164 & 133 & 93 & 63 & 43 \\
\hline 7 & 66 & 1347 & 1123 & 1324 & 1205 & 614 & 591 & 480 & 439 & 366 & 297 & 207 & 140 & 94 \\
\hline 7 & 67 & 1396 & 50 & 1076 & 1061 & 593 & 532 & 442 & 408 & 339 & 275 & 194 & 130 & 89 \\
\hline 7 & 68 & 1909 & 514 & 1833 & 1582 & 905 & 888 & 653 & 610 & 504 & 411 & 291 & 195 & 141 \\
\hline 7 & 69 & 1620 & 1034 & 1582 & 1366 & 741 & 693 & 555 & 509 & 424 & 344 & 240 & 162 & 112 \\
\hline 7 & 70 & 1940 & 1273 & 1796 & 1683 & 861 & 829 & 679 & 638 & 526 & 429 & 306 & 204 & 139 \\
\hline 7 & 71 & 1449 & 14404 & 1096 & 1477 & 655 & 633 & 539 & 461 & 396 & 314 & 207 & 145 & 90 \\
\hline 7 & 72 & 3639 & 7924 & 3275 & 3037 & 1897 & 1864 & 1298 & 1170 & 982 & 792 & 546 & 371 & 266 \\
\hline 7 & 73 & 2577 & 3387 & 2382 & 2376 & 1241 & 1249 & 916 & 844 & 701 & 569 & 399 & 269 & 190 \\
\hline 7 & 74 & 1832 & 1721 & 1625 & 1609 & 909 & 886 & 647 & 590 & 492 & 398 & 277 & 187 & 132 \\
\hline 7 & 75 & 1267 & 6397 & 1227 & 1233 & 651 & 606 & 474 & 427 & 358 & 289 & 199 & 135 & 93 \\
\hline 7 & 76 & 1544 & 1667 & 1374 & 1406 & 733 & 707 & 562 & 528 & 435 & 355 & 253 & 169 & 116 \\
\hline 7 & 77 & 1937 & 15608 & 1949 & 1746 & 1010 & 930 & 766 & 708 & 588 & 477 & 335 & 225 & 154 \\
\hline 7 & 78 & 2426 & 3461 & 2254 & 1984 & 1153 & 1044 & 853 & 801 & 660 & 539 & 383 & 256 & 179 \\
\hline 7 & 79 & 1451 & 4601 & 1284 & 1288 & 674 & 753 & 509 & 467 & 389 & 315 & 221 & 149 & 106 \\
\hline 7 & 80 & 2242 & 4897 & 2086 & 2035 & 1050 & 983 & 815 & 756 & 627 & 510 & 359 & 241 & 163 \\
\hline 7 & 81 & 2652 & 14547 & 2203 & 2322 & 1225 & 1193 & 977 & 925 & 760 & 621 & 445 & 296 & 200 \\
\hline 7 & 82 & 818 & 2216 & 667 & 721 & 381 & 377 & 298 & 286 & 233 & 192 & 138 & 91 & 63 \\
\hline 7 & 83 & 3974 & 4590 & 3489 & 3360 & 1818 & 1829 & 1378 & 1287 & 1063 & 866 & 614 & 410 & 288 \\
\hline 7 & 84 & 2219 & 4950 & 1773 & 1914 & 952 & 957 & 749 & 679 & 568 & 459 & 318 & 216 & 144 \\
\hline 7 & 85 & 1418 & 7174 & 1233 & 1326 & 649 & 667 & 528 & 484 & 403 & 327 & 228 & 154 & 103 \\
\hline 7 & 86 & 3331 & 1433 & 2620 & 2760 & 1461 & 1332 & 1118 & 1019 & 851 & 688 & 479 & 324 & 217 \\
\hline 7 & 87 & 2719 & 11144 & 2466 & 2538 & 1286 & 1185 & 990 & 890 & 747 & 603 & 415 & 282 & 187 \\
\hline 7 & 88 & 2524 & 11062 & 2296 & 2146 & 1151 & 1217 & 861 & 801 & 663 & 540 & 381 & 255 & 183 \\
\hline 7 & 89 & 1180 & 1553 & 1150 & 1050 & 554 & 677 & 410 & 370 & 310 & 250 & 173 & 117 & 85 \\
\hline 7 & 90 & 2175 & 7627 & 1743 & 1962 & 999 & 1074 & 776 & 722 & 597 & 486 & 343 & 230 & 160 \\
\hline 7 & 91 & 1462 & 3301 & 1246 & 1366 & 687 & 727 & 535 & 500 & 413 & 337 & 239 & 160 & 111 \\
\hline 7 & 92 & 3062 & 1189 & 2865 & 2443 & 1370 & 1394 & 1015 & 946 & 782 & 637 & 451 & 302 & 216 \\
\hline 7 & 93 & 2392 & 5206 & 2019 & 2076 & 1094 & 1250 & 833 & 768 & 638 & 518 & 364 & 245 & 174 \\
\hline 7 & 94 & 463 & 3564 & 506 & 416 & 258 & 273 & 188 & 167 & 141 & 113 & 77 & 53 & 37 \\
\hline 7 & 95 & 509 & 34 & 441 & 417 & 216 & 301 & 157 & 146 & 121 & 99 & 70 & 47 & 37 \\
\hline 7 & 96 & 5125 & 3565 & 4893 & 4001 & 2368 & 2272 & 1708 & 1525 & 1285 & 1034 & 708 & 483 & 332 \\
\hline 7 & 97 & 2192 & 3133 & 2362 & 1832 & 1107 & 1258 & 766 & 670 & 570 & 456 & 307 & 212 & 150 \\
\hline 7 & 98 & 2060 & 1358 & 2401 & 1673 & 1104 & 1082 & 734 & 638 & 544 & 434 & 291 & 201 & 140 \\
\hline 7 & 99 & 3592 & 3379 & 4064 & 2995 & 1835 & 1649 & 1294 & 1165 & 978 & 789 & 543 & 370 & 257 \\
\hline 7 & 100 & 1768 & 2206 & 1436 & 1262 & 977 & 777 & 611 & 550 & 461 & 372 & 256 & 174 & 126 \\
\hline 7 & 101 & 1484 & 6758 & 1213 & 1286 & 667 & 699 & 526 & 503 & 411 & 337 & 243 & 161 & 112 \\
\hline 7 & 102 & 4004 & 13976 & 3294 & 3539 & 1845 & 2198 & 1397 & 1279 & 1066 & 863 & 603 & 407 & 291 \\
\hline
\end{tabular}




\begin{tabular}{|c|c|c|c|c|c|c|c|c|c|c|c|c|c|c|}
\hline 7 & 103 & 556 & 1042 & 506 & 484 & 255 & 334 & 200 & 182 & 152 & 123 & 86 & 58 & 42 \\
\hline 7 & & 325 & 2078 & 279 & 287 & 146 & 161 & 117 & 108 & 90 & 73 & 51 & 34 & 24 \\
\hline & & 2400 & 6211 & 2229 & 2141 & 1101 & 1299 & 825 & 746 & 625 & 504 & 348 & 237 & 169 \\
\hline 7 & 66 & 264 & 2531 & 1879 & 1837 & 969 & 1083 & 739 & 698 & 574 & 469 & 335 & 223 & 161 \\
\hline 7 & & 1496 & 4101 & 1371 & 1424 & 681 & 809 & 546 & 501 & 417 & 338 & 236 & 159 & 111 \\
\hline 7 & 108 & 754 & 451 & 671 & 650 & 335 & 350 & 272 & 248 & 207 & 167 & 117 & 79 & 53 \\
\hline 7 & 109 & 348 & 503 & 327 & 303 & 157 & 184 & 122 & 110 & 92 & 74 & 51 & 35 & 24 \\
\hline 7 & & 2361 & 1580 & 2403 & 1989 & 1096 & 1328 & 819 & 735 & 618 & 498 & 342 & 233 & 167 \\
\hline 7 & 11 & 048 & 1731 & 1177 & 932 & 494 & 532 & 383 & 347 & 290 & 235 & 162 & 110 & 76 \\
\hline 7 & 12 & 329 & 127 & 317 & 277 & 138 & 154 & 110 & 99 & 83 & 67 & 46 & 31 & 21 \\
\hline 7 & 113 & 1707 & 1924 & 1403 & 1438 & 744 & 780 & 583 & 534 & 445 & 360 & 251 & 170 & 116 \\
\hline 7 & 114 & 570 & 835 & 528 & 479 & 247 & 258 & 205 & 192 & 158 & 129 & 91 & 61 & 41 \\
\hline 7 & 115 & 2077 & 251 & 1714 & 1487 & 864 & 949 & 634 & 608 & 497 & 408 & 295 & 195 & 146 \\
\hline 7 & 116 & 176 & 603 & 2851 & 2396 & 1291 & 1616 & 1012 & 900 & 760 & 611 & 417 & 285 & 198 \\
\hline 7 & 117 & 2574 & 2515 & 3270 & 1993 & 1159 & 1509 & 987 & 831 & 719 & 569 & 371 & 261 & 165 \\
\hline 7 & & 93 & 1 & 69 & 84 & & 46 & 31 & 28 & & & 13 & 0 & \\
\hline 7 & 119 & 3629 & 6950 & 3962 & 3038 & 1713 & 1548 & 1286 & 1169 & 977 & 790 & 548 & 371 & 252 \\
\hline 7 & 120 & 3295 & 4256 & 3243 & 2805 & 1524 & 1543 & 1161 & 1062 & 885 & 71 & 500 & 338 & 233 \\
\hline 7 & & & & 607 & 603 & 335 & & 247 & 236 & & & 114 & 75 & 54 \\
\hline 7 & 122 & 1212 & 2431 & 1001 & 1085 & 576 & 545 & 443 & 415 & 342 & 279 & 198 & 132 & 90 \\
\hline 7 & & & & 598 & & & & & & & & 110 & 75 & 54 \\
\hline 7 & 124 & 419 & 514 & 343 & 384 & 196 & 187 & 160 & 145 & 121 & 98 & 68 & 46 & 30 \\
\hline 7 & 125 & 133 & 167 & 131 & 128 & 62 & 61 & 51 & 48 & 40 & 32 & 23 & 15 & 10 \\
\hline 7 & & & 1358 & 665 & 732 & 412 & & 306 & 283 & & & 134 & 90 & 61 \\
\hline 7 & 127 & 551 & 370 & 516 & 468 & 237 & 235 & 194 & 191 & 155 & 12 & 94 & 61 & 42 \\
\hline 7 & & & & 1488 & & & & 603 & & & & 273 & 182 & 121 \\
\hline 7 & & 1327 & 6210 & 1231 & 1147 & 610 & & 496 & 471 & 386 & & 227 & 151 & 101 \\
\hline 7 & 130 & 558 & 6309 & 480 & 508 & 273 & 249 & 208 & 202 & 164 & 135 & 99 & 65 & 45 \\
\hline 7 & & & & 512 & 527 & 255 & & 205 & & & & 88 & 59 & 39 \\
\hline 7 & 132 & 271 & 1546 & 209 & 217 & 112 & 105 & 90 & 83 & 69 & & 39 & 26 & 17 \\
\hline 7 & & & 55 & 392 & & & & 131 & & & & 88 & 53 & 40 \\
\hline 7 & & & & 2510 & 2370 & 1316 & 1272 & 1062 & 1026 & & & 499 & 329 & 223 \\
\hline 7 & 135 & 1895 & 1559 & 1653 & 1687 & 875 & 789 & 678 & 606 & 510 & 411 & 282 & 192 & 126 \\
\hline 7 & & & 3713 & 2895 & 2656 & 1654 & 1350 & 1159 & 1040 & 874 & 70 & 484 & 330 & 224 \\
\hline 7 & & 2229 & 779 & 2000 & 1921 & 1021 & 922 & 787 & 716 & 598 & 484 & 336 & 228 & 151 \\
\hline 7 & & & & 791 & & & & 286 & 258 & 217 & & 121 & 82 & 54 \\
\hline 7 & & 3226 & & 3040 & 2917 & 1535 & 1395 & 1183 & 1096 & 909 & 739 & 520 & 349 & 236 \\
\hline 7 & & 4245 & 10922 & 3911 & 3567 & 2055 & 1781 & 1520 & 1386 & 1157 & 936 & 651 & 440 & 298 \\
\hline 7 & 141 & 4485 & 10963 & 4472 & 4080 & 2263 & 1967 & 1692 & 1511 & 1272 & 1024 & 701 & 479 & 317 \\
\hline 7 & 142 & 1598 & 0 & 1163 & 852 & 559 & 488 & 417 & 379 & 317 & 256 & 178 & 120 & 81 \\
\hline 7 & & & 1724 & 341 & 333 & 166 & 151 & 130 & 115 & 98 & & 53 & 37 & 24 \\
\hline 7 & 144 & 2458 & 312 & 2415 & 1966 & 1232 & 996 & 860 & 759 & 643 & 515 & 349 & 240 & 160 \\
\hline 7 & & & & 3309 & 2943 & 1700 & 1454 & 1251 & 1114 & 940 & & 516 & 353 & 234 \\
\hline 7 & 146 & & 4842 & 2445 & 2419 & 1251 & 1163 & 990 & 908 & 756 & 613 & 428 & 289 & 191 \\
\hline 7 & 147 & 1540 & 8711 & 1385 & 1597 & 751 & 700 & 596 & 547 & 455 & 369 & 258 & 174 & 115 \\
\hline
\end{tabular}




\begin{tabular}{|c|c|c|c|c|c|c|c|c|c|c|c|c|c|c|}
\hline 7 & 148 & 1125 & 6219 & 941 & 1128 & 539 & 501 & 431 & 384 & 324 & 260 & 178 & 122 & 79 \\
\hline 7 & 149 & 3477 & 7767 & 3315 & 3036 & 1831 & 1534 & 1321 & 1176 & 992 & 798 & 545 & 373 & 248 \\
\hline 7 & 150 & 1622 & 569 & 1599 & 1306 & 837 & 674 & 581 & 515 & 435 & 349 & 237 & 163 & 109 \\
\hline 7 & 151 & 1301 & 23 & 886 & 901 & 486 & 433 & 370 & 336 & 281 & 227 & 158 & 107 & 71 \\
\hline 7 & 152 & 1757 & 1652 & 1770 & 1461 & 882 & 747 & 640 & 577 & 484 & 391 & 270 & 183 & 124 \\
\hline 7 & 153 & 2079 & 1982 & 1939 & 1847 & 1019 & 896 & 764 & 698 & 582 & 471 & 328 & 222 & 150 \\
\hline 7 & 154 & 3139 & 5114 & 2837 & 2761 & 1586 & 1358 & 1154 & 1064 & 884 & 718 & 504 & 339 & 233 \\
\hline 7 & 155 & 656 & 2088 & 570 & 598 & 316 & 283 & 240 & 223 & 184 & 150 & 106 & 71 & 48 \\
\hline 7 & 156 & 1888 & 3701 & 1776 & 1669 & 957 & 806 & 690 & 623 & 522 & 421 & 291 & 198 & 133 \\
\hline 7 & 157 & 1606 & 5749 & 1392 & 1367 & 868 & 712 & 607 & 556 & 463 & 375 & 262 & 177 & 122 \\
\hline 7 & 158 & 2887 & 6449 & 2686 & 2722 & 1431 & 1273 & 1091 & 983 & 825 & 666 & 459 & 312 & 206 \\
\hline 7 & 159 & 4213 & 17542 & 3739 & 3818 & 2064 & 1822 & 1558 & 1413 & 1182 & 955 & 662 & 449 & 299 \\
\hline 7 & 160 & 177 & 129 & 138 & 141 & 80 & 73 & 63 & 57 & 48 & 38 & 26 & 18 & 12 \\
\hline 7 & 161 & 1885 & 9382 & 1547 & 1808 & 870 & 821 & 699 & 640 & 533 & 432 & 301 & 203 & 134 \\
\hline 7 & 162 & 2988 & 3744 & 2649 & 2567 & 1369 & 1223 & 1042 & 954 & 795 & 644 & 450 & 304 & 204 \\
\hline 7 & 163 & 2136 & 5015 & 2045 & 1756 & 1127 & 901 & 780 & 683 & 580 & 464 & 313 & 216 & 142 \\
\hline 7 & 164 & 3694 & 2414 & 3090 & 3021 & 1880 & 1538 & 1312 & 1197 & 999 & 809 & 563 & 381 & 262 \\
\hline 7 & 165 & 3763 & 3477 & 3445 & 3273 & 1851 & 1574 & 1355 & 1206 & 1017 & 818 & 559 & 382 & 253 \\
\hline 7 & 166 & 248 & 1524 & 218 & 225 & 108 & 104 & 90 & 80 & 67 & 54 & 37 & 25 & 16 \\
\hline 7 & 167 & 99 & 15 & 81 & 98 & 47 & 42 & 37 & 32 & 27 & 22 & 15 & 10 & 7 \\
\hline 7 & 168 & 124 & 55 & 101 & 109 & 54 & 50 & 44 & 38 & 33 & 26 & 18 & 12 & 8 \\
\hline 7 & 169 & 49 & 745 & 40 & 45 & 22 & 22 & 19 & 17 & 14 & 12 & 8 & 6 & 4 \\
\hline 7 & 170 & 4328 & 522 & 3377 & 3214 & 2096 & 1662 & 1424 & 1284 & 1077 & 869 & 600 & 408 & 280 \\
\hline 8 & 1 & 2245 & 1064 & 3099 & 1862 & 1014 & 1060 & 749 & 654 & 557 & 445 & 299 & 207 & 138 \\
\hline 8 & 2 & 495 & 313 & 676 & 439 & 276 & 241 & 188 & 174 & 144 & 117 & 83 & 55 & 40 \\
\hline 8 & 3 & 2361 & 239 & 3412 & 2385 & 1104 & 1324 & 832 & 716 & 613 & 488 & 324 & 225 & 149 \\
\hline 8 & 4 & 1657 & 127 & 2072 & 1283 & 724 & 650 & 531 & 473 & 399 & 321 & 219 & 150 & 100 \\
\hline 8 & 5 & 4859 & 2828 & 6605 & 4769 & 2123 & 2241 & 1725 & 1539 & 1297 & 1043 & 713 & 487 & 322 \\
\hline 8 & 6 & 1784 & 777 & 2271 & 1795 & 796 & 987 & 622 & 546 & 463 & 371 & 250 & 172 & 117 \\
\hline 8 & 7 & 3719 & 8922 & 4907 & 3730 & 1742 & 1766 & 1342 & 1212 & 1016 & 820 & 567 & 385 & 261 \\
\hline 8 & 8 & 2665 & 1628 & 3866 & 2754 & 1325 & 1811 & 982 & 842 & 722 & 574 & 380 & 265 & 180 \\
\hline 8 & 9 & 4168 & 1578 & 5723 & 3710 & 2148 & 1988 & 1503 & 1305 & 1113 & 889 & 595 & 412 & 273 \\
\hline 8 & 10 & 961 & 302 & 1348 & 709 & 524 & 471 & 339 & 287 & 248 & 196 & 129 & 90 & 58 \\
\hline 8 & 11 & 2561 & 3337 & 2832 & 2327 & 1333 & 1241 & 916 & 817 & 689 & 554 & 378 & 259 & 178 \\
\hline 8 & 12 & 2773 & 1447 & 2774 & 2439 & 1517 & 1271 & 1024 & 911 & 768 & 618 & 421 & 288 & 196 \\
\hline 8 & 13 & 4036 & 6877 & 4362 & 3984 & 1973 & 1935 & 1473 & 1330 & 1115 & 900 & 621 & 422 & 286 \\
\hline 8 & 14 & 5377 & 1345 & 6361 & 3831 & 2783 & 2149 & 1743 & 1521 & 1294 & 1035 & 695 & 480 & 325 \\
\hline 8 & 15 & 2834 & 1195 & 3674 & 2216 & 1569 & 1296 & 989 & 849 & 728 & 579 & 384 & 267 & 177 \\
\hline 8 & 16 & 3456 & 946 & 4441 & 2719 & 1949 & 1849 & 1214 & 1044 & 894 & 712 & 472 & 329 & 224 \\
\hline 8 & 17 & 5153 & 973 & 5604 & 3570 & 2777 & 2191 & 1739 & 1501 & 1284 & 1023 & 681 & 473 & 315 \\
\hline 8 & 18 & 2574 & 1394 & 2932 & 1782 & 1459 & 1102 & 873 & 744 & 640 & 508 & 334 & 234 & 153 \\
\hline 8 & 19 & 5476 & 908 & 6371 & 4174 & 2997 & 2471 & 1905 & 1651 & 1409 & 1125 & 751 & 521 & 349 \\
\hline 8 & 20 & 2702 & 537 & 3119 & 2075 & 1495 & 1344 & 955 & 819 & 702 & 559 & 370 & 258 & 171 \\
\hline 8 & 21 & 4897 & 1293 & 5478 & 3514 & 2738 & 2427 & 1667 & 1432 & 1227 & 977 & 647 & 451 & 305 \\
\hline 8 & 22 & 2397 & 616 & 2773 & 1772 & 1266 & 1083 & 806 & 684 & 589 & 468 & 307 & 215 & 139 \\
\hline
\end{tabular}




\begin{tabular}{|c|c|c|c|c|c|c|c|c|c|c|c|c|c|c|}
\hline 8 & 23 & 5831 & 2796 & 7303 & 5169 & 2608 & 2550 & 1974 & 1747 & 1477 & 1186 & 806 & 553 & 367 \\
\hline$\varepsilon$ & 24 & 4681 & 1487 & 6289 & 4124 & 2337 & 2019 & 1642 & 1455 & 1230 & 987 & 671 & 460 & 309 \\
\hline 8 & 5 & 92 & 3527 & 7042 & 5859 & 2907 & 3503 & 2066 & 1848 & 1555 & 1252 & 858 & 586 & 423 \\
\hline 8 & 26 & 2599 & 819 & 2842 & 2539 & 1132 & 1233 & 866 & 801 & 665 & 540 & 380 & 255 & 180 \\
\hline 8 & 27 & 75 & 646 & 4301 & 4065 & 2128 & 2206 & 1524 & 1410 & 1169 & 950 & 669 & 449 & 322 \\
\hline 8 & 28 & 105 & 6076 & 3248 & 2679 & 1311 & 1518 & 984 & 912 & 756 & 615 & 433 & 291 & 210 \\
\hline 8 & 29 & 1323 & 1362 & 1308 & 1199 & 586 & 626 & 468 & 444 & 364 & 298 & 214 & 142 & 98 \\
\hline 8 & 30 & 994 & 429 & 6714 & 5006 & 2281 & 2536 & 1781 & 1568 & 1329 & 1065 & 721 & 496 & 331 \\
\hline 8 & 31 & 3696 & 1112 & 4318 & 3397 & 1533 & 1777 & 1187 & 1067 & 896 & 722 & 497 & 338 & 233 \\
\hline 8 & 32 & 3395 & 8128 & 3002 & 3380 & 1583 & 1596 & 1209 & 1116 & 926 & 752 & 529 & 355 & 243 \\
\hline 8 & 33 & 501 & 1376 & 2208 & 2412 & 1095 & 1091 & 825 & 758 & 630 & 511 & 358 & 241 & 165 \\
\hline 8 & 34 & 2344 & 4235 & 2240 & 1912 & 1207 & 1205 & 794 & 709 & 597 & 480 & 328 & 224 & 160 \\
\hline 8 & 35 & 583 & 5946 & 2686 & 2211 & 1305 & 1614 & 845 & 757 & 637 & 513 & 352 & 240 & 184 \\
\hline 8 & 36 & 35 & 2750 & 7791 & 6475 & 3829 & 3436 & 2586 & 2297 & 1939 & 1558 & 1061 & 727 & 499 \\
\hline 8 & 37 & 972 & 1172 & 1079 & 931 & 560 & 466 & 370 & 326 & 277 & 222 & 150 & 103 & 70 \\
\hline 8 & 38 & 271 & 124 & 237 & 277 & 128 & 147 & 97 & 88 & 74 & 60 & 42 & 28 & 20 \\
\hline 8 & 39 & 524 & 3388 & 3289 & 3123 & 1766 & 1723 & 1206 & 1055 & 897 & 718 & 483 & 333 & 226 \\
\hline 8 & 40 & 563 & 2967 & 604 & 513 & 276 & 285 & 207 & 187 & 157 & 127 & 88 & 60 & 41 \\
\hline 8 & 41 & 3086 & 7376 & 2890 & 2938 & 1610 & 1492 & 1103 & 1000 & 837 & 676 & 468 & 317 & 222 \\
\hline 8 & 42 & 403 & 600 & 338 & 448 & 184 & 194 & 144 & 131 & 110 & 89 & 61 & 42 & 28 \\
\hline 8 & 43 & 1003 & 10387 & 1042 & 994 & 472 & 456 & 366 & 340 & 282 & 229 & 162 & 108 & 74 \\
\hline 8 & 44 & 1758 & 286 & 1390 & 1568 & 809 & 728 & 587 & 540 & 449 & 364 & 255 & 172 & 118 \\
\hline 8 & 45 & 763 & 391 & 763 & 741 & 370 & 389 & 270 & 242 & 204 & 164 & 113 & 77 & 53 \\
\hline 8 & 46 & 570 & 3133 & 548 & 591 & 279 & 271 & 217 & 206 & 169 & 138 & 99 & 66 & 45 \\
\hline 8 & 47 & 1735 & 5542 & 1380 & 1579 & 813 & 869 & 574 & 531 & 441 & 358 & 252 & 169 & 122 \\
\hline 8 & 48 & 297 & 2620 & 290 & 297 & 161 & 143 & 113 & 101 & 85 & 69 & 47 & 32 & 22 \\
\hline 8 & 49 & 05 & 8854 & 1560 & 2054 & 883 & 1081 & 680 & 603 & 510 & 409 & 279 & 191 & 130 \\
\hline 8 & 50 & 1440 & 9343 & 1604 & 1520 & 879 & 999 & 569 & 511 & 429 & 346 & 237 & 162 & 121 \\
\hline 8 & 51 & 752 & 1638 & 687 & 790 & 345 & 510 & 260 & 233 & 196 & 158 & 108 & 74 & 54 \\
\hline 8 & 52 & & 436 & 1948 & 2065 & 976 & 1208 & 713 & 662 & & & 315 & 211 & 156 \\
\hline 8 & 53 & 1911 & 2039 & 1967 & 1882 & 1060 & 1603 & 676 & 587 & 501 & 400 & 267 & 185 & 142 \\
\hline 8 & 54 & 016 & 2094 & 920 & 923 & 472 & 431 & 349 & 332 & 272 & 223 & 160 & 106 & 74 \\
\hline 8 & 55 & 14 & 12973 & 5977 & 6061 & 3867 & 3124 & 2482 & 2236 & 1876 & 1513 & 1043 & 709 & 496 \\
\hline 8 & 56 & 1757 & 11482 & 1897 & 1813 & 861 & 801 & 654 & 616 & 507 & 414 & 295 & 197 & 135 \\
\hline 8 & 57 & 771 & 3802 & 874 & 844 & 388 & 358 & 299 & 273 & 228 & 184 & 129 & 87 & 58 \\
\hline 8 & 58 & 658 & 990 & 654 & 681 & 322 & 320 & 236 & 218 & 181 & 147 & 103 & 69 & 49 \\
\hline 8 & 59 & 1844 & 1783 & 1506 & 1891 & 804 & 791 & 632 & 575 & 480 & 388 & 270 & 183 & 121 \\
\hline 8 & 60 & & 438 & 516 & 517 & 227 & 213 & 176 & 164 & 136 & 110 & 78 & 52 & 36 \\
\hline 8 & 61 & 343 & 0 & 1906 & 2383 & 1033 & 982 & 800 & 734 & 611 & 495 & 346 & 234 & 156 \\
\hline 8 & 62 & 676 & 1847 & 2827 & 2601 & 1296 & 1203 & 956 & 911 & 746 & 611 & 440 & 291 & 206 \\
\hline 8 & 63 & 2426 & 4269 & 2192 & 2090 & 1159 & 1106 & 800 & 731 & 610 & 494 & 344 & 233 & 165 \\
\hline 8 & 64 & 1834 & 6379 & 1760 & 1914 & 886 & 931 & 668 & 623 & 515 & 419 & 297 & 199 & 139 \\
\hline 8 & 65 & 579 & 1136 & 589 & 561 & 295 & 279 & 214 & 197 & 164 & 133 & 93 & 63 & 43 \\
\hline 8 & 66 & 1347 & 1190 & 1457 & 1342 & 624 & 595 & 480 & 439 & 366 & 297 & 207 & 140 & 94 \\
\hline 8 & 67 & 1422 & 32 & 1162 & 1173 & 606 & 533 & 442 & 408 & 339 & 275 & 194 & 130 & 89 \\
\hline
\end{tabular}




\begin{tabular}{|c|c|c|c|c|c|c|c|c|c|c|c|c|c|c|}
\hline 8 & 68 & 1904 & 453 & 2023 & 1753 & 929 & 905 & 653 & 610 & 504 & 411 & 291 & 195 & 141 \\
\hline 8 & 69 & 1627 & 1055 & 1751 & 1518 & 759 & 699 & 555 & 509 & 424 & 344 & 240 & 162 & 112 \\
\hline & 70 & 42 & 1299 & 1968 & 1876 & 872 & 832 & 679 & 638 & 526 & 429 & 306 & 204 & 139 \\
\hline 8 & 71 & 1453 & 23046 & 1142 & 1654 & 661 & 635 & 539 & 461 & 396 & 314 & 207 & 145 & 90 \\
\hline 8 & 72 & 614 & 9825 & 3539 & 3325 & 1961 & 1920 & 1298 & 1170 & 982 & 792 & 546 & 371 & 266 \\
\hline 8 & 73 & 2564 & 3861 & 2593 & 2656 & 1267 & 1275 & 916 & 844 & 701 & 569 & 399 & 269 & 190 \\
\hline 8 & 74 & 1825 & 1858 & 1757 & 1783 & 934 & 905 & 647 & 590 & 492 & 398 & 277 & 187 & 132 \\
\hline 8 & 75 & 1256 & 9083 & 1335 & 1377 & 668 & 614 & 474 & 427 & 358 & 289 & 199 & 135 & 93 \\
\hline 8 & 76 & 1533 & 1833 & 1485 & 1567 & 744 & 713 & 562 & 528 & 435 & 355 & 253 & 169 & 116 \\
\hline 8 & 77 & 1901 & 23725 & 2119 & 1914 & 1030 & 934 & 766 & 708 & 588 & 477 & 335 & 225 & 154 \\
\hline 8 & 78 & 2419 & 4015 & 2469 & 2189 & 1181 & 1049 & 853 & 801 & 660 & 539 & 383 & 256 & 179 \\
\hline 8 & 79 & 1446 & 6073 & 1387 & 1431 & 684 & 778 & 509 & 467 & 389 & 315 & 221 & 149 & 106 \\
\hline 8 & 80 & 2233 & 6072 & 2271 & 2267 & 1066 & 986 & 815 & 756 & 627 & 510 & 359 & 241 & 163 \\
\hline 8 & 81 & 2635 & 20947 & 2352 & 2567 & 1234 & 1197 & 977 & 925 & 760 & 621 & 445 & 296 & 200 \\
\hline 8 & 82 & 811 & 2836 & 712 & 800 & 385 & 380 & 298 & 286 & 233 & 192 & 138 & 91 & 63 \\
\hline 8 & 83 & 3973 & 5140 & 3786 & 3723 & 1848 & 1858 & 1378 & 1287 & 1063 & 866 & 614 & 410 & 288 \\
\hline 8 & 84 & 242 & 6237 & 1901 & 2130 & 963 & 969 & 749 & 679 & 568 & 459 & 318 & 216 & 144 \\
\hline 8 & 85 & 409 & 10187 & 1320 & 1474 & 653 & 674 & 528 & 484 & 403 & 327 & 228 & 154 & 103 \\
\hline 8 & 86 & 3369 & 1376 & 2809 & 3061 & 1487 & 1335 & 1118 & 1019 & 851 & 688 & 479 & 324 & 217 \\
\hline 8 & 87 & 717 & 15379 & 2666 & 2829 & 1310 & 1189 & 990 & 890 & 747 & & 415 & 282 & 187 \\
\hline 8 & 88 & 2524 & 15486 & 2509 & 2385 & 1171 & 1247 & 861 & 801 & 663 & 540 & 381 & 255 & 183 \\
\hline 8 & 89 & 1176 & 1771 & 1261 & 1165 & 563 & 710 & 410 & 370 & 310 & 250 & 173 & 117 & 85 \\
\hline 8 & 90 & 2166 & 10221 & 1852 & 2181 & 1008 & 1097 & 776 & 722 & 597 & 486 & 343 & 230 & 160 \\
\hline 8 & 91 & 1450 & 4093 & 1332 & 1523 & 693 & 741 & 535 & 500 & 413 & 337 & 239 & 160 & 111 \\
\hline 8 & 92 & & & 3164 & 2704 & 1400 & 1423 & 1015 & 946 & 782 & & 451 & 302 & 216 \\
\hline 8 & 93 & 2386 & 6455 & 2168 & 2299 & 1105 & 1292 & 833 & 768 & 638 & 518 & 364 & 245 & 174 \\
\hline 8 & 94 & 452 & 5346 & 553 & 451 & 264 & 283 & 188 & 167 & 141 & 113 & 77 & 53 & 37 \\
\hline 8 & 95 & & 24 & 482 & 465 & 218 & 320 & 157 & 146 & 121 & & 70 & 47 & 37 \\
\hline 8 & 96 & 5176 & 3707 & 5405 & 4390 & 2438 & 2316 & 1708 & 1525 & 1285 & 1034 & 708 & 483 & 332 \\
\hline 8 & 97 & 190 & & 2630 & 2008 & 1144 & 1324 & 766 & 670 & 570 & 456 & 307 & 212 & 150 \\
\hline 8 & 98 & 057 & 1385 & 2704 & 1822 & 1154 & 1122 & 734 & 638 & 544 & 434 & 291 & 201 & 140 \\
\hline 8 & 99 & 3575 & 3649 & 4564 & 3287 & 1901 & 1670 & 1294 & 1165 & 978 & 789 & 543 & 370 & 257 \\
\hline 8 & & & & 1537 & 1355 & 1027 & 787 & 611 & 550 & 461 & & 256 & 174 & 126 \\
\hline 8 & 101 & 1478 & 9461 & 1298 & 1428 & 671 & 709 & 526 & 503 & 411 & 337 & 243 & 161 & 112 \\
\hline 8 & 102 & 3991 & 18728 & 3514 & 3921 & 1863 & 2288 & 1397 & 1279 & 1066 & 863 & 603 & 407 & 291 \\
\hline 8 & 103 & 552 & & 545 & 532 & 256 & 350 & 200 & 182 & 152 & 123 & 86 & 58 & 42 \\
\hline 8 & 104 & 324 & 3075 & 300 & 317 & 147 & 165 & 117 & 108 & 90 & 73 & 51 & 34 & 24 \\
\hline 8 & 105 & 100 & 7950 & 2432 & 2383 & 1119 & 1354 & 825 & 746 & 625 & 504 & 348 & 237 & 169 \\
\hline 8 & 106 & 2276 & 2834 & 2037 & 2041 & 980 & 1114 & 739 & 698 & 574 & 469 & 335 & 223 & 161 \\
\hline 8 & 107 & 1485 & 5249 & 1481 & 1589 & 683 & 835 & 546 & 501 & 417 & 338 & 236 & 159 & 111 \\
\hline 8 & 108 & 754 & 451 & 725 & 717 & 337 & 354 & 272 & 248 & 207 & 167 & 117 & 79 & 53 \\
\hline 8 & 109 & 348 & & 356 & 334 & 159 & 191 & 122 & 110 & 92 & 74 & 51 & 35 & 24 \\
\hline 8 & 110 & 2357 & 1612 & 2655 & 2190 & 1116 & 1391 & 819 & 735 & 618 & 498 & 342 & 233 & 167 \\
\hline 8 & 111 & 1041 & 2042 & 1315 & 1030 & 503 & 545 & 383 & 347 & 290 & 235 & 162 & 110 & 76 \\
\hline 8 & 112 & 332 & 119 & 350 & 307 & 139 & 158 & 110 & 99 & 83 & 67 & 46 & 31 & 21 \\
\hline
\end{tabular}




\begin{tabular}{|c|c|c|c|c|c|c|c|c|c|c|c|c|c|c|}
\hline 8 & 113 & 1717 & 2155 & 1508 & 1592 & 752 & 794 & 583 & 534 & 445 & 360 & 251 & 170 & 116 \\
\hline 8 & & 569 & 969 & 575 & 528 & 248 & 260 & 205 & 192 & 158 & 129 & 91 & 61 & 41 \\
\hline 8 & & 2101 & 196 & 1878 & 1639 & 879 & 977 & 634 & 608 & 497 & 408 & 295 & 195 & 146 \\
\hline 8 & 16 & 227 & 506 & 3130 & 2624 & 1304 & 1691 & 1012 & 900 & 760 & 611 & 417 & 285 & 198 \\
\hline 8 & 17 & 568 & 2717 & 3687 & 2121 & 1164 & 1580 & 987 & 831 & 719 & 569 & 371 & 261 & 165 \\
\hline 8 & & 94 & 1 & 73 & 94 & 40 & 48 & 31 & 28 & 24 & 19 & 13 & 9 & 6 \\
\hline 8 & 19 & 3630 & 8479 & 4442 & 3352 & 1758 & 1555 & 1286 & 1169 & 977 & 790 & 548 & 371 & 252 \\
\hline$\varepsilon$ & & 3293 & 4851 & 3573 & 3101 & 1552 & 1570 & 1161 & 1062 & 885 & 717 & 500 & 338 & 233 \\
\hline 8 & & 757 & 105 & 657 & 669 & 341 & 334 & 247 & 236 & 193 & & 114 & 75 & 54 \\
\hline 8 & 122 & 1205 & 2966 & 1068 & 1204 & 584 & 548 & 443 & 415 & 342 & 279 & 198 & 132 & 90 \\
\hline 8 & 123 & 878 & 1310 & 630 & 850 & 356 & 506 & 268 & 238 & 201 & 161 & 110 & 75 & 54 \\
\hline 8 & 124 & 416 & 576 & 362 & 423 & 197 & 187 & 160 & 145 & 121 & 98 & 68 & 46 & 30 \\
\hline 8 & 125 & 132 & 187 & 143 & 143 & 62 & 61 & 51 & 48 & 40 & 32 & 23 & 15 & 10 \\
\hline 8 & 126 & & 1603 & 705 & 808 & 421 & 361 & 306 & 283 & 235 & 191 & 134 & 90 & 61 \\
\hline 8 & 127 & 550 & 377 & 569 & 523 & 239 & 235 & 194 & 191 & 155 & 128 & 94 & 61 & 42 \\
\hline 8 & & & 5630 & 1613 & 1519 & 742 & & 603 & 569 & & & 273 & 182 & 121 \\
\hline 8 & 129 & 1316 & 8694 & 1336 & 1265 & 615 & 590 & 496 & 471 & 386 & 316 & 227 & 151 & 101 \\
\hline 8 & 130 & 551 & 10221 & 516 & 567 & 277 & 249 & 208 & 202 & 164 & 13 & 99 & 65 & 45 \\
\hline 8 & & & 1887 & 555 & 589 & 258 & & 205 & 187 & & & 88 & 59 & 39 \\
\hline 8 & 132 & 276 & 2288 & 224 & 240 & 113 & 105 & 90 & 83 & 69 & 56 & 39 & 26 & 17 \\
\hline 8 & & & 43 & 451 & & & & 131 & 161 & & & 88 & 53 & 40 \\
\hline 8 & 134 & 3064 & 7159 & 2707 & 2603 & 1324 & 1272 & 1062 & 1026 & 835 & 687 & 499 & 329 & 223 \\
\hline 8 & 135 & 1902 & 1652 & 1782 & 1870 & 891 & 789 & 678 & 606 & 510 & 411 & 282 & 192 & 126 \\
\hline 8 & & & & 3133 & & 1712 & 1350 & 1159 & 1040 & & & 484 & 330 & 224 \\
\hline 8 & 137 & 2237 & 717 & 2173 & 2128 & 1040 & 922 & 787 & 716 & 598 & 484 & 336 & 228 & 151 \\
\hline 8 & & & & & & & & 286 & 258 & 217 & & 121 & 82 & 54 \\
\hline 8 & & 3209 & 6974 & 3314 & 3245 & 1563 & 1395 & 1183 & 1096 & 909 & & 520 & 349 & 236 \\
\hline 8 & 140 & 4241 & 13980 & 4258 & 3927 & 2108 & 1781 & 1520 & 1386 & 1157 & 936 & 651 & 440 & 298 \\
\hline 8 & & & & 4883 & 4502 & 2320 & 1967 & 1692 & 1511 & 1272 & & 701 & 479 & 317 \\
\hline 8 & 142 & 1684 & 0 & 1282 & 917 & 573 & 488 & 417 & 379 & 317 & 256 & 178 & 120 & 81 \\
\hline 8 & & & & & & & & 130 & & & & 53 & 37 & 24 \\
\hline 8 & & & 243 & 2662 & 2147 & 1279 & 996 & 860 & 759 & & & 349 & 240 & 160 \\
\hline 8 & 145 & 3483 & 3944 & 3612 & 3233 & 1748 & 1454 & 1251 & 1114 & 940 & 755 & 516 & 353 & 234 \\
\hline 8 & & & & 2645 & 2682 & 1267 & 1163 & 990 & 908 & & 6 & 428 & 289 & 191 \\
\hline 8 & & 523 & 12544 & 1484 & 1796 & 760 & 700 & 596 & 547 & 455 & 36 & 258 & 174 & 115 \\
\hline 8 & & & & & & & & 431 & 384 & & 260 & 178 & 122 & 79 \\
\hline 8 & & & 9631 & 3588 & 3321 & 1887 & 1534 & 1321 & 1176 & 992 & 798 & 545 & 373 & 248 \\
\hline 8 & & 1623 & 523 & 1756 & 1424 & 870 & 674 & 581 & 515 & 435 & 349 & 237 & 163 & 109 \\
\hline 8 & & & 13 & 952 & 997 & 496 & 433 & 370 & 336 & 281 & 227 & 158 & 107 & 71 \\
\hline 8 & 152 & 751 & 1785 & 1949 & 1600 & 909 & 747 & 640 & 577 & 484 & 391 & 270 & 183 & 124 \\
\hline 8 & & & 2141 & 2107 & 2043 & 1043 & 896 & 764 & 698 & 582 & 471 & 328 & 222 & 150 \\
\hline 8 & 154 & 3116 & 6035 & 3068 & 3052 & 1629 & 1358 & 1154 & 1064 & 884 & 718 & 504 & 339 & 233 \\
\hline 8 & & & 2756 & 613 & 666 & 322 & 283 & 240 & 223 & 184 & & 106 & 71 & 48 \\
\hline 8 & 156 & & 4516 & 1932 & 1844 & 987 & 806 & 690 & 623 & 522 & 421 & 291 & 198 & 133 \\
\hline 8 & 157 & 1587 & 7704 & 1487 & 1494 & 897 & 712 & 607 & 556 & 463 & 375 & 262 & 177 & 122 \\
\hline
\end{tabular}




\begin{tabular}{|c|c|c|c|c|c|c|c|c|c|c|c|c|c|c|}
\hline 8 & 158 & 2867 & 7997 & 2900 & 3021 & 1459 & 1273 & 1091 & 983 & 825 & 666 & 459 & 312 & 206 \\
\hline 8 & 159 & 4195 & 24207 & 4023 & 4227 & 2107 & 1822 & 1558 & 1413 & 1182 & 955 & 662 & 449 & 299 \\
\hline 8 & 160 & 178 & 135 & 147 & 154 & 81 & 73 & 63 & 57 & 48 & 38 & 26 & 18 & 12 \\
\hline 8 & 161 & 1879 & 13322 & 1643 & 2019 & 878 & 821 & 699 & 640 & 533 & 432 & 301 & 203 & 134 \\
\hline 8 & 162 & 3001 & 4269 & 2879 & 2850 & 1396 & 1223 & 1042 & 954 & 795 & 644 & 450 & 304 & 204 \\
\hline 8 & 163 & 2135 & 6319 & 2226 & 1911 & 1171 & 901 & 780 & 683 & 580 & 464 & 313 & 216 & 142 \\
\hline 8 & 164 & 3688 & 2463 & 3315 & 3312 & 1944 & 1538 & 1312 & 1197 & 999 & 809 & 563 & 381 & 262 \\
\hline 8 & 165 & 3767 & 3755 & 3738 & 3610 & 1904 & 1574 & 1355 & 1206 & 1017 & 818 & 559 & 382 & 253 \\
\hline 8 & 166 & 249 & 2255 & 235 & 250 & 109 & 104 & 90 & 80 & 67 & 54 & 37 & 25 & 16 \\
\hline 8 & 167 & 99 & 12 & 86 & 109 & 48 & 42 & 37 & 32 & 27 & 22 & 15 & 10 & 7 \\
\hline 8 & 168 & 125 & 53 & 107 & 121 & 55 & 50 & 44 & 38 & 33 & 26 & 18 & 12 & 8 \\
\hline 8 & 169 & 48 & 1266 & 42 & 50 & 22 & 22 & 19 & 17 & 14 & 12 & 8 & 6 & 4 \\
\hline 8 & 170 & 4379 & 407 & 3624 & 3508 & 2179 & 1662 & 1424 & 1284 & 1077 & 869 & 600 & 408 & 280 \\
\hline 9 & 1 & 2271 & 1043 & 3552 & 2031 & 1039 & 1088 & 749 & 654 & 557 & 445 & 299 & 207 & 138 \\
\hline 9 & 2 & 488 & 313 & 763 & 478 & 286 & 243 & 188 & 174 & 144 & 117 & 83 & 55 & 40 \\
\hline 9 & 3 & 2372 & 187 & 3899 & 2648 & 1127 & 1382 & 832 & 716 & 613 & 488 & 324 & 225 & 149 \\
\hline 9 & 4 & 1686 & 96 & 2361 & 1398 & 744 & 654 & 531 & 473 & 399 & 321 & 219 & 150 & 100 \\
\hline 9 & 5 & 4876 & 2828 & 7501 & 5296 & 2147 & 2273 & 1725 & 1539 & 1297 & 1043 & 713 & 487 & 322 \\
\hline 9 & 6 & 1791 & 746 & 2554 & 1998 & 807 & 1028 & 622 & 546 & 463 & 371 & 250 & 172 & 117 \\
\hline 9 & 7 & 3712 & 10885 & 5539 & 4150 & 1773 & 1792 & 1342 & 1212 & 1016 & 820 & 567 & 385 & 261 \\
\hline 9 & 8 & 2655 & 1628 & 4387 & 3045 & 1353 & 1928 & 982 & 842 & 722 & 574 & 380 & 265 & 180 \\
\hline 9 & 9 & 4173 & 1483 & 6484 & 4041 & 2219 & 2024 & 1503 & 1305 & 1113 & 889 & 595 & 412 & 273 \\
\hline 9 & 10 & 968 & 278 & 1534 & 753 & 547 & 484 & 339 & 287 & 248 & 196 & 129 & 90 & 58 \\
\hline 9 & 11 & 2556 & 3738 & 3118 & 2548 & 1376 & 1266 & 916 & 817 & 689 & 554 & 378 & 259 & 178 \\
\hline 9 & 12 & 2760 & 1418 & 2999 & 2645 & 1570 & 1282 & 1024 & 911 & 768 & 618 & 421 & 288 & 196 \\
\hline 9 & 13 & 4021 & 7977 & 4777 & 4407 & 2012 & 1963 & 1473 & 1330 & 1115 & 900 & 621 & 422 & 286 \\
\hline 9 & 14 & 5455 & 1210 & 7169 & 4107 & 2921 & 2168 & 1743 & 1521 & 1294 & 1035 & 695 & 480 & 325 \\
\hline 9 & 15 & 2852 & 1147 & 4146 & 2377 & 1645 & 1319 & 989 & 849 & 728 & 579 & 384 & 267 & 177 \\
\hline 9 & 16 & 3465 & 851 & 4995 & 2915 & 2043 & 1920 & 1214 & 1044 & 894 & 712 & 472 & 329 & 224 \\
\hline 9 & 17 & 5207 & 836 & 6201 & 3784 & 2912 & 2219 & 1739 & 1501 & 1284 & 1023 & 681 & 473 & 315 \\
\hline 9 & 18 & 2603 & 1394 & 3261 & 1885 & 1541 & 1117 & 873 & 744 & 640 & 508 & 334 & 234 & 153 \\
\hline 9 & 19 & 5505 & 762 & 7086 & 4466 & 3136 & 2511 & 1905 & 1651 & 1409 & 1125 & 751 & 521 & 349 \\
\hline 9 & 20 & 2713 & 461 & 3453 & 2214 & 1562 & 1382 & 955 & 819 & 702 & 559 & 370 & 258 & 171 \\
\hline 9 & 21 & 4935 & 1163 & 6070 & 3734 & 2880 & 2505 & 1667 & 1432 & 1227 & 977 & 647 & 451 & 305 \\
\hline 9 & 22 & 2431 & 555 & 3093 & 1894 & 1325 & 1108 & 806 & 684 & 589 & 468 & 307 & 215 & 139 \\
\hline 9 & 23 & 5888 & 2740 & 8249 & 5689 & 2663 & 2585 & 1974 & 1747 & 1477 & 1186 & 806 & 553 & 367 \\
\hline 9 & 24 & 4698 & 1368 & 7143 & 4515 & 2415 & 2035 & 1642 & 1455 & 1230 & 987 & 671 & 460 & 309 \\
\hline 9 & 25 & 6213 & 3527 & 7838 & 6506 & 2976 & 3670 & 2066 & 1848 & 1555 & 1252 & 858 & 586 & 423 \\
\hline 9 & 26 & 2612 & 754 & 3159 & 2848 & 1149 & 1261 & 866 & 801 & 665 & 540 & 380 & 255 & 180 \\
\hline 9 & 27 & 4705 & 530 & 4677 & 4489 & 2174 & 2261 & 1524 & 1410 & 1169 & 950 & 669 & 449 & 322 \\
\hline 9 & 28 & 3135 & 7291 & 3609 & 2970 & 1331 & 1567 & 984 & 912 & 756 & 615 & 433 & 291 & 210 \\
\hline 9 & 29 & 1319 & 1471 & 1425 & 1321 & 590 & 635 & 468 & 444 & 364 & 298 & 214 & 142 & 98 \\
\hline 9 & 30 & 5004 & 326 & 7595 & 5559 & 2317 & 2604 & 1781 & 1568 & 1329 & 1065 & 721 & 496 & 331 \\
\hline 9 & 31 & 3745 & 1023 & 4860 & 3787 & 1556 & 1833 & 1187 & 1067 & 896 & 722 & 497 & 338 & 233 \\
\hline 9 & 32 & 3390 & 9916 & 3211 & 3758 & 1602 & 1618 & 1209 & 1116 & 926 & 752 & 529 & 355 & 243 \\
\hline
\end{tabular}




\begin{tabular}{|c|c|c|c|c|c|c|c|c|c|c|c|c|c|c|}
\hline 9 & 33 & 2524 & 1376 & 2385 & 2699 & 1112 & 1107 & 825 & 758 & 630 & 511 & 358 & 241 & 165 \\
\hline 9 & 34 & 2350 & 4997 & 2430 & 2073 & 1250 & 1246 & 794 & 709 & 597 & 480 & 328 & 224 & 160 \\
\hline 9 & 35 & 583 & 7254 & 2953 & 2421 & 1348 & 1714 & 845 & 757 & 637 & 513 & 352 & 240 & 184 \\
\hline 9 & 36 & 7684 & 2585 & 8545 & 7072 & 3963 & 3496 & 2586 & 2297 & 1939 & 1558 & 1061 & 727 & 499 \\
\hline 9 & 37 & 963 & 1289 & 1178 & 1017 & 582 & 471 & 370 & 326 & 277 & 222 & 150 & 103 & 70 \\
\hline 9 & 38 & 270 & 119 & 252 & 307 & 129 & 152 & 97 & 88 & 74 & 60 & 42 & 28 & 20 \\
\hline 9 & 39 & 3546 & 3659 & 3551 & 3419 & 1822 & 1772 & 1206 & 1055 & 897 & 718 & 483 & 333 & 226 \\
\hline 9 & 40 & 559 & 4035 & 660 & 560 & 281 & 291 & 207 & 187 & 157 & 127 & 88 & 60 & 41 \\
\hline 9 & 41 & 3074 & 8998 & 3110 & 3239 & 1658 & 1519 & 1103 & 1000 & 837 & 676 & 468 & 317 & 222 \\
\hline 9 & 42 & 403 & 684 & 358 & 504 & 185 & 197 & 144 & 131 & 110 & 89 & 61 & 42 & 28 \\
\hline 9 & 43 & 998 & 15581 & 1138 & 1103 & 478 & 459 & 366 & 340 & 282 & 229 & 162 & 108 & 74 \\
\hline 9 & 44 & 1772 & 240 & 1478 & 1732 & 826 & 733 & 587 & 540 & 449 & 364 & 255 & 172 & 118 \\
\hline 9 & 45 & 762 & 383 & 829 & 820 & 378 & 400 & 270 & 242 & 204 & 164 & 113 & 77 & 53 \\
\hline 9 & 46 & 564 & 4260 & 589 & 657 & 282 & 273 & 217 & 206 & 169 & 138 & 99 & 66 & 45 \\
\hline 9 & 47 & 1741 & 7093 & 1465 & 1747 & 828 & 896 & 574 & 531 & 441 & 358 & 252 & 169 & 122 \\
\hline 9 & 48 & & 3825 & & 326 & 166 & 145 & 113 & 101 & 85 & 69 & 47 & 32 & 22 \\
\hline 9 & 49 & 1904 & 11864 & 1642 & 2295 & 892 & 1124 & 680 & 603 & 510 & 409 & 279 & 191 & 130 \\
\hline 9 & 50 & 1405 & 12894 & 1737 & 1669 & 908 & 1051 & 569 & 511 & 429 & 346 & 237 & 162 & 121 \\
\hline 9 & 51 & & 1966 & 736 & 883 & 347 & 543 & & 233 & 196 & & 108 & 74 & 54 \\
\hline 9 & 52 & 2273 & 375 & 2103 & 2300 & 988 & 1261 & 713 & 662 & 548 & 446 & 315 & 211 & 156 \\
\hline 9 & 53 & & & & 2069 & 1094 & & 676 & & & 00 & 267 & 185 & 142 \\
\hline 9 & 54 & 1018 & 2512 & 995 & 1022 & 480 & 432 & 349 & 332 & 272 & 223 & 160 & 106 & 74 \\
\hline 9 & 55 & 6887 & 15308 & 6358 & 6598 & 4021 & 3154 & 2482 & 2236 & 1876 & 1513 & 1043 & 709 & 496 \\
\hline 9 & 56 & & 16075 & 2079 & 2023 & & & & 616 & & & 295 & 197 & 135 \\
\hline 9 & 57 & 763 & 5095 & 957 & 942 & 394 & 359 & 299 & 273 & 228 & 184 & 129 & 87 & 58 \\
\hline 9 & 58 & & & 710 & & & 325 & 236 & 218 & 181 & & 103 & 69 & 49 \\
\hline 9 & 59 & 1857 & 1926 & 1601 & 2121 & 812 & 798 & 632 & 575 & 480 & & 270 & 183 & 121 \\
\hline 9 & 60 & 511 & 464 & 566 & 581 & 230 & 214 & 176 & 164 & 136 & 110 & 78 & 52 & 36 \\
\hline 9 & 61 & & 0 & 2028 & 2674 & & & & 734 & & & 346 & 234 & 156 \\
\hline 9 & 62 & 2660 & 1884 & 3108 & 2898 & 1322 & 1211 & 956 & 911 & 746 & 611 & 440 & 291 & 206 \\
\hline 9 & 63 & & & & 2299 & 1192 & & 800 & 731 & 610 & & 344 & 233 & 165 \\
\hline 9 & 64 & & & 1897 & 2136 & & & & 623 & 515 & & 297 & 199 & 139 \\
\hline 9 & 65 & 575 & 1340 & 639 & 618 & 301 & 283 & 214 & 197 & 164 & 133 & 93 & 63 & 43 \\
\hline 9 & 66 & & 1262 & 1604 & 1495 & 634 & 599 & 480 & 439 & 366 & & 207 & 140 & 94 \\
\hline 9 & 67 & 447 & 21 & 1254 & 1297 & 619 & 535 & 442 & 408 & 339 & 275 & 194 & 130 & 89 \\
\hline 9 & 68 & & 398 & 2232 & 1943 & & 922 & & 610 & 504 & & 291 & 195 & 141 \\
\hline 9 & 69 & & 1076 & 1938 & 1687 & 777 & 705 & 555 & 509 & 424 & & 240 & 162 & 112 \\
\hline 9 & 70 & 1943 & 1325 & 2157 & 2092 & 884 & 836 & 679 & 638 & 526 & 429 & 306 & 204 & $\begin{array}{l}4 \\
+\end{array}$ \\
\hline 9 & 71 & & 36873 & 1190 & 1853 & 667 & 638 & 539 & 461 & 396 & 314 & 207 & 145 & $5 \quad 90$ \\
\hline 9 & 72 & 3590 & 12183 & 3825 & 3641 & 2027 & 1977 & 1298 & 1170 & 982 & 792 & 546 & 371 & 266 \\
\hline 9 & 73 & & 4402 & 2823 & 2970 & 1295 & 1301 & 916 & 844 & 701 & 569 & 399 & 269 & 190 \\
\hline 9 & 74 & 1818 & 2007 & 1899 & 1975 & 960 & 925 & 647 & 590 & 492 & 398 & 277 & 187 & 132 \\
\hline 9 & 75 & 1245 & 12898 & 1452 & 1538 & 686 & 623 & 474 & 427 & 358 & & 199 & 135 & \\
\hline 9 & 76 & & & 1605 & 1747 & 755 & 719 & 562 & 528 & 435 & 355 & 253 & 169 & 7 116 \\
\hline 9 & 77 & 1866 & 36061 & 2304 & 2099 & 1051 & 938 & 766 & 708 & 588 & 477 & 335 & 225 & 5154 \\
\hline
\end{tabular}




\begin{tabular}{|c|c|c|c|c|c|c|c|c|c|c|c|c|c|c|}
\hline 9 & 78 & 2412 & 4657 & 2704 & 2414 & 1209 & 1053 & 853 & 801 & 660 & 539 & 383 & 256 & 179 \\
\hline 9 & 79 & 441 & 8016 & 1499 & 1589 & 693 & 803 & 509 & 467 & 389 & 315 & 221 & 149 & 106 \\
\hline 9 & 30 & 225 & 7529 & 2473 & 2525 & 1083 & 989 & 815 & 756 & 627 & 510 & 359 & 241 & 163 \\
\hline s & 81 & 2618 & 30164 & 2511 & 2837 & 1243 & 1202 & 977 & 925 & 760 & 621 & 445 & 296 & 200 \\
\hline 9 & 82 & 805 & 3631 & 759 & 887 & 388 & 383 & 298 & 286 & 233 & 192 & 138 & 91 & 63 \\
\hline 9 & 83 & 3971 & 5757 & 4108 & 4126 & 1878 & 1887 & 1378 & 1287 & 1063 & 866 & 614 & 410 & 288 \\
\hline 9 & 84 & 2265 & 7859 & 2037 & 2371 & 974 & 981 & 749 & 679 & 568 & 459 & 318 & 216 & 144 \\
\hline 9 & 85 & 101 & 14465 & 1413 & 1638 & 656 & 681 & 528 & 484 & 403 & 327 & 228 & 154 & 103 \\
\hline 9 & 86 & 407 & 1321 & 3010 & 3394 & 1514 & 1338 & 1118 & 1019 & 851 & 688 & 479 & 324 & 217 \\
\hline 9 & 87 & 715 & 21223 & 2883 & 3153 & 1334 & 1194 & 990 & 890 & 747 & 603 & 415 & 282 & 187 \\
\hline 9 & 88 & 524 & 21681 & 2742 & 2650 & 1192 & 1277 & 861 & 801 & 663 & 540 & 381 & 255 & 183 \\
\hline 9 & 89 & 1172 & 2019 & 1382 & 1293 & 573 & 745 & 410 & 370 & 310 & 250 & 173 & 117 & 85 \\
\hline 9 & 90 & 2158 & 13696 & 1968 & 2424 & 1016 & 1121 & 776 & 722 & 597 & 486 & 343 & 230 & 160 \\
\hline 9 & 91 & 438 & 5076 & 1425 & 1698 & 699 & 754 & 535 & 500 & 413 & 337 & 239 & 160 & 111 \\
\hline 9 & 92 & 089 & 1050 & 3493 & 2992 & 1431 & 1452 & 1015 & 946 & 782 & 637 & 451 & 302 & 216 \\
\hline 9 & 93 & 2380 & 8004 & 2327 & 2545 & 1117 & 1336 & 833 & 768 & 638 & 518 & 364 & 245 & 174 \\
\hline 9 & 94 & 441 & 8019 & 603 & 489 & 271 & 292 & 188 & 167 & 141 & 113 & 77 & 53 & 37 \\
\hline 9 & 95 & 513 & 17 & 527 & 519 & 220 & 341 & 157 & 146 & 121 & 99 & 70 & 47 & 37 \\
\hline 9 & 96 & 5226 & 3855 & 5971 & 4815 & 2510 & 2362 & 1708 & 1525 & 1285 & 1034 & 708 & 483 & 332 \\
\hline 9 & 97 & & 4216 & 2928 & 2201 & 1183 & 1394 & 766 & 670 & & 456 & 307 & 212 & 150 \\
\hline 9 & 98 & 2054 & 1413 & 3045 & 1984 & 1206 & 1164 & 734 & 638 & 544 & 434 & 291 & 201 & 140 \\
\hline 9 & 99 & 57 & 3941 & 5125 & 3608 & 1969 & 1691 & 1294 & 1165 & 978 & 789 & 543 & 370 & 257 \\
\hline 9 & 100 & 761 & 2866 & 1645 & 1455 & 1080 & 797 & 611 & 550 & 461 & 37 & 256 & 174 & 126 \\
\hline 9 & 101 & 1472 & 13245 & 1389 & 1585 & 675 & 720 & 526 & 503 & 411 & 337 & 243 & 161 & 112 \\
\hline 9 & 102 & & 25095 & 3750 & 4343 & 1882 & 2381 & 1397 & 1279 & 1066 & & 603 & 407 & 291 \\
\hline 9 & 103 & 547 & 1500 & 588 & 584 & 257 & 367 & 200 & 182 & 152 & & 86 & 58 & 42 \\
\hline 9 & 1 & 323 & 4551 & 322 & 351 & 148 & 169 & 117 & 108 & 90 & 3 & 51 & 34 & 24 \\
\hline 9 & 105 & 2399 & 10176 & 2653 & 2653 & 1137 & 1411 & 825 & 746 & & & 348 & 237 & 169 \\
\hline 9 & 106 & 2289 & 3174 & 2208 & 2267 & 991 & 1145 & 739 & 698 & 574 & 469 & 335 & 223 & 161 \\
\hline 9 & & & 6719 & 1599 & 1773 & 686 & 862 & 546 & 501 & & 338 & 236 & 159 & 111 \\
\hline 9 & 108 & 754 & 451 & 783 & 791 & 339 & 359 & 272 & 248 & 207 & 167 & 117 & 79 & 53 \\
\hline 9 & 109 & & & 388 & 369 & 161 & 198 & 122 & 110 & 92 & 74 & 51 & 35 & 24 \\
\hline 9 & & & 1644 & 2935 & 2411 & 1136 & 1457 & 819 & 735 & & 498 & 342 & 233 & 167 \\
\hline 9 & 111 & 1034 & 2410 & 1470 & 1139 & 511 & 558 & 383 & 347 & 290 & 235 & 162 & 110 & 76 \\
\hline 9 & 112 & & 112 & 386 & 341 & 141 & 162 & 110 & 99 & 83 & 67 & 46 & 31 & 21 \\
\hline 9 & 113 & 1728 & 2414 & 1620 & 1763 & 760 & 808 & 583 & 534 & 445 & 360 & 251 & 170 & 116 \\
\hline 9 & 114 & & & & 581 & 249 & 262 & 205 & 192 & & 129 & 91 & 61 & 41 \\
\hline 9 & & & 153 & 2057 & 1807 & 894 & 1005 & 634 & 608 & 497 & 408 & 295 & 195 & 146 \\
\hline 9 & 116 & 3280 & 425 & 3436 & 2874 & 1318 & 1769 & 1012 & 900 & 760 & 611 & 417 & 285 & 198 \\
\hline 9 & 117 & 2561 & 2934 & 4157 & 2256 & 1169 & 1656 & 987 & 831 & 719 & 569 & 371 & 261 & 165 \\
\hline 9 & 118 & 95 & 0 & 77 & 105 & 41 & 50 & 31 & 28 & 24 & 19 & 13 & 9 & 6 \\
\hline 9 & 119 & & & 4981 & 3699 & 1804 & 1561 & 1286 & 1169 & 977 & 790 & 548 & 371 & 252 \\
\hline 9 & 120 & 3290 & 5531 & 3936 & 3428 & 1582 & 1598 & 1161 & 1062 & 885 & 717 & 500 & 338 & 233 \\
\hline 9 & 121 & 761 & 86 & 710 & 743 & 347 & 339 & 247 & 236 & 193 & 158 & 114 & 75 & 54 \\
\hline 9 & 122 & 1198 & 3619 & 1139 & 1336 & 591 & 551 & 443 & 415 & 342 & 279 & 198 & 132 & 90 \\
\hline
\end{tabular}




\begin{tabular}{|c|c|c|c|c|c|c|c|c|c|c|c|c|c|c|}
\hline 9 & 123 & 893 & 1519 & 664 & 954 & 359 & 538 & 268 & 238 & 201 & 161 & 110 & 75 & 54 \\
\hline 9 & 124 & 413 & 645 & 383 & 466 & 198 & 187 & 160 & 145 & 121 & 98 & 68 & 46 & 30 \\
\hline s & & 130 & 210 & 156 & 160 & 62 & 61 & 51 & 48 & 40 & 32 & 23 & 15 & 10 \\
\hline 9 & 26 & 810 & 1891 & 747 & 893 & 430 & 361 & 306 & 283 & 235 & 191 & 134 & 90 & 61 \\
\hline 9 & 127 & 548 & 385 & 627 & 584 & 241 & 235 & 194 & 191 & 155 & 128 & 94 & 61 & 42 \\
\hline 9 & 128 & 1784 & 7206 & 1748 & 1672 & 747 & 716 & 603 & 569 & 468 & 382 & 273 & 182 & 121 \\
\hline 9 & 129 & 1305 & 12172 & 1450 & 1395 & 620 & 590 & 496 & 471 & 386 & 316 & 227 & 151 & 101 \\
\hline 9 & 30 & 544 & 16558 & 555 & 632 & 281 & 249 & 208 & 202 & 164 & 135 & 99 & 65 & 45 \\
\hline 9 & 31 & 584 & 2415 & 602 & 658 & 261 & 240 & 205 & 187 & 156 & & 88 & 59 & 39 \\
\hline 9 & 32 & 280 & 3386 & 240 & 266 & 114 & 105 & 90 & 83 & 69 & 56 & 39 & 26 & 17 \\
\hline 9 & 33 & 393 & 34 & 518 & 193 & 146 & 173 & 131 & 161 & 119 & 104 & 88 & 53 & 40 \\
\hline 9 & 34 & 3071 & 8734 & 2920 & 2858 & 1333 & 1272 & 1062 & 1026 & 835 & 687 & 499 & 329 & 223 \\
\hline 9 & 35 & 1909 & 1751 & 1920 & 2073 & 907 & 789 & 678 & 606 & 510 & 411 & 282 & 192 & 126 \\
\hline 9 & 36 & 3192 & 4658 & 3390 & 3180 & 1773 & 1350 & 1159 & 1040 & 874 & 705 & 484 & 330 & 224 \\
\hline 9 & 37 & 2244 & 659 & 2361 & 2357 & 1060 & 922 & 787 & 716 & 598 & 484 & 336 & 228 & 151 \\
\hline 9 & & & 2003 & & & & & 286 & 258 & 217 & & 121 & 82 & 54 \\
\hline 9 & 39 & 3192 & 8369 & 3612 & 3609 & 1592 & 1395 & 1183 & 1096 & 909 & 739 & 520 & 349 & 236 \\
\hline 9 & 40 & 4238 & 17894 & 4636 & 4323 & 2163 & 1781 & 1520 & 1386 & 1157 & 936 & 651 & 440 & 298 \\
\hline 9 & & 428 & 17406 & 5333 & 4968 & 2378 & & & 1511 & 1272 & 1024 & 701 & 479 & 317 \\
\hline 9 & 142 & 1775 & 0 & 1415 & 987 & 588 & 488 & 417 & 379 & 317 & 256 & 178 & 120 & 81 \\
\hline 9 & & & & & & & & & & & & 53 & 37 & 24 \\
\hline 9 & 144 & 2486 & 190 & 2933 & 2346 & 1328 & 996 & 860 & 759 & 643 & 515 & 349 & 240 & 160 \\
\hline 9 & 145 & 3488 & 4339 & 3944 & 3551 & 1797 & 1454 & 1251 & 1114 & 940 & 755 & 516 & 353 & 234 \\
\hline 9 & & & 6972 & 2861 & & 1284 & 1163 & & 908 & 756 & & 428 & 289 & 191 \\
\hline 9 & 147 & 1505 & 18064 & 1589 & 2020 & 768 & 700 & 596 & 547 & 455 & 369 & 258 & 174 & 115 \\
\hline 9 & & & & & & & & 431 & 384 & & & 178 & 122 & 79 \\
\hline 9 & & & 11943 & 3883 & 3632 & 1944 & 1534 & 1321 & 1176 & 992 & & 545 & 373 & 248 \\
\hline 9 & 150 & 1624 & 481 & 1930 & 1551 & 903 & 674 & 581 & 515 & 435 & 349 & 237 & 163 & 109 \\
\hline 9 & & & 8 & & & 506 & & & 336 & 281 & & 158 & 107 & 71 \\
\hline 9 & 152 & 1746 & 1927 & 2146 & 1752 & 936 & 747 & 640 & 577 & 484 & 391 & 270 & 183 & 124 \\
\hline 9 & & & & & & & & 764 & 698 & 582 & & 328 & 222 & 150 \\
\hline 9 & & 3094 & 7121 & 3319 & 3374 & 1673 & 1358 & 1154 & & 884 & & 504 & 339 & 233 \\
\hline 9 & 155 & 649 & 3638 & 660 & 742 & 329 & 283 & 240 & 223 & 184 & 150 & 106 & 71 & 48 \\
\hline 9 & & & 5509 & 2102 & & 1017 & & 690 & 623 & 522 & 421 & 291 & 198 & 133 \\
\hline 9 & 157 & 1567 & 10324 & 1589 & 1632 & 926 & 712 & 607 & 556 & 463 & 375 & 262 & 177 & 122 \\
\hline 9 & & & & 3132 & & & & & 983 & 825 & 666 & 459 & 312 & 206 \\
\hline 9 & & 4176 & 33406 & 4328 & 4680 & 2152 & 1822 & 1558 & 1413 & 1182 & 955 & 662 & 449 & 299 \\
\hline 9 & 160 & 179 & 140 & 156 & 167 & 83 & 73 & 63 & 57 & 48 & 38 & 26 & 18 & 12 \\
\hline 9 & & 1872 & 18918 & 1745 & 2256 & 885 & 821 & 699 & 640 & 533 & 432 & 301 & 203 & 134 \\
\hline 9 & 162 & 3013 & 4866 & 3130 & 3164 & 1425 & 1223 & 1042 & 954 & 795 & 644 & 450 & 304 & 204 \\
\hline 9 & & 2134 & 7962 & 2424 & 2080 & 1216 & 901 & 780 & 683 & 580 & 464 & 313 & 216 & 142 \\
\hline 9 & 164 & 3683 & 2512 & 3557 & 3631 & 2010 & 1538 & 1312 & 1197 & 999 & 809 & 563 & 381 & 262 \\
\hline 9 & & 3772 & 4056 & 4055 & 3981 & 1958 & 1574 & 1355 & 1206 & 1017 & 818 & 559 & 382 & 253 \\
\hline 9 & & 250 & 3338 & 253 & & 110 & 104 & 90 & 80 & 67 & 54 & 37 & 25 & 16 \\
\hline 9 & 167 & 99 & 10 & 91 & 122 & 49 & 42 & 37 & 32 & 27 & 22 & 15 & 10 & \\
\hline
\end{tabular}




\begin{tabular}{|c|c|c|c|c|c|c|c|c|c|c|c|c|c|c|}
\hline 9 & 168 & 127 & 51 & 114 & 134 & 55 & 50 & 44 & 38 & 33 & 26 & 18 & 12 & 8 \\
\hline 9 & 169 & 48 & 2152 & 45 & 55 & 22 & 22 & 19 & 17 & 14 & 12 & 8 & 6 & 4 \\
\hline 9 & 170 & 4431 & 317 & 3889 & 3829 & 2265 & 1662 & 1424 & 1284 & 1077 & 869 & 600 & 408 & 280 \\
\hline 10 & 1 & 2298 & 1022 & 4070 & 2216 & 1064 & 1117 & 749 & 654 & 557 & 445 & 299 & 207 & 138 \\
\hline 10 & 2 & 480 & 313 & 861 & 520 & 297 & 246 & 188 & 174 & 144 & 117 & 83 & 55 & 40 \\
\hline 10 & 3 & 2384 & 146 & 4456 & 2941 & 1150 & 1443 & 832 & 716 & 613 & 488 & 324 & 225 & 149 \\
\hline 10 & 4 & 1714 & 73 & 2689 & 1523 & 764 & 658 & 531 & 473 & 399 & 321 & 219 & 150 & 100 \\
\hline 10 & 5 & 4892 & 2828 & 8518 & 5880 & 2171 & 2305 & 1725 & 1539 & 1297 & 1043 & 713 & 487 & 322 \\
\hline 10 & 6 & 1797 & 717 & 2872 & 2224 & 818 & 1070 & 622 & 546 & 463 & 371 & 250 & 172 & 117 \\
\hline 10 & 7 & 3705 & 13280 & 6253 & 4616 & 1805 & 1819 & 1342 & 1212 & 1016 & 820 & 567 & 385 & 261 \\
\hline 10 & 8 & 2646 & 1628 & 4977 & 3367 & 1382 & 2052 & 982 & 842 & 722 & 574 & 380 & 265 & 180 \\
\hline 10 & 9 & 4179 & 1394 & 7347 & 4401 & 2292 & 2061 & 1503 & 1305 & 1113 & 889 & 595 & 412 & 273 \\
\hline 10 & 10 & 975 & 256 & 1747 & 799 & 572 & 497 & 339 & 287 & 248 & 196 & 129 & 90 & 58 \\
\hline 10 & 11 & 2551 & 4186 & 3432 & 2790 & 1420 & 1290 & 916 & 817 & 689 & 554 & 378 & 259 & 178 \\
\hline 10 & 12 & 2746 & 1390 & 3243 & 2869 & 1625 & 1293 & 1024 & 911 & 768 & 618 & 421 & 288 & 196 \\
\hline 10 & 13 & 4007 & 9253 & 5232 & 4874 & 2051 & 1992 & 1473 & 1330 & 1115 & 900 & 621 & 422 & 286 \\
\hline 10 & 14 & 5535 & 1089 & 8080 & 4402 & 3067 & 2187 & 1743 & 1521 & 1294 & 1035 & 695 & 480 & 325 \\
\hline 10 & 15 & 2870 & 1101 & 4678 & 2550 & 1725 & 1343 & 989 & 849 & 728 & 579 & 384 & 267 & 177 \\
\hline 10 & 16 & 3474 & 766 & 5618 & 3125 & 2143 & 1993 & 1214 & 1044 & 894 & 712 & 472 & 329 & 224 \\
\hline 10 & 17 & 5262 & 719 & 6860 & 4010 & 3054 & 2246 & 1739 & 1501 & 1284 & 1023 & 681 & 473 & 315 \\
\hline 10 & 18 & 2634 & 1394 & 3627 & 1994 & 1628 & 1133 & 873 & 744 & 640 & 508 & 334 & 234 & 153 \\
\hline 10 & 19 & 5535 & 640 & 7881 & 4778 & 3281 & 2551 & 1905 & 1651 & 1409 & 1125 & 751 & 521 & 349 \\
\hline 10 & 20 & 2725 & 397 & 3823 & 2362 & 1632 & 1420 & 955 & 819 & 702 & 559 & 370 & 258 & 171 \\
\hline 10 & 21 & 4974 & 1047 & 6725 & 3967 & 3029 & 2586 & 1667 & 1432 & 1227 & 977 & 647 & 451 & 305 \\
\hline 10 & 22 & 2465 & 499 & 3450 & 2023 & 1387 & 1132 & 806 & 684 & 589 & 468 & 307 & 215 & 139 \\
\hline 10 & 23 & 5946 & 2685 & 9318 & 6262 & 2719 & 2622 & 1974 & 1747 & 1477 & 1186 & 806 & 553 & 367 \\
\hline 10 & 24 & 4715 & 1259 & 8114 & 4943 & 2495 & 2050 & 1642 & 1455 & 1230 & 987 & 671 & 460 & 309 \\
\hline 10 & 25 & 6235 & 3527 & 8723 & 7224 & 3047 & 3844 & 2066 & 1848 & 1555 & 1252 & 858 & 586 & 423 \\
\hline 10 & 26 & 2626 & 693 & 3511 & 3194 & 1166 & 1290 & 866 & 801 & 665 & 540 & 380 & 255 & 180 \\
\hline 10 & 27 & 4735 & 434 & 5087 & 4958 & 2220 & 2318 & 1524 & 1410 & 1169 & 950 & 669 & 449 & 322 \\
\hline 10 & 28 & 3165 & 8750 & 4009 & 3293 & 1352 & 1618 & 984 & 912 & 756 & 615 & 433 & 291 & 210 \\
\hline 10 & 29 & 1316 & 1589 & 1552 & 1455 & 594 & 643 & 468 & 444 & 364 & 298 & 214 & 142 & 98 \\
\hline 10 & 30 & 5014 & 248 & 8591 & 6172 & 2353 & 2675 & 1781 & 1568 & 1329 & 1065 & 721 & 496 & 331 \\
\hline 10 & 31 & 3794 & 941 & 5471 & 4221 & 1579 & 1891 & 1187 & 1067 & 896 & 722 & 497 & 338 & 233 \\
\hline 10 & 32 & 3385 & 12097 & 3433 & 4178 & 1622 & 1641 & 1209 & 1116 & 926 & 752 & 529 & 355 & 243 \\
\hline 10 & 33 & 2548 & 1376 & 2577 & 3020 & 1130 & 1124 & 825 & 758 & 630 & 511 & 358 & 241 & 165 \\
\hline 10 & 34 & 2355 & 5897 & 2637 & 2248 & 1294 & 1289 & 794 & 709 & 597 & 480 & 328 & 224 & 160 \\
\hline 10 & 35 & 2583 & 8850 & 3248 & 2651 & 1393 & 1820 & 845 & 757 & 637 & 513 & 352 & 240 & 184 \\
\hline 10 & 36 & 7734 & 2430 & 9372 & 7724 & 4101 & 3557 & 2586 & 2297 & 1939 & 1558 & 1061 & 727 & 499 \\
\hline 10 & 37 & 955 & 1418 & 1286 & 1111 & 605 & 476 & 370 & 326 & 277 & 222 & 150 & 103 & 70 \\
\hline 10 & 38 & 269 & 114 & 268 & 342 & 130 & 157 & 97 & 88 & 74 & 60 & 42 & 28 & 20 \\
\hline 10 & 39 & 3569 & 3952 & 3835 & 3743 & 1881 & 1821 & 1206 & 1055 & 897 & 718 & 483 & 333 & 226 \\
\hline 10 & 40 & 556 & 5487 & 722 & 611 & 286 & 297 & 207 & 187 & 157 & 127 & 88 & 60 & 41 \\
\hline 10 & 41 & 3062 & 10978 & 3346 & 3571 & 1707 & 1547 & 1103 & 1000 & 837 & 676 & 468 & 317 & 222 \\
\hline 10 & 42 & 403 & 780 & 380 & 567 & 186 & 201 & 144 & 131 & 110 & 89 & 61 & 42 & 28 \\
\hline
\end{tabular}




\begin{tabular}{|c|c|c|c|c|c|c|c|c|c|c|c|c|c|c|}
\hline 10 & 43 & 994 & 23372 & 1243 & 1225 & 485 & 462 & 366 & 340 & 282 & 229 & 162 & 108 & 74 \\
\hline 10 & 44 & 1787 & 202 & 1571 & 1914 & 843 & 738 & 587 & 540 & 449 & 364 & 255 & 172 & 118 \\
\hline 10 & 45 & 761 & 376 & 900 & 907 & 385 & 411 & 270 & 242 & 204 & 164 & 113 & 77 & 53 \\
\hline 10 & 46 & 557 & 5794 & 633 & 730 & 284 & 274 & 217 & 206 & 169 & 138 & 99 & 66 & 45 \\
\hline 10 & 47 & 1747 & 9080 & 1556 & 1933 & 844 & 923 & 574 & 531 & 441 & 358 & 252 & 169 & 122 \\
\hline 10 & 48 & 292 & 5584 & 333 & 358 & 171 & 147 & 113 & 101 & 85 & 69 & 47 & 32 & 22 \\
\hline 10 & 49 & 1903 & 15898 & 1729 & 2564 & 900 & 1170 & 680 & 603 & 510 & 409 & 279 & 191 & 130 \\
\hline 10 & 50 & 1371 & 17793 & 1880 & 1834 & 939 & 1105 & 569 & 511 & 429 & 346 & 237 & 162 & 121 \\
\hline 10 & 51 & 747 & 2359 & 788 & 985 & 350 & 578 & 260 & 233 & 196 & 158 & 108 & 74 & 54 \\
\hline 10 & 52 & 2290 & 323 & 2270 & 2563 & 1001 & 1316 & 713 & 662 & 548 & 446 & 315 & 211 & 156 \\
\hline 10 & 53 & 1872 & 2378 & 2303 & 2273 & 1130 & 1919 & 676 & 587 & 501 & 400 & 267 & 185 & 142 \\
\hline 10 & 54 & 1019 & 3015 & 1076 & 1133 & 489 & 434 & 349 & 332 & 272 & 223 & 160 & 106 & 74 \\
\hline 10 & 55 & 6859 & 18063 & 6764 & 7183 & 4182 & 3185 & 2482 & 2236 & 1876 & 1513 & 1043 & 709 & 496 \\
\hline 10 & 56 & 1726 & 22505 & 2279 & 2256 & 891 & 807 & 654 & 616 & 507 & 414 & 295 & 197 & 135 \\
\hline 10 & 57 & 755 & 6827 & 1049 & 1050 & 400 & 360 & 299 & 273 & 228 & 184 & 129 & 87 & 58 \\
\hline 10 & 58 & 653 & 1287 & 771 & 852 & 335 & 331 & 236 & 218 & 181 & 147 & 103 & 69 & 49 \\
\hline 10 & 59 & 1871 & 2080 & 1702 & 2379 & 820 & 804 & 632 & 575 & 480 & 388 & 270 & 183 & 121 \\
\hline 10 & 60 & 513 & 492 & 621 & 654 & 234 & 215 & 176 & 164 & 136 & 110 & 78 & 52 & 36 \\
\hline 10 & 61 & 3753 & 0 & 2157 & 3000 & 1057 & 992 & 800 & 734 & 611 & 495 & 346 & 234 & 156 \\
\hline 10 & 62 & 2644 & 1922 & 3417 & 3228 & 1349 & 1218 & 956 & 911 & 746 & 611 & 440 & 291 & 206 \\
\hline 10 & 63 & 2456 & 5945 & 2572 & 2530 & 1225 & 1152 & 800 & 731 & 610 & 494 & 344 & 233 & 165 \\
\hline 10 & 64 & 1807 & 10451 & 2045 & 2384 & 909 & 968 & 668 & 623 & 515 & 419 & 297 & 199 & 139 \\
\hline 10 & 65 & 570 & 1581 & 694 & 681 & 308 & 286 & 214 & 197 & 164 & 133 & 93 & 63 & 43 \\
\hline 10 & 66 & 1347 & 1337 & 1765 & 1665 & 644 & 603 & 480 & 439 & 366 & 297 & 207 & 140 & 94 \\
\hline 10 & 67 & 1474 & 13 & 1354 & 1433 & 633 & 537 & 442 & 408 & 339 & 275 & 194 & 130 & 89 \\
\hline 10 & 68 & 1896 & 351 & 2463 & 2153 & 979 & 939 & 653 & 610 & 504 & 411 & 291 & 195 & 141 \\
\hline 10 & 69 & 1641 & 1097 & 2146 & 1874 & 796 & 711 & 555 & 509 & 424 & 344 & 240 & 162 & 112 \\
\hline 10 & 70 & 1945 & 1351 & 2363 & 2333 & 896 & 839 & 679 & 638 & 526 & 429 & 306 & 204 & 139 \\
\hline 10 & 71 & 1462 & 58997 & 1239 & 2076 & 673 & 640 & 539 & 461 & 396 & 314 & 207 & 145 & 90 \\
\hline 10 & 72 & 3565 & 15107 & 4133 & 3987 & 2095 & 2036 & 1298 & 1170 & 982 & 792 & 546 & 371 & 266 \\
\hline 10 & 73 & 2539 & 5018 & 3073 & 3320 & 1322 & 1328 & 916 & 844 & 701 & 569 & 399 & 269 & 190 \\
\hline 10 & 74 & 1811 & 2168 & 2053 & 2189 & 986 & 945 & 647 & 590 & 492 & 398 & 277 & 187 & 132 \\
\hline 10 & 75 & 1233 & 18315 & 1579 & 1718 & 704 & 631 & 474 & 427 & 358 & 289 & 199 & 135 & 93 \\
\hline 10 & 76 & 1511 & 2218 & 1735 & 1947 & 767 & 725 & 562 & 528 & 435 & 355 & 253 & 169 & 116 \\
\hline 10 & 77 & 1831 & 54813 & 2505 & 2301 & 1071 & 942 & 766 & 708 & 588 & 477 & 335 & 225 & 154 \\
\hline 10 & 78 & 2406 & 5402 & 2962 & 2663 & 1239 & 1058 & 853 & 801 & 660 & 539 & 383 & 256 & 179 \\
\hline 10 & 79 & 1436 & 10581 & 1620 & 1765 & 703 & 829 & 509 & 467 & 389 & 315 & 221 & 149 & 106 \\
\hline 10 & 80 & 2216 & 9336 & 2693 & 2813 & 1101 & 992 & 815 & 756 & 627 & 510 & 359 & 241 & 163 \\
\hline 10 & 81 & 2600 & 43436 & 2680 & 3137 & 1252 & 1206 & 977 & 925 & 760 & 621 & 445 & 296 & 200 \\
\hline 10 & 82 & 799 & 4647 & 810 & 984 & 391 & 386 & 298 & 286 & 233 & 192 & 138 & 91 & 63 \\
\hline 10 & 83 & 3970 & 6448 & 4459 & 4572 & 1909 & 1917 & 1378 & 1287 & 1063 & 866 & 614 & 410 & 288 \\
\hline 10 & 84 & 2288 & 9902 & 2183 & 2640 & 985 & 993 & 749 & 679 & 568 & 459 & 318 & 216 & 144 \\
\hline 10 & 85 & 1393 & 20540 & 1513 & 1821 & 660 & 688 & 528 & 484 & 403 & 327 & 228 & 154 & 103 \\
\hline 10 & 86 & 3446 & 1268 & 3226 & 3763 & 1541 & 1342 & 1118 & 1019 & 851 & 688 & 479 & 324 & 217 \\
\hline 10 & 87 & 2712 & 29288 & 3118 & 3514 & 1359 & 1198 & 990 & 890 & 747 & 603 & 415 & 282 & 187 \\
\hline
\end{tabular}




\begin{tabular}{|c|c|c|c|c|c|c|c|c|c|c|c|c|c|c|}
\hline 10 & 88 & 2524 & 30353 & 2996 & 2945 & 1213 & 1309 & 861 & 801 & 663 & 540 & 381 & 255 & 183 \\
\hline 10 & 89 & 1167 & 2301 & 1516 & 1435 & 583 & 781 & 410 & 370 & 310 & 250 & 173 & 117 & 85 \\
\hline 10 & 90 & 2149 & 18352 & 2091 & 2695 & 1024 & 1145 & 776 & 722 & 597 & 486 & 343 & 230 & 160 \\
\hline 10 & 91 & 1426 & 6294 & 1524 & 1892 & 706 & 769 & 535 & 500 & 413 & 337 & 239 & 160 & 111 \\
\hline 10 & 92 & 3102 & 987 & 3857 & 3312 & 1462 & 1482 & 1015 & 946 & 782 & 637 & 451 & 302 & 216 \\
\hline 10 & 93 & 2374 & 9925 & 2499 & 2818 & 1129 & 1381 & 833 & 768 & 638 & 518 & 364 & 245 & 174 \\
\hline 10 & 94 & 431 & 12028 & 659 & 530 & 278 & 302 & 188 & 167 & 141 & 113 & 77 & 53 & 37 \\
\hline 10 & 95 & 515 & 12 & 576 & 578 & 223 & 362 & 157 & 146 & 121 & 99 & 70 & 47 & 37 \\
\hline 10 & 96 & 5277 & 4010 & 6595 & 5282 & 2584 & 2408 & 1708 & 1525 & 1285 & 1034 & 708 & 483 & 332 \\
\hline 10 & 97 & 2184 & 4891 & 3261 & 2412 & 1223 & 1467 & 766 & 670 & 570 & 456 & 307 & 212 & 150 \\
\hline 10 & 98 & 2050 & 1441 & 3430 & 2160 & 1260 & 1208 & 734 & 638 & 544 & 434 & 291 & 201 & 140 \\
\hline 10 & 99 & 3540 & 4257 & 5756 & 3961 & 2040 & 1713 & 1294 & 1165 & 978 & 789 & 543 & 370 & 257 \\
\hline 10 & 100 & 1757 & 3268 & 1760 & 1562 & 1136 & 807 & 611 & 550 & 461 & 372 & 256 & 174 & 126 \\
\hline 10 & 101 & 1466 & 18543 & 1487 & 1760 & 679 & 730 & 526 & 503 & 411 & 337 & 243 & 161 & 112 \\
\hline 10 & 102 & 3964 & 33628 & 4001 & 4811 & 1901 & 2478 & 1397 & 1279 & 1066 & 863 & 603 & 407 & 291 \\
\hline 10 & 103 & 542 & 1800 & 634 & 642 & 258 & 386 & 200 & 182 & 152 & 123 & 86 & 58 & 42 \\
\hline 10 & 104 & 321 & 6736 & 345 & 388 & 149 & 172 & 117 & 108 & 90 & 73 & 51 & 34 & 24 \\
\hline 10 & 105 & 2399 & 13025 & 2895 & 2954 & 1156 & 1471 & 825 & 746 & 625 & 504 & 348 & 237 & 169 \\
\hline 10 & 106 & 2302 & 3555 & 2394 & 2518 & 1002 & 1178 & 739 & 698 & 574 & 469 & 335 & 223 & 161 \\
\hline 10 & 107 & 1463 & 8600 & 1727 & 1979 & 689 & 890 & 546 & 501 & 417 & 338 & 236 & 159 & 111 \\
\hline 10 & 108 & 753 & 451 & 846 & 872 & 341 & 364 & 272 & 248 & 207 & 167 & 117 & 79 & 53 \\
\hline 10 & 109 & 348 & 786 & 423 & 408 & 162 & 205 & 122 & 110 & 92 & 74 & 51 & 35 & 24 \\
\hline 10 & 110 & 2347 & 1677 & 3243 & 2655 & 1156 & 1526 & 819 & 735 & 618 & 498 & 342 & 233 & 167 \\
\hline 10 & 111 & 1027 & 2844 & 1643 & 1260 & 520 & 572 & 383 & 347 & 290 & 235 & 162 & 110 & 76 \\
\hline 10 & 112 & 339 & 105 & 426 & 378 & 142 & 167 & 110 & 99 & 83 & 67 & 46 & 31 & 21 \\
\hline 10 & 113 & 1738 & 2704 & 1741 & 1952 & 768 & 823 & 583 & 534 & 445 & 360 & 251 & 170 & 116 \\
\hline 10 & 114 & 567 & 1303 & 683 & 640 & 249 & 265 & 205 & 192 & 158 & 129 & 91 & 61 & 41 \\
\hline 10 & 115 & 2150 & 119 & 2254 & 1992 & 910 & 1035 & 634 & 608 & 497 & 408 & 295 & 195 & 146 \\
\hline 10 & 116 & 3333 & 357 & 3772 & 3147 & 1331 & 1851 & 1012 & 900 & 760 & 611 & 417 & 285 & 198 \\
\hline 10 & 117 & 2554 & 3169 & 4686 & 2400 & 1175 & 1734 & 987 & 831 & 719 & 569 & 371 & 261 & 165 \\
\hline 10 & 118 & 96 & 0 & 81 & 117 & 41 & 51 & 31 & 28 & 24 & 19 & 13 & 9 & 6 \\
\hline 10 & 119 & 3633 & 12620 & 5585 & 4082 & 1852 & 1567 & 1286 & 1169 & 977 & 790 & 548 & 371 & 252 \\
\hline 10 & 120 & 3287 & 6305 & 4336 & 3789 & 1611 & 1626 & 1161 & 1062 & 885 & 717 & 500 & 338 & 233 \\
\hline 10 & 121 & 765 & 71 & 768 & 825 & 354 & 344 & 247 & 236 & 193 & 158 & 114 & 75 & 54 \\
\hline 10 & 122 & 1191 & 4415 & 1215 & 1482 & 599 & 554 & 443 & 415 & 342 & 279 & 198 & 132 & 90 \\
\hline 10 & 123 & 909 & 1762 & 700 & 1071 & 362 & 571 & 268 & 238 & 201 & 161 & 110 & 75 & 54 \\
\hline 10 & 124 & 410 & 722 & 405 & 514 & 200 & 187 & 160 & 145 & 121 & 98 & 68 & 46 & 30 \\
\hline 10 & 125 & 129 & 235 & 170 & 178 & 63 & 61 & 51 & 48 & 40 & 32 & 23 & 15 & 10 \\
\hline 10 & 126 & 804 & 2232 & 792 & 986 & 439 & 361 & 306 & 283 & 235 & 191 & 134 & 90 & 61 \\
\hline 10 & 127 & 547 & 392 & 690 & 652 & 243 & 235 & 194 & 191 & 155 & 128 & 94 & 61 & 42 \\
\hline 10 & 128 & 1796 & 9224 & 1895 & 1839 & 752 & 716 & 603 & 569 & 468 & 382 & 273 & 182 & 121 \\
\hline 10 & 129 & 1294 & 17041 & 1574 & 1539 & 624 & 590 & 496 & 471 & 386 & 316 & 227 & 151 & 101 \\
\hline 10 & 130 & 537 & 26823 & 597 & 705 & 285 & 249 & 208 & 202 & 164 & 135 & 99 & 65 & 45 \\
\hline 10 & 131 & 587 & 3092 & 652 & 735 & 264 & 240 & 205 & 187 & 156 & 126 & 88 & 59 & 39 \\
\hline 10 & 132 & 284 & 5012 & 257 & 294 & 115 & 105 & 90 & 83 & 69 & 56 & 39 & 26 & 17 \\
\hline
\end{tabular}




\begin{tabular}{|c|c|c|c|c|c|c|c|c|c|c|c|c|c|c|}
\hline 10 & 33 & 388 & 26 & 596 & 206 & 146 & 173 & 131 & 161 & 119 & 104 & 88 & 53 & 40 \\
\hline 10 & 34 & 3077 & 10655 & 3148 & 3138 & 1341 & 1272 & 1062 & 1026 & 835 & 687 & 499 & 329 & 223 \\
\hline 10 & & & 1856 & 2069 & 2299 & 923 & 789 & 678 & 606 & 510 & & 282 & 192 & 126 \\
\hline 10 & & & 5217 & 3669 & 3480 & 1835 & 1350 & 1159 & 1040 & 874 & 705 & 484 & 330 & 224 \\
\hline 10 & 37 & & 607 & 2565 & 2610 & 1079 & 922 & 787 & 716 & 598 & 484 & 336 & 228 & 151 \\
\hline 10 & & 740 & 2403 & 1056 & 978 & 395 & 334 & 286 & 258 & 217 & 175 & 121 & 82 & 54 \\
\hline 10 & 39 & 3175 & 10043 & 3937 & 4014 & 1621 & 1395 & 1183 & 1096 & 909 & 739 & 520 & 349 & 236 \\
\hline 10 & 40 & 4234 & 22904 & 5048 & 4759 & 2219 & 1781 & 1520 & 1386 & 1157 & 936 & 651 & 440 & 298 \\
\hline 10 & 41 & 399 & 21931 & 5823 & 5481 & 2438 & 1967 & 1692 & 1511 & 1272 & 1024 & 701 & 479 & 317 \\
\hline 10 & 42 & 70 & 0 & 1560 & 1062 & 603 & 488 & 417 & 379 & 317 & 256 & 178 & 120 & 81 \\
\hline 10 & 3 & 338 & 4935 & 441 & 459 & 175 & 151 & 130 & 115 & 98 & 78 & 53 & 37 & 24 \\
\hline 10 & & & 148 & & 2562 & & 996 & & 759 & 643 & & 349 & 240 & 160 \\
\hline 10 & 45 & & 4773 & 4306 & 3900 & 1847 & 1454 & 1251 & 1114 & 940 & 755 & 516 & 353 & 234 \\
\hline 10 & 46 & & 8367 & 3095 & 3295 & 1301 & 1163 & 990 & 908 & 756 & 613 & 428 & 289 & 191 \\
\hline 10 & & & 26012 & 1702 & 2272 & 777 & 700 & 596 & 547 & & & 258 & 174 & 115 \\
\hline 10 & 148 & 104 & 18569 & 1113 & 1569 & 557 & 501 & 431 & 384 & 324 & 260 & 178 & 122 & 79 \\
\hline 10 & & & & & 3973 & 2004 & 1534 & 1321 & 1176 & & & 45 & 373 & 248 \\
\hline 10 & 150 & 1626 & 443 & 2120 & 1691 & 938 & 674 & 581 & 515 & 435 & 349 & 237 & 163 & 109 \\
\hline 10 & 151 & 463 & 4 & 1101 & 1220 & 515 & 433 & 370 & 336 & 281 & 227 & 158 & 107 & 71 \\
\hline 10 & & & & & & & & & & & & 270 & 183 & 124 \\
\hline 10 & 153 & & 2497 & 2488 & 2501 & 1092 & 896 & 764 & 698 & 582 & 471 & 328 & 222 & 150 \\
\hline 10 & & & & & 3730 & 1719 & 1358 & 1154 & 1064 & 884 & 8 & 504 & 339 & 233 \\
\hline 10 & & & 4802 & 710 & 827 & & & 240 & 223 & 184 & & 106 & 71 & 48 \\
\hline 10 & 156 & 367 & 6721 & 2287 & 2252 & 1048 & 806 & 690 & 623 & 522 & 421 & 291 & 198 & 133 \\
\hline 10 & & & & 1698 & 1783 & & & 607 & 556 & 463 & & 262 & 177 & 122 \\
\hline 10 & & & 12297 & 3381 & 3723 & 1519 & 1273 & 1091 & 983 & 825 & 666 & 459 & 312 & 206 \\
\hline 10 & & & & & 5182 & 2198 & & 1558 & 1413 & 1182 & & 662 & 449 & 299 \\
\hline 10 & & & & 165 & & & & 63 & 57 & 48 & 38 & 26 & 18 & 12 \\
\hline 10 & 161 & 1866 & 26863 & 1853 & 2521 & 893 & 821 & 699 & 640 & 533 & 432 & 301 & 203 & 134 \\
\hline 10 & & 3026 & & 3402 & 3513 & 1454 & 1223 & 1042 & 954 & 795 & 644 & 450 & 304 & 204 \\
\hline 10 & 163 & 2133 & 10032 & 2639 & 2263 & 1264 & 901 & 780 & 683 & 580 & 464 & 313 & 216 & 142 \\
\hline 10 & & & & & & & & 1312 & 1197 & 999 & & 563 & 381 & 262 \\
\hline 10 & & & 4380 & 4398 & 4392 & 2014 & 1574 & 1355 & 1206 & 1017 & 818 & 559 & 382 & 253 \\
\hline 10 & 166 & 251 & 4940 & 272 & 308 & 110 & 104 & 90 & 80 & 67 & 54 & 37 & 25 & 16 \\
\hline 10 & & 99 & 8 & 96 & 137 & 50 & 42 & 37 & 32 & 27 & 22 & 15 & 10 & 7 \\
\hline 10 & 168 & 128 & 49 & 122 & 148 & 56 & 50 & 44 & 38 & 33 & 26 & 18 & 12 & 8 \\
\hline 10 & & & & 47 & 61 & 22 & 22 & 19 & 17 & 14 & 12 & 8 & 6 & 4 \\
\hline 10 & 170 & 4483 & 248 & 4174 & 4179 & 2354 & 1662 & 1424 & 1284 & 1077 & 869 & 600 & 408 & 280 \\
\hline
\end{tabular}


Table 50 Competitors with highest attraction with respect to demand point INDEXJ INDEXT COMPETITORSATTRACTION

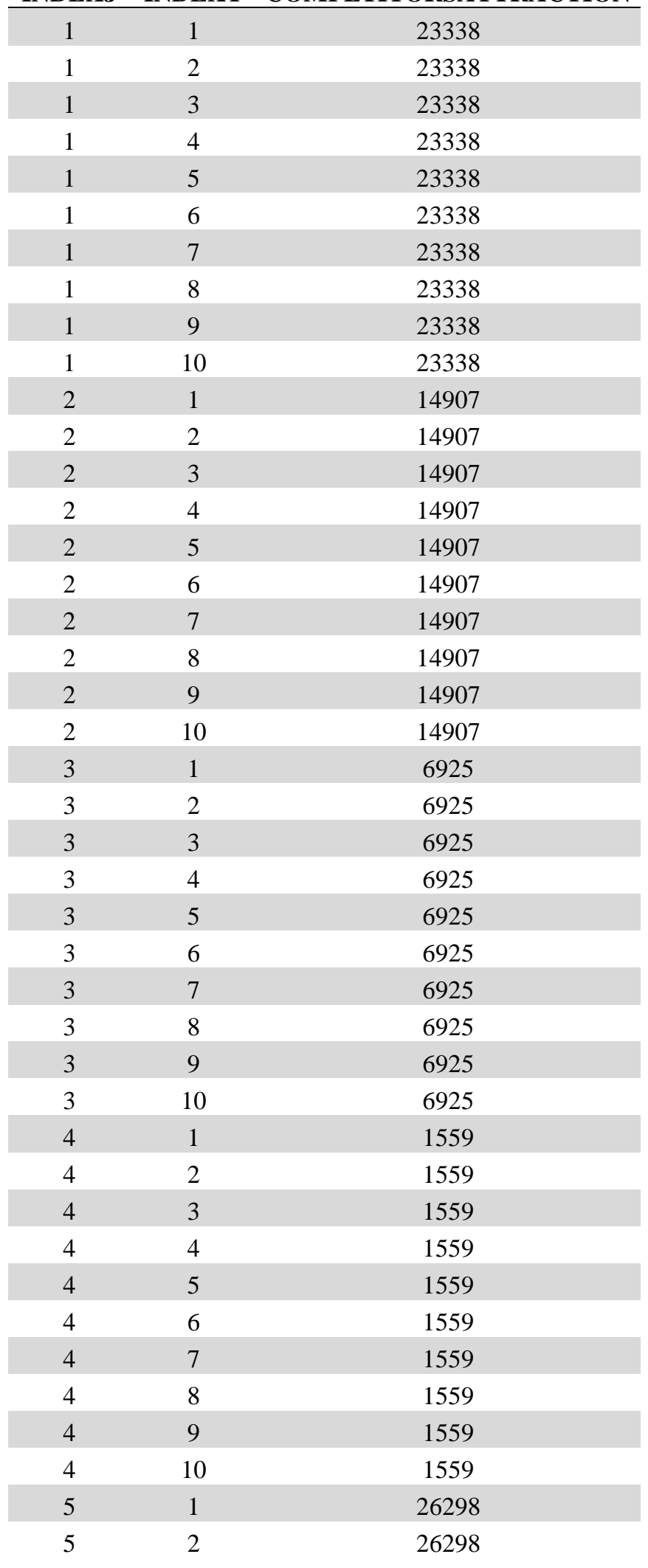




\begin{tabular}{|c|c|c|}
\hline 5 & 3 & 26298 \\
\hline 5 & 4 & 26298 \\
\hline 5 & 5 & 26298 \\
\hline 5 & 6 & 26298 \\
\hline 5 & 7 & 26298 \\
\hline 5 & 8 & 26298 \\
\hline 5 & 9 & 26298 \\
\hline 5 & 10 & 26298 \\
\hline 6 & 1 & 88 \\
\hline 6 & 2 & 88 \\
\hline 6 & 3 & 88 \\
\hline 6 & 4 & 88 \\
\hline 6 & 5 & 88 \\
\hline 6 & 6 & 88 \\
\hline 6 & 7 & 88 \\
\hline 6 & 8 & 88 \\
\hline 6 & 9 & 88 \\
\hline 6 & 10 & 88 \\
\hline 7 & 1 & 681 \\
\hline 7 & 2 & 681 \\
\hline 7 & 3 & 681 \\
\hline 7 & 4 & 681 \\
\hline 7 & 5 & 681 \\
\hline 7 & 6 & 681 \\
\hline 7 & 7 & 681 \\
\hline 7 & 8 & 681 \\
\hline 7 & 9 & 681 \\
\hline 7 & 10 & 681 \\
\hline 8 & 1 & 1179 \\
\hline 8 & 2 & 1179 \\
\hline 8 & 3 & 1179 \\
\hline 8 & 4 & 1179 \\
\hline 8 & 5 & 1179 \\
\hline 8 & 6 & 1179 \\
\hline 8 & 7 & 1179 \\
\hline 8 & 8 & 1179 \\
\hline 8 & 9 & 1179 \\
\hline 8 & 10 & 1179 \\
\hline 9 & 1 & 4938 \\
\hline 9 & 2 & 4938 \\
\hline 9 & 3 & 4938 \\
\hline 9 & 4 & 4938 \\
\hline 9 & 5 & 4938 \\
\hline 9 & 6 & 4938 \\
\hline 9 & 7 & 4938 \\
\hline
\end{tabular}




\begin{tabular}{|c|c|c|}
\hline 9 & 8 & 4938 \\
\hline 9 & 9 & 4938 \\
\hline 9 & 10 & 4938 \\
\hline 10 & 1 & 417 \\
\hline 10 & 2 & 417 \\
\hline 10 & 3 & 417 \\
\hline 10 & 4 & 417 \\
\hline 10 & 5 & 417 \\
\hline 10 & 6 & 417 \\
\hline 10 & 7 & 417 \\
\hline 10 & 8 & 417 \\
\hline 10 & 9 & 417 \\
\hline 10 & 10 & 417 \\
\hline 11 & 1 & 258 \\
\hline 11 & 2 & 258 \\
\hline 11 & 3 & 258 \\
\hline 11 & 4 & 258 \\
\hline 11 & 5 & 258 \\
\hline 11 & 6 & 258 \\
\hline 11 & 7 & 258 \\
\hline 11 & 8 & 258 \\
\hline 11 & 9 & 258 \\
\hline 11 & 10 & 258 \\
\hline 12 & 1 & 930 \\
\hline 12 & 2 & 930 \\
\hline 12 & 3 & 930 \\
\hline 12 & 4 & 930 \\
\hline 12 & 5 & 930 \\
\hline 12 & 6 & 930 \\
\hline 12 & 7 & 930 \\
\hline 12 & 8 & 930 \\
\hline 12 & 9 & 930 \\
\hline 12 & 10 & 930 \\
\hline 13 & 1 & 232 \\
\hline 13 & 2 & 232 \\
\hline 13 & 3 & 232 \\
\hline 13 & 4 & 232 \\
\hline 13 & 5 & 232 \\
\hline 13 & 6 & 232 \\
\hline 13 & 7 & 232 \\
\hline 13 & 8 & 232 \\
\hline 13 & 9 & 232 \\
\hline 13 & 10 & 232 \\
\hline 14 & 1 & 218 \\
\hline 14 & 2 & 218 \\
\hline \multicolumn{3}{|c|}{247} \\
\hline
\end{tabular}




\begin{tabular}{|c|c|c|}
\hline 14 & 3 & 218 \\
\hline 14 & 4 & 218 \\
\hline 14 & 5 & 218 \\
\hline 14 & 6 & 218 \\
\hline 14 & 7 & 218 \\
\hline 14 & 8 & 218 \\
\hline 14 & 9 & 218 \\
\hline 14 & 10 & 218 \\
\hline 15 & 1 & 380 \\
\hline 15 & 2 & 380 \\
\hline 15 & 3 & 380 \\
\hline 15 & 4 & 380 \\
\hline 15 & 5 & 380 \\
\hline 15 & 6 & 380 \\
\hline 15 & 7 & 380 \\
\hline 15 & 8 & 380 \\
\hline 15 & 9 & 380 \\
\hline 15 & 10 & 380 \\
\hline 16 & 1 & 2560 \\
\hline 16 & 2 & 2560 \\
\hline 16 & 3 & 2560 \\
\hline 16 & 4 & 2560 \\
\hline 16 & 5 & 2560 \\
\hline 16 & 6 & 2560 \\
\hline 16 & 7 & 2560 \\
\hline 16 & 8 & 2560 \\
\hline 16 & 9 & 2560 \\
\hline 16 & 10 & 2560 \\
\hline 17 & 1 & 1502 \\
\hline 17 & 2 & 1502 \\
\hline 17 & 3 & 1502 \\
\hline 17 & 4 & 1502 \\
\hline 17 & 5 & 1502 \\
\hline 17 & 6 & 1502 \\
\hline 17 & 7 & 1502 \\
\hline 17 & 8 & 1502 \\
\hline 17 & 9 & 1502 \\
\hline 17 & 10 & 1502 \\
\hline 18 & 1 & 861 \\
\hline 18 & 2 & 861 \\
\hline 18 & 3 & 861 \\
\hline 18 & 4 & 861 \\
\hline 18 & 5 & 861 \\
\hline 18 & 6 & 861 \\
\hline 18 & 7 & 861 \\
\hline
\end{tabular}




\begin{tabular}{|c|c|c|}
\hline 18 & 8 & 861 \\
\hline 18 & 9 & 861 \\
\hline 18 & 10 & 861 \\
\hline 19 & 1 & 457 \\
\hline 19 & 2 & 457 \\
\hline 19 & 3 & 457 \\
\hline 19 & 4 & 457 \\
\hline 19 & 5 & 457 \\
\hline 19 & 6 & 457 \\
\hline 19 & 7 & 457 \\
\hline 19 & 8 & 457 \\
\hline 19 & 9 & 457 \\
\hline 19 & 10 & 457 \\
\hline 20 & 1 & 897 \\
\hline 20 & 2 & 897 \\
\hline 20 & 3 & 897 \\
\hline 20 & 4 & 897 \\
\hline 20 & 5 & 897 \\
\hline 20 & 6 & 897 \\
\hline 20 & 7 & 897 \\
\hline 20 & 8 & 897 \\
\hline 20 & 9 & 897 \\
\hline 20 & 10 & 897 \\
\hline 21 & 1 & 74316 \\
\hline 21 & 2 & 74316 \\
\hline 21 & 3 & 74316 \\
\hline 21 & 4 & 74316 \\
\hline 21 & 5 & 74316 \\
\hline 21 & 6 & 74316 \\
\hline 21 & 7 & 74316 \\
\hline 21 & 8 & 74316 \\
\hline 21 & 9 & 74316 \\
\hline 21 & 10 & 74316 \\
\hline 22 & 1 & 4451 \\
\hline 22 & 2 & 4451 \\
\hline 22 & 3 & 4451 \\
\hline 22 & 4 & 4451 \\
\hline 22 & 5 & 4451 \\
\hline 22 & 6 & 4451 \\
\hline 22 & 7 & 4451 \\
\hline 22 & 8 & 4451 \\
\hline 22 & 9 & 4451 \\
\hline 22 & 10 & 4451 \\
\hline 23 & 1 & 2221 \\
\hline 23 & 2 & 2221 \\
\hline
\end{tabular}




\begin{tabular}{|c|c|c|}
\hline 23 & 3 & 2221 \\
\hline 23 & 4 & 2221 \\
\hline 23 & 5 & 2221 \\
\hline 23 & 6 & 2221 \\
\hline 23 & 7 & 2221 \\
\hline 23 & 8 & 2221 \\
\hline 23 & 9 & 2221 \\
\hline 23 & 10 & 2221 \\
\hline 24 & 1 & 1727 \\
\hline 24 & 2 & 1727 \\
\hline 24 & 3 & 1727 \\
\hline 24 & 4 & 1727 \\
\hline 24 & 5 & 1727 \\
\hline 24 & 6 & 1727 \\
\hline 24 & 7 & 1727 \\
\hline 24 & 8 & 1727 \\
\hline 24 & 9 & 1727 \\
\hline 24 & 10 & 1727 \\
\hline 25 & 1 & 5751 \\
\hline 25 & 2 & 5751 \\
\hline 25 & 3 & 5751 \\
\hline 25 & 4 & 5751 \\
\hline 25 & 5 & 5751 \\
\hline 25 & 6 & 5751 \\
\hline 25 & 7 & 5751 \\
\hline 25 & 8 & 5751 \\
\hline 25 & 9 & 5751 \\
\hline 25 & 10 & 5751 \\
\hline 26 & 1 & 1517 \\
\hline 26 & 2 & 1517 \\
\hline 26 & 3 & 1517 \\
\hline 26 & 4 & 1517 \\
\hline 26 & 5 & 1517 \\
\hline 26 & 6 & 1517 \\
\hline 26 & 7 & 1517 \\
\hline 26 & 8 & 1517 \\
\hline 26 & 9 & 1517 \\
\hline 26 & 10 & 1517 \\
\hline 27 & 1 & 3258 \\
\hline 27 & 2 & 3258 \\
\hline 27 & 3 & 3258 \\
\hline 27 & 4 & 3258 \\
\hline 27 & 5 & 3258 \\
\hline 27 & 6 & 3258 \\
\hline 27 & 7 & 3258 \\
\hline
\end{tabular}




\begin{tabular}{|c|c|c|}
\hline 27 & 8 & 3258 \\
\hline 27 & 9 & 3258 \\
\hline 27 & 10 & 3258 \\
\hline 28 & 1 & 1041 \\
\hline 28 & 2 & 1041 \\
\hline 28 & 3 & 1041 \\
\hline 28 & 4 & 1041 \\
\hline 28 & 5 & 1041 \\
\hline 28 & 6 & 1041 \\
\hline 28 & 7 & 1041 \\
\hline 28 & 8 & 1041 \\
\hline 28 & 9 & 1041 \\
\hline 28 & 10 & 1041 \\
\hline 29 & 1 & 1404 \\
\hline 29 & 2 & 1404 \\
\hline 29 & 3 & 1404 \\
\hline 29 & 4 & 1404 \\
\hline 29 & 5 & 1404 \\
\hline 29 & 6 & 1404 \\
\hline 29 & 7 & 1404 \\
\hline 29 & 8 & 1404 \\
\hline 29 & 9 & 1404 \\
\hline 29 & 10 & 1404 \\
\hline 30 & 1 & 443 \\
\hline 30 & 2 & 443 \\
\hline 30 & 3 & 443 \\
\hline 30 & 4 & 443 \\
\hline 30 & 5 & 443 \\
\hline 30 & 6 & 443 \\
\hline 30 & 7 & 443 \\
\hline 30 & 8 & 443 \\
\hline 30 & 9 & 443 \\
\hline 30 & 10 & 443 \\
\hline 31 & 1 & 517 \\
\hline 31 & 2 & 517 \\
\hline 31 & 3 & 517 \\
\hline 31 & 4 & 517 \\
\hline 31 & 5 & 517 \\
\hline 31 & 6 & 517 \\
\hline 31 & 7 & 517 \\
\hline 31 & 8 & 517 \\
\hline 31 & 9 & 517 \\
\hline 31 & 10 & 517 \\
\hline 32 & 1 & 47562 \\
\hline 32 & 2 & 47562 \\
\hline
\end{tabular}




\begin{tabular}{|c|c|c|}
\hline 32 & 3 & 47562 \\
\hline 32 & 4 & 47562 \\
\hline 32 & 5 & 47562 \\
\hline 32 & 6 & 47562 \\
\hline 32 & 7 & 47562 \\
\hline 32 & 8 & 47562 \\
\hline 32 & 9 & 47562 \\
\hline 32 & 10 & 47562 \\
\hline 33 & 1 & 785 \\
\hline 33 & 2 & 785 \\
\hline 33 & 3 & 785 \\
\hline 33 & 4 & 785 \\
\hline 33 & 5 & 785 \\
\hline 33 & 6 & 785 \\
\hline 33 & 7 & 785 \\
\hline 33 & 8 & 785 \\
\hline 33 & 9 & 785 \\
\hline 33 & 10 & 785 \\
\hline 34 & 1 & 1087 \\
\hline 34 & 2 & 1087 \\
\hline 34 & 3 & 1087 \\
\hline 34 & 4 & 1087 \\
\hline 34 & 5 & 1087 \\
\hline 34 & 6 & 1087 \\
\hline 34 & 7 & 1087 \\
\hline 34 & 8 & 1087 \\
\hline 34 & 9 & 1087 \\
\hline 34 & 10 & 1087 \\
\hline 35 & 1 & 1532 \\
\hline 35 & 2 & 1532 \\
\hline 35 & 3 & 1532 \\
\hline 35 & 4 & 1532 \\
\hline 35 & 5 & 1532 \\
\hline 35 & 6 & 1532 \\
\hline 35 & 7 & 1532 \\
\hline 35 & 8 & 1532 \\
\hline 35 & 9 & 1532 \\
\hline 35 & 10 & 1532 \\
\hline 36 & 1 & 145159 \\
\hline 36 & 2 & 145159 \\
\hline 36 & 3 & 145159 \\
\hline 36 & 4 & 145159 \\
\hline 36 & 5 & 145159 \\
\hline 36 & 6 & 145159 \\
\hline 36 & 7 & 145159 \\
\hline
\end{tabular}




\begin{tabular}{|c|c|c|}
\hline 36 & 8 & 145159 \\
\hline 36 & 9 & 145159 \\
\hline 36 & 10 & 145159 \\
\hline 37 & 1 & 538 \\
\hline 37 & 2 & 538 \\
\hline 37 & 3 & 538 \\
\hline 37 & 4 & 538 \\
\hline 37 & 5 & 538 \\
\hline 37 & 6 & 538 \\
\hline 37 & 7 & 538 \\
\hline 37 & 8 & 538 \\
\hline 37 & 9 & 538 \\
\hline 37 & 10 & 538 \\
\hline 38 & 1 & 141 \\
\hline 38 & 2 & 141 \\
\hline 38 & 3 & 141 \\
\hline 38 & 4 & 141 \\
\hline 38 & 5 & 141 \\
\hline 38 & 6 & 141 \\
\hline 38 & 7 & 141 \\
\hline 38 & 8 & 141 \\
\hline 38 & 9 & 141 \\
\hline 38 & 10 & 141 \\
\hline 39 & 1 & 135 \\
\hline 39 & 2 & 135 \\
\hline 39 & 3 & 135 \\
\hline 39 & 4 & 135 \\
\hline 39 & 5 & 135 \\
\hline 39 & 6 & 135 \\
\hline 39 & 7 & 135 \\
\hline 39 & 8 & 135 \\
\hline 39 & 9 & 135 \\
\hline 39 & 10 & 135 \\
\hline 40 & 1 & 278 \\
\hline 40 & 2 & 278 \\
\hline 40 & 3 & 278 \\
\hline 40 & 4 & 278 \\
\hline 40 & 5 & 278 \\
\hline 40 & 6 & 278 \\
\hline 40 & 7 & 278 \\
\hline 40 & 8 & 278 \\
\hline 40 & 9 & 278 \\
\hline 40 & 10 & 278 \\
\hline 41 & 1 & 2520 \\
\hline 41 & 2 & 2520 \\
\hline
\end{tabular}




\begin{tabular}{|c|c|c|}
\hline 41 & 3 & 2520 \\
\hline 41 & 4 & 2520 \\
\hline 41 & 5 & 2520 \\
\hline 41 & 6 & 2520 \\
\hline 41 & 7 & 2520 \\
\hline 41 & 8 & 2520 \\
\hline 41 & 9 & 2520 \\
\hline 41 & 10 & 2520 \\
\hline 42 & 1 & 209 \\
\hline 42 & 2 & 209 \\
\hline 42 & 3 & 209 \\
\hline 42 & 4 & 209 \\
\hline 42 & 5 & 209 \\
\hline 42 & 6 & 209 \\
\hline 42 & 7 & 209 \\
\hline 42 & 8 & 209 \\
\hline 42 & 9 & 209 \\
\hline 42 & 10 & 209 \\
\hline 43 & 1 & 361 \\
\hline 43 & 2 & 361 \\
\hline 43 & 3 & 361 \\
\hline 43 & 4 & 361 \\
\hline 43 & 5 & 361 \\
\hline 43 & 6 & 361 \\
\hline 43 & 7 & 361 \\
\hline 43 & 8 & 361 \\
\hline 43 & 9 & 361 \\
\hline 43 & 10 & 361 \\
\hline 44 & 1 & 278 \\
\hline 44 & 2 & 278 \\
\hline 44 & 3 & 278 \\
\hline 44 & 4 & 278 \\
\hline 44 & 5 & 278 \\
\hline 44 & 6 & 278 \\
\hline 44 & 7 & 278 \\
\hline 44 & 8 & 278 \\
\hline 44 & 9 & 278 \\
\hline 44 & 10 & 278 \\
\hline 45 & 1 & 231 \\
\hline 45 & 2 & 231 \\
\hline 45 & 3 & 231 \\
\hline 45 & 4 & 231 \\
\hline 45 & 5 & 231 \\
\hline 45 & 6 & 231 \\
\hline 45 & 7 & 231 \\
\hline
\end{tabular}




\begin{tabular}{|c|c|c|}
\hline 45 & 8 & 231 \\
\hline 45 & 9 & 231 \\
\hline 45 & 10 & 231 \\
\hline 46 & 1 & 284 \\
\hline 46 & 2 & 284 \\
\hline 46 & 3 & 284 \\
\hline 46 & 4 & 284 \\
\hline 46 & 5 & 284 \\
\hline 46 & 6 & 284 \\
\hline 46 & 7 & 284 \\
\hline 46 & 8 & 284 \\
\hline 46 & 9 & 284 \\
\hline 46 & 10 & 284 \\
\hline 47 & 1 & 151 \\
\hline 47 & 2 & 151 \\
\hline 47 & 3 & 151 \\
\hline 47 & 4 & 151 \\
\hline 47 & 5 & 151 \\
\hline 47 & 6 & 151 \\
\hline 47 & 7 & 151 \\
\hline 47 & 8 & 151 \\
\hline 47 & 9 & 151 \\
\hline 47 & 10 & 151 \\
\hline 48 & 1 & 3442 \\
\hline 48 & 2 & 3442 \\
\hline 48 & 3 & 3442 \\
\hline 48 & 4 & 3442 \\
\hline 48 & 5 & 3442 \\
\hline 48 & 6 & 3442 \\
\hline 48 & 7 & 3442 \\
\hline 48 & 8 & 3442 \\
\hline 48 & 9 & 3442 \\
\hline 48 & 10 & 3442 \\
\hline 49 & 1 & 2360 \\
\hline 49 & 2 & 2360 \\
\hline 49 & 3 & 2360 \\
\hline 49 & 4 & 2360 \\
\hline 49 & 5 & 2360 \\
\hline 49 & 6 & 2360 \\
\hline 49 & 7 & 2360 \\
\hline 49 & 8 & 2360 \\
\hline 49 & 9 & 2360 \\
\hline 49 & 10 & 2360 \\
\hline 50 & 1 & 1479290 \\
\hline 50 & 2 & 1479290 \\
\hline
\end{tabular}




\begin{tabular}{|c|c|c|}
\hline 50 & 3 & 1479290 \\
\hline 50 & 4 & 1479290 \\
\hline 50 & 5 & 1479290 \\
\hline 50 & 6 & 1479290 \\
\hline 50 & 7 & 1479290 \\
\hline 50 & 8 & 1479290 \\
\hline 50 & 9 & 1479290 \\
\hline 50 & 10 & 1479290 \\
\hline 51 & 1 & 120 \\
\hline 51 & 2 & 120 \\
\hline 51 & 3 & 120 \\
\hline 51 & 4 & 120 \\
\hline 51 & 5 & 120 \\
\hline 51 & 6 & 120 \\
\hline 51 & 7 & 120 \\
\hline 51 & 8 & 120 \\
\hline 51 & 9 & 120 \\
\hline 51 & 10 & 120 \\
\hline 52 & 1 & 45654 \\
\hline 52 & 2 & 45654 \\
\hline 52 & 3 & 45654 \\
\hline 52 & 4 & 45654 \\
\hline 52 & 5 & 45654 \\
\hline 52 & 6 & 45654 \\
\hline 52 & 7 & 45654 \\
\hline 52 & 8 & 45654 \\
\hline 52 & 9 & 45654 \\
\hline 52 & 10 & 45654 \\
\hline 53 & 1 & 319 \\
\hline 53 & 2 & 319 \\
\hline 53 & 3 & 319 \\
\hline 53 & 4 & 319 \\
\hline 53 & 5 & 319 \\
\hline 53 & 6 & 319 \\
\hline 53 & 7 & 319 \\
\hline 53 & 8 & 319 \\
\hline 53 & 9 & 319 \\
\hline 53 & 10 & 319 \\
\hline 54 & 1 & 1783 \\
\hline 54 & 2 & 1783 \\
\hline 54 & 3 & 1783 \\
\hline 54 & 4 & 1783 \\
\hline 54 & 5 & 1783 \\
\hline 54 & 6 & 1783 \\
\hline 54 & 7 & 1783 \\
\hline
\end{tabular}




\begin{tabular}{|c|c|c|}
\hline 54 & 8 & 1783 \\
\hline 54 & 9 & 1783 \\
\hline 54 & 10 & 1783 \\
\hline 55 & 1 & 935 \\
\hline 55 & 2 & 935 \\
\hline 55 & 3 & 935 \\
\hline 55 & 4 & 935 \\
\hline 55 & 5 & 935 \\
\hline 55 & 6 & 935 \\
\hline 55 & 7 & 935 \\
\hline 55 & 8 & 935 \\
\hline 55 & 9 & 935 \\
\hline 55 & 10 & 935 \\
\hline 56 & 1 & 92 \\
\hline 56 & 2 & 92 \\
\hline 56 & 3 & 92 \\
\hline 56 & 4 & 92 \\
\hline 56 & 5 & 92 \\
\hline 56 & 6 & 92 \\
\hline 56 & 7 & 92 \\
\hline 56 & 8 & 92 \\
\hline 56 & 9 & 92 \\
\hline 56 & 10 & 92 \\
\hline 57 & 1 & 3507 \\
\hline 57 & 2 & 3507 \\
\hline 57 & 3 & 3507 \\
\hline 57 & 4 & 3507 \\
\hline 57 & 5 & 3507 \\
\hline 57 & 6 & 3507 \\
\hline 57 & 7 & 3507 \\
\hline 57 & 8 & 3507 \\
\hline 57 & 9 & 3507 \\
\hline 57 & 10 & 3507 \\
\hline 58 & 1 & 4322 \\
\hline 58 & 2 & 4322 \\
\hline 58 & 3 & 4322 \\
\hline 58 & 4 & 4322 \\
\hline 58 & 5 & 4322 \\
\hline 58 & 6 & 4322 \\
\hline 58 & 7 & 4322 \\
\hline 58 & 8 & 4322 \\
\hline 58 & 9 & 4322 \\
\hline 58 & 10 & 4322 \\
\hline 59 & 1 & 748 \\
\hline 59 & 2 & 748 \\
\hline
\end{tabular}




\begin{tabular}{|c|c|c|}
\hline 59 & 3 & 748 \\
\hline 59 & 4 & 748 \\
\hline 59 & 5 & 748 \\
\hline 59 & 6 & 748 \\
\hline 59 & 7 & 748 \\
\hline 59 & 8 & 748 \\
\hline 59 & 9 & 748 \\
\hline 59 & 10 & 748 \\
\hline 60 & 1 & 205 \\
\hline 60 & 2 & 205 \\
\hline 60 & 3 & 205 \\
\hline 60 & 4 & 205 \\
\hline 60 & 5 & 205 \\
\hline 60 & 6 & 205 \\
\hline 60 & 7 & 205 \\
\hline 60 & 8 & 205 \\
\hline 60 & 9 & 205 \\
\hline 60 & 10 & 205 \\
\hline 61 & 1 & 124 \\
\hline 61 & 2 & 124 \\
\hline 61 & 3 & 124 \\
\hline 61 & 4 & 124 \\
\hline 61 & 5 & 124 \\
\hline 61 & 6 & 124 \\
\hline 61 & 7 & 124 \\
\hline 61 & 8 & 124 \\
\hline 61 & 9 & 124 \\
\hline 61 & 10 & 124 \\
\hline 62 & 1 & 249 \\
\hline 62 & 2 & 249 \\
\hline 62 & 3 & 249 \\
\hline 62 & 4 & 249 \\
\hline 62 & 5 & 249 \\
\hline 62 & 6 & 249 \\
\hline 62 & 7 & 249 \\
\hline 62 & 8 & 249 \\
\hline 62 & 9 & 249 \\
\hline 62 & 10 & 249 \\
\hline 63 & 1 & 1826 \\
\hline 63 & 2 & 1826 \\
\hline 63 & 3 & 1826 \\
\hline 63 & 4 & 1826 \\
\hline 63 & 5 & 1826 \\
\hline 63 & 6 & 1826 \\
\hline 63 & 7 & 1826 \\
\hline
\end{tabular}




\begin{tabular}{|c|c|c|}
\hline 63 & 8 & 1826 \\
\hline 63 & 9 & 1826 \\
\hline 63 & 10 & 1826 \\
\hline 64 & 1 & 1851 \\
\hline 64 & 2 & 1851 \\
\hline 64 & 3 & 1851 \\
\hline 64 & 4 & 1851 \\
\hline 64 & 5 & 1851 \\
\hline 64 & 6 & 1851 \\
\hline 64 & 7 & 1851 \\
\hline 64 & 8 & 1851 \\
\hline 64 & 9 & 1851 \\
\hline 64 & 10 & 1851 \\
\hline 65 & 1 & 1547 \\
\hline 65 & 2 & 1547 \\
\hline 65 & 3 & 1547 \\
\hline 65 & 4 & 1547 \\
\hline 65 & 5 & 1547 \\
\hline 65 & 6 & 1547 \\
\hline 65 & 7 & 1547 \\
\hline 65 & 8 & 1547 \\
\hline 65 & 9 & 1547 \\
\hline 65 & 10 & 1547 \\
\hline 66 & 1 & 2750 \\
\hline 66 & 2 & 2750 \\
\hline 66 & 3 & 2750 \\
\hline 66 & 4 & 2750 \\
\hline 66 & 5 & 2750 \\
\hline 66 & 6 & 2750 \\
\hline 66 & 7 & 2750 \\
\hline 66 & 8 & 2750 \\
\hline 66 & 9 & 2750 \\
\hline 66 & 10 & 2750 \\
\hline 67 & 1 & 140 \\
\hline 67 & 2 & 140 \\
\hline 67 & 3 & 140 \\
\hline 67 & 4 & 140 \\
\hline 67 & 5 & 140 \\
\hline 67 & 6 & 140 \\
\hline 67 & 7 & 140 \\
\hline 67 & 8 & 140 \\
\hline 67 & 9 & 140 \\
\hline 67 & 10 & 140 \\
\hline 68 & 1 & 362 \\
\hline 68 & 2 & 362 \\
\hline
\end{tabular}




\begin{tabular}{|c|c|c|}
\hline 68 & 3 & 362 \\
\hline 68 & 4 & 362 \\
\hline 68 & 5 & 362 \\
\hline 68 & 6 & 362 \\
\hline 68 & 7 & 362 \\
\hline 68 & 8 & 362 \\
\hline 68 & 9 & 362 \\
\hline 68 & 10 & 362 \\
\hline 69 & 1 & 1866 \\
\hline 69 & 2 & 1866 \\
\hline 69 & 3 & 1866 \\
\hline 69 & 4 & 1866 \\
\hline 69 & 5 & 1866 \\
\hline 69 & 6 & 1866 \\
\hline 69 & 7 & 1866 \\
\hline 69 & 8 & 1866 \\
\hline 69 & 9 & 1866 \\
\hline 69 & 10 & 1866 \\
\hline 70 & 1 & 3587 \\
\hline 70 & 2 & 3587 \\
\hline 70 & 3 & 3587 \\
\hline 70 & 4 & 3587 \\
\hline 70 & 5 & 3587 \\
\hline 70 & 6 & 3587 \\
\hline 70 & 7 & 3587 \\
\hline 70 & 8 & 3587 \\
\hline 70 & 9 & 3587 \\
\hline 70 & 10 & 3587 \\
\hline 71 & 1 & 1171 \\
\hline 71 & 2 & 1171 \\
\hline 71 & 3 & 1171 \\
\hline 71 & 4 & 1171 \\
\hline 71 & 5 & 1171 \\
\hline 71 & 6 & 1171 \\
\hline 71 & 7 & 1171 \\
\hline 71 & 8 & 1171 \\
\hline 71 & 9 & 1171 \\
\hline 71 & 10 & 1171 \\
\hline 72 & 1 & 298 \\
\hline 72 & 2 & 298 \\
\hline 72 & 3 & 298 \\
\hline 72 & 4 & 298 \\
\hline 72 & 5 & 298 \\
\hline 72 & 6 & 298 \\
\hline 72 & 7 & 298 \\
\hline
\end{tabular}




\begin{tabular}{|c|c|c|}
\hline 72 & 8 & 298 \\
\hline 72 & 9 & 298 \\
\hline 72 & 10 & 298 \\
\hline 73 & 1 & 7111 \\
\hline 73 & 2 & 7111 \\
\hline 73 & 3 & 7111 \\
\hline 73 & 4 & 7111 \\
\hline 73 & 5 & 7111 \\
\hline 73 & 6 & 7111 \\
\hline 73 & 7 & 7111 \\
\hline 73 & 8 & 7111 \\
\hline 73 & 9 & 7111 \\
\hline 73 & 10 & 7111 \\
\hline 74 & 1 & 822 \\
\hline 74 & 2 & 822 \\
\hline 74 & 3 & 822 \\
\hline 74 & 4 & 822 \\
\hline 74 & 5 & 822 \\
\hline 74 & 6 & 822 \\
\hline 74 & 7 & 822 \\
\hline 74 & 8 & 822 \\
\hline 74 & 9 & 822 \\
\hline 74 & 10 & 822 \\
\hline 75 & 1 & 608 \\
\hline 75 & 2 & 608 \\
\hline 75 & 3 & 608 \\
\hline 75 & 4 & 608 \\
\hline 75 & 5 & 608 \\
\hline 75 & 6 & 608 \\
\hline 75 & 7 & 608 \\
\hline 75 & 8 & 608 \\
\hline 75 & 9 & 608 \\
\hline 75 & 10 & 608 \\
\hline 76 & 1 & 202 \\
\hline 76 & 2 & 202 \\
\hline 76 & 3 & 202 \\
\hline 76 & 4 & 202 \\
\hline 76 & 5 & 202 \\
\hline 76 & 6 & 202 \\
\hline 76 & 7 & 202 \\
\hline 76 & 8 & 202 \\
\hline 76 & 9 & 202 \\
\hline 76 & 10 & 202 \\
\hline 77 & 1 & 231 \\
\hline 77 & 2 & 231 \\
\hline
\end{tabular}




\begin{tabular}{|c|c|c|}
\hline 77 & 3 & 231 \\
\hline 77 & 4 & 231 \\
\hline 77 & 5 & 231 \\
\hline 77 & 6 & 231 \\
\hline 77 & 7 & 231 \\
\hline 77 & 8 & 231 \\
\hline 77 & 9 & 231 \\
\hline 77 & 10 & 231 \\
\hline 78 & 1 & 5102041 \\
\hline 78 & 2 & 5102041 \\
\hline 78 & 3 & 5102041 \\
\hline 78 & 4 & 5102041 \\
\hline 78 & 5 & 5102041 \\
\hline 78 & 6 & 5102041 \\
\hline 78 & 7 & 5102041 \\
\hline 78 & 8 & 5102041 \\
\hline 78 & 9 & 5102041 \\
\hline 78 & 10 & 5102041 \\
\hline 79 & 1 & 462 \\
\hline 79 & 2 & 462 \\
\hline 79 & 3 & 462 \\
\hline 79 & 4 & 462 \\
\hline 79 & 5 & 462 \\
\hline 79 & 6 & 462 \\
\hline 79 & 7 & 462 \\
\hline 79 & 8 & 462 \\
\hline 79 & 9 & 462 \\
\hline 79 & 10 & 462 \\
\hline 80 & 1 & 978 \\
\hline 80 & 2 & 978 \\
\hline 80 & 3 & 978 \\
\hline 80 & 4 & 978 \\
\hline 80 & 5 & 978 \\
\hline 80 & 6 & 978 \\
\hline 80 & 7 & 978 \\
\hline 80 & 8 & 978 \\
\hline 80 & 9 & 978 \\
\hline 80 & 10 & 978 \\
\hline 81 & 1 & 564 \\
\hline 81 & 2 & 564 \\
\hline 81 & 3 & 564 \\
\hline 81 & 4 & 564 \\
\hline 81 & 5 & 564 \\
\hline 81 & 6 & 564 \\
\hline 81 & 7 & 564 \\
\hline
\end{tabular}




\begin{tabular}{|c|c|c|}
\hline 81 & 8 & 564 \\
\hline 81 & 9 & 564 \\
\hline 81 & 10 & 564 \\
\hline 82 & 1 & 564 \\
\hline 82 & 2 & 564 \\
\hline 82 & 3 & 564 \\
\hline 82 & 4 & 564 \\
\hline 82 & 5 & 564 \\
\hline 82 & 6 & 564 \\
\hline 82 & 7 & 564 \\
\hline 82 & 8 & 564 \\
\hline 82 & 9 & 564 \\
\hline 82 & 10 & 564 \\
\hline 83 & 1 & 978 \\
\hline 83 & 2 & 978 \\
\hline 83 & 3 & 978 \\
\hline 83 & 4 & 978 \\
\hline 83 & 5 & 978 \\
\hline 83 & 6 & 978 \\
\hline 83 & 7 & 978 \\
\hline 83 & 8 & 978 \\
\hline 83 & 9 & 978 \\
\hline 83 & 10 & 978 \\
\hline 84 & 1 & 1424 \\
\hline 84 & 2 & 1424 \\
\hline 84 & 3 & 1424 \\
\hline 84 & 4 & 1424 \\
\hline 84 & 5 & 1424 \\
\hline 84 & 6 & 1424 \\
\hline 84 & 7 & 1424 \\
\hline 84 & 8 & 1424 \\
\hline 84 & 9 & 1424 \\
\hline 84 & 10 & 1424 \\
\hline 85 & 1 & 271 \\
\hline 85 & 2 & 271 \\
\hline 85 & 3 & 271 \\
\hline 85 & 4 & 271 \\
\hline 85 & 5 & 271 \\
\hline 85 & 6 & 271 \\
\hline 85 & 7 & 271 \\
\hline 85 & 8 & 271 \\
\hline 85 & 9 & 271 \\
\hline 85 & 10 & 271 \\
\hline 86 & 1 & 156 \\
\hline 86 & 2 & 156 \\
\hline
\end{tabular}




\begin{tabular}{|c|c|c|}
\hline 86 & 3 & 156 \\
\hline 86 & 4 & 156 \\
\hline 86 & 5 & 156 \\
\hline 86 & 6 & 156 \\
\hline 86 & 7 & 156 \\
\hline 86 & 8 & 156 \\
\hline 86 & 9 & 156 \\
\hline 86 & 10 & 156 \\
\hline 87 & 1 & 1438 \\
\hline 87 & 2 & 1438 \\
\hline 87 & 3 & 1438 \\
\hline 87 & 4 & 1438 \\
\hline 87 & 5 & 1438 \\
\hline 87 & 6 & 1438 \\
\hline 87 & 7 & 1438 \\
\hline 87 & 8 & 1438 \\
\hline 87 & 9 & 1438 \\
\hline 87 & 10 & 1438 \\
\hline 88 & 1 & 8071 \\
\hline 88 & 2 & 8071 \\
\hline 88 & 3 & 8071 \\
\hline 88 & 4 & 8071 \\
\hline 88 & 5 & 8071 \\
\hline 88 & 6 & 8071 \\
\hline 88 & 7 & 8071 \\
\hline 88 & 8 & 8071 \\
\hline 88 & 9 & 8071 \\
\hline 88 & 10 & 8071 \\
\hline 89 & 1 & 3815 \\
\hline 89 & 2 & 3815 \\
\hline 89 & 3 & 3815 \\
\hline 89 & 4 & 3815 \\
\hline 89 & 5 & 3815 \\
\hline 89 & 6 & 3815 \\
\hline 89 & 7 & 3815 \\
\hline 89 & 8 & 3815 \\
\hline 89 & 9 & 3815 \\
\hline 89 & 10 & 3815 \\
\hline 90 & 1 & 604 \\
\hline 90 & 2 & 604 \\
\hline 90 & 3 & 604 \\
\hline 90 & 4 & 604 \\
\hline 90 & 5 & 604 \\
\hline 90 & 6 & 604 \\
\hline 90 & 7 & 604 \\
\hline
\end{tabular}




\begin{tabular}{|c|c|c|}
\hline 90 & 8 & 604 \\
\hline 90 & 9 & 604 \\
\hline 90 & 10 & 604 \\
\hline 91 & 1 & 1035 \\
\hline 91 & 2 & 1035 \\
\hline 91 & 3 & 1035 \\
\hline 91 & 4 & 1035 \\
\hline 91 & 5 & 1035 \\
\hline 91 & 6 & 1035 \\
\hline 91 & 7 & 1035 \\
\hline 91 & 8 & 1035 \\
\hline 91 & 9 & 1035 \\
\hline 91 & 10 & 1035 \\
\hline 92 & 1 & 3698 \\
\hline 92 & 2 & 3698 \\
\hline 92 & 3 & 3698 \\
\hline 92 & 4 & 3698 \\
\hline 92 & 5 & 3698 \\
\hline 92 & 6 & 3698 \\
\hline 92 & 7 & 3698 \\
\hline 92 & 8 & 3698 \\
\hline 92 & 9 & 3698 \\
\hline 92 & 10 & 3698 \\
\hline 93 & 1 & 1740 \\
\hline 93 & 2 & 1740 \\
\hline 93 & 3 & 1740 \\
\hline 93 & 4 & 1740 \\
\hline 93 & 5 & 1740 \\
\hline 93 & 6 & 1740 \\
\hline 93 & 7 & 1740 \\
\hline 93 & 8 & 1740 \\
\hline 93 & 9 & 1740 \\
\hline 93 & 10 & 1740 \\
\hline 94 & 1 & 452 \\
\hline 94 & 2 & 452 \\
\hline 94 & 3 & 452 \\
\hline 94 & 4 & 452 \\
\hline 94 & 5 & 452 \\
\hline 94 & 6 & 452 \\
\hline 94 & 7 & 452 \\
\hline 94 & 8 & 452 \\
\hline 94 & 9 & 452 \\
\hline 94 & 10 & 452 \\
\hline 95 & 1 & 703 \\
\hline 95 & 2 & 703 \\
\hline
\end{tabular}




\begin{tabular}{|c|c|c|}
\hline 95 & 3 & 703 \\
\hline 95 & 4 & 703 \\
\hline 95 & 5 & 703 \\
\hline 95 & 6 & 703 \\
\hline 95 & 7 & 703 \\
\hline 95 & 8 & 703 \\
\hline 95 & 9 & 703 \\
\hline 95 & 10 & 703 \\
\hline 96 & 1 & 8117 \\
\hline 96 & 2 & 8117 \\
\hline 96 & 3 & 8117 \\
\hline 96 & 4 & 8117 \\
\hline 96 & 5 & 8117 \\
\hline 96 & 6 & 8117 \\
\hline 96 & 7 & 8117 \\
\hline 96 & 8 & 8117 \\
\hline 96 & 9 & 8117 \\
\hline 96 & 10 & 8117 \\
\hline 97 & 1 & 18579 \\
\hline 97 & 2 & 18579 \\
\hline 97 & 3 & 18579 \\
\hline 97 & 4 & 18579 \\
\hline 97 & 5 & 18579 \\
\hline 97 & 6 & 18579 \\
\hline 97 & 7 & 18579 \\
\hline 97 & 8 & 18579 \\
\hline 97 & 9 & 18579 \\
\hline 97 & 10 & 18579 \\
\hline 98 & 1 & 354 \\
\hline 98 & 2 & 354 \\
\hline 98 & 3 & 354 \\
\hline 98 & 4 & 354 \\
\hline 98 & 5 & 354 \\
\hline 98 & 6 & 354 \\
\hline 98 & 7 & 354 \\
\hline 98 & 8 & 354 \\
\hline 98 & 9 & 354 \\
\hline 98 & 10 & 354 \\
\hline 99 & 1 & 3258 \\
\hline 99 & 2 & 3258 \\
\hline 99 & 3 & 3258 \\
\hline 99 & 4 & 3258 \\
\hline 99 & 5 & 3258 \\
\hline 99 & 6 & 3258 \\
\hline 99 & 7 & 3258 \\
\hline
\end{tabular}




\begin{tabular}{|c|c|c|}
\hline 99 & 8 & 3258 \\
\hline 99 & 9 & 3258 \\
\hline 99 & 10 & 3258 \\
\hline 100 & 1 & 1669 \\
\hline 100 & 2 & 1669 \\
\hline 100 & 3 & 1669 \\
\hline 100 & 4 & 1669 \\
\hline 100 & 5 & 1669 \\
\hline 100 & 6 & 1669 \\
\hline 100 & 7 & 1669 \\
\hline 100 & 8 & 1669 \\
\hline 100 & 9 & 1669 \\
\hline 100 & 10 & 1669 \\
\hline 101 & 1 & 919 \\
\hline 101 & 2 & 919 \\
\hline 101 & 3 & 919 \\
\hline 101 & 4 & 919 \\
\hline 101 & 5 & 919 \\
\hline 101 & 6 & 919 \\
\hline 101 & 7 & 919 \\
\hline 101 & 8 & 919 \\
\hline 101 & 9 & 919 \\
\hline 101 & 10 & 919 \\
\hline 102 & 1 & 4269 \\
\hline 102 & 2 & 4269 \\
\hline 102 & 3 & 4269 \\
\hline 102 & 4 & 4269 \\
\hline 102 & 5 & 4269 \\
\hline 102 & 6 & 4269 \\
\hline 102 & 7 & 4269 \\
\hline 102 & 8 & 4269 \\
\hline 102 & 9 & 4269 \\
\hline 102 & 10 & 4269 \\
\hline 103 & 1 & 32 \\
\hline 103 & 2 & 32 \\
\hline 103 & 3 & 32 \\
\hline 103 & 4 & 32 \\
\hline 103 & 5 & 32 \\
\hline 103 & 6 & 32 \\
\hline 103 & 7 & 32 \\
\hline 103 & 8 & 32 \\
\hline 103 & 9 & 32 \\
\hline 103 & 10 & 32 \\
\hline 104 & 1 & 101 \\
\hline 104 & 2 & 101 \\
\hline \\
\hline
\end{tabular}




\begin{tabular}{|c|c|c|}
\hline 104 & 3 & 101 \\
\hline 104 & 4 & 101 \\
\hline 104 & 5 & 101 \\
\hline 104 & 6 & 101 \\
\hline 104 & 7 & 101 \\
\hline 104 & 8 & 101 \\
\hline 104 & 9 & 101 \\
\hline 104 & 10 & 101 \\
\hline 105 & 1 & 493827 \\
\hline 105 & 2 & 493827 \\
\hline 105 & 3 & 493827 \\
\hline 105 & 4 & 493827 \\
\hline 105 & 5 & 493827 \\
\hline 105 & 6 & 493827 \\
\hline 105 & 7 & 493827 \\
\hline 105 & 8 & 493827 \\
\hline 105 & 9 & 493827 \\
\hline 105 & 10 & 493827 \\
\hline 106 & 1 & 877 \\
\hline 106 & 2 & 877 \\
\hline 106 & 3 & 877 \\
\hline 106 & 4 & 877 \\
\hline 106 & 5 & 877 \\
\hline 106 & 6 & 877 \\
\hline 106 & 7 & 877 \\
\hline 106 & 8 & 877 \\
\hline 106 & 9 & 877 \\
\hline 106 & 10 & 877 \\
\hline 107 & 1 & 174 \\
\hline 107 & 2 & 174 \\
\hline 107 & 3 & 174 \\
\hline 107 & 4 & 174 \\
\hline 107 & 5 & 174 \\
\hline 107 & 6 & 174 \\
\hline 107 & 7 & 174 \\
\hline 107 & 8 & 174 \\
\hline 107 & 9 & 174 \\
\hline 107 & 10 & 174 \\
\hline 108 & 1 & 227 \\
\hline 108 & 2 & 227 \\
\hline 108 & 3 & 227 \\
\hline 108 & 4 & 227 \\
\hline 108 & 5 & 227 \\
\hline 108 & 6 & 227 \\
\hline 108 & 7 & 227 \\
\hline
\end{tabular}




\begin{tabular}{|c|c|c|}
\hline 108 & 8 & 227 \\
\hline 108 & 9 & 227 \\
\hline 108 & 10 & 227 \\
\hline 109 & 1 & 1397 \\
\hline 109 & 2 & 1397 \\
\hline 109 & 3 & 1397 \\
\hline 109 & 4 & 1397 \\
\hline 109 & 5 & 1397 \\
\hline 109 & 6 & 1397 \\
\hline 109 & 7 & 1397 \\
\hline 109 & 8 & 1397 \\
\hline 109 & 9 & 1397 \\
\hline 109 & 10 & 1397 \\
\hline 110 & 1 & 539 \\
\hline 110 & 2 & 539 \\
\hline 110 & 3 & 539 \\
\hline 110 & 4 & 539 \\
\hline 110 & 5 & 539 \\
\hline 110 & 6 & 539 \\
\hline 110 & 7 & 539 \\
\hline 110 & 8 & 539 \\
\hline 110 & 9 & 539 \\
\hline 110 & 10 & 539 \\
\hline 111 & 1 & 1821 \\
\hline 111 & 2 & 1821 \\
\hline 111 & 3 & 1821 \\
\hline 111 & 4 & 1821 \\
\hline 111 & 5 & 1821 \\
\hline 111 & 6 & 1821 \\
\hline 111 & 7 & 1821 \\
\hline 111 & 8 & 1821 \\
\hline 111 & 9 & 1821 \\
\hline 111 & 10 & 1821 \\
\hline 112 & 1 & 7846 \\
\hline 112 & 2 & 7846 \\
\hline 112 & 3 & 7846 \\
\hline 112 & 4 & 7846 \\
\hline 112 & 5 & 7846 \\
\hline 112 & 6 & 7846 \\
\hline 112 & 7 & 7846 \\
\hline 112 & 8 & 7846 \\
\hline 112 & 9 & 7846 \\
\hline 112 & 10 & 7846 \\
\hline 113 & 1 & 603 \\
\hline 113 & 2 & 603 \\
\hline \multicolumn{3}{|c|}{269} \\
\hline
\end{tabular}




\begin{tabular}{|c|c|c|}
\hline 113 & 3 & 603 \\
\hline 113 & 4 & 603 \\
\hline 113 & 5 & 603 \\
\hline 113 & 6 & 603 \\
\hline 113 & 7 & 603 \\
\hline 113 & 8 & 603 \\
\hline 113 & 9 & 603 \\
\hline 113 & 10 & 603 \\
\hline 114 & 1 & 632 \\
\hline 114 & 2 & 632 \\
\hline 114 & 3 & 632 \\
\hline 114 & 4 & 632 \\
\hline 114 & 5 & 632 \\
\hline 114 & 6 & 632 \\
\hline 114 & 7 & 632 \\
\hline 114 & 8 & 632 \\
\hline 114 & 9 & 632 \\
\hline 114 & 10 & 632 \\
\hline 115 & 1 & 1539 \\
\hline 115 & 2 & 1539 \\
\hline 115 & 3 & 1539 \\
\hline 115 & 4 & 1539 \\
\hline 115 & 5 & 1539 \\
\hline 115 & 6 & 1539 \\
\hline 115 & 7 & 1539 \\
\hline 115 & 8 & 1539 \\
\hline 115 & 9 & 1539 \\
\hline 115 & 10 & 1539 \\
\hline 116 & 1 & 2661 \\
\hline 116 & 2 & 2661 \\
\hline 116 & 3 & 2661 \\
\hline 116 & 4 & 2661 \\
\hline 116 & 5 & 2661 \\
\hline 116 & 6 & 2661 \\
\hline 116 & 7 & 2661 \\
\hline 116 & 8 & 2661 \\
\hline 116 & 9 & 2661 \\
\hline 116 & 10 & 2661 \\
\hline 117 & 1 & 233 \\
\hline 117 & 2 & 233 \\
\hline 117 & 3 & 233 \\
\hline 117 & 4 & 233 \\
\hline 117 & 5 & 233 \\
\hline 117 & 6 & 233 \\
\hline 117 & 7 & 233 \\
\hline
\end{tabular}




\begin{tabular}{|c|c|c|}
\hline 117 & 8 & 233 \\
\hline 117 & 9 & 233 \\
\hline 117 & 10 & 233 \\
\hline 118 & 1 & 293 \\
\hline 118 & 2 & 293 \\
\hline 118 & 3 & 293 \\
\hline 118 & 4 & 293 \\
\hline 118 & 5 & 293 \\
\hline 118 & 6 & 293 \\
\hline 118 & 7 & 293 \\
\hline 118 & 8 & 293 \\
\hline 118 & 9 & 293 \\
\hline 118 & 10 & 293 \\
\hline 119 & 1 & 9352 \\
\hline 119 & 2 & 9352 \\
\hline 119 & 3 & 9352 \\
\hline 119 & 4 & 9352 \\
\hline 119 & 5 & 9352 \\
\hline 119 & 6 & 9352 \\
\hline 119 & 7 & 9352 \\
\hline 119 & 8 & 9352 \\
\hline 119 & 9 & 9352 \\
\hline 119 & 10 & 9352 \\
\hline 120 & 1 & 1524 \\
\hline 120 & 2 & 1524 \\
\hline 120 & 3 & 1524 \\
\hline 120 & 4 & 1524 \\
\hline 120 & 5 & 1524 \\
\hline 120 & 6 & 1524 \\
\hline 120 & 7 & 1524 \\
\hline 120 & 8 & 1524 \\
\hline 120 & 9 & 1524 \\
\hline 120 & 10 & 1524 \\
\hline 121 & 1 & 757 \\
\hline 121 & 2 & 757 \\
\hline 121 & 3 & 757 \\
\hline 121 & 4 & 757 \\
\hline 121 & 5 & 757 \\
\hline 121 & 6 & 757 \\
\hline 121 & 7 & 757 \\
\hline 121 & 8 & 757 \\
\hline 121 & 9 & 757 \\
\hline 121 & 10 & 757 \\
\hline 122 & 1 & 129 \\
\hline 122 & 2 & 129 \\
\hline \multicolumn{3}{|c|}{271} \\
\hline
\end{tabular}




\begin{tabular}{|c|c|c|}
\hline 122 & 3 & 129 \\
\hline 122 & 4 & 129 \\
\hline 122 & 5 & 129 \\
\hline 122 & 6 & 129 \\
\hline 122 & 7 & 129 \\
\hline 122 & 8 & 129 \\
\hline 122 & 9 & 129 \\
\hline 122 & 10 & 129 \\
\hline 123 & 1 & 73 \\
\hline 123 & 2 & 73 \\
\hline 123 & 3 & 73 \\
\hline 123 & 4 & 73 \\
\hline 123 & 5 & 73 \\
\hline 123 & 6 & 73 \\
\hline 123 & 7 & 73 \\
\hline 123 & 8 & 73 \\
\hline 123 & 9 & 73 \\
\hline 123 & 10 & 73 \\
\hline 124 & 1 & 80 \\
\hline 124 & 2 & 80 \\
\hline 124 & 3 & 80 \\
\hline 124 & 4 & 80 \\
\hline 124 & 5 & 80 \\
\hline 124 & 6 & 80 \\
\hline 124 & 7 & 80 \\
\hline 124 & 8 & 80 \\
\hline 124 & 9 & 80 \\
\hline 124 & 10 & 80 \\
\hline 125 & 1 & 128 \\
\hline 125 & 2 & 128 \\
\hline 125 & 3 & 128 \\
\hline 125 & 4 & 128 \\
\hline 125 & 5 & 128 \\
\hline 125 & 6 & 128 \\
\hline 125 & 7 & 128 \\
\hline 125 & 8 & 128 \\
\hline 125 & 9 & 128 \\
\hline 125 & 10 & 128 \\
\hline 126 & 1 & 437 \\
\hline 126 & 2 & 437 \\
\hline 126 & 3 & 437 \\
\hline 126 & 4 & 437 \\
\hline 126 & 5 & 437 \\
\hline 126 & 6 & 437 \\
\hline 126 & 7 & 437 \\
\hline
\end{tabular}




\begin{tabular}{|c|c|c|}
\hline 126 & 8 & 437 \\
\hline 126 & 9 & 437 \\
\hline 126 & 10 & 437 \\
\hline 127 & 1 & 3520 \\
\hline 127 & 2 & 3520 \\
\hline 127 & 3 & 3520 \\
\hline 127 & 4 & 3520 \\
\hline 127 & 5 & 3520 \\
\hline 127 & 6 & 3520 \\
\hline 127 & 7 & 3520 \\
\hline 127 & 8 & 3520 \\
\hline 127 & 9 & 3520 \\
\hline 127 & 10 & 3520 \\
\hline 128 & 1 & 5358 \\
\hline 128 & 2 & 5358 \\
\hline 128 & 3 & 5358 \\
\hline 128 & 4 & 5358 \\
\hline 128 & 5 & 5358 \\
\hline 128 & 6 & 5358 \\
\hline 128 & 7 & 5358 \\
\hline 128 & 8 & 5358 \\
\hline 128 & 9 & 5358 \\
\hline 128 & 10 & 5358 \\
\hline 129 & 1 & 4665 \\
\hline 129 & 2 & 4665 \\
\hline 129 & 3 & 4665 \\
\hline 129 & 4 & 4665 \\
\hline 129 & 5 & 4665 \\
\hline 129 & 6 & 4665 \\
\hline 129 & 7 & 4665 \\
\hline 129 & 8 & 4665 \\
\hline 129 & 9 & 4665 \\
\hline 129 & 10 & 4665 \\
\hline 130 & 1 & 158 \\
\hline 130 & 2 & 158 \\
\hline 130 & 3 & 158 \\
\hline 130 & 4 & 158 \\
\hline 130 & 5 & 158 \\
\hline 130 & 6 & 158 \\
\hline 130 & 7 & 158 \\
\hline 130 & 8 & 158 \\
\hline 130 & 9 & 158 \\
\hline 130 & 10 & 158 \\
\hline 131 & 1 & 187 \\
\hline 131 & 2 & 187 \\
\hline \multicolumn{3}{|c|}{273} \\
\hline
\end{tabular}




\begin{tabular}{|c|c|c|}
\hline 131 & 3 & 187 \\
\hline 131 & 4 & 187 \\
\hline 131 & 5 & 187 \\
\hline 131 & 6 & 187 \\
\hline 131 & 7 & 187 \\
\hline 131 & 8 & 187 \\
\hline 131 & 9 & 187 \\
\hline 131 & 10 & 187 \\
\hline 132 & 1 & 244 \\
\hline 132 & 2 & 244 \\
\hline 132 & 3 & 244 \\
\hline 132 & 4 & 244 \\
\hline 132 & 5 & 244 \\
\hline 132 & 6 & 244 \\
\hline 132 & 7 & 244 \\
\hline 132 & 8 & 244 \\
\hline 132 & 9 & 244 \\
\hline 132 & 10 & 244 \\
\hline 133 & 1 & 255 \\
\hline 133 & 2 & 255 \\
\hline 133 & 3 & 255 \\
\hline 133 & 4 & 255 \\
\hline 133 & 5 & 255 \\
\hline 133 & 6 & 255 \\
\hline 133 & 7 & 255 \\
\hline 133 & 8 & 255 \\
\hline 133 & 9 & 255 \\
\hline 133 & 10 & 255 \\
\hline 134 & 1 & 623 \\
\hline 134 & 2 & 623 \\
\hline 134 & 3 & 623 \\
\hline 134 & 4 & 623 \\
\hline 134 & 5 & 623 \\
\hline 134 & 6 & 623 \\
\hline 134 & 7 & 623 \\
\hline 134 & 8 & 623 \\
\hline 134 & 9 & 623 \\
\hline 134 & 10 & 623 \\
\hline 135 & 1 & 215 \\
\hline 135 & 2 & 215 \\
\hline 135 & 3 & 215 \\
\hline 135 & 4 & 215 \\
\hline 135 & 5 & 215 \\
\hline 135 & 6 & 215 \\
\hline 135 & 7 & 215 \\
\hline
\end{tabular}




\begin{tabular}{|c|c|c|}
\hline 135 & 8 & 215 \\
\hline 135 & 9 & 215 \\
\hline 135 & 10 & 215 \\
\hline 136 & 1 & 11973 \\
\hline 136 & 2 & 11973 \\
\hline 136 & 3 & 11973 \\
\hline 136 & 4 & 11973 \\
\hline 136 & 5 & 11973 \\
\hline 136 & 6 & 11973 \\
\hline 136 & 7 & 11973 \\
\hline 136 & 8 & 11973 \\
\hline 136 & 9 & 11973 \\
\hline 136 & 10 & 11973 \\
\hline 137 & 1 & 343 \\
\hline 137 & 2 & 343 \\
\hline 137 & 3 & 343 \\
\hline 137 & 4 & 343 \\
\hline 137 & 5 & 343 \\
\hline 137 & 6 & 343 \\
\hline 137 & 7 & 343 \\
\hline 137 & 8 & 343 \\
\hline 137 & 9 & 343 \\
\hline 137 & 10 & 343 \\
\hline 138 & 1 & 228 \\
\hline 138 & 2 & 228 \\
\hline 138 & 3 & 228 \\
\hline 138 & 4 & 228 \\
\hline 138 & 5 & 228 \\
\hline 138 & 6 & 228 \\
\hline 138 & 7 & 228 \\
\hline 138 & 8 & 228 \\
\hline 138 & 9 & 228 \\
\hline 138 & 10 & 228 \\
\hline 139 & 1 & 507 \\
\hline 139 & 2 & 507 \\
\hline 139 & 3 & 507 \\
\hline 139 & 4 & 507 \\
\hline 139 & 5 & 507 \\
\hline 139 & 6 & 507 \\
\hline 139 & 7 & 507 \\
\hline 139 & 8 & 507 \\
\hline 139 & 9 & 507 \\
\hline 139 & 10 & 507 \\
\hline 140 & 1 & 1462 \\
\hline 140 & 2 & 1462 \\
\hline \multicolumn{3}{|c|}{275} \\
\hline
\end{tabular}




\begin{tabular}{|c|c|c|}
\hline 140 & 3 & 1462 \\
\hline 140 & 4 & 1462 \\
\hline 140 & 5 & 1462 \\
\hline 140 & 6 & 1462 \\
\hline 140 & 7 & 1462 \\
\hline 140 & 8 & 1462 \\
\hline 140 & 9 & 1462 \\
\hline 140 & 10 & 1462 \\
\hline 141 & 1 & 672 \\
\hline 141 & 2 & 672 \\
\hline 141 & 3 & 672 \\
\hline 141 & 4 & 672 \\
\hline 141 & 5 & 672 \\
\hline 141 & 6 & 672 \\
\hline 141 & 7 & 672 \\
\hline 141 & 8 & 672 \\
\hline 141 & 9 & 672 \\
\hline 141 & 10 & 672 \\
\hline 142 & 1 & 661 \\
\hline 142 & 2 & 661 \\
\hline 142 & 3 & 661 \\
\hline 142 & 4 & 661 \\
\hline 142 & 5 & 661 \\
\hline 142 & 6 & 661 \\
\hline 142 & 7 & 661 \\
\hline 142 & 8 & 661 \\
\hline 142 & 9 & 661 \\
\hline 142 & 10 & 661 \\
\hline 143 & 1 & 266 \\
\hline 143 & 2 & 266 \\
\hline 143 & 3 & 266 \\
\hline 143 & 4 & 266 \\
\hline 143 & 5 & 266 \\
\hline 143 & 6 & 266 \\
\hline 143 & 7 & 266 \\
\hline 143 & 8 & 266 \\
\hline 143 & 9 & 266 \\
\hline 143 & 10 & 266 \\
\hline 144 & 1 & 300 \\
\hline 144 & 2 & 300 \\
\hline 144 & 3 & 300 \\
\hline 144 & 4 & 300 \\
\hline 144 & 5 & 300 \\
\hline 144 & 6 & 300 \\
\hline 144 & 7 & 300 \\
\hline
\end{tabular}




\begin{tabular}{|c|c|c|}
\hline 144 & 8 & 300 \\
\hline 144 & 9 & 300 \\
\hline 144 & 10 & 300 \\
\hline 145 & 1 & 9409 \\
\hline 145 & 2 & 9409 \\
\hline 145 & 3 & 9409 \\
\hline 145 & 4 & 9409 \\
\hline 145 & 5 & 9409 \\
\hline 145 & 6 & 9409 \\
\hline 145 & 7 & 9409 \\
\hline 145 & 8 & 9409 \\
\hline 145 & 9 & 9409 \\
\hline 145 & 10 & 9409 \\
\hline 146 & 1 & 1452 \\
\hline 146 & 2 & 1452 \\
\hline 146 & 3 & 1452 \\
\hline 146 & 4 & 1452 \\
\hline 146 & 5 & 1452 \\
\hline 146 & 6 & 1452 \\
\hline 146 & 7 & 1452 \\
\hline 146 & 8 & 1452 \\
\hline 146 & 9 & 1452 \\
\hline 146 & 10 & 1452 \\
\hline 147 & 1 & 324 \\
\hline 147 & 2 & 324 \\
\hline 147 & 3 & 324 \\
\hline 147 & 4 & 324 \\
\hline 147 & 5 & 324 \\
\hline 147 & 6 & 324 \\
\hline 147 & 7 & 324 \\
\hline 147 & 8 & 324 \\
\hline 147 & 9 & 324 \\
\hline 147 & 10 & 324 \\
\hline 148 & 1 & 111 \\
\hline 148 & 2 & 111 \\
\hline 148 & 3 & 111 \\
\hline 148 & 4 & 111 \\
\hline 148 & 5 & 111 \\
\hline 148 & 6 & 111 \\
\hline 148 & 7 & 111 \\
\hline 148 & 8 & 111 \\
\hline 148 & 9 & 111 \\
\hline 148 & 10 & 111 \\
\hline 149 & 1 & 495 \\
\hline 149 & 2 & 495 \\
\hline \multicolumn{3}{|c|}{277} \\
\hline
\end{tabular}




\begin{tabular}{|c|c|c|}
\hline 149 & 3 & 495 \\
\hline 149 & 4 & 495 \\
\hline 149 & 5 & 495 \\
\hline 149 & 6 & 495 \\
\hline 149 & 7 & 495 \\
\hline 149 & 8 & 495 \\
\hline 149 & 9 & 495 \\
\hline 149 & 10 & 495 \\
\hline 150 & 1 & 198 \\
\hline 150 & 2 & 198 \\
\hline 150 & 3 & 198 \\
\hline 150 & 4 & 198 \\
\hline 150 & 5 & 198 \\
\hline 150 & 6 & 198 \\
\hline 150 & 7 & 198 \\
\hline 150 & 8 & 198 \\
\hline 150 & 9 & 198 \\
\hline 150 & 10 & 198 \\
\hline 151 & 1 & 132 \\
\hline 151 & 2 & 132 \\
\hline 151 & 3 & 132 \\
\hline 151 & 4 & 132 \\
\hline 151 & 5 & 132 \\
\hline 151 & 6 & 132 \\
\hline 151 & 7 & 132 \\
\hline 151 & 8 & 132 \\
\hline 151 & 9 & 132 \\
\hline 151 & 10 & 132 \\
\hline 152 & 1 & 305 \\
\hline 152 & 2 & 305 \\
\hline 152 & 3 & 305 \\
\hline 152 & 4 & 305 \\
\hline 152 & 5 & 305 \\
\hline 152 & 6 & 305 \\
\hline 152 & 7 & 305 \\
\hline 152 & 8 & 305 \\
\hline 152 & 9 & 305 \\
\hline 152 & 10 & 305 \\
\hline 153 & 1 & 1297 \\
\hline 153 & 2 & 1297 \\
\hline 153 & 3 & 1297 \\
\hline 153 & 4 & 1297 \\
\hline 153 & 5 & 1297 \\
\hline 153 & 6 & 1297 \\
\hline 153 & 7 & 1297 \\
\hline
\end{tabular}




\begin{tabular}{|c|c|c|}
\hline 153 & 8 & 1297 \\
\hline 153 & 9 & 1297 \\
\hline 153 & 10 & 1297 \\
\hline 154 & 1 & 760 \\
\hline 154 & 2 & 760 \\
\hline 154 & 3 & 760 \\
\hline 154 & 4 & 760 \\
\hline 154 & 5 & 760 \\
\hline 154 & 6 & 760 \\
\hline 154 & 7 & 760 \\
\hline 154 & 8 & 760 \\
\hline 154 & 9 & 760 \\
\hline 154 & 10 & 760 \\
\hline 155 & 1 & 274 \\
\hline 155 & 2 & 274 \\
\hline 155 & 3 & 274 \\
\hline 155 & 4 & 274 \\
\hline 155 & 5 & 274 \\
\hline 155 & 6 & 274 \\
\hline 155 & 7 & 274 \\
\hline 155 & 8 & 274 \\
\hline 155 & 9 & 274 \\
\hline 155 & 10 & 274 \\
\hline 156 & 1 & 393 \\
\hline 156 & 2 & 393 \\
\hline 156 & 3 & 393 \\
\hline 156 & 4 & 393 \\
\hline 156 & 5 & 393 \\
\hline 156 & 6 & 393 \\
\hline 156 & 7 & 393 \\
\hline 156 & 8 & 393 \\
\hline 156 & 9 & 393 \\
\hline 156 & 10 & 393 \\
\hline 157 & 1 & 590 \\
\hline 157 & 2 & 590 \\
\hline 157 & 3 & 590 \\
\hline 157 & 4 & 590 \\
\hline 157 & 5 & 590 \\
\hline 157 & 6 & 590 \\
\hline 157 & 7 & 590 \\
\hline 157 & 8 & 590 \\
\hline 157 & 9 & 590 \\
\hline 157 & 10 & 590 \\
\hline 158 & 1 & 1106 \\
\hline 158 & 2 & 1106 \\
\hline \multicolumn{3}{|c|}{279} \\
\hline
\end{tabular}




\begin{tabular}{|c|c|c|}
\hline 158 & 3 & 1106 \\
\hline 158 & 4 & 1106 \\
\hline 158 & 5 & 1106 \\
\hline 158 & 6 & 1106 \\
\hline 158 & 7 & 1106 \\
\hline 158 & 8 & 1106 \\
\hline 158 & 9 & 1106 \\
\hline 158 & 10 & 1106 \\
\hline 159 & 1 & 7305 \\
\hline 159 & 2 & 7305 \\
\hline 159 & 3 & 7305 \\
\hline 159 & 4 & 7305 \\
\hline 159 & 5 & 7305 \\
\hline 159 & 6 & 7305 \\
\hline 159 & 7 & 7305 \\
\hline 159 & 8 & 7305 \\
\hline 159 & 9 & 7305 \\
\hline 159 & 10 & 7305 \\
\hline 160 & 1 & 207 \\
\hline 160 & 2 & 207 \\
\hline 160 & 3 & 207 \\
\hline 160 & 4 & 207 \\
\hline 160 & 5 & 207 \\
\hline 160 & 6 & 207 \\
\hline 160 & 7 & 207 \\
\hline 160 & 8 & 207 \\
\hline 160 & 9 & 207 \\
\hline 160 & 10 & 207 \\
\hline 161 & 1 & 241 \\
\hline 161 & 2 & 241 \\
\hline 161 & 3 & 241 \\
\hline 161 & 4 & 241 \\
\hline 161 & 5 & 241 \\
\hline 161 & 6 & 241 \\
\hline 161 & 7 & 241 \\
\hline 161 & 8 & 241 \\
\hline 161 & 9 & 241 \\
\hline 161 & 10 & 241 \\
\hline 162 & 1 & 3200 \\
\hline 162 & 2 & 3200 \\
\hline 162 & 3 & 3200 \\
\hline 162 & 4 & 3200 \\
\hline 162 & 5 & 3200 \\
\hline 162 & 6 & 3200 \\
\hline 162 & 7 & 3200 \\
\hline
\end{tabular}




\begin{tabular}{|c|c|c|}
\hline 162 & 8 & 3200 \\
\hline 162 & 9 & 3200 \\
\hline 162 & 10 & 3200 \\
\hline 163 & 1 & 3830 \\
\hline 163 & 2 & 3830 \\
\hline 163 & 3 & 3830 \\
\hline 163 & 4 & 3830 \\
\hline 163 & 5 & 3830 \\
\hline 163 & 6 & 3830 \\
\hline 163 & 7 & 3830 \\
\hline 163 & 8 & 3830 \\
\hline 163 & 9 & 3830 \\
\hline 163 & 10 & 3830 \\
\hline 164 & 1 & 292 \\
\hline 164 & 2 & 292 \\
\hline 164 & 3 & 292 \\
\hline 164 & 4 & 292 \\
\hline 164 & 5 & 292 \\
\hline 164 & 6 & 292 \\
\hline 164 & 7 & 292 \\
\hline 164 & 8 & 292 \\
\hline 164 & 9 & 292 \\
\hline 164 & 10 & 292 \\
\hline 165 & 1 & 31 \\
\hline 165 & 2 & 31 \\
\hline 165 & 3 & 31 \\
\hline 165 & 4 & 31 \\
\hline 165 & 5 & 31 \\
\hline 165 & 6 & 31 \\
\hline 165 & 7 & 31 \\
\hline 165 & 8 & 31 \\
\hline 165 & 9 & 31 \\
\hline 165 & 10 & 31 \\
\hline 166 & 1 & 100 \\
\hline 166 & 2 & 100 \\
\hline 166 & 3 & 100 \\
\hline 166 & 4 & 100 \\
\hline 166 & 5 & 100 \\
\hline 166 & 6 & 100 \\
\hline 166 & 7 & 100 \\
\hline 166 & 8 & 100 \\
\hline 166 & 9 & 100 \\
\hline 166 & 10 & 100 \\
\hline 167 & 1 & 54 \\
\hline 167 & 2 & 54 \\
\hline
\end{tabular}




\begin{tabular}{|c|c|c|}
\hline 167 & 3 & 54 \\
\hline 167 & 4 & 54 \\
\hline 167 & 5 & 54 \\
\hline 167 & 6 & 54 \\
\hline 167 & 7 & 54 \\
\hline 167 & 8 & 54 \\
\hline 167 & 9 & 54 \\
\hline 167 & 10 & 54 \\
\hline 168 & 1 & 82 \\
\hline 168 & 2 & 82 \\
\hline 168 & 3 & 82 \\
\hline 168 & 4 & 82 \\
\hline 168 & 5 & 82 \\
\hline 168 & 6 & 82 \\
\hline 168 & 7 & 82 \\
\hline 168 & 8 & 82 \\
\hline 168 & 9 & 82 \\
\hline 168 & 10 & 82 \\
\hline 169 & 1 & 84 \\
\hline 169 & 2 & 84 \\
\hline 169 & 3 & 84 \\
\hline 169 & 4 & 84 \\
\hline 169 & 5 & 84 \\
\hline 169 & 6 & 84 \\
\hline 169 & 7 & 84 \\
\hline 169 & 8 & 84 \\
\hline 169 & 9 & 84 \\
\hline 169 & 10 & 84 \\
\hline 170 & 1 & 1513 \\
\hline 170 & 2 & 1513 \\
\hline 170 & 3 & 1513 \\
\hline 170 & 4 & 1513 \\
\hline 170 & 5 & 1513 \\
\hline 170 & 6 & 1513 \\
\hline 170 & 7 & 1513 \\
\hline 170 & 8 & 1513 \\
\hline 170 & 9 & 1513 \\
\hline 170 & 10 & 1513 \\
\hline
\end{tabular}


Table 51 Attraction value for Dortmunder Volksbank's facilities with respect to demand points

\begin{tabular}{|c|c|c|}
\hline INDEXI & INDEXJ & ATTRACTIVITY \\
\hline 1 & 1 & 41091 \\
\hline 1 & 2 & 468 \\
\hline 1 & 3 & 639 \\
\hline 1 & 4 & 607 \\
\hline 1 & 5 & 179 \\
\hline 1 & 6 & 20 \\
\hline 1 & 7 & 94 \\
\hline 1 & 8 & 186 \\
\hline 1 & 9 & 168 \\
\hline 1 & 10 & 80 \\
\hline 1 & 11 & 36 \\
\hline 1 & 12 & 47 \\
\hline 1 & 13 & 31 \\
\hline 1 & 14 & 44 \\
\hline 1 & 15 & 77 \\
\hline 1 & 16 & 156 \\
\hline 1 & 17 & 191 \\
\hline 1 & 18 & 268 \\
\hline 1 & 19 & 69 \\
\hline 1 & 20 & 77 \\
\hline 1 & 21 & 222 \\
\hline 1 & 22 & 40 \\
\hline 1 & 23 & 440 \\
\hline 1 & 24 & 172 \\
\hline 1 & 25 & 100 \\
\hline 1 & 26 & 178 \\
\hline 1 & 27 & 47 \\
\hline 1 & 28 & 124 \\
\hline 1 & 29 & 65 \\
\hline 1 & 30 & 172 \\
\hline 1 & 31 & 221 \\
\hline 1 & 32 & 5 \\
\hline 1 & 33 & 5 \\
\hline 1 & 34 & 27 \\
\hline 1 & 35 & 30 \\
\hline 1 & 36 & 26 \\
\hline 1 & 37 & 18 \\
\hline 1 & 38 & 17 \\
\hline 1 & 39 & 32 \\
\hline 1 & 40 & 10 \\
\hline 1 & 41 & 8 \\
\hline 1 & 42 & 6 \\
\hline
\end{tabular}




\begin{tabular}{|c|c|c|}
\hline 1 & 43 & 10 \\
\hline 1 & 44 & 23 \\
\hline 1 & 45 & 17 \\
\hline 1 & 46 & 6 \\
\hline 1 & 47 & 9 \\
\hline 1 & 48 & 5 \\
\hline 1 & 49 & 7 \\
\hline 1 & 50 & 4 \\
\hline 1 & 51 & 5 \\
\hline 1 & 52 & 12 \\
\hline 1 & 53 & 21 \\
\hline 1 & 54 & 13 \\
\hline 1 & 55 & 10 \\
\hline 1 & 56 & 9 \\
\hline 1 & 57 & 8 \\
\hline 1 & 58 & 8 \\
\hline 1 & 59 & 7 \\
\hline 1 & 60 & 10 \\
\hline 1 & 61 & 11 \\
\hline 1 & 62 & 24 \\
\hline 1 & 63 & 28 \\
\hline 1 & 64 & 11 \\
\hline 1 & 65 & 11 \\
\hline 1 & 66 & 17 \\
\hline 1 & 67 & 25 \\
\hline 1 & 68 & 15 \\
\hline 1 & 69 & 39 \\
\hline 1 & 70 & 45 \\
\hline 1 & 71 & 22 \\
\hline 1 & 72 & 5 \\
\hline 1 & 73 & 6 \\
\hline 1 & 74 & 4 \\
\hline 1 & 75 & 7 \\
\hline 1 & 76 & 10 \\
\hline 1 & 77 & 9 \\
\hline 1 & 78 & 11 \\
\hline 1 & 79 & 9 \\
\hline 1 & 80 & 7 \\
\hline 1 & 81 & 8 \\
\hline 1 & 82 & 8 \\
\hline 1 & 83 & 13 \\
\hline 1 & 84 & 11 \\
\hline 1 & 85 & 8 \\
\hline 1 & 86 & 21 \\
\hline 1 & 87 & 27 \\
\hline
\end{tabular}




\begin{tabular}{|c|c|c|}
\hline 1 & 88 & 9 \\
\hline 1 & 89 & 8 \\
\hline 1 & 90 & 6 \\
\hline 1 & 91 & 3 \\
\hline 1 & 92 & 15 \\
\hline 1 & 93 & 14 \\
\hline 1 & 94 & 37 \\
\hline 1 & 95 & 41 \\
\hline 1 & 96 & 83 \\
\hline 1 & 97 & 43 \\
\hline 1 & 98 & 54 \\
\hline 1 & 99 & 32 \\
\hline 1 & 100 & 28 \\
\hline 1 & 101 & 10 \\
\hline 1 & 102 & 5 \\
\hline 1 & 103 & 8 \\
\hline 1 & 104 & 4 \\
\hline 1 & 105 & 25 \\
\hline 1 & 106 & 21 \\
\hline 1 & 107 & 14 \\
\hline 1 & 108 & 32 \\
\hline 1 & 109 & 19 \\
\hline 1 & 110 & 44 \\
\hline 1 & 111 & 22 \\
\hline 1 & 112 & 27 \\
\hline 1 & 113 & 15 \\
\hline 1 & 114 & 51 \\
\hline 1 & 115 & 37 \\
\hline 1 & 116 & 12 \\
\hline 1 & 117 & 23 \\
\hline 1 & 118 & 9 \\
\hline 1 & 119 & 26 \\
\hline 1 & 120 & 26 \\
\hline 1 & 121 & 13 \\
\hline 1 & 122 & 11 \\
\hline 1 & 123 & 11 \\
\hline 1 & 124 & 7 \\
\hline 1 & 125 & 15 \\
\hline 1 & 126 & 12 \\
\hline 1 & 127 & 15 \\
\hline 1 & 128 & 14 \\
\hline 1 & 129 & 11 \\
\hline 1 & 130 & 15 \\
\hline 1 & 131 & 9 \\
\hline 1 & 132 & 10 \\
\hline
\end{tabular}




\begin{tabular}{|c|c|c|}
\hline 1 & 133 & 25 \\
\hline 1 & 134 & 20 \\
\hline 1 & 135 & 14 \\
\hline 1 & 136 & 4 \\
\hline 1 & 137 & 9 \\
\hline 1 & 138 & 4 \\
\hline 1 & 139 & 5 \\
\hline 1 & 140 & 5 \\
\hline 1 & 141 & 9 \\
\hline 1 & 142 & 6 \\
\hline 1 & 143 & 8 \\
\hline 1 & 144 & 10 \\
\hline 1 & 145 & 12 \\
\hline 1 & 146 & 14 \\
\hline 1 & 147 & 11 \\
\hline 1 & 148 & 22 \\
\hline 1 & 149 & 20 \\
\hline 1 & 150 & 18 \\
\hline 1 & 151 & 30 \\
\hline 1 & 152 & 18 \\
\hline 1 & 153 & 40 \\
\hline 1 & 154 & 10 \\
\hline 1 & 155 & 8 \\
\hline 1 & 156 & 10 \\
\hline 1 & 157 & 10 \\
\hline 1 & 158 & 11 \\
\hline 1 & 159 & 9 \\
\hline 1 & 160 & 2 \\
\hline 1 & 161 & 2 \\
\hline 1 & 162 & 8 \\
\hline 1 & 163 & 9 \\
\hline 1 & 164 & 11 \\
\hline 1 & 165 & 2 \\
\hline 1 & 166 & 2 \\
\hline 1 & 167 & 8 \\
\hline 1 & 168 & 7 \\
\hline 1 & 169 & 11 \\
\hline 1 & 170 & 8 \\
\hline 2 & 1 & 17 \\
\hline 2 & 2 & 14 \\
\hline 2 & 3 & 14 \\
\hline 2 & 4 & 22 \\
\hline 2 & 5 & 6 \\
\hline 2 & 6 & 5 \\
\hline 2 & 7 & 5 \\
\hline
\end{tabular}




\begin{tabular}{|c|c|c|}
\hline 2 & 8 & 12 \\
\hline 2 & 9 & 12 \\
\hline 2 & 10 & 10 \\
\hline 2 & 11 & 7 \\
\hline 2 & 12 & 5 \\
\hline 2 & 13 & 4 \\
\hline 2 & 14 & 15 \\
\hline 2 & 15 & 11 \\
\hline 2 & 16 & 16 \\
\hline 2 & 17 & 16 \\
\hline 2 & 18 & 22 \\
\hline 2 & 19 & 23 \\
\hline 2 & 20 & 29 \\
\hline 2 & 21 & 30 \\
\hline 2 & 22 & 112 \\
\hline 2 & 23 & 21 \\
\hline 2 & 24 & 25 \\
\hline 2 & 25 & 44 \\
\hline 2 & 26 & 18 \\
\hline 2 & 27 & 37 \\
\hline 2 & 28 & 12 \\
\hline 2 & 29 & 20 \\
\hline 2 & 30 & 10 \\
\hline 2 & 31 & 8 \\
\hline 2 & 32 & 10 \\
\hline 2 & 33 & 8 \\
\hline 2 & 34 & 24 \\
\hline 2 & 35 & 35 \\
\hline 2 & 36 & 30 \\
\hline 2 & 37 & 79 \\
\hline 2 & 38 & 6 \\
\hline 2 & 39 & 16 \\
\hline 2 & 40 & 23 \\
\hline 2 & 41 & 49 \\
\hline 2 & 42 & 40 \\
\hline 2 & 43 & 72 \\
\hline 2 & 44 & 190 \\
\hline 2 & 45 & 153 \\
\hline 2 & 46 & 28 \\
\hline 2 & 47 & 16 \\
\hline 2 & 48 & 8 \\
\hline 2 & 49 & 11 \\
\hline 2 & 50 & 19 \\
\hline 2 & 51 & 8 \\
\hline 2 & 52 & 417 \\
\hline
\end{tabular}




\begin{tabular}{|c|c|c|}
\hline 2 & 53 & 1094 \\
\hline 2 & 54 & 452 \\
\hline 2 & 55 & 73 \\
\hline 2 & 56 & 17 \\
\hline 2 & 57 & 15 \\
\hline 2 & 58 & 15 \\
\hline 2 & 59 & 12 \\
\hline 2 & 60 & 12 \\
\hline 2 & 61 & 22 \\
\hline 2 & 62 & 50 \\
\hline 2 & 63 & 50 \\
\hline 2 & 64 & 39 \\
\hline 2 & 65 & 37 \\
\hline 2 & 66 & 42 \\
\hline 2 & 67 & 20 \\
\hline 2 & 68 & 13 \\
\hline 2 & 69 & 63 \\
\hline 2 & 70 & 71 \\
\hline 2 & 71 & 19 \\
\hline 2 & 72 & 8 \\
\hline 2 & 73 & 10 \\
\hline 2 & 74 & 8 \\
\hline 2 & 75 & 6 \\
\hline 2 & 76 & 9 \\
\hline 2 & 77 & 10 \\
\hline 2 & 78 & 10 \\
\hline 2 & 79 & 8 \\
\hline 2 & 80 & 7 \\
\hline 2 & 81 & 8 \\
\hline 2 & 82 & 8 \\
\hline 2 & 83 & 11 \\
\hline 2 & 84 & 7 \\
\hline 2 & 85 & 7 \\
\hline 2 & 86 & 18 \\
\hline 2 & 87 & 17 \\
\hline 2 & 88 & 8 \\
\hline 2 & 89 & 7 \\
\hline 2 & 90 & 5 \\
\hline 2 & 91 & 4 \\
\hline 2 & 92 & 9 \\
\hline 2 & 93 & 8 \\
\hline 2 & 94 & 6 \\
\hline 2 & 95 & 6 \\
\hline 2 & 96 & 13 \\
\hline 2 & 97 & 9 \\
\hline
\end{tabular}




\begin{tabular}{|c|c|c|}
\hline 2 & 98 & 7 \\
\hline 2 & 99 & 8 \\
\hline 2 & 100 & 8 \\
\hline 2 & 101 & 6 \\
\hline 2 & 102 & 5 \\
\hline 2 & 103 & 3 \\
\hline 2 & 104 & 3 \\
\hline 2 & 105 & 7 \\
\hline 2 & 106 & 6 \\
\hline 2 & 107 & 4 \\
\hline 2 & 108 & 6 \\
\hline 2 & 109 & 4 \\
\hline 2 & 110 & 6 \\
\hline 2 & 111 & 5 \\
\hline 2 & 112 & 5 \\
\hline 2 & 113 & 4 \\
\hline 2 & 114 & 6 \\
\hline 2 & 115 & 5 \\
\hline 2 & 116 & 4 \\
\hline 2 & 117 & 5 \\
\hline 2 & 118 & 3 \\
\hline 2 & 119 & 5 \\
\hline 2 & 120 & 4 \\
\hline 2 & 121 & 4 \\
\hline 2 & 122 & 3 \\
\hline 2 & 123 & 3 \\
\hline 2 & 124 & 3 \\
\hline 2 & 125 & 3 \\
\hline 2 & 126 & 4 \\
\hline 2 & 127 & 5 \\
\hline 2 & 128 & 4 \\
\hline 2 & 129 & 4 \\
\hline 2 & 130 & 3 \\
\hline 2 & 131 & 3 \\
\hline 2 & 132 & 3 \\
\hline 2 & 133 & 5 \\
\hline 2 & 134 & 6 \\
\hline 2 & 135 & 4 \\
\hline 2 & 136 & 2 \\
\hline 2 & 137 & 3 \\
\hline 2 & 138 & 2 \\
\hline 2 & 139 & 2 \\
\hline 2 & 140 & 2 \\
\hline 2 & 141 & 3 \\
\hline 2 & 142 & 2 \\
\hline
\end{tabular}




\begin{tabular}{|c|c|c|}
\hline 2 & 143 & 3 \\
\hline 2 & 144 & 3 \\
\hline 2 & 145 & 4 \\
\hline 2 & 146 & 4 \\
\hline 2 & 147 & 4 \\
\hline 2 & 148 & 11 \\
\hline 2 & 149 & 3 \\
\hline 2 & 150 & 2 \\
\hline 2 & 151 & 8 \\
\hline 2 & 152 & 2 \\
\hline 2 & 153 & 9 \\
\hline 2 & 154 & 4 \\
\hline 2 & 155 & 2 \\
\hline 2 & 156 & 4 \\
\hline 2 & 157 & 4 \\
\hline 2 & 158 & 5 \\
\hline 2 & 159 & 2 \\
\hline 2 & 160 & 3 \\
\hline 2 & 161 & 4 \\
\hline 2 & 162 & 4 \\
\hline 2 & 163 & 3 \\
\hline 2 & 164 & 3 \\
\hline 2 & 165 & 3 \\
\hline 2 & 166 & 4 \\
\hline 2 & 167 & 3 \\
\hline 2 & 168 & 4 \\
\hline 2 & 169 & 3 \\
\hline 2 & 170 & 2 \\
\hline 3 & 1 & 9 \\
\hline 3 & 2 & 9 \\
\hline 3 & 3 & 10 \\
\hline 3 & 4 & 7 \\
\hline 3 & 5 & 8 \\
\hline 3 & 6 & 6 \\
\hline 3 & 7 & 7 \\
\hline 3 & 8 & 7 \\
\hline 3 & 9 & 7 \\
\hline 3 & 10 & 5 \\
\hline 3 & 11 & 4 \\
\hline 3 & 12 & 6 \\
\hline 3 & 13 & 5 \\
\hline 3 & 14 & 5 \\
\hline 3 & 15 & 6 \\
\hline 3 & 16 & 7 \\
\hline 3 & 17 & 6 \\
\hline
\end{tabular}




\begin{tabular}{|c|c|c|}
\hline 3 & 18 & 7 \\
\hline 3 & 19 & 6 \\
\hline 3 & 20 & 6 \\
\hline 3 & 21 & 9 \\
\hline 3 & 22 & 17 \\
\hline 3 & 23 & 16 \\
\hline 3 & 24 & 11 \\
\hline 3 & 25 & 20 \\
\hline 3 & 26 & 20 \\
\hline 3 & 27 & 29 \\
\hline 3 & 28 & 18 \\
\hline 3 & 29 & 32 \\
\hline 3 & 30 & 14 \\
\hline 3 & 31 & 11 \\
\hline 3 & 32 & 3 \\
\hline 3 & 33 & 3 \\
\hline 3 & 34 & 4 \\
\hline 3 & 35 & 4 \\
\hline 3 & 36 & 4 \\
\hline 3 & 37 & 5 \\
\hline 3 & 38 & 3 \\
\hline 3 & 39 & 4 \\
\hline 3 & 40 & 6 \\
\hline 3 & 41 & 5 \\
\hline 3 & 42 & 8 \\
\hline 3 & 43 & 13 \\
\hline 3 & 44 & 5 \\
\hline 3 & 45 & 7 \\
\hline 3 & 46 & 13 \\
\hline 3 & 47 & 20 \\
\hline 3 & 48 & 10 \\
\hline 3 & 49 & 13 \\
\hline 3 & 50 & 7 \\
\hline 3 & 51 & 9 \\
\hline 3 & 52 & 17 \\
\hline 3 & 53 & 10 \\
\hline 3 & 54 & 18 \\
\hline 3 & 55 & 13 \\
\hline 3 & 56 & 21 \\
\hline 3 & 57 & 37 \\
\hline 3 & 58 & 42 \\
\hline 3 & 59 & 32 \\
\hline 3 & 60 & 88 \\
\hline 3 & 61 & 18 \\
\hline 3 & 62 & 31 \\
\hline
\end{tabular}




\begin{tabular}{|c|c|c|}
\hline 3 & 63 & 49 \\
\hline 3 & 64 & 37 \\
\hline 3 & 65 & 42 \\
\hline 3 & 66 & 49 \\
\hline 3 & 67 & 40 \\
\hline 3 & 68 & 91 \\
\hline 3 & 69 & 27 \\
\hline 3 & 70 & 26 \\
\hline 3 & 71 & 45 \\
\hline 3 & 72 & 9 \\
\hline 3 & 73 & 19 \\
\hline 3 & 74 & 11 \\
\hline 3 & 75 & 36 \\
\hline 3 & 76 & 184 \\
\hline 3 & 77 & 194 \\
\hline 3 & 78 & 692521 \\
\hline 3 & 79 & 750 \\
\hline 3 & 80 & 529 \\
\hline 3 & 81 & 102 \\
\hline 3 & 82 & 102 \\
\hline 3 & 83 & 55 \\
\hline 3 & 84 & 251 \\
\hline 3 & 85 & 79 \\
\hline 3 & 86 & 52 \\
\hline 3 & 87 & 107 \\
\hline 3 & 88 & 146 \\
\hline 3 & 89 & 108 \\
\hline 3 & 90 & 63 \\
\hline 3 & 91 & 31 \\
\hline 3 & 92 & 30 \\
\hline 3 & 93 & 34 \\
\hline 3 & 94 & 17 \\
\hline 3 & 95 & 19 \\
\hline 3 & 96 & 19 \\
\hline 3 & 97 & 42 \\
\hline 3 & 98 & 9 \\
\hline 3 & 99 & 24 \\
\hline 3 & 100 & 27 \\
\hline 3 & 101 & 37 \\
\hline 3 & 102 & 22 \\
\hline 3 & 103 & 10 \\
\hline 3 & 104 & 11 \\
\hline 3 & 105 & 18 \\
\hline 3 & 106 & 19 \\
\hline 3 & 107 & 16 \\
\hline
\end{tabular}




\begin{tabular}{|c|c|c|}
\hline 3 & 108 & 7 \\
\hline 3 & 109 & 5 \\
\hline 3 & 110 & 8 \\
\hline 3 & 111 & 6 \\
\hline 3 & 112 & 7 \\
\hline 3 & 113 & 10 \\
\hline 3 & 114 & 8 \\
\hline 3 & 115 & 10 \\
\hline 3 & 116 & 4 \\
\hline 3 & 117 & 6 \\
\hline 3 & 118 & 4 \\
\hline 3 & 119 & 7 \\
\hline 3 & 120 & 9 \\
\hline 3 & 121 & 9 \\
\hline 3 & 122 & 4 \\
\hline 3 & 123 & 5 \\
\hline 3 & 124 & 6 \\
\hline 3 & 125 & 6 \\
\hline 3 & 126 & 9 \\
\hline 3 & 127 & 10 \\
\hline 3 & 128 & 10 \\
\hline 3 & 129 & 8 \\
\hline 3 & 130 & 6 \\
\hline 3 & 131 & 7 \\
\hline 3 & 132 & 7 \\
\hline 3 & 133 & 8 \\
\hline 3 & 134 & 13 \\
\hline 3 & 135 & 5 \\
\hline 3 & 136 & 2 \\
\hline 3 & 137 & 4 \\
\hline 3 & 138 & 2 \\
\hline 3 & 139 & 2 \\
\hline 3 & 140 & 2 \\
\hline 3 & 141 & 4 \\
\hline 3 & 142 & 3 \\
\hline 3 & 143 & 3 \\
\hline 3 & 144 & 4 \\
\hline 3 & 145 & 4 \\
\hline 3 & 146 & 5 \\
\hline 3 & 147 & 3 \\
\hline 3 & 148 & 1 \\
\hline 3 & 149 & 3 \\
\hline 3 & 150 & 3 \\
\hline 3 & 151 & 4 \\
\hline 3 & 152 & 3 \\
\hline
\end{tabular}




\begin{tabular}{|c|c|c|}
\hline 3 & 153 & 4 \\
\hline 3 & 154 & 3 \\
\hline 3 & 155 & 3 \\
\hline 3 & 156 & 3 \\
\hline 3 & 157 & 3 \\
\hline 3 & 158 & 3 \\
\hline 3 & 159 & 1 \\
\hline 3 & 160 & 2 \\
\hline 3 & 161 & 2 \\
\hline 3 & 162 & 2 \\
\hline 3 & 163 & 2 \\
\hline 3 & 164 & 2 \\
\hline 3 & 165 & 2 \\
\hline 3 & 166 & 2 \\
\hline 3 & 167 & 2 \\
\hline 3 & 168 & 2 \\
\hline 3 & 169 & 2 \\
\hline 3 & 170 & 2 \\
\hline 4 & 1 & 8 \\
\hline 4 & 2 & 7 \\
\hline 4 & 3 & 7 \\
\hline 4 & 4 & 9 \\
\hline 4 & 5 & 6 \\
\hline 4 & 6 & 4 \\
\hline 4 & 7 & 5 \\
\hline 4 & 8 & 6 \\
\hline 4 & 9 & 6 \\
\hline 4 & 10 & 5 \\
\hline 4 & 11 & 4 \\
\hline 4 & 12 & 4 \\
\hline 4 & 13 & 4 \\
\hline 4 & 14 & 6 \\
\hline 4 & 15 & 6 \\
\hline 4 & 16 & 7 \\
\hline 4 & 17 & 7 \\
\hline 4 & 18 & 9 \\
\hline 4 & 19 & 7 \\
\hline 4 & 20 & 8 \\
\hline 4 & 21 & 11 \\
\hline 4 & 22 & 21 \\
\hline 4 & 23 & 9 \\
\hline 4 & 24 & 10 \\
\hline 4 & 25 & 13 \\
\hline 4 & 26 & 12 \\
\hline 4 & 27 & 26 \\
\hline
\end{tabular}




\begin{tabular}{|c|c|c|}
\hline 4 & 28 & 11 \\
\hline 4 & 29 & 17 \\
\hline 4 & 30 & 9 \\
\hline 4 & 31 & 7 \\
\hline 4 & 32 & 4 \\
\hline 4 & 33 & 4 \\
\hline 4 & 34 & 4 \\
\hline 4 & 35 & 5 \\
\hline 4 & 36 & 5 \\
\hline 4 & 37 & 7 \\
\hline 4 & 38 & 3 \\
\hline 4 & 39 & 5 \\
\hline 4 & 40 & 7 \\
\hline 4 & 41 & 6 \\
\hline 4 & 42 & 18 \\
\hline 4 & 43 & 14 \\
\hline 4 & 44 & 10 \\
\hline 4 & 45 & 10 \\
\hline 4 & 46 & 36 \\
\hline 4 & 47 & 83 \\
\hline 4 & 48 & 32 \\
\hline 4 & 49 & 49 \\
\hline 4 & 50 & 16 \\
\hline 4 & 51 & 21 \\
\hline 4 & 52 & 18 \\
\hline 4 & 53 & 14 \\
\hline 4 & 54 & 20 \\
\hline 4 & 55 & 14 \\
\hline 4 & 56 & 93 \\
\hline 4 & 57 & 3845 \\
\hline 4 & 58 & 4788 \\
\hline 4 & 59 & 780 \\
\hline 4 & 60 & 209 \\
\hline 4 & 61 & 70 \\
\hline 4 & 62 & 34 \\
\hline 4 & 63 & 67 \\
\hline 4 & 64 & 156 \\
\hline 4 & 65 & 104 \\
\hline 4 & 66 & 72 \\
\hline 4 & 67 & 8 \\
\hline 4 & 68 & 99 \\
\hline 4 & 69 & 33 \\
\hline 4 & 70 & 36 \\
\hline 4 & 71 & 38 \\
\hline 4 & 72 & 86 \\
\hline
\end{tabular}




\begin{tabular}{|c|c|c|}
\hline 4 & 73 & 263 \\
\hline 4 & 74 & 139 \\
\hline 4 & 75 & 22 \\
\hline 4 & 76 & 45 \\
\hline 4 & 77 & 23 \\
\hline 4 & 78 & 32 \\
\hline 4 & 79 & 34 \\
\hline 4 & 80 & 18 \\
\hline 4 & 81 & 7 \\
\hline 4 & 82 & 7 \\
\hline 4 & 83 & 9 \\
\hline 4 & 84 & 18 \\
\hline 4 & 85 & 6 \\
\hline 4 & 86 & 24 \\
\hline 4 & 87 & 14 \\
\hline 4 & 88 & 35 \\
\hline 4 & 89 & 26 \\
\hline 4 & 90 & 16 \\
\hline 4 & 91 & 12 \\
\hline 4 & 92 & 8 \\
\hline 4 & 93 & 7 \\
\hline 4 & 94 & 5 \\
\hline 4 & 95 & 6 \\
\hline 4 & 96 & 12 \\
\hline 4 & 97 & 9 \\
\hline 4 & 98 & 6 \\
\hline 4 & 99 & 7 \\
\hline 4 & 100 & 7 \\
\hline 4 & 101 & 5 \\
\hline 4 & 102 & 4 \\
\hline 4 & 103 & 3 \\
\hline 4 & 104 & 3 \\
\hline 4 & 105 & 6 \\
\hline 4 & 106 & 4 \\
\hline 4 & 107 & 3 \\
\hline 4 & 108 & 5 \\
\hline 4 & 109 & 4 \\
\hline 4 & 110 & 6 \\
\hline 4 & 111 & 5 \\
\hline 4 & 112 & 5 \\
\hline 4 & 113 & 4 \\
\hline 4 & 114 & 6 \\
\hline 4 & 115 & 5 \\
\hline 4 & 116 & 3 \\
\hline 4 & 117 & 5 \\
\hline
\end{tabular}




\begin{tabular}{|c|c|c|}
\hline 4 & 118 & 3 \\
\hline 4 & 119 & 5 \\
\hline 4 & 120 & 3 \\
\hline 4 & 121 & 3 \\
\hline 4 & 122 & 3 \\
\hline 4 & 123 & 2 \\
\hline 4 & 124 & 2 \\
\hline 4 & 125 & 3 \\
\hline 4 & 126 & 3 \\
\hline 4 & 127 & 4 \\
\hline 4 & 128 & 4 \\
\hline 4 & 129 & 3 \\
\hline 4 & 130 & 3 \\
\hline 4 & 131 & 3 \\
\hline 4 & 132 & 3 \\
\hline 4 & 133 & 4 \\
\hline 4 & 134 & 4 \\
\hline 4 & 135 & 4 \\
\hline 4 & 136 & 2 \\
\hline 4 & 137 & 3 \\
\hline 4 & 138 & 2 \\
\hline 4 & 139 & 2 \\
\hline 4 & 140 & 2 \\
\hline 4 & 141 & 3 \\
\hline 4 & 142 & 2 \\
\hline 4 & 143 & 3 \\
\hline 4 & 144 & 3 \\
\hline 4 & 145 & 3 \\
\hline 4 & 146 & 4 \\
\hline 4 & 147 & 3 \\
\hline 4 & 148 & 2 \\
\hline 4 & 149 & 2 \\
\hline 4 & 150 & 1 \\
\hline 4 & 151 & 4 \\
\hline 4 & 152 & 3 \\
\hline 4 & 153 & 5 \\
\hline 4 & 154 & 3 \\
\hline 4 & 155 & 3 \\
\hline 4 & 156 & 3 \\
\hline 4 & 157 & 3 \\
\hline 4 & 158 & 3 \\
\hline 4 & 159 & 1 \\
\hline 4 & 160 & 2 \\
\hline 4 & 161 & 2 \\
\hline 4 & 162 & 2 \\
\hline
\end{tabular}




\begin{tabular}{|c|c|c|}
\hline 4 & 163 & 2 \\
\hline 4 & 164 & 2 \\
\hline 4 & 165 & 2 \\
\hline 4 & 166 & 2 \\
\hline 4 & 167 & 2 \\
\hline 4 & 168 & 2 \\
\hline 4 & 169 & 2 \\
\hline 4 & 170 & 1 \\
\hline 5 & 1 & 32 \\
\hline 5 & 2 & 48 \\
\hline 5 & 3 & 70 \\
\hline 5 & 4 & 26 \\
\hline 5 & 5 & 87 \\
\hline 5 & 6 & 31 \\
\hline 5 & 7 & 55 \\
\hline 5 & 8 & 62 \\
\hline 5 & 9 & 46 \\
\hline 5 & 10 & 31 \\
\hline 5 & 11 & 27 \\
\hline 5 & 12 & 32 \\
\hline 5 & 13 & 33 \\
\hline 5 & 14 & 18 \\
\hline 5 & 15 & 30 \\
\hline 5 & 16 & 34 \\
\hline 5 & 17 & 27 \\
\hline 5 & 18 & 21 \\
\hline 5 & 19 & 16 \\
\hline 5 & 20 & 14 \\
\hline 5 & 21 & 20 \\
\hline 5 & 22 & 9 \\
\hline 5 & 23 & 18 \\
\hline 5 & 24 & 16 \\
\hline 5 & 25 & 14 \\
\hline 5 & 26 & 32 \\
\hline 5 & 27 & 17 \\
\hline 5 & 28 & 30 \\
\hline 5 & 29 & 21 \\
\hline 5 & 30 & 42 \\
\hline 5 & 31 & 44 \\
\hline 5 & 32 & 3 \\
\hline 5 & 33 & 2 \\
\hline 5 & 34 & 10 \\
\hline 5 & 35 & 9 \\
\hline 5 & 36 & 8 \\
\hline 5 & 37 & 4 \\
\hline
\end{tabular}




\begin{tabular}{|c|c|c|}
\hline 5 & 38 & 5 \\
\hline 5 & 39 & 11 \\
\hline 5 & 40 & 4 \\
\hline 5 & 41 & 4 \\
\hline 5 & 42 & 3 \\
\hline 5 & 43 & 4 \\
\hline 5 & 44 & 4 \\
\hline 5 & 45 & 5 \\
\hline 5 & 46 & 3 \\
\hline 5 & 47 & 3 \\
\hline 5 & 48 & 3 \\
\hline 5 & 49 & 3 \\
\hline 5 & 50 & 2 \\
\hline 5 & 51 & 2 \\
\hline 5 & 52 & 5 \\
\hline 5 & 53 & 6 \\
\hline 5 & 54 & 5 \\
\hline 5 & 55 & 4 \\
\hline 5 & 56 & 4 \\
\hline 5 & 57 & 5 \\
\hline 5 & 58 & 5 \\
\hline 5 & 59 & 4 \\
\hline 5 & 60 & 6 \\
\hline 5 & 61 & 4 \\
\hline 5 & 62 & 7 \\
\hline 5 & 63 & 8 \\
\hline 5 & 64 & 6 \\
\hline 5 & 65 & 7 \\
\hline 5 & 66 & 9 \\
\hline 5 & 67 & 11 \\
\hline 5 & 68 & 8 \\
\hline 5 & 69 & 10 \\
\hline 5 & 70 & 12 \\
\hline 5 & 71 & 11 \\
\hline 5 & 72 & 2 \\
\hline 5 & 73 & 4 \\
\hline 5 & 74 & 3 \\
\hline 5 & 75 & 4 \\
\hline 5 & 76 & 6 \\
\hline 5 & 77 & 6 \\
\hline 5 & 78 & 7 \\
\hline 5 & 79 & 6 \\
\hline 5 & 80 & 9 \\
\hline 5 & 81 & 5 \\
\hline 5 & 82 & 5 \\
\hline
\end{tabular}




\begin{tabular}{|c|c|c|}
\hline 5 & 83 & 18 \\
\hline 5 & 84 & 12 \\
\hline 5 & 85 & 10 \\
\hline 5 & 86 & 10 \\
\hline 5 & 87 & 10 \\
\hline 5 & 88 & 6 \\
\hline 5 & 89 & 5 \\
\hline 5 & 90 & 4 \\
\hline 5 & 91 & 2 \\
\hline 5 & 92 & 24 \\
\hline 5 & 93 & 20 \\
\hline 5 & 94 & 34 \\
\hline 5 & 95 & 38 \\
\hline 5 & 96 & 20 \\
\hline 5 & 97 & 21 \\
\hline 5 & 98 & 22 \\
\hline 5 & 99 & 35 \\
\hline 5 & 100 & 26 \\
\hline 5 & 101 & 14 \\
\hline 5 & 102 & 8 \\
\hline 5 & 103 & 11 \\
\hline 5 & 104 & 5 \\
\hline 5 & 105 & 42 \\
\hline 5 & 106 & 34 \\
\hline 5 & 107 & 20 \\
\hline 5 & 108 & 230 \\
\hline 5 & 109 & 1559 \\
\hline 5 & 110 & 554 \\
\hline 5 & 111 & 2064 \\
\hline 5 & 112 & 8702 \\
\hline 5 & 113 & 31 \\
\hline 5 & 114 & 98 \\
\hline 5 & 115 & 149 \\
\hline 5 & 116 & 224 \\
\hline 5 & 117 & 106 \\
\hline 5 & 118 & 85 \\
\hline 5 & 119 & 1381 \\
\hline 5 & 120 & 180 \\
\hline 5 & 121 & 152 \\
\hline 5 & 122 & 86 \\
\hline 5 & 123 & 52 \\
\hline 5 & 124 & 30 \\
\hline 5 & 125 & 67 \\
\hline 5 & 126 & 100 \\
\hline 5 & 127 & 117 \\
\hline
\end{tabular}




\begin{tabular}{|c|c|c|}
\hline 5 & 128 & 48 \\
\hline 5 & 129 & 45 \\
\hline 5 & 130 & 70 \\
\hline 5 & 131 & 42 \\
\hline 5 & 132 & 24 \\
\hline 5 & 133 & 71 \\
\hline 5 & 134 & 41 \\
\hline 5 & 135 & 182 \\
\hline 5 & 136 & 5 \\
\hline 5 & 137 & 35 \\
\hline 5 & 138 & 6 \\
\hline 5 & 139 & 6 \\
\hline 5 & 140 & 6 \\
\hline 5 & 141 & 12 \\
\hline 5 & 142 & 7 \\
\hline 5 & 143 & 15 \\
\hline 5 & 144 & 14 \\
\hline 5 & 145 & 17 \\
\hline 5 & 146 & 52 \\
\hline 5 & 147 & 9 \\
\hline 5 & 148 & 9 \\
\hline 5 & 149 & 11 \\
\hline 5 & 150 & 6 \\
\hline 5 & 151 & 15 \\
\hline 5 & 152 & 7 \\
\hline 5 & 153 & 18 \\
\hline 5 & 154 & 9 \\
\hline 5 & 155 & 13 \\
\hline 5 & 156 & 8 \\
\hline 5 & 157 & 9 \\
\hline 5 & 158 & 10 \\
\hline 5 & 159 & 6 \\
\hline 5 & 160 & 3 \\
\hline 5 & 161 & 2 \\
\hline 5 & 162 & 3 \\
\hline 5 & 163 & 4 \\
\hline 5 & 164 & 7 \\
\hline 5 & 165 & 2 \\
\hline 5 & 166 & 3 \\
\hline 5 & 167 & 2 \\
\hline 5 & 168 & 2 \\
\hline 5 & 169 & 6 \\
\hline 5 & 170 & 7 \\
\hline 6 & 1 & 12 \\
\hline 6 & 2 & 11 \\
\hline
\end{tabular}




\begin{tabular}{|c|c|c|}
\hline 6 & 3 & 12 \\
\hline 6 & 4 & 9 \\
\hline 6 & 5 & 10 \\
\hline 6 & 6 & 7 \\
\hline 6 & 7 & 8 \\
\hline 6 & 8 & 9 \\
\hline 6 & 9 & 8 \\
\hline 6 & 10 & 5 \\
\hline 6 & 11 & 5 \\
\hline 6 & 12 & 7 \\
\hline 6 & 13 & 6 \\
\hline 6 & 14 & 6 \\
\hline 6 & 15 & 6 \\
\hline 6 & 16 & 7 \\
\hline 6 & 17 & 7 \\
\hline 6 & 18 & 9 \\
\hline 6 & 19 & 7 \\
\hline 6 & 20 & 8 \\
\hline 6 & 21 & 11 \\
\hline 6 & 22 & 24 \\
\hline 6 & 23 & 21 \\
\hline 6 & 24 & 15 \\
\hline 6 & 25 & 16 \\
\hline 6 & 26 & 27 \\
\hline 6 & 27 & 34 \\
\hline 6 & 28 & 24 \\
\hline 6 & 29 & 47 \\
\hline 6 & 30 & 18 \\
\hline 6 & 31 & 14 \\
\hline 6 & 32 & 4 \\
\hline 6 & 33 & 4 \\
\hline 6 & 34 & 4 \\
\hline 6 & 35 & 5 \\
\hline 6 & 36 & 5 \\
\hline 6 & 37 & 6 \\
\hline 6 & 38 & 3 \\
\hline 6 & 39 & 5 \\
\hline 6 & 40 & 7 \\
\hline 6 & 41 & 6 \\
\hline 6 & 42 & 4 \\
\hline 6 & 43 & 7 \\
\hline 6 & 44 & 6 \\
\hline 6 & 45 & 9 \\
\hline 6 & 46 & 5 \\
\hline 6 & 47 & 6 \\
\hline
\end{tabular}




\begin{tabular}{|c|c|c|}
\hline 6 & 48 & 4 \\
\hline 6 & 49 & 5 \\
\hline 6 & 50 & 3 \\
\hline 6 & 51 & 4 \\
\hline 6 & 52 & 9 \\
\hline 6 & 53 & 13 \\
\hline 6 & 54 & 8 \\
\hline 6 & 55 & 7 \\
\hline 6 & 56 & 6 \\
\hline 6 & 57 & 8 \\
\hline 6 & 58 & 9 \\
\hline 6 & 59 & 8 \\
\hline 6 & 60 & 12 \\
\hline 6 & 61 & 7 \\
\hline 6 & 62 & 16 \\
\hline 6 & 63 & 19 \\
\hline 6 & 64 & 13 \\
\hline 6 & 65 & 14 \\
\hline 6 & 66 & 22 \\
\hline 6 & 67 & 32 \\
\hline 6 & 68 & 19 \\
\hline 6 & 69 & 16 \\
\hline 6 & 70 & 17 \\
\hline 6 & 71 & 29 \\
\hline 6 & 72 & 4 \\
\hline 6 & 73 & 6 \\
\hline 6 & 74 & 4 \\
\hline 6 & 75 & 7 \\
\hline 6 & 76 & 12 \\
\hline 6 & 77 & 35 \\
\hline 6 & 78 & 61 \\
\hline 6 & 79 & 58 \\
\hline 6 & 80 & 213 \\
\hline 6 & 81 & 134 \\
\hline 6 & 82 & 134 \\
\hline 6 & 83 & 573 \\
\hline 6 & 84 & 1821 \\
\hline 6 & 85 & 301 \\
\hline 6 & 86 & 33 \\
\hline 6 & 87 & 136 \\
\hline 6 & 88 & 30 \\
\hline 6 & 89 & 41 \\
\hline 6 & 90 & 40 \\
\hline 6 & 91 & 21 \\
\hline 6 & 92 & 129 \\
\hline
\end{tabular}




\begin{tabular}{|c|c|c|}
\hline 6 & 93 & 121 \\
\hline 6 & 94 & 46 \\
\hline 6 & 95 & 52 \\
\hline 6 & 96 & 27 \\
\hline 6 & 97 & 103 \\
\hline 6 & 98 & 30 \\
\hline 6 & 99 & 82 \\
\hline 6 & 100 & 100 \\
\hline 6 & 101 & 82 \\
\hline 6 & 102 & 40 \\
\hline 6 & 103 & 14 \\
\hline 6 & 104 & 16 \\
\hline 6 & 105 & 56 \\
\hline 6 & 106 & 32 \\
\hline 6 & 107 & 26 \\
\hline 6 & 108 & 9 \\
\hline 6 & 109 & 12 \\
\hline 6 & 110 & 10 \\
\hline 6 & 111 & 12 \\
\hline 6 & 112 & 14 \\
\hline 6 & 113 & 19 \\
\hline 6 & 114 & 15 \\
\hline 6 & 115 & 22 \\
\hline 6 & 116 & 9 \\
\hline 6 & 117 & 7 \\
\hline 6 & 118 & 7 \\
\hline 6 & 119 & 17 \\
\hline 6 & 120 & 17 \\
\hline 6 & 121 & 18 \\
\hline 6 & 122 & 7 \\
\hline 6 & 123 & 8 \\
\hline 6 & 124 & 9 \\
\hline 6 & 125 & 11 \\
\hline 6 & 126 & 16 \\
\hline 6 & 127 & 21 \\
\hline 6 & 128 & 18 \\
\hline 6 & 129 & 15 \\
\hline 6 & 130 & 11 \\
\hline 6 & 131 & 11 \\
\hline 6 & 132 & 12 \\
\hline 6 & 133 & 15 \\
\hline 6 & 134 & 31 \\
\hline 6 & 135 & 9 \\
\hline 6 & 136 & 2 \\
\hline 6 & 137 & 4 \\
\hline
\end{tabular}




\begin{tabular}{|c|c|c|}
\hline 6 & 138 & 2 \\
\hline 6 & 139 & 3 \\
\hline 6 & 140 & 3 \\
\hline 6 & 141 & 4 \\
\hline 6 & 142 & 3 \\
\hline 6 & 143 & 4 \\
\hline 6 & 144 & 4 \\
\hline 6 & 145 & 5 \\
\hline 6 & 146 & 5 \\
\hline 6 & 147 & 3 \\
\hline 6 & 148 & 2 \\
\hline 6 & 149 & 2 \\
\hline 6 & 150 & 4 \\
\hline 6 & 151 & 5 \\
\hline 6 & 152 & 4 \\
\hline 6 & 153 & 5 \\
\hline 6 & 154 & 3 \\
\hline 6 & 155 & 4 \\
\hline 6 & 156 & 3 \\
\hline 6 & 157 & 3 \\
\hline 6 & 158 & 3 \\
\hline 6 & 159 & 1 \\
\hline 6 & 160 & 2 \\
\hline 6 & 161 & 2 \\
\hline 6 & 162 & 2 \\
\hline 6 & 163 & 2 \\
\hline 6 & 164 & 2 \\
\hline 6 & 165 & 2 \\
\hline 6 & 166 & 2 \\
\hline 6 & 167 & 2 \\
\hline 6 & 168 & 2 \\
\hline 6 & 169 & 2 \\
\hline 6 & 170 & 3 \\
\hline 7 & 1 & 30 \\
\hline 7 & 2 & 21 \\
\hline 7 & 3 & 10 \\
\hline 7 & 4 & 17 \\
\hline 7 & 5 & 8 \\
\hline 7 & 6 & 6 \\
\hline 7 & 7 & 7 \\
\hline 7 & 8 & 7 \\
\hline 7 & 9 & 10 \\
\hline 7 & 10 & 9 \\
\hline 7 & 11 & 4 \\
\hline 7 & 12 & 6 \\
\hline
\end{tabular}




\begin{tabular}{|c|c|c|}
\hline 7 & 13 & 5 \\
\hline 7 & 14 & 10 \\
\hline 7 & 15 & 10 \\
\hline 7 & 16 & 13 \\
\hline 7 & 17 & 13 \\
\hline 7 & 18 & 18 \\
\hline 7 & 19 & 12 \\
\hline 7 & 20 & 14 \\
\hline 7 & 21 & 24 \\
\hline 7 & 22 & 284 \\
\hline 7 & 23 & 48 \\
\hline 7 & 24 & 61 \\
\hline 7 & 25 & 114 \\
\hline 7 & 26 & 40 \\
\hline 7 & 27 & 96 \\
\hline 7 & 28 & 18 \\
\hline 7 & 29 & 32 \\
\hline 7 & 30 & 14 \\
\hline 7 & 31 & 11 \\
\hline 7 & 32 & 7 \\
\hline 7 & 33 & 6 \\
\hline 7 & 34 & 7 \\
\hline 7 & 35 & 8 \\
\hline 7 & 36 & 8 \\
\hline 7 & 37 & 12 \\
\hline 7 & 38 & 4 \\
\hline 7 & 39 & 8 \\
\hline 7 & 40 & 13 \\
\hline 7 & 41 & 11 \\
\hline 7 & 42 & 23 \\
\hline 7 & 43 & 35 \\
\hline 7 & 44 & 25 \\
\hline 7 & 45 & 23 \\
\hline 7 & 46 & 19 \\
\hline 7 & 47 & 33 \\
\hline 7 & 48 & 14 \\
\hline 7 & 49 & 20 \\
\hline 7 & 50 & 13 \\
\hline 7 & 51 & 12 \\
\hline 7 & 52 & 55 \\
\hline 7 & 53 & 44 \\
\hline 7 & 54 & 65 \\
\hline 7 & 55 & 36 \\
\hline 7 & 56 & 35 \\
\hline 7 & 57 & 98 \\
\hline
\end{tabular}




\begin{tabular}{|c|c|c|}
\hline 7 & 58 & 98 \\
\hline 7 & 59 & 58 \\
\hline 7 & 60 & 131 \\
\hline 7 & 61 & 91 \\
\hline 7 & 62 & 564 \\
\hline 7 & 63 & 3770 \\
\hline 7 & 64 & 680 \\
\hline 7 & 65 & 608 \\
\hline 7 & 66 & 6999 \\
\hline 7 & 67 & 79 \\
\hline 7 & 68 & 219 \\
\hline 7 & 69 & 220 \\
\hline 7 & 70 & 268 \\
\hline 7 & 71 & 230 \\
\hline 7 & 72 & 22 \\
\hline 7 & 73 & 36 \\
\hline 7 & 74 & 28 \\
\hline 7 & 75 & 18 \\
\hline 7 & 76 & 51 \\
\hline 7 & 77 & 38 \\
\hline 7 & 78 & 60 \\
\hline 7 & 79 & 55 \\
\hline 7 & 80 & 9 \\
\hline 7 & 81 & 10 \\
\hline 7 & 82 & 10 \\
\hline 7 & 83 & 14 \\
\hline 7 & 84 & 27 \\
\hline 7 & 85 & 9 \\
\hline 7 & 86 & 82 \\
\hline 7 & 87 & 24 \\
\hline 7 & 88 & 40 \\
\hline 7 & 89 & 29 \\
\hline 7 & 90 & 18 \\
\hline 7 & 91 & 13 \\
\hline 7 & 92 & 12 \\
\hline 7 & 93 & 9 \\
\hline 7 & 94 & 7 \\
\hline 7 & 95 & 8 \\
\hline 7 & 96 & 19 \\
\hline 7 & 97 & 18 \\
\hline 7 & 98 & 9 \\
\hline 7 & 99 & 10 \\
\hline 7 & 100 & 11 \\
\hline 7 & 101 & 7 \\
\hline 7 & 102 & 5 \\
\hline
\end{tabular}




\begin{tabular}{|c|c|c|}
\hline 7 & 103 & 3 \\
\hline 7 & 104 & 4 \\
\hline 7 & 105 & 9 \\
\hline 7 & 106 & 6 \\
\hline 7 & 107 & 4 \\
\hline 7 & 108 & 7 \\
\hline 7 & 109 & 5 \\
\hline 7 & 110 & 8 \\
\hline 7 & 111 & 6 \\
\hline 7 & 112 & 7 \\
\hline 7 & 113 & 5 \\
\hline 7 & 114 & 8 \\
\hline 7 & 115 & 7 \\
\hline 7 & 116 & 4 \\
\hline 7 & 117 & 6 \\
\hline 7 & 118 & 4 \\
\hline 7 & 119 & 7 \\
\hline 7 & 120 & 4 \\
\hline 7 & 121 & 4 \\
\hline 7 & 122 & 4 \\
\hline 7 & 123 & 3 \\
\hline 7 & 124 & 3 \\
\hline 7 & 125 & 3 \\
\hline 7 & 126 & 4 \\
\hline 7 & 127 & 5 \\
\hline 7 & 128 & 5 \\
\hline 7 & 129 & 4 \\
\hline 7 & 130 & 3 \\
\hline 7 & 131 & 4 \\
\hline 7 & 132 & 4 \\
\hline 7 & 133 & 6 \\
\hline 7 & 134 & 6 \\
\hline 7 & 135 & 5 \\
\hline 7 & 136 & 2 \\
\hline 7 & 137 & 4 \\
\hline 7 & 138 & 2 \\
\hline 7 & 139 & 2 \\
\hline 7 & 140 & 2 \\
\hline 7 & 141 & 4 \\
\hline 7 & 142 & 3 \\
\hline 7 & 143 & 3 \\
\hline 7 & 144 & 4 \\
\hline 7 & 145 & 4 \\
\hline 7 & 146 & 5 \\
\hline 7 & 147 & 3 \\
\hline
\end{tabular}




\begin{tabular}{|c|c|c|}
\hline 7 & 148 & 2 \\
\hline 7 & 149 & 2 \\
\hline 7 & 150 & 2 \\
\hline 7 & 151 & 7 \\
\hline 7 & 152 & 3 \\
\hline 7 & 153 & 4 \\
\hline 7 & 154 & 3 \\
\hline 7 & 155 & 3 \\
\hline 7 & 156 & 3 \\
\hline 7 & 157 & 3 \\
\hline 7 & 158 & 3 \\
\hline 7 & 159 & 2 \\
\hline 7 & 160 & 2 \\
\hline 7 & 161 & 3 \\
\hline 7 & 162 & 3 \\
\hline 7 & 163 & 3 \\
\hline 7 & 164 & 2 \\
\hline 7 & 165 & 3 \\
\hline 7 & 166 & 3 \\
\hline 7 & 167 & 3 \\
\hline 7 & 168 & 3 \\
\hline 7 & 169 & 2 \\
\hline 7 & 170 & 2 \\
\hline 8 & 1 & 4 \\
\hline 8 & 2 & 7 \\
\hline 8 & 3 & 2 \\
\hline 8 & 4 & 4 \\
\hline 8 & 5 & 2 \\
\hline 8 & 6 & 2 \\
\hline 8 & 7 & 5 \\
\hline 8 & 8 & 5 \\
\hline 8 & 9 & 5 \\
\hline 8 & 10 & 7 \\
\hline 8 & 11 & 6 \\
\hline 8 & 12 & 5 \\
\hline 8 & 13 & 3 \\
\hline 8 & 14 & 12 \\
\hline 8 & 15 & 6 \\
\hline 8 & 16 & 10 \\
\hline 8 & 17 & 10 \\
\hline 8 & 18 & 4 \\
\hline 8 & 19 & 14 \\
\hline 8 & 20 & 12 \\
\hline 8 & 21 & 5 \\
\hline 8 & 22 & 6 \\
\hline
\end{tabular}




\begin{tabular}{|c|c|c|}
\hline 8 & 23 & 4 \\
\hline 8 & 24 & 4 \\
\hline 8 & 25 & 4 \\
\hline 8 & 26 & 3 \\
\hline 8 & 27 & 3 \\
\hline 8 & 28 & 3 \\
\hline 8 & 29 & 4 \\
\hline 8 & 30 & 3 \\
\hline 8 & 31 & 2 \\
\hline 8 & 32 & 141 \\
\hline 8 & 33 & 75 \\
\hline 8 & 34 & 24 \\
\hline 8 & 35 & 26 \\
\hline 8 & 36 & 20 \\
\hline 8 & 37 & 21 \\
\hline 8 & 38 & 36 \\
\hline 8 & 39 & 23 \\
\hline 8 & 40 & 13 \\
\hline 8 & 41 & 11 \\
\hline 8 & 42 & 8 \\
\hline 8 & 43 & 8 \\
\hline 8 & 44 & 10 \\
\hline 8 & 45 & 9 \\
\hline 8 & 46 & 5 \\
\hline 8 & 47 & 3 \\
\hline 8 & 48 & 4 \\
\hline 8 & 49 & 3 \\
\hline 8 & 50 & 6 \\
\hline 8 & 51 & 4 \\
\hline 8 & 52 & 6 \\
\hline 8 & 53 & 6 \\
\hline 8 & 54 & 4 \\
\hline 8 & 55 & 6 \\
\hline 8 & 56 & 4 \\
\hline 8 & 57 & 3 \\
\hline 8 & 58 & 3 \\
\hline 8 & 59 & 3 \\
\hline 8 & 60 & 3 \\
\hline 8 & 61 & 4 \\
\hline 8 & 62 & 5 \\
\hline 8 & 63 & 5 \\
\hline 8 & 64 & 4 \\
\hline 8 & 65 & 4 \\
\hline 8 & 66 & 4 \\
\hline 8 & 67 & 4 \\
\hline
\end{tabular}




\begin{tabular}{|c|c|c|}
\hline 8 & 68 & 3 \\
\hline 8 & 69 & 5 \\
\hline 8 & 70 & 5 \\
\hline 8 & 71 & 4 \\
\hline 8 & 72 & 2 \\
\hline 8 & 73 & 3 \\
\hline 8 & 74 & 2 \\
\hline 8 & 75 & 2 \\
\hline 8 & 76 & 3 \\
\hline 8 & 77 & 3 \\
\hline 8 & 78 & 3 \\
\hline 8 & 79 & 2 \\
\hline 8 & 80 & 2 \\
\hline 8 & 81 & 2 \\
\hline 8 & 82 & 2 \\
\hline 8 & 83 & 3 \\
\hline 8 & 84 & 2 \\
\hline 8 & 85 & 2 \\
\hline 8 & 86 & 4 \\
\hline 8 & 87 & 4 \\
\hline 8 & 88 & 2 \\
\hline 8 & 89 & 2 \\
\hline 8 & 90 & 2 \\
\hline 8 & 91 & 2 \\
\hline 8 & 92 & 3 \\
\hline 8 & 93 & 2 \\
\hline 8 & 94 & 2 \\
\hline 8 & 95 & 2 \\
\hline 8 & 96 & 3 \\
\hline 8 & 97 & 3 \\
\hline 8 & 98 & 2 \\
\hline 8 & 99 & 2 \\
\hline 8 & 100 & 3 \\
\hline 8 & 101 & 2 \\
\hline 8 & 102 & 2 \\
\hline 8 & 103 & 1 \\
\hline 8 & 104 & 1 \\
\hline 8 & 105 & 2 \\
\hline 8 & 106 & 2 \\
\hline 8 & 107 & 2 \\
\hline 8 & 108 & 2 \\
\hline 8 & 109 & 2 \\
\hline 8 & 110 & 2 \\
\hline 8 & 111 & 2 \\
\hline 8 & 112 & 2 \\
\hline
\end{tabular}




\begin{tabular}{|c|c|c|}
\hline 8 & 113 & 2 \\
\hline 8 & 114 & 2 \\
\hline 8 & 115 & 2 \\
\hline 8 & 116 & 2 \\
\hline 8 & 117 & 3 \\
\hline 8 & 118 & 2 \\
\hline 8 & 119 & 2 \\
\hline 8 & 120 & 2 \\
\hline 8 & 121 & 2 \\
\hline 8 & 122 & 2 \\
\hline 8 & 123 & 2 \\
\hline 8 & 124 & 1 \\
\hline 8 & 125 & 2 \\
\hline 8 & 126 & 2 \\
\hline 8 & 127 & 2 \\
\hline 8 & 128 & 2 \\
\hline 8 & 129 & 2 \\
\hline 8 & 130 & 2 \\
\hline 8 & 131 & 1 \\
\hline 8 & 132 & 1 \\
\hline 8 & 133 & 1 \\
\hline 8 & 134 & 2 \\
\hline 8 & 135 & 2 \\
\hline 8 & 136 & 6 \\
\hline 8 & 137 & 3 \\
\hline 8 & 138 & 2 \\
\hline 8 & 139 & 2 \\
\hline 8 & 140 & 3 \\
\hline 8 & 141 & 3 \\
\hline 8 & 142 & 2 \\
\hline 8 & 143 & 2 \\
\hline 8 & 144 & 3 \\
\hline 8 & 145 & 4 \\
\hline 8 & 146 & 3 \\
\hline 8 & 147 & 4 \\
\hline 8 & 148 & 13 \\
\hline 8 & 149 & 13 \\
\hline 8 & 150 & 9 \\
\hline 8 & 151 & 6 \\
\hline 8 & 152 & 8 \\
\hline 8 & 153 & 9 \\
\hline 8 & 154 & 3 \\
\hline 8 & 155 & 5 \\
\hline 8 & 156 & 6 \\
\hline 8 & 157 & 3 \\
\hline
\end{tabular}




\begin{tabular}{|c|c|c|}
\hline 8 & 158 & 4 \\
\hline 8 & 159 & 9 \\
\hline 8 & 160 & 18 \\
\hline 8 & 161 & 28 \\
\hline 8 & 162 & 37 \\
\hline 8 & 163 & 25 \\
\hline 8 & 164 & 18 \\
\hline 8 & 165 & 16 \\
\hline 8 & 166 & 179 \\
\hline 8 & 167 & 75 \\
\hline 8 & 168 & 54 \\
\hline 8 & 169 & 19 \\
\hline 8 & 170 & 9 \\
\hline 9 & 1 & 5 \\
\hline 9 & 2 & 12 \\
\hline 9 & 3 & 4 \\
\hline 9 & 4 & 6 \\
\hline 9 & 5 & 3 \\
\hline 9 & 6 & 1 \\
\hline 9 & 7 & 9 \\
\hline 9 & 8 & 11 \\
\hline 9 & 9 & 11 \\
\hline 9 & 10 & 11 \\
\hline 9 & 11 & 8 \\
\hline 9 & 12 & 7 \\
\hline 9 & 13 & 1 \\
\hline 9 & 14 & 24 \\
\hline 9 & 15 & 11 \\
\hline 9 & 16 & 19 \\
\hline 9 & 17 & 19 \\
\hline 9 & 18 & 6 \\
\hline 9 & 19 & 28 \\
\hline 9 & 20 & 23 \\
\hline 9 & 21 & 7 \\
\hline 9 & 22 & 9 \\
\hline 9 & 23 & 6 \\
\hline 9 & 24 & 6 \\
\hline 9 & 25 & 6 \\
\hline 9 & 26 & 4 \\
\hline 9 & 27 & 5 \\
\hline 9 & 28 & 4 \\
\hline 9 & 29 & 5 \\
\hline 9 & 30 & 4 \\
\hline 9 & 31 & 3 \\
\hline 9 & 32 & 61035 \\
\hline
\end{tabular}




\begin{tabular}{|c|c|c|}
\hline 9 & 33 & 809 \\
\hline 9 & 34 & 68 \\
\hline 9 & 35 & 74 \\
\hline 9 & 36 & 48 \\
\hline 9 & 37 & 51 \\
\hline 9 & 38 & 143 \\
\hline 9 & 39 & 35 \\
\hline 9 & 40 & 25 \\
\hline 9 & 41 & 20 \\
\hline 9 & 42 & 13 \\
\hline 9 & 43 & 14 \\
\hline 9 & 44 & 19 \\
\hline 9 & 45 & 16 \\
\hline 9 & 46 & 8 \\
\hline 9 & 47 & 5 \\
\hline 9 & 48 & 6 \\
\hline 9 & 49 & 4 \\
\hline 9 & 50 & 8 \\
\hline 9 & 51 & 6 \\
\hline 9 & 52 & 10 \\
\hline 9 & 53 & 9 \\
\hline 9 & 54 & 7 \\
\hline 9 & 55 & 9 \\
\hline 9 & 56 & 5 \\
\hline 9 & 57 & 5 \\
\hline 9 & 58 & 5 \\
\hline 9 & 59 & 4 \\
\hline 9 & 60 & 4 \\
\hline 9 & 61 & 6 \\
\hline 9 & 62 & 7 \\
\hline 9 & 63 & 7 \\
\hline 9 & 64 & 6 \\
\hline 9 & 65 & 6 \\
\hline 9 & 66 & 6 \\
\hline 9 & 67 & 5 \\
\hline 9 & 68 & 4 \\
\hline 9 & 69 & 7 \\
\hline 9 & 70 & 7 \\
\hline 9 & 71 & 5 \\
\hline 9 & 72 & 3 \\
\hline 9 & 73 & 4 \\
\hline 9 & 74 & 3 \\
\hline 9 & 75 & 3 \\
\hline 9 & 76 & 3 \\
\hline 9 & 77 & 4 \\
\hline
\end{tabular}




\begin{tabular}{|c|c|c|}
\hline 9 & 78 & 4 \\
\hline 9 & 79 & 3 \\
\hline 9 & 80 & 3 \\
\hline 9 & 81 & 3 \\
\hline 9 & 82 & 3 \\
\hline 9 & 83 & 4 \\
\hline 9 & 84 & 3 \\
\hline 9 & 85 & 3 \\
\hline 9 & 86 & 5 \\
\hline 9 & 87 & 5 \\
\hline 9 & 88 & 3 \\
\hline 9 & 89 & 3 \\
\hline 9 & 90 & 2 \\
\hline 9 & 91 & 2 \\
\hline 9 & 92 & 3 \\
\hline 9 & 93 & 3 \\
\hline 9 & 94 & 3 \\
\hline 9 & 95 & 3 \\
\hline 9 & 96 & 4 \\
\hline 9 & 97 & 3 \\
\hline 9 & 98 & 3 \\
\hline 9 & 99 & 3 \\
\hline 9 & 100 & 3 \\
\hline 9 & 101 & 3 \\
\hline 9 & 102 & 2 \\
\hline 9 & 103 & 2 \\
\hline 9 & 104 & 2 \\
\hline 9 & 105 & 3 \\
\hline 9 & 106 & 3 \\
\hline 9 & 107 & 2 \\
\hline 9 & 108 & 3 \\
\hline 9 & 109 & 2 \\
\hline 9 & 110 & 3 \\
\hline 9 & 111 & 2 \\
\hline 9 & 112 & 2 \\
\hline 9 & 113 & 2 \\
\hline 9 & 114 & 3 \\
\hline 9 & 115 & 2 \\
\hline 9 & 116 & 1 \\
\hline 9 & 117 & 2 \\
\hline 9 & 118 & 1 \\
\hline 9 & 119 & 2 \\
\hline 9 & 120 & 2 \\
\hline 9 & 121 & 2 \\
\hline 9 & 122 & 1 \\
\hline
\end{tabular}




\begin{tabular}{|c|c|c|}
\hline 9 & 123 & 1 \\
\hline 9 & 124 & 2 \\
\hline 9 & 125 & 2 \\
\hline 9 & 126 & 2 \\
\hline 9 & 127 & 2 \\
\hline 9 & 128 & 2 \\
\hline 9 & 129 & 2 \\
\hline 9 & 130 & 2 \\
\hline 9 & 131 & 2 \\
\hline 9 & 132 & 2 \\
\hline 9 & 133 & 2 \\
\hline 9 & 134 & 3 \\
\hline 9 & 135 & 1 \\
\hline 9 & 136 & 2 \\
\hline 9 & 137 & 1 \\
\hline 9 & 138 & 1 \\
\hline 9 & 139 & 1 \\
\hline 9 & 140 & 1 \\
\hline 9 & 141 & 1 \\
\hline 9 & 142 & 1 \\
\hline 9 & 143 & 1 \\
\hline 9 & 144 & 1 \\
\hline 9 & 145 & 2 \\
\hline 9 & 146 & 1 \\
\hline 9 & 147 & 2 \\
\hline 9 & 148 & 22 \\
\hline 9 & 149 & 10 \\
\hline 9 & 150 & 8 \\
\hline 9 & 151 & 9 \\
\hline 9 & 152 & 7 \\
\hline 9 & 153 & 12 \\
\hline 9 & 154 & 2 \\
\hline 9 & 155 & 2 \\
\hline 9 & 156 & 5 \\
\hline 9 & 157 & 2 \\
\hline 9 & 158 & 2 \\
\hline 9 & 159 & 8 \\
\hline 9 & 160 & 10 \\
\hline 9 & 161 & 14 \\
\hline 9 & 162 & 17 \\
\hline 9 & 163 & 13 \\
\hline 9 & 164 & 14 \\
\hline 9 & 165 & 4 \\
\hline 9 & 166 & 42 \\
\hline 9 & 167 & 37 \\
\hline
\end{tabular}




\begin{tabular}{|c|c|c|}
\hline 9 & 168 & 29 \\
\hline 9 & 169 & 20 \\
\hline 9 & 170 & 7 \\
\hline 10 & 1 & 46 \\
\hline 10 & 2 & 51 \\
\hline 10 & 3 & 75 \\
\hline 10 & 4 & 36 \\
\hline 10 & 5 & 82 \\
\hline 10 & 6 & 16 \\
\hline 10 & 7 & 41 \\
\hline 10 & 8 & 48 \\
\hline 10 & 9 & 34 \\
\hline 10 & 10 & 24 \\
\hline 10 & 11 & 10 \\
\hline 10 & 12 & 17 \\
\hline 10 & 13 & 13 \\
\hline 10 & 14 & 17 \\
\hline 10 & 15 & 23 \\
\hline 10 & 16 & 33 \\
\hline 10 & 17 & 25 \\
\hline 10 & 18 & 29 \\
\hline 10 & 19 & 16 \\
\hline 10 & 20 & 17 \\
\hline 10 & 21 & 27 \\
\hline 10 & 22 & 11 \\
\hline 10 & 23 & 33 \\
\hline 10 & 24 & 24 \\
\hline 10 & 25 & 18 \\
\hline 10 & 26 & 42 \\
\hline 10 & 27 & 21 \\
\hline 10 & 28 & 40 \\
\hline 10 & 29 & 27 \\
\hline 10 & 30 & 104 \\
\hline 10 & 31 & 68 \\
\hline 10 & 32 & 3 \\
\hline 10 & 33 & 3 \\
\hline 10 & 34 & 10 \\
\hline 10 & 35 & 11 \\
\hline 10 & 36 & 10 \\
\hline 10 & 37 & 4 \\
\hline 10 & 38 & 8 \\
\hline 10 & 39 & 11 \\
\hline 10 & 40 & 4 \\
\hline 10 & 41 & 4 \\
\hline 10 & 42 & 3 \\
\hline
\end{tabular}




\begin{tabular}{|c|c|c|}
\hline 10 & 43 & 4 \\
\hline 10 & 44 & 4 \\
\hline 10 & 45 & 5 \\
\hline 10 & 46 & 3 \\
\hline 10 & 47 & 4 \\
\hline 10 & 48 & 3 \\
\hline 10 & 49 & 3 \\
\hline 10 & 50 & 2 \\
\hline 10 & 51 & 3 \\
\hline 10 & 52 & 5 \\
\hline 10 & 53 & 7 \\
\hline 10 & 54 & 5 \\
\hline 10 & 55 & 4 \\
\hline 10 & 56 & 4 \\
\hline 10 & 57 & 5 \\
\hline 10 & 58 & 5 \\
\hline 10 & 59 & 5 \\
\hline 10 & 60 & 7 \\
\hline 10 & 61 & 4 \\
\hline 10 & 62 & 8 \\
\hline 10 & 63 & 10 \\
\hline 10 & 64 & 7 \\
\hline 10 & 65 & 7 \\
\hline 10 & 66 & 10 \\
\hline 10 & 67 & 14 \\
\hline 10 & 68 & 9 \\
\hline 10 & 69 & 12 \\
\hline 10 & 70 & 14 \\
\hline 10 & 71 & 13 \\
\hline 10 & 72 & 3 \\
\hline 10 & 73 & 4 \\
\hline 10 & 74 & 3 \\
\hline 10 & 75 & 5 \\
\hline 10 & 76 & 7 \\
\hline 10 & 77 & 9 \\
\hline 10 & 78 & 12 \\
\hline 10 & 79 & 6 \\
\hline 10 & 80 & 13 \\
\hline 10 & 81 & 10 \\
\hline 10 & 82 & 10 \\
\hline 10 & 83 & 32 \\
\hline 10 & 84 & 19 \\
\hline 10 & 85 & 14 \\
\hline 10 & 86 & 12 \\
\hline 10 & 87 & 18 \\
\hline
\end{tabular}




\begin{tabular}{|c|c|c|}
\hline 10 & 88 & 6 \\
\hline 10 & 89 & 6 \\
\hline 10 & 90 & 7 \\
\hline 10 & 91 & 4 \\
\hline 10 & 92 & 45 \\
\hline 10 & 93 & 35 \\
\hline 10 & 94 & 75 \\
\hline 10 & 95 & 89 \\
\hline 10 & 96 & 26 \\
\hline 10 & 97 & 39 \\
\hline 10 & 98 & 34 \\
\hline 10 & 99 & 77 \\
\hline 10 & 100 & 52 \\
\hline 10 & 101 & 23 \\
\hline 10 & 102 & 11 \\
\hline 10 & 103 & 17 \\
\hline 10 & 104 & 7 \\
\hline 10 & 105 & 103 \\
\hline 10 & 106 & 76 \\
\hline 10 & 107 & 36 \\
\hline 10 & 108 & 66 \\
\hline 10 & 109 & 112 \\
\hline 10 & 110 & 105 \\
\hline 10 & 111 & 120 \\
\hline 10 & 112 & 194 \\
\hline 10 & 113 & 67 \\
\hline 10 & 114 & 337 \\
\hline 10 & 115 & 6219 \\
\hline 10 & 116 & 54 \\
\hline 10 & 117 & 36 \\
\hline 10 & 118 & 32 \\
\hline 10 & 119 & 177 \\
\hline 10 & 120 & 198 \\
\hline 10 & 121 & 209 \\
\hline 10 & 122 & 32 \\
\hline 10 & 123 & 40 \\
\hline 10 & 124 & 39 \\
\hline 10 & 125 & 57 \\
\hline 10 & 126 & 154 \\
\hline 10 & 127 & 406 \\
\hline 10 & 128 & 127 \\
\hline 10 & 129 & 115 \\
\hline 10 & 130 & 79 \\
\hline 10 & 131 & 57 \\
\hline 10 & 132 & 47 \\
\hline
\end{tabular}




\begin{tabular}{|c|c|c|}
\hline 10 & 133 & 402 \\
\hline 10 & 134 & 101 \\
\hline 10 & 135 & 49 \\
\hline 10 & 136 & 4 \\
\hline 10 & 137 & 8 \\
\hline 10 & 138 & 4 \\
\hline 10 & 139 & 4 \\
\hline 10 & 140 & 5 \\
\hline 10 & 141 & 8 \\
\hline 10 & 142 & 5 \\
\hline 10 & 143 & 7 \\
\hline 10 & 144 & 9 \\
\hline 10 & 145 & 10 \\
\hline 10 & 146 & 23 \\
\hline 10 & 147 & 5 \\
\hline 10 & 148 & 11 \\
\hline 10 & 149 & 7 \\
\hline 10 & 150 & 7 \\
\hline 10 & 151 & 9 \\
\hline 10 & 152 & 7 \\
\hline 10 & 153 & 11 \\
\hline 10 & 154 & 5 \\
\hline 10 & 155 & 7 \\
\hline 10 & 156 & 5 \\
\hline 10 & 157 & 5 \\
\hline 10 & 158 & 5 \\
\hline 10 & 159 & 4 \\
\hline 10 & 160 & 2 \\
\hline 10 & 161 & 2 \\
\hline 10 & 162 & 2 \\
\hline 10 & 163 & 3 \\
\hline 10 & 164 & 5 \\
\hline 10 & 165 & 2 \\
\hline 10 & 166 & 2 \\
\hline 10 & 167 & 2 \\
\hline 10 & 168 & 2 \\
\hline 10 & 169 & 7 \\
\hline 10 & 170 & 4 \\
\hline 11 & 1 & 59 \\
\hline 11 & 2 & 97 \\
\hline 11 & 3 & 79 \\
\hline 11 & 4 & 62 \\
\hline 11 & 5 & 73 \\
\hline 11 & 6 & 92 \\
\hline 11 & 7 & 168 \\
\hline
\end{tabular}




\begin{tabular}{|c|c|c|}
\hline 11 & 8 & 189 \\
\hline 11 & 9 & 271 \\
\hline 11 & 10 & 243 \\
\hline 11 & 11 & 249 \\
\hline 11 & 12 & 1087 \\
\hline 11 & 13 & 251 \\
\hline 11 & 14 & 32 \\
\hline 11 & 15 & 82 \\
\hline 11 & 16 & 65 \\
\hline 11 & 17 & 44 \\
\hline 11 & 18 & 45 \\
\hline 11 & 19 & 35 \\
\hline 11 & 20 & 22 \\
\hline 11 & 21 & 28 \\
\hline 11 & 22 & 8 \\
\hline 11 & 23 & 41 \\
\hline 11 & 24 & 29 \\
\hline 11 & 25 & 12 \\
\hline 11 & 26 & 24 \\
\hline 11 & 27 & 14 \\
\hline 11 & 28 & 23 \\
\hline 11 & 29 & 17 \\
\hline 11 & 30 & 31 \\
\hline 11 & 31 & 32 \\
\hline 11 & 32 & 9 \\
\hline 11 & 33 & 9 \\
\hline 11 & 34 & 17 \\
\hline 11 & 35 & 15 \\
\hline 11 & 36 & 13 \\
\hline 11 & 37 & 10 \\
\hline 11 & 38 & 13 \\
\hline 11 & 39 & 20 \\
\hline 11 & 40 & 3 \\
\hline 11 & 41 & 3 \\
\hline 11 & 42 & 3 \\
\hline 11 & 43 & 3 \\
\hline 11 & 44 & 11 \\
\hline 11 & 45 & 9 \\
\hline 11 & 46 & 3 \\
\hline 11 & 47 & 3 \\
\hline 11 & 48 & 2 \\
\hline 11 & 49 & 3 \\
\hline 11 & 50 & 2 \\
\hline 11 & 51 & 2 \\
\hline 11 & 52 & 4 \\
\hline
\end{tabular}




\begin{tabular}{|c|c|c|}
\hline 11 & 53 & 5 \\
\hline 11 & 54 & 4 \\
\hline 11 & 55 & 3 \\
\hline 11 & 56 & 3 \\
\hline 11 & 57 & 4 \\
\hline 11 & 58 & 4 \\
\hline 11 & 59 & 4 \\
\hline 11 & 60 & 5 \\
\hline 11 & 61 & 4 \\
\hline 11 & 62 & 6 \\
\hline 11 & 63 & 7 \\
\hline 11 & 64 & 6 \\
\hline 11 & 65 & 6 \\
\hline 11 & 66 & 8 \\
\hline 11 & 67 & 10 \\
\hline 11 & 68 & 7 \\
\hline 11 & 69 & 9 \\
\hline 11 & 70 & 10 \\
\hline 11 & 71 & 9 \\
\hline 11 & 72 & 2 \\
\hline 11 & 73 & 3 \\
\hline 11 & 74 & 2 \\
\hline 11 & 75 & 4 \\
\hline 11 & 76 & 5 \\
\hline 11 & 77 & 5 \\
\hline 11 & 78 & 6 \\
\hline 11 & 79 & 5 \\
\hline 11 & 80 & 4 \\
\hline 11 & 81 & 5 \\
\hline 11 & 82 & 5 \\
\hline 11 & 83 & 7 \\
\hline 11 & 84 & 4 \\
\hline 11 & 85 & 4 \\
\hline 11 & 86 & 9 \\
\hline 11 & 87 & 8 \\
\hline 11 & 88 & 5 \\
\hline 11 & 89 & 4 \\
\hline 11 & 90 & 4 \\
\hline 11 & 91 & 3 \\
\hline 11 & 92 & 8 \\
\hline 11 & 93 & 7 \\
\hline 11 & 94 & 14 \\
\hline 11 & 95 & 15 \\
\hline 11 & 96 & 16 \\
\hline 11 & 97 & 11 \\
\hline
\end{tabular}




\begin{tabular}{|c|c|c|}
\hline 11 & 98 & 17 \\
\hline 11 & 99 & 13 \\
\hline 11 & 100 & 12 \\
\hline 11 & 101 & 6 \\
\hline 11 & 102 & 3 \\
\hline 11 & 103 & 5 \\
\hline 11 & 104 & 3 \\
\hline 11 & 105 & 11 \\
\hline 11 & 106 & 10 \\
\hline 11 & 107 & 7 \\
\hline 11 & 108 & 78 \\
\hline 11 & 109 & 80 \\
\hline 11 & 110 & 77 \\
\hline 11 & 111 & 46 \\
\hline 11 & 112 & 45 \\
\hline 11 & 113 & 7 \\
\hline 11 & 114 & 20 \\
\hline 11 & 115 & 16 \\
\hline 11 & 116 & 48 \\
\hline 11 & 117 & 167 \\
\hline 11 & 118 & 29 \\
\hline 11 & 119 & 40 \\
\hline 11 & 120 & 24 \\
\hline 11 & 121 & 21 \\
\hline 11 & 122 & 22 \\
\hline 11 & 123 & 16 \\
\hline 11 & 124 & 10 \\
\hline 11 & 125 & 12 \\
\hline 11 & 126 & 19 \\
\hline 11 & 127 & 12 \\
\hline 11 & 128 & 7 \\
\hline 11 & 129 & 9 \\
\hline 11 & 130 & 16 \\
\hline 11 & 131 & 5 \\
\hline 11 & 132 & 6 \\
\hline 11 & 133 & 12 \\
\hline 11 & 134 & 9 \\
\hline 11 & 135 & 26 \\
\hline 11 & 136 & 11 \\
\hline 11 & 137 & 22 \\
\hline 11 & 138 & 8 \\
\hline 11 & 139 & 9 \\
\hline 11 & 140 & 12 \\
\hline 11 & 141 & 30 \\
\hline 11 & 142 & 11 \\
\hline
\end{tabular}




\begin{tabular}{|c|c|c|}
\hline 11 & 143 & 22 \\
\hline 11 & 144 & 38 \\
\hline 11 & 145 & 66 \\
\hline 11 & 146 & 46 \\
\hline 11 & 147 & 23 \\
\hline 11 & 148 & 37 \\
\hline 11 & 149 & 74 \\
\hline 11 & 150 & 58 \\
\hline 11 & 151 & 129 \\
\hline 11 & 152 & 72 \\
\hline 11 & 153 & 254 \\
\hline 11 & 154 & 45 \\
\hline 11 & 155 & 36 \\
\hline 11 & 156 & 20 \\
\hline 11 & 157 & 22 \\
\hline 11 & 158 & 24 \\
\hline 11 & 159 & 18 \\
\hline 11 & 160 & 5 \\
\hline 11 & 161 & 3 \\
\hline 11 & 162 & 15 \\
\hline 11 & 163 & 17 \\
\hline 11 & 164 & 24 \\
\hline 11 & 165 & 6 \\
\hline 11 & 166 & 10 \\
\hline 11 & 167 & 11 \\
\hline 11 & 168 & 13 \\
\hline 11 & 169 & 15 \\
\hline 11 & 170 & 15 \\
\hline 12 & 1 & 7 \\
\hline 12 & 2 & 23 \\
\hline 12 & 3 & 29 \\
\hline 12 & 4 & 7 \\
\hline 12 & 5 & 34 \\
\hline 12 & 6 & 14 \\
\hline 12 & 7 & 25 \\
\hline 12 & 8 & 27 \\
\hline 12 & 9 & 29 \\
\hline 12 & 10 & 9 \\
\hline 12 & 11 & 33 \\
\hline 12 & 12 & 41 \\
\hline 12 & 13 & 64 \\
\hline 12 & 14 & 6 \\
\hline 12 & 15 & 6 \\
\hline 12 & 16 & 8 \\
\hline 12 & 17 & 7 \\
\hline
\end{tabular}




\begin{tabular}{|c|c|c|}
\hline 12 & 18 & 6 \\
\hline 12 & 19 & 6 \\
\hline 12 & 20 & 5 \\
\hline 12 & 21 & 5 \\
\hline 12 & 22 & 4 \\
\hline 12 & 23 & 5 \\
\hline 12 & 24 & 5 \\
\hline 12 & 25 & 5 \\
\hline 12 & 26 & 7 \\
\hline 12 & 27 & 5 \\
\hline 12 & 28 & 7 \\
\hline 12 & 29 & 6 \\
\hline 12 & 30 & 8 \\
\hline 12 & 31 & 9 \\
\hline 12 & 32 & 1 \\
\hline 12 & 33 & 1 \\
\hline 12 & 34 & 4 \\
\hline 12 & 35 & 4 \\
\hline 12 & 36 & 4 \\
\hline 12 & 37 & 1 \\
\hline 12 & 38 & 4 \\
\hline 12 & 39 & 5 \\
\hline 12 & 40 & 1 \\
\hline 12 & 41 & 1 \\
\hline 12 & 42 & 1 \\
\hline 12 & 43 & 1 \\
\hline 12 & 44 & 3 \\
\hline 12 & 45 & 1 \\
\hline 12 & 46 & 2 \\
\hline 12 & 47 & 2 \\
\hline 12 & 48 & 1 \\
\hline 12 & 49 & 2 \\
\hline 12 & 50 & 1 \\
\hline 12 & 51 & 1 \\
\hline 12 & 52 & 2 \\
\hline 12 & 53 & 3 \\
\hline 12 & 54 & 2 \\
\hline 12 & 55 & 2 \\
\hline 12 & 56 & 2 \\
\hline 12 & 57 & 2 \\
\hline 12 & 58 & 2 \\
\hline 12 & 59 & 2 \\
\hline 12 & 60 & 3 \\
\hline 12 & 61 & 2 \\
\hline 12 & 62 & 3 \\
\hline
\end{tabular}




\begin{tabular}{|c|c|c|}
\hline 12 & 63 & 3 \\
\hline 12 & 64 & 3 \\
\hline 12 & 65 & 3 \\
\hline 12 & 66 & 4 \\
\hline 12 & 67 & 4 \\
\hline 12 & 68 & 3 \\
\hline 12 & 69 & 4 \\
\hline 12 & 70 & 4 \\
\hline 12 & 71 & 4 \\
\hline 12 & 72 & 1 \\
\hline 12 & 73 & 2 \\
\hline 12 & 74 & 1 \\
\hline 12 & 75 & 2 \\
\hline 12 & 76 & 3 \\
\hline 12 & 77 & 3 \\
\hline 12 & 78 & 3 \\
\hline 12 & 79 & 3 \\
\hline 12 & 80 & 2 \\
\hline 12 & 81 & 3 \\
\hline 12 & 82 & 3 \\
\hline 12 & 83 & 11 \\
\hline 12 & 84 & 2 \\
\hline 12 & 85 & 3 \\
\hline 12 & 86 & 4 \\
\hline 12 & 87 & 4 \\
\hline 12 & 88 & 3 \\
\hline 12 & 89 & 2 \\
\hline 12 & 90 & 2 \\
\hline 12 & 91 & 2 \\
\hline 12 & 92 & 14 \\
\hline 12 & 93 & 12 \\
\hline 12 & 94 & 5 \\
\hline 12 & 95 & 5 \\
\hline 12 & 96 & 6 \\
\hline 12 & 97 & 13 \\
\hline 12 & 98 & 6 \\
\hline 12 & 99 & 18 \\
\hline 12 & 100 & 15 \\
\hline 12 & 101 & 4 \\
\hline 12 & 102 & 4 \\
\hline 12 & 103 & 6 \\
\hline 12 & 104 & 4 \\
\hline 12 & 105 & 21 \\
\hline 12 & 106 & 18 \\
\hline 12 & 107 & 5 \\
\hline
\end{tabular}




\begin{tabular}{|c|c|c|}
\hline 12 & 108 & 100 \\
\hline 12 & 109 & 223 \\
\hline 12 & 110 & 86 \\
\hline 12 & 111 & 227 \\
\hline 12 & 112 & 173 \\
\hline 12 & 113 & 17 \\
\hline 12 & 114 & 36 \\
\hline 12 & 115 & 46 \\
\hline 12 & 116 & 4470 \\
\hline 12 & 117 & 166 \\
\hline 12 & 118 & 343 \\
\hline 12 & 119 & 117 \\
\hline 12 & 120 & 56 \\
\hline 12 & 121 & 47 \\
\hline 12 & 122 & 143 \\
\hline 12 & 123 & 76 \\
\hline 12 & 124 & 16 \\
\hline 12 & 125 & 40 \\
\hline 12 & 126 & 37 \\
\hline 12 & 127 & 40 \\
\hline 12 & 128 & 23 \\
\hline 12 & 129 & 22 \\
\hline 12 & 130 & 26 \\
\hline 12 & 131 & 20 \\
\hline 12 & 132 & 14 \\
\hline 12 & 133 & 29 \\
\hline 12 & 134 & 6 \\
\hline 12 & 135 & 217 \\
\hline 12 & 136 & 8 \\
\hline 12 & 137 & 92 \\
\hline 12 & 138 & 9 \\
\hline 12 & 139 & 10 \\
\hline 12 & 140 & 11 \\
\hline 12 & 141 & 53 \\
\hline 12 & 142 & 13 \\
\hline 12 & 143 & 25 \\
\hline 12 & 144 & 73 \\
\hline 12 & 145 & 51 \\
\hline 12 & 146 & 182 \\
\hline 12 & 147 & 15 \\
\hline 12 & 148 & 6 \\
\hline 12 & 149 & 7 \\
\hline 12 & 150 & 9 \\
\hline 12 & 151 & 8 \\
\hline 12 & 152 & 10 \\
\hline
\end{tabular}




\begin{tabular}{|c|c|c|}
\hline 12 & 153 & 10 \\
\hline 12 & 154 & 13 \\
\hline 12 & 155 & 22 \\
\hline 12 & 156 & 13 \\
\hline 12 & 157 & 14 \\
\hline 12 & 158 & 15 \\
\hline 12 & 159 & 8 \\
\hline 12 & 160 & 4 \\
\hline 12 & 161 & 3 \\
\hline 12 & 162 & 4 \\
\hline 12 & 163 & 5 \\
\hline 12 & 164 & 4 \\
\hline 12 & 165 & 2 \\
\hline 12 & 166 & 3 \\
\hline 12 & 167 & 3 \\
\hline 12 & 168 & 3 \\
\hline 12 & 169 & 2 \\
\hline 12 & 170 & 10 \\
\hline 13 & 1 & 36 \\
\hline 13 & 2 & 34 \\
\hline 13 & 3 & 30 \\
\hline 13 & 4 & 50 \\
\hline 13 & 5 & 23 \\
\hline 13 & 6 & 10 \\
\hline 13 & 7 & 22 \\
\hline 13 & 8 & 31 \\
\hline 13 & 9 & 32 \\
\hline 13 & 10 & 32 \\
\hline 13 & 11 & 17 \\
\hline 13 & 12 & 15 \\
\hline 13 & 13 & 12 \\
\hline 13 & 14 & 132 \\
\hline 13 & 15 & 29 \\
\hline 13 & 16 & 77 \\
\hline 13 & 17 & 81 \\
\hline 13 & 18 & 50 \\
\hline 13 & 19 & 194 \\
\hline 13 & 20 & 123 \\
\hline 13 & 21 & 49 \\
\hline 13 & 22 & 9 \\
\hline 13 & 23 & 30 \\
\hline 13 & 24 & 29 \\
\hline 13 & 25 & 20 \\
\hline 13 & 26 & 22 \\
\hline 13 & 27 & 5 \\
\hline
\end{tabular}




\begin{tabular}{|c|c|c|}
\hline 13 & 28 & 17 \\
\hline 13 & 29 & 6 \\
\hline 13 & 30 & 19 \\
\hline 13 & 31 & 21 \\
\hline 13 & 32 & 75 \\
\hline 13 & 33 & 88 \\
\hline 13 & 34 & 1029 \\
\hline 13 & 35 & 488 \\
\hline 13 & 36 & 232 \\
\hline 13 & 37 & 122 \\
\hline 13 & 38 & 113 \\
\hline 13 & 39 & 224 \\
\hline 13 & 40 & 35 \\
\hline 13 & 41 & 27 \\
\hline 13 & 42 & 15 \\
\hline 13 & 43 & 16 \\
\hline 13 & 44 & 56 \\
\hline 13 & 45 & 36 \\
\hline 13 & 46 & 9 \\
\hline 13 & 47 & 5 \\
\hline 13 & 48 & 6 \\
\hline 13 & 49 & 7 \\
\hline 13 & 50 & 9 \\
\hline 13 & 51 & 7 \\
\hline 13 & 52 & 11 \\
\hline 13 & 53 & 19 \\
\hline 13 & 54 & 16 \\
\hline 13 & 55 & 10 \\
\hline 13 & 56 & 5 \\
\hline 13 & 57 & 5 \\
\hline 13 & 58 & 5 \\
\hline 13 & 59 & 4 \\
\hline 13 & 60 & 4 \\
\hline 13 & 61 & 6 \\
\hline 13 & 62 & 7 \\
\hline 13 & 63 & 7 \\
\hline 13 & 64 & 7 \\
\hline 13 & 65 & 6 \\
\hline 13 & 66 & 7 \\
\hline 13 & 67 & 6 \\
\hline 13 & 68 & 5 \\
\hline 13 & 69 & 8 \\
\hline 13 & 70 & 8 \\
\hline 13 & 71 & 6 \\
\hline 13 & 72 & 3 \\
\hline
\end{tabular}




\begin{tabular}{|c|c|c|}
\hline 13 & 73 & 4 \\
\hline 13 & 74 & 3 \\
\hline 13 & 75 & 3 \\
\hline 13 & 76 & 4 \\
\hline 13 & 77 & 4 \\
\hline 13 & 78 & 4 \\
\hline 13 & 79 & 3 \\
\hline 13 & 80 & 3 \\
\hline 13 & 81 & 3 \\
\hline 13 & 82 & 3 \\
\hline 13 & 83 & 4 \\
\hline 13 & 84 & 3 \\
\hline 13 & 85 & 3 \\
\hline 13 & 86 & 5 \\
\hline 13 & 87 & 5 \\
\hline 13 & 88 & 3 \\
\hline 13 & 89 & 3 \\
\hline 13 & 90 & 3 \\
\hline 13 & 91 & 2 \\
\hline 13 & 92 & 4 \\
\hline 13 & 93 & 3 \\
\hline 13 & 94 & 10 \\
\hline 13 & 95 & 11 \\
\hline 13 & 96 & 14 \\
\hline 13 & 97 & 11 \\
\hline 13 & 98 & 12 \\
\hline 13 & 99 & 9 \\
\hline 13 & 100 & 3 \\
\hline 13 & 101 & 3 \\
\hline 13 & 102 & 2 \\
\hline 13 & 103 & 4 \\
\hline 13 & 104 & 3 \\
\hline 13 & 105 & 8 \\
\hline 13 & 106 & 7 \\
\hline 13 & 107 & 6 \\
\hline 13 & 108 & 9 \\
\hline 13 & 109 & 9 \\
\hline 13 & 110 & 15 \\
\hline 13 & 111 & 10 \\
\hline 13 & 112 & 11 \\
\hline 13 & 113 & 6 \\
\hline 13 & 114 & 13 \\
\hline 13 & 115 & 11 \\
\hline 13 & 116 & 5 \\
\hline 13 & 117 & 10 \\
\hline
\end{tabular}




\begin{tabular}{|c|c|c|}
\hline 13 & 118 & 4 \\
\hline 13 & 119 & 11 \\
\hline 13 & 120 & 9 \\
\hline 13 & 121 & 8 \\
\hline 13 & 122 & 4 \\
\hline 13 & 123 & 3 \\
\hline 13 & 124 & 5 \\
\hline 13 & 125 & 6 \\
\hline 13 & 126 & 7 \\
\hline 13 & 127 & 9 \\
\hline 13 & 128 & 6 \\
\hline 13 & 129 & 7 \\
\hline 13 & 130 & 6 \\
\hline 13 & 131 & 4 \\
\hline 13 & 132 & 5 \\
\hline 13 & 133 & 8 \\
\hline 13 & 134 & 7 \\
\hline 13 & 135 & 4 \\
\hline 13 & 136 & 5 \\
\hline 13 & 137 & 6 \\
\hline 13 & 138 & 3 \\
\hline 13 & 139 & 5 \\
\hline 13 & 140 & 6 \\
\hline 13 & 141 & 5 \\
\hline 13 & 142 & 4 \\
\hline 13 & 143 & 5 \\
\hline 13 & 144 & 7 \\
\hline 13 & 145 & 10 \\
\hline 13 & 146 & 5 \\
\hline 13 & 147 & 9 \\
\hline 13 & 148 & 60 \\
\hline 13 & 149 & 15 \\
\hline 13 & 150 & 11 \\
\hline 13 & 151 & 21 \\
\hline 13 & 152 & 19 \\
\hline 13 & 153 & 33 \\
\hline 13 & 154 & 8 \\
\hline 13 & 155 & 11 \\
\hline 13 & 156 & 8 \\
\hline 13 & 157 & 8 \\
\hline 13 & 158 & 9 \\
\hline 13 & 159 & 11 \\
\hline 13 & 160 & 11 \\
\hline 13 & 161 & 13 \\
\hline 13 & 162 & 23 \\
\hline
\end{tabular}




\begin{tabular}{|c|c|c|}
\hline 13 & 163 & 17 \\
\hline 13 & 164 & 23 \\
\hline 13 & 165 & 8 \\
\hline 13 & 166 & 14 \\
\hline 13 & 167 & 25 \\
\hline 13 & 168 & 20 \\
\hline 13 & 169 & 48 \\
\hline 13 & 170 & 10 \\
\hline 14 & 1 & 36 \\
\hline 14 & 2 & 31 \\
\hline 14 & 3 & 38 \\
\hline 14 & 4 & 29 \\
\hline 14 & 5 & 27 \\
\hline 14 & 6 & 15 \\
\hline 14 & 7 & 21 \\
\hline 14 & 8 & 22 \\
\hline 14 & 9 & 18 \\
\hline 14 & 10 & 11 \\
\hline 14 & 11 & 9 \\
\hline 14 & 12 & 15 \\
\hline 14 & 13 & 12 \\
\hline 14 & 14 & 13 \\
\hline 14 & 15 & 16 \\
\hline 14 & 16 & 22 \\
\hline 14 & 17 & 21 \\
\hline 14 & 18 & 40 \\
\hline 14 & 19 & 14 \\
\hline 14 & 20 & 15 \\
\hline 14 & 21 & 14 \\
\hline 14 & 22 & 32 \\
\hline 14 & 23 & 126 \\
\hline 14 & 24 & 106 \\
\hline 14 & 25 & 244 \\
\hline 14 & 26 & 238 \\
\hline 14 & 27 & 476 \\
\hline 14 & 28 & 160 \\
\hline 14 & 29 & 2912 \\
\hline 14 & 30 & 80 \\
\hline 14 & 31 & 49 \\
\hline 14 & 32 & 5 \\
\hline 14 & 33 & 4 \\
\hline 14 & 34 & 5 \\
\hline 14 & 35 & 6 \\
\hline 14 & 36 & 5 \\
\hline 14 & 37 & 7 \\
\hline
\end{tabular}




\begin{tabular}{|c|c|c|}
\hline 14 & 38 & 3 \\
\hline 14 & 39 & 10 \\
\hline 14 & 40 & 8 \\
\hline 14 & 41 & 7 \\
\hline 14 & 42 & 5 \\
\hline 14 & 43 & 8 \\
\hline 14 & 44 & 7 \\
\hline 14 & 45 & 11 \\
\hline 14 & 46 & 5 \\
\hline 14 & 47 & 7 \\
\hline 14 & 48 & 4 \\
\hline 14 & 49 & 5 \\
\hline 14 & 50 & 4 \\
\hline 14 & 51 & 4 \\
\hline 14 & 52 & 11 \\
\hline 14 & 53 & 16 \\
\hline 14 & 54 & 10 \\
\hline 14 & 55 & 8 \\
\hline 14 & 56 & 7 \\
\hline 14 & 57 & 10 \\
\hline 14 & 58 & 11 \\
\hline 14 & 59 & 9 \\
\hline 14 & 60 & 15 \\
\hline 14 & 61 & 8 \\
\hline 14 & 62 & 20 \\
\hline 14 & 63 & 42 \\
\hline 14 & 64 & 17 \\
\hline 14 & 65 & 17 \\
\hline 14 & 66 & 51 \\
\hline 14 & 67 & 48 \\
\hline 14 & 68 & 25 \\
\hline 14 & 69 & 68 \\
\hline 14 & 70 & 116 \\
\hline 14 & 71 & 80 \\
\hline 14 & 72 & 4 \\
\hline 14 & 73 & 7 \\
\hline 14 & 74 & 5 \\
\hline 14 & 75 & 9 \\
\hline 14 & 76 & 16 \\
\hline 14 & 77 & 32 \\
\hline 14 & 78 & 18 \\
\hline 14 & 79 & 13 \\
\hline 14 & 80 & 10 \\
\hline 14 & 81 & 12 \\
\hline 14 & 82 & 12 \\
\hline
\end{tabular}




\begin{tabular}{|c|c|c|}
\hline 14 & 83 & 19 \\
\hline 14 & 84 & 11 \\
\hline 14 & 85 & 11 \\
\hline 14 & 86 & 289 \\
\hline 14 & 87 & 113 \\
\hline 14 & 88 & 13 \\
\hline 14 & 89 & 11 \\
\hline 14 & 90 & 8 \\
\hline 14 & 91 & 6 \\
\hline 14 & 92 & 50 \\
\hline 14 & 93 & 33 \\
\hline 14 & 94 & 23 \\
\hline 14 & 95 & 25 \\
\hline 14 & 96 & 220 \\
\hline 14 & 97 & 76 \\
\hline 14 & 98 & 31 \\
\hline 14 & 99 & 21 \\
\hline 14 & 100 & 57 \\
\hline 14 & 101 & 22 \\
\hline 14 & 102 & 6 \\
\hline 14 & 103 & 7 \\
\hline 14 & 104 & 4 \\
\hline 14 & 105 & 17 \\
\hline 14 & 106 & 15 \\
\hline 14 & 107 & 10 \\
\hline 14 & 108 & 22 \\
\hline 14 & 109 & 14 \\
\hline 14 & 110 & 29 \\
\hline 14 & 111 & 16 \\
\hline 14 & 112 & 19 \\
\hline 14 & 113 & 11 \\
\hline 14 & 114 & 25 \\
\hline 14 & 115 & 21 \\
\hline 14 & 116 & 10 \\
\hline 14 & 117 & 17 \\
\hline 14 & 118 & 8 \\
\hline 14 & 119 & 19 \\
\hline 14 & 120 & 9 \\
\hline 14 & 121 & 10 \\
\hline 14 & 122 & 9 \\
\hline 14 & 123 & 5 \\
\hline 14 & 124 & 6 \\
\hline 14 & 125 & 7 \\
\hline 14 & 126 & 9 \\
\hline 14 & 127 & 11 \\
\hline
\end{tabular}




\begin{tabular}{|c|c|c|}
\hline 14 & 128 & 11 \\
\hline 14 & 129 & 8 \\
\hline 14 & 130 & 7 \\
\hline 14 & 131 & 7 \\
\hline 14 & 132 & 8 \\
\hline 14 & 133 & 15 \\
\hline 14 & 134 & 14 \\
\hline 14 & 135 & 11 \\
\hline 14 & 136 & 4 \\
\hline 14 & 137 & 8 \\
\hline 14 & 138 & 4 \\
\hline 14 & 139 & 4 \\
\hline 14 & 140 & 4 \\
\hline 14 & 141 & 7 \\
\hline 14 & 142 & 5 \\
\hline 14 & 143 & 6 \\
\hline 14 & 144 & 8 \\
\hline 14 & 145 & 9 \\
\hline 14 & 146 & 11 \\
\hline 14 & 147 & 5 \\
\hline 14 & 148 & 6 \\
\hline 14 & 149 & 7 \\
\hline 14 & 150 & 6 \\
\hline 14 & 151 & 9 \\
\hline 14 & 152 & 7 \\
\hline 14 & 153 & 10 \\
\hline 14 & 154 & 5 \\
\hline 14 & 155 & 6 \\
\hline 14 & 156 & 5 \\
\hline 14 & 157 & 5 \\
\hline 14 & 158 & 5 \\
\hline 14 & 159 & 3 \\
\hline 14 & 160 & 2 \\
\hline 14 & 161 & 2 \\
\hline 14 & 162 & 2 \\
\hline 14 & 163 & 2 \\
\hline 14 & 164 & 5 \\
\hline 14 & 165 & 2 \\
\hline 14 & 166 & 2 \\
\hline 14 & 167 & 2 \\
\hline 14 & 168 & 2 \\
\hline 14 & 169 & 2 \\
\hline 14 & 170 & 4 \\
\hline 15 & 1 & 5 \\
\hline 15 & 2 & 5 \\
\hline
\end{tabular}




\begin{tabular}{|c|c|c|}
\hline 15 & 3 & 5 \\
\hline 15 & 4 & 5 \\
\hline 15 & 5 & 5 \\
\hline 15 & 6 & 2 \\
\hline 15 & 7 & 5 \\
\hline 15 & 8 & 5 \\
\hline 15 & 9 & 4 \\
\hline 15 & 10 & 2 \\
\hline 15 & 11 & 3 \\
\hline 15 & 12 & 4 \\
\hline 15 & 13 & 3 \\
\hline 15 & 14 & 4 \\
\hline 15 & 15 & 4 \\
\hline 15 & 16 & 4 \\
\hline 15 & 17 & 5 \\
\hline 15 & 18 & 5 \\
\hline 15 & 19 & 4 \\
\hline 15 & 20 & 5 \\
\hline 15 & 21 & 6 \\
\hline 15 & 22 & 11 \\
\hline 15 & 23 & 10 \\
\hline 15 & 24 & 8 \\
\hline 15 & 25 & 8 \\
\hline 15 & 26 & 12 \\
\hline 15 & 27 & 14 \\
\hline 15 & 28 & 11 \\
\hline 15 & 29 & 17 \\
\hline 15 & 30 & 9 \\
\hline 15 & 31 & 6 \\
\hline 15 & 32 & 3 \\
\hline 15 & 33 & 3 \\
\hline 15 & 34 & 3 \\
\hline 15 & 35 & 3 \\
\hline 15 & 36 & 3 \\
\hline 15 & 37 & 4 \\
\hline 15 & 38 & 2 \\
\hline 15 & 39 & 3 \\
\hline 15 & 40 & 4 \\
\hline 15 & 41 & 4 \\
\hline 15 & 42 & 3 \\
\hline 15 & 43 & 4 \\
\hline 15 & 44 & 4 \\
\hline 15 & 45 & 5 \\
\hline 15 & 46 & 3 \\
\hline 15 & 47 & 4 \\
\hline
\end{tabular}




\begin{tabular}{|c|c|c|}
\hline 15 & 48 & 3 \\
\hline 15 & 49 & 3 \\
\hline 15 & 50 & 2 \\
\hline 15 & 51 & 3 \\
\hline 15 & 52 & 5 \\
\hline 15 & 53 & 7 \\
\hline 15 & 54 & 5 \\
\hline 15 & 55 & 4 \\
\hline 15 & 56 & 4 \\
\hline 15 & 57 & 5 \\
\hline 15 & 58 & 5 \\
\hline 15 & 59 & 5 \\
\hline 15 & 60 & 7 \\
\hline 15 & 61 & 4 \\
\hline 15 & 62 & 8 \\
\hline 15 & 63 & 9 \\
\hline 15 & 64 & 7 \\
\hline 15 & 65 & 7 \\
\hline 15 & 66 & 10 \\
\hline 15 & 67 & 13 \\
\hline 15 & 68 & 9 \\
\hline 15 & 69 & 8 \\
\hline 15 & 70 & 9 \\
\hline 15 & 71 & 12 \\
\hline 15 & 72 & 3 \\
\hline 15 & 73 & 4 \\
\hline 15 & 74 & 3 \\
\hline 15 & 75 & 5 \\
\hline 15 & 76 & 19 \\
\hline 15 & 77 & 22 \\
\hline 15 & 78 & 34 \\
\hline 15 & 79 & 32 \\
\hline 15 & 80 & 79 \\
\hline 15 & 81 & 59 \\
\hline 15 & 82 & 59 \\
\hline 15 & 83 & 65 \\
\hline 15 & 84 & 98 \\
\hline 15 & 85 & 227 \\
\hline 15 & 86 & 13 \\
\hline 15 & 87 & 26 \\
\hline 15 & 88 & 19 \\
\hline 15 & 89 & 25 \\
\hline 15 & 90 & 24 \\
\hline 15 & 91 & 22 \\
\hline 15 & 92 & 75 \\
\hline
\end{tabular}




\begin{tabular}{|c|c|c|}
\hline 15 & 93 & 148 \\
\hline 15 & 94 & 10 \\
\hline 15 & 95 & 9 \\
\hline 15 & 96 & 12 \\
\hline 15 & 97 & 31 \\
\hline 15 & 98 & 14 \\
\hline 15 & 99 & 27 \\
\hline 15 & 100 & 30 \\
\hline 15 & 101 & 161 \\
\hline 15 & 102 & 159 \\
\hline 15 & 103 & 28 \\
\hline 15 & 104 & 34 \\
\hline 15 & 105 & 47 \\
\hline 15 & 106 & 30 \\
\hline 15 & 107 & 48 \\
\hline 15 & 108 & 5 \\
\hline 15 & 109 & 6 \\
\hline 15 & 110 & 6 \\
\hline 15 & 111 & 6 \\
\hline 15 & 112 & 6 \\
\hline 15 & 113 & 17 \\
\hline 15 & 114 & 5 \\
\hline 15 & 115 & 8 \\
\hline 15 & 116 & 4 \\
\hline 15 & 117 & 5 \\
\hline 15 & 118 & 4 \\
\hline 15 & 119 & 7 \\
\hline 15 & 120 & 7 \\
\hline 15 & 121 & 7 \\
\hline 15 & 122 & 4 \\
\hline 15 & 123 & 7 \\
\hline 15 & 124 & 9 \\
\hline 15 & 125 & 8 \\
\hline 15 & 126 & 11 \\
\hline 15 & 127 & 14 \\
\hline 15 & 128 & 16 \\
\hline 15 & 129 & 12 \\
\hline 15 & 130 & 5 \\
\hline 15 & 131 & 10 \\
\hline 15 & 132 & 11 \\
\hline 15 & 133 & 7 \\
\hline 15 & 134 & 11 \\
\hline 15 & 135 & 3 \\
\hline 15 & 136 & 2 \\
\hline 15 & 137 & 3 \\
\hline
\end{tabular}




\begin{tabular}{|c|c|c|}
\hline 15 & 138 & 2 \\
\hline 15 & 139 & 2 \\
\hline 15 & 140 & 2 \\
\hline 15 & 141 & 3 \\
\hline 15 & 142 & 2 \\
\hline 15 & 143 & 3 \\
\hline 15 & 144 & 3 \\
\hline 15 & 145 & 3 \\
\hline 15 & 146 & 4 \\
\hline 15 & 147 & 2 \\
\hline 15 & 148 & 1 \\
\hline 15 & 149 & 2 \\
\hline 15 & 150 & 2 \\
\hline 15 & 151 & 2 \\
\hline 15 & 152 & 2 \\
\hline 15 & 153 & 2 \\
\hline 15 & 154 & 2 \\
\hline 15 & 155 & 3 \\
\hline 15 & 156 & 2 \\
\hline 15 & 157 & 2 \\
\hline 15 & 158 & 2 \\
\hline 15 & 159 & 2 \\
\hline 15 & 160 & 1 \\
\hline 15 & 161 & 2 \\
\hline 15 & 162 & 2 \\
\hline 15 & 163 & 2 \\
\hline 15 & 164 & 1 \\
\hline 15 & 165 & 1 \\
\hline 15 & 166 & 2 \\
\hline 15 & 167 & 1 \\
\hline 15 & 168 & 2 \\
\hline 15 & 169 & 1 \\
\hline 15 & 170 & 2 \\
\hline 16 & 1 & 37 \\
\hline 16 & 2 & 32 \\
\hline 16 & 3 & 13 \\
\hline 16 & 4 & 29 \\
\hline 16 & 5 & 13 \\
\hline 16 & 6 & 12 \\
\hline 16 & 7 & 16 \\
\hline 16 & 8 & 24 \\
\hline 16 & 9 & 23 \\
\hline 16 & 10 & 17 \\
\hline 16 & 11 & 7 \\
\hline 16 & 12 & 12 \\
\hline
\end{tabular}




\begin{tabular}{|c|c|c|}
\hline 16 & 13 & 10 \\
\hline 16 & 14 & 13 \\
\hline 16 & 15 & 17 \\
\hline 16 & 16 & 23 \\
\hline 16 & 17 & 21 \\
\hline 16 & 18 & 9 \\
\hline 16 & 19 & 6 \\
\hline 16 & 20 & 7 \\
\hline 16 & 21 & 10 \\
\hline 16 & 22 & 21 \\
\hline 16 & 23 & 35 \\
\hline 16 & 24 & 13 \\
\hline 16 & 25 & 15 \\
\hline 16 & 26 & 63 \\
\hline 16 & 27 & 29 \\
\hline 16 & 28 & 95 \\
\hline 16 & 29 & 39 \\
\hline 16 & 30 & 49 \\
\hline 16 & 31 & 15 \\
\hline 16 & 32 & 4 \\
\hline 16 & 33 & 3 \\
\hline 16 & 34 & 4 \\
\hline 16 & 35 & 5 \\
\hline 16 & 36 & 4 \\
\hline 16 & 37 & 6 \\
\hline 16 & 38 & 3 \\
\hline 16 & 39 & 10 \\
\hline 16 & 40 & 6 \\
\hline 16 & 41 & 6 \\
\hline 16 & 42 & 4 \\
\hline 16 & 43 & 6 \\
\hline 16 & 44 & 6 \\
\hline 16 & 45 & 8 \\
\hline 16 & 46 & 4 \\
\hline 16 & 47 & 5 \\
\hline 16 & 48 & 4 \\
\hline 16 & 49 & 4 \\
\hline 16 & 50 & 3 \\
\hline 16 & 51 & 3 \\
\hline 16 & 52 & 8 \\
\hline 16 & 53 & 12 \\
\hline 16 & 54 & 8 \\
\hline 16 & 55 & 6 \\
\hline 16 & 56 & 6 \\
\hline 16 & 57 & 8 \\
\hline
\end{tabular}




\begin{tabular}{|c|c|c|}
\hline 16 & 58 & 8 \\
\hline 16 & 59 & 7 \\
\hline 16 & 60 & 11 \\
\hline 16 & 61 & 7 \\
\hline 16 & 62 & 14 \\
\hline 16 & 63 & 17 \\
\hline 16 & 64 & 12 \\
\hline 16 & 65 & 12 \\
\hline 16 & 66 & 19 \\
\hline 16 & 67 & 27 \\
\hline 16 & 68 & 17 \\
\hline 16 & 69 & 14 \\
\hline 16 & 70 & 15 \\
\hline 16 & 71 & 25 \\
\hline 16 & 72 & 3 \\
\hline 16 & 73 & 5 \\
\hline 16 & 74 & 4 \\
\hline 16 & 75 & 7 \\
\hline 16 & 76 & 11 \\
\hline 16 & 77 & 28 \\
\hline 16 & 78 & 45 \\
\hline 16 & 79 & 34 \\
\hline 16 & 80 & 43 \\
\hline 16 & 81 & 30 \\
\hline 16 & 82 & 30 \\
\hline 16 & 83 & 137 \\
\hline 16 & 84 & 86 \\
\hline 16 & 85 & 49 \\
\hline 16 & 86 & 28 \\
\hline 16 & 87 & 101 \\
\hline 16 & 88 & 10 \\
\hline 16 & 89 & 17 \\
\hline 16 & 90 & 17 \\
\hline 16 & 91 & 10 \\
\hline 16 & 92 & 488 \\
\hline 16 & 93 & 168 \\
\hline 16 & 94 & 90 \\
\hline 16 & 95 & 109 \\
\hline 16 & 96 & 218 \\
\hline 16 & 97 & 2058 \\
\hline 16 & 98 & 237 \\
\hline 16 & 99 & 329 \\
\hline 16 & 100 & 778 \\
\hline 16 & 101 & 72 \\
\hline 16 & 102 & 30 \\
\hline
\end{tabular}




\begin{tabular}{|c|c|c|}
\hline 16 & 103 & 11 \\
\hline 16 & 104 & 13 \\
\hline 16 & 105 & 123 \\
\hline 16 & 106 & 65 \\
\hline 16 & 107 & 30 \\
\hline 16 & 108 & 17 \\
\hline 16 & 109 & 17 \\
\hline 16 & 110 & 21 \\
\hline 16 & 111 & 17 \\
\hline 16 & 112 & 20 \\
\hline 16 & 113 & 29 \\
\hline 16 & 114 & 21 \\
\hline 16 & 115 & 34 \\
\hline 16 & 116 & 12 \\
\hline 16 & 117 & 13 \\
\hline 16 & 118 & 9 \\
\hline 16 & 119 & 25 \\
\hline 16 & 120 & 26 \\
\hline 16 & 121 & 26 \\
\hline 16 & 122 & 9 \\
\hline 16 & 123 & 11 \\
\hline 16 & 124 & 12 \\
\hline 16 & 125 & 15 \\
\hline 16 & 126 & 24 \\
\hline 16 & 127 & 33 \\
\hline 16 & 128 & 28 \\
\hline 16 & 129 & 21 \\
\hline 16 & 130 & 15 \\
\hline 16 & 131 & 16 \\
\hline 16 & 132 & 17 \\
\hline 16 & 133 & 22 \\
\hline 16 & 134 & 53 \\
\hline 16 & 135 & 12 \\
\hline 16 & 136 & 3 \\
\hline 16 & 137 & 6 \\
\hline 16 & 138 & 3 \\
\hline 16 & 139 & 4 \\
\hline 16 & 140 & 4 \\
\hline 16 & 141 & 6 \\
\hline 16 & 142 & 4 \\
\hline 16 & 143 & 5 \\
\hline 16 & 144 & 7 \\
\hline 16 & 145 & 8 \\
\hline 16 & 146 & 9 \\
\hline 16 & 147 & 4 \\
\hline
\end{tabular}




\begin{tabular}{|c|c|c|}
\hline 16 & 148 & 2 \\
\hline 16 & 149 & 6 \\
\hline 16 & 150 & 5 \\
\hline 16 & 151 & 7 \\
\hline 16 & 152 & 6 \\
\hline 16 & 153 & 8 \\
\hline 16 & 154 & 4 \\
\hline 16 & 155 & 5 \\
\hline 16 & 156 & 4 \\
\hline 16 & 157 & 4 \\
\hline 16 & 158 & 4 \\
\hline 16 & 159 & 1 \\
\hline 16 & 160 & 2 \\
\hline 16 & 161 & 2 \\
\hline 16 & 162 & 2 \\
\hline 16 & 163 & 2 \\
\hline 16 & 164 & 2 \\
\hline 16 & 165 & 2 \\
\hline 16 & 166 & 2 \\
\hline 16 & 167 & 2 \\
\hline 16 & 168 & 2 \\
\hline 16 & 169 & 2 \\
\hline 16 & 170 & 4 \\
\hline 17 & 1 & 5 \\
\hline 17 & 2 & 4 \\
\hline 17 & 3 & 5 \\
\hline 17 & 4 & 4 \\
\hline 17 & 5 & 5 \\
\hline 17 & 6 & 2 \\
\hline 17 & 7 & 4 \\
\hline 17 & 8 & 4 \\
\hline 17 & 9 & 4 \\
\hline 17 & 10 & 2 \\
\hline 17 & 11 & 2 \\
\hline 17 & 12 & 3 \\
\hline 17 & 13 & 3 \\
\hline 17 & 14 & 1 \\
\hline 17 & 15 & 3 \\
\hline 17 & 16 & 4 \\
\hline 17 & 17 & 4 \\
\hline 17 & 18 & 4 \\
\hline 17 & 19 & 3 \\
\hline 17 & 20 & 4 \\
\hline 17 & 21 & 5 \\
\hline 17 & 22 & 7 \\
\hline
\end{tabular}




\begin{tabular}{|c|c|c|}
\hline 17 & 23 & 7 \\
\hline 17 & 24 & 5 \\
\hline 17 & 25 & 6 \\
\hline 17 & 26 & 8 \\
\hline 17 & 27 & 8 \\
\hline 17 & 28 & 7 \\
\hline 17 & 29 & 10 \\
\hline 17 & 30 & 6 \\
\hline 17 & 31 & 5 \\
\hline 17 & 32 & 2 \\
\hline 17 & 33 & 2 \\
\hline 17 & 34 & 2 \\
\hline 17 & 35 & 3 \\
\hline 17 & 36 & 2 \\
\hline 17 & 37 & 3 \\
\hline 17 & 38 & 2 \\
\hline 17 & 39 & 3 \\
\hline 17 & 40 & 3 \\
\hline 17 & 41 & 3 \\
\hline 17 & 42 & 2 \\
\hline 17 & 43 & 3 \\
\hline 17 & 44 & 3 \\
\hline 17 & 45 & 4 \\
\hline 17 & 46 & 2 \\
\hline 17 & 47 & 3 \\
\hline 17 & 48 & 2 \\
\hline 17 & 49 & 2 \\
\hline 17 & 50 & 2 \\
\hline 17 & 51 & 2 \\
\hline 17 & 52 & 4 \\
\hline 17 & 53 & 5 \\
\hline 17 & 54 & 4 \\
\hline 17 & 55 & 3 \\
\hline 17 & 56 & 3 \\
\hline 17 & 57 & 4 \\
\hline 17 & 58 & 4 \\
\hline 17 & 59 & 4 \\
\hline 17 & 60 & 5 \\
\hline 17 & 61 & 3 \\
\hline 17 & 62 & 6 \\
\hline 17 & 63 & 6 \\
\hline 17 & 64 & 5 \\
\hline 17 & 65 & 5 \\
\hline 17 & 66 & 7 \\
\hline 17 & 67 & 8 \\
\hline
\end{tabular}




\begin{tabular}{|c|c|c|}
\hline 17 & 68 & 6 \\
\hline 17 & 69 & 6 \\
\hline 17 & 70 & 6 \\
\hline 17 & 71 & 8 \\
\hline 17 & 72 & 2 \\
\hline 17 & 73 & 3 \\
\hline 17 & 74 & 2 \\
\hline 17 & 75 & 3 \\
\hline 17 & 76 & 11 \\
\hline 17 & 77 & 12 \\
\hline 17 & 78 & 17 \\
\hline 17 & 79 & 16 \\
\hline 17 & 80 & 29 \\
\hline 17 & 81 & 24 \\
\hline 17 & 82 & 24 \\
\hline 17 & 83 & 40 \\
\hline 17 & 84 & 34 \\
\hline 17 & 85 & 52 \\
\hline 17 & 86 & 8 \\
\hline 17 & 87 & 14 \\
\hline 17 & 88 & 11 \\
\hline 17 & 89 & 14 \\
\hline 17 & 90 & 13 \\
\hline 17 & 91 & 18 \\
\hline 17 & 92 & 49 \\
\hline 17 & 93 & 83 \\
\hline 17 & 94 & 9 \\
\hline 17 & 95 & 22 \\
\hline 17 & 96 & 21 \\
\hline 17 & 97 & 26 \\
\hline 17 & 98 & 15 \\
\hline 17 & 99 & 29 \\
\hline 17 & 100 & 32 \\
\hline 17 & 101 & 92 \\
\hline 17 & 102 & 32283 \\
\hline 17 & 103 & 55 \\
\hline 17 & 104 & 101 \\
\hline 17 & 105 & 33 \\
\hline 17 & 106 & 25 \\
\hline 17 & 107 & 39 \\
\hline 17 & 108 & 4 \\
\hline 17 & 109 & 5 \\
\hline 17 & 110 & 4 \\
\hline 17 & 111 & 5 \\
\hline 17 & 112 & 6 \\
\hline
\end{tabular}




\begin{tabular}{|c|c|c|}
\hline 17 & 113 & 15 \\
\hline 17 & 114 & 5 \\
\hline 17 & 115 & 7 \\
\hline 17 & 116 & 4 \\
\hline 17 & 117 & 3 \\
\hline 17 & 118 & 4 \\
\hline 17 & 119 & 7 \\
\hline 17 & 120 & 7 \\
\hline 17 & 121 & 7 \\
\hline 17 & 122 & 4 \\
\hline 17 & 123 & 6 \\
\hline 17 & 124 & 8 \\
\hline 17 & 125 & 7 \\
\hline 17 & 126 & 10 \\
\hline 17 & 127 & 12 \\
\hline 17 & 128 & 14 \\
\hline 17 & 129 & 11 \\
\hline 17 & 130 & 5 \\
\hline 17 & 131 & 9 \\
\hline 17 & 132 & 10 \\
\hline 17 & 133 & 6 \\
\hline 17 & 134 & 10 \\
\hline 17 & 135 & 3 \\
\hline 17 & 136 & 2 \\
\hline 17 & 137 & 3 \\
\hline 17 & 138 & 2 \\
\hline 17 & 139 & 2 \\
\hline 17 & 140 & 2 \\
\hline 17 & 141 & 3 \\
\hline 17 & 142 & 2 \\
\hline 17 & 143 & 2 \\
\hline 17 & 144 & 3 \\
\hline 17 & 145 & 2 \\
\hline 17 & 146 & 4 \\
\hline 17 & 147 & 2 \\
\hline 17 & 148 & 1 \\
\hline 17 & 149 & 2 \\
\hline 17 & 150 & 2 \\
\hline 17 & 151 & 2 \\
\hline 17 & 152 & 2 \\
\hline 17 & 153 & 2 \\
\hline 17 & 154 & 2 \\
\hline 17 & 155 & 3 \\
\hline 17 & 156 & 2 \\
\hline 17 & 157 & 2 \\
\hline
\end{tabular}




\begin{tabular}{|c|c|c|}
\hline 17 & 158 & 2 \\
\hline 17 & 159 & 2 \\
\hline 17 & 160 & 1 \\
\hline 17 & 161 & 1 \\
\hline 17 & 162 & 1 \\
\hline 17 & 163 & 1 \\
\hline 17 & 164 & 1 \\
\hline 17 & 165 & 1 \\
\hline 17 & 166 & 1 \\
\hline 17 & 167 & 1 \\
\hline 17 & 168 & 1 \\
\hline 17 & 169 & 1 \\
\hline 17 & 170 & 2 \\
\hline 18 & 1 & 14 \\
\hline 18 & 2 & 31 \\
\hline 18 & 3 & 42 \\
\hline 18 & 4 & 12 \\
\hline 18 & 5 & 55 \\
\hline 18 & 6 & 23 \\
\hline 18 & 7 & 38 \\
\hline 18 & 8 & 42 \\
\hline 18 & 9 & 33 \\
\hline 18 & 10 & 23 \\
\hline 18 & 11 & 13 \\
\hline 18 & 12 & 24 \\
\hline 18 & 13 & 18 \\
\hline 18 & 14 & 13 \\
\hline 18 & 15 & 22 \\
\hline 18 & 16 & 22 \\
\hline 18 & 17 & 10 \\
\hline 18 & 18 & 10 \\
\hline 18 & 19 & 7 \\
\hline 18 & 20 & 7 \\
\hline 18 & 21 & 10 \\
\hline 18 & 22 & 7 \\
\hline 18 & 23 & 11 \\
\hline 18 & 24 & 9 \\
\hline 18 & 25 & 12 \\
\hline 18 & 26 & 24 \\
\hline 18 & 27 & 14 \\
\hline 18 & 28 & 22 \\
\hline 18 & 29 & 17 \\
\hline 18 & 30 & 20 \\
\hline 18 & 31 & 16 \\
\hline 18 & 32 & 2 \\
\hline
\end{tabular}




\begin{tabular}{|c|c|c|}
\hline 18 & 33 & 2 \\
\hline 18 & 34 & 5 \\
\hline 18 & 35 & 5 \\
\hline 18 & 36 & 5 \\
\hline 18 & 37 & 3 \\
\hline 18 & 38 & 4 \\
\hline 18 & 39 & 5 \\
\hline 18 & 40 & 3 \\
\hline 18 & 41 & 3 \\
\hline 18 & 42 & 2 \\
\hline 18 & 43 & 3 \\
\hline 18 & 44 & 3 \\
\hline 18 & 45 & 4 \\
\hline 18 & 46 & 2 \\
\hline 18 & 47 & 3 \\
\hline 18 & 48 & 2 \\
\hline 18 & 49 & 2 \\
\hline 18 & 50 & 2 \\
\hline 18 & 51 & 2 \\
\hline 18 & 52 & 4 \\
\hline 18 & 53 & 5 \\
\hline 18 & 54 & 4 \\
\hline 18 & 55 & 3 \\
\hline 18 & 56 & 3 \\
\hline 18 & 57 & 4 \\
\hline 18 & 58 & 4 \\
\hline 18 & 59 & 4 \\
\hline 18 & 60 & 5 \\
\hline 18 & 61 & 3 \\
\hline 18 & 62 & 6 \\
\hline 18 & 63 & 7 \\
\hline 18 & 64 & 6 \\
\hline 18 & 65 & 6 \\
\hline 18 & 66 & 8 \\
\hline 18 & 67 & 10 \\
\hline 18 & 68 & 7 \\
\hline 18 & 69 & 8 \\
\hline 18 & 70 & 10 \\
\hline 18 & 71 & 9 \\
\hline 18 & 72 & 2 \\
\hline 18 & 73 & 3 \\
\hline 18 & 74 & 2 \\
\hline 18 & 75 & 4 \\
\hline 18 & 76 & 5 \\
\hline 18 & 77 & 8 \\
\hline
\end{tabular}




\begin{tabular}{|c|c|c|}
\hline 18 & 78 & 10 \\
\hline 18 & 79 & 9 \\
\hline 18 & 80 & 11 \\
\hline 18 & 81 & 9 \\
\hline 18 & 82 & 9 \\
\hline 18 & 83 & 24 \\
\hline 18 & 84 & 15 \\
\hline 18 & 85 & 12 \\
\hline 18 & 86 & 9 \\
\hline 18 & 87 & 15 \\
\hline 18 & 88 & 5 \\
\hline 18 & 89 & 4 \\
\hline 18 & 90 & 7 \\
\hline 18 & 91 & 3 \\
\hline 18 & 92 & 33 \\
\hline 18 & 93 & 26 \\
\hline 18 & 94 & 50 \\
\hline 18 & 95 & 57 \\
\hline 18 & 96 & 16 \\
\hline 18 & 97 & 29 \\
\hline 18 & 98 & 22 \\
\hline 18 & 99 & 51 \\
\hline 18 & 100 & 37 \\
\hline 18 & 101 & 18 \\
\hline 18 & 102 & 9 \\
\hline 18 & 103 & 14 \\
\hline 18 & 104 & 6 \\
\hline 18 & 105 & 64 \\
\hline 18 & 106 & 50 \\
\hline 18 & 107 & 27 \\
\hline 18 & 108 & 113 \\
\hline 18 & 109 & 231 \\
\hline 18 & 110 & 201 \\
\hline 18 & 111 & 256 \\
\hline 18 & 112 & 539 \\
\hline 18 & 113 & 45 \\
\hline 18 & 114 & 129 \\
\hline 18 & 115 & 197 \\
\hline 18 & 116 & 87 \\
\hline 18 & 117 & 53 \\
\hline 18 & 118 & 45 \\
\hline 18 & 119 & 5408 \\
\hline 18 & 120 & 1627 \\
\hline 18 & 121 & 514 \\
\hline 18 & 122 & 36 \\
\hline
\end{tabular}




\begin{tabular}{|c|c|c|}
\hline 18 & 123 & 60 \\
\hline 18 & 124 & 47 \\
\hline 18 & 125 & 134 \\
\hline 18 & 126 & 241 \\
\hline 18 & 127 & 256 \\
\hline 18 & 128 & 76 \\
\hline 18 & 129 & 79 \\
\hline 18 & 130 & 143 \\
\hline 18 & 131 & 72 \\
\hline 18 & 132 & 41 \\
\hline 18 & 133 & 82 \\
\hline 18 & 134 & 63 \\
\hline 18 & 135 & 76 \\
\hline 18 & 136 & 5 \\
\hline 18 & 137 & 10 \\
\hline 18 & 138 & 5 \\
\hline 18 & 139 & 5 \\
\hline 18 & 140 & 5 \\
\hline 18 & 141 & 10 \\
\hline 18 & 142 & 6 \\
\hline 18 & 143 & 8 \\
\hline 18 & 144 & 12 \\
\hline 18 & 145 & 13 \\
\hline 18 & 146 & 31 \\
\hline 18 & 147 & 6 \\
\hline 18 & 148 & 8 \\
\hline 18 & 149 & 9 \\
\hline 18 & 150 & 8 \\
\hline 18 & 151 & 12 \\
\hline 18 & 152 & 9 \\
\hline 18 & 153 & 15 \\
\hline 18 & 154 & 6 \\
\hline 18 & 155 & 8 \\
\hline 18 & 156 & 6 \\
\hline 18 & 157 & 6 \\
\hline 18 & 158 & 7 \\
\hline 18 & 159 & 4 \\
\hline 18 & 160 & 2 \\
\hline 18 & 161 & 2 \\
\hline 18 & 162 & 3 \\
\hline 18 & 163 & 3 \\
\hline 18 & 164 & 6 \\
\hline 18 & 165 & 2 \\
\hline 18 & 166 & 2 \\
\hline 18 & 167 & 2 \\
\hline
\end{tabular}




\begin{tabular}{|c|c|c|}
\hline 18 & 168 & 2 \\
\hline 18 & 169 & 5 \\
\hline 18 & 170 & 5 \\
\hline 19 & 1 & 6 \\
\hline 19 & 2 & 5 \\
\hline 19 & 3 & 5 \\
\hline 19 & 4 & 7 \\
\hline 19 & 5 & 4 \\
\hline 19 & 6 & 2 \\
\hline 19 & 7 & 4 \\
\hline 19 & 8 & 5 \\
\hline 19 & 9 & 5 \\
\hline 19 & 10 & 4 \\
\hline 19 & 11 & 3 \\
\hline 19 & 12 & 2 \\
\hline 19 & 13 & 2 \\
\hline 19 & 14 & 4 \\
\hline 19 & 15 & 4 \\
\hline 19 & 16 & 6 \\
\hline 19 & 17 & 6 \\
\hline 19 & 18 & 7 \\
\hline 19 & 19 & 5 \\
\hline 19 & 20 & 8 \\
\hline 19 & 21 & 8 \\
\hline 19 & 22 & 13 \\
\hline 19 & 23 & 7 \\
\hline 19 & 24 & 7 \\
\hline 19 & 25 & 9 \\
\hline 19 & 26 & 6 \\
\hline 19 & 27 & 8 \\
\hline 19 & 28 & 4 \\
\hline 19 & 29 & 6 \\
\hline 19 & 30 & 4 \\
\hline 19 & 31 & 3 \\
\hline 19 & 32 & 6 \\
\hline 19 & 33 & 5 \\
\hline 19 & 34 & 7 \\
\hline 19 & 35 & 8 \\
\hline 19 & 36 & 8 \\
\hline 19 & 37 & 12 \\
\hline 19 & 38 & 4 \\
\hline 19 & 39 & 5 \\
\hline 19 & 40 & 14 \\
\hline 19 & 41 & 22 \\
\hline 19 & 42 & 63 \\
\hline
\end{tabular}




\begin{tabular}{|c|c|c|}
\hline 19 & 43 & 30 \\
\hline 19 & 44 & 16 \\
\hline 19 & 45 & 18 \\
\hline 19 & 46 & 311 \\
\hline 19 & 47 & 161 \\
\hline 19 & 48 & 4216 \\
\hline 19 & 49 & 3100 \\
\hline 19 & 50 & 50 \\
\hline 19 & 51 & 85 \\
\hline 19 & 52 & 28 \\
\hline 19 & 53 & 10 \\
\hline 19 & 54 & 21 \\
\hline 19 & 55 & 35 \\
\hline 19 & 56 & 70 \\
\hline 19 & 57 & 43 \\
\hline 19 & 58 & 39 \\
\hline 19 & 59 & 31 \\
\hline 19 & 60 & 25 \\
\hline 19 & 61 & 32 \\
\hline 19 & 62 & 19 \\
\hline 19 & 63 & 15 \\
\hline 19 & 64 & 23 \\
\hline 19 & 65 & 19 \\
\hline 19 & 66 & 14 \\
\hline 19 & 67 & 6 \\
\hline 19 & 68 & 19 \\
\hline 19 & 69 & 11 \\
\hline 19 & 70 & 11 \\
\hline 19 & 71 & 6 \\
\hline 19 & 72 & 139 \\
\hline 19 & 73 & 95 \\
\hline 19 & 74 & 53 \\
\hline 19 & 75 & 14 \\
\hline 19 & 76 & 13 \\
\hline 19 & 77 & 9 \\
\hline 19 & 78 & 11 \\
\hline 19 & 79 & 11 \\
\hline 19 & 80 & 3 \\
\hline 19 & 81 & 3 \\
\hline 19 & 82 & 3 \\
\hline 19 & 83 & 4 \\
\hline 19 & 84 & 3 \\
\hline 19 & 85 & 3 \\
\hline 19 & 86 & 9 \\
\hline 19 & 87 & 5 \\
\hline
\end{tabular}




\begin{tabular}{|c|c|c|}
\hline 19 & 88 & 11 \\
\hline 19 & 89 & 9 \\
\hline 19 & 90 & 7 \\
\hline 19 & 91 & 6 \\
\hline 19 & 92 & 4 \\
\hline 19 & 93 & 3 \\
\hline 19 & 94 & 3 \\
\hline 19 & 95 & 3 \\
\hline 19 & 96 & 5 \\
\hline 19 & 97 & 4 \\
\hline 19 & 98 & 3 \\
\hline 19 & 99 & 3 \\
\hline 19 & 100 & 4 \\
\hline 19 & 101 & 3 \\
\hline 19 & 102 & 2 \\
\hline 19 & 103 & 2 \\
\hline 19 & 104 & 2 \\
\hline 19 & 105 & 3 \\
\hline 19 & 106 & 3 \\
\hline 19 & 107 & 2 \\
\hline 19 & 108 & 3 \\
\hline 19 & 109 & 2 \\
\hline 19 & 110 & 3 \\
\hline 19 & 111 & 2 \\
\hline 19 & 112 & 3 \\
\hline 19 & 113 & 2 \\
\hline 19 & 114 & 3 \\
\hline 19 & 115 & 2 \\
\hline 19 & 116 & 2 \\
\hline 19 & 117 & 2 \\
\hline 19 & 118 & 2 \\
\hline 19 & 119 & 3 \\
\hline 19 & 120 & 2 \\
\hline 19 & 121 & 2 \\
\hline 19 & 122 & 2 \\
\hline 19 & 123 & 2 \\
\hline 19 & 124 & 2 \\
\hline 19 & 125 & 2 \\
\hline 19 & 126 & 2 \\
\hline 19 & 127 & 2 \\
\hline 19 & 128 & 2 \\
\hline 19 & 129 & 2 \\
\hline 19 & 130 & 2 \\
\hline 19 & 131 & 2 \\
\hline 19 & 132 & 2 \\
\hline
\end{tabular}




\begin{tabular}{|c|c|c|}
\hline 19 & 133 & 2 \\
\hline 19 & 134 & 3 \\
\hline 19 & 135 & 2 \\
\hline 19 & 136 & 1 \\
\hline 19 & 137 & 2 \\
\hline 19 & 138 & 1 \\
\hline 19 & 139 & 1 \\
\hline 19 & 140 & 1 \\
\hline 19 & 141 & 2 \\
\hline 19 & 142 & 1 \\
\hline 19 & 143 & 2 \\
\hline 19 & 144 & 2 \\
\hline 19 & 145 & 1 \\
\hline 19 & 146 & 2 \\
\hline 19 & 147 & 1 \\
\hline 19 & 148 & 2 \\
\hline 19 & 149 & 2 \\
\hline 19 & 150 & 2 \\
\hline 19 & 151 & 4 \\
\hline 19 & 152 & 2 \\
\hline 19 & 153 & 4 \\
\hline 19 & 154 & 1 \\
\hline 19 & 155 & 1 \\
\hline 19 & 156 & 1 \\
\hline 19 & 157 & 1 \\
\hline 19 & 158 & 1 \\
\hline 19 & 159 & 2 \\
\hline 19 & 160 & 2 \\
\hline 19 & 161 & 3 \\
\hline 19 & 162 & 3 \\
\hline 19 & 163 & 3 \\
\hline 19 & 164 & 2 \\
\hline 19 & 165 & 3 \\
\hline 19 & 166 & 3 \\
\hline 19 & 167 & 3 \\
\hline 19 & 168 & 3 \\
\hline 19 & 169 & 2 \\
\hline 19 & 170 & 1 \\
\hline 20 & 1 & 1074 \\
\hline 20 & 2 & 256 \\
\hline 20 & 3 & 321 \\
\hline 20 & 4 & 895 \\
\hline 20 & 5 & 121 \\
\hline 20 & 6 & 17 \\
\hline 20 & 7 & 70 \\
\hline
\end{tabular}




\begin{tabular}{|c|c|c|}
\hline 20 & 8 & 124 \\
\hline 20 & 9 & 115 \\
\hline 20 & 10 & 63 \\
\hline 20 & 11 & 30 \\
\hline 20 & 12 & 38 \\
\hline 20 & 13 & 26 \\
\hline 20 & 14 & 52 \\
\hline 20 & 15 & 69 \\
\hline 20 & 16 & 168 \\
\hline 20 & 17 & 272 \\
\hline 20 & 18 & 544 \\
\hline 20 & 19 & 85 \\
\hline 20 & 20 & 97 \\
\hline 20 & 21 & 419 \\
\hline 20 & 22 & 66 \\
\hline 20 & 23 & 718 \\
\hline 20 & 24 & 608 \\
\hline 20 & 25 & 240 \\
\hline 20 & 26 & 279 \\
\hline 20 & 27 & 75 \\
\hline 20 & 28 & 124 \\
\hline 20 & 29 & 99 \\
\hline 20 & 30 & 117 \\
\hline 20 & 31 & 143 \\
\hline 20 & 32 & 6 \\
\hline 20 & 33 & 5 \\
\hline 20 & 34 & 30 \\
\hline 20 & 35 & 35 \\
\hline 20 & 36 & 29 \\
\hline 20 & 37 & 20 \\
\hline 20 & 38 & 19 \\
\hline 20 & 39 & 36 \\
\hline 20 & 40 & 11 \\
\hline 20 & 41 & 9 \\
\hline 20 & 42 & 6 \\
\hline 20 & 43 & 11 \\
\hline 20 & 44 & 26 \\
\hline 20 & 45 & 16 \\
\hline 20 & 46 & 7 \\
\hline 20 & 47 & 10 \\
\hline 20 & 48 & 6 \\
\hline 20 & 49 & 7 \\
\hline 20 & 50 & 5 \\
\hline 20 & 51 & 5 \\
\hline 20 & 52 & 14 \\
\hline
\end{tabular}




\begin{tabular}{|c|c|c|}
\hline 20 & 53 & 26 \\
\hline 20 & 54 & 15 \\
\hline 20 & 55 & 11 \\
\hline 20 & 56 & 10 \\
\hline 20 & 57 & 9 \\
\hline 20 & 58 & 9 \\
\hline 20 & 59 & 8 \\
\hline 20 & 60 & 12 \\
\hline 20 & 61 & 12 \\
\hline 20 & 62 & 35 \\
\hline 20 & 63 & 43 \\
\hline 20 & 64 & 14 \\
\hline 20 & 65 & 26 \\
\hline 20 & 66 & 42 \\
\hline 20 & 67 & 31 \\
\hline 20 & 68 & 18 \\
\hline 20 & 69 & 65 \\
\hline 20 & 70 & 77 \\
\hline 20 & 71 & 38 \\
\hline 20 & 72 & 5 \\
\hline 20 & 73 & 6 \\
\hline 20 & 74 & 6 \\
\hline 20 & 75 & 7 \\
\hline 20 & 76 & 12 \\
\hline 20 & 77 & 11 \\
\hline 20 & 78 & 13 \\
\hline 20 & 79 & 11 \\
\hline 20 & 80 & 9 \\
\hline 20 & 81 & 9 \\
\hline 20 & 82 & 9 \\
\hline 20 & 83 & 14 \\
\hline 20 & 84 & 9 \\
\hline 20 & 85 & 9 \\
\hline 20 & 86 & 27 \\
\hline 20 & 87 & 24 \\
\hline 20 & 88 & 11 \\
\hline 20 & 89 & 9 \\
\hline 20 & 90 & 7 \\
\hline 20 & 91 & 5 \\
\hline 20 & 92 & 31 \\
\hline 20 & 93 & 22 \\
\hline 20 & 94 & 31 \\
\hline 20 & 95 & 34 \\
\hline 20 & 96 & 82 \\
\hline 20 & 97 & 43 \\
\hline
\end{tabular}




\begin{tabular}{|c|c|c|}
\hline 20 & 98 & 43 \\
\hline 20 & 99 & 27 \\
\hline 20 & 100 & 35 \\
\hline 20 & 101 & 9 \\
\hline 20 & 102 & 4 \\
\hline 20 & 103 & 8 \\
\hline 20 & 104 & 4 \\
\hline 20 & 105 & 21 \\
\hline 20 & 106 & 18 \\
\hline 20 & 107 & 12 \\
\hline 20 & 108 & 27 \\
\hline 20 & 109 & 16 \\
\hline 20 & 110 & 36 \\
\hline 20 & 111 & 19 \\
\hline 20 & 112 & 23 \\
\hline 20 & 113 & 13 \\
\hline 20 & 114 & 41 \\
\hline 20 & 115 & 31 \\
\hline 20 & 116 & 11 \\
\hline 20 & 117 & 20 \\
\hline 20 & 118 & 9 \\
\hline 20 & 119 & 22 \\
\hline 20 & 120 & 22 \\
\hline 20 & 121 & 11 \\
\hline 20 & 122 & 10 \\
\hline 20 & 123 & 10 \\
\hline 20 & 124 & 7 \\
\hline 20 & 125 & 13 \\
\hline 20 & 126 & 16 \\
\hline 20 & 127 & 13 \\
\hline 20 & 128 & 13 \\
\hline 20 & 129 & 10 \\
\hline 20 & 130 & 13 \\
\hline 20 & 131 & 8 \\
\hline 20 & 132 & 9 \\
\hline 20 & 133 & 21 \\
\hline 20 & 134 & 18 \\
\hline 20 & 135 & 13 \\
\hline 20 & 136 & 4 \\
\hline 20 & 137 & 8 \\
\hline 20 & 138 & 4 \\
\hline 20 & 139 & 5 \\
\hline 20 & 140 & 5 \\
\hline 20 & 141 & 8 \\
\hline 20 & 142 & 5 \\
\hline
\end{tabular}




\begin{tabular}{|c|c|c|}
\hline 20 & 143 & 7 \\
\hline 20 & 144 & 9 \\
\hline 20 & 145 & 11 \\
\hline 20 & 146 & 12 \\
\hline 20 & 147 & 10 \\
\hline 20 & 148 & 22 \\
\hline 20 & 149 & 18 \\
\hline 20 & 150 & 15 \\
\hline 20 & 151 & 27 \\
\hline 20 & 152 & 15 \\
\hline 20 & 153 & 32 \\
\hline 20 & 154 & 10 \\
\hline 20 & 155 & 14 \\
\hline 20 & 156 & 9 \\
\hline 20 & 157 & 10 \\
\hline 20 & 158 & 11 \\
\hline 20 & 159 & 6 \\
\hline 20 & 160 & 3 \\
\hline 20 & 161 & 3 \\
\hline 20 & 162 & 8 \\
\hline 20 & 163 & 8 \\
\hline 20 & 164 & 10 \\
\hline 20 & 165 & 2 \\
\hline 20 & 166 & 3 \\
\hline 20 & 167 & 9 \\
\hline 20 & 168 & 3 \\
\hline 20 & 169 & 12 \\
\hline 20 & 170 & 8 \\
\hline 21 & 1 & 14 \\
\hline 21 & 2 & 25 \\
\hline 21 & 3 & 32 \\
\hline 21 & 4 & 12 \\
\hline 21 & 5 & 34 \\
\hline 21 & 6 & 7 \\
\hline 21 & 7 & 21 \\
\hline 21 & 8 & 24 \\
\hline 21 & 9 & 19 \\
\hline 21 & 10 & 14 \\
\hline 21 & 11 & 5 \\
\hline 21 & 12 & 7 \\
\hline 21 & 13 & 6 \\
\hline 21 & 14 & 11 \\
\hline 21 & 15 & 14 \\
\hline 21 & 16 & 18 \\
\hline 21 & 17 & 10 \\
\hline
\end{tabular}




\begin{tabular}{|c|c|c|}
\hline 21 & 18 & 10 \\
\hline 21 & 19 & 7 \\
\hline 21 & 20 & 8 \\
\hline 21 & 21 & 10 \\
\hline 21 & 22 & 7 \\
\hline 21 & 23 & 11 \\
\hline 21 & 24 & 9 \\
\hline 21 & 25 & 8 \\
\hline 21 & 26 & 13 \\
\hline 21 & 27 & 9 \\
\hline 21 & 28 & 13 \\
\hline 21 & 29 & 10 \\
\hline 21 & 30 & 20 \\
\hline 21 & 31 & 17 \\
\hline 21 & 32 & 2 \\
\hline 21 & 33 & 2 \\
\hline 21 & 34 & 5 \\
\hline 21 & 35 & 5 \\
\hline 21 & 36 & 5 \\
\hline 21 & 37 & 3 \\
\hline 21 & 38 & 4 \\
\hline 21 & 39 & 5 \\
\hline 21 & 40 & 3 \\
\hline 21 & 41 & 3 \\
\hline 21 & 42 & 2 \\
\hline 21 & 43 & 3 \\
\hline 21 & 44 & 3 \\
\hline 21 & 45 & 4 \\
\hline 21 & 46 & 2 \\
\hline 21 & 47 & 3 \\
\hline 21 & 48 & 2 \\
\hline 21 & 49 & 2 \\
\hline 21 & 50 & 2 \\
\hline 21 & 51 & 2 \\
\hline 21 & 52 & 4 \\
\hline 21 & 53 & 5 \\
\hline 21 & 54 & 4 \\
\hline 21 & 55 & 3 \\
\hline 21 & 56 & 3 \\
\hline 21 & 57 & 3 \\
\hline 21 & 58 & 3 \\
\hline 21 & 59 & 3 \\
\hline 21 & 60 & 4 \\
\hline 21 & 61 & 3 \\
\hline 21 & 62 & 6 \\
\hline
\end{tabular}




\begin{tabular}{|c|c|c|}
\hline 21 & 63 & 5 \\
\hline 21 & 64 & 4 \\
\hline 21 & 65 & 4 \\
\hline 21 & 66 & 5 \\
\hline 21 & 67 & 6 \\
\hline 21 & 68 & 5 \\
\hline 21 & 69 & 6 \\
\hline 21 & 70 & 7 \\
\hline 21 & 71 & 6 \\
\hline 21 & 72 & 2 \\
\hline 21 & 73 & 3 \\
\hline 21 & 74 & 2 \\
\hline 21 & 75 & 3 \\
\hline 21 & 76 & 4 \\
\hline 21 & 77 & 8 \\
\hline 21 & 78 & 11 \\
\hline 21 & 79 & 9 \\
\hline 21 & 80 & 11 \\
\hline 21 & 81 & 9 \\
\hline 21 & 82 & 9 \\
\hline 21 & 83 & 24 \\
\hline 21 & 84 & 15 \\
\hline 21 & 85 & 13 \\
\hline 21 & 86 & 8 \\
\hline 21 & 87 & 15 \\
\hline 21 & 88 & 4 \\
\hline 21 & 89 & 7 \\
\hline 21 & 90 & 7 \\
\hline 21 & 91 & 3 \\
\hline 21 & 92 & 33 \\
\hline 21 & 93 & 27 \\
\hline 21 & 94 & 51 \\
\hline 21 & 95 & 59 \\
\hline 21 & 96 & 10 \\
\hline 21 & 97 & 29 \\
\hline 21 & 98 & 18 \\
\hline 21 & 99 & 52 \\
\hline 21 & 100 & 38 \\
\hline 21 & 101 & 25 \\
\hline 21 & 102 & 12 \\
\hline 21 & 103 & 14 \\
\hline 21 & 104 & 10 \\
\hline 21 & 105 & 66 \\
\hline 21 & 106 & 88 \\
\hline 21 & 107 & 40 \\
\hline
\end{tabular}




\begin{tabular}{|c|c|c|}
\hline 21 & 108 & 38 \\
\hline 21 & 109 & 55 \\
\hline 21 & 110 & 53 \\
\hline 21 & 111 & 58 \\
\hline 21 & 112 & 79 \\
\hline 21 & 113 & 75 \\
\hline 21 & 114 & 75 \\
\hline 21 & 115 & 208 \\
\hline 21 & 116 & 32 \\
\hline 21 & 117 & 23 \\
\hline 21 & 118 & 21 \\
\hline 21 & 119 & 126 \\
\hline 21 & 120 & 129 \\
\hline 21 & 121 & 283 \\
\hline 21 & 122 & 33 \\
\hline 21 & 123 & 52 \\
\hline 21 & 124 & 57 \\
\hline 21 & 125 & 73 \\
\hline 21 & 126 & 199 \\
\hline 21 & 127 & 626 \\
\hline 21 & 128 & 1294 \\
\hline 21 & 129 & 941 \\
\hline 21 & 130 & 95 \\
\hline 21 & 131 & 112 \\
\hline 21 & 132 & 137 \\
\hline 21 & 133 & 85 \\
\hline 21 & 134 & 199 \\
\hline 21 & 135 & 30 \\
\hline 21 & 136 & 3 \\
\hline 21 & 137 & 4 \\
\hline 21 & 138 & 3 \\
\hline 21 & 139 & 3 \\
\hline 21 & 140 & 3 \\
\hline 21 & 141 & 4 \\
\hline 21 & 142 & 3 \\
\hline 21 & 143 & 4 \\
\hline 21 & 144 & 5 \\
\hline 21 & 145 & 5 \\
\hline 21 & 146 & 16 \\
\hline 21 & 147 & 3 \\
\hline 21 & 148 & 7 \\
\hline 21 & 149 & 4 \\
\hline 21 & 150 & 4 \\
\hline 21 & 151 & 5 \\
\hline 21 & 152 & 4 \\
\hline
\end{tabular}




\begin{tabular}{|c|c|c|}
\hline 21 & 153 & 6 \\
\hline 21 & 154 & 3 \\
\hline 21 & 155 & 4 \\
\hline 21 & 156 & 3 \\
\hline 21 & 157 & 3 \\
\hline 21 & 158 & 3 \\
\hline 21 & 159 & 2 \\
\hline 21 & 160 & 2 \\
\hline 21 & 161 & 1 \\
\hline 21 & 162 & 2 \\
\hline 21 & 163 & 2 \\
\hline 21 & 164 & 3 \\
\hline 21 & 165 & 1 \\
\hline 21 & 166 & 1 \\
\hline 21 & 167 & 1 \\
\hline 21 & 168 & 1 \\
\hline 21 & 169 & 5 \\
\hline 21 & 170 & 3 \\
\hline 22 & 1 & 80 \\
\hline 22 & 2 & 48 \\
\hline 22 & 3 & 53 \\
\hline 22 & 4 & 84 \\
\hline 22 & 5 & 34 \\
\hline 22 & 6 & 10 \\
\hline 22 & 7 & 25 \\
\hline 22 & 8 & 34 \\
\hline 22 & 9 & 35 \\
\hline 22 & 10 & 25 \\
\hline 22 & 11 & 12 \\
\hline 22 & 12 & 10 \\
\hline 22 & 13 & 8 \\
\hline 22 & 14 & 22 \\
\hline 22 & 15 & 26 \\
\hline 22 & 16 & 43 \\
\hline 22 & 17 & 54 \\
\hline 22 & 18 & 71 \\
\hline 22 & 19 & 29 \\
\hline 22 & 20 & 37 \\
\hline 22 & 21 & 103 \\
\hline 22 & 22 & 491 \\
\hline 22 & 23 & 191 \\
\hline 22 & 24 & 346 \\
\hline 22 & 25 & 1473 \\
\hline 22 & 26 & 127 \\
\hline 22 & 27 & 125 \\
\hline
\end{tabular}




\begin{tabular}{|c|c|c|}
\hline 22 & 28 & 51 \\
\hline 22 & 29 & 35 \\
\hline 22 & 30 & 34 \\
\hline 22 & 31 & 37 \\
\hline 22 & 32 & 7 \\
\hline 22 & 33 & 6 \\
\hline 22 & 34 & 7 \\
\hline 22 & 35 & 9 \\
\hline 22 & 36 & 8 \\
\hline 22 & 37 & 13 \\
\hline 22 & 38 & 5 \\
\hline 22 & 39 & 17 \\
\hline 22 & 40 & 15 \\
\hline 22 & 41 & 12 \\
\hline 22 & 42 & 13 \\
\hline 22 & 43 & 18 \\
\hline 22 & 44 & 30 \\
\hline 22 & 45 & 28 \\
\hline 22 & 46 & 11 \\
\hline 22 & 47 & 17 \\
\hline 22 & 48 & 9 \\
\hline 22 & 49 & 12 \\
\hline 22 & 50 & 8 \\
\hline 22 & 51 & 8 \\
\hline 22 & 52 & 29 \\
\hline 22 & 53 & 55 \\
\hline 22 & 54 & 28 \\
\hline 22 & 55 & 18 \\
\hline 22 & 56 & 18 \\
\hline 22 & 57 & 30 \\
\hline 22 & 58 & 29 \\
\hline 22 & 59 & 22 \\
\hline 22 & 60 & 34 \\
\hline 22 & 61 & 24 \\
\hline 22 & 62 & 88 \\
\hline 22 & 63 & 151 \\
\hline 22 & 64 & 68 \\
\hline 22 & 65 & 65 \\
\hline 22 & 66 & 143 \\
\hline 22 & 67 & 37 \\
\hline 22 & 68 & 44 \\
\hline 22 & 69 & 352 \\
\hline 22 & 70 & 521 \\
\hline 22 & 71 & 117 \\
\hline 22 & 72 & 8 \\
\hline
\end{tabular}




\begin{tabular}{|c|c|c|}
\hline 22 & 73 & 16 \\
\hline 22 & 74 & 13 \\
\hline 22 & 75 & 8 \\
\hline 22 & 76 & 13 \\
\hline 22 & 77 & 14 \\
\hline 22 & 78 & 22 \\
\hline 22 & 79 & 11 \\
\hline 22 & 80 & 10 \\
\hline 22 & 81 & 11 \\
\hline 22 & 82 & 11 \\
\hline 22 & 83 & 17 \\
\hline 22 & 84 & 10 \\
\hline 22 & 85 & 10 \\
\hline 22 & 86 & 43 \\
\hline 22 & 87 & 29 \\
\hline 22 & 88 & 12 \\
\hline 22 & 89 & 10 \\
\hline 22 & 90 & 7 \\
\hline 22 & 91 & 5 \\
\hline 22 & 92 & 13 \\
\hline 22 & 93 & 11 \\
\hline 22 & 94 & 14 \\
\hline 22 & 95 & 15 \\
\hline 22 & 96 & 60 \\
\hline 22 & 97 & 13 \\
\hline 22 & 98 & 17 \\
\hline 22 & 99 & 11 \\
\hline 22 & 100 & 12 \\
\hline 22 & 101 & 8 \\
\hline 22 & 102 & 6 \\
\hline 22 & 103 & 5 \\
\hline 22 & 104 & 4 \\
\hline 22 & 105 & 10 \\
\hline 22 & 106 & 10 \\
\hline 22 & 107 & 5 \\
\hline 22 & 108 & 13 \\
\hline 22 & 109 & 9 \\
\hline 22 & 110 & 16 \\
\hline 22 & 111 & 11 \\
\hline 22 & 112 & 12 \\
\hline 22 & 113 & 7 \\
\hline 22 & 114 & 15 \\
\hline 22 & 115 & 13 \\
\hline 22 & 116 & 7 \\
\hline 22 & 117 & 11 \\
\hline
\end{tabular}




\begin{tabular}{|c|c|c|}
\hline 22 & 118 & 6 \\
\hline 22 & 119 & 12 \\
\hline 22 & 120 & 5 \\
\hline 22 & 121 & 6 \\
\hline 22 & 122 & 6 \\
\hline 22 & 123 & 4 \\
\hline 22 & 124 & 4 \\
\hline 22 & 125 & 4 \\
\hline 22 & 126 & 5 \\
\hline 22 & 127 & 6 \\
\hline 22 & 128 & 7 \\
\hline 22 & 129 & 6 \\
\hline 22 & 130 & 4 \\
\hline 22 & 131 & 5 \\
\hline 22 & 132 & 6 \\
\hline 22 & 133 & 10 \\
\hline 22 & 134 & 7 \\
\hline 22 & 135 & 8 \\
\hline 22 & 136 & 3 \\
\hline 22 & 137 & 6 \\
\hline 22 & 138 & 3 \\
\hline 22 & 139 & 3 \\
\hline 22 & 140 & 3 \\
\hline 22 & 141 & 5 \\
\hline 22 & 142 & 4 \\
\hline 22 & 143 & 5 \\
\hline 22 & 144 & 6 \\
\hline 22 & 145 & 7 \\
\hline 22 & 146 & 7 \\
\hline 22 & 147 & 7 \\
\hline 22 & 148 & 12 \\
\hline 22 & 149 & 2 \\
\hline 22 & 150 & 9 \\
\hline 22 & 151 & 14 \\
\hline 22 & 152 & 9 \\
\hline 22 & 153 & 16 \\
\hline 22 & 154 & 6 \\
\hline 22 & 155 & 5 \\
\hline 22 & 156 & 6 \\
\hline 22 & 157 & 6 \\
\hline 22 & 158 & 7 \\
\hline 22 & 159 & 2 \\
\hline 22 & 160 & 3 \\
\hline 22 & 161 & 3 \\
\hline 22 & 162 & 3 \\
\hline
\end{tabular}




\begin{tabular}{|c|c|c|}
\hline 22 & 163 & 3 \\
\hline 22 & 164 & 3 \\
\hline 22 & 165 & 3 \\
\hline 22 & 166 & 3 \\
\hline 22 & 167 & 3 \\
\hline 22 & 168 & 3 \\
\hline 22 & 169 & 3 \\
\hline 22 & 170 & 5 \\
\hline 23 & 1 & 4 \\
\hline 23 & 2 & 3 \\
\hline 23 & 3 & 3 \\
\hline 23 & 4 & 4 \\
\hline 23 & 5 & 2 \\
\hline 23 & 6 & 1 \\
\hline 23 & 7 & 2 \\
\hline 23 & 8 & 3 \\
\hline 23 & 9 & 3 \\
\hline 23 & 10 & 1 \\
\hline 23 & 11 & 1 \\
\hline 23 & 12 & 2 \\
\hline 23 & 13 & 1 \\
\hline 23 & 14 & 7 \\
\hline 23 & 15 & 3 \\
\hline 23 & 16 & 4 \\
\hline 23 & 17 & 4 \\
\hline 23 & 18 & 4 \\
\hline 23 & 19 & 10 \\
\hline 23 & 20 & 11 \\
\hline 23 & 21 & 5 \\
\hline 23 & 22 & 6 \\
\hline 23 & 23 & 4 \\
\hline 23 & 24 & 5 \\
\hline 23 & 25 & 5 \\
\hline 23 & 26 & 3 \\
\hline 23 & 27 & 4 \\
\hline 23 & 28 & 3 \\
\hline 23 & 29 & 4 \\
\hline 23 & 30 & 3 \\
\hline 23 & 31 & 3 \\
\hline 23 & 32 & 11 \\
\hline 23 & 33 & 9 \\
\hline 23 & 34 & 10 \\
\hline 23 & 35 & 13 \\
\hline 23 & 36 & 11 \\
\hline 23 & 37 & 20 \\
\hline
\end{tabular}




\begin{tabular}{|c|c|c|}
\hline 23 & 38 & 7 \\
\hline 23 & 39 & 2 \\
\hline 23 & 40 & 24 \\
\hline 23 & 41 & 41 \\
\hline 23 & 42 & 197 \\
\hline 23 & 43 & 69 \\
\hline 23 & 44 & 30 \\
\hline 23 & 45 & 35 \\
\hline 23 & 46 & 123 \\
\hline 23 & 47 & 64 \\
\hline 23 & 48 & 48 \\
\hline 23 & 49 & 57 \\
\hline 23 & 50 & 1731 \\
\hline 23 & 51 & 179 \\
\hline 23 & 52 & 35 \\
\hline 23 & 53 & 21 \\
\hline 23 & 54 & 25 \\
\hline 23 & 55 & 38 \\
\hline 23 & 56 & 37 \\
\hline 23 & 57 & 25 \\
\hline 23 & 58 & 24 \\
\hline 23 & 59 & 20 \\
\hline 23 & 60 & 17 \\
\hline 23 & 61 & 20 \\
\hline 23 & 62 & 13 \\
\hline 23 & 63 & 11 \\
\hline 23 & 64 & 15 \\
\hline 23 & 65 & 13 \\
\hline 23 & 66 & 10 \\
\hline 23 & 67 & 4 \\
\hline 23 & 68 & 13 \\
\hline 23 & 69 & 5 \\
\hline 23 & 70 & 5 \\
\hline 23 & 71 & 4 \\
\hline 23 & 72 & 25 \\
\hline 23 & 73 & 21 \\
\hline 23 & 74 & 11 \\
\hline 23 & 75 & 6 \\
\hline 23 & 76 & 9 \\
\hline 23 & 77 & 7 \\
\hline 23 & 78 & 8 \\
\hline 23 & 79 & 8 \\
\hline 23 & 80 & 2 \\
\hline 23 & 81 & 2 \\
\hline 23 & 82 & 2 \\
\hline
\end{tabular}




\begin{tabular}{|c|c|c|}
\hline 23 & 83 & 3 \\
\hline 23 & 84 & 2 \\
\hline 23 & 85 & 2 \\
\hline 23 & 86 & 4 \\
\hline 23 & 87 & 4 \\
\hline 23 & 88 & 8 \\
\hline 23 & 89 & 7 \\
\hline 23 & 90 & 6 \\
\hline 23 & 91 & 5 \\
\hline 23 & 92 & 3 \\
\hline 23 & 93 & 2 \\
\hline 23 & 94 & 2 \\
\hline 23 & 95 & 2 \\
\hline 23 & 96 & 3 \\
\hline 23 & 97 & 3 \\
\hline 23 & 98 & 2 \\
\hline 23 & 99 & 3 \\
\hline 23 & 100 & 3 \\
\hline 23 & 101 & 2 \\
\hline 23 & 102 & 2 \\
\hline 23 & 103 & 1 \\
\hline 23 & 104 & 1 \\
\hline 23 & 105 & 2 \\
\hline 23 & 106 & 2 \\
\hline 23 & 107 & 2 \\
\hline 23 & 108 & 2 \\
\hline 23 & 109 & 2 \\
\hline 23 & 110 & 2 \\
\hline 23 & 111 & 2 \\
\hline 23 & 112 & 2 \\
\hline 23 & 113 & 2 \\
\hline 23 & 114 & 2 \\
\hline 23 & 115 & 2 \\
\hline 23 & 116 & 1 \\
\hline 23 & 117 & 1 \\
\hline 23 & 118 & 1 \\
\hline 23 & 119 & 2 \\
\hline 23 & 120 & 2 \\
\hline 23 & 121 & 2 \\
\hline 23 & 122 & 1 \\
\hline 23 & 123 & 1 \\
\hline 23 & 124 & 1 \\
\hline 23 & 125 & 1 \\
\hline 23 & 126 & 2 \\
\hline 23 & 127 & 2 \\
\hline
\end{tabular}




\begin{tabular}{|c|c|c|}
\hline 23 & 128 & 2 \\
\hline 23 & 129 & 2 \\
\hline 23 & 130 & 2 \\
\hline 23 & 131 & 1 \\
\hline 23 & 132 & 1 \\
\hline 23 & 133 & 2 \\
\hline 23 & 134 & 2 \\
\hline 23 & 135 & 1 \\
\hline 23 & 136 & 2 \\
\hline 23 & 137 & 1 \\
\hline 23 & 138 & 1 \\
\hline 23 & 139 & 1 \\
\hline 23 & 140 & 1 \\
\hline 23 & 141 & 1 \\
\hline 23 & 142 & 1 \\
\hline 23 & 143 & 1 \\
\hline 23 & 144 & 1 \\
\hline 23 & 145 & 2 \\
\hline 23 & 146 & 1 \\
\hline 23 & 147 & 2 \\
\hline 23 & 148 & 3 \\
\hline 23 & 149 & 3 \\
\hline 23 & 150 & 2 \\
\hline 23 & 151 & 1 \\
\hline 23 & 152 & 2 \\
\hline 23 & 153 & 2 \\
\hline 23 & 154 & 1 \\
\hline 23 & 155 & 2 \\
\hline 23 & 156 & 1 \\
\hline 23 & 157 & 2 \\
\hline 23 & 158 & 2 \\
\hline 23 & 159 & 2 \\
\hline 23 & 160 & 3 \\
\hline 23 & 161 & 4 \\
\hline 23 & 162 & 4 \\
\hline 23 & 163 & 4 \\
\hline 23 & 164 & 3 \\
\hline 23 & 165 & 4 \\
\hline 23 & 166 & 4 \\
\hline 23 & 167 & 4 \\
\hline 23 & 168 & 4 \\
\hline 23 & 169 & 3 \\
\hline 23 & 170 & 2 \\
\hline 24 & 1 & 5 \\
\hline 24 & 2 & 4 \\
\hline
\end{tabular}




\begin{tabular}{|c|c|c|}
\hline 24 & 3 & 5 \\
\hline 24 & 4 & 4 \\
\hline 24 & 5 & 4 \\
\hline 24 & 6 & 3 \\
\hline 24 & 7 & 4 \\
\hline 24 & 8 & 4 \\
\hline 24 & 9 & 4 \\
\hline 24 & 10 & 3 \\
\hline 24 & 11 & 3 \\
\hline 24 & 12 & 3 \\
\hline 24 & 13 & 1 \\
\hline 24 & 14 & 3 \\
\hline 24 & 15 & 3 \\
\hline 24 & 16 & 4 \\
\hline 24 & 17 & 3 \\
\hline 24 & 18 & 4 \\
\hline 24 & 19 & 3 \\
\hline 24 & 20 & 3 \\
\hline 24 & 21 & 5 \\
\hline 24 & 22 & 7 \\
\hline 24 & 23 & 7 \\
\hline 24 & 24 & 5 \\
\hline 24 & 25 & 8 \\
\hline 24 & 26 & 8 \\
\hline 24 & 27 & 10 \\
\hline 24 & 28 & 7 \\
\hline 24 & 29 & 10 \\
\hline 24 & 30 & 6 \\
\hline 24 & 31 & 5 \\
\hline 24 & 32 & 2 \\
\hline 24 & 33 & 2 \\
\hline 24 & 34 & 2 \\
\hline 24 & 35 & 3 \\
\hline 24 & 36 & 2 \\
\hline 24 & 37 & 3 \\
\hline 24 & 38 & 2 \\
\hline 24 & 39 & 3 \\
\hline 24 & 40 & 3 \\
\hline 24 & 41 & 3 \\
\hline 24 & 42 & 5 \\
\hline 24 & 43 & 6 \\
\hline 24 & 44 & 3 \\
\hline 24 & 45 & 4 \\
\hline 24 & 46 & 7 \\
\hline 24 & 47 & 10 \\
\hline
\end{tabular}




\begin{tabular}{|c|c|c|}
\hline 24 & 48 & 6 \\
\hline 24 & 49 & 8 \\
\hline 24 & 50 & 5 \\
\hline 24 & 51 & 6 \\
\hline 24 & 52 & 7 \\
\hline 24 & 53 & 5 \\
\hline 24 & 54 & 7 \\
\hline 24 & 55 & 6 \\
\hline 24 & 56 & 11 \\
\hline 24 & 57 & 16 \\
\hline 24 & 58 & 17 \\
\hline 24 & 59 & 14 \\
\hline 24 & 60 & 26 \\
\hline 24 & 61 & 10 \\
\hline 24 & 62 & 10 \\
\hline 24 & 63 & 13 \\
\hline 24 & 64 & 17 \\
\hline 24 & 65 & 19 \\
\hline 24 & 66 & 13 \\
\hline 24 & 67 & 12 \\
\hline 24 & 68 & 26 \\
\hline 24 & 69 & 9 \\
\hline 24 & 70 & 9 \\
\hline 24 & 71 & 12 \\
\hline 24 & 72 & 7 \\
\hline 24 & 73 & 9 \\
\hline 24 & 74 & 9 \\
\hline 24 & 75 & 24 \\
\hline 24 & 76 & 44 \\
\hline 24 & 77 & 22 \\
\hline 24 & 78 & 41 \\
\hline 24 & 79 & 72 \\
\hline 24 & 80 & 52 \\
\hline 24 & 81 & 48 \\
\hline 24 & 82 & 48 \\
\hline 24 & 83 & 20 \\
\hline 24 & 84 & 44 \\
\hline 24 & 85 & 25 \\
\hline 24 & 86 & 13 \\
\hline 24 & 87 & 9 \\
\hline 24 & 88 & 118 \\
\hline 24 & 89 & 239 \\
\hline 24 & 90 & 648 \\
\hline 24 & 91 & 1455 \\
\hline 24 & 92 & 14 \\
\hline
\end{tabular}




\begin{tabular}{|c|c|c|}
\hline 24 & 93 & 15 \\
\hline 24 & 94 & 9 \\
\hline 24 & 95 & 10 \\
\hline 24 & 96 & 8 \\
\hline 24 & 97 & 13 \\
\hline 24 & 98 & 4 \\
\hline 24 & 99 & 12 \\
\hline 24 & 100 & 13 \\
\hline 24 & 101 & 16 \\
\hline 24 & 102 & 13 \\
\hline 24 & 103 & 4 \\
\hline 24 & 104 & 10 \\
\hline 24 & 105 & 10 \\
\hline 24 & 106 & 10 \\
\hline 24 & 107 & 9 \\
\hline 24 & 108 & 4 \\
\hline 24 & 109 & 3 \\
\hline 24 & 110 & 4 \\
\hline 24 & 111 & 3 \\
\hline 24 & 112 & 4 \\
\hline 24 & 113 & 6 \\
\hline 24 & 114 & 4 \\
\hline 24 & 115 & 3 \\
\hline 24 & 116 & 2 \\
\hline 24 & 117 & 3 \\
\hline 24 & 118 & 2 \\
\hline 24 & 119 & 4 \\
\hline 24 & 120 & 3 \\
\hline 24 & 121 & 3 \\
\hline 24 & 122 & 2 \\
\hline 24 & 123 & 2 \\
\hline 24 & 124 & 3 \\
\hline 24 & 125 & 2 \\
\hline 24 & 126 & 3 \\
\hline 24 & 127 & 3 \\
\hline 24 & 128 & 6 \\
\hline 24 & 129 & 5 \\
\hline 24 & 130 & 2 \\
\hline 24 & 131 & 3 \\
\hline 24 & 132 & 3 \\
\hline 24 & 133 & 2 \\
\hline 24 & 134 & 3 \\
\hline 24 & 135 & 2 \\
\hline 24 & 136 & 1 \\
\hline 24 & 137 & 2 \\
\hline
\end{tabular}




\begin{tabular}{|c|c|c|}
\hline 24 & 138 & 1 \\
\hline 24 & 139 & 1 \\
\hline 24 & 140 & 1 \\
\hline 24 & 141 & 1 \\
\hline 24 & 142 & 1 \\
\hline 24 & 143 & 1 \\
\hline 24 & 144 & 2 \\
\hline 24 & 145 & 1 \\
\hline 24 & 146 & 2 \\
\hline 24 & 147 & 1 \\
\hline 24 & 148 & 1 \\
\hline 24 & 149 & 2 \\
\hline 24 & 150 & 1 \\
\hline 24 & 151 & 2 \\
\hline 24 & 152 & 1 \\
\hline 24 & 153 & 3 \\
\hline 24 & 154 & 1 \\
\hline 24 & 155 & 1 \\
\hline 24 & 156 & 1 \\
\hline 24 & 157 & 1 \\
\hline 24 & 158 & 1 \\
\hline 24 & 159 & 1 \\
\hline 24 & 160 & 1 \\
\hline 24 & 161 & 1 \\
\hline 24 & 162 & 1 \\
\hline 24 & 163 & 1 \\
\hline 24 & 164 & 1 \\
\hline 24 & 165 & 1 \\
\hline 24 & 166 & 1 \\
\hline 24 & 167 & 1 \\
\hline 24 & 168 & 1 \\
\hline 24 & 169 & 1 \\
\hline 24 & 170 & 1 \\
\hline 25 & 1 & 12 \\
\hline 25 & 2 & 27 \\
\hline 25 & 3 & 18 \\
\hline 25 & 4 & 11 \\
\hline 25 & 5 & 20 \\
\hline 25 & 6 & 27 \\
\hline 25 & 7 & 35 \\
\hline 25 & 8 & 37 \\
\hline 25 & 9 & 43 \\
\hline 25 & 10 & 21 \\
\hline 25 & 11 & 73 \\
\hline 25 & 12 & 61 \\
\hline
\end{tabular}




\begin{tabular}{|c|c|c|}
\hline 25 & 13 & 150 \\
\hline 25 & 14 & 12 \\
\hline 25 & 15 & 13 \\
\hline 25 & 16 & 17 \\
\hline 25 & 17 & 14 \\
\hline 25 & 18 & 11 \\
\hline 25 & 19 & 12 \\
\hline 25 & 20 & 9 \\
\hline 25 & 21 & 11 \\
\hline 25 & 22 & 5 \\
\hline 25 & 23 & 8 \\
\hline 25 & 24 & 8 \\
\hline 25 & 25 & 7 \\
\hline 25 & 26 & 12 \\
\hline 25 & 27 & 8 \\
\hline 25 & 28 & 11 \\
\hline 25 & 29 & 9 \\
\hline 25 & 30 & 14 \\
\hline 25 & 31 & 14 \\
\hline 25 & 32 & 2 \\
\hline 25 & 33 & 2 \\
\hline 25 & 34 & 8 \\
\hline 25 & 35 & 7 \\
\hline 25 & 36 & 6 \\
\hline 25 & 37 & 5 \\
\hline 25 & 38 & 6 \\
\hline 25 & 39 & 8 \\
\hline 25 & 40 & 2 \\
\hline 25 & 41 & 2 \\
\hline 25 & 42 & 2 \\
\hline 25 & 43 & 2 \\
\hline 25 & 44 & 6 \\
\hline 25 & 45 & 2 \\
\hline 25 & 46 & 2 \\
\hline 25 & 47 & 2 \\
\hline 25 & 48 & 2 \\
\hline 25 & 49 & 2 \\
\hline 25 & 50 & 1 \\
\hline 25 & 51 & 2 \\
\hline 25 & 52 & 3 \\
\hline 25 & 53 & 4 \\
\hline 25 & 54 & 3 \\
\hline 25 & 55 & 3 \\
\hline 25 & 56 & 2 \\
\hline 25 & 57 & 3 \\
\hline
\end{tabular}




\begin{tabular}{|c|c|c|}
\hline 25 & 58 & 3 \\
\hline 25 & 59 & 3 \\
\hline 25 & 60 & 4 \\
\hline 25 & 61 & 3 \\
\hline 25 & 62 & 4 \\
\hline 25 & 63 & 5 \\
\hline 25 & 64 & 4 \\
\hline 25 & 65 & 4 \\
\hline 25 & 66 & 5 \\
\hline 25 & 67 & 6 \\
\hline 25 & 68 & 5 \\
\hline 25 & 69 & 5 \\
\hline 25 & 70 & 6 \\
\hline 25 & 71 & 6 \\
\hline 25 & 72 & 2 \\
\hline 25 & 73 & 2 \\
\hline 25 & 74 & 2 \\
\hline 25 & 75 & 3 \\
\hline 25 & 76 & 4 \\
\hline 25 & 77 & 3 \\
\hline 25 & 78 & 4 \\
\hline 25 & 79 & 3 \\
\hline 25 & 80 & 3 \\
\hline 25 & 81 & 3 \\
\hline 25 & 82 & 3 \\
\hline 25 & 83 & 5 \\
\hline 25 & 84 & 3 \\
\hline 25 & 85 & 3 \\
\hline 25 & 86 & 6 \\
\hline 25 & 87 & 5 \\
\hline 25 & 88 & 3 \\
\hline 25 & 89 & 3 \\
\hline 25 & 90 & 3 \\
\hline 25 & 91 & 1 \\
\hline 25 & 92 & 5 \\
\hline 25 & 93 & 5 \\
\hline 25 & 94 & 8 \\
\hline 25 & 95 & 8 \\
\hline 25 & 96 & 9 \\
\hline 25 & 97 & 6 \\
\hline 25 & 98 & 9 \\
\hline 25 & 99 & 7 \\
\hline 25 & 100 & 7 \\
\hline 25 & 101 & 4 \\
\hline 25 & 102 & 2 \\
\hline
\end{tabular}




\begin{tabular}{|c|c|c|}
\hline 25 & 103 & 3 \\
\hline 25 & 104 & 2 \\
\hline 25 & 105 & 7 \\
\hline 25 & 106 & 6 \\
\hline 25 & 107 & 3 \\
\hline 25 & 108 & 23 \\
\hline 25 & 109 & 34 \\
\hline 25 & 110 & 15 \\
\hline 25 & 111 & 22 \\
\hline 25 & 112 & 20 \\
\hline 25 & 113 & 5 \\
\hline 25 & 114 & 10 \\
\hline 25 & 115 & 9 \\
\hline 25 & 116 & 39 \\
\hline 25 & 117 & 64 \\
\hline 25 & 118 & 25 \\
\hline 25 & 119 & 11 \\
\hline 25 & 120 & 13 \\
\hline 25 & 121 & 7 \\
\hline 25 & 122 & 19 \\
\hline 25 & 123 & 15 \\
\hline 25 & 124 & 7 \\
\hline 25 & 125 & 11 \\
\hline 25 & 126 & 7 \\
\hline 25 & 127 & 7 \\
\hline 25 & 128 & 5 \\
\hline 25 & 129 & 6 \\
\hline 25 & 130 & 9 \\
\hline 25 & 131 & 4 \\
\hline 25 & 132 & 4 \\
\hline 25 & 133 & 7 \\
\hline 25 & 134 & 6 \\
\hline 25 & 135 & 22 \\
\hline 25 & 136 & 33 \\
\hline 25 & 137 & 73 \\
\hline 25 & 138 & 29 \\
\hline 25 & 139 & 44 \\
\hline 25 & 140 & 57 \\
\hline 25 & 141 & 112 \\
\hline 25 & 142 & 35 \\
\hline 25 & 143 & 62 \\
\hline 25 & 144 & 240 \\
\hline 25 & 145 & 5536 \\
\hline 25 & 146 & 73 \\
\hline 25 & 147 & 118 \\
\hline
\end{tabular}




\begin{tabular}{|c|c|c|}
\hline 25 & 148 & 12 \\
\hline 25 & 149 & 39 \\
\hline 25 & 150 & 74 \\
\hline 25 & 151 & 19 \\
\hline 25 & 152 & 96 \\
\hline 25 & 153 & 28 \\
\hline 25 & 154 & 83 \\
\hline 25 & 155 & 117 \\
\hline 25 & 156 & 80 \\
\hline 25 & 157 & 95 \\
\hline 25 & 158 & 140 \\
\hline 25 & 159 & 28 \\
\hline 25 & 160 & 7 \\
\hline 25 & 161 & 4 \\
\hline 25 & 162 & 7 \\
\hline 25 & 163 & 9 \\
\hline 25 & 164 & 9 \\
\hline 25 & 165 & 4 \\
\hline 25 & 166 & 5 \\
\hline 25 & 167 & 4 \\
\hline 25 & 168 & 4 \\
\hline 25 & 169 & 7 \\
\hline 25 & 170 & 47 \\
\hline 26 & 1 & 293 \\
\hline 26 & 2 & 477 \\
\hline 26 & 3 & 996 \\
\hline 26 & 4 & 165 \\
\hline 26 & 5 & 1035 \\
\hline 26 & 6 & 34 \\
\hline 26 & 7 & 504 \\
\hline 26 & 8 & 2268 \\
\hline 26 & 9 & 639 \\
\hline 26 & 10 & 187 \\
\hline 26 & 11 & 62 \\
\hline 26 & 12 & 87 \\
\hline 26 & 13 & 51 \\
\hline 26 & 14 & 60 \\
\hline 26 & 15 & 176 \\
\hline 26 & 16 & 248 \\
\hline 26 & 17 & 144 \\
\hline 26 & 18 & 103 \\
\hline 26 & 19 & 52 \\
\hline 26 & 20 & 47 \\
\hline 26 & 21 & 92 \\
\hline 26 & 22 & 13 \\
\hline
\end{tabular}




\begin{tabular}{|c|c|c|}
\hline 26 & 23 & 138 \\
\hline 26 & 24 & 78 \\
\hline 26 & 25 & 21 \\
\hline 26 & 26 & 59 \\
\hline 26 & 27 & 27 \\
\hline 26 & 28 & 54 \\
\hline 26 & 29 & 34 \\
\hline 26 & 30 & 88 \\
\hline 26 & 31 & 93 \\
\hline 26 & 32 & 3 \\
\hline 26 & 33 & 3 \\
\hline 26 & 34 & 22 \\
\hline 26 & 35 & 22 \\
\hline 26 & 36 & 19 \\
\hline 26 & 37 & 14 \\
\hline 26 & 38 & 15 \\
\hline 26 & 39 & 26 \\
\hline 26 & 40 & 5 \\
\hline 26 & 41 & 4 \\
\hline 26 & 42 & 3 \\
\hline 26 & 43 & 5 \\
\hline 26 & 44 & 17 \\
\hline 26 & 45 & 6 \\
\hline 26 & 46 & 3 \\
\hline 26 & 47 & 4 \\
\hline 26 & 48 & 3 \\
\hline 26 & 49 & 3 \\
\hline 26 & 50 & 3 \\
\hline 26 & 51 & 3 \\
\hline 26 & 52 & 6 \\
\hline 26 & 53 & 8 \\
\hline 26 & 54 & 6 \\
\hline 26 & 55 & 5 \\
\hline 26 & 56 & 4 \\
\hline 26 & 57 & 6 \\
\hline 26 & 58 & 6 \\
\hline 26 & 59 & 5 \\
\hline 26 & 60 & 8 \\
\hline 26 & 61 & 5 \\
\hline 26 & 62 & 9 \\
\hline 26 & 63 & 11 \\
\hline 26 & 64 & 8 \\
\hline 26 & 65 & 9 \\
\hline 26 & 66 & 12 \\
\hline 26 & 67 & 16 \\
\hline
\end{tabular}




\begin{tabular}{|c|c|c|}
\hline 26 & 68 & 11 \\
\hline 26 & 69 & 14 \\
\hline 26 & 70 & 17 \\
\hline 26 & 71 & 15 \\
\hline 26 & 72 & 3 \\
\hline 26 & 73 & 4 \\
\hline 26 & 74 & 3 \\
\hline 26 & 75 & 5 \\
\hline 26 & 76 & 8 \\
\hline 26 & 77 & 7 \\
\hline 26 & 78 & 9 \\
\hline 26 & 79 & 7 \\
\hline 26 & 80 & 6 \\
\hline 26 & 81 & 6 \\
\hline 26 & 82 & 6 \\
\hline 26 & 83 & 10 \\
\hline 26 & 84 & 6 \\
\hline 26 & 85 & 6 \\
\hline 26 & 86 & 14 \\
\hline 26 & 87 & 13 \\
\hline 26 & 88 & 7 \\
\hline 26 & 89 & 6 \\
\hline 26 & 90 & 5 \\
\hline 26 & 91 & 4 \\
\hline 26 & 92 & 12 \\
\hline 26 & 93 & 11 \\
\hline 26 & 94 & 27 \\
\hline 26 & 95 & 29 \\
\hline 26 & 96 & 33 \\
\hline 26 & 97 & 18 \\
\hline 26 & 98 & 36 \\
\hline 26 & 99 & 23 \\
\hline 26 & 100 & 21 \\
\hline 26 & 101 & 9 \\
\hline 26 & 102 & 4 \\
\hline 26 & 103 & 7 \\
\hline 26 & 104 & 4 \\
\hline 26 & 105 & 19 \\
\hline 26 & 106 & 16 \\
\hline 26 & 107 & 11 \\
\hline 26 & 108 & 88 \\
\hline 26 & 109 & 51 \\
\hline 26 & 110 & 150 \\
\hline 26 & 111 & 50 \\
\hline 26 & 112 & 67 \\
\hline
\end{tabular}




\begin{tabular}{|c|c|c|}
\hline 26 & 113 & 12 \\
\hline 26 & 114 & 65 \\
\hline 26 & 115 & 46 \\
\hline 26 & 116 & 29 \\
\hline 26 & 117 & 43 \\
\hline 26 & 118 & 19 \\
\hline 26 & 119 & 64 \\
\hline 26 & 120 & 40 \\
\hline 26 & 121 & 31 \\
\hline 26 & 122 & 19 \\
\hline 26 & 123 & 15 \\
\hline 26 & 124 & 13 \\
\hline 26 & 125 & 21 \\
\hline 26 & 126 & 25 \\
\hline 26 & 127 & 29 \\
\hline 26 & 128 & 20 \\
\hline 26 & 129 & 19 \\
\hline 26 & 130 & 21 \\
\hline 26 & 131 & 16 \\
\hline 26 & 132 & 8 \\
\hline 26 & 133 & 29 \\
\hline 26 & 134 & 16 \\
\hline 26 & 135 & 27 \\
\hline 26 & 136 & 5 \\
\hline 26 & 137 & 13 \\
\hline 26 & 138 & 6 \\
\hline 26 & 139 & 6 \\
\hline 26 & 140 & 6 \\
\hline 26 & 141 & 13 \\
\hline 26 & 142 & 7 \\
\hline 26 & 143 & 10 \\
\hline 26 & 144 & 15 \\
\hline 26 & 145 & 18 \\
\hline 26 & 146 & 21 \\
\hline 26 & 147 & 14 \\
\hline 26 & 148 & 33 \\
\hline 26 & 149 & 29 \\
\hline 26 & 150 & 25 \\
\hline 26 & 151 & 48 \\
\hline 26 & 152 & 25 \\
\hline 26 & 153 & 71 \\
\hline 26 & 154 & 13 \\
\hline 26 & 155 & 10 \\
\hline 26 & 156 & 13 \\
\hline 26 & 157 & 14 \\
\hline
\end{tabular}




\begin{tabular}{|c|c|c|}
\hline 26 & 158 & 15 \\
\hline 26 & 159 & 12 \\
\hline 26 & 160 & 3 \\
\hline 26 & 161 & 2 \\
\hline 26 & 162 & 10 \\
\hline 26 & 163 & 11 \\
\hline 26 & 164 & 15 \\
\hline 26 & 165 & 2 \\
\hline 26 & 166 & 2 \\
\hline 26 & 167 & 8 \\
\hline 26 & 168 & 9 \\
\hline 26 & 169 & 16 \\
\hline 26 & 170 & 10 \\
\hline 27 & 1 & 177 \\
\hline 27 & 2 & 158 \\
\hline 27 & 3 & 122 \\
\hline 27 & 4 & 407 \\
\hline 27 & 5 & 72 \\
\hline 27 & 6 & 19 \\
\hline 27 & 7 & 73 \\
\hline 27 & 8 & 132 \\
\hline 27 & 9 & 131 \\
\hline 27 & 10 & 116 \\
\hline 27 & 11 & 39 \\
\hline 27 & 12 & 33 \\
\hline 27 & 13 & 24 \\
\hline 27 & 14 & 148 \\
\hline 27 & 15 & 84 \\
\hline 27 & 16 & 1179 \\
\hline 27 & 17 & 2576 \\
\hline 27 & 18 & 341 \\
\hline 27 & 19 & 397 \\
\hline 27 & 20 & 201 \\
\hline 27 & 21 & 296 \\
\hline 27 & 22 & 23 \\
\hline 27 & 23 & 125 \\
\hline 27 & 24 & 97 \\
\hline 27 & 25 & 64 \\
\hline 27 & 26 & 70 \\
\hline 27 & 27 & 32 \\
\hline 27 & 28 & 44 \\
\hline 27 & 29 & 29 \\
\hline 27 & 30 & 53 \\
\hline 27 & 31 & 61 \\
\hline 27 & 32 & 21 \\
\hline
\end{tabular}




\begin{tabular}{|c|c|c|}
\hline 27 & 33 & 23 \\
\hline 27 & 34 & 65 \\
\hline 27 & 35 & 52 \\
\hline 27 & 36 & 42 \\
\hline 27 & 37 & 27 \\
\hline 27 & 38 & 35 \\
\hline 27 & 39 & 87 \\
\hline 27 & 40 & 20 \\
\hline 27 & 41 & 16 \\
\hline 27 & 42 & 12 \\
\hline 27 & 43 & 11 \\
\hline 27 & 44 & 36 \\
\hline 27 & 45 & 24 \\
\hline 27 & 46 & 7 \\
\hline 27 & 47 & 9 \\
\hline 27 & 48 & 6 \\
\hline 27 & 49 & 7 \\
\hline 27 & 50 & 8 \\
\hline 27 & 51 & 5 \\
\hline 27 & 52 & 14 \\
\hline 27 & 53 & 25 \\
\hline 27 & 54 & 15 \\
\hline 27 & 55 & 11 \\
\hline 27 & 56 & 10 \\
\hline 27 & 57 & 9 \\
\hline 27 & 58 & 9 \\
\hline 27 & 59 & 8 \\
\hline 27 & 60 & 8 \\
\hline 27 & 61 & 12 \\
\hline 27 & 62 & 15 \\
\hline 27 & 63 & 15 \\
\hline 27 & 64 & 14 \\
\hline 27 & 65 & 12 \\
\hline 27 & 66 & 14 \\
\hline 27 & 67 & 11 \\
\hline 27 & 68 & 8 \\
\hline 27 & 69 & 29 \\
\hline 27 & 70 & 33 \\
\hline 27 & 71 & 10 \\
\hline 27 & 72 & 5 \\
\hline 27 & 73 & 6 \\
\hline 27 & 74 & 6 \\
\hline 27 & 75 & 4 \\
\hline 27 & 76 & 6 \\
\hline 27 & 77 & 6 \\
\hline
\end{tabular}




\begin{tabular}{|c|c|c|}
\hline 27 & 78 & 6 \\
\hline 27 & 79 & 5 \\
\hline 27 & 80 & 5 \\
\hline 27 & 81 & 5 \\
\hline 27 & 82 & 5 \\
\hline 27 & 83 & 7 \\
\hline 27 & 84 & 5 \\
\hline 27 & 85 & 5 \\
\hline 27 & 86 & 10 \\
\hline 27 & 87 & 10 \\
\hline 27 & 88 & 5 \\
\hline 27 & 89 & 5 \\
\hline 27 & 90 & 4 \\
\hline 27 & 91 & 3 \\
\hline 27 & 92 & 10 \\
\hline 27 & 93 & 9 \\
\hline 27 & 94 & 20 \\
\hline 27 & 95 & 21 \\
\hline 27 & 96 & 34 \\
\hline 27 & 97 & 22 \\
\hline 27 & 98 & 26 \\
\hline 27 & 99 & 18 \\
\hline 27 & 100 & 16 \\
\hline 27 & 101 & 7 \\
\hline 27 & 102 & 4 \\
\hline 27 & 103 & 6 \\
\hline 27 & 104 & 3 \\
\hline 27 & 105 & 15 \\
\hline 27 & 106 & 13 \\
\hline 27 & 107 & 9 \\
\hline 27 & 108 & 17 \\
\hline 27 & 109 & 16 \\
\hline 27 & 110 & 35 \\
\hline 27 & 111 & 19 \\
\hline 27 & 112 & 23 \\
\hline 27 & 113 & 10 \\
\hline 27 & 114 & 27 \\
\hline 27 & 115 & 22 \\
\hline 27 & 116 & 7 \\
\hline 27 & 117 & 19 \\
\hline 27 & 118 & 6 \\
\hline 27 & 119 & 22 \\
\hline 27 & 120 & 16 \\
\hline 27 & 121 & 15 \\
\hline 27 & 122 & 5 \\
\hline
\end{tabular}




\begin{tabular}{|c|c|c|}
\hline 27 & 123 & 5 \\
\hline 27 & 124 & 8 \\
\hline 27 & 125 & 10 \\
\hline 27 & 126 & 12 \\
\hline 27 & 127 & 16 \\
\hline 27 & 128 & 9 \\
\hline 27 & 129 & 11 \\
\hline 27 & 130 & 11 \\
\hline 27 & 131 & 7 \\
\hline 27 & 132 & 7 \\
\hline 27 & 133 & 16 \\
\hline 27 & 134 & 12 \\
\hline 27 & 135 & 6 \\
\hline 27 & 136 & 8 \\
\hline 27 & 137 & 9 \\
\hline 27 & 138 & 4 \\
\hline 27 & 139 & 8 \\
\hline 27 & 140 & 9 \\
\hline 27 & 141 & 8 \\
\hline 27 & 142 & 5 \\
\hline 27 & 143 & 7 \\
\hline 27 & 144 & 12 \\
\hline 27 & 145 & 17 \\
\hline 27 & 146 & 8 \\
\hline 27 & 147 & 16 \\
\hline 27 & 148 & 31 \\
\hline 27 & 149 & 31 \\
\hline 27 & 150 & 25 \\
\hline 27 & 151 & 57 \\
\hline 27 & 152 & 25 \\
\hline 27 & 153 & 71 \\
\hline 27 & 154 & 14 \\
\hline 27 & 155 & 23 \\
\hline 27 & 156 & 14 \\
\hline 27 & 157 & 15 \\
\hline 27 & 158 & 16 \\
\hline 27 & 159 & 8 \\
\hline 27 & 160 & 4 \\
\hline 27 & 161 & 3 \\
\hline 27 & 162 & 12 \\
\hline 27 & 163 & 12 \\
\hline 27 & 164 & 16 \\
\hline 27 & 165 & 2 \\
\hline 27 & 166 & 3 \\
\hline 27 & 167 & 13 \\
\hline
\end{tabular}




\begin{tabular}{|c|c|c|}
\hline 27 & 168 & 10 \\
\hline 27 & 169 & 19 \\
\hline 27 & 170 & 11 \\
\hline 28 & 1 & 20 \\
\hline 28 & 2 & 26 \\
\hline 28 & 3 & 34 \\
\hline 28 & 4 & 17 \\
\hline 28 & 5 & 39 \\
\hline 28 & 6 & 64 \\
\hline 28 & 7 & 28 \\
\hline 28 & 8 & 31 \\
\hline 28 & 9 & 72 \\
\hline 28 & 10 & 68 \\
\hline 28 & 11 & 89 \\
\hline 28 & 12 & 195 \\
\hline 28 & 13 & 214 \\
\hline 28 & 14 & 16 \\
\hline 28 & 15 & 36 \\
\hline 28 & 16 & 25 \\
\hline 28 & 17 & 20 \\
\hline 28 & 18 & 14 \\
\hline 28 & 19 & 17 \\
\hline 28 & 20 & 12 \\
\hline 28 & 21 & 14 \\
\hline 28 & 22 & 7 \\
\hline 28 & 23 & 12 \\
\hline 28 & 24 & 11 \\
\hline 28 & 25 & 10 \\
\hline 28 & 26 & 20 \\
\hline 28 & 27 & 12 \\
\hline 28 & 28 & 19 \\
\hline 28 & 29 & 14 \\
\hline 28 & 30 & 25 \\
\hline 28 & 31 & 26 \\
\hline 28 & 32 & 2 \\
\hline 28 & 33 & 2 \\
\hline 28 & 34 & 10 \\
\hline 28 & 35 & 9 \\
\hline 28 & 36 & 8 \\
\hline 28 & 37 & 3 \\
\hline 28 & 38 & 8 \\
\hline 28 & 39 & 11 \\
\hline 28 & 40 & 3 \\
\hline 28 & 41 & 3 \\
\hline 28 & 42 & 2 \\
\hline
\end{tabular}




\begin{tabular}{|c|c|c|}
\hline 28 & 43 & 3 \\
\hline 28 & 44 & 3 \\
\hline 28 & 45 & 4 \\
\hline 28 & 46 & 2 \\
\hline 28 & 47 & 3 \\
\hline 28 & 48 & 2 \\
\hline 28 & 49 & 2 \\
\hline 28 & 50 & 2 \\
\hline 28 & 51 & 2 \\
\hline 28 & 52 & 4 \\
\hline 28 & 53 & 5 \\
\hline 28 & 54 & 4 \\
\hline 28 & 55 & 3 \\
\hline 28 & 56 & 3 \\
\hline 28 & 57 & 4 \\
\hline 28 & 58 & 4 \\
\hline 28 & 59 & 4 \\
\hline 28 & 60 & 5 \\
\hline 28 & 61 & 3 \\
\hline 28 & 62 & 6 \\
\hline 28 & 63 & 6 \\
\hline 28 & 64 & 5 \\
\hline 28 & 65 & 5 \\
\hline 28 & 66 & 7 \\
\hline 28 & 67 & 9 \\
\hline 28 & 68 & 6 \\
\hline 28 & 69 & 8 \\
\hline 28 & 70 & 9 \\
\hline 28 & 71 & 8 \\
\hline 28 & 72 & 2 \\
\hline 28 & 73 & 3 \\
\hline 28 & 74 & 2 \\
\hline 28 & 75 & 4 \\
\hline 28 & 76 & 5 \\
\hline 28 & 77 & 5 \\
\hline 28 & 78 & 5 \\
\hline 28 & 79 & 4 \\
\hline 28 & 80 & 4 \\
\hline 28 & 81 & 4 \\
\hline 28 & 82 & 4 \\
\hline 28 & 83 & 6 \\
\hline 28 & 84 & 4 \\
\hline 28 & 85 & 4 \\
\hline 28 & 86 & 8 \\
\hline 28 & 87 & 7 \\
\hline
\end{tabular}




\begin{tabular}{|c|c|c|}
\hline 28 & 88 & 5 \\
\hline 28 & 89 & 4 \\
\hline 28 & 90 & 3 \\
\hline 28 & 91 & 2 \\
\hline 28 & 92 & 7 \\
\hline 28 & 93 & 6 \\
\hline 28 & 94 & 12 \\
\hline 28 & 95 & 13 \\
\hline 28 & 96 & 14 \\
\hline 28 & 97 & 9 \\
\hline 28 & 98 & 15 \\
\hline 28 & 99 & 11 \\
\hline 28 & 100 & 10 \\
\hline 28 & 101 & 5 \\
\hline 28 & 102 & 3 \\
\hline 28 & 103 & 4 \\
\hline 28 & 104 & 3 \\
\hline 28 & 105 & 9 \\
\hline 28 & 106 & 9 \\
\hline 28 & 107 & 6 \\
\hline 28 & 108 & 49 \\
\hline 28 & 109 & 94 \\
\hline 28 & 110 & 27 \\
\hline 28 & 111 & 48 \\
\hline 28 & 112 & 42 \\
\hline 28 & 113 & 7 \\
\hline 28 & 114 & 16 \\
\hline 28 & 115 & 14 \\
\hline 28 & 116 & 116 \\
\hline 28 & 117 & 304 \\
\hline 28 & 118 & 58 \\
\hline 28 & 119 & 18 \\
\hline 28 & 120 & 22 \\
\hline 28 & 121 & 10 \\
\hline 28 & 122 & 38 \\
\hline 28 & 123 & 27 \\
\hline 28 & 124 & 9 \\
\hline 28 & 125 & 18 \\
\hline 28 & 126 & 10 \\
\hline 28 & 127 & 11 \\
\hline 28 & 128 & 7 \\
\hline 28 & 129 & 8 \\
\hline 28 & 130 & 14 \\
\hline 28 & 131 & 5 \\
\hline 28 & 132 & 5 \\
\hline
\end{tabular}




\begin{tabular}{|c|c|c|}
\hline 28 & 133 & 11 \\
\hline 28 & 134 & 8 \\
\hline 28 & 135 & 47 \\
\hline 28 & 136 & 11 \\
\hline 28 & 137 & 48 \\
\hline 28 & 138 & 12 \\
\hline 28 & 139 & 14 \\
\hline 28 & 140 & 14 \\
\hline 28 & 141 & 45 \\
\hline 28 & 142 & 18 \\
\hline 28 & 143 & 31 \\
\hline 28 & 144 & 60 \\
\hline 28 & 145 & 146 \\
\hline 28 & 146 & 164 \\
\hline 28 & 147 & 19 \\
\hline 28 & 148 & 17 \\
\hline 28 & 149 & 24 \\
\hline 28 & 150 & 25 \\
\hline 28 & 151 & 38 \\
\hline 28 & 152 & 29 \\
\hline 28 & 153 & 53 \\
\hline 28 & 154 & 17 \\
\hline 28 & 155 & 31 \\
\hline 28 & 156 & 17 \\
\hline 28 & 157 & 18 \\
\hline 28 & 158 & 20 \\
\hline 28 & 159 & 10 \\
\hline 28 & 160 & 5 \\
\hline 28 & 161 & 3 \\
\hline 28 & 162 & 5 \\
\hline 28 & 163 & 6 \\
\hline 28 & 164 & 13 \\
\hline 28 & 165 & 3 \\
\hline 28 & 166 & 4 \\
\hline 28 & 167 & 3 \\
\hline 28 & 168 & 3 \\
\hline 28 & 169 & 9 \\
\hline 28 & 170 & 13 \\
\hline 29 & 1 & 556 \\
\hline 29 & 2 & 477 \\
\hline 29 & 3 & 2705 \\
\hline 29 & 4 & 261 \\
\hline 29 & 5 & 666 \\
\hline 29 & 6 & 25 \\
\hline 29 & 7 & 248 \\
\hline
\end{tabular}




\begin{tabular}{|c|c|c|}
\hline 29 & 8 & 240 \\
\hline 29 & 9 & 170 \\
\hline 29 & 10 & 81 \\
\hline 29 & 11 & 37 \\
\hline 29 & 12 & 26 \\
\hline 29 & 13 & 19 \\
\hline 29 & 14 & 48 \\
\hline 29 & 15 & 77 \\
\hline 29 & 16 & 158 \\
\hline 29 & 17 & 114 \\
\hline 29 & 18 & 147 \\
\hline 29 & 19 & 50 \\
\hline 29 & 20 & 54 \\
\hline 29 & 21 & 128 \\
\hline 29 & 22 & 18 \\
\hline 29 & 23 & 253 \\
\hline 29 & 24 & 105 \\
\hline 29 & 25 & 68 \\
\hline 29 & 26 & 229 \\
\hline 29 & 27 & 48 \\
\hline 29 & 28 & 153 \\
\hline 29 & 29 & 67 \\
\hline 29 & 30 & 400 \\
\hline 29 & 31 & 594 \\
\hline 29 & 32 & 4 \\
\hline 29 & 33 & 3 \\
\hline 29 & 34 & 22 \\
\hline 29 & 35 & 24 \\
\hline 29 & 36 & 21 \\
\hline 29 & 37 & 15 \\
\hline 29 & 38 & 15 \\
\hline 29 & 39 & 25 \\
\hline 29 & 40 & 6 \\
\hline 29 & 41 & 5 \\
\hline 29 & 42 & 4 \\
\hline 29 & 43 & 9 \\
\hline 29 & 44 & 19 \\
\hline 29 & 45 & 14 \\
\hline 29 & 46 & 6 \\
\hline 29 & 47 & 8 \\
\hline 29 & 48 & 5 \\
\hline 29 & 49 & 6 \\
\hline 29 & 50 & 3 \\
\hline 29 & 51 & 4 \\
\hline 29 & 52 & 11 \\
\hline
\end{tabular}




\begin{tabular}{|c|c|c|}
\hline 29 & 53 & 11 \\
\hline 29 & 54 & 11 \\
\hline 29 & 55 & 9 \\
\hline 29 & 56 & 8 \\
\hline 29 & 57 & 7 \\
\hline 29 & 58 & 8 \\
\hline 29 & 59 & 7 \\
\hline 29 & 60 & 10 \\
\hline 29 & 61 & 9 \\
\hline 29 & 62 & 13 \\
\hline 29 & 63 & 16 \\
\hline 29 & 64 & 11 \\
\hline 29 & 65 & 12 \\
\hline 29 & 66 & 18 \\
\hline 29 & 67 & 25 \\
\hline 29 & 68 & 15 \\
\hline 29 & 69 & 31 \\
\hline 29 & 70 & 27 \\
\hline 29 & 71 & 23 \\
\hline 29 & 72 & 4 \\
\hline 29 & 73 & 5 \\
\hline 29 & 74 & 4 \\
\hline 29 & 75 & 7 \\
\hline 29 & 76 & 11 \\
\hline 29 & 77 & 9 \\
\hline 29 & 78 & 12 \\
\hline 29 & 79 & 9 \\
\hline 29 & 80 & 8 \\
\hline 29 & 81 & 8 \\
\hline 29 & 82 & 8 \\
\hline 29 & 83 & 16 \\
\hline 29 & 84 & 13 \\
\hline 29 & 85 & 8 \\
\hline 29 & 86 & 21 \\
\hline 29 & 87 & 20 \\
\hline 29 & 88 & 9 \\
\hline 29 & 89 & 8 \\
\hline 29 & 90 & 6 \\
\hline 29 & 91 & 3 \\
\hline 29 & 92 & 19 \\
\hline 29 & 93 & 17 \\
\hline 29 & 94 & 53 \\
\hline 29 & 95 & 60 \\
\hline 29 & 96 & 98 \\
\hline 29 & 97 & 32 \\
\hline
\end{tabular}




\begin{tabular}{|c|c|c|}
\hline 29 & 98 & 83 \\
\hline 29 & 99 & 44 \\
\hline 29 & 100 & 37 \\
\hline 29 & 101 & 12 \\
\hline 29 & 102 & 5 \\
\hline 29 & 103 & 10 \\
\hline 29 & 104 & 5 \\
\hline 29 & 105 & 33 \\
\hline 29 & 106 & 27 \\
\hline 29 & 107 & 17 \\
\hline 29 & 108 & 79 \\
\hline 29 & 109 & 47 \\
\hline 29 & 110 & 130 \\
\hline 29 & 111 & 46 \\
\hline 29 & 112 & 61 \\
\hline 29 & 113 & 18 \\
\hline 29 & 114 & 96 \\
\hline 29 & 115 & 63 \\
\hline 29 & 116 & 15 \\
\hline 29 & 117 & 31 \\
\hline 29 & 118 & 11 \\
\hline 29 & 119 & 58 \\
\hline 29 & 120 & 39 \\
\hline 29 & 121 & 36 \\
\hline 29 & 122 & 18 \\
\hline 29 & 123 & 14 \\
\hline 29 & 124 & 13 \\
\hline 29 & 125 & 20 \\
\hline 29 & 126 & 25 \\
\hline 29 & 127 & 38 \\
\hline 29 & 128 & 18 \\
\hline 29 & 129 & 23 \\
\hline 29 & 130 & 21 \\
\hline 29 & 131 & 11 \\
\hline 29 & 132 & 12 \\
\hline 29 & 133 & 38 \\
\hline 29 & 134 & 26 \\
\hline 29 & 135 & 25 \\
\hline 29 & 136 & 5 \\
\hline 29 & 137 & 11 \\
\hline 29 & 138 & 5 \\
\hline 29 & 139 & 6 \\
\hline 29 & 140 & 6 \\
\hline 29 & 141 & 11 \\
\hline 29 & 142 & 6 \\
\hline
\end{tabular}




\begin{tabular}{|c|c|c|}
\hline 29 & 143 & 9 \\
\hline 29 & 144 & 12 \\
\hline 29 & 145 & 14 \\
\hline 29 & 146 & 17 \\
\hline 29 & 147 & 7 \\
\hline 29 & 148 & 22 \\
\hline 29 & 149 & 20 \\
\hline 29 & 150 & 18 \\
\hline 29 & 151 & 30 \\
\hline 29 & 152 & 18 \\
\hline 29 & 153 & 41 \\
\hline 29 & 154 & 6 \\
\hline 29 & 155 & 9 \\
\hline 29 & 156 & 6 \\
\hline 29 & 157 & 6 \\
\hline 29 & 158 & 7 \\
\hline 29 & 159 & 4 \\
\hline 29 & 160 & 3 \\
\hline 29 & 161 & 2 \\
\hline 29 & 162 & 8 \\
\hline 29 & 163 & 3 \\
\hline 29 & 164 & 11 \\
\hline 29 & 165 & 2 \\
\hline 29 & 166 & 2 \\
\hline 29 & 167 & 7 \\
\hline 29 & 168 & 7 \\
\hline 29 & 169 & 12 \\
\hline 29 & 170 & 5 \\
\hline 30 & 1 & 13 \\
\hline 30 & 2 & 10 \\
\hline 30 & 3 & 11 \\
\hline 30 & 4 & 15 \\
\hline 30 & 5 & 8 \\
\hline 30 & 6 & 4 \\
\hline 30 & 7 & 8 \\
\hline 30 & 8 & 9 \\
\hline 30 & 9 & 9 \\
\hline 30 & 10 & 8 \\
\hline 30 & 11 & 6 \\
\hline 30 & 12 & 4 \\
\hline 30 & 13 & 3 \\
\hline 30 & 14 & 9 \\
\hline 30 & 15 & 9 \\
\hline 30 & 16 & 12 \\
\hline 30 & 17 & 12 \\
\hline
\end{tabular}




\begin{tabular}{|c|c|c|}
\hline 30 & 18 & 16 \\
\hline 30 & 19 & 13 \\
\hline 30 & 20 & 15 \\
\hline 30 & 21 & 20 \\
\hline 30 & 22 & 39 \\
\hline 30 & 23 & 15 \\
\hline 30 & 24 & 17 \\
\hline 30 & 25 & 21 \\
\hline 30 & 26 & 13 \\
\hline 30 & 27 & 19 \\
\hline 30 & 28 & 9 \\
\hline 30 & 29 & 13 \\
\hline 30 & 30 & 7 \\
\hline 30 & 31 & 6 \\
\hline 30 & 32 & 11 \\
\hline 30 & 33 & 9 \\
\hline 30 & 34 & 12 \\
\hline 30 & 35 & 17 \\
\hline 30 & 36 & 15 \\
\hline 30 & 37 & 28 \\
\hline 30 & 38 & 7 \\
\hline 30 & 39 & 9 \\
\hline 30 & 40 & 36 \\
\hline 30 & 41 & 78 \\
\hline 30 & 42 & 119 \\
\hline 30 & 43 & 377 \\
\hline 30 & 44 & 46 \\
\hline 30 & 45 & 57 \\
\hline 30 & 46 & 78 \\
\hline 30 & 47 & 37 \\
\hline 30 & 48 & 31 \\
\hline 30 & 49 & 34 \\
\hline 30 & 50 & 39 \\
\hline 30 & 51 & 30 \\
\hline 30 & 52 & 623 \\
\hline 30 & 53 & 119 \\
\hline 30 & 54 & 203 \\
\hline 30 & 55 & 457 \\
\hline 30 & 56 & 24 \\
\hline 30 & 57 & 21 \\
\hline 30 & 58 & 21 \\
\hline 30 & 59 & 16 \\
\hline 30 & 60 & 24 \\
\hline 30 & 61 & 48 \\
\hline 30 & 62 & 78 \\
\hline
\end{tabular}




\begin{tabular}{|c|c|c|}
\hline 30 & 63 & 50 \\
\hline 30 & 64 & 42 \\
\hline 30 & 65 & 40 \\
\hline 30 & 66 & 45 \\
\hline 30 & 67 & 13 \\
\hline 30 & 68 & 29 \\
\hline 30 & 69 & 27 \\
\hline 30 & 70 & 29 \\
\hline 30 & 71 & 26 \\
\hline 30 & 72 & 18 \\
\hline 30 & 73 & 15 \\
\hline 30 & 74 & 12 \\
\hline 30 & 75 & 5 \\
\hline 30 & 76 & 15 \\
\hline 30 & 77 & 13 \\
\hline 30 & 78 & 16 \\
\hline 30 & 79 & 16 \\
\hline 30 & 80 & 5 \\
\hline 30 & 81 & 6 \\
\hline 30 & 82 & 6 \\
\hline 30 & 83 & 8 \\
\hline 30 & 84 & 6 \\
\hline 30 & 85 & 6 \\
\hline 30 & 86 & 12 \\
\hline 30 & 87 & 11 \\
\hline 30 & 88 & 13 \\
\hline 30 & 89 & 11 \\
\hline 30 & 90 & 8 \\
\hline 30 & 91 & 3 \\
\hline 30 & 92 & 7 \\
\hline 30 & 93 & 6 \\
\hline 30 & 94 & 5 \\
\hline 30 & 95 & 5 \\
\hline 30 & 96 & 9 \\
\hline 30 & 97 & 7 \\
\hline 30 & 98 & 5 \\
\hline 30 & 99 & 6 \\
\hline 30 & 100 & 6 \\
\hline 30 & 101 & 4 \\
\hline 30 & 102 & 4 \\
\hline 30 & 103 & 2 \\
\hline 30 & 104 & 3 \\
\hline 30 & 105 & 5 \\
\hline 30 & 106 & 5 \\
\hline 30 & 107 & 3 \\
\hline
\end{tabular}




\begin{tabular}{|c|c|c|}
\hline 30 & 108 & 4 \\
\hline 30 & 109 & 4 \\
\hline 30 & 110 & 5 \\
\hline 30 & 111 & 4 \\
\hline 30 & 112 & 4 \\
\hline 30 & 113 & 3 \\
\hline 30 & 114 & 5 \\
\hline 30 & 115 & 4 \\
\hline 30 & 116 & 3 \\
\hline 30 & 117 & 4 \\
\hline 30 & 118 & 3 \\
\hline 30 & 119 & 4 \\
\hline 30 & 120 & 3 \\
\hline 30 & 121 & 3 \\
\hline 30 & 122 & 3 \\
\hline 30 & 123 & 2 \\
\hline 30 & 124 & 3 \\
\hline 30 & 125 & 3 \\
\hline 30 & 126 & 3 \\
\hline 30 & 127 & 4 \\
\hline 30 & 128 & 3 \\
\hline 30 & 129 & 3 \\
\hline 30 & 130 & 3 \\
\hline 30 & 131 & 3 \\
\hline 30 & 132 & 3 \\
\hline 30 & 133 & 4 \\
\hline 30 & 134 & 4 \\
\hline 30 & 135 & 3 \\
\hline 30 & 136 & 2 \\
\hline 30 & 137 & 3 \\
\hline 30 & 138 & 2 \\
\hline 30 & 139 & 2 \\
\hline 30 & 140 & 2 \\
\hline 30 & 141 & 3 \\
\hline 30 & 142 & 2 \\
\hline 30 & 143 & 2 \\
\hline 30 & 144 & 3 \\
\hline 30 & 145 & 3 \\
\hline 30 & 146 & 3 \\
\hline 30 & 147 & 4 \\
\hline 30 & 148 & 7 \\
\hline 30 & 149 & 3 \\
\hline 30 & 150 & 2 \\
\hline 30 & 151 & 6 \\
\hline 30 & 152 & 2 \\
\hline
\end{tabular}




\begin{tabular}{|c|c|c|}
\hline 30 & 153 & 7 \\
\hline 30 & 154 & 4 \\
\hline 30 & 155 & 2 \\
\hline 30 & 156 & 4 \\
\hline 30 & 157 & 4 \\
\hline 30 & 158 & 4 \\
\hline 30 & 159 & 2 \\
\hline 30 & 160 & 3 \\
\hline 30 & 161 & 4 \\
\hline 30 & 162 & 4 \\
\hline 30 & 163 & 4 \\
\hline 30 & 164 & 3 \\
\hline 30 & 165 & 3 \\
\hline 30 & 166 & 4 \\
\hline 30 & 167 & 4 \\
\hline 30 & 168 & 4 \\
\hline 30 & 169 & 3 \\
\hline 30 & 170 & 2 \\
\hline 31 & 1 & 7 \\
\hline 31 & 2 & 6 \\
\hline 31 & 3 & 7 \\
\hline 31 & 4 & 6 \\
\hline 31 & 5 & 6 \\
\hline 31 & 6 & 4 \\
\hline 31 & 7 & 5 \\
\hline 31 & 8 & 6 \\
\hline 31 & 9 & 5 \\
\hline 31 & 10 & 4 \\
\hline 31 & 11 & 3 \\
\hline 31 & 12 & 4 \\
\hline 31 & 13 & 4 \\
\hline 31 & 14 & 4 \\
\hline 31 & 15 & 5 \\
\hline 31 & 16 & 5 \\
\hline 31 & 17 & 5 \\
\hline 31 & 18 & 6 \\
\hline 31 & 19 & 4 \\
\hline 31 & 20 & 5 \\
\hline 31 & 21 & 7 \\
\hline 31 & 22 & 11 \\
\hline 31 & 23 & 11 \\
\hline 31 & 24 & 8 \\
\hline 31 & 25 & 13 \\
\hline 31 & 26 & 13 \\
\hline 31 & 27 & 17 \\
\hline
\end{tabular}




\begin{tabular}{|c|c|c|}
\hline 31 & 28 & 12 \\
\hline 31 & 29 & 18 \\
\hline 31 & 30 & 9 \\
\hline 31 & 31 & 8 \\
\hline 31 & 32 & 3 \\
\hline 31 & 33 & 3 \\
\hline 31 & 34 & 3 \\
\hline 31 & 35 & 3 \\
\hline 31 & 36 & 3 \\
\hline 31 & 37 & 4 \\
\hline 31 & 38 & 2 \\
\hline 31 & 39 & 3 \\
\hline 31 & 40 & 4 \\
\hline 31 & 41 & 4 \\
\hline 31 & 42 & 8 \\
\hline 31 & 43 & 9 \\
\hline 31 & 44 & 4 \\
\hline 31 & 45 & 5 \\
\hline 31 & 46 & 12 \\
\hline 31 & 47 & 18 \\
\hline 31 & 48 & 9 \\
\hline 31 & 49 & 13 \\
\hline 31 & 50 & 7 \\
\hline 31 & 51 & 9 \\
\hline 31 & 52 & 11 \\
\hline 31 & 53 & 7 \\
\hline 31 & 54 & 12 \\
\hline 31 & 55 & 9 \\
\hline 31 & 56 & 20 \\
\hline 31 & 57 & 34 \\
\hline 31 & 58 & 38 \\
\hline 31 & 59 & 29 \\
\hline 31 & 60 & 76 \\
\hline 31 & 61 & 17 \\
\hline 31 & 62 & 18 \\
\hline 31 & 63 & 26 \\
\hline 31 & 64 & 38 \\
\hline 31 & 65 & 44 \\
\hline 31 & 66 & 26 \\
\hline 31 & 67 & 22 \\
\hline 31 & 68 & 73 \\
\hline 31 & 69 & 16 \\
\hline 31 & 70 & 16 \\
\hline 31 & 71 & 24 \\
\hline 31 & 72 & 12 \\
\hline
\end{tabular}




\begin{tabular}{|c|c|c|}
\hline 31 & 73 & 14 \\
\hline 31 & 74 & 15 \\
\hline 31 & 75 & 64 \\
\hline 31 & 76 & 196 \\
\hline 31 & 77 & 43 \\
\hline 31 & 78 & 109 \\
\hline 31 & 79 & 179 \\
\hline 31 & 80 & 91 \\
\hline 31 & 81 & 54 \\
\hline 31 & 82 & 54 \\
\hline 31 & 83 & 22 \\
\hline 31 & 84 & 51 \\
\hline 31 & 85 & 28 \\
\hline 31 & 86 & 27 \\
\hline 31 & 87 & 15 \\
\hline 31 & 88 & 6747 \\
\hline 31 & 89 & 4358 \\
\hline 31 & 90 & 230 \\
\hline 31 & 91 & 89 \\
\hline 31 & 92 & 8 \\
\hline 31 & 93 & 16 \\
\hline 31 & 94 & 6 \\
\hline 31 & 95 & 6 \\
\hline 31 & 96 & 13 \\
\hline 31 & 97 & 9 \\
\hline 31 & 98 & 6 \\
\hline 31 & 99 & 7 \\
\hline 31 & 100 & 8 \\
\hline 31 & 101 & 17 \\
\hline 31 & 102 & 12 \\
\hline 31 & 103 & 3 \\
\hline 31 & 104 & 7 \\
\hline 31 & 105 & 6 \\
\hline 31 & 106 & 10 \\
\hline 31 & 107 & 9 \\
\hline 31 & 108 & 6 \\
\hline 31 & 109 & 4 \\
\hline 31 & 110 & 6 \\
\hline 31 & 111 & 5 \\
\hline 31 & 112 & 5 \\
\hline 31 & 113 & 4 \\
\hline 31 & 114 & 6 \\
\hline 31 & 115 & 5 \\
\hline 31 & 116 & 3 \\
\hline 31 & 117 & 5 \\
\hline
\end{tabular}




\begin{tabular}{|c|c|c|}
\hline 31 & 118 & 3 \\
\hline 31 & 119 & 5 \\
\hline 31 & 120 & 3 \\
\hline 31 & 121 & 3 \\
\hline 31 & 122 & 3 \\
\hline 31 & 123 & 2 \\
\hline 31 & 124 & 3 \\
\hline 31 & 125 & 3 \\
\hline 31 & 126 & 3 \\
\hline 31 & 127 & 4 \\
\hline 31 & 128 & 4 \\
\hline 31 & 129 & 3 \\
\hline 31 & 130 & 3 \\
\hline 31 & 131 & 3 \\
\hline 31 & 132 & 3 \\
\hline 31 & 133 & 5 \\
\hline 31 & 134 & 4 \\
\hline 31 & 135 & 4 \\
\hline 31 & 136 & 2 \\
\hline 31 & 137 & 3 \\
\hline 31 & 138 & 2 \\
\hline 31 & 139 & 2 \\
\hline 31 & 140 & 2 \\
\hline 31 & 141 & 3 \\
\hline 31 & 142 & 2 \\
\hline 31 & 143 & 3 \\
\hline 31 & 144 & 3 \\
\hline 31 & 145 & 3 \\
\hline 31 & 146 & 4 \\
\hline 31 & 147 & 2 \\
\hline 31 & 148 & 1 \\
\hline 31 & 149 & 3 \\
\hline 31 & 150 & 3 \\
\hline 31 & 151 & 3 \\
\hline 31 & 152 & 3 \\
\hline 31 & 153 & 4 \\
\hline 31 & 154 & 2 \\
\hline 31 & 155 & 3 \\
\hline 31 & 156 & 2 \\
\hline 31 & 157 & 2 \\
\hline 31 & 158 & 2 \\
\hline 31 & 159 & 1 \\
\hline 31 & 160 & 1 \\
\hline 31 & 161 & 2 \\
\hline 31 & 162 & 2 \\
\hline
\end{tabular}




\begin{tabular}{|c|c|c|}
\hline 31 & 163 & 2 \\
\hline 31 & 164 & 1 \\
\hline 31 & 165 & 1 \\
\hline 31 & 166 & 2 \\
\hline 31 & 167 & 2 \\
\hline 31 & 168 & 2 \\
\hline 31 & 169 & 1 \\
\hline 31 & 170 & 2 \\
\hline 32 & 1 & 27 \\
\hline 32 & 2 & 38 \\
\hline 32 & 3 & 51 \\
\hline 32 & 4 & 22 \\
\hline 32 & 5 & 61 \\
\hline 32 & 6 & 115 \\
\hline 32 & 7 & 53 \\
\hline 32 & 8 & 46 \\
\hline 32 & 9 & 68 \\
\hline 32 & 10 & 64 \\
\hline 32 & 11 & 83 \\
\hline 32 & 12 & 119 \\
\hline 32 & 13 & 123 \\
\hline 32 & 14 & 15 \\
\hline 32 & 15 & 34 \\
\hline 32 & 16 & 23 \\
\hline 32 & 17 & 18 \\
\hline 32 & 18 & 19 \\
\hline 32 & 19 & 15 \\
\hline 32 & 20 & 11 \\
\hline 32 & 21 & 18 \\
\hline 32 & 22 & 9 \\
\hline 32 & 23 & 16 \\
\hline 32 & 24 & 14 \\
\hline 32 & 25 & 13 \\
\hline 32 & 26 & 27 \\
\hline 32 & 27 & 15 \\
\hline 32 & 28 & 26 \\
\hline 32 & 29 & 18 \\
\hline 32 & 30 & 35 \\
\hline 32 & 31 & 36 \\
\hline 32 & 32 & 2 \\
\hline 32 & 33 & 2 \\
\hline 32 & 34 & 9 \\
\hline 32 & 35 & 8 \\
\hline 32 & 36 & 7 \\
\hline 32 & 37 & 3 \\
\hline
\end{tabular}




\begin{tabular}{|c|c|c|}
\hline 32 & 38 & 8 \\
\hline 32 & 39 & 10 \\
\hline 32 & 40 & 4 \\
\hline 32 & 41 & 3 \\
\hline 32 & 42 & 3 \\
\hline 32 & 43 & 4 \\
\hline 32 & 44 & 3 \\
\hline 32 & 45 & 4 \\
\hline 32 & 46 & 3 \\
\hline 32 & 47 & 3 \\
\hline 32 & 48 & 2 \\
\hline 32 & 49 & 3 \\
\hline 32 & 50 & 2 \\
\hline 32 & 51 & 2 \\
\hline 32 & 52 & 5 \\
\hline 32 & 53 & 6 \\
\hline 32 & 54 & 4 \\
\hline 32 & 55 & 4 \\
\hline 32 & 56 & 3 \\
\hline 32 & 57 & 4 \\
\hline 32 & 58 & 5 \\
\hline 32 & 59 & 4 \\
\hline 32 & 60 & 6 \\
\hline 32 & 61 & 4 \\
\hline 32 & 62 & 7 \\
\hline 32 & 63 & 8 \\
\hline 32 & 64 & 6 \\
\hline 32 & 65 & 6 \\
\hline 32 & 66 & 8 \\
\hline 32 & 67 & 10 \\
\hline 32 & 68 & 7 \\
\hline 32 & 69 & 9 \\
\hline 32 & 70 & 11 \\
\hline 32 & 71 & 10 \\
\hline 32 & 72 & 2 \\
\hline 32 & 73 & 3 \\
\hline 32 & 74 & 3 \\
\hline 32 & 75 & 4 \\
\hline 32 & 76 & 6 \\
\hline 32 & 77 & 5 \\
\hline 32 & 78 & 6 \\
\hline 32 & 79 & 5 \\
\hline 32 & 80 & 4 \\
\hline 32 & 81 & 5 \\
\hline 32 & 82 & 5 \\
\hline
\end{tabular}




\begin{tabular}{|c|c|c|}
\hline 32 & 83 & 7 \\
\hline 32 & 84 & 5 \\
\hline 32 & 85 & 5 \\
\hline 32 & 86 & 9 \\
\hline 32 & 87 & 9 \\
\hline 32 & 88 & 5 \\
\hline 32 & 89 & 5 \\
\hline 32 & 90 & 4 \\
\hline 32 & 91 & 2 \\
\hline 32 & 92 & 8 \\
\hline 32 & 93 & 8 \\
\hline 32 & 94 & 15 \\
\hline 32 & 95 & 16 \\
\hline 32 & 96 & 18 \\
\hline 32 & 97 & 11 \\
\hline 32 & 98 & 19 \\
\hline 32 & 99 & 14 \\
\hline 32 & 100 & 13 \\
\hline 32 & 101 & 6 \\
\hline 32 & 102 & 3 \\
\hline 32 & 103 & 5 \\
\hline 32 & 104 & 3 \\
\hline 32 & 105 & 12 \\
\hline 32 & 106 & 10 \\
\hline 32 & 107 & 8 \\
\hline 32 & 108 & 186 \\
\hline 32 & 109 & 131 \\
\hline 32 & 110 & 183 \\
\hline 32 & 111 & 65 \\
\hline 32 & 112 & 76 \\
\hline 32 & 113 & 8 \\
\hline 32 & 114 & 22 \\
\hline 32 & 115 & 18 \\
\hline 32 & 116 & 95 \\
\hline 32 & 117 & 5459 \\
\hline 32 & 118 & 50 \\
\hline 32 & 119 & 72 \\
\hline 32 & 120 & 44 \\
\hline 32 & 121 & 34 \\
\hline 32 & 122 & 34 \\
\hline 32 & 123 & 24 \\
\hline 32 & 124 & 14 \\
\hline 32 & 125 & 17 \\
\hline 32 & 126 & 27 \\
\hline 32 & 127 & 30 \\
\hline
\end{tabular}




\begin{tabular}{|c|c|c|}
\hline 32 & 128 & 8 \\
\hline 32 & 129 & 17 \\
\hline 32 & 130 & 23 \\
\hline 32 & 131 & 17 \\
\hline 32 & 132 & 6 \\
\hline 32 & 133 & 13 \\
\hline 32 & 134 & 10 \\
\hline 32 & 135 & 41 \\
\hline 32 & 136 & 8 \\
\hline 32 & 137 & 24 \\
\hline 32 & 138 & 8 \\
\hline 32 & 139 & 9 \\
\hline 32 & 140 & 10 \\
\hline 32 & 141 & 23 \\
\hline 32 & 142 & 11 \\
\hline 32 & 143 & 17 \\
\hline 32 & 144 & 28 \\
\hline 32 & 145 & 70 \\
\hline 32 & 146 & 101 \\
\hline 32 & 147 & 12 \\
\hline 32 & 148 & 16 \\
\hline 32 & 149 & 22 \\
\hline 32 & 150 & 24 \\
\hline 32 & 151 & 33 \\
\hline 32 & 152 & 28 \\
\hline 32 & 153 & 46 \\
\hline 32 & 154 & 11 \\
\hline 32 & 155 & 18 \\
\hline 32 & 156 & 11 \\
\hline 32 & 157 & 11 \\
\hline 32 & 158 & 12 \\
\hline 32 & 159 & 7 \\
\hline 32 & 160 & 4 \\
\hline 32 & 161 & 3 \\
\hline 32 & 162 & 9 \\
\hline 32 & 163 & 4 \\
\hline 32 & 164 & 12 \\
\hline 32 & 165 & 2 \\
\hline 32 & 166 & 3 \\
\hline 32 & 167 & 7 \\
\hline 32 & 168 & 8 \\
\hline 32 & 169 & 9 \\
\hline 32 & 170 & 9 \\
\hline 33 & 1 & 263 \\
\hline 33 & 2 & 416 \\
\hline
\end{tabular}




\begin{tabular}{|c|c|c|}
\hline 33 & 3 & 1691 \\
\hline 33 & 4 & 145 \\
\hline 33 & 5 & 5309 \\
\hline 33 & 6 & 42 \\
\hline 33 & 7 & 299 \\
\hline 33 & 8 & 410 \\
\hline 33 & 9 & 203 \\
\hline 33 & 10 & 93 \\
\hline 33 & 11 & 19 \\
\hline 33 & 12 & 43 \\
\hline 33 & 13 & 29 \\
\hline 33 & 14 & 45 \\
\hline 33 & 15 & 89 \\
\hline 33 & 16 & 146 \\
\hline 33 & 17 & 95 \\
\hline 33 & 18 & 93 \\
\hline 33 & 19 & 50 \\
\hline 33 & 20 & 41 \\
\hline 33 & 21 & 83 \\
\hline 33 & 22 & 14 \\
\hline 33 & 23 & 142 \\
\hline 33 & 24 & 71 \\
\hline 33 & 25 & 25 \\
\hline 33 & 26 & 78 \\
\hline 33 & 27 & 32 \\
\hline 33 & 28 & 71 \\
\hline 33 & 29 & 43 \\
\hline 33 & 30 & 126 \\
\hline 33 & 31 & 298 \\
\hline 33 & 32 & 3 \\
\hline 33 & 33 & 3 \\
\hline 33 & 34 & 22 \\
\hline 33 & 35 & 20 \\
\hline 33 & 36 & 17 \\
\hline 33 & 37 & 5 \\
\hline 33 & 38 & 15 \\
\hline 33 & 39 & 26 \\
\hline 33 & 40 & 5 \\
\hline 33 & 41 & 5 \\
\hline 33 & 42 & 4 \\
\hline 33 & 43 & 5 \\
\hline 33 & 44 & 16 \\
\hline 33 & 45 & 6 \\
\hline 33 & 46 & 4 \\
\hline 33 & 47 & 4 \\
\hline
\end{tabular}




\begin{tabular}{|c|c|c|}
\hline 33 & 48 & 3 \\
\hline 33 & 49 & 4 \\
\hline 33 & 50 & 3 \\
\hline 33 & 51 & 3 \\
\hline 33 & 52 & 7 \\
\hline 33 & 53 & 9 \\
\hline 33 & 54 & 6 \\
\hline 33 & 55 & 5 \\
\hline 33 & 56 & 5 \\
\hline 33 & 57 & 6 \\
\hline 33 & 58 & 7 \\
\hline 33 & 59 & 6 \\
\hline 33 & 60 & 8 \\
\hline 33 & 61 & 5 \\
\hline 33 & 62 & 10 \\
\hline 33 & 63 & 13 \\
\hline 33 & 64 & 9 \\
\hline 33 & 65 & 9 \\
\hline 33 & 66 & 14 \\
\hline 33 & 67 & 19 \\
\hline 33 & 68 & 12 \\
\hline 33 & 69 & 16 \\
\hline 33 & 70 & 20 \\
\hline 33 & 71 & 17 \\
\hline 33 & 72 & 3 \\
\hline 33 & 73 & 5 \\
\hline 33 & 74 & 3 \\
\hline 33 & 75 & 6 \\
\hline 33 & 76 & 9 \\
\hline 33 & 77 & 8 \\
\hline 33 & 78 & 9 \\
\hline 33 & 79 & 8 \\
\hline 33 & 80 & 6 \\
\hline 33 & 81 & 7 \\
\hline 33 & 82 & 7 \\
\hline 33 & 83 & 12 \\
\hline 33 & 84 & 7 \\
\hline 33 & 85 & 7 \\
\hline 33 & 86 & 16 \\
\hline 33 & 87 & 15 \\
\hline 33 & 88 & 8 \\
\hline 33 & 89 & 7 \\
\hline 33 & 90 & 5 \\
\hline 33 & 91 & 4 \\
\hline 33 & 92 & 14 \\
\hline
\end{tabular}




\begin{tabular}{|c|c|c|}
\hline 33 & 93 & 12 \\
\hline 33 & 94 & 32 \\
\hline 33 & 95 & 35 \\
\hline 33 & 96 & 41 \\
\hline 33 & 97 & 21 \\
\hline 33 & 98 & 45 \\
\hline 33 & 99 & 28 \\
\hline 33 & 100 & 24 \\
\hline 33 & 101 & 10 \\
\hline 33 & 102 & 4 \\
\hline 33 & 103 & 8 \\
\hline 33 & 104 & 4 \\
\hline 33 & 105 & 22 \\
\hline 33 & 106 & 19 \\
\hline 33 & 107 & 13 \\
\hline 33 & 108 & 125 \\
\hline 33 & 109 & 67 \\
\hline 33 & 110 & 242 \\
\hline 33 & 111 & 65 \\
\hline 33 & 112 & 91 \\
\hline 33 & 113 & 13 \\
\hline 33 & 114 & 109 \\
\hline 33 & 115 & 70 \\
\hline 33 & 116 & 35 \\
\hline 33 & 117 & 55 \\
\hline 33 & 118 & 23 \\
\hline 33 & 119 & 86 \\
\hline 33 & 120 & 50 \\
\hline 33 & 121 & 39 \\
\hline 33 & 122 & 23 \\
\hline 33 & 123 & 17 \\
\hline 33 & 124 & 14 \\
\hline 33 & 125 & 24 \\
\hline 33 & 126 & 30 \\
\hline 33 & 127 & 41 \\
\hline 33 & 128 & 26 \\
\hline 33 & 129 & 25 \\
\hline 33 & 130 & 25 \\
\hline 33 & 131 & 17 \\
\hline 33 & 132 & 16 \\
\hline 33 & 133 & 41 \\
\hline 33 & 134 & 18 \\
\hline 33 & 135 & 32 \\
\hline 33 & 136 & 6 \\
\hline 33 & 137 & 15 \\
\hline
\end{tabular}




\begin{tabular}{|c|c|c|}
\hline 33 & 138 & 6 \\
\hline 33 & 139 & 7 \\
\hline 33 & 140 & 7 \\
\hline 33 & 141 & 15 \\
\hline 33 & 142 & 8 \\
\hline 33 & 143 & 12 \\
\hline 33 & 144 & 17 \\
\hline 33 & 145 & 21 \\
\hline 33 & 146 & 25 \\
\hline 33 & 147 & 9 \\
\hline 33 & 148 & 24 \\
\hline 33 & 149 & 13 \\
\hline 33 & 150 & 11 \\
\hline 33 & 151 & 18 \\
\hline 33 & 152 & 13 \\
\hline 33 & 153 & 23 \\
\hline 33 & 154 & 8 \\
\hline 33 & 155 & 12 \\
\hline 33 & 156 & 8 \\
\hline 33 & 157 & 8 \\
\hline 33 & 158 & 9 \\
\hline 33 & 159 & 5 \\
\hline 33 & 160 & 3 \\
\hline 33 & 161 & 2 \\
\hline 33 & 162 & 3 \\
\hline 33 & 163 & 4 \\
\hline 33 & 164 & 8 \\
\hline 33 & 165 & 2 \\
\hline 33 & 166 & 2 \\
\hline 33 & 167 & 2 \\
\hline 33 & 168 & 2 \\
\hline 33 & 169 & 13 \\
\hline 33 & 170 & 7 \\
\hline 34 & 1 & 39 \\
\hline 34 & 2 & 27 \\
\hline 34 & 3 & 12 \\
\hline 34 & 4 & 40 \\
\hline 34 & 5 & 10 \\
\hline 34 & 6 & 7 \\
\hline 34 & 7 & 8 \\
\hline 34 & 8 & 9 \\
\hline 34 & 9 & 21 \\
\hline 34 & 10 & 15 \\
\hline 34 & 11 & 5 \\
\hline 34 & 12 & 7 \\
\hline
\end{tabular}




\begin{tabular}{|c|c|c|}
\hline 34 & 13 & 6 \\
\hline 34 & 14 & 17 \\
\hline 34 & 15 & 17 \\
\hline 34 & 16 & 26 \\
\hline 34 & 17 & 29 \\
\hline 34 & 18 & 42 \\
\hline 34 & 19 & 22 \\
\hline 34 & 20 & 15 \\
\hline 34 & 21 & 65 \\
\hline 34 & 22 & 24752 \\
\hline 34 & 23 & 68 \\
\hline 34 & 24 & 117 \\
\hline 34 & 25 & 396 \\
\hline 34 & 26 & 52 \\
\hline 34 & 27 & 252 \\
\hline 34 & 28 & 24 \\
\hline 34 & 29 & 49 \\
\hline 34 & 30 & 18 \\
\hline 34 & 31 & 14 \\
\hline 34 & 32 & 9 \\
\hline 34 & 33 & 7 \\
\hline 34 & 34 & 9 \\
\hline 34 & 35 & 12 \\
\hline 34 & 36 & 11 \\
\hline 34 & 37 & 18 \\
\hline 34 & 38 & 6 \\
\hline 34 & 39 & 7 \\
\hline 34 & 40 & 20 \\
\hline 34 & 41 & 16 \\
\hline 34 & 42 & 18 \\
\hline 34 & 43 & 26 \\
\hline 34 & 44 & 47 \\
\hline 34 & 45 & 42 \\
\hline 34 & 46 & 15 \\
\hline 34 & 47 & 24 \\
\hline 34 & 48 & 11 \\
\hline 34 & 49 & 15 \\
\hline 34 & 50 & 11 \\
\hline 34 & 51 & 10 \\
\hline 34 & 52 & 44 \\
\hline 34 & 53 & 103 \\
\hline 34 & 54 & 42 \\
\hline 34 & 55 & 26 \\
\hline 34 & 56 & 25 \\
\hline 34 & 57 & 23 \\
\hline
\end{tabular}




\begin{tabular}{|c|c|c|}
\hline 34 & 58 & 23 \\
\hline 34 & 59 & 17 \\
\hline 34 & 60 & 51 \\
\hline 34 & 61 & 35 \\
\hline 34 & 62 & 205 \\
\hline 34 & 63 & 392 \\
\hline 34 & 64 & 122 \\
\hline 34 & 65 & 116 \\
\hline 34 & 66 & 362 \\
\hline 34 & 67 & 52 \\
\hline 34 & 68 & 70 \\
\hline 34 & 69 & 4747 \\
\hline 34 & 70 & 3294 \\
\hline 34 & 71 & 228 \\
\hline 34 & 72 & 10 \\
\hline 34 & 73 & 13 \\
\hline 34 & 74 & 11 \\
\hline 34 & 75 & 9 \\
\hline 34 & 76 & 16 \\
\hline 34 & 77 & 17 \\
\hline 34 & 78 & 18 \\
\hline 34 & 79 & 14 \\
\hline 34 & 80 & 11 \\
\hline 34 & 81 & 13 \\
\hline 34 & 82 & 13 \\
\hline 34 & 83 & 21 \\
\hline 34 & 84 & 12 \\
\hline 34 & 85 & 12 \\
\hline 34 & 86 & 42 \\
\hline 34 & 87 & 40 \\
\hline 34 & 88 & 14 \\
\hline 34 & 89 & 12 \\
\hline 34 & 90 & 8 \\
\hline 34 & 91 & 6 \\
\hline 34 & 92 & 16 \\
\hline 34 & 93 & 13 \\
\hline 34 & 94 & 9 \\
\hline 34 & 95 & 10 \\
\hline 34 & 96 & 27 \\
\hline 34 & 97 & 16 \\
\hline 34 & 98 & 11 \\
\hline 34 & 99 & 13 \\
\hline 34 & 100 & 15 \\
\hline 34 & 101 & 9 \\
\hline 34 & 102 & 7 \\
\hline
\end{tabular}




\begin{tabular}{|c|c|c|}
\hline 34 & 103 & 4 \\
\hline 34 & 104 & 4 \\
\hline 34 & 105 & 12 \\
\hline 34 & 106 & 9 \\
\hline 34 & 107 & 6 \\
\hline 34 & 108 & 9 \\
\hline 34 & 109 & 7 \\
\hline 34 & 110 & 10 \\
\hline 34 & 111 & 7 \\
\hline 34 & 112 & 8 \\
\hline 34 & 113 & 5 \\
\hline 34 & 114 & 9 \\
\hline 34 & 115 & 7 \\
\hline 34 & 116 & 5 \\
\hline 34 & 117 & 7 \\
\hline 34 & 118 & 4 \\
\hline 34 & 119 & 8 \\
\hline 34 & 120 & 6 \\
\hline 34 & 121 & 6 \\
\hline 34 & 122 & 5 \\
\hline 34 & 123 & 4 \\
\hline 34 & 124 & 4 \\
\hline 34 & 125 & 5 \\
\hline 34 & 126 & 6 \\
\hline 34 & 127 & 7 \\
\hline 34 & 128 & 5 \\
\hline 34 & 129 & 6 \\
\hline 34 & 130 & 5 \\
\hline 34 & 131 & 4 \\
\hline 34 & 132 & 4 \\
\hline 34 & 133 & 7 \\
\hline 34 & 134 & 9 \\
\hline 34 & 135 & 6 \\
\hline 34 & 136 & 2 \\
\hline 34 & 137 & 4 \\
\hline 34 & 138 & 2 \\
\hline 34 & 139 & 3 \\
\hline 34 & 140 & 3 \\
\hline 34 & 141 & 4 \\
\hline 34 & 142 & 3 \\
\hline 34 & 143 & 4 \\
\hline 34 & 144 & 4 \\
\hline 34 & 145 & 5 \\
\hline 34 & 146 & 5 \\
\hline 34 & 147 & 3 \\
\hline
\end{tabular}




\begin{tabular}{|c|c|c|}
\hline 34 & 148 & 2 \\
\hline 34 & 149 & 2 \\
\hline 34 & 150 & 2 \\
\hline 34 & 151 & 11 \\
\hline 34 & 152 & 4 \\
\hline 34 & 153 & 5 \\
\hline 34 & 154 & 3 \\
\hline 34 & 155 & 4 \\
\hline 34 & 156 & 3 \\
\hline 34 & 157 & 3 \\
\hline 34 & 158 & 3 \\
\hline 34 & 159 & 2 \\
\hline 34 & 160 & 3 \\
\hline 34 & 161 & 3 \\
\hline 34 & 162 & 4 \\
\hline 34 & 163 & 3 \\
\hline 34 & 164 & 3 \\
\hline 34 & 165 & 3 \\
\hline 34 & 166 & 4 \\
\hline 34 & 167 & 3 \\
\hline 34 & 168 & 3 \\
\hline 34 & 169 & 3 \\
\hline 34 & 170 & 2 \\
\hline 35 & 1 & 21 \\
\hline 35 & 2 & 19 \\
\hline 35 & 3 & 22 \\
\hline 35 & 4 & 18 \\
\hline 35 & 5 & 23 \\
\hline 35 & 6 & 10 \\
\hline 35 & 7 & 13 \\
\hline 35 & 8 & 14 \\
\hline 35 & 9 & 15 \\
\hline 35 & 10 & 12 \\
\hline 35 & 11 & 7 \\
\hline 35 & 12 & 10 \\
\hline 35 & 13 & 8 \\
\hline 35 & 14 & 9 \\
\hline 35 & 15 & 11 \\
\hline 35 & 16 & 14 \\
\hline 35 & 17 & 14 \\
\hline 35 & 18 & 15 \\
\hline 35 & 19 & 10 \\
\hline 35 & 20 & 10 \\
\hline 35 & 21 & 14 \\
\hline 35 & 22 & 13 \\
\hline
\end{tabular}




\begin{tabular}{|c|c|c|}
\hline 35 & 23 & 17 \\
\hline 35 & 24 & 14 \\
\hline 35 & 25 & 10 \\
\hline 35 & 26 & 20 \\
\hline 35 & 27 & 12 \\
\hline 35 & 28 & 19 \\
\hline 35 & 29 & 14 \\
\hline 35 & 30 & 35 \\
\hline 35 & 31 & 27 \\
\hline 35 & 32 & 3 \\
\hline 35 & 33 & 3 \\
\hline 35 & 34 & 7 \\
\hline 35 & 35 & 7 \\
\hline 35 & 36 & 6 \\
\hline 35 & 37 & 4 \\
\hline 35 & 38 & 5 \\
\hline 35 & 39 & 7 \\
\hline 35 & 40 & 5 \\
\hline 35 & 41 & 4 \\
\hline 35 & 42 & 3 \\
\hline 35 & 43 & 5 \\
\hline 35 & 44 & 4 \\
\hline 35 & 45 & 6 \\
\hline 35 & 46 & 3 \\
\hline 35 & 47 & 4 \\
\hline 35 & 48 & 3 \\
\hline 35 & 49 & 3 \\
\hline 35 & 50 & 3 \\
\hline 35 & 51 & 3 \\
\hline 35 & 52 & 6 \\
\hline 35 & 53 & 8 \\
\hline 35 & 54 & 6 \\
\hline 35 & 55 & 5 \\
\hline 35 & 56 & 4 \\
\hline 35 & 57 & 6 \\
\hline 35 & 58 & 6 \\
\hline 35 & 59 & 5 \\
\hline 35 & 60 & 7 \\
\hline 35 & 61 & 5 \\
\hline 35 & 62 & 9 \\
\hline 35 & 63 & 11 \\
\hline 35 & 64 & 8 \\
\hline 35 & 65 & 8 \\
\hline 35 & 66 & 12 \\
\hline 35 & 67 & 16 \\
\hline
\end{tabular}




\begin{tabular}{|c|c|c|}
\hline 35 & 68 & 11 \\
\hline 35 & 69 & 9 \\
\hline 35 & 70 & 9 \\
\hline 35 & 71 & 15 \\
\hline 35 & 72 & 3 \\
\hline 35 & 73 & 4 \\
\hline 35 & 74 & 3 \\
\hline 35 & 75 & 5 \\
\hline 35 & 76 & 8 \\
\hline 35 & 77 & 16 \\
\hline 35 & 78 & 23 \\
\hline 35 & 79 & 18 \\
\hline 35 & 80 & 23 \\
\hline 35 & 81 & 17 \\
\hline 35 & 82 & 17 \\
\hline 35 & 83 & 156 \\
\hline 35 & 84 & 52 \\
\hline 35 & 85 & 37 \\
\hline 35 & 86 & 16 \\
\hline 35 & 87 & 39 \\
\hline 35 & 88 & 7 \\
\hline 35 & 89 & 11 \\
\hline 35 & 90 & 11 \\
\hline 35 & 91 & 5 \\
\hline 35 & 92 & 336 \\
\hline 35 & 93 & 195 \\
\hline 35 & 94 & 429 \\
\hline 35 & 95 & 659 \\
\hline 35 & 96 & 59 \\
\hline 35 & 97 & 130 \\
\hline 35 & 98 & 65 \\
\hline 35 & 99 & 590 \\
\hline 35 & 100 & 229 \\
\hline 35 & 101 & 113 \\
\hline 35 & 102 & 31 \\
\hline 35 & 103 & 26 \\
\hline 35 & 104 & 9 \\
\hline 35 & 105 & 976563 \\
\hline 35 & 106 & 763 \\
\hline 35 & 107 & 163 \\
\hline 35 & 108 & 14 \\
\hline 35 & 109 & 31 \\
\hline 35 & 110 & 17 \\
\hline 35 & 111 & 33 \\
\hline 35 & 112 & 41 \\
\hline
\end{tabular}




\begin{tabular}{|c|c|c|}
\hline 35 & 113 & 100 \\
\hline 35 & 114 & 43 \\
\hline 35 & 115 & 90 \\
\hline 35 & 116 & 21 \\
\hline 35 & 117 & 11 \\
\hline 35 & 118 & 5 \\
\hline 35 & 119 & 57 \\
\hline 35 & 120 & 58 \\
\hline 35 & 121 & 61 \\
\hline 35 & 122 & 15 \\
\hline 35 & 123 & 18 \\
\hline 35 & 124 & 21 \\
\hline 35 & 125 & 27 \\
\hline 35 & 126 & 52 \\
\hline 35 & 127 & 85 \\
\hline 35 & 128 & 95 \\
\hline 35 & 129 & 49 \\
\hline 35 & 130 & 28 \\
\hline 35 & 131 & 37 \\
\hline 35 & 132 & 41 \\
\hline 35 & 133 & 48 \\
\hline 35 & 134 & 201 \\
\hline 35 & 135 & 19 \\
\hline 35 & 136 & 3 \\
\hline 35 & 137 & 6 \\
\hline 35 & 138 & 3 \\
\hline 35 & 139 & 3 \\
\hline 35 & 140 & 3 \\
\hline 35 & 141 & 6 \\
\hline 35 & 142 & 4 \\
\hline 35 & 143 & 5 \\
\hline 35 & 144 & 6 \\
\hline 35 & 145 & 7 \\
\hline 35 & 146 & 8 \\
\hline 35 & 147 & 3 \\
\hline 35 & 148 & 4 \\
\hline 35 & 149 & 5 \\
\hline 35 & 150 & 5 \\
\hline 35 & 151 & 6 \\
\hline 35 & 152 & 5 \\
\hline 35 & 153 & 7 \\
\hline 35 & 154 & 2 \\
\hline 35 & 155 & 3 \\
\hline 35 & 156 & 2 \\
\hline 35 & 157 & 3 \\
\hline
\end{tabular}




\begin{tabular}{|c|c|c|}
\hline 35 & 158 & 3 \\
\hline 35 & 159 & 2 \\
\hline 35 & 160 & 1 \\
\hline 35 & 161 & 1 \\
\hline 35 & 162 & 1 \\
\hline 35 & 163 & 2 \\
\hline 35 & 164 & 4 \\
\hline 35 & 165 & 2 \\
\hline 35 & 166 & 1 \\
\hline 35 & 167 & 1 \\
\hline 35 & 168 & 1 \\
\hline 35 & 169 & 3 \\
\hline 35 & 170 & 2 \\
\hline 36 & 1 & 6 \\
\hline 36 & 2 & 5 \\
\hline 36 & 3 & 5 \\
\hline 36 & 4 & 7 \\
\hline 36 & 5 & 5 \\
\hline 36 & 6 & 3 \\
\hline 36 & 7 & 4 \\
\hline 36 & 8 & 5 \\
\hline 36 & 9 & 5 \\
\hline 36 & 10 & 4 \\
\hline 36 & 11 & 3 \\
\hline 36 & 12 & 3 \\
\hline 36 & 13 & 3 \\
\hline 36 & 14 & 5 \\
\hline 36 & 15 & 5 \\
\hline 36 & 16 & 6 \\
\hline 36 & 17 & 6 \\
\hline 36 & 18 & 7 \\
\hline 36 & 19 & 5 \\
\hline 36 & 20 & 6 \\
\hline 36 & 21 & 8 \\
\hline 36 & 22 & 13 \\
\hline 36 & 23 & 7 \\
\hline 36 & 24 & 7 \\
\hline 36 & 25 & 9 \\
\hline 36 & 26 & 9 \\
\hline 36 & 27 & 16 \\
\hline 36 & 28 & 8 \\
\hline 36 & 29 & 11 \\
\hline 36 & 30 & 7 \\
\hline 36 & 31 & 6 \\
\hline 36 & 32 & 3 \\
\hline
\end{tabular}




\begin{tabular}{|c|c|c|}
\hline 36 & 33 & 3 \\
\hline 36 & 34 & 4 \\
\hline 36 & 35 & 4 \\
\hline 36 & 36 & 4 \\
\hline 36 & 37 & 5 \\
\hline 36 & 38 & 3 \\
\hline 36 & 39 & 4 \\
\hline 36 & 40 & 5 \\
\hline 36 & 41 & 10 \\
\hline 36 & 42 & 20 \\
\hline 36 & 43 & 13 \\
\hline 36 & 44 & 7 \\
\hline 36 & 45 & 9 \\
\hline 36 & 46 & 42 \\
\hline 36 & 47 & 58 \\
\hline 36 & 48 & 66 \\
\hline 36 & 49 & 125 \\
\hline 36 & 50 & 17 \\
\hline 36 & 51 & 24 \\
\hline 36 & 52 & 12 \\
\hline 36 & 53 & 10 \\
\hline 36 & 54 & 13 \\
\hline 36 & 55 & 14 \\
\hline 36 & 56 & 40 \\
\hline 36 & 57 & 201 \\
\hline 36 & 58 & 211 \\
\hline 36 & 59 & 68 \\
\hline 36 & 60 & 65 \\
\hline 36 & 61 & 33 \\
\hline 36 & 62 & 20 \\
\hline 36 & 63 & 32 \\
\hline 36 & 64 & 55 \\
\hline 36 & 65 & 43 \\
\hline 36 & 66 & 34 \\
\hline 36 & 67 & 6 \\
\hline 36 & 68 & 42 \\
\hline 36 & 69 & 19 \\
\hline 36 & 70 & 20 \\
\hline 36 & 71 & 21 \\
\hline 36 & 72 & 352 \\
\hline 36 & 73 & 13717 \\
\hline 36 & 74 & 1092 \\
\hline 36 & 75 & 40 \\
\hline 36 & 76 & 24 \\
\hline 36 & 77 & 14 \\
\hline
\end{tabular}




\begin{tabular}{|c|c|c|}
\hline 36 & 78 & 19 \\
\hline 36 & 79 & 20 \\
\hline 36 & 80 & 12 \\
\hline 36 & 81 & 5 \\
\hline 36 & 82 & 5 \\
\hline 36 & 83 & 7 \\
\hline 36 & 84 & 12 \\
\hline 36 & 85 & 5 \\
\hline 36 & 86 & 15 \\
\hline 36 & 87 & 10 \\
\hline 36 & 88 & 17 \\
\hline 36 & 89 & 14 \\
\hline 36 & 90 & 10 \\
\hline 36 & 91 & 8 \\
\hline 36 & 92 & 6 \\
\hline 36 & 93 & 5 \\
\hline 36 & 94 & 4 \\
\hline 36 & 95 & 4 \\
\hline 36 & 96 & 8 \\
\hline 36 & 97 & 6 \\
\hline 36 & 98 & 5 \\
\hline 36 & 99 & 5 \\
\hline 36 & 100 & 6 \\
\hline 36 & 101 & 4 \\
\hline 36 & 102 & 3 \\
\hline 36 & 103 & 1 \\
\hline 36 & 104 & 2 \\
\hline 36 & 105 & 5 \\
\hline 36 & 106 & 3 \\
\hline 36 & 107 & 3 \\
\hline 36 & 108 & 4 \\
\hline 36 & 109 & 3 \\
\hline 36 & 110 & 5 \\
\hline 36 & 111 & 4 \\
\hline 36 & 112 & 4 \\
\hline 36 & 113 & 3 \\
\hline 36 & 114 & 4 \\
\hline 36 & 115 & 4 \\
\hline 36 & 116 & 3 \\
\hline 36 & 117 & 4 \\
\hline 36 & 118 & 2 \\
\hline 36 & 119 & 4 \\
\hline 36 & 120 & 3 \\
\hline 36 & 121 & 3 \\
\hline 36 & 122 & 3 \\
\hline
\end{tabular}




\begin{tabular}{|c|c|c|}
\hline 36 & 123 & 2 \\
\hline 36 & 124 & 2 \\
\hline 36 & 125 & 2 \\
\hline 36 & 126 & 3 \\
\hline 36 & 127 & 3 \\
\hline 36 & 128 & 3 \\
\hline 36 & 129 & 3 \\
\hline 36 & 130 & 2 \\
\hline 36 & 131 & 2 \\
\hline 36 & 132 & 2 \\
\hline 36 & 133 & 3 \\
\hline 36 & 134 & 3 \\
\hline 36 & 135 & 3 \\
\hline 36 & 136 & 2 \\
\hline 36 & 137 & 2 \\
\hline 36 & 138 & 2 \\
\hline 36 & 139 & 2 \\
\hline 36 & 140 & 2 \\
\hline 36 & 141 & 2 \\
\hline 36 & 142 & 2 \\
\hline 36 & 143 & 2 \\
\hline 36 & 144 & 3 \\
\hline 36 & 145 & 3 \\
\hline 36 & 146 & 3 \\
\hline 36 & 147 & 2 \\
\hline 36 & 148 & 1 \\
\hline 36 & 149 & 1 \\
\hline 36 & 150 & 1 \\
\hline 36 & 151 & 4 \\
\hline 36 & 152 & 3 \\
\hline 36 & 153 & 4 \\
\hline 36 & 154 & 2 \\
\hline 36 & 155 & 2 \\
\hline 36 & 156 & 2 \\
\hline 36 & 157 & 2 \\
\hline 36 & 158 & 2 \\
\hline 36 & 159 & 1 \\
\hline 36 & 160 & 2 \\
\hline 36 & 161 & 2 \\
\hline 36 & 162 & 2 \\
\hline 36 & 163 & 2 \\
\hline 36 & 164 & 2 \\
\hline 36 & 165 & 2 \\
\hline 36 & 166 & 2 \\
\hline 36 & 167 & 2 \\
\hline
\end{tabular}




\begin{tabular}{|l|l|r|}
\hline 36 & 168 & 2 \\
\hline 36 & 169 & 2 \\
\hline 36 & 170 & 1 \\
\hline
\end{tabular}




\section{Appendix I LINGO file for case study model}

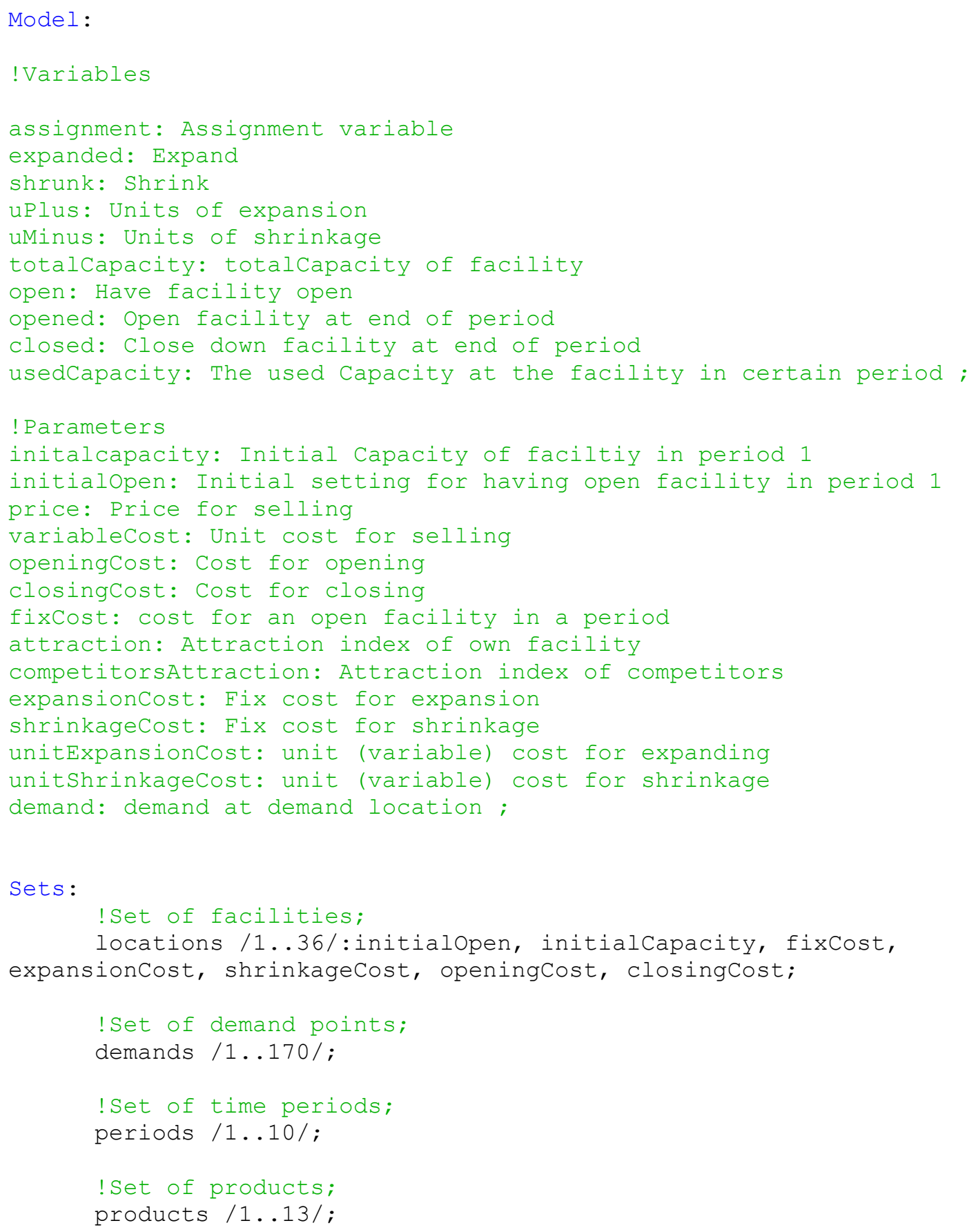




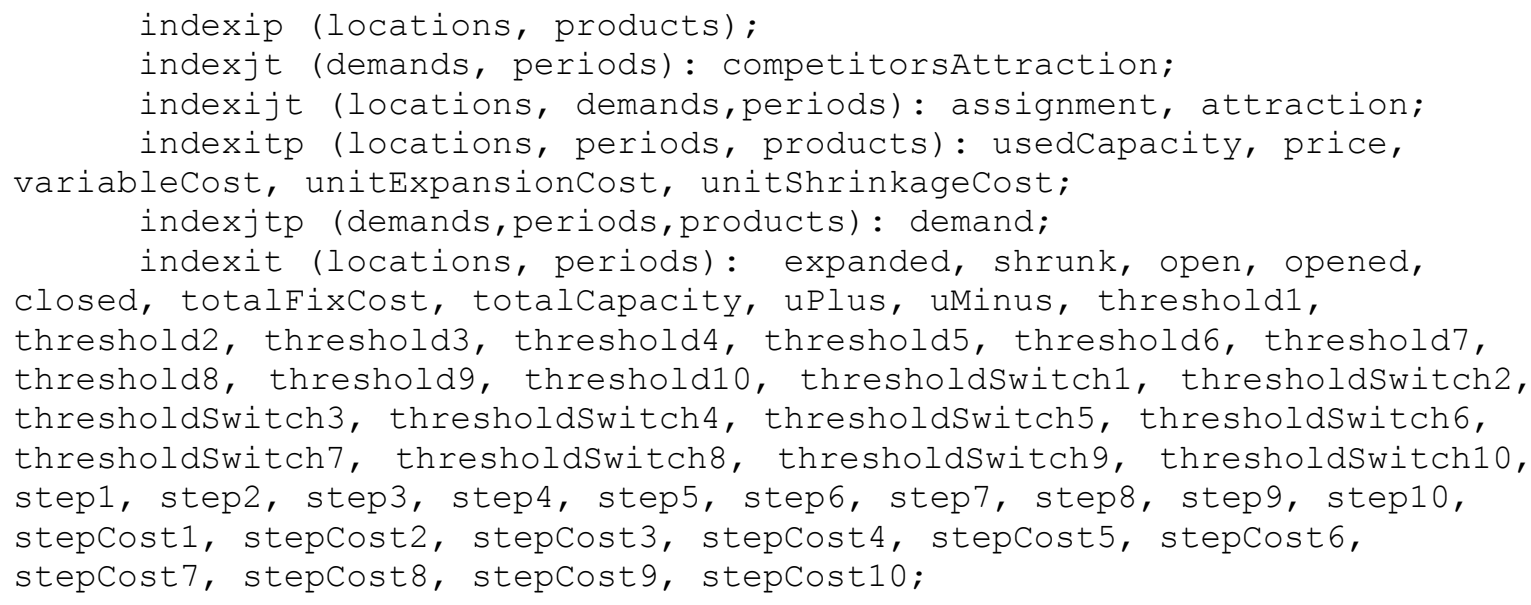

Endsets

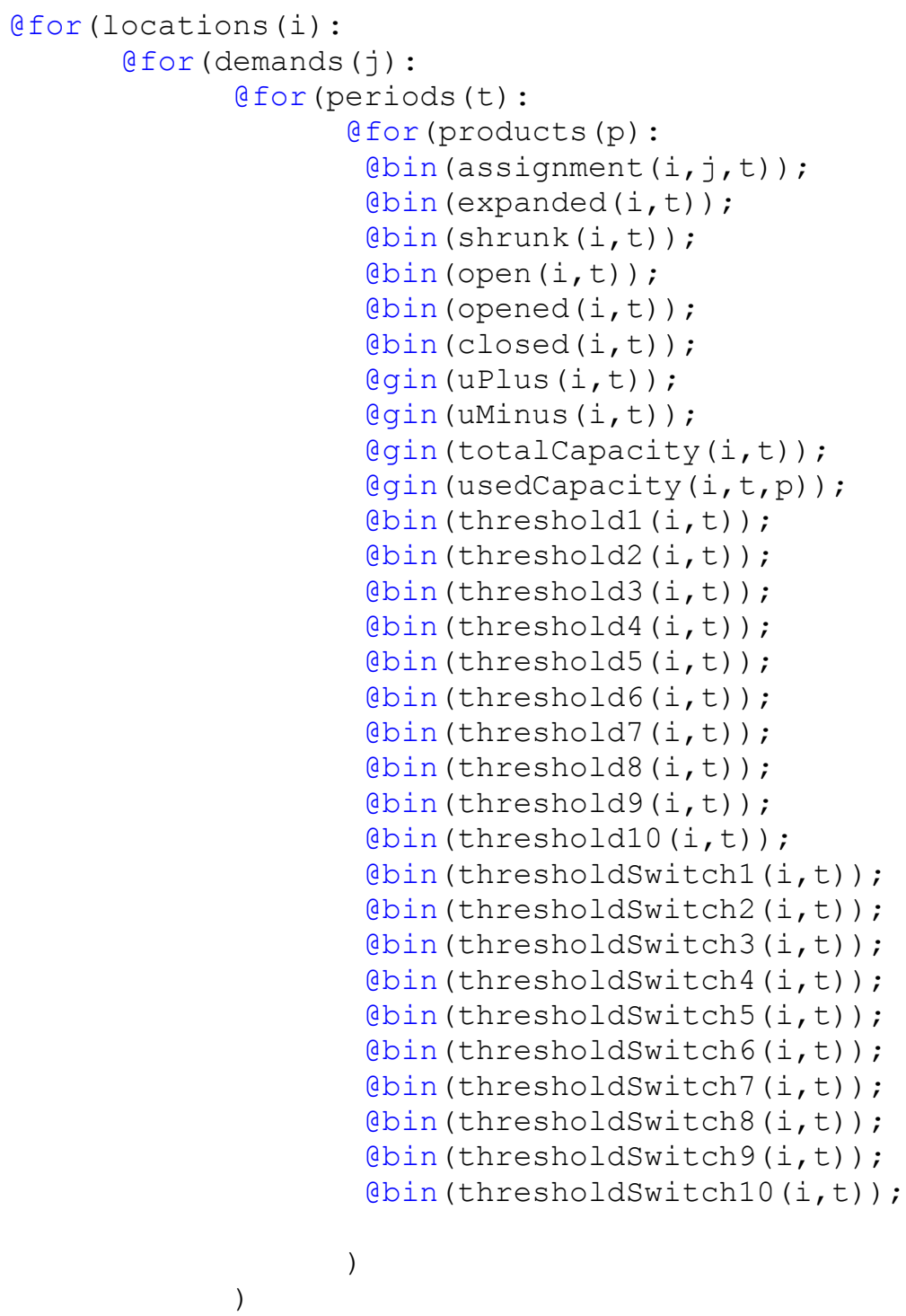


) ;

Data:

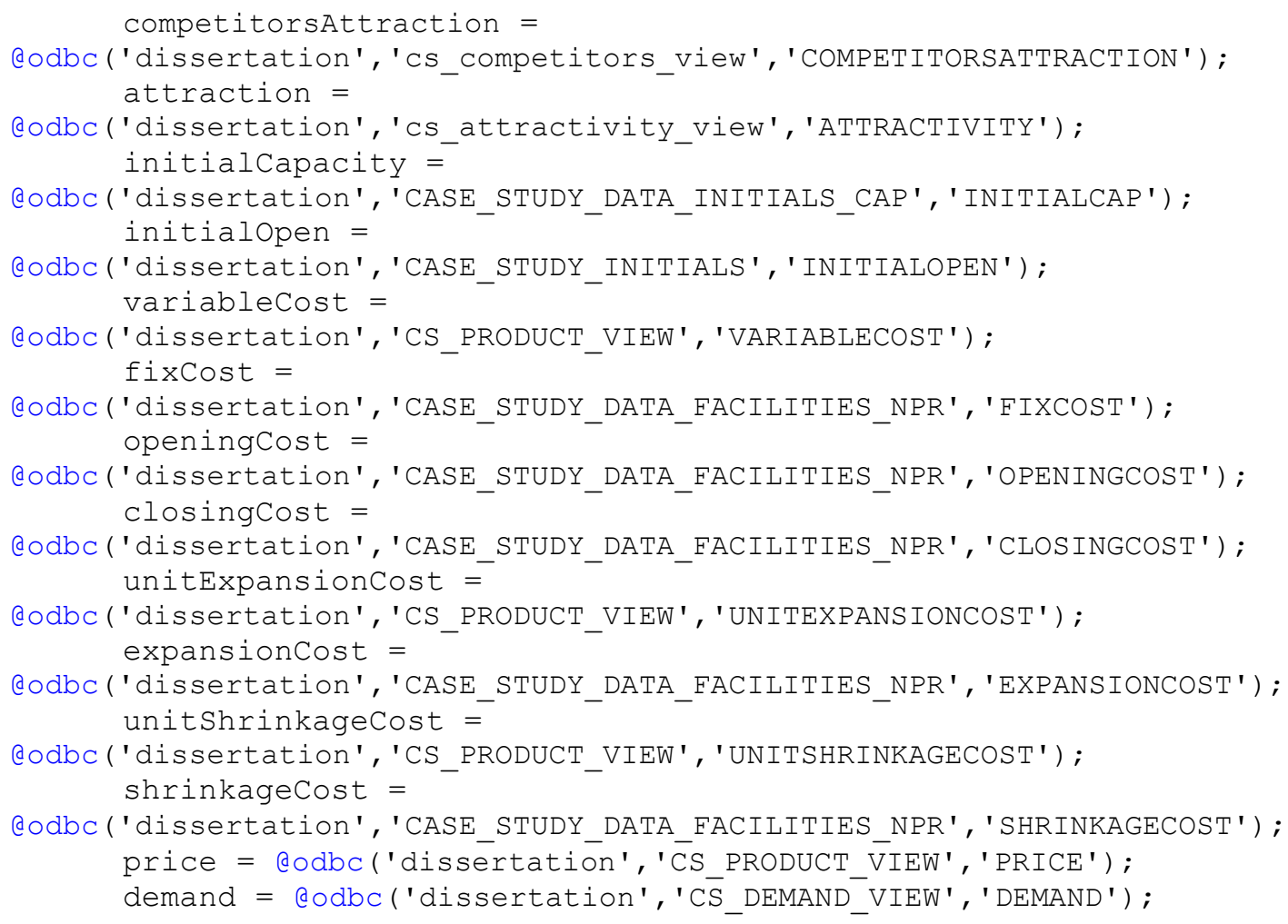

enddata

$\max =$ @sum(indexitp $(i, t, p): \operatorname{usedCapacity}(i, t, p) *(p r i c e(i, t, p) \quad-$ variableCost $(i, t, p))$ - esum(indexit $(i, t)$ : totalfixCost $(i, t)+$ closingcost $(i){ }^{*}$ closed $(i, t)+$ openingcost $\left.(i) * o p e n e d(i, t)\right)$ ¿sum (indexit $(i, t)$ : expansionCost $(i) *$ expanded $(i, t))$ @sum(indexitp (i,t,p) : unitExpansionCost $(i, t, p) * u P l u s(i, t)) \quad-$ @sum(indexit (i,t): shrinkageCost $\left.(i){ }^{*} \operatorname{shrunk}(i, t)\right)$ - asum(indexitp $(i, t, p)$ : unitShrinkageCost (i,t,p) *uMinus $(i, t))$;

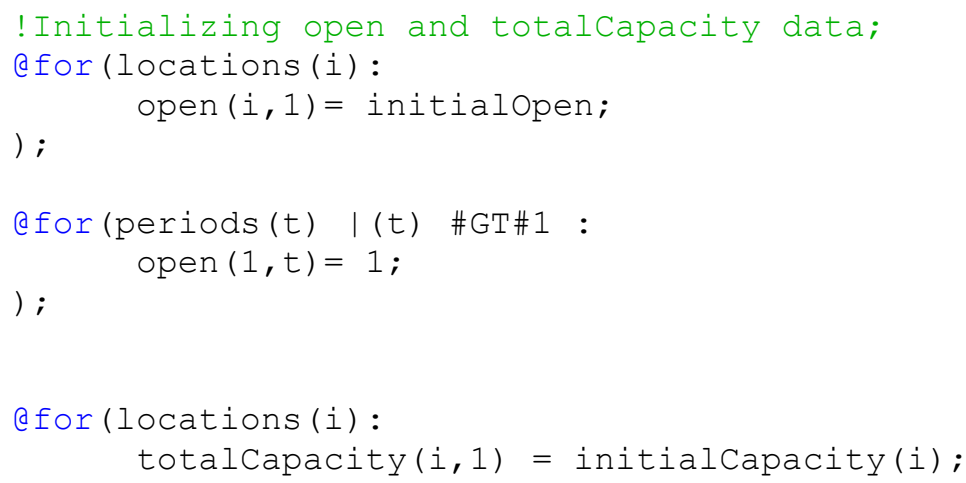




\section{) ;}

efor(locations (i):

efor (periods (t):

afor (products $(p)$ :

$\operatorname{step} 1(i, t)=600 ;$

$\operatorname{step} 2(i, t)=1200 ;$

$\operatorname{step} 3(i, t)=1800 ;$

step $4(i, t)=2400 ;$

$\operatorname{step} 5(i, t)=3000 ;$

$\operatorname{step} 6(i, t)=3600 ;$

step $7(i, t)=4200 ;$

$\operatorname{step} 8(i, t)=4800 ;$

$\operatorname{step} 9(i, t)=5400 ;$

step10(i,t) =6000;

stepCost1 $(i, t)=100000 ;$

stepCost2 $(i, t)=100000$;

stepCost3 $(i, t)=100000$;

stepCost4 $(i, t)=100000$;

stepCost5 $(i, t)=100000$;

stepCost $6(i, t)=100000 ;$

stepCost7 $(i, t)=100000 ;$

stepCost8 $(i, t)=100000 ;$

stepCost9 $(i, t)=100000$;

stepCost10 $(i, t)=100000$;

)

)

) ；

Modification of fix cost in terms of change in facility size; efor(locations(i):

afor (periods (t):

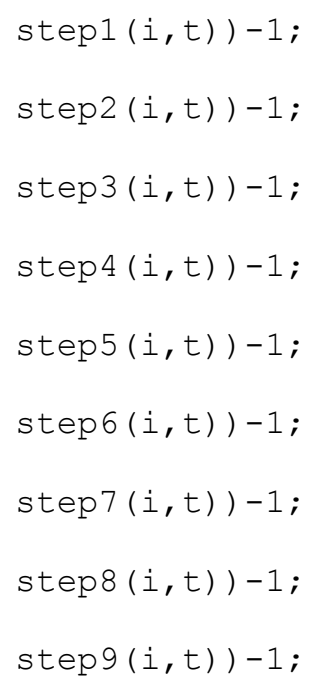




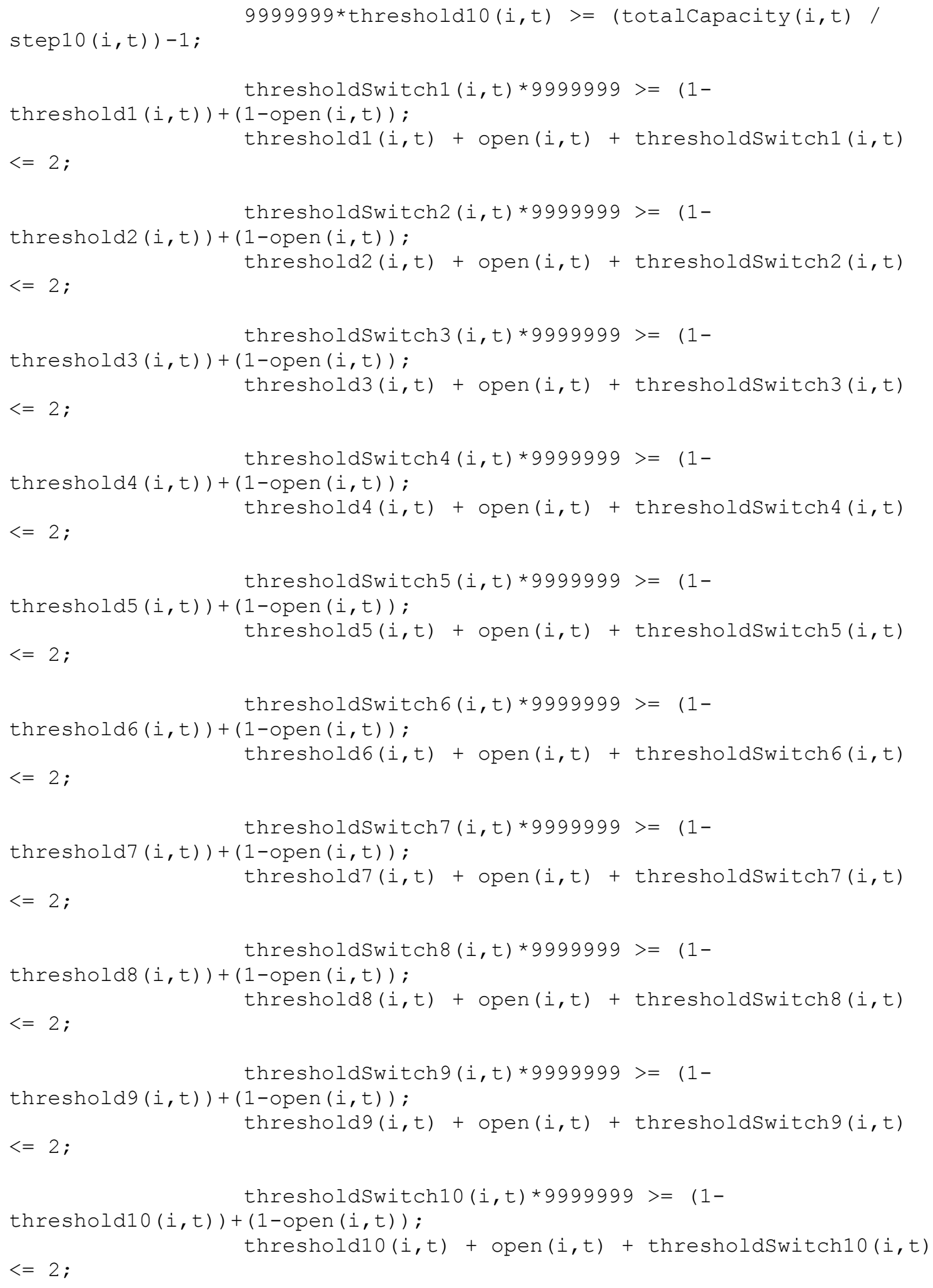




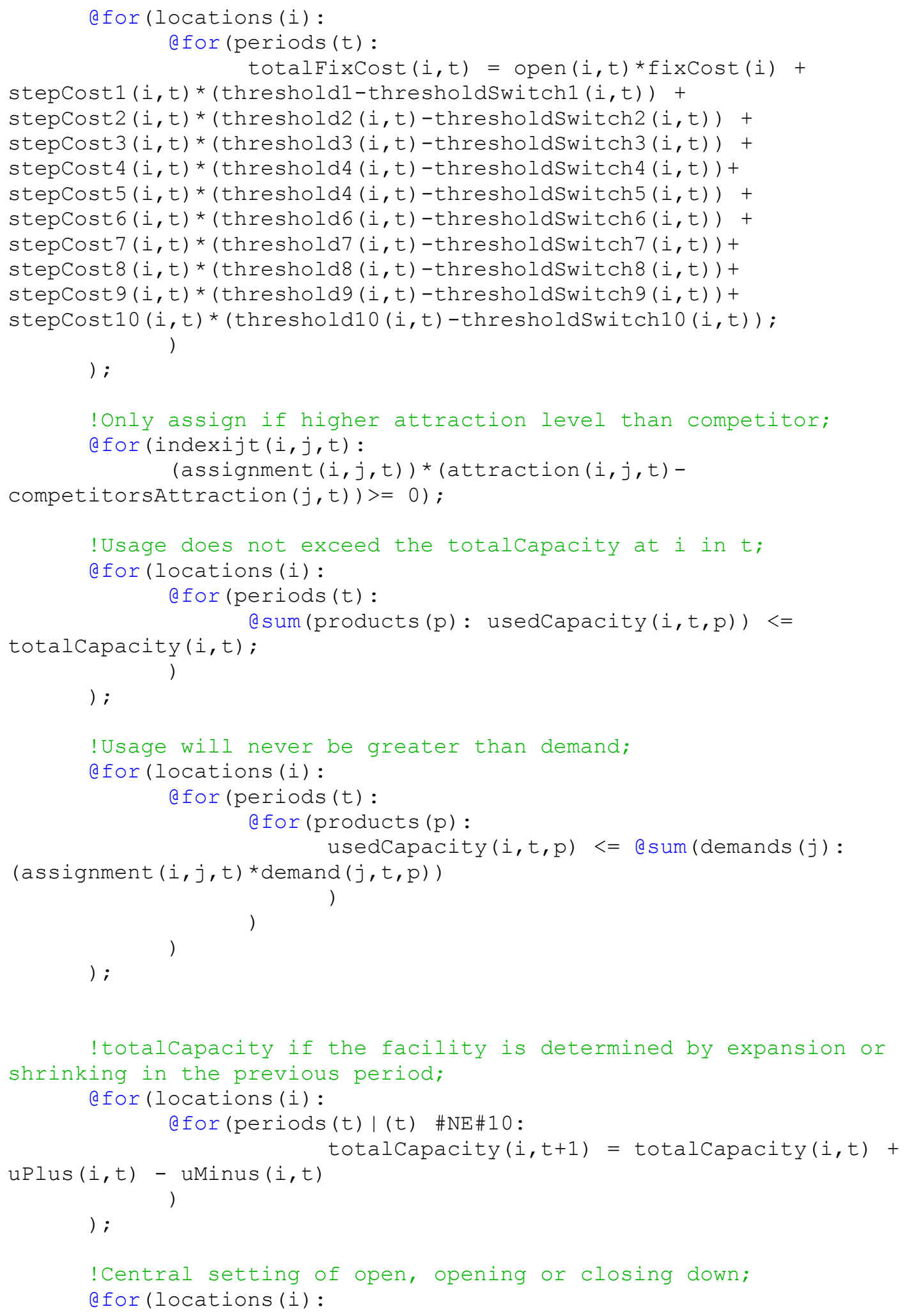




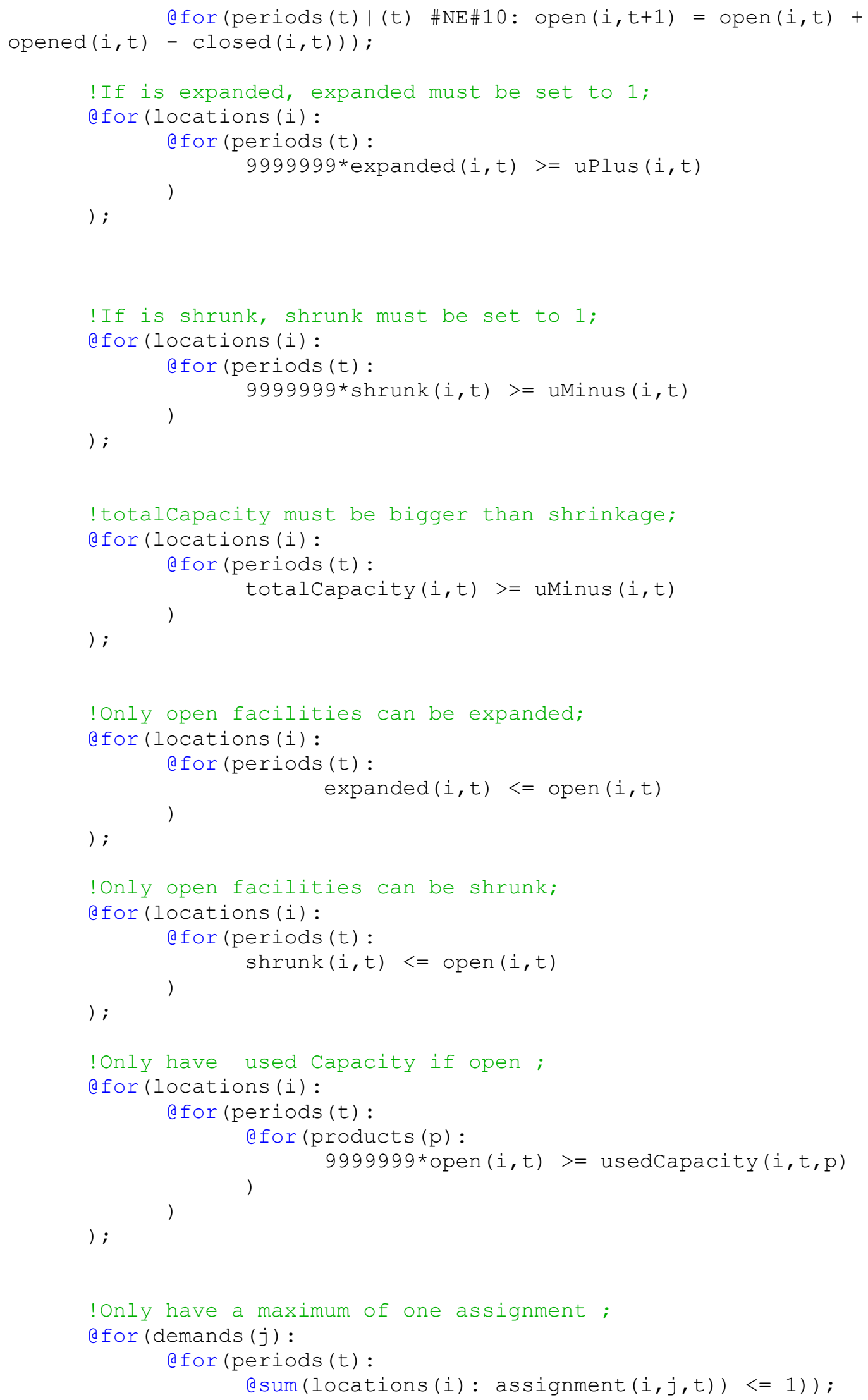


end 


\section{Appendix J JAVA Source Code of annealing algorithm for case}

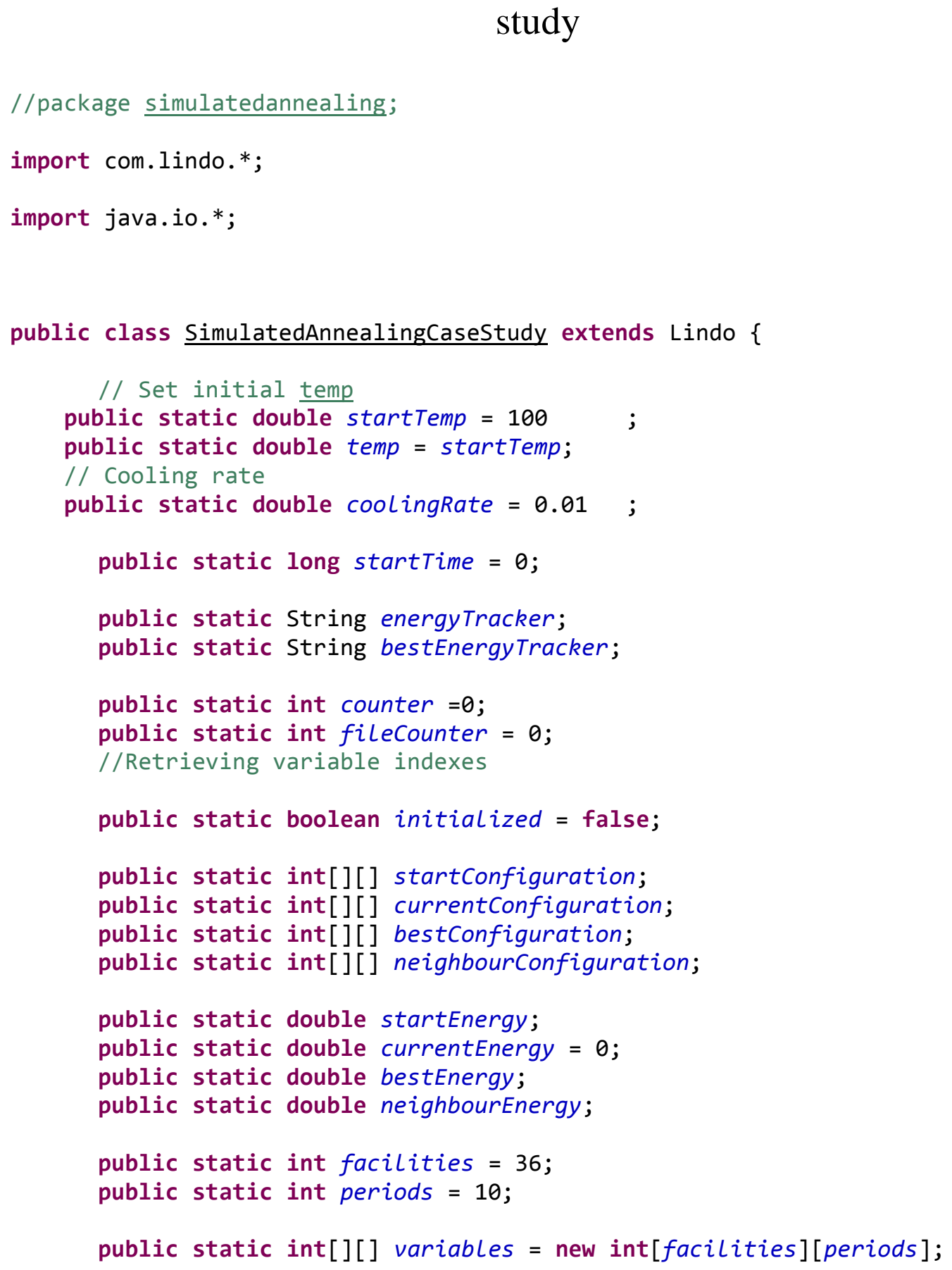


public static String License = "D:/Dropbox/PhD/Dissertation Sven Brose/09_Development/workspace/lib/lindoapi/license/lndapi100.lic";

private static int nErrorCode[] = new int[1];

private static StringBuffer cErrorMessage = new

StringBuffer (LS_MAX_ERROR_MESSAGE_LENGTH);

private static StringBuffer $C L$ icenseKey = new

StringBuffer(LS_MAX_ERROR_MESSAGE_LENGTH);

double[][] array = null;

double[] adC = null;

double[] adB = null;

/* Number of constraints, Number of variables */static int $n M=0, n N=0$;

/* The length of each column. Since we aren't leaving any blanks in our matrix, we can set this to null */

static int pnLenCol []$=$ null;

/* The nonzero coefficients $* /$ static double adA[] = null;

/* The row indices of the nonzero coefficients $* /$ static int anRow $X[]=$ null;

/* Simple upper and lower bounds on the variables.

By default, all variables have a lower bound of zero and an upper bound of infinity. Therefore pass null pointers in order to use these default values. */

static double pdLower [] = null, pdUpper [] = null;

$I^{*}$ The number of nonzeros in the constraint matrix */static int $n N Z=0$;

/* The indices of the first nonzero in each column */static int anBegCol[] $=$ null;

/* The length of each column. Since there are no balnks can be null

*/static int []

Alencol $=$ null;

/* LB and UB of the variable, null means $\theta$ LB and infinity UB */static double[]

$L b=$ null, $u b=$ null;

static String varnames[] = null, connames [] = null;

public static void main(String[] args) \{

startTime $=$ System.currentTimeMillis () ;

System.out.println("Runtime in Milliseconds: " +

getELapsedTime());

System.LoadLibrary("lindojni");

nErrorCode $[\theta]=$ LSLoadLicenseString(License, CLicenseKey);

// Create startConfiguration (all Facilities open) 


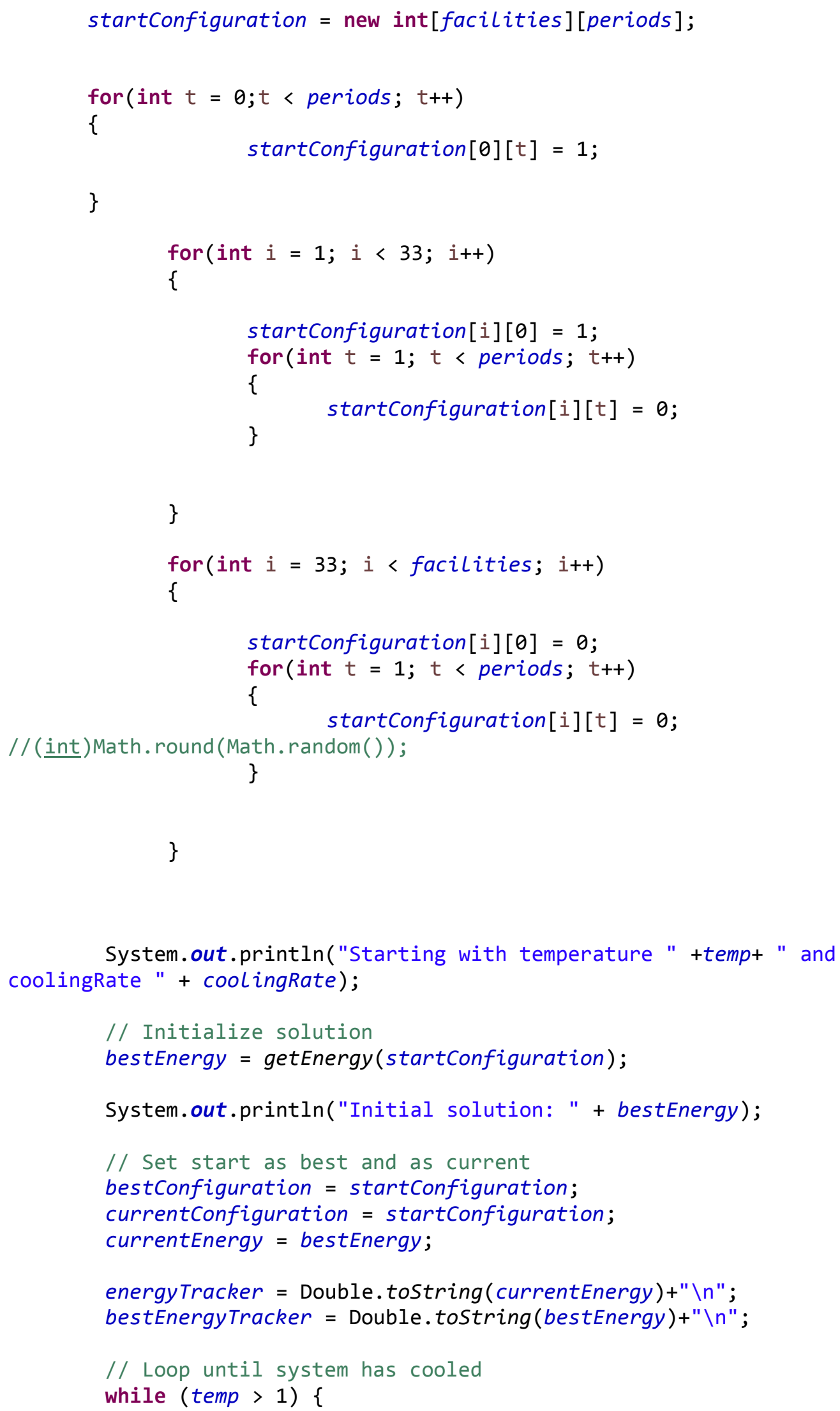


// Create new configuration

neighbourConfiguration =

createNewConfiguration(currentConfiguration);

// Get energy of solutions

//double currentEnergy = getEnergy (currentConfiguration);

double neighbourEnergy = getEnergy(neighbourConfiguration);

// Decide if we should accept the neighbour

if (acceptanceProbability(currentEnergy, neighbourEnergy, temp) >

Math.random( )) \{

currentConfiguration = neighbourConfiguration;

currentEnergy = neighbourEnergy;

\}

System.out.println("accepted neighbour"+ neighbourEnergy);

// Keep track of the best solution found

if (currentEnergy > bestEnergy)

\{

bestConfiguration = currentConfiguration;

//writeFile(input, fileName);

//System.out.println(configurationToString(bestConfiguration));

$\operatorname{try}\{$

writeFile(configurationToString(bestConfiguration)+" $\backslash r \backslash n "$,

"D:/Dropbox/PhD/Dissertation Sven Brose/11_Case_Study/Volksbank/Szenario

3/Best2.txt");

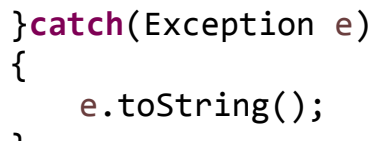

\}

bestEnergy = currentEnergy;

System.out.println("New Best "+ bestEnergy);

//printConfiguration(bestConfiguration);

\}

energyTracker $=$ getELapsedTime ()$+" ; "+$

Double.toString(currentEnergy)+ ";" + temp;

bestEnergyTracker $=$ getELapsedTime ()$+" ; "+$

Double.toString(bestEnergy)+ ";" + temp;

// Cool system

temp $*=1$-coolingRate;

//temp = temp-coolingRate;

System.out.println("Getting colder... ");

System.out.println("New temperature is: "+temp);

$\operatorname{try}\{$

writeFile(bestEnergyTracker, "D:/Dropbox/PhD/Dissertation Sven Brose/11_Case_Study/Volksbank/Simulated annealing/"+facilities+"x"+periods+"-

"+"bestEnergies-T"+startTemp+"-C"+coolingRate+".csv"); 
writeFile(energyTracker, "D:/Dropbox/PhD/Dissertation Sven Brose/11_Case_Study/Volksbank/Simulated annealing/"+ facilities+"x"+periods+" "+"allEnergies-T"+startTemp+"-C"+coolingRate+".csv");

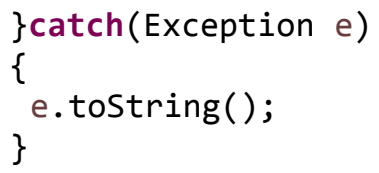


public static void writeFile (String input, String fileName) throws IOException

\{

BufferedWriter out = new BufferedWriter (

new OutputStreamWriter(

new FileOutputStream(fileName, true)));

out.write(input);

out.newLine ( );

out.close ();

\}

public static long getElapsedTime() \{

long elapsed;

elapsed $=$ (System.currentTimeMillis() - startTime);

return elapsed;

\}

public static double getEnergy(int[][] configuration)\{

Object pEnv = null;

Object pModel = null;

pEnv = LScreateEnv(nErrorCode, cLicenseKey.toString ());

APIErrorCheck (pEnv);

pModel = LScreateModel (pEnv, nErrorCode);

APIErrorCheck (pEnv);

String fileName = "D:/Dropbox/PhD/Dissertation Sven

Brose/11_Case_Study/Volksbank/Szenario 3/model_voba_S3.mps";

//System.out.println("Loading file ... ");

//System.out.println(fileName);

nErrorCode [0] = LSreadMPSFiLe (pModel, fileName, LS_UNFORMATTED_MPS);

APIErrorCheck (pEnv);

int [] iVar = new int [1];

$\operatorname{iVar}[0]=1$; 


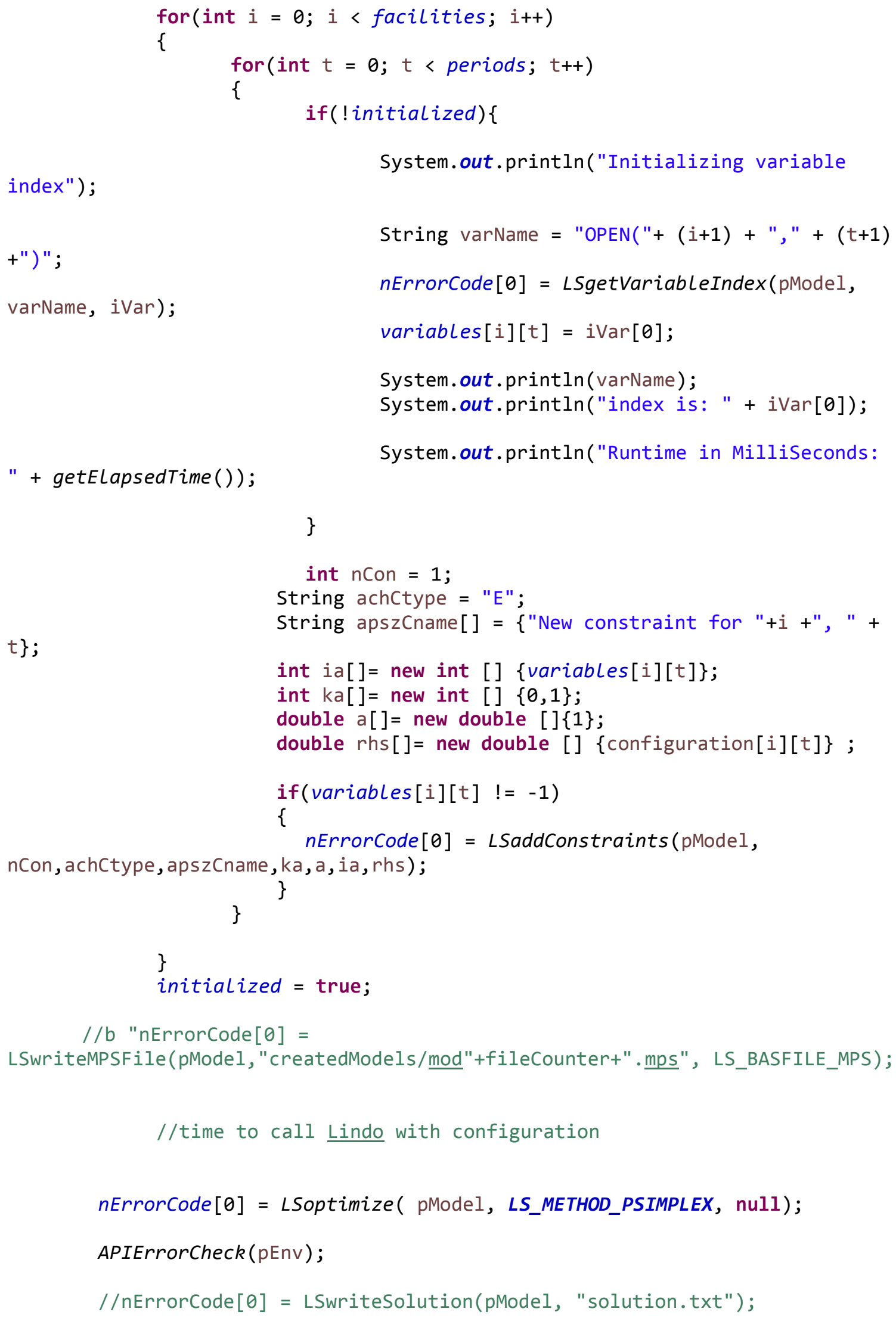


double dobj[]= new double[1];

nErrorCode $[\theta]=$ LSgetInfo $($ pModel,LS_DINFO_POBJ, dobj $) ;$

APIErrorCheck(pEnv);

System.out.println("Current Configuration");

//printConfiguration(configuration);

System.out.println("Runtime in Milliseconds: " + getELapsedTime()); $\operatorname{dobj}[\theta])$;

System.out.println( "Objective Value for configuration = "+ " " +

nErrorCode $[\theta]=$ LSdeleteModel $(\mathrm{pModel})$;

$n$ ErrorCode $[\theta]=$ LSdeLeteEnv (pEnv);

//System.out.print(nErrorCode[0]);

APIErrorCheck(pEnv);

filecounter++;

return $-\operatorname{dobj}[\theta]$;

\}

public static int[][] createNewConfiguration(int[][] currentConfiguration)

\{

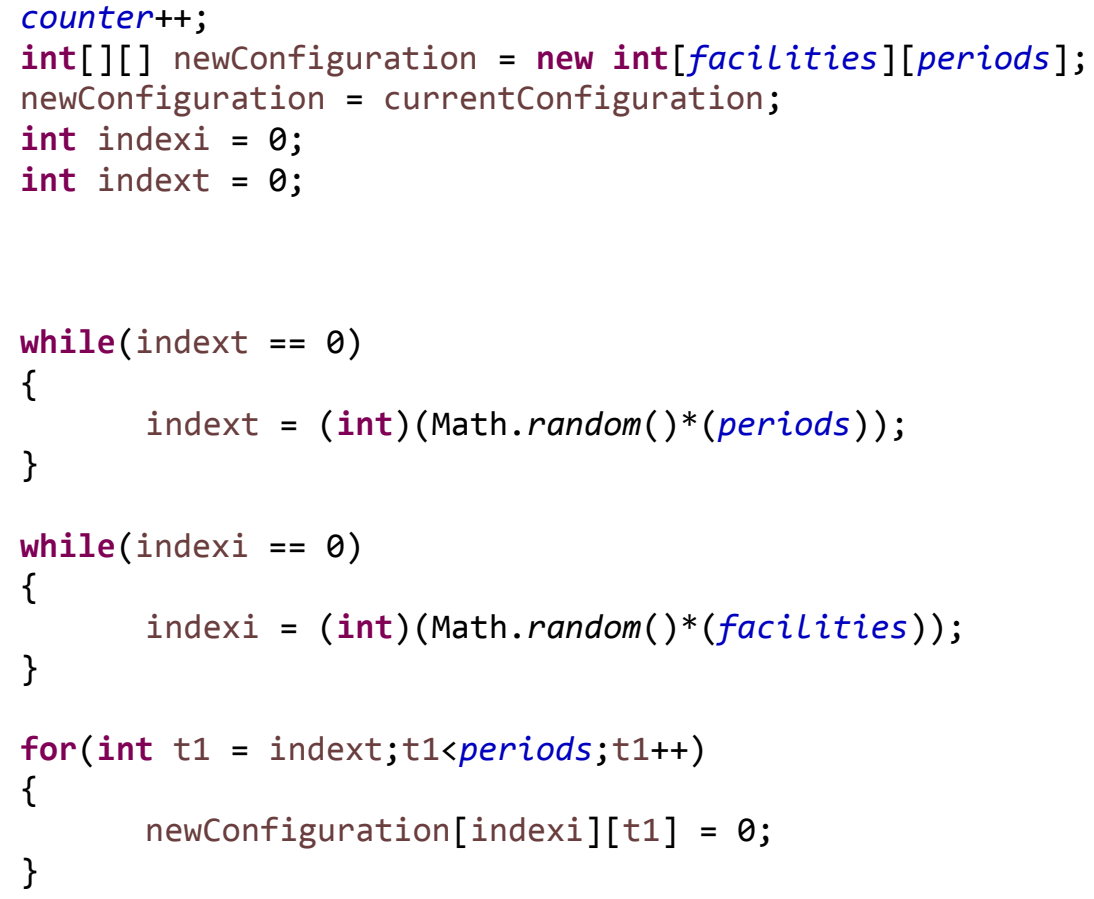




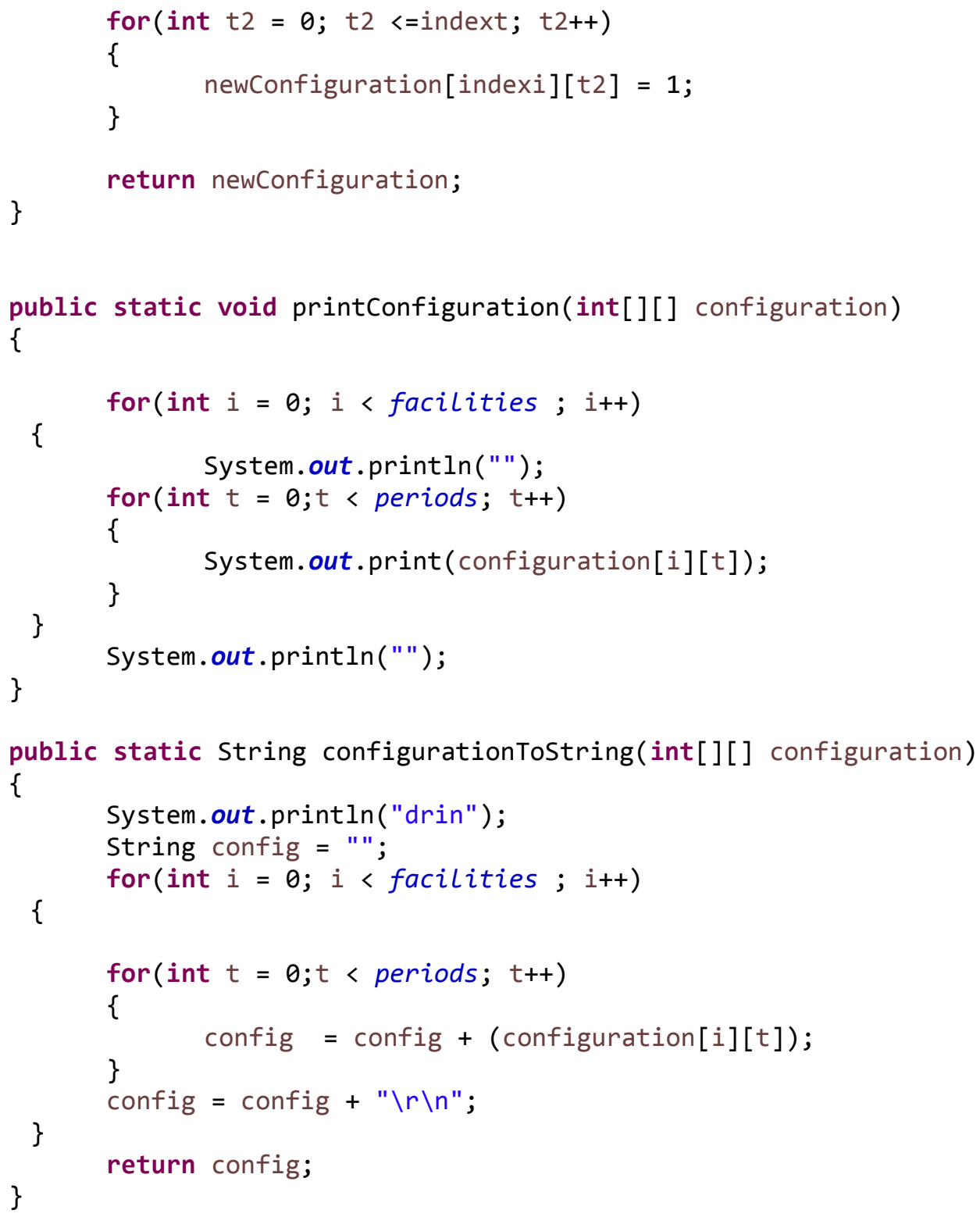




\title{
CURRICULUM VITA
}

\author{
Sven W. Brose
}

\section{Contact Information}

Address: Tiefe Mark 47a, 44287 Dortmund, Germany

Email: mail@svenbrose.de

\section{$\underline{\text { Research Interests }}$}

Applied Operations Research, Supply Chain Management, Logistics, Management

Information Systems, Decision Support Systems, , Software Engineering, Software Development, Design of Experiments

\section{$\underline{\text { Education }}$}

Ph.D in Industrial Engineering at University of Louisville, USA (since September, 2009)

Diploma in Industrial Engineering at Technische Universitat Dortmund, Germany

(October, 2003 - September, 2008) 
Vocational Training in Software Development at adesso AG, Dortmund, Germany

(August, 2000 - February, 2003)

\section{Working Experience}

Manager in Business Consultancy at Sopra Steria GmbH, Hamburg, Germany (since February 2014)

Senior Consultant in Requirements Engineering at adesso AG, Dortmund, Germany (October 2010 - January 2014)

Financial Controller at Eurowings Luftverkehrs AG, Dortmund, Germany (June 2007 June 2010)

\section{Professional Skills}

Analysis Software: Lingo (mathematical programming), MiniTab (statistics analysis), Arena (simulation), Excel, Gephi BIC Design

Common Software: Microsoft Word, PowerPoint, Project, Visio, Access, etc.

Programming Languages: Java, Visual Basic, UML, SQL, LATEX, PHP

OS Platforms: Microsoft Windows, Linux

Database Platforms: Oracle, MySql 
Languages: Fluent in German and English, basics in Spanish

\section{Awards and Honors}

Golden Key International Honour Society (2012)

Intra-corparate award for paperless business models (2013)

ISTQB Certified Tester (2011)

Lean Six Sigma Green Belt (2012)

Lean Six Sigma Black Belt (2013)

Lean Six Sigma Master Black Belt (2015)

\section{$\underline{\text { Affiliations }}$}

Verein Deutscher Ingenieure (VDI) 